\title{
Direct C-H carboxylation forming polyfunctionalized aromatic carboxylic acids by combined Brønsted bases
}

Masanori Shigeno, ${ }^{* 1}$ Kazuya Hanasaka, ${ }^{1}$ Itsuki Tohara, ${ }^{1}$ Koki Izumi, ${ }^{1}$ Hiroyuki Yamakoshi, ${ }^{2}$ Eunsang Kwon, ${ }^{3}$ Kanako Nozawa-Kumada, ${ }^{1}$ and Yoshinori Kondo*1

${ }^{1}$ Department of Biophysical Chemistry, Graduate School of Pharmaceutical Science, Tohoku University, Aoba, Sendai, 980-8578, Japan

${ }^{2}$ Central Analytical Center, Graduate School of Pharmaceutical Science,

Tohoku University, Aoba, Sendai, 980-8578, Japan

${ }^{3}$ Research and Analytical Center for Giant Molecules, Graduate School of Science,

Tohoku University, Aoba, Sendai 980-8578, Japan

*E-mail: masanori.shigeno.e5@tohoku.ac.jp, yoshinori.kondo.a7@tohoku.ac.jp

Supporting Information

Table of Contents

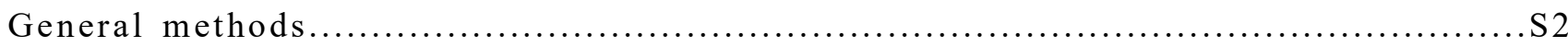

Effects of amounts of reagents, solvents, and temperatures in the carboxylation of 1a (Table S1)..........S3

Reaction of $1 \mathbf{a}$ with $\mathrm{LiOCO}_{2}-t$-Bu, CsF, and 18-crown-6 under Ar atmosphere (Scheme S1).......................S4

Experimental procedures and spectra data for obtained products.............................................S4-S35

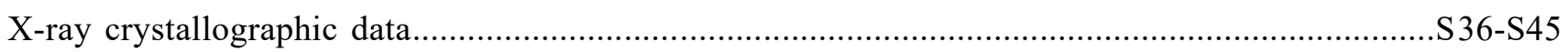

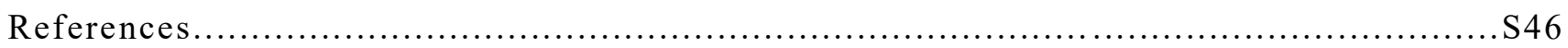

${ }^{1} \mathrm{H},{ }^{13} \mathrm{C},{ }^{19} \mathrm{~F}$, COSY, NOESY, HMQC, HMBC, and ${ }^{1} \mathrm{H}-{ }^{19} \mathrm{~F}$ HETCOR NMR spectra.............S47-S139 
General methods. All reactions were carried out under $\mathrm{N}_{2}$ or Ar atmosphere. Flash column chromatography was performed with Kanto silica gel $60 \mathrm{~N}$ (spherical, neutral, 40-50 $\mu \mathrm{m}$ ). Preparative thin-layer chromatography was performed with silica gel (Wakogel $\left.{ }^{\circledR} \mathrm{B}-5 \mathrm{~F}\right)$. Melting points (Mp) were determined with a Yazawa micro melting point apparatus without correction. Infrared (IR) data were recorded on SensIR ATR (Attenuated Total Reflectance) FT-IR, and absorbance frequencies are reported in reciprocal centimeters $\left(\mathrm{cm}^{-1}\right)$. NMR data were recorded on a JEOL AL400 spectrometer (395.75 MHz for ${ }^{1} \mathrm{H}, 99.50 \mathrm{MHz}$ for ${ }^{13} \mathrm{C}$ ), a Varian Mercury $\left({ }^{1} \mathrm{H}\right.$ at $399.17 \mathrm{MHz},{ }^{19} \mathrm{~F}$ at $375.56 \mathrm{MHz}$ ), or a JEOL ECA600 spectrometer $\left(597.17 \mathrm{MHz}\right.$ for ${ }^{1} \mathrm{H}, 150.91 \mathrm{MHz}$ for ${ }^{13} \mathrm{C},{ }^{19} \mathrm{~F}$ at $\left.561.81 \mathrm{MHz}\right)$. Chemical shifts are expressed in $\delta$ (parts per million, ppm) values, and coupling constants are expressed in herts $(\mathrm{Hz}) .{ }^{1} \mathrm{H}$ NMR spectra were referenced to tetramethylsilane as an internal standard or to a solvent signal $\left(\mathrm{CDCl}_{3}: 7.26 \mathrm{ppm}, \mathrm{DMSO}-d_{6}: 2.49 \mathrm{ppm}\right) .{ }^{13} \mathrm{C}$ NMR spectra were referenced to a solvent signal $\left(\mathrm{CDCl}_{3}: 77.0 \mathrm{ppm}, \mathrm{DMSO}-d_{6}: 39.5 \mathrm{ppm}\right) .{ }^{19} \mathrm{~F}$ NMR spectra were referenced to 4 fluorotoluene ( $\delta=-118.0 \mathrm{ppm}$ ) as an internal standard. Low and high resolution mass spectra (LRMS and HRMS) were obtained from Mass Spectrometry Resource, Graduate School of Pharmaceutical Sciences, Tohoku University, on a JEOL JMS-DX 303 and JMS-700/JMS-T 100 GC spectrometer, respectively. Gel permeation chromatography (GPC) was conducted with a Recycling Preparative HPLC LC-9210 (Japan Analytical Industry, Co. Ltd.). 
Table S1. Effects of amounts of reagents, solvents, and temperatures. ${ }^{a}$

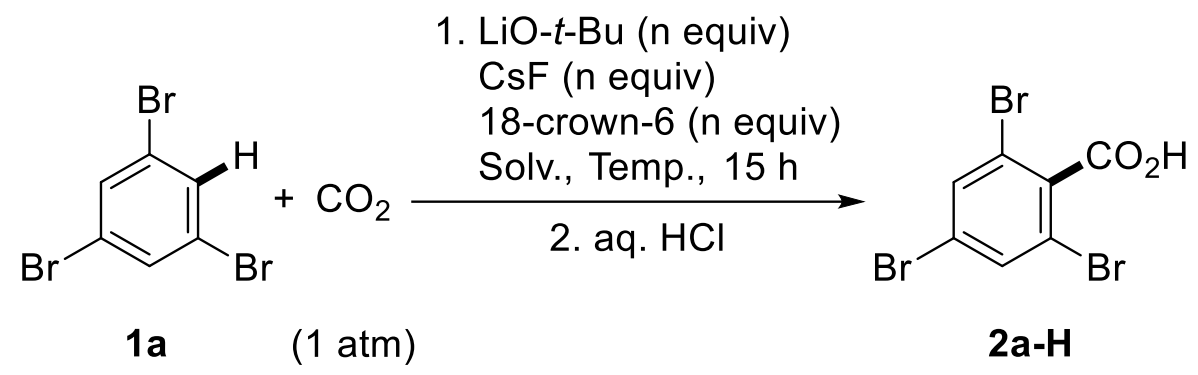

\begin{tabular}{|c|c|c|c|c|}
\hline Entry & $\begin{array}{c}\text { Amounts of reagents } \\
\text { (n eq.) }\end{array}$ & Solv. & $\begin{array}{l}\text { Temp. } \\
\left({ }^{\circ} \mathrm{C}\right)\end{array}$ & $\begin{array}{c}\text { Yield of } \\
\mathbf{2} \mathbf{a}-\mathbf{H}(\%)^{b}\end{array}$ \\
\hline 1 & 2 & DMI & 100 & 74 \\
\hline 2 & 3 & DMI & 100 & 86 \\
\hline 3 & 4 & DMI & 100 & 51 \\
\hline 4 & 3 & DMF & 100 & 81 \\
\hline 5 & 3 & DMPU & 100 & 83 \\
\hline 6 & 3 & diglyme & 100 & 59 \\
\hline 7 & 3 & $o$-xylene & 100 & 28 \\
\hline 8 & 3 & dioxane & 100 & 60 \\
\hline 9 & 3 & DMI (0.5 mL) & 100 & 82 \\
\hline 10 & 3 & DMI (1.5 mL) & 100 & 75 \\
\hline 11 & 3 & DMI & 80 & 77 \\
\hline 12 & 3 & DMI & 120 & 52 \\
\hline
\end{tabular}

${ }^{a} \mathbf{1 a}(0.30 \mathrm{mmol}), \mathrm{CO}_{2}(1 \mathrm{~atm}), \mathrm{LiO}-t-\mathrm{Bu}$, and $\mathrm{CsF}(0.60 \mathrm{mmol}$ for $\mathrm{n}=2 ; 0.90 \mathrm{mmol}$ for $\mathrm{n}=3 ; 1.20$ mmol for $\mathrm{n}=4)$, solvent $(1.0 \mathrm{~mL}), 100{ }^{\circ} \mathrm{C}, 15 \mathrm{~h} .{ }^{b}$ Yields were determined by ${ }^{1} \mathrm{H}$ NMR spectroscopy using 1,1,2-trichloroethane as the internal standard. 
Scheme S1. Reaction of 1a with $\mathrm{LiOCO}_{2}-t-\mathrm{Bu}, \mathrm{CsF}$, and 18-crown-6 under Ar atmosphere ${ }^{a, b}$<smiles>Brc1cc(Br)cc(Br)c1</smiles>

1a
1. $\mathrm{LiOCO}_{2}$-t-Bu (3.0 equiv)

CsF (3.0 equiv)

18-crown-6 (3.0 equiv)

DMI, $100{ }^{\circ} \mathrm{C}, 15 \mathrm{~h}$

2. Mel (3.0 equiv)

$60{ }^{\circ} \mathrm{C}, 2 \mathrm{~h}$<smiles>CC(=O)c1c(Br)cc(Br)cc1Br</smiles>

$2 a, 82 \%$

${ }^{a}$ Reaction was conducted on a $0.3 \mathrm{mmol} \mathrm{scale.}{ }^{b}$ Isolated yield.

Materials. Unless otherwise noted, materials were purchased from Tokyo Kasei Co., Aldrich Inc. and other commercial suppliers and were used as received. $\mathbf{3 k},{ }^{1} \mathbf{S 8},{ }^{2} \mathrm{LiO}-t-\mathrm{Am},{ }^{3}$ and $\mathrm{LiOCO}_{2}-t-\mathrm{Bu}^{4}$ were prepared according to the literature procedure. 1i, $1 \mathbf{r}, \mathbf{1 t}, \mathbf{1 y}, \mathbf{1 z}, \mathbf{3 d}, \mathbf{3 e}, \mathbf{3 f}$, and $\mathbf{3 h}$ were prepared according to the following Schemes S2, S3, S4, S5, S6, S7, S8, S9, and S10, respectively. 1j-1q and 1s were prepared according to the procedure similar to that described for $\mathbf{1 i}$ (Scheme S2). 1u and 1v were prepared according to the procedure similar to that described for $\mathbf{1 t}$ (Scheme S4). 1w and $\mathbf{1 x}$ was prepared according to the procedure similar to that described for 1y (Scheme S5). 3g was prepared according to the procedure similar to that described for $\mathbf{1 z}$ (Scheme S6). DMI was distilled over $4 \AA$ molecular sieves under a reduced pressure.

Scheme S2. Preparation of $1 \mathbf{i}$<smiles>Fc1cc(F)cc(Br)c1</smiles>

S1

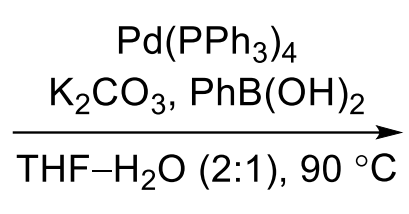

P

$1 \mathrm{i}$

3,5-Difluoro-1,1'-biphenyl (1i). A mixture of S1 (1.54 g, $7.98 \mathrm{mmol})$, phenyl boronic acid (1.17 g, $9.59 \mathrm{mmol}), \mathrm{Pd}\left(\mathrm{PPh}_{3}\right)_{4}(571.8 \mathrm{mg}, 0.495 \mathrm{mmol})$, and $\mathrm{K}_{2} \mathrm{CO}_{3}$ in THF $(20 \mathrm{~mL})$ and $\mathrm{H}_{2} \mathrm{O}(10 \mathrm{~mL})$ was refluxed at $90{ }^{\circ} \mathrm{C}$ in an oil bath for $13 \mathrm{~h}$. The reaction mixture was extracted with $\mathrm{CH}_{2} \mathrm{Cl}_{2}$. The combined organic extracts were washed with $\mathrm{H}_{2} \mathrm{O}$ and brine, dried over $\mathrm{MgSO}_{4}$, and concentrated. The residue was purified by column chromatography on silica gel (hexane) to afford $\mathbf{1 i}(1.14 \mathrm{~g}, 5.99 \mathrm{mmol}$, $75 \%$ ) as a colorless oil. ${ }^{1} \mathrm{H} \mathrm{NMR}\left(600 \mathrm{MHz}, \mathrm{CDCl}_{3} / \mathrm{TMS}\right) \delta 7.55-7.50(\mathrm{~m}, 2 \mathrm{H}), 7.46-7.42(\mathrm{~m}, 2 \mathrm{H})$, $7.38(\mathrm{tt}, 1 \mathrm{H}, J=7.4,1.5 \mathrm{~Hz}), 7.12-7.06(\mathrm{~m}, 2 \mathrm{H}), 6.77(\mathrm{tt}, J=8.9,2.3 \mathrm{~Hz}, 1 \mathrm{H}) .{ }^{13} \mathrm{C} \mathrm{NMR}(150 \mathrm{MHz}$, $\left.\mathrm{CDCl}_{3} / \mathrm{TMS}\right) \delta 163.3\left(\mathrm{dd},{ }^{1} J_{\mathrm{CF}}=247.7 \mathrm{~Hz},{ }^{3} J_{\mathrm{CF}}=12.9 \mathrm{~Hz}\right), 144.5\left(\mathrm{t},{ }^{3} J_{\mathrm{CF}}=9.3 \mathrm{~Hz}\right), 138.9,129.0$, 128.4, 127.0, $109.9\left(\mathrm{dd},{ }^{2} J_{\mathrm{CF}}=20.8 \mathrm{~Hz},{ }^{4} J_{\mathrm{CF}}=5.0 \mathrm{~Hz}\right), 102.4\left(\mathrm{t},{ }^{2} J_{\mathrm{CF}}=25.1 \mathrm{~Hz}\right) .{ }^{19} \mathrm{~F}$ NMR $(564.5$ $\mathrm{MHz}, \mathrm{CDCl}_{3} / 4$-fluorotoluene) $\delta$-109.2. LRMS (EI) $m / z: 190\left(\mathrm{M}^{+}\right)$. HRMS (EI-TOF) $m / z$ : $\left(\mathrm{M}^{+}\right.$) Calcd. for $\mathrm{C}_{12} \mathrm{H}_{8} \mathrm{~F}_{2}$ : 190.0594, found: 190.0624. IR (neat): 3091, 1620, 1600, 1580, 1457, 1421, $1339 \mathrm{~cm}^{-1}$. 
The spectra data matched those reported in the literature. ${ }^{5}$<smiles>Clc1cc(Cl)cc(-c2ccccc2)c1</smiles>

3,5-Dichloro-1,1'-biphenyl (1j). According to the procedure analogous to that described for $\mathbf{1 i}, \mathbf{1} \mathbf{j}$ (742.3 mg, $3.33 \mathrm{mmol}, 67 \%$ ) was obtained from 3,5-dichlorobromobenzene (1.12 g, $4.96 \mathrm{mmol})$ and phenyl boronic acid $(611.7 \mathrm{mg}, 5.01 \mathrm{mmol})$ as a colorless oil. ${ }^{1} \mathrm{H}$ NMR (600 $\mathrm{MHz}, \mathrm{CDCl}_{3} / \mathrm{TMS}$ ) $\delta 7.53-7.50(\mathrm{~m}, 2 \mathrm{H}), 7.46-7.42(\mathrm{~m}, 4 \mathrm{H}), 7.38(\mathrm{tt}, 1 \mathrm{H}, J=7.4,1.6 \mathrm{~Hz}), 7.32(\mathrm{t}, 1 \mathrm{H}, J=1.9 \mathrm{~Hz}) .{ }^{13} \mathrm{C}$ NMR (150 MHz, $\left.\mathrm{CDCl}_{3} / \mathrm{TMS}\right) \delta 144.2,138.5,135.2,129.0,128.4,127.1,127.0,125.6 . \mathrm{LRMS}$ (EI) $m / z: 222\left(\mathrm{M}^{+}\right)$. HRMS (EI-TOF) $\mathrm{m} / z$ : $\left(\mathrm{M}^{+}\right)$Calcd. for $\mathrm{C}_{12} \mathrm{H}_{8} \mathrm{Cl}_{2}: 222.0003$, found: 222.0003. IR (neat): $3065,1585,1558,1497,1430,1407,1281 \mathrm{~cm}^{-1}$. The spectra data matched those reported in the literature. ${ }^{6}$<smiles>Brc1cc(Br)cc(-c2ccccc2)c1</smiles>

3,5-Dibromo-1,1'-biphenyl (1k). According to the procedure analogous to that described for $\mathbf{1 i}, \mathbf{1 k}$ (1.39 g, $4.45 \mathrm{mmol}, 45 \%)$ was obtained from 1,3,5-tribromobenzene (3.14 g, $9.97 \mathrm{mmol})$ and phenyl boronic acid (1.22 g, $10.0 \mathrm{mmol})$ as a colorless oil. ${ }^{1} \mathrm{H} \mathrm{NMR}\left(400 \mathrm{MHz}, \mathrm{CDCl}_{3} / \mathrm{TMS}\right) \delta 7.68-7.62(\mathrm{~m}$, 3H), 7.54-7.50 (m, 2H), 7.48-7.42 (m, 2H), 7.42-7.36 (m, 1H). $\left.{ }^{13} \mathrm{C} \mathrm{NMR} \mathrm{(150} \mathrm{MHz,} \mathrm{CDCl}_{3} / \mathrm{TMS}\right)$ $\delta 144.7,138.3,132.5,129.0,128.9,128.4,127.0,123.2$. LRMS (EI) $m / z: 310\left(\mathrm{M}^{+}\right)$. HRMS (EI-TOF) $m / z:\left(\mathrm{M}^{+}\right)$Calcd. for $\mathrm{C}_{12} \mathrm{H}_{8} \mathrm{Br}_{2}$ : 309.8993, found: 309.8998. IR (neat): 3061, 1583, 1547, 1495, 1423, $1400 \mathrm{~cm}^{-1}$. The spectra data matched those reported in the literature. ${ }^{7}$<smiles>Cc1ccc(-c2cc(F)cc(F)c2)cc1</smiles>

3,5-Difluoro-4'-methyl-1,1'-biphenyl (11). According to the procedure analogous to that described for 1i, 11 (1.58 g, $7.73 \mathrm{mmol}, 98 \%$ ) was obtained from 3,5-difluorobromobenzene (1.52 g, $7.88 \mathrm{mmol}$ ) and 4-methlphenyl boronic acid (1.30 g, $9.57 \mathrm{mmol})$ as a white solid: Mp 69-70 ${ }^{\circ} \mathrm{C}$ (hexane). ${ }^{1} \mathrm{H}$ NMR (600 MHz, $\left.\mathrm{CDCl}_{3} / \mathrm{TMS}\right) \delta 7.44(\mathrm{dt}, 2 \mathrm{H}, J=8.2,1.9 \mathrm{~Hz}), 7.27-7.24(\mathrm{~m}, 2 \mathrm{H}), 7.11-7.05(\mathrm{~m}, 2 \mathrm{H}), 6.75$ $(\mathrm{tt}, 1 \mathrm{H}, J=8.9,2.4 \mathrm{~Hz}), 2.40(\mathrm{~s}, 3 \mathrm{H}) .{ }^{13} \mathrm{C} \mathrm{NMR}\left(150 \mathrm{MHz}, \mathrm{CDCl}_{3} / \mathrm{TMS}\right) \delta 163.3\left(\mathrm{dd},{ }^{1} J_{\mathrm{CF}}=245.6 \mathrm{~Hz}\right.$, 
$\left.{ }^{3} J_{\mathrm{CF}}=12.9 \mathrm{~Hz}\right), 144.5\left(\mathrm{t},{ }^{3} J_{\mathrm{CF}}=10.1 \mathrm{~Hz}\right), 138.4,136.0,129.7,126.8,109.6\left(\mathrm{dd},{ }^{2} J_{\mathrm{CF}}=20.1 \mathrm{~Hz},{ }^{4} J_{\mathrm{CF}}=\right.$ $4.3 \mathrm{~Hz}), 102.1\left(\mathrm{t},{ }^{2} J_{\mathrm{CF}}=25.1 \mathrm{~Hz}\right), 21.1 .{ }^{19} \mathrm{~F} \mathrm{NMR}\left(564.5 \mathrm{MHz}, \mathrm{CDCl}_{3} / 4\right.$-fluorotoluene) $\delta$-109.4. LRMS (EI) $m / z$ : $204\left(\mathrm{M}^{+}\right)$. HRMS (EI-TOF) $m / z$ : $\left(\mathrm{M}^{+}\right)$Calcd. for $\mathrm{C}_{13} \mathrm{H}_{10} \mathrm{~F}_{2}$ : 204.0751, found: 204.0744. IR (neat): $3101,2925,1622,1597,1520,1332 \mathrm{~cm}^{-1}$. The spectra data matched those reported in the literature. $^{8}$<smiles>COc1ccc(-c2cc(F)cc(F)c2)cc1</smiles>

3,5-Difluoro-4'-methoxy-1,1'-biphenyl (1m). According to the procedure analogous to that described for $1 \mathbf{i}, 1 \mathrm{~m}$ (1.06 g, $4.81 \mathrm{mmol}, 61 \%$ ) was obtained from 3,5-difluorobromobenzene (1.53 g, $7.92 \mathrm{mmol})$ and 4-methoxyphenyl boronic acid (1.39 g, $9.14 \mathrm{mmol})$ as a white solid: Mp 69-70 ${ }^{\circ} \mathrm{C}$ (hexane/AcOEt) (lit. $\left.74-75^{\circ} \mathrm{C}^{9}\right) .{ }^{1} \mathrm{H} \mathrm{NMR}\left(600 \mathrm{MHz}, \mathrm{CDCl}_{3} / \mathrm{TMS}\right) \delta 7.48(\mathrm{~d}, 2 \mathrm{H}, J=8.2 \mathrm{~Hz}), 7.05(\mathrm{~d}, 2 \mathrm{H}, J=7.3 \mathrm{~Hz})$, $6.97(\mathrm{~d}, 2 \mathrm{H}, J=7.8 \mathrm{~Hz}), 6.73(\mathrm{t}, 1 \mathrm{H}, J=8.9 \mathrm{~Hz}), 3.85(\mathrm{~s}, 3 \mathrm{H}) .{ }^{13} \mathrm{C} \mathrm{NMR}\left(150 \mathrm{MHz}, \mathrm{CDCl}_{3} / \mathrm{TMS}\right)$ $\delta 163.3\left(\mathrm{dd},{ }^{1} J_{\mathrm{CF}}=245.5 \mathrm{~Hz},{ }^{3} J_{\mathrm{CF}}=12.9 \mathrm{~Hz}\right), 159.9,144.1\left(\mathrm{t},{ }^{3} J_{\mathrm{CF}}=9.3 \mathrm{~Hz}\right), 131.3,128.0,114.4$, $109.3\left(\mathrm{dd},{ }^{2} J_{\mathrm{CF}}=20.1 \mathrm{~Hz},{ }^{4} J_{\mathrm{CF}}=5.7 \mathrm{~Hz}\right), 101.8\left(\mathrm{t},{ }^{2} J_{\mathrm{CF}}=25.8 \mathrm{~Hz}\right), 55.4 .{ }^{19} \mathrm{~F} \mathrm{NMR}(564.5 \mathrm{MHz}$, $\mathrm{CDCl}_{3} / 4$-fluorotoluene) $\delta$-109.4. LRMS (EI) $\mathrm{m} / z$ : $220\left(\mathrm{M}^{+}\right)$. HRMS (EI-TOF) $\mathrm{m} / z:\left(\mathrm{M}^{+}\right)$Calcd. for $\mathrm{C}_{13} \mathrm{H}_{10} \mathrm{~F}_{2} \mathrm{O}: 220.0700$, found: 220.0708. IR (neat): 3082, 2976, 2936, 1622, 1596, $1520 \mathrm{~cm}^{-1}$. The spectra data matched those reported in the literature. ${ }^{9}$<smiles>CSc1ccc(-c2cc(F)cc(F)c2)cc1</smiles>

(3',5'-Difluoro-[1,1'-biphenyl]-4-yl)(methyl)sulfane (1n). According to the procedure analogous to that described for 1i, 1 n $(269.7 \mathrm{mg}, 1.14 \mathrm{mmol}$, 97\%) was obtained from 3,5-difluorobromobenzene (226.5 mg, $1.17 \mathrm{mmol})$ and thiomethoxyphenyl boronic acid (202.1 $\mathrm{mg}, 1.20 \mathrm{mmol})$ as a white solid: Mp 51-52 ${ }^{\circ} \mathrm{C}$ (hexane). ${ }^{1} \mathrm{H} \mathrm{NMR}\left(600 \mathrm{MHz}, \mathrm{CDCl}_{3} / \mathrm{TMS}\right) \delta 7.47(\mathrm{~d}, 2 \mathrm{H}, J=8.2 \mathrm{~Hz}), 7.32(\mathrm{~d}, 2 \mathrm{H}, J=$ $8.3 \mathrm{~Hz}), 7.11-7.04(\mathrm{~m}, 2 \mathrm{H}), 6.76(\mathrm{tt}, J=8.9,2.2 \mathrm{~Hz}), 2.52$ (s, 3H). ${ }^{13} \mathrm{C} \mathrm{NMR}\left(150 \mathrm{MHz}, \mathrm{CDCl}_{3} / \mathrm{TMS}\right)$ $\delta 163.3\left(\mathrm{dd},{ }^{1} J_{\mathrm{CF}}=246.9 \mathrm{~Hz},{ }^{3} J_{\mathrm{CF}}=12.9 \mathrm{~Hz}\right), 143.8\left(\mathrm{t},{ }^{3} J_{\mathrm{CF}}=9.3 \mathrm{~Hz}\right), 139.4,135.4,127.2,126.7$, $109.5\left(\mathrm{dd},{ }^{2} J_{\mathrm{CF}}=20.8 \mathrm{~Hz},{ }^{4} J_{\mathrm{CF}}=5.0 \mathrm{~Hz}\right), 102.3\left(\mathrm{t},{ }^{2} J_{\mathrm{CF}}=25.1 \mathrm{~Hz}\right), 15.6 .{ }^{19} \mathrm{~F} \mathrm{NMR}(564.5 \mathrm{MHz}$, $\mathrm{CDCl}_{3} / 4$-fluorotoluene) $\delta$-109.1. LRMS (EI) $\mathrm{m} / \mathrm{z}: 236\left(\mathrm{M}^{+}\right)$. HRMS (EI-TOF) $\mathrm{m} / \mathrm{z}:\left(\mathrm{M}^{+}\right.$) Calcd. for $\mathrm{C}_{13} \mathrm{H}_{10} \mathrm{~F}_{2} \mathrm{~S}: 236.0471$, found: 236.0462. IR (neat): 3083, 2924, 1616, 1591, 1554, 1430, $1331 \mathrm{~cm}^{-1}$. 
<smiles>Fc1cc(F)cc(-c2ccc(Cl)cc2)c1</smiles>

4'-Chloro-3,5-difluoro-1,1'-biphenyl (10). According to the procedure analogous to that described for 1i, 10 (228.3 mg, $1.02 \mathrm{mmol}, 86 \%)$ was obtained from 3,5-difluorobromobenzene (244.7 mg, 1.26 mmol) and 4-chlorophenyl boronic acid $(184.7 \mathrm{mg}, 1.18 \mathrm{mmol})$ as a white solid: $\mathrm{Mp} 74-75{ }^{\circ} \mathrm{C}$ (hexane). ${ }^{1} \mathrm{H}$ NMR (600 MHz, $\left.\mathrm{CDCl}_{3} / \mathrm{TMS}\right) \delta 7.48$ (dt, $2 \mathrm{H}, J=8.6,2.3 \mathrm{~Hz}$ ), 7.43 (dt, $2 \mathrm{H}, J=8.6,2.2$ $\mathrm{Hz}), 7.09-7.04(\mathrm{~m}, 2 \mathrm{H}), 6.80(\mathrm{tt}, 1 \mathrm{H}, J=8.9,2.3 \mathrm{~Hz}) .{ }^{13} \mathrm{C} \mathrm{NMR}\left(150 \mathrm{MHz}, \mathrm{CDCl}_{3} / \mathrm{TMS}\right) \delta 163.4(\mathrm{dd}$, $\left.{ }^{1} J_{\mathrm{CF}}=247.0 \mathrm{~Hz},{ }^{3} J_{\mathrm{CF}}=12.9 \mathrm{~Hz}\right), 143.3\left(\mathrm{t},{ }^{3} J_{\mathrm{CF}}=9.3 \mathrm{~Hz}\right), 137.4\left(\mathrm{~d},{ }^{4} J_{\mathrm{CF}}=2.9 \mathrm{~Hz}\right), 134.7,129.2,128.3$, $109.9\left(\mathrm{dd},{ }^{2} J_{\mathrm{CF}}=20.1 \mathrm{~Hz},{ }^{4} J_{\mathrm{CF}}=5.7 \mathrm{~Hz}\right), 102.9\left(\mathrm{t},{ }^{2} J_{\mathrm{CF}}=25.1 \mathrm{~Hz}\right) .{ }^{19} \mathrm{~F} \mathrm{NMR}\left(564.5 \mathrm{MHz}, \mathrm{CDCl}_{3} / 4-\right.$ fluorotoluene) $\delta$-108.7. LRMS (EI) $m / z: 224\left(\mathrm{M}^{+}\right.$). HRMS (EI-TOF) $m / z:\left(\mathrm{M}^{+}\right.$) Calcd. for $\mathrm{C}_{12} \mathrm{H}_{7} \mathrm{ClF}_{2}$ : 224.0204, found: 224.0213. IR (neat): 3095, 1622, 1593, 1569, 1451, $1394 \mathrm{~cm}^{-1}$.<smiles>Fc1cc(F)cc(-c2ccc(Br)cc2)c1</smiles>

4'-Bromo-3,5-difluoro-1,1'-biphenyl (1p). According to the procedure analogous to that described for 1i, 1p (916.8 mg, $3.41 \mathrm{mmol}, 43 \%$ ) was obtained from 3,5-difluorobromobenzene (1.53 g, 7.93 $\mathrm{mmol}$ ) and 4-bromophenyl boronic acid (1.96 mg, $9.76 \mathrm{mmol}$ ) as a white solid: $\mathrm{Mp} 70-71{ }^{\circ} \mathrm{C}$ (hexane). ${ }^{1} \mathrm{H}$ NMR (600 MHz, CDCl $\left.3 / \mathrm{TMS}\right) \delta 7.61-7.57$ (m, 2H), 7.44-7.38 (m, 2H), 7.10-7.04 (m, 2H), 6.81 $(\mathrm{tt}, 1 \mathrm{H}, J=8.8,2.2 \mathrm{~Hz}) .{ }^{13} \mathrm{C} \mathrm{NMR}\left(150 \mathrm{MHz}, \mathrm{CDCl}_{3} / \mathrm{TMS}\right) \delta 163.4\left(\mathrm{dd},{ }^{1} J_{\mathrm{CF}}=247.0 \mathrm{~Hz},{ }^{3} J_{\mathrm{CF}}=12.9\right.$ $\mathrm{Hz}), 143.2\left(\mathrm{t},{ }^{3} J_{\mathrm{CF}}=7.9 \mathrm{~Hz}\right), 137.8,132.2,128.5,122.8,109.8\left(\mathrm{~d},{ }^{2} J_{\mathrm{CF}}=23.0 \mathrm{~Hz}\right), 102.9\left(\mathrm{t},{ }^{2} J_{\mathrm{CF}}=25.1\right.$ Hz). ${ }^{19} \mathrm{~F}$ NMR (564.5 MHz, $\mathrm{CDCl}_{3} / 4$-fluorotoluene) $\delta$-108.7. LRMS (EI) $m / z: 268\left(\mathrm{M}^{+}\right)$. HRMS (EITOF) $m / z:\left(\mathrm{M}^{+}\right)$Calcd. for $\mathrm{C}_{12} \mathrm{H}_{7} \mathrm{BrF}_{2}: 267.9699$, found: 267.9709. IR (neat): 3090, 1622, 1589, 1564, $1449,1386 \mathrm{~cm}^{-1}$.<smiles>N#Cc1ccc(-c2cc(F)cc(F)c2)cc1</smiles> 
3',5'-Difluoro-[1,1'-biphenyl]-4-carbonitrile (1q). According to the procedure analogous to that described for 1i, 1q (1.21 g, $5.62 \mathrm{mmol}, 71 \%)$ was obtained from 3,5-difluorobromobenzene (1.52 g, $7.88 \mathrm{mmol})$ and 4-cyanophenyl boronic acid $(1.40 \mathrm{~g}, 9.53 \mathrm{mmol})$ as a white solid: $\mathrm{Mp} 125-126{ }^{\circ} \mathrm{C}$ (hexane/AcOEt). ${ }^{1} \mathrm{H}$ NMR (600 MHz, $\left.\mathrm{CDCl}_{3} / \mathrm{TMS}\right) \delta 7.76(\mathrm{dt}, 2 \mathrm{H}, J=8.6,1.8 \mathrm{~Hz}), 7.65(\mathrm{dt}, 2 \mathrm{H}, J=$ 8.6, $1.9 \mathrm{~Hz}), 7.13-7.09(\mathrm{~m}, 2 \mathrm{H}), 6.88(\mathrm{tt}, 1 \mathrm{H}, J=8.8,2.4 \mathrm{~Hz}) .{ }^{13} \mathrm{C} \mathrm{NMR}\left(150 \mathrm{MHz}, \mathrm{CDCl}_{3} / \mathrm{TMS}\right)$ $\delta 163.5\left(\mathrm{dd},{ }^{1} J_{\mathrm{CF}}=248.5 \mathrm{~Hz},{ }^{3} J_{\mathrm{CF}}=12.9 \mathrm{~Hz}\right), 143.2,142.4\left(\mathrm{t},{ }^{3} J_{\mathrm{CF}}=7.9 \mathrm{~Hz}\right), 132.8,127.7,118.5$, $112.3,110.3\left(\mathrm{dd},{ }^{2} J_{\mathrm{CF}}=20.1 \mathrm{~Hz},{ }^{4} J_{\mathrm{CF}}=5.7 \mathrm{~Hz}\right), 104.0\left(\mathrm{t},{ }^{2} J_{\mathrm{CF}}=25.1 \mathrm{~Hz}\right) .{ }^{19} \mathrm{~F}$ NMR $(564.5 \mathrm{MHz}$, $\mathrm{CDCl}_{3} / 4$-fluorotoluene) $\delta$-107.9. LRMS (EI) $\mathrm{m} / \mathrm{z}: 215\left(\mathrm{M}^{+}\right)$. HRMS (EI-TOF) $\mathrm{m} / \mathrm{z}:\left(\mathrm{M}^{+}\right)$Calcd. for $\mathrm{C}_{13} \mathrm{H}_{7} \mathrm{~F}_{2} \mathrm{~N}$ : 215.0547, found: 215.0550. IR (neat): 3100, 2223, 1621, 1597, 1451, $1398 \mathrm{~cm}^{-1}$. The spectra data matched those reported in the literature. ${ }^{10}$<smiles>O=Cc1ccc(-c2cc(F)cc(F)c2)cc1</smiles>

3',5'-Difluoro-[1,1'-biphenyl]-4-carbaldehyde (1s). According to the procedure analogous to that described for 1i, $1 \mathrm{~s}(2.84 \mathrm{~g}, 13.0 \mathrm{mmol}, 65 \%)$ was obtained from 3,5-difluorobromobenzene (4.05 g, $20.9 \mathrm{mmol})$ and 4-formylphenyl boronic acid (3.00 g, $20.0 \mathrm{mmol})$ as a white solid: $\mathrm{Mp} 73-74{ }^{\circ} \mathrm{C}$ (hexane/AcOEt) (lit. $78^{\circ} \mathrm{C}^{11}$ ). ${ }^{1} \mathrm{H}$ NMR (600 MHz, $\left.\mathrm{CDCl}_{3} / \mathrm{TMS}\right) \delta 10.1$ (s, 1H), 7.98 (dt, $2 \mathrm{H}, J=8.3$, $1.8 \mathrm{~Hz}), 7.72(\mathrm{dt}, 2 \mathrm{H}, J=8.2,1.8 \mathrm{~Hz}), 7.17-7.13(\mathrm{~m}, 2 \mathrm{H}), 6.87(\mathrm{tt}, 1 \mathrm{H}, J=8.8,2.3 \mathrm{~Hz}) .{ }^{13} \mathrm{C} \mathrm{NMR}$ $\left(150 \mathrm{MHz}, \mathrm{CDCl}_{3} / \mathrm{TMS}\right) \delta 191.5,163.3\left(\mathrm{dd},{ }^{1} J_{\mathrm{CF}}=247.7 \mathrm{~Hz},{ }^{3} J_{\mathrm{CF}}=12.9 \mathrm{~Hz}\right), 144.6,142.9\left(\mathrm{t},{ }^{3} J_{\mathrm{CF}}=\right.$ $9.3 \mathrm{~Hz}), 136.0,130.3,127.6,110.3\left(\mathrm{dd},{ }^{2} J_{\mathrm{CF}}=20.1 \mathrm{~Hz},{ }^{4} J_{\mathrm{CF}}=5.7 \mathrm{~Hz}\right), 103.7\left(\mathrm{t},{ }^{2} J_{\mathrm{CF}}=25.8 \mathrm{~Hz}\right) .{ }^{19} \mathrm{~F}$ $\operatorname{NMR}\left(564.5 \mathrm{MHz}, \mathrm{CDCl}_{3} / 4\right.$-fluorotoluene) $\delta$-108.2. LRMS (EI) $m / z: 218\left(\mathrm{M}^{+}\right.$). HRMS (EI-TOF) $m / z$ : $\left(\mathrm{M}^{+}\right)$Calcd. for $\mathrm{C}_{13} \mathrm{H}_{8} \mathrm{~F}_{2} \mathrm{O}: 218.0543$, found: 218.0532. IR (neat): 3090, 2839, 2750, 1699, 1622, 1599, $1454 \mathrm{~cm}^{-1}$. The spectra data matched those reported in the literature. ${ }^{12}$ 
Scheme S3. Preparation of 1r<smiles>COc1ccc(C2OCCO2)cc1</smiles>

S2

S3

$\mathrm{Pd}\left(\mathrm{PPh}_{3}\right)_{4}, \mathrm{~K}_{2} \mathrm{CO}_{3}$,

1-Bromo-3,5-difluorobenzene

$\mathrm{THF}-\mathrm{H}_{2} \mathrm{O}(2: 1), 90{ }^{\circ} \mathrm{C}$<smiles>Fc1cc(F)cc(-c2ccc(C3OCCO3)cc2)c1</smiles>

2-(3',5'-Difluoro-[1,1'-biphenyl]-4-yl)-1,3-dioxolane (1r). To the solution of S2 (1.39 g, $6.07 \mathrm{mmol})$ in THF $(12 \mathrm{~mL})$ was slowly added $n$-BuLi $(1.56 \mathrm{M}$ in hexane $3.91 \mathrm{~mL}, 6.10 \mathrm{mmol})$ at $-78^{\circ} \mathrm{C}$, and the mixture was stirred at the same temperature for $2 \mathrm{~h}$. Subsequently, $\mathrm{B}(\mathrm{OMe})_{3}(728.4 \mathrm{mg}, 7.01 \mathrm{mmol})$ was added, and the mixture was stirred at room temperature for $1 \mathrm{~h}$. Then, $\mathrm{H}_{2} \mathrm{O}(10 \mathrm{~mL})$ was added, and the mixture was stirred at room temperature for $5 \mathrm{~min}$. The mixture was added to a mixture of 3,5difluorobromobenzene (1.15 g, $5.95 \mathrm{mmol}), \mathrm{Pd}\left(\mathrm{PPh}_{3}\right)_{4}(339.4 \mathrm{mg}, 0.293 \mathrm{mmol})$, and $\mathrm{K}_{2} \mathrm{CO}_{3}(432.0$ $\mathrm{mg}, 3.12 \mathrm{mmol})$ in THF $(8 \mathrm{~mL})$. The reaction mixture was refluxed at $90{ }^{\circ} \mathrm{C}$ in an oil bath for $15 \mathrm{~h}$. The mixture was extracted with $\mathrm{Et}_{2} \mathrm{O}$. The combined organic extracts were washed with $\mathrm{H}_{2} \mathrm{O}$ and brine, dried over $\mathrm{MgSO}_{4}$, and concentrated. The residue was purified by column chromatography on silica gel (hexane:AcOEt = 40:1) to afford 1r $(774.3 \mathrm{mg}, 2.95 \mathrm{mmol}, 50 \%)$ as a white solid: $\mathrm{Mp} 78-80{ }^{\circ} \mathrm{C}$ (hexane). ${ }^{1} \mathrm{H}$ NMR (600 MHz, $\left.\mathrm{CDCl}_{3} / \mathrm{TMS}\right) \delta 7.59-7.53(\mathrm{~m}, 4 \mathrm{H}), 7.13-7.07$ (m, 2H), 6.79 (tt, $1 \mathrm{H}, J$ $=8.9,2.4 \mathrm{~Hz}), 5.87(\mathrm{~s}, 1 \mathrm{H}), 4.16-4.14(\mathrm{~m}, 2 \mathrm{H}), 4.08-4.05(\mathrm{~m}, 2 \mathrm{H}) .{ }^{13} \mathrm{C} \mathrm{NMR}\left(150 \mathrm{MHz}, \mathrm{CDCl}_{3} / \mathrm{TMS}\right)$ $\delta 163.3\left(\mathrm{dd},{ }^{1} J_{\mathrm{CF}}=245.6 \mathrm{~Hz},{ }^{3} J_{\mathrm{CF}}=12.9 \mathrm{~Hz}\right), 144.1\left(\mathrm{t},{ }^{3} J_{\mathrm{CF}}=9.3 \mathrm{~Hz}\right), 139.7,138.2,127.1,127.0$, $110.0\left(\mathrm{dd},{ }^{2} J_{\mathrm{CF}}=20.1 \mathrm{~Hz},{ }^{4} J_{\mathrm{CF}}=5.7 \mathrm{~Hz}\right), 103.3,102.6\left(\mathrm{t},{ }^{2} J_{\mathrm{CF}}=25.1 \mathrm{~Hz}\right), 65.4 .{ }^{19} \mathrm{~F} \mathrm{NMR}(564.5 \mathrm{MHz}$, $\mathrm{CDCl}_{3} / 4$-fluorotoluene) $\delta$-109.0. LRMS (EI) $\mathrm{m} / z: 262\left(\mathrm{M}^{+}\right)$. HRMS (EI-TOF) $\mathrm{m} / z:\left(\mathrm{M}^{+}\right)$Calcd. for $\mathrm{C}_{15} \mathrm{H}_{12} \mathrm{~F}_{2} \mathrm{O}_{2}: 262.0805$, found: 262.0831. IR (neat): 3093, 2959, 2891, 1622, 1593, 1571, $1411 \mathrm{~cm}^{-1}$.

Scheme S4. Preparation of $\mathbf{1 t}$<smiles>CC(C)(C)c1cc(F)cc(C(=O)O)c1</smiles>

S4<smiles>CCCCOC(=O)c1cc(F)cc(F)c1</smiles>

$1 t$

tert-Butyl 3,5-difluorobenzoate (1t). A mixture of $\mathbf{S 4}(956.5 \mathrm{mg}, 6.05 \mathrm{mmol}), \mathrm{Boc}_{2} \mathrm{O}(1.58 \mathrm{~g}, 7.23$ 
mmol), and DMAP (366.5 mg, $3.00 \mathrm{mmol})$ in $t$ - $\mathrm{BuOH}(10 \mathrm{~mL})$ was heated at $40{ }^{\circ} \mathrm{C}$ in an oil bath for $13 \mathrm{~h}$ and concentrated in vacuo. The residue was purified by column chromatography on silica gel (hexane:AcOEt $=40: 1)$ to afford $1 \mathrm{t}(1.14 \mathrm{~g}, 5.32 \mathrm{mmol}, 88 \%)$ as a colorless oil. ${ }^{1} \mathrm{H} \mathrm{NMR}(400 \mathrm{MHz}$, $\left.\mathrm{CDCl}_{3} / \mathrm{TMS}\right) \delta 7.52-7.44(\mathrm{~m}, 2 \mathrm{H}), 6.97(\mathrm{tt}, 1 \mathrm{H}, J=8.5,2.4 \mathrm{~Hz}), 1.59(\mathrm{~s}, 9 \mathrm{H}) .{ }^{13} \mathrm{C} \mathrm{NMR}(150 \mathrm{MHz}$, $\left.\mathrm{CDCl}_{3} / \mathrm{TMS}\right) \delta 163.4,162.7\left(\mathrm{dd},{ }^{1} J_{\mathrm{CF}}=248.5 \mathrm{~Hz},{ }^{3} J_{\mathrm{CF}}=11.5 \mathrm{~Hz}\right), 135.4\left(\mathrm{t},{ }^{3} J_{\mathrm{CF}}=8.6 \mathrm{~Hz}\right), 112.4(\mathrm{dd}$, $\left.{ }^{2} J_{\mathrm{CF}}=21.5 \mathrm{~Hz},{ }^{4} J_{\mathrm{CF}}=5.7 \mathrm{~Hz}\right), 107.8\left(\mathrm{t},{ }^{2} J_{\mathrm{CF}}=25.1 \mathrm{~Hz}\right), 82.2,28.1 .{ }^{19} \mathrm{~F} \mathrm{NMR}\left(564.5 \mathrm{MHz}, \mathrm{CDCl}_{3} / 4-\right.$ fluorotoluene) $\delta$-108.5. LRMS (EI) $m / z: 214\left(\mathrm{M}^{+}\right)$. HRMS (EI-TOF) $m / z:\left(\mathrm{M}^{+}\right)$Calcd. for $\mathrm{C}_{11} \mathrm{H}_{12} \mathrm{~F}_{2} \mathrm{O}_{2}$ : 214.0805, found: 214.0802. IR (neat): 2981, 1723, 1622, 1599, 1442, 1370, $1328 \mathrm{~cm}^{-1}$. The spectra data matched those reported in the literature. ${ }^{13}$<smiles>CCCCOC(=O)c1cc(Cl)cc(Cl)c1</smiles>

tert-Butyl 3,5-dichlorobenzoate (1u). According to the procedure analogous to that described for 1t, 1u (1.35 g, $5.46 \mathrm{mmol}, 92 \%)$ was obtained from 3,5-dichlorobenzoic acid (1.13 g, $5.91 \mathrm{mmol})$ as a colorless oil. ${ }^{1} \mathrm{H}$ NMR (400 MHz, $\left.\mathrm{CDCl}_{3} / \mathrm{TMS}\right) \delta 7.84$ (d, 2H, $J=2.0 \mathrm{~Hz}$ ), 7.50 (t, 1H, $J=2.0 \mathrm{~Hz}$ ), 1.59 (s, 9H). ${ }^{13} \mathrm{C} \mathrm{NMR}\left(100 \mathrm{MHz}, \mathrm{CDCl}_{3} / \mathrm{TMS}\right) \delta 163.2,135.0,134.8,132.2,127.9,82.4,28.1 . \mathrm{RMS}$ (EI) $m / z: 246\left(\mathrm{M}^{+}\right)$. HRMS (EI-TOF) $\mathrm{m} / z:\left(\mathrm{M}^{+}\right)$Calcd. for $\mathrm{C}_{11} \mathrm{H}_{12} \mathrm{Cl}_{2} \mathrm{O}_{2}: 246.0214$, found: 246.0232 . IR (neat): 2980, 1721, 1569, 1430, 1369, $1284 \mathrm{~cm}^{-1}$.<smiles>CC(C)(C)OC(=O)c1cc(Br)cc(Br)c1</smiles>

tert-Butyl 3,5-dibromobenzoate (1v). According to the procedure analogous to that described for 1t, $1 \mathbf{v}(1.42 \mathrm{~g}, 4.23 \mathrm{mmol}, 70 \%)$ was obtained from 3,5-dibromobenzoic acid (1.69 $\mathrm{g}, 6.03 \mathrm{mmol})$ as a white solid: $\mathrm{Mp} 54-55^{\circ} \mathrm{C}$ (hexane) (lit. 58-61 $\left.{ }^{\circ} \mathrm{C}^{14}\right) .{ }^{1} \mathrm{H} \mathrm{NMR}\left(400 \mathrm{MHz}, \mathrm{CDCl}_{3} / \mathrm{TMS}\right) \delta 8.03(\mathrm{~d}, 2 \mathrm{H}$, $J=2.0 \mathrm{~Hz}), 7.81(\mathrm{t}, 1 \mathrm{H}, J=1.7 \mathrm{~Hz}), 1.59(\mathrm{~s}, 9 \mathrm{H}) .{ }^{13} \mathrm{C} \mathrm{NMR}\left(150 \mathrm{MHz}, \mathrm{CDCl}_{3} / \mathrm{TMS}\right) \delta 163.0,137.6$, 135.2, 131.2, 122.8, 82.5, 28.1. LRMS (EI) $m / z: 334\left(\mathrm{M}^{+}\right)$. HRMS (EI-TOF) $m / z:\left(\mathrm{M}^{+}\right)$Calcd. for $\mathrm{C}_{11} \mathrm{H}_{12} \mathrm{Br}_{2} \mathrm{O}_{2}$ : 333.9204, found: 333.9203. IR (neat): 3082, 2992, 1712, 1557, $1283 \mathrm{~cm}^{-1}$. The spectra data matched those reported in the literature. ${ }^{14}$ 
Scheme S5. Preparation of $1 y$<smiles>O=C(O)c1cc(Br)cc(Br)c1</smiles>

S5<smiles>CNC(=O)c1cc(Br)cc(Br)c1</smiles>

$1 y$

3,5-Dibromo- $N$-methyl- $N$-phenylbenzamide (1y). A mixture of S5 $(2.04 \mathrm{~g}, 7.28 \mathrm{mmol})$ in thionyl chloride $(10 \mathrm{~mL})$ was refluxed at $70{ }^{\circ} \mathrm{C}$ in an oil bath for $4 \mathrm{~h}$ and concentrated in vacuo. To the residue was added anhydrous $\mathrm{CH}_{2} \mathrm{Cl}_{2}(20 \mathrm{~mL}), \mathrm{NEt}_{3}(2.20 \mathrm{~mL}, 16.0 \mathrm{mmol})$, and $N$-methylaniline $(1.30 \mathrm{~mL}$, $12.0 \mathrm{mmol}$ ). The reaction mixture was stirred at room temperature for $24 \mathrm{~h}$. The reaction mixture was washed with $1 \mathrm{M} \mathrm{HCl}$ aqueous solution $(10 \mathrm{~mL})$ and brine $(10 \mathrm{~mL})$, dried over $\mathrm{Na}_{2} \mathrm{SO}_{4}$, and concentrated. The residue was purified by column chromatography on silica gel (hexane:AcOEt $=4: 1$ ) to afford $1 \mathbf{y}(2.41 \mathrm{~g}, 6.53 \mathrm{mmol}, 90 \%)$ as a white solid. Mp $104-105^{\circ} \mathrm{C}$ (AcOEt). ${ }^{1} \mathrm{H} \mathrm{NMR}(400 \mathrm{MHz}$, $\left.\mathrm{CDCl}_{3} / \mathrm{TMS}\right) \delta 7.52(\mathrm{~s}, 1 \mathrm{H}), 7.35(\mathrm{~s}, 2 \mathrm{H}), 7.29(\mathrm{t}, 2 \mathrm{H}, J=7.6 \mathrm{~Hz}), 7.22(\mathrm{t}, 1 \mathrm{H}, J=7.3 \mathrm{~Hz}), 7.04(\mathrm{~d}$, $2 \mathrm{H}, J=7.3 \mathrm{~Hz}$ ), 3.47 (s, 3H). ${ }^{13} \mathrm{C} \mathrm{NMR}\left(100 \mathrm{MHz}, \mathrm{CDCl}_{3} / \mathrm{TMS}\right) \delta 167.3,143.9,139.1,135.0,130.4$, 129.5, 127.3, 126.8, 122.2, 38.4. LRMS (EI) $\mathrm{m} / z$ : $367\left(\mathrm{M}^{+}\right)$. HRMS (EI-TOF) $\mathrm{m} / \mathrm{z}:\left(\mathrm{M}^{+}\right)$Calcd. for $\mathrm{C}_{14} \mathrm{H}_{11} \mathrm{Br}_{2} \mathrm{NO}: 366.9207$, found: 366.9207. IR (neat): 3066, 1643, 1593, 1554, 1495, 1412, $1368 \mathrm{~cm}^{-1}$.<smiles>CNC(=O)c1cc(F)cc(F)c1</smiles>

3,5-Difluoro- $N$-methyl- $N$-phenylbenzamide (1w). According to the procedure analogous to that described for 1y, $1 \mathbf{w}(848.6 \mathrm{mg}, 3.43 \mathrm{mmol}, 86 \%$ ) was obtained from 3,5-difluorobenzoic acid (633.5 mg, $4.01 \mathrm{mmol}$ ) as a white solid: $\mathrm{Mp} 79-80{ }^{\circ} \mathrm{C}$ (hexane). ${ }^{1} \mathrm{H}$ NMR (600 MHz, $\mathrm{CDCl}_{3} / \mathrm{TMS}$ ) $\delta 7.27(\mathrm{t}, 2 \mathrm{H}, J=7.8 \mathrm{~Hz}), 7.20(\mathrm{t}, 1 \mathrm{H}, J=7.6 \mathrm{~Hz}), 7.04(\mathrm{~d}, 2 \mathrm{H}, J=7.8 \mathrm{~Hz}), 6.81(\mathrm{~d}, 2 \mathrm{H}, J=5.9 \mathrm{~Hz})$, $6.68(\mathrm{t}, 1 \mathrm{H}, J=8.7 \mathrm{~Hz}), 3.48(\mathrm{~s}, 3 \mathrm{H}) .{ }^{13} \mathrm{C} \mathrm{NMR}\left(150 \mathrm{MHz}, \mathrm{CDCl}_{3} / \mathrm{TMS}\right) \delta 168.0,162.2\left(\mathrm{dd},{ }^{1} J_{\mathrm{CF}}=\right.$ $\left.247.8 \mathrm{~Hz},{ }^{3} J_{\mathrm{CF}}=12.2 \mathrm{~Hz}\right), 144.0,139.1\left(\mathrm{t},{ }^{3} J_{\mathrm{CF}}=8.6 \mathrm{~Hz}\right), 129.5,127.3,126.8,111.9\left(\mathrm{dd},{ }^{2} J_{\mathrm{CF}}=20.1\right.$ $\left.\mathrm{Hz},{ }^{4} J_{\mathrm{CF}}=5.7 \mathrm{~Hz}\right), 105.1\left(\mathrm{t},{ }^{2} J_{\mathrm{CF}}=25.1 \mathrm{~Hz}\right), 38.5 .{ }^{19} \mathrm{~F} \mathrm{NMR}\left(564.5 \mathrm{MHz}, \mathrm{CDCl}_{3} / 4\right.$-fluorotoluene $) \delta$ 108.6. LRMS (EI) $m / z: 247\left(\mathrm{M}^{+}\right)$. HRMS (EI-TOF) $m / z$ : $\left(\mathrm{M}^{+}\right)$Calcd. for $\mathrm{C}_{14} \mathrm{H}_{11} \mathrm{~F}_{2} \mathrm{NO}$ : 247.0809, found: 247.0808 . IR (neat): 3079, 1641, 1620, 1585, 1498, $1440 \mathrm{~cm}^{-1}$. 
<smiles>CNC(=O)c1cc(Cl)cc(Cl)c1</smiles>

3,5-Dichloro- $N$-methyl- $N$-phenylbenzamide (1x). According to the procedure analogous to that described for $1 \mathbf{y}, \mathbf{1 x}(1.01 \mathrm{~g}, 0.361 \mathrm{mmol}, 90 \%)$ was obtained from 3,5-dichlorobenzoic acid (0.766 g, $4.01 \mathrm{mmol}$ ) as a white solid: Mp 90-92 ${ }^{\circ} \mathrm{C}$ (hexane). ${ }^{1} \mathrm{H}$ NMR (600 MHz, $\left.\mathrm{CDCl}_{3} / \mathrm{TMS}\right) \delta 7.29(\mathrm{t}, 2 \mathrm{H}$, $J=7.1 \mathrm{~Hz}), 7.23-7.20(\mathrm{~m}, 2 \mathrm{H}), 7.16(\mathrm{~s}, 2 \mathrm{H}), 7.05(\mathrm{~d}, 2 \mathrm{H}, J=7.9 \mathrm{~Hz}), 3.48(\mathrm{~s}, 3 \mathrm{H}) .{ }^{13} \mathrm{C}$ NMR $(150$ $\left.\mathrm{MHz}, \mathrm{CDCl}_{3} / \mathrm{TMS}\right) \delta 167.6,143.9,138.7,134.4,129.6,129.5,127.3,127.1,126.8,38.4$. LRMS (EI) $m / z: 279\left(\mathrm{M}^{+}\right)$. HRMS (EI-TOF) $m / z$ : $\left(\mathrm{M}^{+}\right)$Calcd. for $\mathrm{C}_{14} \mathrm{H}_{11} \mathrm{Cl}_{2} \mathrm{NO}_{2}$ : 279.0218, found: 279.0197. IR (neat): $3076,3054,1646,1595,1561,1496 \mathrm{~cm}^{-1}$.

Scheme S6. Preparation of $1 z$<smiles>O=Cc1cc(F)cc(F)c1</smiles>

S6

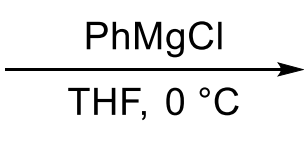<smiles>OC(/C=C/CF)c1ccccc1</smiles>

S7

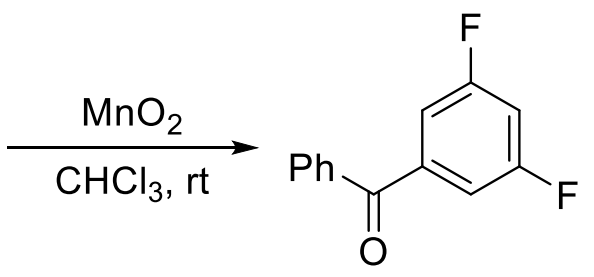

$1 z$

3,5-Difluorobenzophenone (1z). To the solution of $\mathbf{S 6}(760.3 \mathrm{mg}, 5.35 \mathrm{mmol})$ in THF (10 mL) was slowly added $\mathrm{PhMgCl}(2.0 \mathrm{M}$ in THF, $3.0 \mathrm{~mL}, 6.0 \mathrm{mmol})$ at $0{ }^{\circ} \mathrm{C}$. After stirring at the same temperature for $2 \mathrm{~h}$, the reaction mixture was quenched with saturated $\mathrm{NH}_{4} \mathrm{Cl}$ aqueous solution and extracted with $\mathrm{Et}_{2} \mathrm{O}$. The combined organic layer was washed with $\mathrm{H}_{2} \mathrm{O}$, dried over $\mathrm{Na}_{2} \mathrm{SO}_{4}$, and concentrated. The residue was purified by column chromatography on silica gel (hexane:AcOEt $=20: 1$ ) to afford $\mathbf{S 7}$. The reaction mixture of $\mathbf{S} 7$ and $\mathrm{MnO}_{2}(5.29 \mathrm{~g}, 60.8 \mathrm{mmol})$ in $\mathrm{CHCl}_{3}(30 \mathrm{~mL})$ was stirred at room temperature for $17 \mathrm{~h}$. The resulting mixture was filtrated $\left(\mathrm{Celite}{ }^{\circledR}, \mathrm{CH}_{2} \mathrm{Cl}_{2}\right)$ and concentrated. The residue was purified by column chromatography on silica gel (hexane:AcOEt $=20: 1$ ) to afford $\mathbf{~} \mathbf{z}$ (849.7 mg, $3.89 \mathrm{mmol}, 73 \%$ ) as a white solid : Mp 46-48 ${ }^{\circ} \mathrm{C}$ (hexane) (lit. $\left.51-53{ }^{\circ} \mathrm{C}^{15}\right) .{ }^{1} \mathrm{H}$ NMR $(400$ $\left.\mathrm{MHz}, \mathrm{CDCl}_{3} / \mathrm{TMS}\right) \delta 7.85-7.75(\mathrm{~m}, 2 \mathrm{H}), 7.68-7.59(\mathrm{~m}, 1 \mathrm{H}), 7.56-7.47(\mathrm{~m}, 2 \mathrm{H}), 7.37-7.28(\mathrm{~m}, 2 \mathrm{H})$, $7.04(\mathrm{tt}, 1 \mathrm{H}, J=8.5,2.4 \mathrm{~Hz}) .{ }^{13} \mathrm{C} \mathrm{NMR}\left(150 \mathrm{MHz}, \mathrm{CDCl}_{3} / \mathrm{TMS}\right) \delta 193.8,162.8\left(\mathrm{dd},{ }^{1} J_{\mathrm{CF}}=251.3 \mathrm{~Hz}\right.$, $\left.{ }^{3} J_{\mathrm{CF}}=12.2 \mathrm{~Hz}\right), 140.7\left(\mathrm{t},{ }^{3} J_{\mathrm{CF}}=7.2 \mathrm{~Hz}\right), 136.5,133.2,130.1,128.7,113.0\left(\mathrm{dd},{ }^{2} J_{\mathrm{CF}}=21.5 \mathrm{~Hz},{ }^{4} J_{\mathrm{CF}}=\right.$ 
$5.7 \mathrm{~Hz}), 107.7\left(\mathrm{t},{ }^{2} J_{\mathrm{CF}}=25.1 \mathrm{~Hz}\right) .{ }^{19} \mathrm{~F}$ NMR (564.5 MHz, $\mathrm{CDCl}_{3} / 4$-fluorotoluene) $\delta$-107.5. LRMS (EI) $m / z: 218\left(\mathrm{M}^{+}\right)$. HRMS (EI-TOF) $\mathrm{m} / z:\left(\mathrm{M}^{+}\right)$Calcd. for $\mathrm{C}_{13} \mathrm{H}_{8} \mathrm{~F}_{2} \mathrm{O}: 218.0543$, found: 218.0548 . IR (neat): $3073,1663,1592,1442,1306 \mathrm{~cm}^{-1}$. The spectra data matched those reported in the literature. ${ }^{16}$<smiles>O=C(c1ccccc1)c1cccc(F)c1</smiles>

3-Fluorobenzophenone (3g). According to the procedure analogous to that described for $\mathbf{1 z}, \mathbf{3 g}$ ( 1.46 g, $7.29 \mathrm{mmol}, 76 \%)$ was obtained from 3-fluorobenzaldehyde (1.19 g, $9.59 \mathrm{mmol})$ as a white solid: Mp 49-50 ${ }^{\circ} \mathrm{C}$ (hexane) (lit. 50-52 ${ }^{\circ} \mathrm{C}^{17}$ ). ${ }^{1} \mathrm{H}$ NMR (600 MHz, $\left.\mathrm{CDCl}_{3} / \mathrm{TMS}\right) \delta 7.82-7.79$ (m, 2H), $7.63-7.59(\mathrm{~m}, 1 \mathrm{H}), 7.59-7.56(\mathrm{~m}, 1 \mathrm{H}), 7.53-7.49(\mathrm{~m}, 3 \mathrm{H}), 7.46(\mathrm{td}, 1 \mathrm{H}, J=8.1,5.5 \mathrm{~Hz}), 7.32-7.28$ (m, 1H). ${ }^{13} \mathrm{C}$ NMR $\left(150 \mathrm{MHz}, \mathrm{CDCl}_{3} / \mathrm{TMS}\right) \delta 195.2,162.4\left(\mathrm{~d},{ }^{1} J_{\mathrm{CF}}=247.7 \mathrm{~Hz}\right), 139.6\left(\mathrm{~d},{ }^{3} J_{\mathrm{CF}}=7.2\right.$ $\mathrm{Hz}), 137.0,132.7,129.96,128.89,128.4,125.8\left(\mathrm{~d},{ }^{4} J_{\mathrm{CF}}=2.9 \mathrm{~Hz}\right), 119.4\left(\mathrm{~d},{ }^{2} J_{\mathrm{CF}}=21.5 \mathrm{~Hz}\right), 116.7(\mathrm{~d}$, $\left.{ }^{2} J_{\mathrm{CF}}=22.9 \mathrm{~Hz}\right) .{ }^{19} \mathrm{~F}$ NMR (564.5 MHz, $\mathrm{CDCl}_{3} / 4$-fluorotoluene) $\delta$-111.3. LRMS (EI) $m / z: 200\left(\mathrm{M}^{+}\right)$. HRMS (EI-TOF) $m / z:\left(\mathrm{M}^{+}\right.$) Calcd. for $\mathrm{C}_{13} \mathrm{H}_{9} \mathrm{FO}$ : 200.0637, found: 200.0637. IR (neat): 3069, 1653, $1586,1576,1441,1280 \mathrm{~cm}^{-1}$. The spectra data matched those reported in the literature. ${ }^{17}$

Scheme S7. Preparation of 3d

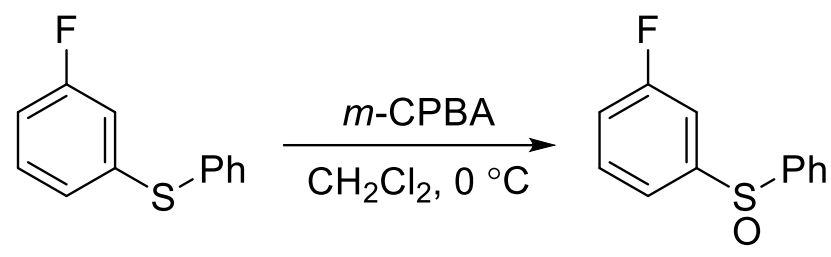

S8

3d

1-Fluoro-3-(phenylsulfinyl)benzene (3d). To the solution of $\mathbf{S 8}(319.7 \mathrm{~g}, 1.57 \mathrm{mmol})$ in $\mathrm{CH}_{2} \mathrm{Cl}_{2}(10$ $\mathrm{mL})$ was slowly added $m$-CPBA $(399.8 \mathrm{mg}, 1.62 \mathrm{mmol})$ at $0{ }^{\circ} \mathrm{C}$. After stirring at $0{ }^{\circ} \mathrm{C}$ for $3 \mathrm{~h}$, saturated $\mathrm{NaHCO}_{3}$ aqueous solution was added, and the mixture was extracted with $\mathrm{CH}_{2} \mathrm{Cl}_{2}$. The combined organic layer was washed with $1 \mathrm{M} \mathrm{NaOH}$ aqueous solution and brine, dried over $\mathrm{MgSO}_{4}$, and concentrated. The residue was purified by column chromatography on silica gel (hexane:AcOEt $=4: 1$ ) to afford 3d (240.9 mg, $1.09 \mathrm{mmol}, 70 \%$ ) as a white solid. Mp 60-61 ${ }^{\circ} \mathrm{C}$ (hexane/AcOEt) (lit. $\left.62-63{ }^{\circ} \mathrm{C}^{18}\right) .{ }^{1} \mathrm{H}$ NMR (600 MHz, $\left.\mathrm{CDCl}_{3} / \mathrm{TMS}\right) \delta 7.68-7.63(\mathrm{~m}, 2 \mathrm{H}), 7.51-7.41(\mathrm{~m}, 5 \mathrm{H}), 7.40-7.38$ (m, 1H), 7.14-7.10 (m, 1H). ${ }^{13} \mathrm{C}$ NMR (150 MHz, CDCl $\left.3 / \mathrm{TMS}\right) \delta 163.0\left(\mathrm{~d},{ }^{1} J_{\mathrm{CF}}=251.4 \mathrm{~Hz}\right), 148.2$ $\left(\mathrm{d},{ }^{3} J_{\mathrm{CF}}=5.7 \mathrm{~Hz}\right), 145.2,131.4,131.0\left(\mathrm{~d},{ }^{3} J_{\mathrm{CF}}=7.9 \mathrm{~Hz}\right), 129.5,124.8,120.3\left(\mathrm{~d},{ }^{4} J_{\mathrm{CF}}=3.6 \mathrm{~Hz}\right), 118.1$ $\left(\mathrm{d},{ }^{2} J_{\mathrm{CF}}=21.5 \mathrm{~Hz}\right), 111.7\left(\mathrm{~d},{ }^{2} J_{\mathrm{CF}}=24.3 \mathrm{~Hz}\right) .{ }^{19} \mathrm{~F}$ NMR $\left(564.5 \mathrm{MHz}, \mathrm{CDCl}_{3} / 4\right.$-fluorotoluene) $\delta-108.8$. LRMS (EI) $m / z: 236\left(\mathrm{M}^{+}\right)$. HRMS (EI-TOF) $\mathrm{m} / z$ : $\left(\mathrm{M}^{+}\right)$Calcd. for $\mathrm{C}_{12} \mathrm{H}_{9} \mathrm{FOS}: 220.0358$, found: 220.0361. IR (neat): 3059, 1589, 1476, 1445, 1429, 1265, $1213 \mathrm{~cm}^{-1}$. 
Scheme S8. Preparation of 3e<smiles>O=[SH](Cl)c1cccc(F)c1</smiles>

S9

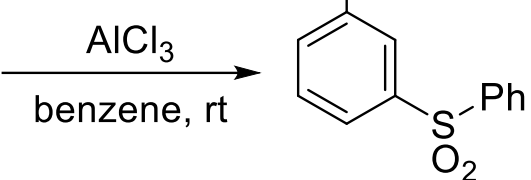

$3 e$

1-Fluoro-3-(phenylsulfonyl)benzene (3e). A mixture of $\mathbf{S 9}(1.41 \mathrm{~g}, 7.25 \mathrm{mmol})$ and $\mathrm{AlCl}_{3}(1.32 \mathrm{~g}$, $9.90 \mathrm{mmol})$ in benzene $(10 \mathrm{~mL})$ was stirred at room temperature for $24 \mathrm{~h}$. The reaction mixture was poured into $\mathrm{H}_{2} \mathrm{O}$ at $0{ }^{\circ} \mathrm{C}$ and extracted with AcOEt. The combined organic extracts were washed with $1 \mathrm{M} \mathrm{NaOH}$ aqueous solution and brine, dried over $\mathrm{NaSO}_{4}$, and concentrated. The residue was purified by column chromatography on silica gel (hexane:AcOEt $=4: 1)$ to afford $3 \mathbf{e}(1.64 \mathrm{~g}, 6.94 \mathrm{mmol}, 96 \%)$ as a white solid. Mp 94-95 ${ }^{\circ} \mathrm{C}$ (hexane/AcOEt) $\left(96-97{ }^{\circ} \mathrm{C}^{18}\right) .{ }^{1} \mathrm{H}$ NMR $\left(600 \mathrm{MHz}, \mathrm{CDCl}_{3} / \mathrm{TMS}\right)$ $\delta 7.97-7.94(\mathrm{~m}, 2 \mathrm{H}), 7.74(\mathrm{ddd}, 1 \mathrm{H}, J=7.9,1.7,1.0 \mathrm{~Hz}), 7.64(\mathrm{ddd}, 1 \mathrm{H}, J=8.1,2.6,1.7 \mathrm{~Hz}), 7.56-7.52$ $(\mathrm{m}, 2 \mathrm{H}), 7.50(\mathrm{td}, 1 \mathrm{H}, J=8.3,5.1 \mathrm{~Hz}), 7.28-7.24(\mathrm{~m}, 1 \mathrm{H}) .{ }^{13} \mathrm{C} \mathrm{NMR}\left(150 \mathrm{MHz}, \mathrm{CDCl}_{3} / \mathrm{TMS}\right) \delta 162.5$ $\left(\mathrm{d},{ }^{1} J_{\mathrm{CF}}=252.2 \mathrm{~Hz}\right), 143.7\left(\mathrm{~d},{ }^{3} J_{\mathrm{CF}}=7.2 \mathrm{~Hz}\right), 140.9,133.5,131.1\left(\mathrm{~d},{ }^{3} J_{\mathrm{CF}}=8.6 \mathrm{~Hz}\right), 129.4,127.8$, $123.4\left(\mathrm{~d},{ }^{4} J_{\mathrm{CF}}=4.3 \mathrm{~Hz}\right), 120.4\left(\mathrm{~d},{ }^{2} J_{\mathrm{CF}}=21.5 \mathrm{~Hz}\right), 115.0\left(\mathrm{~d},{ }^{2} J_{\mathrm{CF}}=24.4 \mathrm{~Hz}\right) .{ }^{19} \mathrm{~F}$ NMR $(564.5 \mathrm{MHz}$, $\mathrm{CDCl}_{3} / 4$-fluorotoluene) $\delta$-108.5. LRMS (EI) $m / z: 236\left(\mathrm{M}^{+}\right)$. HRMS (EI-TOF) $m / z$ : $\left(\mathrm{M}^{+}\right)$Calcd. for $\mathrm{C}_{12} \mathrm{H}_{9} \mathrm{FO}_{2} \mathrm{~S}: 236.0307$, found: 236.0293. IR (neat): 3077, 1590, 1475, 1448, 1304, 1231, $1150 \mathrm{~cm}^{-1}$. The spectra data matched those reported in the literature. ${ }^{19}$

Scheme S9. Preparation of $\mathbf{3 f}$<smiles>O=[SH](Cl)c1cccc(F)c1</smiles>

S10<smiles>O=C(OCc1ccccc1)ONc1ccccc1</smiles>

$\mathrm{CH}_{2} \mathrm{Cl}_{2}, 0{ }^{\circ} \mathrm{C}$ to rt<smiles>CNS(=O)c1cccc(F)c1</smiles>

$3 \mathbf{f}$

3-Fluoro- $N$-methyl- $N$-phenylbenzenesulfonamide (3f). To the solution of $N$-methylaniline (634.3 $\mathrm{mg}, 5.92 \mathrm{mmol})$ and triethylamine $(1.21 \mathrm{~g}, 12.0 \mathrm{mmol})$ in $\mathrm{CH}_{2} \mathrm{Cl}_{2}(20 \mathrm{~mL})$ was slowly added $\mathbf{S 1 0}$ (1.29 $\mathrm{g}, 6.63 \mathrm{mmol}$ ) at $0{ }^{\circ} \mathrm{C}$. The reaction mixture was warmed to room temperature and stirred for $4 \mathrm{~h}$. The mixture was neutralized with $1 \mathrm{M} \mathrm{HCl}$ aqueous solution, extracted with $\mathrm{Et}_{2} \mathrm{O}$. The combined organic layer was washed with brine, dried over $\mathrm{Na}_{2} \mathrm{SO}_{4}$, and concentrated. The residue was purified by column chromatography on silica gel (hexane:AcOEt $=20: 1)$ to afford $\mathbf{3 f}(1.47 \mathrm{~g}, 5.54 \mathrm{mmol}, 94 \%)$ as a white solid. Mp 74-76 ${ }^{\circ} \mathrm{C}$ (hexane/AcOEt). ${ }^{1} \mathrm{H}$ NMR (600 MHz, $\left.\mathrm{CDCl}_{3} / \mathrm{TMS}\right) \delta 7.47-7.41$ (m, 1H), $7.36-7.26(\mathrm{~m}, 6 \mathrm{H}), 7.10(\mathrm{~d}, 2 \mathrm{H}, J=7.6 \mathrm{~Hz}), 3.21(\mathrm{~s}, 3 \mathrm{H}) .{ }^{13} \mathrm{C} \mathrm{NMR}\left(150 \mathrm{MHz}, \mathrm{CDCl}_{3} / \mathrm{TMS}\right) \delta 162.2$ $\left(\mathrm{d},{ }^{1} J_{\mathrm{CF}}=250.7 \mathrm{~Hz}\right), 141.1,138.6\left(\mathrm{~d},{ }^{3} J_{\mathrm{CF}}=5.7 \mathrm{~Hz}\right), 130.5\left(\mathrm{~d},{ }^{3} J_{\mathrm{CF}}=7.2 \mathrm{~Hz}\right), 129.0,127.7,126.6$, 
$123.6\left(\mathrm{~d},{ }^{4} J_{\mathrm{CF}}=2.9 \mathrm{~Hz}\right), 120.0\left(\mathrm{~d},{ }^{2} J_{\mathrm{CF}}=21.5 \mathrm{~Hz}\right), 115.1\left(\mathrm{~d},{ }^{2} J_{\mathrm{CF}}=24.3 \mathrm{~Hz}\right), 38.3 .{ }^{19} \mathrm{~F}$ NMR $(564.5$ $\mathrm{MHz}, \mathrm{CDCl}_{3} / 4$-fluorotoluene) $\delta$-109.3. LRMS (EI) $\mathrm{m} / z$ : $265\left(\mathrm{M}^{+}\right)$. HRMS (EI-TOF) $\mathrm{m} / z$ : $\left(\mathrm{M}^{+}\right.$) Calcd. for $\mathrm{C}_{13} \mathrm{H}_{12} \mathrm{FNO}_{2} \mathrm{~S}: 265.0573$, found: 265.0548. IR (neat): 3078, 1589, 1492, 1345, 1227, $1172 \mathrm{~cm}^{-1}$.

Scheme S10. Preparation of $3 \mathrm{~h}$

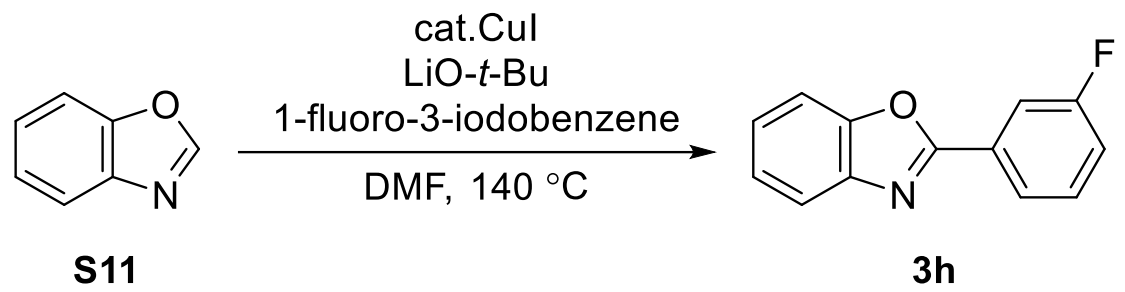

2-(3-Fluorophenyl)benzoxazole (3h). A mixture of S11 (154.6 mg, $1.30 \mathrm{mmol}$ ), 1-fluoro-3-iodobenzene (664.7 mg, $2.99 \mathrm{mmol}$ ), CuI (19.9 mg, $0.104 \mathrm{mmol}$ ), and LiO-t-Bu (163.2 mg, $2.04 \mathrm{mmol})$ in DMF (1 $\mathrm{mL}$ ) was stirred at room temperature for $5 \mathrm{~min}$ and at $140{ }^{\circ} \mathrm{C}$ in an oil bath for $10 \mathrm{~min}$. The mixture was allowed to cool to room temperature and diluted with AcOEt $(50 \mathrm{~mL})$. The resulting solution was washed with brine ( $3 \times 15 \mathrm{~mL}$ ), dried over $\mathrm{MgSO}_{4}$, and concentrated. The residue was purified by column chromatography on silica gel (hexane:AcOEt $=40: 1)$ to afford $\mathbf{3 h}(190.2 \mathrm{mg}, 0.892 \mathrm{mmol}$, $69 \%$ ) as a white solid. Mp $94-95{ }^{\circ} \mathrm{C}$ (hexane/AcOEt) (lit. 91-93 $\left.{ }^{\circ} \mathrm{C}\right)^{20} .{ }^{1} \mathrm{H}$ NMR $(400 \mathrm{MHz}$, $\left.\mathrm{CDCl}_{3} / \mathrm{TMS}\right) \delta 8.06(\mathrm{~d}, 1 \mathrm{H}, J=7.8 \mathrm{~Hz}), 7.96(\mathrm{~d}, 1 \mathrm{H}, J=9.3 \mathrm{~Hz}), 7.83-7.75(\mathrm{~m}, 1 \mathrm{H}), 7.64-7.58(\mathrm{~m}$, $1 \mathrm{H}), 7.55-7.48(\mathrm{~m}, 1 \mathrm{H}), 7.42-7.38(\mathrm{~m}, 2 \mathrm{H}), 7.25-7.22(\mathrm{~m}, 1 \mathrm{H}) .{ }^{13} \mathrm{C} \mathrm{NMR}\left(150 \mathrm{MHz}, \mathrm{CDCl}_{3} / \mathrm{TMS}\right)$ $\delta 162.8\left(\mathrm{~d},{ }^{1} J_{\mathrm{CF}}=246.3 \mathrm{~Hz}\right), 161.7\left(\mathrm{~d},{ }^{4} J_{\mathrm{CF}}=4.3 \mathrm{~Hz}\right), 150.7,141.9,130.5\left(\mathrm{~d},{ }^{3} J_{\mathrm{CF}}=7.2 \mathrm{~Hz}\right), 129.1(\mathrm{~d}$, $\left.{ }^{3} J_{\mathrm{CF}}=8.6 \mathrm{~Hz}\right), 125.4,124.7,123.2\left(\mathrm{~d},{ }^{4} J_{\mathrm{CF}} J=2.9 \mathrm{~Hz}\right), 120.2,118.4\left(\mathrm{~d},{ }^{2} J_{\mathrm{CF}}=21.5 \mathrm{~Hz}\right), 114.5\left(\mathrm{~d},{ }^{2} J_{\mathrm{CF}}\right.$ $=24.3 \mathrm{~Hz}), 110.6 .{ }^{19} \mathrm{~F} \mathrm{NMR}\left(564.5 \mathrm{MHz}, \mathrm{CDCl}_{3} / 4\right.$-fluorotoluene) $\delta$-111.2. LRMS (EI) $\mathrm{m} / \mathrm{z}: 213\left(\mathrm{M}^{+}\right)$. HRMS (EI-TOF) $m / z:\left(\mathrm{M}^{+}\right)$Calcd. for $\mathrm{C}_{13} \mathrm{H}_{8} \mathrm{FNO}: 213.0590$, found: 213.0598. IR (neat): 3078, 1591, $1554,1486,1474,1449 \mathrm{~cm}^{-1}$. The spectra data matched those reported in the literature. ${ }^{20}$

\section{Procedure of combined Bronsted base promoted carboxylation of aromatic C-H bonds (Entry} 3 of Table 1, Figure 2, and Figure 3).

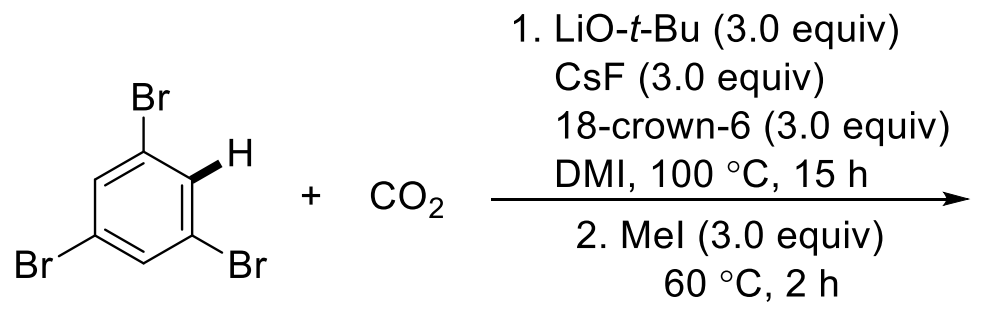

$1 \mathrm{a} \quad(1 \mathrm{~atm})$

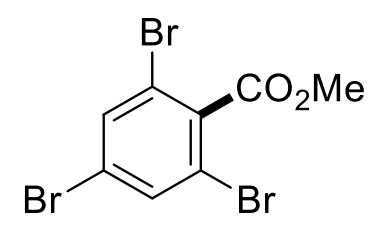

$2 a$

Methyl 2,4,6-tribromobenzoate (2a). In a glove box under an Ar atmosphere, a solution of $\mathrm{LiO}-t$ - $\mathrm{Bu}$ (72.3 mg, $0.903 \mathrm{mmol}$ ), CsF (138.8 mg, $0.914 \mathrm{mmol}$ ), and 18-crown-6 (240.9 mg, $0.911 \mathrm{mmol})$ in DMI $(1.0 \mathrm{~mL})$ was prepared in an oven-dried glass screw tube $(\phi=1.65 \mathrm{~cm}, 10.5 \mathrm{~cm})$ equipped with 
a stirrer bar. The tube was sealed with a rubber cap and taken out from the glove box. The tube was evacuated and refilled with $\mathrm{CO}_{2}$ gas, and this procedure was repeated three times. The tube was further flushed with $\mathrm{CO}_{2}$ gas for 5 min with stirring at room temperature. The rubber cap was removed from the tube, and $1 \mathbf{a}(94.0 \mathrm{mg}, 0.299 \mathrm{mmol})$ was added. The tube was then sealed with a cap containing an inner Teflom film. After stirring at $100{ }^{\circ} \mathrm{C}$ in a heat block for $15 \mathrm{~h}$, the mixture was cooled to $60{ }^{\circ} \mathrm{C}$, and MeI (128.4 mg, $0.904 \mathrm{mmol})$ was added. The mixture was stirred at $60{ }^{\circ} \mathrm{C}$ in a heat block for $2 \mathrm{~h}$, and $\mathrm{H}_{2} \mathrm{O}(5 \mathrm{~mL})$ was added. The mixture was extracted with AcOEt $(10 \mathrm{~mL} \times 3)$. The combined organic layers were collected, washed with $\mathrm{H}_{2} \mathrm{O}(10 \mathrm{~mL})$ and brine $(10 \mathrm{~mL})$, dried over $\mathrm{Na}_{2} \mathrm{SO}_{4}$, and concentrated. The residue was purified by column chromatography on silica gel (hexane:AcOEt = 40:1) to afford 2a (93.2 $\mathrm{mg}, 0.250 \mathrm{mmol}, 84 \%$ ) as a white solid: Mp $65-66{ }^{\circ} \mathrm{C}$ (hexane). ${ }^{1} \mathrm{H}$ NMR (400 $\left.\mathrm{MHz}, \quad \mathrm{CDCl}_{3} / \mathrm{TMS}\right) \quad \delta 7.71(\mathrm{~s}, \quad 2 \mathrm{H}), \quad 3.97 \quad(\mathrm{~s}, \quad 3 \mathrm{H}) .{ }^{13} \mathrm{C} \quad \mathrm{NMR} \quad\left(150 \mathrm{MHz}, \mathrm{CDCl}_{3} / \mathrm{TMS}\right)$ $\delta$ 165.9, 136.6, 134.0, 124.0, 120.3, 53.2. LRMS (EI) $m / z: 370\left(\mathrm{M}^{+}\right)$. HRMS (EI-TOF) $m / z:\left(\mathrm{M}^{+}\right)$ Calcd. for $\mathrm{C}_{8} \mathrm{H}_{5} \mathrm{Br}_{3} \mathrm{O}_{2}: 369.7840$, found: 369.7846 . IR (neat): 2949, 1738, 1539, 1270, 1192, 1135, 831 $\mathrm{cm}^{-1}$.<smiles>COC(=O)c1c(Cl)cc(Cl)cc1Cl</smiles>

Methyl 2,4,6-trichlorobenzoate (2b). In a glove box under an Ar atmosphere, a solution of $\mathbf{1 b}$ (53.6 $\mathrm{mg}, 0.295 \mathrm{mmol}$ ), LiO- $t$-Bu (73.3 mg, $0.915 \mathrm{mmol}$ ), CsF (139.6 mg, $0.919 \mathrm{mmol}$ ), and 18-crown-6 (240.2 $\mathrm{mg}, 0.910 \mathrm{mmol})$ in DMI $(1.0 \mathrm{~mL})$ was prepared in an oven-dried glass screw tube $(\phi=1.65$ $\mathrm{cm}, 10.5 \mathrm{~cm}$ ) equipped with a stirrer bar. The tube was sealed with a rubber cap and taken out from the glove box. The tube was evacuated and refilled with $\mathrm{CO}_{2}$ gas, and this procedure was repeated three times. The tube was further flushed with $\mathrm{CO}_{2}$ gas for 5 min with stirring at room temperature. The rubber cap was then replaced with a cap containing an inner Teflom film. After stirring $120^{\circ} \mathrm{C}$ in a heat block for $15 \mathrm{~h}$, the mixture was cooled to $60{ }^{\circ} \mathrm{C}$, and $\mathrm{MeI}(128.8 \mathrm{mg}, 0.903 \mathrm{mmol})$ was added. The mixture was stirred at $60{ }^{\circ} \mathrm{C}$ in a heat block for $2 \mathrm{~h}$, and $\mathrm{H}_{2} \mathrm{O}(5 \mathrm{~mL})$ was added. The mixture was extracted with AcOEt (10 mL x 3). The combined organic layers were collected, washed with $\mathrm{H}_{2} \mathrm{O}$ (10 $\mathrm{mL})$ and brine $(10 \mathrm{~mL})$, dried over $\mathrm{Na}_{2} \mathrm{SO}_{4}$, and concentrated. The residue was purified by column chromatography on silica gel (hexane:AcOEt $=40: 1)$ to afford $\mathbf{2 b}(51.1 \mathrm{mg}, 0.213 \mathrm{mmol}, 72 \%)$ as a white solid: $\mathrm{Mp} 42-43{ }^{\circ} \mathrm{C}$ (hexane) (lit. $43-44{ }^{\circ} \mathrm{C}^{21}$ ). ${ }^{1} \mathrm{H}$ NMR (400 MHz, $\left.\mathrm{CDCl}_{3} / \mathrm{TMS}\right) \delta 7.35$ (s, 2H), 3.97 (s, 3H). $\left.{ }^{13} \mathrm{C} \mathrm{NMR} \mathrm{(100} \mathrm{MHz,} \mathrm{CDCl}_{3} / \mathrm{TMS}\right) \delta 164.5,136.2,132.6,132.1,128.0,53.2$. LRMS (EI) $m / z: 238\left(\mathrm{M}^{+}\right)$. HRMS (EI-TOF) $m / z:\left(\mathrm{M}^{+}\right)$Calcd. for $\mathrm{C}_{8} \mathrm{H}_{5} \mathrm{Cl}_{3} \mathrm{O}_{2}: 237.9355$, found: 237.9374. IR (neat): $2956,1743,1573,1383,1272,1114,846 \mathrm{~cm}^{-1}$. 
<smiles>CC(=O)c1c(F)ccc(Cl)c1F</smiles>

Methyl 3-chloro-2,6-difluorobenzoate (2c). According to the procedure analogous to that described for $\mathbf{2 b}, \mathbf{2 c}$ (35.6 mg, $0.172 \mathrm{mmol}, 57 \%)$ was obtained from $\mathbf{1 c}(45.1 \mathrm{mg}, 0.303 \mathrm{mmol})$ as a white solid: Mp 37-38 ${ }^{\circ} \mathrm{C}$ (hexane). ${ }^{1} \mathrm{H}$ NMR (600 MHz, $\left.\mathrm{CDCl}_{3} / \mathrm{TMS}\right) \delta 7.48$ (dt, $1 \mathrm{H}, J=5.5,8.6 \mathrm{~Hz}$ ), 6.94 (dt, $1 \mathrm{H}, J=1.4,8.9 \mathrm{~Hz}), 3.97$ (s, 3H). ${ }^{13} \mathrm{C} \mathrm{NMR}\left(150 \mathrm{MHz}, \mathrm{CDCl}_{3} / \mathrm{TMS}\right) \delta 161.1,158.8\left(\mathrm{dd},{ }^{1} J_{\mathrm{CF}}=257.1\right.$ $\left.\mathrm{Hz},{ }^{3} J_{\mathrm{CF}}=5.0 \mathrm{~Hz}\right), 155.9\left(\mathrm{dd},{ }^{1} J_{\mathrm{CF}}=257.8 \mathrm{~Hz},{ }^{3} J_{\mathrm{CF}}=7.2 \mathrm{~Hz}\right), 132.9\left(\mathrm{~d},{ }^{3} J_{\mathrm{CF}}=10.0 \mathrm{~Hz}\right), 117.5(\mathrm{dd}$, $\left.{ }^{2} J_{\mathrm{CF}}=18.6 \mathrm{~Hz},{ }^{4} J_{\mathrm{CF}}=4.3 \mathrm{~Hz}\right), 112.6\left(\mathrm{dd},{ }^{2} J_{\mathrm{CF}}=22.9 \mathrm{~Hz},{ }^{4} J_{\mathrm{CF}}=4.3 \mathrm{~Hz}\right), 112.3\left(\mathrm{t},{ }^{2} J_{\mathrm{CF}}=19.3 \mathrm{~Hz}\right)$, 53.1. ${ }^{19} \mathrm{~F}$ NMR (564.5 MHz, $\mathrm{CDCl}_{3} / 4$-fluorotoluene) $\delta$-109.5, -110.8. LRMS (EI) m/z: $206\left(\mathrm{M}^{+}\right)$. HRMS (EI-TOF) $m / z:\left(\mathrm{M}^{+}\right)$Calcd. for $\mathrm{C}_{8} \mathrm{H}_{5} \mathrm{ClF}_{2} \mathrm{O}_{2}: 205.9946$, found: 205.9953. IR (neat): 3071, 2966, $1730,1616,1479,1430,1293 \mathrm{~cm}^{-1}$.<smiles>CC(=O)c1c(F)ccc(Br)c1F</smiles>

Methyl 3-bromo-2,6-difluorobenzoate (2d). According to the procedure analogous to that described for $\mathbf{2 b}, \mathbf{2 d}(47.6 \mathrm{mg}, 0.190 \mathrm{mmol}, 59 \%)$ was obtained from $\mathbf{1 d}(61.9 \mathrm{mg}, 0.321 \mathrm{mmol})$ as a white solid: Mp 45-46 ${ }^{\circ} \mathrm{C}$ (hexane). ${ }^{1} \mathrm{H}$ NMR (400 MHz, $\left.\mathrm{CDCl}_{3} / \mathrm{TMS}\right) \delta 7.70-7.58(\mathrm{~m}, 1 \mathrm{H}), 6.94-6.86(\mathrm{~m}, 1 \mathrm{H})$, $3.97(\mathrm{~s}, 3 \mathrm{H}) .{ }^{13} \mathrm{C} \mathrm{NMR}\left(150 \mathrm{MHz}, \mathrm{CDCl}_{3} / \mathrm{TMS}\right) \delta 161.1,159.6\left(\mathrm{dd},{ }^{1} J_{\mathrm{CF}}=255.6 \mathrm{~Hz},{ }^{3} J_{\mathrm{CF}}=5.7 \mathrm{~Hz}\right)$, $156.8\left(\mathrm{dd},{ }^{1} J_{\mathrm{CF}}=254.9 \mathrm{~Hz},{ }^{3} J_{\mathrm{CF}}=6.5 \mathrm{~Hz}\right), 135.7\left(\mathrm{dd},{ }^{3} J_{\mathrm{CF}}=10.1,{ }^{3} J_{\mathrm{CF}}=4.0 \mathrm{~Hz}\right), 113.2\left(\mathrm{dd},{ }^{2} J_{\mathrm{CF}}=\right.$ $\left.23.0 \mathrm{~Hz},{ }^{4} J_{\mathrm{CF}}=4.3 \mathrm{~Hz}\right), 112.3\left(\mathrm{t},{ }^{2} J_{\mathrm{CF}}=19.4 \mathrm{~Hz}\right), 104.7\left(\mathrm{dd},{ }^{2} J_{\mathrm{CF}}=22.3 \mathrm{~Hz},{ }^{4} J_{\mathrm{CF}}=3.6 \mathrm{~Hz}\right), 53.0 .{ }^{19} \mathrm{~F}$ NMR (564.5 MHz, $\mathrm{CDCl}_{3} / 4$-fluorotoluene) $\delta$-101.3, -110.2. LRMS (EI) $m / z: 250\left(\mathrm{M}^{+}\right.$). HRMS (EITOF) $m / z:\left(\mathrm{M}^{+}\right)$Calcd. for $\mathrm{C}_{8} \mathrm{H}_{5} \mathrm{BrF}_{2} \mathrm{O}_{2}: 249.9441$, found: 249.9468. IR (neat): 3099, 3070, 1730, 1615, $1473,1426,1289,822 \mathrm{~cm}^{-1}$. The spectra data matched those reported in the literature. ${ }^{22}$<smiles>COC(=O)c1c(Cl)ccc(Br)c1Cl</smiles>

Methyl 3-bromo-2,6-dichlorobenzoate (2e). According to the procedure analogous to that described for $2 \mathrm{a}, 2 \mathrm{e}(52.9 \mathrm{mg}, 0.186 \mathrm{mmol}, 64 \%)$ was obtained from $1 \mathrm{e}(65.9 \mathrm{mg}, 0.292 \mathrm{mmol})$ as a colorless oil. ${ }^{1} \mathrm{H}$ NMR (600 MHz, $\left.\mathrm{CDCl}_{3} / \mathrm{TMS}\right) \delta 7.60(\mathrm{~d}, 1 \mathrm{H}, J=8.9 \mathrm{~Hz}), 7.20(\mathrm{~d}, 1 \mathrm{H}, J=8.2 \mathrm{~Hz}), 3.99$ (s, $3 \mathrm{H}) .{ }^{13} \mathrm{C}$ NMR (150 MHz, $\left.\mathrm{CDCl}_{3} / \mathrm{TMS}\right) \delta 164.5,135.1,134.7,132.3,130.7,128.7,121.6,53.2$. LRMS (EI) $m / z: 282\left(\mathrm{M}^{+}\right.$). HRMS (EI-TOF) $\mathrm{m} / z$ : $\left(\mathrm{M}^{+}\right.$) Calcd. for $\mathrm{C}_{8} \mathrm{H}_{5} \mathrm{BrCl}_{2} \mathrm{O}_{2}$ : 281.8850, found: 281.8853. IR (neat): 2954, 1740, 1568, 1442, 1429, 1380, 1266, $1249 \mathrm{~cm}^{-1}$. 
<smiles>COC(=O)c1c(Br)cccc1Br</smiles>

Methyl 2,6-dibromobenzoate (2f). According to the procedure analogous to that described for 2a, except that the carboxylation was conducted at $130{ }^{\circ} \mathrm{C}$ in a heat block with LiO- $t$-Am $(85.6 \mathrm{mg}, 0.910$ $\mathrm{mmol}$ ) instead of LiO- $t$-Bu and that the crude material was purified by column chromatography on silica gel (hexane:AcOEt = 60:1), $2 \mathbf{f}(49.1 \mathrm{mg}, 0.167 \mathrm{mmol}, 56 \%)$ was obtained from $1 \mathbf{f}(70.6 \mathrm{mg}$, $0.299 \mathrm{mmol}$ ) as a white solid: $\mathrm{Mp} 57-58{ }^{\circ} \mathrm{C}$ (hexane). ${ }^{1} \mathrm{H}$ NMR $\left(400 \mathrm{MHz}, \mathrm{CDCl}_{3} / \mathrm{TMS}\right) \delta 7.53(\mathrm{~d}$, $2 \mathrm{H}, J=8.3 \mathrm{~Hz}), 7.14(\mathrm{t}, 1 \mathrm{H}, J=8.0 \mathrm{~Hz}), 3.98(\mathrm{~s}, 3 \mathrm{H}) .{ }^{13} \mathrm{C}$ NMR (100 MHz, $\left.\mathrm{CDCl}_{3} / \mathrm{TMS}\right)$ $\delta$ 166.6, 137.6, 131.5, 131.4, 119.7, 53.1. LRMS (EI) $m / z: 294\left(\mathrm{M}^{+}\right)$. HRMS (EI-TOF) $m / z:\left(\mathrm{M}^{+}\right)$ Calcd. for $\mathrm{C}_{8} \mathrm{H}_{6} \mathrm{Br}_{2} \mathrm{O}_{2}$ : 291.8735, found: 291.8729. IR (neat): 2961, 1719, 1550, 1424, 1286, 1138, 949, $778 \mathrm{~cm}^{-1}$. The spectra data matched those reported in the literature. ${ }^{23}$<smiles>CC(=O)c1c(Br)c(Br)cc(Br)c1Br</smiles>

Methyl 2,3,5,6-tetrabromobenzoate (2g). According to the procedure analogous to that described for 2a, except that the carboxylation was conducted with $\mathrm{LiO}-t$-Bu $(49.3 \mathrm{mg}, 0.616 \mathrm{mmol}), \mathrm{CsF}(92.1 \mathrm{mg}$, $0.606 \mathrm{mmol})$, and 18 -crown-6 (162.4 mg, $0.614 \mathrm{mmol})$ at $80{ }^{\circ} \mathrm{C}$ in a heat block, $2 \mathrm{~g}(112.6 \mathrm{mg}, 0.249$ mmol, 82\%) was obtained from $1 \mathrm{~g}(119.2 \mathrm{mg}, 0.303 \mathrm{mmol})$ as a white solid: Mp 100-101 ${ }^{\circ} \mathrm{C}$ (hexane). ${ }^{1} \mathrm{H}$ NMR (600 MHz, $\left.\mathrm{CDCl}_{3} / \mathrm{TMS}\right) \delta 7.94$ (s, 1H), 3.99 (s, 3H). ${ }^{13} \mathrm{C} \mathrm{NMR}\left(150 \mathrm{MHz}, \mathrm{CDCl}_{3} / \mathrm{TMS}\right)$ $\delta$ 165.4, 140.8, 137.3, 125.2, 121.3, 53.5. LRMS (EI) $m / z: 448\left(\mathrm{M}^{+}\right)$. HRMS (EI-TOF) $m / z:\left(\mathrm{M}^{+}\right)$ Calcd. for $\mathrm{C}_{8} \mathrm{H}_{4} \mathrm{Br}_{4} \mathrm{O}_{2}$ : 447.6945, found: 447.6962. IR (neat): 3090, 2963, 1750, 1739, 1539, 1434, $1383 \mathrm{~cm}^{-1}$.<smiles>COC(=O)c1c(Br)cc(Cl)cc1Br</smiles>

Methyl 2,4-dibromo-6-chlorobenzoate (2h-1), Methyl 2,6-dibromo-4-chlorobenzoate (2h-2). According to the procedure analogous to that described for $\mathbf{2 b}$, except that the carboxylation was conducted at $100{ }^{\circ} \mathrm{C}$ in a heat block, mixture of $\mathbf{2 h - 1}$ and $\mathbf{2 h - 2}(80.6 \mathrm{mg}, 0.245 \mathrm{mmol}, 81 \%)$ was obtained from $\mathbf{1 h}(82.0 \mathrm{mg}, 0.303 \mathrm{mmol})$ as a white solid (2h-1:2h-2 = 69:31). ${ }^{1} \mathrm{H}$ NMR $(600 \mathrm{MHz}$, $\left.\mathrm{CDCl}_{3}\right)$ 2h-1: $\delta 7.66(\mathrm{~d}, 1 \mathrm{H}, J=1.7 \mathrm{~Hz}), 7.54(\mathrm{~d}, 1 \mathrm{H}, J=1.4 \mathrm{~Hz}), 3.97(\mathrm{~s}, 3 \mathrm{H}) .2 \mathrm{~h}-2: \delta 7.55(\mathrm{~s}, 2 \mathrm{H})$, 
3.973 (s, 3H). ${ }^{13} \mathrm{C}$ NMR $\left(150 \mathrm{MHz}, \mathrm{CDCl}_{3}\right)$ 2h-1: $\delta 165.2,133.6,131.4,131.2,123.8,120.4,120.1$, 53.12. 2h-2: $\delta 165.9,136.4,136.2,134.6,132.6$, 53.15. LRMS (EI) $\mathrm{m} / z$ : $326\left(\mathrm{M}^{+}\right)$. HRMS (EI-TOF) $m / z$ : $\left(\mathrm{M}^{+}\right)$Calcd. for $\mathrm{C}_{8} \mathrm{H}_{5} \mathrm{Br}_{2} \mathrm{ClO}_{2}: 325.8345$, found: 325.8340 . The spectra data matched those reported in the literature. ${ }^{24}$<smiles>CC(=O)c1c(F)cc(-c2ccccc2)cc1F</smiles>

Methyl 3,5-difluoro-[1,1'-biphenyl]-4-carboxylate (2i). According to the procedure analogous to that described for $\mathbf{2 b}$, except that the carboxylation was conducted with LiO-t-Bu (120.0 mg, 1.50 mmol) and CsF (227.2 mg, $1.50 \mathrm{mmol})$ at $150{ }^{\circ} \mathrm{C}$ in a heat block, $2 \mathbf{i}(57.3 \mathrm{mg}, 0.231 \mathrm{mmol}, 77 \%)$ was obtained from $1 \mathbf{i}(56.7 \mathrm{mg}, 0.298 \mathrm{mmol})$ as a white solid: $\mathrm{Mp} 57-59{ }^{\circ} \mathrm{C}$ (hexane/AcOEt). ${ }^{1} \mathrm{H}$ NMR $\left(600 \mathrm{MHz}\right.$, DMSO- $\left.d_{6}\right) \delta 7.81(\mathrm{~d}, 2 \mathrm{H}, J=7.2 \mathrm{~Hz}), 7.63(\mathrm{~d}, 2 \mathrm{H}, J=10.0 \mathrm{~Hz}), 7.51(\mathrm{t}, 2 \mathrm{H}, J=7.6 \mathrm{~Hz})$, $7.47(\mathrm{t}, 1 \mathrm{H}, J=7.4 \mathrm{~Hz}), 3.90(\mathrm{~s}, 3 \mathrm{H}) .{ }^{13} \mathrm{C}$ NMR $\left(150 \mathrm{MHz}\right.$, DMSO- $\left.d_{6}\right) \delta 161.2,160.2\left(\mathrm{dd},{ }^{1} J_{\mathrm{CF}}=253.5\right.$ $\left.\mathrm{Hz},{ }^{3} J_{\mathrm{CF}}=7.2 \mathrm{~Hz}\right), 145.9\left(\mathrm{t},{ }^{3} J_{\mathrm{CF}}=10.0 \mathrm{~Hz}\right), 136.5,129.5,129.2,127.1,110.4\left(\mathrm{~d},{ }^{2} J_{\mathrm{CF}}=22.9 \mathrm{~Hz}\right)$, $108.5\left(\mathrm{t},{ }^{2} J_{\mathrm{CF}}=18.6 \mathrm{~Hz}\right), 53.0 .{ }^{19} \mathrm{~F}$ NMR $\left(564.5 \mathrm{MHz}\right.$, DMSO- $d_{6} / 4$-fluorotoluene) $\delta$-110.2. LRMS (EI) $m / z: 248\left(\mathrm{M}^{+}\right)$. HRMS (EI-TOF) $m / z:\left(\mathrm{M}^{+}\right)$Calcd. for $\mathrm{C}_{14} \mathrm{H}_{10} \mathrm{~F}_{2} \mathrm{O}_{2}: 248.0649$, found: 248.0627. IR (neat): $3070,2960,1718,1623,1560,1410 \mathrm{~cm}^{-1}$.<smiles>CC(=O)c1c(Cl)cc(-c2ccccc2)cc1Cl</smiles>

Methyl 3,5-dichloro-[1,1'-biphenyl]-4-carboxylate (2j). According to the procedure analogous to that described for $\mathbf{2 b}$, except that the carboxylation was conducted at $150{ }^{\circ} \mathrm{C}$ in a heat block and that the crude material was purified by column chromatography on silica gel (hexane: $\mathrm{AcOEt}=20: 1$ ), $\mathbf{2} \mathbf{j}$ $(64.7 \mathrm{mg}, 0.230 \mathrm{mmol}, 74 \%)$ was obtained from $\mathbf{1 j}(69.0 \mathrm{mg}, 0.309 \mathrm{mmol})$ as a white solid: $\mathrm{Mp}$ 60-61 ${ }^{\circ} \mathrm{C}$ (hexane/AcOEt). ${ }^{1} \mathrm{H}$ NMR (600 MHz, CDCl $/$ TMS) $\delta 7.55-7.51$ (m, 4H), 7.47 (t, $2 \mathrm{H}, J=$ $7.5 \mathrm{~Hz}), 7.42(\mathrm{t}, 1 \mathrm{H}, J=7.6 \mathrm{~Hz}), 4.00(\mathrm{~s}, 3 \mathrm{H}) .{ }^{13} \mathrm{C}$ NMR $\left(150 \mathrm{MHz}, \mathrm{CDCl}_{3} / \mathrm{TMS}\right) \delta 165.3,144.6,137.7$, 132.2, 131.8, 129.1, 128.9, 127.1, 126.5, 53.0. LRMS (EI) $m / z: 280\left(\mathrm{M}^{+}\right)$. HRMS (EI-TOF) $\mathrm{m} / z:\left(\mathrm{M}^{+}\right)$ Calcd. for $\mathrm{C}_{14} \mathrm{H}_{10} \mathrm{Cl}_{2} \mathrm{O}_{2}: 280.0058$, found: 280.0049. IR (neat): 2955, 1736, 1726, 1596, 1540, 1375, $1276 \mathrm{~cm}^{-1}$.<smiles>CC(=O)c1c(Br)cc(-c2ccccc2)cc1Br</smiles> 
Methyl 3,5-dibromo-[1,1'-biphenyl]-4-carboxylate (2k). According to the procedure analogous to that described for $\mathbf{2 b}$, except that the carboxylation was conducted with LiO- $t$-Am (141.2 $\mathrm{mg}, 1.50$ $\mathrm{mmol})$ instead of LiO-t-Bu and $\mathrm{CsF}(228.7 \mathrm{mg}, 1.51 \mathrm{mmol})$ at $130{ }^{\circ} \mathrm{C}$ in a heat block, $2 \mathbf{k}(72.4 \mathrm{mg}$, $0.196 \mathrm{mmol}, 66 \%)$ was obtained from $1 \mathbf{k}(92.8 \mathrm{mg}, 0.297 \mathrm{mmol})$ as a white solid: $\mathrm{Mp} 66-67{ }^{\circ} \mathrm{C}$ (hexane). ${ }^{1} \mathrm{H}$ NMR (400 MHz, $\left.\mathrm{CDCl}_{3} / \mathrm{TMS}\right) \delta 7.74(\mathrm{~s}, 2 \mathrm{H}), 7.56-7.50(\mathrm{~m}, 2 \mathrm{H}), 7.50-7.44(\mathrm{~m}, 2 \mathrm{H})$, 7.44-7.40 (m, 1H), $4.00(\mathrm{~s}, 3 \mathrm{H}) .{ }^{13} \mathrm{C} \mathrm{NMR}\left(150 \mathrm{MHz}, \mathrm{CDCl}_{3} / \mathrm{TMS}\right) \delta 166.6,145.0,137.5,135.9$, 130.0, 129.1, 128.8, 127.1, 120.0, 53.1. LRMS (EI) m/z: $368\left(\mathrm{M}^{+}\right)$. HRMS (EI-TOF) m/z: $\left(\mathrm{M}^{+}\right.$) Calcd. for $\mathrm{C}_{14} \mathrm{H}_{10} \mathrm{Br}_{2} \mathrm{O}_{2}$ : 367.9058, found: 367.9048. IR (neat): 2951, 1725, 1591, 1531, 1272, 1137, $737 \mathrm{~cm}^{-}$ 1 .<smiles>CC(=O)c1c(F)cc(-c2ccc(C)cc2)cc1F</smiles>

Methyl 3,5-difluoro-4'-methyl-[1,1'-biphenyl]-4-carboxylate (2I). According to the procedure analogous to that described for $\mathbf{2} \mathbf{b}$, except that the carboxylation was conducted at $100{ }^{\circ} \mathrm{C}$ in a heat block, 21 (50.3 mg, $0.192 \mathrm{mmol}, 64 \%)$ was obtained from 11 (61.6 mg, $0.301 \mathrm{mmol})$ as a white solid: Mp 55-57 ${ }^{\circ} \mathrm{C}$ (hexane/AcOEt). ${ }^{1} \mathrm{H}$ NMR (600 MHz, $\left.\mathrm{CDCl}_{3} / \mathrm{TMS}\right) \delta 7.44$ (d, 2H, J=7.6 Hz), 7.26 (d, $2 \mathrm{H}, J=7.5 \mathrm{~Hz}), 7.15(\mathrm{~d}, 2 \mathrm{H}, J=9.6 \mathrm{~Hz}), 3.96(\mathrm{~s}, 3 \mathrm{H}), 2.39$ (s, 3H). ${ }^{13} \mathrm{C} \mathrm{NMR}\left(150 \mathrm{MHz}, \mathrm{CDCl}_{3} / \mathrm{TMS}\right)$ $\delta 162.1,161.1\left(\mathrm{dd},{ }^{1} J_{\mathrm{CF}}=254.2 \mathrm{~Hz},{ }^{3} J_{\mathrm{CF}}=7.2 \mathrm{~Hz}\right), 146.6\left(\mathrm{t},{ }^{3} J_{\mathrm{CF}}=11.5 \mathrm{~Hz}\right), 139.4,134.9,129.9$, $126.8,110.2\left(\mathrm{dd},{ }^{2} J_{\mathrm{CF}}=22.3 \mathrm{~Hz},{ }^{4} J_{\mathrm{CF}}=3.6 \mathrm{~Hz}\right), 108.7\left(\mathrm{t},{ }^{2} J_{\mathrm{CF}}=18.0 \mathrm{~Hz}\right), 52.7,21.2 .{ }^{19} \mathrm{~F} \mathrm{NMR}(564.5$ $\mathrm{MHz}, \mathrm{CDCl}_{3} / 4$-fluorotoluene) $\delta$-109.1. LRMS (EI) $\mathrm{m} / z: 262\left(\mathrm{M}^{+}\right)$. HRMS (EI-TOF) $\mathrm{m} / \mathrm{z}:\left(\mathrm{M}^{+}\right.$) Calcd. for $\mathrm{C}_{15} \mathrm{H}_{12} \mathrm{~F}_{2} \mathrm{O}_{2}$ : 262.0805, found: 262.0832. IR (neat): 2957, 2926, 1723, 1634, 1622, $1559 \mathrm{~cm}^{-1}$.<smiles>COc1ccc(-c2cc(F)c(C(C)=O)c(F)c2)cc1</smiles>

Methyl 3,5-difluoro-4'-methoxy-[1,1'-biphenyl]-4-carboxylate (2m). According to the procedure analogous to that described for $\mathbf{2 b}$, except that the carboxylation was conducted with LiO- $t$-Am (86.0 $\mathrm{mg}, 0.914 \mathrm{mmol}$ ) instead of $\mathrm{LiO}-t-\mathrm{Bu}$ at $150{ }^{\circ} \mathrm{C}$ in a heat block and that the crude material was purified by column chromatography on silica gel (hexane:AcOEt $=20: 1), 2 \mathrm{~m}(63.6 \mathrm{mg}, 0.229 \mathrm{mmol}, 76 \%)$ was obtained from $1 \mathrm{~m}(66.1 \mathrm{mg}, 0.300 \mathrm{mmol})$ as a white solid: $\mathrm{Mp} 81-82{ }^{\circ} \mathrm{C}$ (hexane/AcOEt). ${ }^{1} \mathrm{H}$ NMR (600 MHz, DMSO- $\left.d_{6}\right) \delta 7.78(\mathrm{~d}, 2 \mathrm{H}, J=8.9 \mathrm{~Hz}), 7.57(\mathrm{~d}, 2 \mathrm{H}, J=10.3 \mathrm{~Hz}), 7.04(\mathrm{~d}, 2 \mathrm{H}, J=8.2$ 
$\mathrm{Hz}), 3.88(\mathrm{~s}, 3 \mathrm{H}), 3.81(\mathrm{~s}, 3 \mathrm{H}) .{ }^{13} \mathrm{C} \mathrm{NMR}\left(150 \mathrm{MHz}, \mathrm{DMSO}-d_{6}\right) \delta 161.3,160.5,160.4\left(\mathrm{dd},{ }^{1} J_{\mathrm{CF}}=\right.$ $\left.252.1 \mathrm{~Hz},{ }^{3} J_{\mathrm{CF}}=7.2 \mathrm{~Hz}\right), 145.7\left(\mathrm{t},{ }^{3} J_{\mathrm{CF}}=10.7 \mathrm{~Hz}\right), 128.7,128.5,114.6,109.6\left(\mathrm{~d},{ }^{2} J_{\mathrm{CF}}=22.9 \mathrm{~Hz}\right)$,

$107.6\left(\mathrm{t},{ }^{2} J_{\mathrm{CF}}=19.3 \mathrm{~Hz}\right), 55.4,52.9 .{ }^{19} \mathrm{~F}$ NMR (564.5 MHz, DMSO- $d_{6} / 4$-fluorotoluene) $\delta$-110.2. LRMS (EI) $m / z$ : $278\left(\mathrm{M}^{+}\right)$. HRMS (EI-TOF) $m / z$ : $\left(\mathrm{M}^{+}\right)$Calcd. for $\mathrm{C}_{15} \mathrm{H}_{12} \mathrm{~F}_{2} \mathrm{O}_{3}: 278.0755$, found: 278.0728. IR (neat): 2960, 2845, 1723, 1602, 1558, 1527, $1437 \mathrm{~cm}^{-1}$.<smiles>CC(=O)c1c(F)cc(-c2ccc(S(C)(=O)=O)cc2)cc1F</smiles>

Methyl 3,5-difluoro-4'-(methylthio)-[1,1'-biphenyl]-4-carboxylate (2n). According to the procedure analogous to that described for $\mathbf{2} \mathbf{b}$, except that the carboxylation was conducted with $\mathrm{LiO}$ $t$-Bu (122.2 mg, $1.52 \mathrm{mmol})$ and $\mathrm{CsF}(232.4 \mathrm{mg}, 1.53 \mathrm{mmol})$ at $150{ }^{\circ} \mathrm{C}$ in a heat block and that the crude material was purified by column chromatography on silica gel (hexane:AcOEt = 20:1), 2n (69.2 $\mathrm{mg}, 0.235 \mathrm{mmol}, 83 \%)$ was obtained from 1 n $(67.2 \mathrm{mg}, 0.284 \mathrm{mmol})$ as a white solid: $\mathrm{Mp} 76-77{ }^{\circ} \mathrm{C}$ (hexane/AcOEt). ${ }^{1} \mathrm{H}$ NMR (400 MHz, $\left.\mathrm{CDCl}_{3} / \mathrm{TMS}\right) \delta 7.49(\mathrm{~d}, 2 \mathrm{H}, J=8.3 \mathrm{~Hz}), 7.32(\mathrm{~d}, 2 \mathrm{H}, J=8.3$ $\mathrm{Hz}), 7.16(\mathrm{~d}, 2 \mathrm{H}, J=9.3 \mathrm{~Hz}), 3.96(\mathrm{~s}, 3 \mathrm{H}), 2.53(\mathrm{~s}, 3 \mathrm{H}) .{ }^{13} \mathrm{C} \mathrm{NMR}\left(150 \mathrm{MHz}, \mathrm{CDCl}_{3} / \mathrm{TMS}\right) \delta 162.1$, $161.2\left(\mathrm{dd},{ }^{1} J_{\mathrm{CF}}=247.7 \mathrm{~Hz},{ }^{3} J_{\mathrm{CF}}=6.5 \mathrm{~Hz}\right), 145.9\left(\mathrm{t},{ }^{3} J_{\mathrm{CF}}=10.8 \mathrm{~Hz}\right), 140.6,134.2,127.2,126.6,110.0$ $\left(\mathrm{dd},{ }^{2} J_{\mathrm{CF}}=21.5 \mathrm{~Hz},{ }^{4} J_{\mathrm{CF}}=4.3 \mathrm{~Hz}\right), 108.8\left(\mathrm{t},{ }^{2} J_{\mathrm{CF}}=16.5 \mathrm{~Hz}\right), 52.7,15.4 .{ }^{19} \mathrm{~F}$ NMR $(564.5 \mathrm{MHz}$, $\mathrm{CDCl}_{3} / 4$-fluorotoluene) $\delta$-108.8. LRMS (EI) $\mathrm{m} / z: 294\left(\mathrm{M}^{+}\right)$. HRMS (EI-TOF) $\mathrm{m} / z:\left(\mathrm{M}^{+}\right)$Calcd. for $\mathrm{C}_{15} \mathrm{H}_{12} \mathrm{~F}_{2} \mathrm{O}_{2} \mathrm{~S}: 294.0526$, found: 294.0518. IR (neat): 2951, 2836, 1725, 1634, 1550, 1425, $1391 \mathrm{~cm}^{-1}$.<smiles>CC(=O)c1c(F)cc(-c2ccc(Cl)cc2)cc1F</smiles>

Methyl 4'-chloro-3,5-difluoro-[1,1'-biphenyl]-4-carboxylate (2o). According to the procedure analogous to that described for $\mathbf{2 b}$, except that the carboxylation was conducted with $\mathrm{LiO}-t-\mathrm{Bu}(120.9$ $\mathrm{mg}, 1.51 \mathrm{mmol})$ and $\mathrm{CsF}(228.0 \mathrm{mg}, 1.50 \mathrm{mmol})$ at $150{ }^{\circ} \mathrm{C}$ in a heat block, $20(65.5 \mathrm{mg}, 0.231 \mathrm{mmol}$, $72 \%)$ was obtained from $10(72.6 \mathrm{mg}, 0.323 \mathrm{mmol})$ as a white solid: Mp $110-11{ }^{\circ} \mathrm{C}$ (hexane/AcOEt). ${ }^{1} \mathrm{H}$ NMR (600 MHz, $\left.\mathrm{CDCl}_{3} / \mathrm{TMS}\right) \delta 7.49(\mathrm{~d}, 2 \mathrm{H}, J=8.2 \mathrm{~Hz}), 7.45(\mathrm{~d}, 2 \mathrm{H}, J=8.3 \mathrm{~Hz}), 7.15(\mathrm{~d}, 2 \mathrm{H}, J$ $=8.9 \mathrm{~Hz}), 3.97(\mathrm{~s}, 3 \mathrm{H}) \cdot{ }^{13} \mathrm{C} \mathrm{NMR}\left(150 \mathrm{MHz}, \mathrm{CDCl}_{3} / \mathrm{TMS}\right) \delta 161.9,161.2\left(\mathrm{dd},{ }^{1} J_{\mathrm{CF}}=254.9 \mathrm{~Hz},{ }^{3} J_{\mathrm{CF}}\right.$ $=6.5 \mathrm{~Hz}), 145.3\left(\mathrm{t},{ }^{3} J_{\mathrm{CF}}=10.1 \mathrm{~Hz}\right), 136.2,135.5,129.4,128.3,110.4\left(\mathrm{dd},{ }^{2} J_{\mathrm{CF}}=23.0 \mathrm{~Hz},{ }^{4} J_{\mathrm{CF}}=4.3\right.$ $\mathrm{Hz}), 109.4\left(\mathrm{t},{ }^{2} J_{\mathrm{CF}}=17.2 \mathrm{~Hz}\right), 52.8 .{ }^{19} \mathrm{~F} \mathrm{NMR}\left(564.5 \mathrm{MHz}, \mathrm{CDCl}_{3} / 4\right.$-fluorotoluene) $\delta$-108.5. LRMS 
(EI) $m / z: 282\left(\mathrm{M}^{+}\right)$. HRMS (EI-TOF) $m / z:\left(\mathrm{M}^{+}\right)$Calcd. for $\mathrm{C}_{14} \mathrm{H}_{9} \mathrm{ClF}_{2} \mathrm{O}_{2}: 282.0259$, found: 282.0249 . IR (neat): 3089, 2971, 1728, 1630, 1559, $1434 \mathrm{~cm}^{-1}$.<smiles>CC(=O)c1c(F)cc(-c2ccc(Br)cc2)cc1F</smiles>

Methyl 4'-bromo-3,5-difluoro-[1,1'-biphenyl]-4-carboxylate (2p). According to the procedure analogous to that described for $\mathbf{2 b}$, except that the carboxylation was conducted with LiO-t-Am (85.5 $\mathrm{mg}, 0.908 \mathrm{mmol})$ instead of LiO-t-Bu at $100{ }^{\circ} \mathrm{C}$ in a heat block, $2 \mathbf{p}(71.7 \mathrm{mg}, 0.219 \mathrm{mmol}, 73 \%)$ was obtained from $1 \mathbf{p}(81.0 \mathrm{mg}, 0.301 \mathrm{mmol})$ as a white solid: $\mathrm{Mp} 108-110{ }^{\circ} \mathrm{C}$ (hexane/AcOEt). ${ }^{1} \mathrm{H} \mathrm{NMR}$ $\left(600 \mathrm{MHz}, \mathrm{CDCl}_{3} / \mathrm{TMS}\right) \delta 7.60(\mathrm{~d}, 2 \mathrm{H}, J=8.2 \mathrm{~Hz}), 7.42(\mathrm{~d}, 2 \mathrm{H}, J=8.2 \mathrm{~Hz}), 7.14(\mathrm{~d}, 2 \mathrm{H}, J=9.6 \mathrm{~Hz})$, $3.97(\mathrm{~s}, 3 \mathrm{H}) .{ }^{13} \mathrm{C} \mathrm{NMR}\left(150 \mathrm{MHz}, \mathrm{CDCl}_{3} / \mathrm{TMS}\right) \delta 161.8,161.1\left(\mathrm{dd},{ }^{1} J_{\mathrm{CF}}=255.5 \mathrm{~Hz},{ }^{3} J_{\mathrm{CF}}=5.7 \mathrm{~Hz}\right)$, $145.2\left(\mathrm{t},{ }^{3} J_{\mathrm{CF}}=10.1 \mathrm{~Hz}\right), 136.6,132.3,128.5,123.7,110.3\left(\mathrm{~d},{ }^{2} J_{\mathrm{CF}}=24.4 \mathrm{~Hz}\right), 109.4\left(\mathrm{t},{ }^{2} J_{\mathrm{CF}}=17.2\right.$ $\mathrm{Hz}$ ), 52.8. ${ }^{19} \mathrm{~F}$ NMR (564.5 MHz, $\mathrm{CDCl}_{3} / 4$-fluorotoluene) $\delta$-108.4. LRMS (EI) $m / z: 326\left(\mathrm{M}^{+}\right.$). HRMS (EI-TOF) $m / z:\left(\mathrm{M}^{+}\right)$Calcd. for $\mathrm{C}_{14} \mathrm{H}_{9} \mathrm{BrF}_{2} \mathrm{O}_{2}: 325.9754$, found: 325.9773. IR (neat): 3087, 2966, 1725, $1627,1559,1432 \mathrm{~cm}^{-1}$.<smiles>CC(=O)c1c(F)cc(-c2ccc(C#N)cc2)cc1F</smiles>

Methyl 4'-cyano-3,5-difluoro-[1,1'-biphenyl]-4-carboxylate (2q). According to the procedure analogous to that described for $\mathbf{2 b}$, except that the carboxylation was conducted at $100{ }^{\circ} \mathrm{C}$ in a heat block and that the crude material was purified by column chromatography on silica gel (hexane:AcOEt $=10: 1)$, 2q (59.2 mg, $0.217 \mathrm{mmol}, 74 \%)$ was obtained from 1q (62.9 $\mathrm{mg}, 0.292 \mathrm{mmol})$ as a white solid: Mp 201-202 ${ }^{\circ} \mathrm{C}$ (hexane/AcOEt). ${ }^{1} \mathrm{H} \mathrm{NMR}\left(400 \mathrm{MHz}, \mathrm{CDCl}_{3} / \mathrm{TMS}\right) \delta 7.78(\mathrm{~d}, 2 \mathrm{H}, J=8.3 \mathrm{~Hz}$ ), $7.66(\mathrm{~d}, 2 \mathrm{H}, J=8.3 \mathrm{~Hz}), 7.19$ (d, 2H, $J=8.8 \mathrm{~Hz}), 3.98(\mathrm{~s}, 3 \mathrm{H}) .{ }^{13} \mathrm{C} \mathrm{NMR}\left(150 \mathrm{MHz}, \mathrm{CDCl}_{3} / \mathrm{TMS}\right) \delta$ $161.5,161.1\left(\mathrm{dd},{ }^{1} J_{\mathrm{CF}}=257.1 \mathrm{~Hz},{ }^{3} J_{\mathrm{CF}}=6.5 \mathrm{~Hz}\right), 144.2\left(\mathrm{t},{ }^{3} J_{\mathrm{CF}}=10.0 \mathrm{~Hz}\right), 142.0,132.9,127.7,118.2$, $112.9,110.8\left(\mathrm{dd},{ }^{2} J_{\mathrm{CF}}=22.9 \mathrm{~Hz},{ }^{4} J_{\mathrm{CF}}=4.3 \mathrm{~Hz}\right), 110.6\left(\mathrm{t},{ }^{2} J_{\mathrm{CF}}=17.9 \mathrm{~Hz}\right), 57.7 .{ }^{19} \mathrm{~F} \mathrm{NMR}(564.5 \mathrm{MHz}$, $\mathrm{CDCl}_{3} / 4$-fluorotoluene) $\delta$-107.8. LRMS (EI) $\mathrm{m} / z: 273\left(\mathrm{M}^{+}\right)$. HRMS (EI-TOF) $\mathrm{m} / \mathrm{z}:\left(\mathrm{M}^{+}\right)$Calcd. for $\mathrm{C}_{15} \mathrm{H}_{9} \mathrm{~F}_{2} \mathrm{NO}_{2}$ : 273.0601, found: 273.0607. IR (neat): 2955, 2228, 1729, 1623, 1558, $1432 \mathrm{~cm}^{-1}$. 
<smiles>CC(=O)c1c(F)cc(-c2ccc(C3OCCO3)cc2)cc1F</smiles>

Methyl 4'-(1,3-dioxolan-2-yl)-3,5-difluoro-[1,1'-biphenyl]-4-carboxylate (2r). According to the procedure analogous to that described for $\mathbf{2 b}$, except that the carboxylation was conducted with LiO$t$-Bu (121.7 mg, $1.52 \mathrm{mmol})$ and $\mathrm{CsF}(228.2 \mathrm{mg}, 1.50 \mathrm{mmol})$ at $150{ }^{\circ} \mathrm{C}$ in a heat block and that the crude material was purified by column chromatography on silica gel (hexane:AcOEt =10:1), 2r $(72.4$ $\mathrm{mg}, 0.226 \mathrm{mmol}, 74 \%)$ was obtained from $1 \mathbf{r}(80.3 \mathrm{mg}, 0.306 \mathrm{mmol})$ as a white solid: $\mathrm{Mp} 92-93{ }^{\circ} \mathrm{C}$ (hexane/AcOEt). ${ }^{1} \mathrm{H}$ NMR (600 MHz, DMSO- $\left.d_{6}\right) \delta 7.82(\mathrm{~d}, 2 \mathrm{H}, J=8.3 \mathrm{~Hz}), 7.64(\mathrm{~d}, 2 \mathrm{H}, J=10.1 \mathrm{~Hz})$, $7.54(\mathrm{~d}, 2 \mathrm{H}, J=8.3 \mathrm{~Hz}), 5.79$ (s, 1H), 4.09-4.03 (m, 2H), 3.99-3.94 (m, 2H), $3.90(\mathrm{~s}, 3 \mathrm{H}) .{ }^{13} \mathrm{C}$ NMR $\left(150 \mathrm{MHz}, \mathrm{DMSO}-d_{6}\right) \delta 161.1,160.1\left(\mathrm{dd},{ }^{1} J_{\mathrm{CF}}=252.8 \mathrm{~Hz},{ }^{3} J_{\mathrm{CF}}=6.5 \mathrm{~Hz}\right), 145.5\left(\mathrm{t},{ }^{3} J_{\mathrm{CF}}=11.5 \mathrm{~Hz}\right)$, 139.3, 137.2, 127.2, 127.0, $110.5\left(\mathrm{~d},{ }^{2} J_{\mathrm{CF}}=22.9 \mathrm{~Hz}\right), 108.6\left(\mathrm{t},{ }^{2} J_{\mathrm{CF}}=20.1 \mathrm{~Hz}\right), 102.3,64.9,52.9 .{ }^{19} \mathrm{~F}$ NMR (564.5 MHz, DMSO-d $/ 4$-fluorotoluene) $\delta$-110.0. LRMS (EI) $m / z: 320\left(\mathrm{M}^{+}\right.$). HRMS (EI-TOF) $m / z:\left(\mathrm{M}^{+}\right)$Calcd. for $\mathrm{C}_{17} \mathrm{H}_{14} \mathrm{~F}_{2} \mathrm{O}_{4}: 320.0860$, found: 320.0857. IR (neat): 2859, 2917, 2874, 1734, 1627, $1559,1436 \mathrm{~cm}^{-1}$.<smiles>CC(=O)c1c(F)cc(-c2ccc(C=O)cc2)cc1F</smiles>

Methyl 3,5-difluoro-4'-formyl-[1,1'-biphenyl]-4-carboxylate (2s). According to the procedure analogous to that described for $\mathbf{2 a}$, except that the carboxylation was conducted in DMF $(1.0 \mathrm{~mL})$ at $90{ }^{\circ} \mathrm{C}$ in a heat block and that the crude material was purified by column chromatography on silica gel (hexane:AcOEt $=40: 1)$ and $\mathrm{GPC}\left(\mathrm{CHCl}_{3}\right), \mathbf{2 s}(41.5 \mathrm{mg}, 0.150 \mathrm{mmol}, 50 \%)$ was obtained from $1 \mathrm{~s}(66.2$ mg, $0.303 \mathrm{mmol}$ ) as a white solid: $\mathrm{Mp} 122-123{ }^{\circ} \mathrm{C}$ (hexane/AcOEt). ${ }^{1} \mathrm{H}$ NMR $\left(600 \mathrm{MHz}, \mathrm{CDCl}_{3} / \mathrm{TMS}\right)$ $\delta 10.09(\mathrm{~s}, 1 \mathrm{H}), 8.00$ (d, 2H, $J=8.3 \mathrm{~Hz}), 7.73$ (d, 2H, $J=8.2 \mathrm{~Hz}$ ), 7.24 (d, 2H, $J=9.2 \mathrm{~Hz}$ ), 3.98 (s, $3 \mathrm{H}) .{ }^{13} \mathrm{C} \mathrm{NMR}\left(150 \mathrm{MHz}, \mathrm{CDCl}_{3} / \mathrm{TMS}\right) \delta 191.5,161.7,161.1\left(\mathrm{dd},{ }^{1} J_{\mathrm{CF}}=257.9 \mathrm{~Hz},{ }^{3} J_{\mathrm{CF}}=7.2 \mathrm{~Hz}\right)$, $144.9\left(\mathrm{t},{ }^{3} J_{\mathrm{CF}}=10.0 \mathrm{~Hz}\right), 143.4,136.5,130.5,127.7,110.9\left(\mathrm{dd},{ }^{2} J_{\mathrm{CF}}=22.9 \mathrm{~Hz},{ }^{4} J_{\mathrm{CF}}=4.3 \mathrm{~Hz}\right), 110.3$ $\left(\mathrm{t},{ }^{2} J_{\mathrm{CF}}=17.9 \mathrm{~Hz}\right), 52.9 .{ }^{19} \mathrm{~F}$ NMR $\left(564.5 \mathrm{MHz}, \mathrm{CDCl}_{3} / 4\right.$-fluorotoluene) $\delta$-108.1. LRMS (EI) $\mathrm{m} / z$ : $276\left(\mathrm{M}^{+}\right)$. HRMS (EI-TOF) $\mathrm{m} / z$ : $\left(\mathrm{M}^{+}\right)$Calcd. for $\mathrm{C}_{15} \mathrm{H}_{10} \mathrm{~F}_{2} \mathrm{O}_{3}: 276.0598$, found: 276.0627. IR (neat): $2963,2860,1719,1689,1605,1429 \mathrm{~cm}^{-1}$. 
<smiles>CCCCC(=O)c1cc(F)c(C(=O)OC)c(F)c1</smiles>

4-(tert-Butyl) 1-methyl 2,6-difluoroterephthalate (2t). According to the procedure analogous to that described for $\mathbf{2 b}$, except that the carboxylation was conducted at $100{ }^{\circ} \mathrm{C}$ in a heat block and that the crude material was purified by preparative thin-layer chromatography of silica gel (hexane:AcOEt $=$ 20:1), $2 \mathbf{t}$ (60.8 $\mathrm{mg}, 0.223 \mathrm{mmol}, 71 \%$ ) was obtained from $\mathbf{1 t}$ (67.5 mg, $0.315 \mathrm{mmol})$ as a white solid: Mp 57-58 ${ }^{\circ} \mathrm{C}$ (hexane/AcOEt). ${ }^{1} \mathrm{H}$ NMR (400 MHz, $\left.\mathrm{CDCl}_{3} / \mathrm{TMS}\right) \delta 7.54$ (d, 2H, $J=8.3 \mathrm{~Hz}$ ), 3.97 (s, $3 \mathrm{H}), 1.60(\mathrm{~s}, 9 \mathrm{H}) .{ }^{13} \mathrm{C} \mathrm{NMR}\left(150 \mathrm{MHz}, \mathrm{CDCl}_{3} / \mathrm{TMS}\right) \delta 162.6\left(\mathrm{~d},{ }^{3} J_{\mathrm{CF}}=2.9 \mathrm{~Hz}\right), 161.5,160.2\left(\mathrm{dd},{ }^{1} J_{\mathrm{CF}}\right.$ $\left.=255.6 \mathrm{~Hz},{ }^{3} J_{\mathrm{CF}}=5.7 \mathrm{~Hz}\right), 136.7\left(\mathrm{t},{ }^{3} J_{\mathrm{CF}}=8.6 \mathrm{~Hz}\right), 114.2\left(\mathrm{t},{ }^{2} J_{\mathrm{CF}}=18.7 \mathrm{~Hz}\right), 112.9\left(\mathrm{dd},{ }^{2} J_{\mathrm{CF}}=22.3\right.$ $\left.\mathrm{Hz},{ }^{4} J_{\mathrm{CF}}=3.6 \mathrm{~Hz}\right), 82.9,53.0,28.0 .{ }^{19} \mathrm{~F} \mathrm{NMR}\left(564.5 \mathrm{MHz}, \mathrm{CDCl}_{3} / 4\right.$-fluorotoluene) $\delta$-108.8. LRMS (EI) $m / z: 272\left(\mathrm{M}^{+}\right)$. HRMS (EI-TOF) $m / z:\left(\mathrm{M}^{+}\right)$Calcd. for $\mathrm{C}_{13} \mathrm{H}_{14} \mathrm{~F}_{2} \mathrm{O}_{4}: 272.0860$, found: 272.0890. IR (neat): $3081,2977,1718,1635,1570,1427,1337 \mathrm{~cm}^{-1}$.<smiles>COC(=O)c1cc(Cl)c(C(=O)OCC(C)(C)C)c(Cl)c1</smiles>

4-(tert-Butyl) 1-methyl 2,6-dichloroterephthalate (2u). According to the procedure analogous to that described for $\mathbf{2 b}$, except that the carboxylation was conducted at $100{ }^{\circ} \mathrm{C}$ in a heat block and that the crude material was purified by preparative thin-layer chromatography of silica gel (hexane:AcOEt $=$ 20:1), 2u (60.9 mg, $0.199 \mathrm{mmol}, 67 \%)$ was obtained from $1 \mathbf{u}(73.8 \mathrm{mg}, 0.299 \mathrm{mmol})$ as a white solid: Mp 98-99 ${ }^{\circ} \mathrm{C}$ (hexane/AcOEt). ${ }^{1} \mathrm{H}$ NMR (400 MHz, $\left.\mathrm{CDCl}_{3} / \mathrm{TMS}\right) \delta 7.91$ (s, 2H), 3.99 (s, 3H), 1.60 (s, 9H). ${ }^{13} \mathrm{C}$ NMR (100 MHz, $\left.\mathrm{CDCl}_{3} / \mathrm{TMS}\right) \delta 164.7,162.6,136.6,134.8,132.0,128.7,82.9,53.1$, 28.0. LRMS (EI) $m / z: 304\left(\mathrm{M}^{+}\right)$. HRMS (EI-TOF) $\mathrm{m} / z$ : $\left(\mathrm{M}^{+}\right)$Calcd. for $\mathrm{C}_{13} \mathrm{H}_{14} \mathrm{Cl}_{2} \mathrm{O}_{4}: 304.0269$, found: 304.0260. IR (neat): 3079, 2983, 1744, 1714, 1559, 1370, 1272, $1126 \mathrm{~cm}^{-1}$.<smiles>COC(=O)c1cc(Br)c(C(=O)OC(C)(C)C)c(Br)c1</smiles>

4-(tert-Butyl) 1-methyl 2,6-dibromoterephthalate (2v). According to the procedure analogous to that described for $\mathbf{2 b}$, except that the carboxylation was conducted at $100{ }^{\circ} \mathrm{C}$ in a heat block and that the crude material was purified by preparative thin-layer chromatography of silica gel (hexane:AcOEt = 20:1), 2v (72.2 mg, $0.183 \mathrm{mmol}, 61 \%)$ was obtained from $\mathbf{1 v}(100.4 \mathrm{mg}, 0.299 \mathrm{mmol})$ as a white solid: Mp $116-117^{\circ} \mathrm{C}$ (hexane/AcOEt). ${ }^{1} \mathrm{H}$ NMR (600 MHz, $\left.\mathrm{CDCl}_{3} / \mathrm{TMS}\right) \delta 8.11$ (s, 2H), 3.99 (s, 3H), 1.59 
(s, 9H). ${ }^{13} \mathrm{C}$ NMR (150 MHz, $\left.\mathrm{CDCl}_{3} / \mathrm{TMS}\right) \delta 166.1,162.4,140.7,135.1,132.3,119.6,82.9,53.2$, 28.0. LRMS (EI) $m / z: 392\left(\mathrm{M}^{+}\right)$. HRMS (EI-TOF) $m / z$ : ( $\mathrm{M}^{+}$) Calcd. for $\mathrm{C}_{13} \mathrm{H}_{14} \mathrm{Br}_{2} \mathrm{O}_{4}: 391.9259$, found: 391.9284. IR (neat): 3072, 2891, 1740, 1719, 1542, 1370, 1270, $1257 \mathrm{~cm}^{-1}$.<smiles>CCCCCCNC(=O)c1cc(F)c(C(C)=O)c(F)c1</smiles>

Methyl 2,6-difluoro-4-(methyl(phenyl)carbamoyl)benzoate (2w). According to the procedure analogous to that described for $\mathbf{2 b}$, except that the crude material was purified by column chromatography on silica gel (hexane:AcOEt $=4: 1), 2 \mathbf{w}(70.1 \mathrm{mg}, 0.230 \mathrm{mmol}, 74 \%)$ was obtained from 1w (77.1 mg, $0.311 \mathrm{mmol}$ ) as a white solid: $\mathrm{Mp} 88-90{ }^{\circ} \mathrm{C}$ (hexane/AcOEt). ${ }^{1} \mathrm{H}$ NMR $(600 \mathrm{MHz}$, $\left.\mathrm{CDCl}_{3} / \mathrm{TMS}\right) \delta 7.29(\mathrm{t}, 2 \mathrm{H}, J=7.9 \mathrm{~Hz}), 7.23(\mathrm{t}, 1 \mathrm{H}, J=7.9 \mathrm{~Hz}), 7.04(\mathrm{~d}, 2 \mathrm{H}, J=7.5 \mathrm{~Hz}), 6.87(\mathrm{~d}, 2 \mathrm{H}$, $J=8.3 \mathrm{~Hz}), 3.90$ (s, $3 \mathrm{H}), 3.48(\mathrm{~s}, 3 \mathrm{H}) .{ }^{13} \mathrm{C}$ NMR $\left(150 \mathrm{MHz}, \mathrm{CDCl}_{3} / \mathrm{TMS}\right) \delta 166.9,161.4,159.9(\mathrm{dd}$, $\left.{ }^{1} J_{\mathrm{CF}}=257.1 \mathrm{~Hz},{ }^{3} J_{\mathrm{CF}}=6.5 \mathrm{~Hz}\right), 143.6,140.9\left(\mathrm{t},{ }^{3} J_{\mathrm{CF}}=8.6 \mathrm{~Hz}\right), 129.8,127.6,126.8,112.5\left(\mathrm{dd},{ }^{2} J_{\mathrm{CF}}=\right.$ $\left.22.9 \mathrm{~Hz},{ }^{4} J_{\mathrm{CF}}=4.3 \mathrm{~Hz}\right), 111.6\left(\mathrm{t},{ }^{2} J_{\mathrm{CF}}=17.9 \mathrm{~Hz}\right), 52.9,38.5 .{ }^{19} \mathrm{~F} \mathrm{NMR}\left(564.5 \mathrm{MHz}, \mathrm{CDCl}_{3} / 4-\right.$ fluorotoluene) $\delta$-108.8. LRMS (EI) $\mathrm{m} / z: 305\left(\mathrm{M}^{+}\right)$. HRMS (EI-TOF) $\mathrm{m} / z:\left(\mathrm{M}^{+}\right)$Calcd. for $\mathrm{C}_{16} \mathrm{H}_{13} \mathrm{~F}_{2} \mathrm{NO}_{3}$ : 305.0863, found: 305.0863. IR (neat): 2951, 1729, 1645, 1635, 1569, 1496, 1420, 1377 $\mathrm{cm}^{-1}$.<smiles>CCCCCCNC(=O)c1cc(Cl)c(C(C)=O)c(Cl)c1</smiles>

Methyl 2,6-dichloro-4-(methyl(phenyl)carbamoyl)benzoate (2x). According to the procedure analogous to that described for $\mathbf{2 b}$, except that the crude material was purified by column chromatography on silica gel (hexane:AcOEt $=4: 1), \mathbf{2 x}(61.4 \mathrm{mg}, 0.182 \mathrm{mmol}, 61 \%)$ was obtained from 1x (83.3 mg, $0.297 \mathrm{mmol}$ ) as a white solid: $\mathrm{Mp} 108-109{ }^{\circ} \mathrm{C}$ (hexane/AcOEt). ${ }^{1} \mathrm{H}$ NMR (600 MHz, DMSO- $\left.d_{6}\right) \delta 7.42(\mathrm{~s}, 2 \mathrm{H}), 7.33(\mathrm{t}, 2 \mathrm{H}, J=7.9 \mathrm{~Hz}), 7.26(\mathrm{~d}, 2 \mathrm{H}, J=6.8 \mathrm{~Hz}), 7.23(\mathrm{t}, 1 \mathrm{H}, J=7.2$ $\mathrm{Hz}), 3.87$ (s, 3H), $3.36(\mathrm{~s}, 3 \mathrm{H}) .{ }^{13} \mathrm{C} \mathrm{NMR}\left(150 \mathrm{MHz}, \mathrm{CDCl}_{3} / \mathrm{TMS}\right) \delta 166.7,164.7,143.6,138.9,134.1$, 131.5, 129.8, 128.1, 127.6, 126.8, 53.1, 38.6. LRMS (EI) $m / z: 337$ (M+). HRMS (EI-TOF) $m / z:\left(\mathrm{M}^{+}\right)$ Calcd. for $\mathrm{C}_{16} \mathrm{H}_{13} \mathrm{Cl}_{2} \mathrm{NO}_{3}: 337.0273$, found: 337.0265. IR (neat): 2957, 1740, 1652, 1595, 1546, 1497 $\mathrm{cm}^{-1}$. 
$\prod_{\mathrm{O}}^{\mathrm{Cr}}$

Methyl 2,6-dibromo-4-(methyl(phenyl)carbamoyl)benzoate (2y). According to the procedure analogous to that described for $\mathbf{2 b}$, except that the carboxylation was conducted in DMI (1.5 mL) at $100{ }^{\circ} \mathrm{C}$ in a heat block for $24 \mathrm{~h}$ and that the crude material was purified by column chromatography on silica gel (hexane:AcOEt = 5:1), $2 \mathbf{y}(77.9 \mathrm{mg}, 0.182 \mathrm{mmol}, 61 \%)$ was obtained from $1 \mathbf{y}(110.6 \mathrm{mg}$, $0.300 \mathrm{mmol}$ ) as a colorless oil. ${ }^{1} \mathrm{H}$ NMR $\left(400 \mathrm{MHz}, \mathrm{CDCl}_{3} / \mathrm{TMS}\right) \delta 7.43(\mathrm{~s}, 2 \mathrm{H}), 7.32(\mathrm{t}, 2 \mathrm{H}, J=7.8$ $\mathrm{Hz}), 7.30-7.20(\mathrm{~m}, 1 \mathrm{H}), 7.05$ (d, 2H, $J=7.3 \mathrm{~Hz}), 3.93$ (s, 3H), 3.48 (s, 3H). ${ }^{13} \mathrm{C}$ NMR (100 MHz, $\mathrm{CDCl}_{3}$ /TMS) $\delta$ 166.2, 165.8, 143.4, 139.0, 138.0, 131.5, 129.6, 127.4, 126.6, 118.9, 52.9, 38.3. LRMS (EI) $m / z$ : $425\left(\mathrm{M}^{+}\right)$. HRMS (EI-TOF) $m / z:\left(\mathrm{M}^{+}\right)$Calcd. for $\mathrm{C}_{16} \mathrm{H}_{13} \mathrm{Br}_{2} \mathrm{NO}_{3}$ : 424.9262, found: 424.9253. IR (neat): 2952, 1739, 1646, 1595, 1496, 1373, 1274, $1136 \mathrm{~cm}^{-1}$.<smiles>COC(=O)c1c(F)cc(C(=O)c2ccccc2)cc1F</smiles>

Methyl 4-benzoyl-2,6-difluorobenzoate (2z). According to the procedure analogous to that described for $\mathbf{2 b}$, except that the carboxylation was conducted at $80{ }^{\circ} \mathrm{C}$ in a heat block, $2 \mathbf{z}(72.6 \mathrm{mg}, 0.263 \mathrm{mmol}$, $89 \%$ ) was obtained from $\mathbf{1 z}(64.3 \mathrm{mg}, 0.295 \mathrm{mmol})$ as a white solid: $\mathrm{Mp} 81-82{ }^{\circ} \mathrm{C}$ (hexane/AcOEt). ${ }^{1} \mathrm{H}$ NMR (400 MHz, $\left.\mathrm{CDCl}_{3} / \mathrm{TMS}\right) \delta 7.79(\mathrm{~d}, 2 \mathrm{H}, J=7.3 \mathrm{~Hz}), 7.65(\mathrm{t}, 1 \mathrm{H}, J=7.6 \mathrm{~Hz}), 7.52(\mathrm{t}, 2 \mathrm{H}, J=$ $7.8 \mathrm{~Hz}), 7.37(\mathrm{~d}, 2 \mathrm{H}, J=7.8 \mathrm{~Hz}), 4.00(\mathrm{~s}, 3 \mathrm{H}) .{ }^{13} \mathrm{C} \mathrm{NMR}\left(150 \mathrm{MHz}, \mathrm{CDCl}_{3} / \mathrm{TMS}\right) \delta 193.0,161.3$, $160.2\left(\mathrm{dd},{ }^{1} J_{\mathrm{CF}}=257.8 \mathrm{~Hz},{ }^{3} J_{\mathrm{CF}}=5.7 \mathrm{~Hz}\right), 141.7\left(\mathrm{t},{ }^{3} J_{\mathrm{CF}}=7.9 \mathrm{~Hz}\right), 135.9,133.4,129.9,128.7,114.1$ $\left(\mathrm{t},{ }^{2} J_{\mathrm{CF}}=18.6 \mathrm{~Hz}\right), 113.3\left(\mathrm{dd},{ }^{2} J_{\mathrm{CF}}=22.9 \mathrm{~Hz},{ }^{4} J_{\mathrm{CF}}=4.3 \mathrm{~Hz}\right), 53.1 .{ }^{19} \mathrm{~F} \mathrm{NMR}\left(564.5 \mathrm{MHz}, \mathrm{CDCl}_{3} / 4-\right.$ fluorotoluene) $\delta$-104.3. LRMS (EI) $m / z: 276\left(\mathrm{M}^{+}\right)$. HRMS (EI-TOF) $m / z:\left(\mathrm{M}^{+}\right.$) Calcd. for $\mathrm{C}_{15} \mathrm{H}_{10} \mathrm{~F}_{2} \mathrm{O}_{3}$ : 276.0598, found: 276.0591. IR (neat): 2960, 1718, 1652, 1565, 1421, 1044, $722 \mathrm{~cm}^{-1}$.<smiles>CC(=O)c1c(F)cc([N+](=O)[O-])cc1F</smiles>

Methyl 2,6-difluoro-4-nitrobenzoate (2aa). According to the procedure analogous to that described for $\mathbf{2 b}$, except that the carboxylation was conducted at $60^{\circ} \mathrm{C}$ in a heat block and that the crude material was purified by preparative thin-layer chromatography of silica gel (hexane:AcOEt = 4:1), $2 \mathbf{a a}(36.8$ $\mathrm{mg}, 0.169 \mathrm{mmol}, 55 \%)$ was obtained from 1 aa $(49.4 \mathrm{mg}, 0.311 \mathrm{mmol})$ as a yellow solid: $\mathrm{Mp} 60-61{ }^{\circ} \mathrm{C}$ 
(hexane). ${ }^{1} \mathrm{H}$ NMR (400 MHz, $\left.\mathrm{CDCl}_{3} / \mathrm{TMS}\right) \delta 7.89-7.83(\mathrm{~m}, 2 \mathrm{H}), 4.01(\mathrm{~s}, 3 \mathrm{H}) .{ }^{13} \mathrm{C} \mathrm{NMR}(150 \mathrm{MHz}$, $\left.\mathrm{CDCl}_{3} / \mathrm{TMS}\right) \delta 160.4\left(\mathrm{dd},{ }^{1} J_{\mathrm{CF}}=259.9 \mathrm{~Hz},{ }^{3} J_{\mathrm{CF}}=6.4 \mathrm{~Hz}\right), 160.3,149.9\left(\mathrm{t},{ }^{3} J_{\mathrm{CF}}=10.7 \mathrm{~Hz}\right), 117.0(\mathrm{t}$, $\left.{ }^{2} J_{\mathrm{CF}}=19.3 \mathrm{~Hz}\right), 108.5-108.1(\mathrm{~m}), 53.6 .{ }^{19} \mathrm{~F} \mathrm{NMR}\left(564.5 \mathrm{MHz}, \mathrm{CDCl}_{3} / 4\right.$-fluorotoluene) $\delta$-107.8. LRMS (EI) $m / z: 217\left(\mathrm{M}^{+}\right)$. HRMS (EI-TOF) $m / z:\left(\mathrm{M}^{+}\right)$Calcd. for $\mathrm{C}_{8} \mathrm{H}_{5} \mathrm{~F}_{2} \mathrm{NO}_{4}:$ 217.0187, found: 217.0178. IR (neat): 3109, 1734, 1623, 1533, 1434, 1281, 1053, $780 \mathrm{~cm}^{-1}$.<smiles>CC(=O)c1ccc(Cl)c(Cl)c1Cl</smiles>

Methyl 2,3,4-trichlorobenzoate (2ab). According to the procedure analogous to that described for $\mathbf{2 b}$, except that the carboxylation was conducted at $160{ }^{\circ} \mathrm{C}$ in a heat block, $2 \mathbf{a b}(57.2 \mathrm{mg}, 0.239 \mathrm{mmol}$, $77 \%$ ) was obtained from 1 ab $\left(56.2 \mathrm{mg}, 0.310 \mathrm{mmol}\right.$ ) as a white solid: $\mathrm{Mp} 63-64{ }^{\circ} \mathrm{C}$ (hexane) (lit. $\left.63-66^{\circ} \mathrm{C}^{25}\right) .{ }^{1} \mathrm{H} \mathrm{NMR}\left(600 \mathrm{MHz}, \mathrm{CDCl}_{3} / \mathrm{TMS}\right) \delta 7.63(\mathrm{~d}, 1 \mathrm{H}, J=8.6 \mathrm{~Hz}), 7.45(\mathrm{~d}, 1 \mathrm{H}, J=8.2 \mathrm{~Hz})$, 3.95 (s, 3H). $\left.{ }^{13} \mathrm{C} \mathrm{NMR} \mathrm{(150} \mathrm{MHz,} \mathrm{CDCl}_{3} / \mathrm{TMS}\right) \delta 165.1,137.5$ 133.7, 133.6, 130.8, 128.9, 128.1, 52.8. LRMS (EI) $\mathrm{m} / z$ : $238\left(\mathrm{M}^{+}\right)$. HRMS (EI-TOF) $\mathrm{m} / z$ : $\left(\mathrm{M}^{+}\right)$Calcd. for $\mathrm{C}_{8} \mathrm{H}_{5} \mathrm{Cl}_{3} \mathrm{O}_{2}: 237.9355$, found: 237.9353. IR (neat): $3102,2952,1735,1577,1425,1361 \mathrm{~cm}^{-1}$. The spectra data matched those reported in the literature. ${ }^{25}$<smiles>COC(=O)c1cccc(Cl)c1Cl</smiles>

Methyl 2,3-dichlorobenzoate (2ac). According to the procedure analogous to that described for $\mathbf{2 b}$, except that the carboxylation was conducted at $180{ }^{\circ} \mathrm{C}$ in a heat block and that the crude material was purified by column chromatography on silica gel (hexane:AcOEt =10:1), 2ac (26.7 mg, 0.130 mmol, $43 \%$ ) was obtained from 1ac $(44.2 \mathrm{mg}, 0.301 \mathrm{mmol})$ as a colorless oil. ${ }^{1} \mathrm{H}$ NMR (400 MHz, $\left.\mathrm{CDCl}_{3} / \mathrm{TMS}\right) \delta 7.66(\mathrm{dd}, 1 \mathrm{H}, J=7.8,1.5 \mathrm{~Hz}), 7.59(\mathrm{dd}, 1 \mathrm{H}, J=8.3,1.5 \mathrm{~Hz}), 7.26(\mathrm{t}, 1 \mathrm{H}, J=7.8 \mathrm{~Hz})$, 3.95 (s, 3H). ${ }^{13} \mathrm{C}$ NMR (100 MHz, $\left.\mathrm{CDCl}_{3} / \mathrm{TMS}\right) \delta 165.8,134.6,133.1,132.8,131.6,129.0,127.1$, 52.7. LRMS (EI) $m / z: 204\left(\mathrm{M}^{+}\right)$. HRMS (EI-TOF) $m / z$ : $\left(\mathrm{M}^{+}\right)$Calcd. for $\mathrm{C}_{8} \mathrm{H}_{6} \mathrm{Cl}_{2} \mathrm{O}_{2}: 203.9745$, found: 203.9751. IR (neat): 2955, 1734, 1414, 1283, 1205, $1143 \mathrm{~cm}^{-1}$. The spectra data matched those reported in the literature. ${ }^{26}$<smiles>COC(=O)c1c(F)cccc1C#N</smiles>

Methyl 2-cyano-6-fluorobenzoate (4a). According to the procedure analogous to that described for 
2b, except that the carboxylation was conducted with LiO- $t$-Am $(83.9 \mathrm{mg}, 0.892 \mathrm{mmol})$ instead of $\mathrm{LiO}-\mathrm{t}$-Bu at $130{ }^{\circ} \mathrm{C}$ in a heat block and that the crude material was purified by column chromatography on silica gel (hexane:AcOEt = 10:1), 4a $(40.0 \mathrm{mg}, 0.223 \mathrm{mmol}, 76 \%)$ was obtained from 3a $(35.7 \mathrm{mg}$, $0.295 \mathrm{mmol}$ ) as a white solid: $\mathrm{Mp} 69-70{ }^{\circ} \mathrm{C}$ (hexane/AcOEt). ${ }^{1} \mathrm{H}$ NMR (600 MHz, $\mathrm{CDCl}_{3} / \mathrm{TMS}$ ) $\delta 7.65-7.56(\mathrm{~m}, 2 \mathrm{H}), 7.43(\mathrm{t}, 1 \mathrm{H}, J=8.7 \mathrm{~Hz}), 4.03(\mathrm{~s}, 3 \mathrm{H}) .{ }^{13} \mathrm{C} \mathrm{NMR}\left(150 \mathrm{MHz}, \mathrm{CDCl}_{3} / \mathrm{TMS}\right) \delta 162.5$ $\left(\mathrm{d},{ }^{3} J_{\mathrm{CF}}=2.9 \mathrm{~Hz}\right), 160.7\left(\mathrm{~d},{ }^{1} J_{\mathrm{CF}}=258.5 \mathrm{~Hz}\right), 133.5\left(\mathrm{~d},{ }^{3} J_{\mathrm{CF}}=10.1 \mathrm{~Hz}\right), 130.1\left(\mathrm{~d},{ }^{3} J_{\mathrm{CF}}=4.3 \mathrm{~Hz}\right), 122.9$ $\left(\mathrm{d},{ }^{2} J_{\mathrm{CF}}=15.8 \mathrm{~Hz}\right), 121.6\left(\mathrm{~d},{ }^{2} J_{\mathrm{CF}}=23.0 \mathrm{~Hz}\right), 116.1\left(\mathrm{~d},{ }^{4} J_{\mathrm{CF}}=2.9 \mathrm{~Hz}\right), 114.1\left(\mathrm{~d},{ }^{4} J_{\mathrm{CF}}=2.9 \mathrm{~Hz}\right), 53.3$. ${ }^{19} \mathrm{~F}$ NMR (564.5 MHz, $\mathrm{CDCl}_{3} / 4$-fluorotoluene) $\delta$-106.8. LRMS (EI) $m / z$ : $179\left(\mathrm{M}^{+}\right.$). HRMS (EI-TOF) $m / z:\left(\mathrm{M}^{+}\right)$Calcd. for $\mathrm{C}_{9} \mathrm{H}_{6} \mathrm{FNO}_{2}: 179.0383$, found: 179.0382. IR (neat): 3092, 2961, 2243, 1724, 1601, $1576 \mathrm{~cm}^{-1}$.<smiles>COC(=O)c1c(Cl)cccc1C#N</smiles>

Methyl 2-chloro-6-cyanobenzoate (4b). According to the procedure analogous to that described for $\mathbf{2 b}$, except that the carboxylation was conducted with $\mathrm{LiO}-t$-Am $(85.0 \mathrm{mg}, 0.903 \mathrm{mmol})$ instead of $\mathrm{LiO}-t$-Bu at $130{ }^{\circ} \mathrm{C}$ in a heat block and that the crude material was purified by column chromatography on silica gel (hexane:AcOEt = 10:1), 4b $(48.0 \mathrm{mg}, 0.245 \mathrm{mmol}, 80 \%)$ was obtained from $\mathbf{3 b}(42.4 \mathrm{mg}$, $0.308 \mathrm{mmol}$ ) as a white solid: $\mathrm{Mp} 72-74{ }^{\circ} \mathrm{C}$ (hexane/AcOEt). ${ }^{1} \mathrm{H} \mathrm{NMR}\left(400 \mathrm{MHz}, \mathrm{CDCl}_{3} / \mathrm{TMS}\right)$ $\delta 7.68(\mathrm{dd}, 1 \mathrm{H}, J=8.3,1.0 \mathrm{~Hz}), 7.65(\mathrm{dd}, 1 \mathrm{H}, J=7.8,1.0 \mathrm{~Hz}), 7.50(\mathrm{t}, 1 \mathrm{H}, J=8.1 \mathrm{~Hz}), 4.04(\mathrm{~s}$, $3 \mathrm{H}) .{ }^{13} \mathrm{C} \mathrm{NMR}\left(100 \mathrm{MHz}, \mathrm{CDCl}_{3} / \mathrm{TMS}\right) \delta$ 164.2, 135.6, 134.4, 133.0, 131.4, 131.3, 115.8, 112.8, 53.4. LRMS (EI) $m / z$ : $195\left(\mathrm{M}^{+}\right)$. HRMS (EI-TOF) $\mathrm{m} / z$ : $\left(\mathrm{M}^{+}\right)$Calcd. for $\mathrm{C}_{9} \mathrm{H}_{6} \mathrm{ClNO}_{2}$ : 195.0087, found: 195.0087. IR (neat): 3079, 2238, 1722, 1559, 1457, 1280, $1109 \mathrm{~cm}^{-1}$.<smiles>COC(=O)c1c(Br)cccc1C#N</smiles>

Methyl 2-bromo-6-cyanobenzoate (4c). According to the procedure analogous to that described for $\mathbf{2 b}$, except that the carboxylation was conducted with LiO- $t$-Am $(85.9 \mathrm{mg}, 0.913 \mathrm{mmol})$ instead of $\mathrm{LiO}-\mathrm{t}-\mathrm{Bu}$ and that the crude material was purified by column chromatography on silica gel (hexane:AcOEt = 10:1), 4c (51.8 mg, $0.216 \mathrm{mmol}, 72 \%)$ was obtained from 3c (54.4 $\mathrm{mg}, 0.299 \mathrm{mmol})$ as a white solid: $\mathrm{Mp} 58-60{ }^{\circ} \mathrm{C}$ (hexane/AcOEt). ${ }^{1} \mathrm{H}$ NMR $\left(400 \mathrm{MHz}, \mathrm{CDCl}_{3} / \mathrm{TMS}\right) \delta 7.85$ (dd, $1 \mathrm{H}, J$ $=8.3,1.0 \mathrm{~Hz}), 7.69(\mathrm{dd}, 1 \mathrm{H}, J=7.8,1.0 \mathrm{~Hz}), 7.41(\mathrm{t}, 1 \mathrm{H}, J=8.1 \mathrm{~Hz}), 4.04(\mathrm{~s}, 3 \mathrm{H}) .{ }^{13} \mathrm{C} \mathrm{NMR}(100$ $\left.\mathrm{MHz}, \mathrm{CDCl}_{3} / \mathrm{TMS}\right) \delta 164.9,137.9,137.5,131.8,131.3,120.7,115.7,112.6,53.4$. LRMS (EI) $m / z$ : $239\left(\mathrm{M}^{+}\right)$. HRMS (EI-TOF) $\mathrm{m} / z$ : $\left(\mathrm{M}^{+}\right)$Calcd. for $\mathrm{C}_{9} \mathrm{H}_{6} \mathrm{BrNO}_{2}:$ 238.9582, found: 238.9577. IR (neat): $3073,2957,2238,1723,1560,1456,1276 \mathrm{~cm}^{-1}$. 
<smiles>CC(=O)c1c(F)cccc1S(=O)c1ccccc1</smiles>

Methyl 2-fluoro-6-(phenylsulfinyl)benzoate (4d). According to the procedure analogous to that described for $\mathbf{2 b}$, except that the carboxylation was conducted at $150{ }^{\circ} \mathrm{C}$ in a heat block and that the crude material was purified by column chromatography on silica gel (hexane:AcOEt = 2:1), 4d (56.1 $\mathrm{mg}, 0.201 \mathrm{mmol}, 69 \%)$ was obtained from $3 \mathbf{d}(64.8 \mathrm{mg}, 0.294 \mathrm{mmol})$ as a white solid: $\mathrm{Mp} 70-71{ }^{\circ} \mathrm{C}$ (hexane/AcOEt). ${ }^{1} \mathrm{H}$ NMR (400 MHz, $\left.\mathrm{CDCl}_{3} / \mathrm{TMS}\right) \delta 8.08(\mathrm{~d}, 1 \mathrm{H}, J=8.3 \mathrm{~Hz}), 7.78-7.67(\mathrm{~m}, 3 \mathrm{H})$, $7.45-7.38(\mathrm{~m}, 3 \mathrm{H}), 7.24(\mathrm{t}, 1 \mathrm{H}, J=9.3 \mathrm{~Hz}), 3.89(\mathrm{~s}, 3 \mathrm{H}) .{ }^{13} \mathrm{C} \mathrm{NMR}\left(150 \mathrm{MHz}, \mathrm{CDCl}_{3} / \mathrm{TMS}\right) \delta 163.8$, $160.7\left(\mathrm{~d},{ }^{1} J_{\mathrm{CF}}=258.5 \mathrm{~Hz}\right), 149.7,145.8,133.8\left(\mathrm{~d},{ }^{3} J_{\mathrm{CF}}=8.6 \mathrm{~Hz}\right), 131.2,129.2,126.1,120.7\left(\mathrm{~d},{ }^{4} J_{\mathrm{CF}}\right.$ $=2.9 \mathrm{~Hz}), 118.9\left(\mathrm{~d},{ }^{2} J_{\mathrm{CF}}=23.0 \mathrm{~Hz}\right), 117.7\left(\mathrm{~d},{ }^{2} J_{\mathrm{CF}}=14.4 \mathrm{~Hz}\right), 52.9 .{ }^{19} \mathrm{~F} \mathrm{NMR}\left(564.5 \mathrm{MHz}, \mathrm{CDCl}_{3} / 4-\right.$ fluorotoluene) $\delta$-107.9. LRMS (EI) $m / z: 278\left(\mathrm{M}^{+}\right)$. HRMS (EI-TOF) $\mathrm{m} / z:\left(\mathrm{M}^{+}\right)$Calcd. for $\mathrm{C}_{14} \mathrm{H}_{11} \mathrm{FO}_{3} \mathrm{~S}: 278.0413$, found: 278.0425. IR (neat): 3073, 2953, 1708, 1597, 1452, 1440, $1292 \mathrm{~cm}^{-1}$.<smiles>CC(=O)c1ccc(S(=O)(=O)c2ccccc2)cc1F</smiles>

Methyl 2-fluoro-4-(phenylsulfonyl)benzoate (4e). According to the procedure analogous to that described for $\mathbf{2 b}$, except that the carboxylation was conducted in DMF $(1.0 \mathrm{~mL})$ at $150{ }^{\circ} \mathrm{C}$ in a heat block and that the crude material was purified by column chromatography on silica gel (hexane:AcOEt $=3: 1)$ and $\mathrm{GPC}\left(\mathrm{CHCl}_{3}\right), 4 \mathrm{e}(42.6 \mathrm{mg}, 0.145 \mathrm{mmol}, 47 \%)$ was obtained from 3e (72.3 mg, $\left.0.306 \mathrm{mmol}\right)$ as a white solid: $\mathrm{Mp} 109-110{ }^{\circ} \mathrm{C}$ (hexane/AcOEt). ${ }^{1} \mathrm{H} \mathrm{NMR}\left(600 \mathrm{MHz}, \mathrm{CDCl}_{3} / \mathrm{TMS}\right) \delta 8.05(\mathrm{dd}, 1 \mathrm{H}$, $J=7.7,7.1 \mathrm{~Hz}), 7.96(\mathrm{~d}, 2 \mathrm{H}, J=7.4 \mathrm{~Hz}), 7.77(\mathrm{dd}, 1 \mathrm{H}, J=8.3,1.6 \mathrm{~Hz}), 7.72(\mathrm{~d}, 1 \mathrm{H}, J=9.4 \mathrm{~Hz}), 7.63$ $(\mathrm{t}, 1 \mathrm{H}, J=7.5 \mathrm{~Hz}), 7.55(\mathrm{t}, 2 \mathrm{H}, J=8.1 \mathrm{~Hz}), 3.94(\mathrm{~s}, 3 \mathrm{H}) .{ }^{13} \mathrm{C} \mathrm{NMR}\left(150 \mathrm{MHz}, \mathrm{CDCl}_{3} / \mathrm{TMS}\right) \delta 163.4$ $\left(\mathrm{d},{ }^{3} J_{\mathrm{CF}}=4.3 \mathrm{~Hz}\right), 161.4\left(\mathrm{~d},{ }^{1} J_{\mathrm{CF}}=264.9 \mathrm{~Hz}\right), 147.1\left(\mathrm{~d},{ }^{3} J_{\mathrm{CF}}=5.7 \mathrm{~Hz}\right), 140.1,133.9,133.3,129.6$, 128.0, $122.94\left(\mathrm{~d},{ }^{3} J_{\mathrm{CF}}=10.0 \mathrm{~Hz}\right), 122.88\left(\mathrm{~d},{ }^{4} J_{\mathrm{CF}}=4.3 \mathrm{~Hz}\right), 116.6\left(\mathrm{~d},{ }^{2} J_{\mathrm{CF}}=25.8 \mathrm{~Hz}\right), 52.9 .{ }^{19} \mathrm{~F} \mathrm{NMR}$ (564.5 MHz, CDCl $3 / 4$-fluorotoluene) -104.8. LRMS (EI) $\mathrm{m} / z: 294\left(\mathrm{M}^{+}\right)$. HRMS (EI-TOF) $m / z:\left(\mathrm{M}^{+}\right)$ Calcd. for $\mathrm{C}_{14} \mathrm{H}_{11} \mathrm{FO}_{4} \mathrm{~S}: 294.0362$, found: 294.0362. IR (neat): 3102, 2953, 1723, 1606, 1433, 1404, $1290 \mathrm{~cm}^{-1}$. 
$\mathrm{PhMeN}_{\mathrm{O}_{2}} \mathrm{Me}$

Methyl 2-fluoro-4-( $N$-methyl- $N$-phenylsulfamoyl)benzoate (4f). According to the procedure analogous to that described for $\mathbf{2 b}$, except that the carboxylation was conducted at $170{ }^{\circ} \mathrm{C}$ in a heat block and that the crude material was purified by column chromatography on silica gel (hexane:AcOEt $=10: 1), \mathbf{4 f}(73.7 \mathrm{mg}, 0.228 \mathrm{mmol}, 75 \%)$ was obtained from $\mathbf{3 f}(80.2 \mathrm{mg}, 0.302 \mathrm{mmol})$ as a white solid: Mp 95-96 ${ }^{\circ} \mathrm{C}$ (hexane/AcOEt). ${ }^{1} \mathrm{H}$ NMR (600 MHz, $\left.\mathrm{CDCl}_{3} / \mathrm{TMS}\right) \delta 8.01$ (dd, $\left.1 \mathrm{H}, J=8.0,6.7 \mathrm{~Hz}\right)$, 7.38-7.28 (m, 5H), 7.11-7.07 (m, 2H), 3.97 (s, 3H), 3.23 (s, 3H). $\left.{ }^{13} \mathrm{C} \mathrm{NMR} \mathrm{(100} \mathrm{MHz,} \mathrm{CDCl}_{3} / \mathrm{TMS}\right)$ $\delta 163.7\left(\mathrm{~d},{ }^{3} J_{\mathrm{CF}}=3.3 \mathrm{~Hz}\right), 161.2\left(\mathrm{~d},{ }^{1} J_{\mathrm{CF}}=264.3 \mathrm{~Hz}\right), 142.1\left(\mathrm{~d},{ }^{3} J_{\mathrm{CF}}=6.6 \mathrm{~Hz}\right), 140.7,132.7,129.2$, $127.9,126.6,123.1\left(\mathrm{~d},{ }^{4} J_{\mathrm{CF}}=4.1 \mathrm{~Hz}\right), 122.5\left(\mathrm{~d},{ }^{3} J_{\mathrm{CF}}=9.9 \mathrm{~Hz}\right), 116.7\left(\mathrm{~d},{ }^{2} J_{\mathrm{CF}}=25.5 \mathrm{~Hz}\right), 52.9,38.4 .{ }^{19} \mathrm{~F}$ $\operatorname{NMR}\left(564.5 \mathrm{MHz}, \mathrm{CDCl}_{3} / 4\right.$-fluorotoluene) $\delta$-105.5. LRMS (EI) $m / z: 323\left(\mathrm{M}^{+}\right.$). HRMS (EI-TOF) $m / z$ : $\left(\mathrm{M}^{+}\right)$Calcd. for $\mathrm{C}_{15} \mathrm{H}_{14} \mathrm{FNO}_{4} \mathrm{~S}: 323.0628$, found: 323.0624. IR (neat): 2957, 1734, 1718, 1609, 1492, $1406,1349 \mathrm{~cm}^{-1}$.<smiles>COC(=O)c1ccc(C(=O)c2ccccc2)cc1F</smiles>

Methyl 4-benzoyl-2-fluorobenzoate (4g). According to the procedure analogous to that described for 2a, except that the carboxylation was conducted with LiO-t-Am $(85.5 \mathrm{mg}, 0.909 \mathrm{mmol})$ instead of $\mathrm{LiO}-\mathrm{t}$-Bu at $180{ }^{\circ} \mathrm{C}$ in a heat block and that the crude material was purified by preparative thin-layer chromatography of silica gel (hexane:AcOEt $=4: 1)$ and $\mathrm{GPC}\left(\mathrm{CHCl}_{3}\right), \mathbf{4 g}(38.2 \mathrm{mg}, 0.148 \mathrm{mmol}, 49 \%)$ was obtained from $3 \mathrm{~g}(61.0 \mathrm{mg}, 0.305 \mathrm{mmol})$ as a white solid: $\mathrm{Mp} 104-105{ }^{\circ} \mathrm{C}$ (hexane/AcOEt). ${ }^{1} \mathrm{H}$ NMR (600 MHz, $\left.\mathrm{CDCl}_{3} / \mathrm{TMS}\right) \delta 8.05(\mathrm{t}, 1 \mathrm{H}, J=7.4 \mathrm{~Hz}), 7.80(\mathrm{~d}, 2 \mathrm{H}, J=8.3 \mathrm{~Hz}), 7.64(\mathrm{t}, 1 \mathrm{H}, J=6.9$ $\mathrm{Hz}), 7.60(\mathrm{~d}, 1 \mathrm{H}, J=7.3 \mathrm{~Hz}), 7.56(\mathrm{~d}, 1 \mathrm{H}, J=11.0 \mathrm{~Hz}), 7.52(\mathrm{t}, 2 \mathrm{H}, J=7.8 \mathrm{~Hz}), 3.98(\mathrm{~s}, 3 \mathrm{H}) .{ }^{13} \mathrm{C}$ NMR (150 MHz, $\left.\mathrm{CDCl}_{3} / \mathrm{TMS}\right) \delta 194.4,164.2\left(\mathrm{~d},{ }^{3} J_{\mathrm{CF}}=2.9 \mathrm{~Hz}\right), 161.4\left(\mathrm{~d},{ }^{1} J_{\mathrm{CF}}=261.3 \mathrm{~Hz}\right), 143.0(\mathrm{~d}$, $\left.{ }^{3} J_{\mathrm{CF}}=7.2 \mathrm{~Hz}\right), 136.4,132.22,132.17,130.0,128.6,125.0\left(\mathrm{~d},{ }^{4} J_{\mathrm{CF}}=2.9 \mathrm{~Hz}\right), 121.7\left(\mathrm{~d},{ }^{3} J_{\mathrm{CF}}=10.1 \mathrm{~Hz}\right)$, $118.2\left(\mathrm{~d},{ }^{2} J_{\mathrm{CF}}=23.0 \mathrm{~Hz}\right), 52.7 .{ }^{19} \mathrm{~F} \mathrm{NMR}\left(564.5 \mathrm{MHz}, \mathrm{CDCl}_{3} / 4\right.$-fluorotoluene) $\delta$-107.7. LRMS (EI) $m / z: 258\left(\mathrm{M}^{+}\right)$. HRMS (EI-TOF) $m / z$ : $\left(\mathrm{M}^{+}\right)$Calcd. for $\mathrm{C}_{15} \mathrm{H}_{11} \mathrm{FO}_{3}$ : 258.0692, found: 258.0696. IR (neat): $3076,2959,1723,1643,1438,1410,1272 \mathrm{~cm}^{-1}$. 
<smiles>CC(=O)c1ccc(-c2nc3ccccc3o2)cc1F</smiles>

Methyl 4-(benzo $[d]$ oxazol-2-yl)-2-fluorobenzoate (4h). According to the procedure analogous to that described for $\mathbf{2 b}$, except that the carboxylation was conducted at $190{ }^{\circ} \mathrm{C}$ in a heat block and that the crude material was purified by column chromatography on silica gel (hexane:AcOEt = 10:1), 4h (46.0 $\mathrm{mg}, 0.170 \mathrm{mmol}, 56 \%)$ was obtained from $3 \mathrm{~h}(64.1 \mathrm{mg}, 0.301 \mathrm{mmol})$ as a white solid: $\mathrm{Mp} 170-172{ }^{\circ} \mathrm{C}$ (hexane/AcOEt). ${ }^{1} \mathrm{H}$ NMR (600 MHz, DMSO- $\left.d_{6}\right) \delta 8.16-8.10(\mathrm{~m}, 2 \mathrm{H}), 8.07$ (d, $\left.1 \mathrm{H}, J=11.3 \mathrm{~Hz}\right), 7.87$ $(\mathrm{d}, 1 \mathrm{H}, J=7.9 \mathrm{~Hz}), 7.83(\mathrm{~d}, 1 \mathrm{H}, J=8.2 \mathrm{~Hz}), 7.51(\mathrm{t}, 1 \mathrm{H}, J=7.5 \mathrm{~Hz}), 7.47$ (t, $1 \mathrm{H}, J=7.6 \mathrm{~Hz}), 3.91(\mathrm{~s}$, $3 \mathrm{H}) .{ }^{13} \mathrm{C}$ NMR $\left(150 \mathrm{MHz}, \mathrm{DMSO}-d_{6}\right) \delta 163.1\left(\mathrm{~d},{ }^{3} J_{\mathrm{CF}}=2.9 \mathrm{~Hz}\right), 160.7\left(\mathrm{~d},{ }^{1} J_{\mathrm{CF}}=257.9 \mathrm{~Hz}\right), 159.9(\mathrm{~d}$, $\left.{ }^{4} J_{\mathrm{CF}}=2.9 \mathrm{~Hz}\right), 150.2,141.1,132.6,132.0\left(\mathrm{~d},{ }^{3} J_{\mathrm{CF}}=10.0 \mathrm{~Hz}\right), 126.2,125.0,122.9\left(\mathrm{~d},{ }^{4} J_{\mathrm{CF}}=2.9 \mathrm{~Hz}\right)$, $120.6\left(\mathrm{~d},{ }^{3} J_{\mathrm{CF}}=10.0 \mathrm{~Hz}\right), 120.1,115.2\left(\mathrm{~d},{ }^{2} J_{\mathrm{CF}}=25.8 \mathrm{~Hz}\right), 110.9,52.3 .{ }^{19} \mathrm{~F}$ NMR $(564.5 \mathrm{MHz}$, DMSO$d_{6} / 4$-fluorotoluene) $\delta$-108.6. LRMS (EI) $m / z: 271\left(\mathrm{M}^{+}\right)$. HRMS (EI-TOF) $m / z:\left(\mathrm{M}^{+}\right)$Calcd. for $\mathrm{C}_{15} \mathrm{H}_{10} \mathrm{FNO}_{3}$ : 271.0645, found: 271.0644. IR (neat): 2965, 1734, 1629, 1608, 1554, 1497, 1435, 1423 $\mathrm{cm}^{-1}$.<smiles>COC(=O)c1c(C#N)cccc1C#N</smiles>

Methyl 2,6-dicyanobenzoate (4i). According to the procedure analogous to that described for $\mathbf{2 b}$, except that the carboxylation was conducted at $130{ }^{\circ} \mathrm{C}$ in a heat block and that the crude material was purified by short column chromatography on silica gel $\left(\mathrm{CHCl}_{3}\right)$ and $\mathrm{GPC}\left(\mathrm{CHCl}_{3}\right), 4 \mathbf{i}(40.8 \mathrm{mg}, 0.219$ mmol, 73\%) was obtained from $3 \mathbf{i}(38.5 \mathrm{mg}, 0.300 \mathrm{mmol})$ as a white solid: $\mathrm{Mp} 106-108{ }^{\circ} \mathrm{C}$ (hexane/AcOEt). ${ }^{1} \mathrm{H}$ NMR (600 MHz, $\left.\mathrm{CDCl}_{3} / \mathrm{TMS}\right) \delta 8.02(\mathrm{~d}, 2 \mathrm{H}, J=7.8 \mathrm{~Hz}), 7.78(\mathrm{t}, 1 \mathrm{H}, J=8.0$ $\mathrm{Hz}), 4.11$ (s, 3H). ${ }^{13} \mathrm{C} \mathrm{NMR}\left(150 \mathrm{MHz}, \mathrm{CDCl}_{3} / \mathrm{TMS}\right) \delta 162.5,137.7,136.5,132.3,115.6,114.6,53.8$. LRMS (EI) $m / z$ : $186\left(\mathrm{M}^{+}\right)$. HRMS (EI-TOF) $m / z$ : $\left(\mathrm{M}^{+}\right)$Calcd. for $\mathrm{C}_{10} \mathrm{H}_{6} \mathrm{~N}_{2} \mathrm{O}_{2}$ : 186.0429, found: 186.0428. IR (neat): 3072, 2243, 2237, 1728, 1579, 1456, $1440 \mathrm{~cm}^{-1}$.<smiles>COC(=O)c1c(C#N)cccc1[N+](=O)[O-]</smiles>

Methyl 2-cyano-6-nitrobenzoate (4j). According to the procedure analogous to that described for $\mathbf{2 a}$, except that the carboxylation was conducted at $90{ }^{\circ} \mathrm{C}$ in a heat block and that the crude material was 
purified by column chromatography on silica gel (hexane: $\left.\mathrm{CH}_{2} \mathrm{Cl}_{2}=1: 10\right), \mathbf{4 j}$ (44.7 $\mathrm{mg}, 0.217 \mathrm{mmol}$, $73 \%$ ) was obtained from $3 \mathbf{j}$ (43.8 $\mathrm{mg}, 0.295 \mathrm{mmol}$ ) as a white solid: Mp 106-108 ${ }^{\circ} \mathrm{C}$ (hexane/AcOEt). ${ }^{1} \mathrm{H}$ NMR (600 MHz, $\left.\mathrm{CDCl}_{3} / \mathrm{TMS}\right) \delta 8.35(\mathrm{dd}, 1 \mathrm{H}, J=9.0,1.4 \mathrm{~Hz}), 8.02(\mathrm{dd}, 1 \mathrm{H}, J=8.3,1.4 \mathrm{~Hz}), 7.77$ (t, $1 \mathrm{H}, J=8.3 \mathrm{~Hz}), 4.07$ (s, 3H). ${ }^{13} \mathrm{C}$ NMR (150 MHz, $\left.\mathrm{CDCl}_{3} / \mathrm{TMS}\right) \delta 163.4,146.6,137.6,132.9$, 131.4, 128.2, 114.6, 113.3, 54.3. LRMS (EI) $m / z: 206\left(\mathrm{M}^{+}\right)$. HRMS (EI-TOF) $\mathrm{m} / z$ : $\left(\mathrm{M}^{+}\right)$Calcd. for $\mathrm{C}_{9} \mathrm{H}_{6} \mathrm{~N}_{2} \mathrm{O}_{4}: 206.0328$, found: 206.0330. IR (neat): 3087, 2240, 1739, 1606, 1539, 1360, $1276 \mathrm{~cm}^{-1}$.<smiles>COC(=O)c1c(C#N)cccc1OC(F)(F)F</smiles>

Methyl 2-cyano-6-(trifluoromethoxy)benzoate (4k). According to the procedure analogous to that described for $\mathbf{2 a}$, except that the carboxylation was conducted at $120{ }^{\circ} \mathrm{C}$ in a heat block and that the crude material was purified by column chromatography on silica gel (hexane:AcOEt =10:1), 4k (49.3 $\mathrm{mg}, 0.201 \mathrm{mmol}, 66 \%)$ was obtained from $3 \mathbf{k}(56.8 \mathrm{mg}, 0.303 \mathrm{mmol})$ as a colorless oil. ${ }^{1} \mathrm{H}$ NMR $(600$ $\left.\mathrm{MHz}, \mathrm{CDCl}_{3} / \mathrm{TMS}\right) \delta 7.71(\mathrm{~d}, 1 \mathrm{H}, J=6.9 \mathrm{~Hz}), 7.64(\mathrm{t}, 1 \mathrm{H}, J=8.3 \mathrm{~Hz}), 7.61-7.57(\mathrm{~m}, 1 \mathrm{H}), 4.03(\mathrm{~s}$, $3 \mathrm{H}) .{ }^{13} \mathrm{C} \mathrm{NMR}\left(150 \mathrm{MHz}, \mathrm{CDCl}_{3} / \mathrm{TMS}\right) \delta 162.8,146.9,132.5,131.9,129.9,126.0,120.1$ (q, ${ }^{1} J_{\mathrm{CF}}=$ $258.9 \mathrm{~Hz}), 115.6,113.9,53.4 .{ }^{19} \mathrm{~F} \mathrm{NMR}\left(564.5 \mathrm{MHz}, \mathrm{CDCl}_{3} / 4\right.$-fluorotoluene) $\delta$-56.8. LRMS (EI) $\mathrm{m} / z$ : $245\left(\mathrm{M}^{+}\right)$. HRMS (EI-TOF) $m / z$ : $\left(\mathrm{M}^{+}\right)$Calcd. for $\mathrm{C}_{10} \mathrm{H}_{6} \mathrm{~F}_{3} \mathrm{NO}_{3}$ : 245.0300, found: 245.0285 . IR (neat): $2962,2234,1739,1600,1577,1464,1435 \mathrm{~cm}^{-1}$.<smiles>COC(=O)c1ccc(C(F)(F)F)cc1C#N</smiles>

Methyl 2-cyano-4-(trifluoromethyl)benzoate (4I). According to the procedure analogous to that described for $\mathbf{2 b}$, except that the carboxylation was conducted at $180{ }^{\circ} \mathrm{C}$ in a heat block and that the crude material was purified by column chromatography on silica gel (hexane:AcOEt $=4: 1$ ) and GPC $\left(\mathrm{CHCl}_{3}\right), 4 \mathrm{l}$ (33.5 mg, $\left.0.146 \mathrm{mmol}, 48 \%\right)$ was obtained from 31 (52.4 mg, $\left.0.306 \mathrm{mmol}\right)$ as a white solid: Mp 56-57 ${ }^{\circ} \mathrm{C}$ (hexane/AcOEt). ${ }^{1} \mathrm{H}$ NMR $\left(600 \mathrm{MHz}, \mathrm{CDCl}_{3} / \mathrm{TMS}\right) \delta 8.29$ (d, $\left.1 \mathrm{H}, J=8.3 \mathrm{~Hz}\right), 8.07$ (d, $1 \mathrm{H}, J=1.4 \mathrm{~Hz}), 7.94(\mathrm{dd}, 1 \mathrm{H}, J=8.2,1.4 \mathrm{~Hz}), 4.05$ (s, 3H). ${ }^{13} \mathrm{C} \mathrm{NMR}\left(150 \mathrm{MHz}, \mathrm{CDCl}_{3} / \mathrm{TMS}\right) \delta 163.3$, $135.5,134.8\left(\mathrm{q},{ }^{2} J_{\mathrm{CF}}=34.4 \mathrm{~Hz}\right), 131.9,131.6\left(\mathrm{q},{ }^{3} J_{\mathrm{CF}}=3.8 \mathrm{~Hz}\right), 129.3\left(\mathrm{q},{ }^{3} J_{\mathrm{CF}}=3.8 \mathrm{~Hz}\right), 122.4(\mathrm{q}$, $\left.{ }^{1} J_{\mathrm{CF}}=273.0 \mathrm{~Hz}\right), 116.1,114.0,53.3 .{ }^{19} \mathrm{~F} \mathrm{NMR}\left(564.5 \mathrm{MHz}, \mathrm{CDCl}_{3} / 4\right.$-fluorotoluene) $\delta$-62.8. LRMS (EI) $m / z: 229\left(\mathrm{M}^{+}\right)$. HRMS (EI-TOF) $m / z:\left(\mathrm{M}^{+}\right)$Calcd. for $\mathrm{C}_{10} \mathrm{H}_{6} \mathrm{~F}_{3} \mathrm{NO}_{2}: 229.0351$, found: 229.0379 . IR (neat): 3100, 3064, 2977, 2232, 1729, 1507, $1330 \mathrm{~cm}^{-1}$. 
<smiles>N#Cc1cc(C(F)(F)F)ccc1C(=O)OCc1ccccc1</smiles>

Benzyl 2-cyano-4-(trifluoromethyl)benzoate (4I-Bn). According to the procedure analogous to that described for $\mathbf{2 b}$, except that the carboxylation was conducted at $180{ }^{\circ} \mathrm{C}$ in a heat block, that $\mathrm{BnBr}$ $(159.1 \mathrm{mg}, 0.930 \mathrm{mmol}$ ) was used for esterification instead of MeI, and that the crude material was purified by column chromatography on silica gel (hexane:AcOEt $=4: 1)$ and $\mathrm{GPC}\left(\mathrm{CHCl}_{3}\right),(38.2 \mathrm{mg}$, $0.125 \mathrm{mmol}, 41 \%)$ was obtained from 31 (52.7 $\mathrm{mg}, 0.308 \mathrm{mmol})$ as a white solid: $\mathrm{Mp} 103-104{ }^{\circ} \mathrm{C}$ (hexane/AcOEt). ${ }^{1} \mathrm{H}$ NMR (600 MHz, DMSO-d $) \delta 8.50(\mathrm{~s}, 1 \mathrm{H}), 8.30(\mathrm{~d}, 1 \mathrm{H}, J=8.2 \mathrm{~Hz}), 8.20(\mathrm{~d}, 1 \mathrm{H}$, $J=8.2 \mathrm{~Hz}), 7.51(\mathrm{~d}, 2 \mathrm{H}, J=7.5 \mathrm{~Hz}), 7.41(\mathrm{t}, 2 \mathrm{H}, J=7.6 \mathrm{~Hz}), 7.36(\mathrm{t}, 1 \mathrm{H}, J=7.2 \mathrm{~Hz}), 5.43(\mathrm{~s}, 2 \mathrm{H}) .{ }^{13} \mathrm{C}$ NMR (150 MHz, DMSO- $\left.d_{6}\right) \delta 162.5,135.3,135.1,133.0$ (q, $\left.J_{\mathrm{CF}}=33.0 \mathrm{~Hz}\right), 132.0,131.9,130.1,128.5$, $128.35,128.29,122.6\left(\mathrm{q}, J_{\mathrm{CF}}=271.3 \mathrm{~Hz}\right), 116.0,112.9,67.7 .{ }^{19} \mathrm{~F}$ NMR $\left(564.5 \mathrm{MHz}\right.$, DMSO- $d_{6} / 4-$ fluorotoluene) $\delta$-61.7. LRMS (EI) $m / z: 305\left(\mathrm{M}^{+}\right)$. HRMS (EI-TOF) $m / z:\left(\mathrm{M}^{+}\right)$Calcd. for $\mathrm{C}_{16} \mathrm{H}_{10} \mathrm{~F}_{3} \mathrm{NO}_{2}$ : 305.0664, found: 305.0662 . IR (neat): 3061, 2235, 1724, 1331, $1279 \mathrm{~cm}^{-1}$.<smiles>COC(OC)c1cccc(C#N)c1C#N</smiles>

Methyl 2,3-dicyanobenzoate (4m). According to the procedure analogous to that described for $\mathbf{2 b}$, except that the carboxylation was conducted at $150{ }^{\circ} \mathrm{C}$ in a heat block and that the crude material was purified by column chromatography on silica gel (hexane:AcOEt = 1:1), 4m (43.7 mg, 0.235 mmol, $78 \%$ ) was obtained from $\mathbf{3 m}(38.8 \mathrm{mg}, 0.303 \mathrm{mmol})$ as a pale yellow solid: $\mathrm{Mp} 178-180{ }^{\circ} \mathrm{C}$ (hexane/ $\mathrm{CH}_{2} \mathrm{Cl}_{2}$ ). ${ }^{1} \mathrm{H}$ NMR (400 MHz, $\left.\mathrm{CDCl}_{3} / \mathrm{TMS}\right) \delta 8.39$ (dd, $\left.1 \mathrm{H}, J=8.0,1.2 \mathrm{~Hz}\right), 8.01$ (dd, $1 \mathrm{H}, J$ $=7.8,1.0 \mathrm{~Hz}), 7.85(\mathrm{t}, 1 \mathrm{H}, J=8.1 \mathrm{~Hz}), 4.06(\mathrm{~s}, 3 \mathrm{H}) .{ }^{13} \mathrm{C} \mathrm{NMR}\left(100 \mathrm{MHz}, \mathrm{CDCl}_{3} / \mathrm{TMS}\right) \delta 162.9,136.6$, 134.8, 134.2, 132.9, 118.7, 116.3, 114.9, 114.0, 53.5. LRMS (EI) $m / z: 186\left(\mathrm{M}^{+}\right.$). HRMS (EI-TOF) $\mathrm{m} / z$ : $\left(\mathrm{M}^{+}\right)$Calcd. for $\mathrm{C}_{10} \mathrm{H}_{6} \mathrm{~N}_{2} \mathrm{O}_{2}: 186.0429$, found: 186.0413. IR (neat): 2233, 1723, 1576, 1437, 1287, 777 $\mathrm{cm}^{-1}$.<smiles>COC(=O)c1ccccc1[N+](=O)[O-]</smiles>

Methyl 2-nitrobenzoate (4n). According to the procedure analogous to that described for $\mathbf{2 b}$, except that the carboxylation was conducted at $160{ }^{\circ} \mathrm{C}$ in a heat block and that the crude material was purified by column chromatography on silica gel (hexane:AcOEt $=2: 1)$ and $\mathrm{GPC}\left(\mathrm{CHCl}_{3}\right), \mathbf{4 n}(14.2 \mathrm{mg}, 0.0784$ mmol, 26\%) was obtained from 3n $(36.9 \mathrm{mg}, 0.300 \mathrm{mmol})$ as a colorless oil. ${ }^{1} \mathrm{H}$ NMR (600 MHz, 
$\left.\mathrm{CDCl}_{3} / \mathrm{TMS}\right) \delta 7.94-7.90(\mathrm{~m}, 1 \mathrm{H}), 7.75(\mathrm{dd}, 1 \mathrm{H}, J=7.6,1.4 \mathrm{~Hz}), 7.68(\mathrm{td}, 1 \mathrm{H}, J=7.6,1.4 \mathrm{~Hz}), 7.64$ (td, $1 \mathrm{H}, J=7.9,1.6 \mathrm{~Hz}), 3.93$ (s, 3H). ${ }^{13} \mathrm{C} \mathrm{NMR}\left(150 \mathrm{MHz}, \mathrm{CDCl}_{3} / \mathrm{TMS}\right) \delta 165.9,148.3,132.9,131.8$, 129.9, 127.6, 123.9, 53.3. LRMS (EI) $\mathrm{m} / z: 181\left(\mathrm{M}^{+}\right)$. HRMS (EI-TOF) $\mathrm{m} / \mathrm{z}:\left(\mathrm{M}^{+}\right.$) Calcd. for $\mathrm{C}_{8} \mathrm{H}_{7} \mathrm{NO}_{4}$ : 181.0375, found: 181.0372 . IR (neat): $2956,1733,1530,1351,1289,1074 \mathrm{~cm}^{-1}$. The spectra data matched those reported in the literature. ${ }^{27}$

\section{Procedure of carboxylation of 1 a on a $1.0 \mathrm{mmol}$ scale (Table 1, entry 18).}

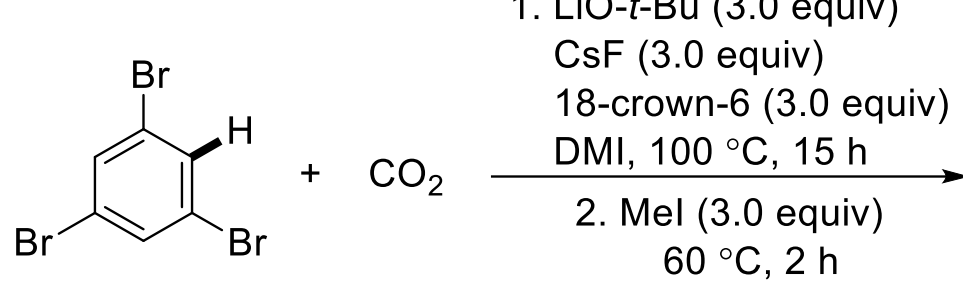

$1 \mathrm{a}$

$1.04 \mathrm{mmol}$
(1 atm)<smiles>COC(OC)c1c(Br)cc(Br)cc1Br</smiles>

2a

$0.853 \mathrm{mmol}, 82 \%$

In a glove box under an $\mathrm{Ar}$ atmosphere, a solution of $\mathrm{LiO}-t$-Bu $(240.8 \mathrm{mg}, 3.01 \mathrm{mmol}), \mathrm{CsF}$ (461.9 mg, $3.04 \mathrm{mmol})$, and 18-crown-6 (795.2 $\mathrm{mg}, 3.01 \mathrm{mmol})$ in DMI $(3.3 \mathrm{~mL})$ was prepared in an oven-dried glass screw tube $(\phi=1.65 \mathrm{~cm}, 10.5 \mathrm{~cm})$ equipped with a stirrer bar. The tube was sealed with a rubber cap and taken out from the glove box. The tube was evacuated and refilled with $\mathrm{CO}_{2}$ gas, and this procedure was repeated three times. The tube was further flushed with $\mathrm{CO}_{2}$ gas for 5 min with stirring at room temperature. The rubber cap was removed from the tube, and 1a (326.8 $\mathrm{mg}, 1.04 \mathrm{mmol}$ ) was added. The tube was then sealed with a cap containing an inner Teflom film. After stirring at $100{ }^{\circ} \mathrm{C}$ in a heat block for $15 \mathrm{~h}$, MeI (429.1 mg, $3.02 \mathrm{mmol})$ was added to the reaction mixture at room temperature. The resulting mixture was stirred at $60^{\circ} \mathrm{C}$ in a heat block for $2 \mathrm{~h}$. The mixture was then quenched with $\mathrm{H}_{2} \mathrm{O}$ and extracted with AcOEt $(10 \mathrm{~mL} \times 3)$. The combined organic layers were collected, washed with $\mathrm{H}_{2} \mathrm{O}(10 \mathrm{~mL})$ and brine $(10 \mathrm{~mL})$, dried over $\mathrm{Na}_{2} \mathrm{SO}_{4}$, and concentrated. The crude material was purified by column chromatography on silica gel (hexane:AcOEt $=10: 1$ ) to afford 2a (317.9 mg, $0.853 \mathrm{mmol}, 82 \%)$.

\section{Procedure of carboxylation of 1a with [ $\left.\mathrm{LiOCO}_{2}-\mathrm{t}-\mathrm{Bu}\right], \mathrm{CsF}$, and 18-crown-6 under an Ar atmosphere (Scheme S1).}

In a glove box under an Ar atmosphere, a solution of $1 \mathrm{a}(100.0 \mathrm{mg}, 0.318 \mathrm{mmol}), \mathrm{CsF}(142.1 \mathrm{mg}$, $0.935 \mathrm{mmol}), 18$-crown-6 (240.5 mg, $0.904 \mathrm{mmol})$ and [LiOCO $2-t-\mathrm{Bu}]$ (110.9 mg, $0.894 \mathrm{mmol}) \mathrm{in}$ DMI $(1.0 \mathrm{~mL})$ was prepared in an oven-dried glass screw tube $(\phi=1.65 \mathrm{~cm}, 10.5 \mathrm{~cm})$ equipped with a stirrer bar. The tube was sealed with a cap containing an inner Teflom film and taken out from the glove box. The mixture was stirred at room temperature for $5 \mathrm{~min}$. After stirring at $100{ }^{\circ} \mathrm{C}$ in a heat block for $15 \mathrm{~h}, \mathrm{MeI}(128.7 \mathrm{mg}, 0.907 \mathrm{mmol})$ was added to the reaction mixture at room temperature. The resulting mixture was stirred at $60^{\circ} \mathrm{C}$ in a heat block for $2 \mathrm{~h}$. The mixture was then quenched with 
$\mathrm{H}_{2} \mathrm{O}$ and extracted with AcOEt (10 mL x 3). The combined organic layers were collected, washed with $\mathrm{H}_{2} \mathrm{O}(10 \mathrm{~mL})$ and brine $(10 \mathrm{~mL})$, dried over $\mathrm{Na}_{2} \mathrm{SO}_{4}$, and concentrated. The crude material was purified by column chromatography on silica gel (hexane:AcOEt $=40: 1)$ to afford $\mathbf{2 a}(97.8 \mathrm{mg}, 0.262 \mathrm{mmol}$, $82 \%)$. 


\section{X-Ray Crystallographic Data for $4 a$}

The structure of $\mathbf{4 a}$ was also determined by x-ray analysis. The single crystal for the analysis was grown by vapor diffusion of hexane to a $\mathrm{CH}_{2} \mathrm{Cl}_{2}$ solution of 4a. X Ray Crystallography: CCDC2120351 contains the supplementary crystallographic data for this paper. The data can be obtained free of charge from the Cambridge Crystallographic Data Centre via www.ccdc.cam.ac.uk/data_request/cif.

\section{Crystal data and structure refinement}

Compound: $\mathbf{4 a}$

Formula: C9 H6 F N O2

Formula weight: 179.15

Wave length: $=0.71073$

Crystal system: triclinic

Space group: $\mathrm{P}-1$

Color of crystal: Colorless

Unit cell parameters: $\quad \mathrm{a}=3.7696(11) \AA \quad \alpha=84.305(10)^{\circ}$

$$
\begin{array}{ll}
\mathrm{b}=7.339(2) \AA & \beta=84.348(10)^{\circ} \\
\mathrm{c}=14.967(5) \AA & \gamma=83.811(9)^{\circ}
\end{array}
$$

Temperature of data collection: $100(\mathrm{~K})$

Values of $\mathrm{Z}, \mathrm{R}, \mathrm{GOF}: \mathrm{Z}=2$

$\mathrm{R}($ reflections $)=0.0447(1258), \mathrm{wR} 2($ reflections $)=0.1160(1445)$

$$
\mathrm{GOF}=1.080
$$

Radiation type: Mo K/a

Radiation source: micro focus sealed tube

Radiation monochromator: Incoatec Helios multilayer confocal mirror

Measurement device type: Bruker D8 goniometer

Computing structure solution: SHELXT (Sheldrick, 2014)

Computing structure refinement: SHELXL-2018 (Sheldrick, 2018) 
ORTEP views of $\mathbf{4 a}$

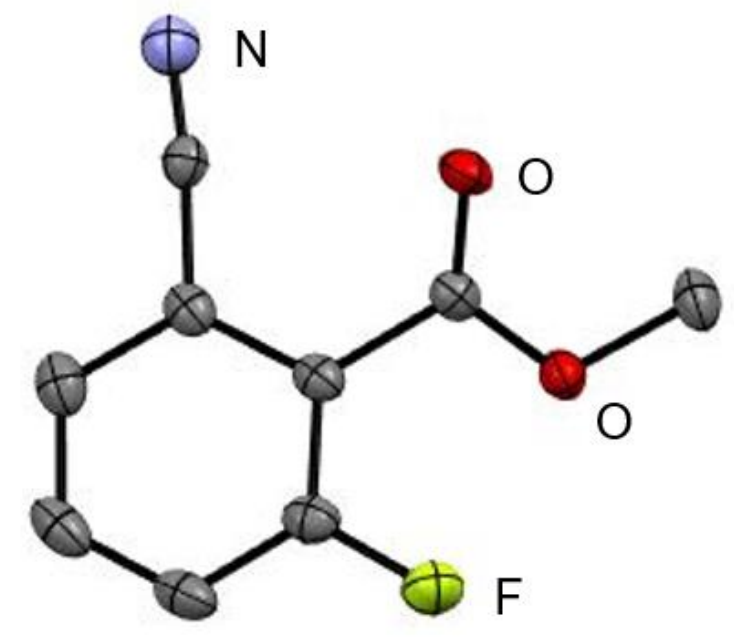

Thermal ellipsoid plot of $\mathbf{4 a}$ at $50 \%$ probability. 


\section{X-Ray Crystallographic Data for 4d}

The structure of $\mathbf{4 d}$ was also determined by $\mathrm{x}$-ray analysis. The single crystal for the analysis was grown by vapor diffusion of hexane to a $\mathrm{CH}_{2} \mathrm{Cl}_{2}$ solution of $\mathbf{4 d}$. X Ray Crystallography: CCDC2120355 contains the supplementary crystallographic data for this paper. The data can be obtained free of charge from the Cambridge Crystallographic Data Centre via www.ccdc.cam.ac.uk/data_request/cif.

\section{Crystal data and structure refinement}

Compound: 4d

Formula: C14 H11 F O3 S

Formula weight: 278.30

Wave length: $=0.71073$

Crystal system: monoclinic

Space group: C 2/c

Color of crystal: Colorless

Unit cell parameters: $\quad \mathrm{a}=23.8780(6) \AA \quad \alpha=90^{\circ}$

$$
\begin{array}{ll}
\mathrm{b}=8.2672(2) \AA & \beta=129.110(1)^{\circ} \\
\mathrm{c}=16.2285(4) \AA & \gamma=90^{\circ}
\end{array}
$$

Temperature of data collection: $100(\mathrm{~K})$

Values of $\mathrm{Z}, \mathrm{R}, \mathrm{GOF}: \mathrm{Z}=8$

$$
\begin{aligned}
& \mathrm{R}(\text { reflections })=0.0293(1856), \mathrm{wR} 2(\text { reflections })=0.0714(2191) \\
& \mathrm{GOF}=1.060
\end{aligned}
$$

Radiation type: Mo K/a

Radiation source: micro focus sealed tube

Radiation monochromator: Incoatec Helios multilayer confocal mirror

Measurement device type: Bruker D8 goniometer

Computing structure solution: SHELXT (Sheldrick, 2013)

Computing structure refinement: SHELXL-2014 (Sheldrick, 2014) 


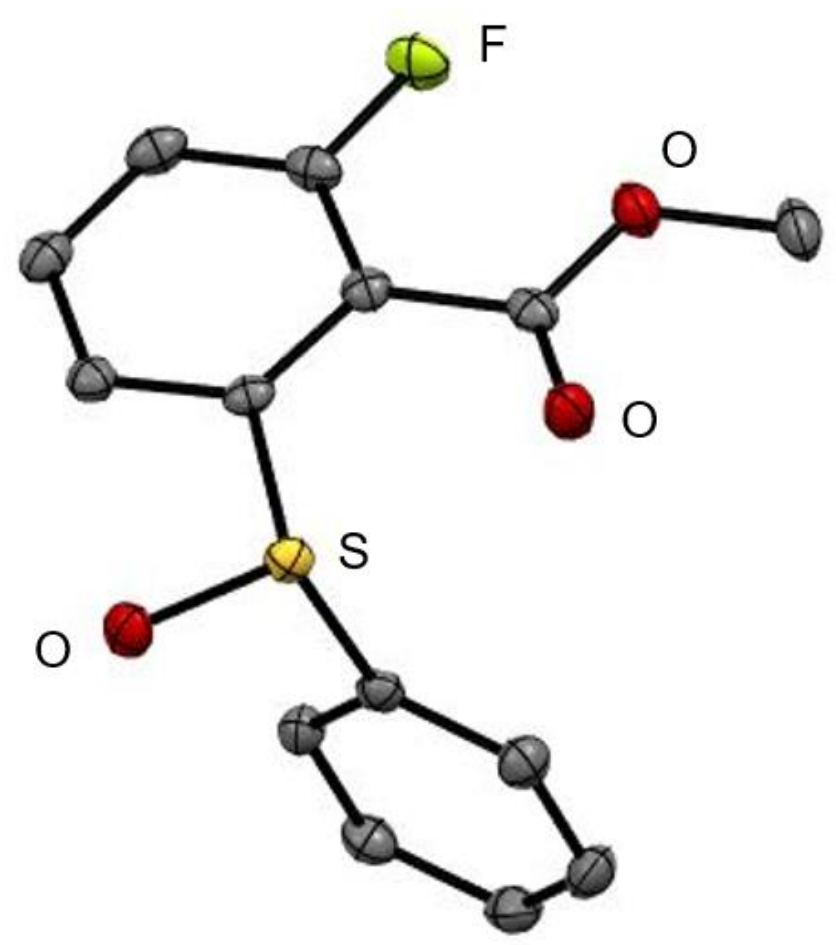

Thermal ellipsoid plot of $\mathbf{4 d}$ at $50 \%$ probability. 


\section{X-Ray Crystallographic Data for 4e}

The structure of $\mathbf{4} \mathbf{e}$ was also determined by $\mathrm{x}$-ray analysis. The single crystal for the analysis was grown by vapor diffusion of hexane to a $\mathrm{Et}_{2} \mathrm{O}$ solution of 4e. X Ray Crystallography: CCDC-2120356 contains the supplementary crystallographic data for this paper. The data can be obtained free of charge from the Cambridge Crystallographic Data Centre via www.ccdc.cam.ac.uk/data_request/cif.

\section{Crystal data and structure refinement}

Compound: 4 e

Formula: C14 H11 F O4 S

Formula weight: 294.30

Wave length: $=0.71073$

Crystal system: monoclinic

Space group: P 21/c

Color of crystal: Colorless

Unit cell parameters: $\quad \mathrm{a}=14.0192(12) \AA \quad \alpha=90^{\circ}$

$$
\begin{array}{ll}
\mathrm{b}=8.1964(6) \AA & \beta=92.309(3)^{\circ} \\
\mathrm{c}=11.0502(8) \AA & \gamma=90^{\circ}
\end{array}
$$

Temperature of data collection: $100(\mathrm{~K})$

Values of $\mathrm{Z}, \mathrm{R}, \mathrm{GOF}: \mathrm{Z}=4$

$$
\mathrm{R}(\text { reflections })=0.0412(1858), \mathrm{wR} 2(\text { reflections })=0.1083(2247)
$$

$$
\mathrm{GOF}=1.036
$$

Radiation type: Mo K/a

Radiation source: micro focus sealed tube

Radiation monochromator: Incoatec Helios multilayer confocal mirror

Measurement device type: Bruker D8 goniometer

Computing structure solution: SHELXT (Sheldrick, 2013)

Computing structure refinement: SHELXL-2014 (Sheldrick, 2014) 


\section{ORTEP views of $\mathbf{4 e}$}

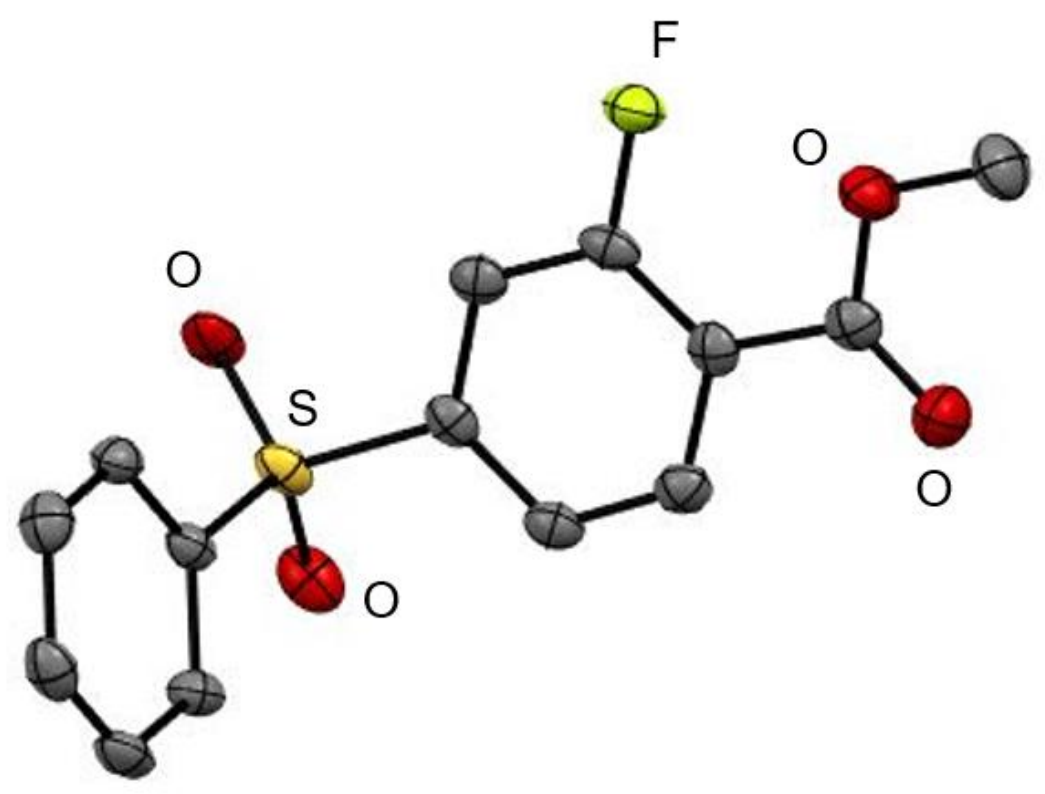

Thermal ellipsoid plot of $\mathbf{4 e}$ at $50 \%$ probability. 


\section{X-Ray Crystallographic Data for $\mathbf{4 g}$}

The structure of $\mathbf{4 g}$ was also determined by $\mathrm{x}$-ray analysis. The single crystal for the analysis was grown by vapor diffusion of hexane to a $\mathrm{CH}_{2} \mathrm{Cl}_{2}$ solution of $\mathbf{4 g}$. X Ray Crystallography: CCDC2120362 contains the supplementary crystallographic data for this paper. The data can be obtained free of charge from the Cambridge Crystallographic Data Centre via www.ccdc.cam.ac.uk/data_request/cif.

\section{Crystal data and structure refinement}

Compound: $\mathbf{4 g}$

Formula: C15 H11 F O3

Formula weight: 258.25

Wave length: $=0.71073$

Crystal system: monoclinic

Space group: P 21/c

Color of crystal: Colorless

Unit cell parameters: $\quad \mathrm{a}=13.5652(8) \AA \quad \alpha=90^{\circ}$

$$
\begin{array}{ll}
\mathrm{b}=12.2770(6) \AA & \beta=97.798(2)^{\circ} \\
\mathrm{c}=7.0299(5) \AA & \gamma=90^{\circ}
\end{array}
$$

Temperature of data collection: $100(\mathrm{~K})$

Values of $\mathrm{Z}, \mathrm{R}, \mathrm{GOF}: \mathrm{Z}=4$

$$
\begin{aligned}
& \mathrm{R}(\text { reflections })=0.0458(1621), \mathrm{wR} 2(\text { reflections })=0.1246(2053) \\
& \mathrm{GOF}=1.070
\end{aligned}
$$

Radiation type: Mo K/a

Radiation source: micro focus sealed tube

Radiation monochromator: Incoatec Helios multilayer confocal mirror

Measurement device type: Bruker D8 goniometer

Computing structure solution: SHELXT (Sheldrick, 2014)

Computing structure refinement: SHELXL-2018 (Sheldrick, 2018) 
ORTEP views of $\mathbf{4 g}$

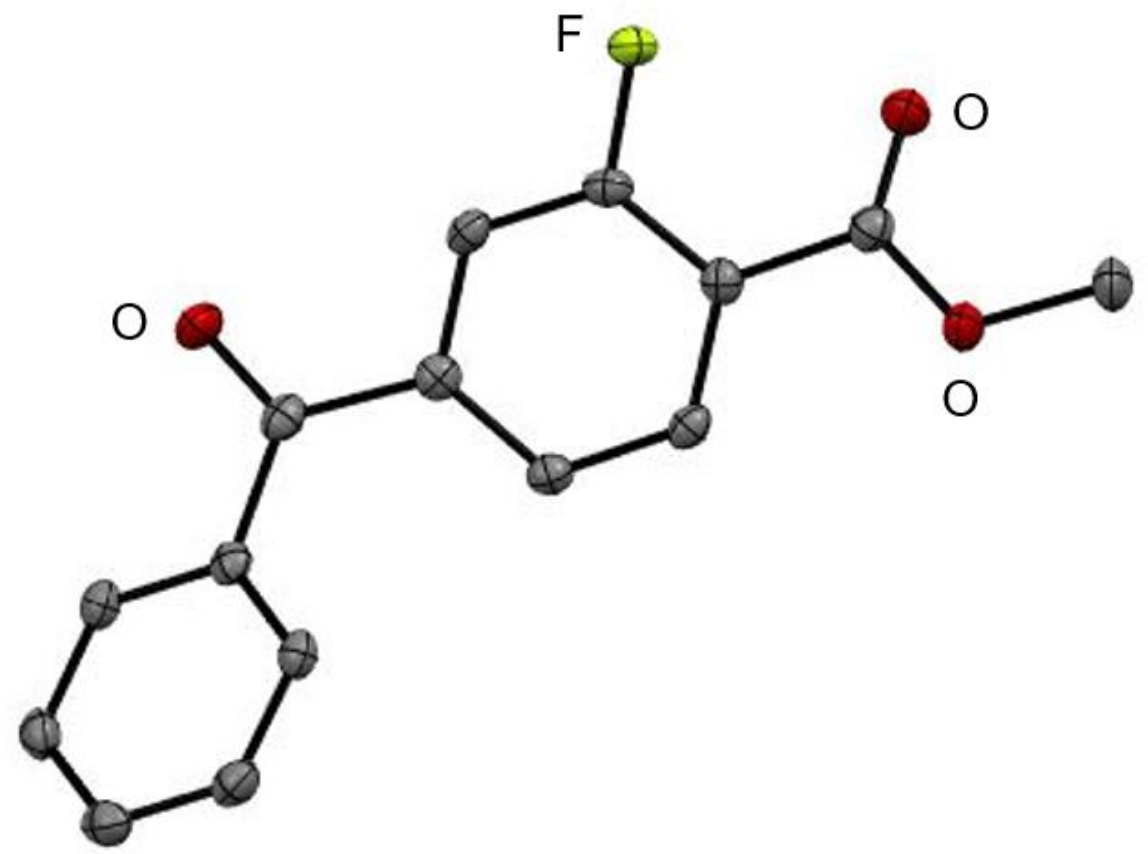

Thermal ellipsoid plot of $\mathbf{4 g}$ at $50 \%$ probability. 


\section{X-Ray Crystallographic Data for 4l-Bn}

The structure of 41-Bn was also determined by x-ray analysis. The single crystal for the analysis was grown by vapor diffusion of hexane to a $\mathrm{Et}_{2} \mathrm{O}$ solution of 4l-Bn. X Ray Crystallography: CCDC2120363 contains the supplementary crystallographic data for this paper. The data can be obtained free of charge from the Cambridge Crystallographic Data Centre via www.ccdc.cam.ac.uk/data_request/cif.

\section{Crystal data and structure refinement}

Compound: 4l-Bn

Formula: C16 H10 F3 N O2

Formula weight: 305.26

Wave length: $=0.71073$

Crystal system: monoclinic

Space group: P 21/c

Color of crystal: Colorless

Unit cell parameters: $\quad \mathrm{a}=5.9014(11) \AA \quad \alpha=90^{\circ}$

$$
\begin{array}{ll}
\mathrm{b}=26.086(5) \AA & \beta=91.987(6)^{\circ} \\
\mathrm{c}=8.7748(18) \AA & \gamma=90^{\circ}
\end{array}
$$

Temperature of data collection: $100(\mathrm{~K})$

Values of $Z, R, G O F: ~ Z=4$

$$
\mathrm{R}(\text { reflections })=0.0537(1838), \mathrm{wR} 2(\text { reflections })=0.1359(2377)
$$

$$
\mathrm{GOF}=1.073
$$

Radiation type: Mo K/a

Radiation source: micro focus sealed tube

Radiation monochromator: Incoatec Helios multilayer confocal mirror

Measurement device type: Bruker D8 goniometer

Computing structure solution: SHELXT (Sheldrick, 2013)

Computing structure refinement: SHELXL-2014 (Sheldrick, 2014) 
ORTEP views of 4l-Bn

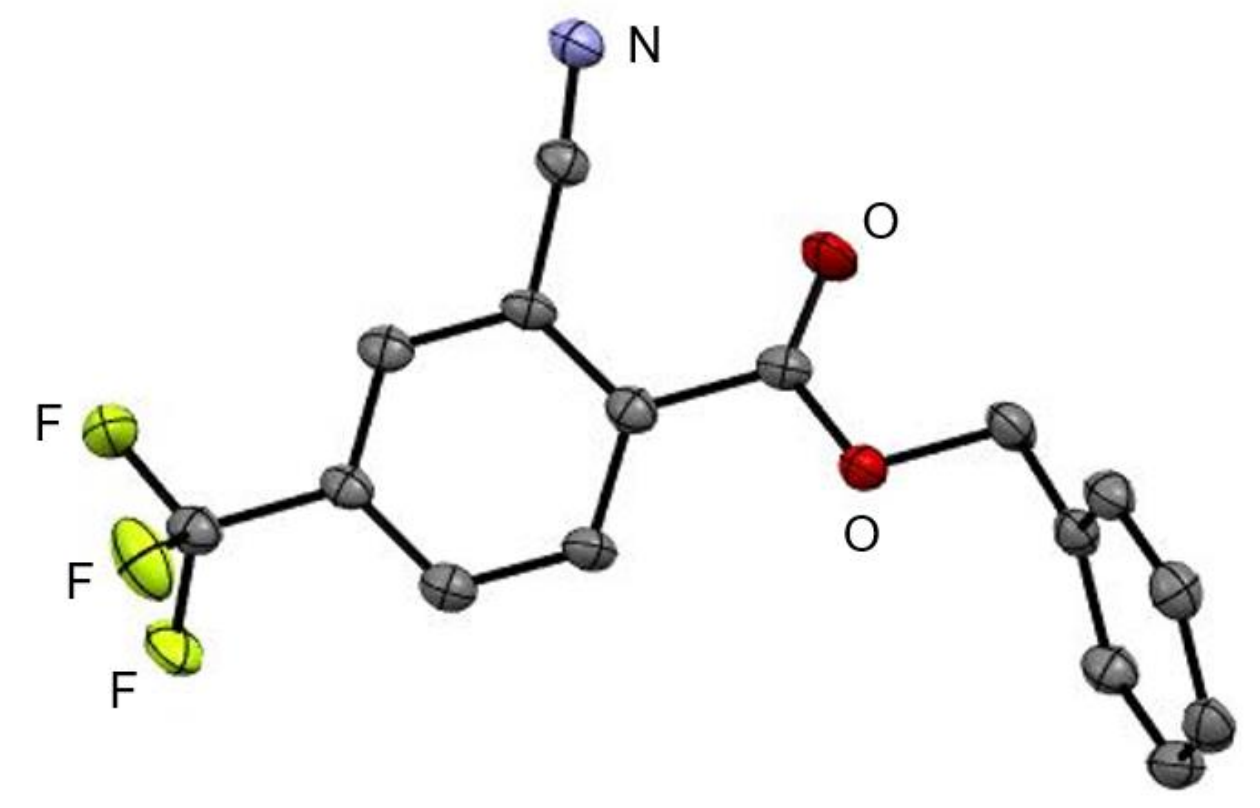

Thermal ellipsoid plot of $\mathbf{4 I - B n}$ at $50 \%$ probability. 


\section{References}

1. C.-T. Yang, J. Han, J. Liu, M. Gu, Y. Li, J. Wen, H.-Z. Yu, S. Hu, X. Wang. Org. Biomol. Chem. 2015, 13, 2541.

2. X. Wu, G. Yan. Synlett 2015, 26, 537.

3. M. V. Joannou, B. S. Moyer, S. J. Meek. J. Am. Chem. Soc. 2015, 137, 6176.

4. (a) M. Shigeno, K. Hanasaka, K. Sasaki, K. Nozawa-Kumada, Y. Kondo. Chem. Eur. J. 2019, 25, 3235. (b) H. Yildirimyan, G. Gattow. Z. Anorg. Allg. Chem. 1984, 519, 204. (c) E. E. Coyle, B. J. Doonan, A. J. Holohan, K. A. Walsh, F. Lavigne, E. H. Krenske, C. J. O’Brien. Angew. Chem. Int. Ed. 2014, 53, 12907.

5. K. Fuchibe, Y. Ohshima, K. Mitomi, T. Akiyama. J. Fluorine Chem. 2007, 128, 1158.

6. J. Yang, Q.-Y. Han, C.-L. Zhao, T. Dong, Z.-Y. Hou, H.-L. Qin, C.-P. Zhang. Org. Biomol. Chem. 2016, 14, 7654 .

7. S. M. Johnson, S. Connelly, I. A. Wilson, J. W. Kelly. J. Med. Chem. 2008, 51, 6348.

8. S. Lindner, S. Bräse, K.-S. Masters. J. Fluorine Chem. 2015, 179, 102.

9. S. Yamada, A. Gavryushin, P. Knochel. Angew. Chem. Int. Ed. 2010, 49, 2215.

10. M. Gholinejad, F. Hamed, P. Bijib. Dalton Trans. 2015, 44, 14293.

11. X. Huanga, J. Tiana, F. Xua, X. Liua, Y. Lia, Y. Guoa, W. Chua, Z. Sun. Tetrahedron Lett. 2018, 59, 681 .

12. S. L. Adamski-Werner, S. K. Palaninathan, J. C. Sacchettini, J. W. Kelly. J. Med. Chem. 2004, 47, 355.

13. H.-Q. Do, O. Daugulis. J. Am. Chem. Soc. 2011, 133, 13577.

14. J. D. Arredondo, H. Li, J. Balsells. Org. Synth. 2012, 89, 460.

15. P. Ghosh, B. Ganguly, E. Perl, S. Das.Tetrahedron Lett. 2017, 58, 2751.

16. C. Liu, G. Meng, Y. Liu, R. Liu, R. Lalancette, R. Szostak, M. Szostak. Org. Lett. 2016, 18, 4194.

17. L. Tang, F. Yang, H. Cheng, C. Tan, C. Jin, H. Chen, Y. Huang, S. Zhang, W. Song, J. Tan. Org. Lett. 2020, 22, 8618.

18. L. J. Kaplan, J. C. Martin. J. Am. Chem. Soc. 1973, 95, 3, 793.

19. N.-W. Liu, K. Hofman, A. Herbert, G. Manolikakes. Org. Lett. 2018, $20,760$.

20. R. R. Putta, S. Chun, S. H. Choi, S. B. Lee, D.-C. Oh, S. Hong. J. Org. Chem. 2020, 85, 15396.

21. W. E. Doering, L. H. Knox, J. Am. Chem. Soc. 1952, 74, 5683.

22. A. P. Crew, K. R. Hornberger, J. Wang, C. M. Crews, S. Jaime-Figueroa, H. Dong, Y. Qian, K. Zimmerman, 2020, WO2020/51564, A1.

23. T. Miyazaki, M. Kawasaki, A. Suzuki, Y. Ito, A. Imanishi, T. Maru, T. Kawamoto, T. Koike. Bioorg. Med. Chem. 2019, 29, 815.

24. J. A. Gladding, J. P. Bacci, S. A. Shaw, A. B. Smith. Tetrahedron 2011, 67, 6697.

25. J. Castañer, J. Riera, J. Carilla, A. Robert, E. Molins, C. Miravitlles. J. Org. Chem. 1991, 56, 103.

26. J. Gassmann, J. Voss. Z. Naturforsch. B 2008, 63, 1291.

27. Q. Xia, X. Liu, Y. Zhang, C. Chen, W. Chen. Org. Lett., 2013, 15, 3326. 
${ }^{1} \mathrm{H}$ NMR spectra of $1 \mathrm{i}\left(\mathrm{CDCl}_{3}, 600 \mathrm{MHz}\right)$

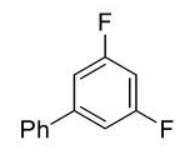

$1 \mathrm{i}$
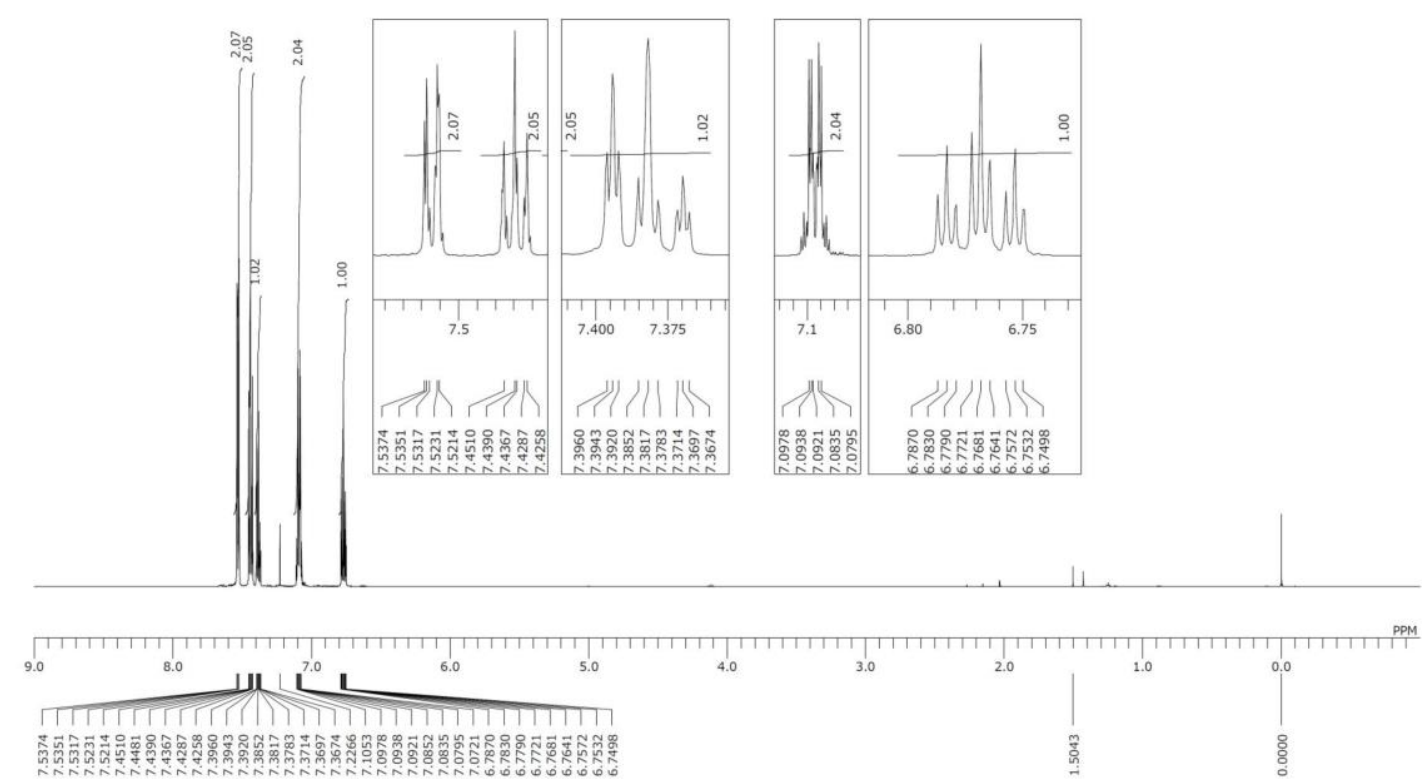

${ }^{13} \mathrm{C}$ NMR spectra of $1 \mathrm{i}\left(\mathrm{CDCl}_{3}, 150 \mathrm{MHz}\right)$

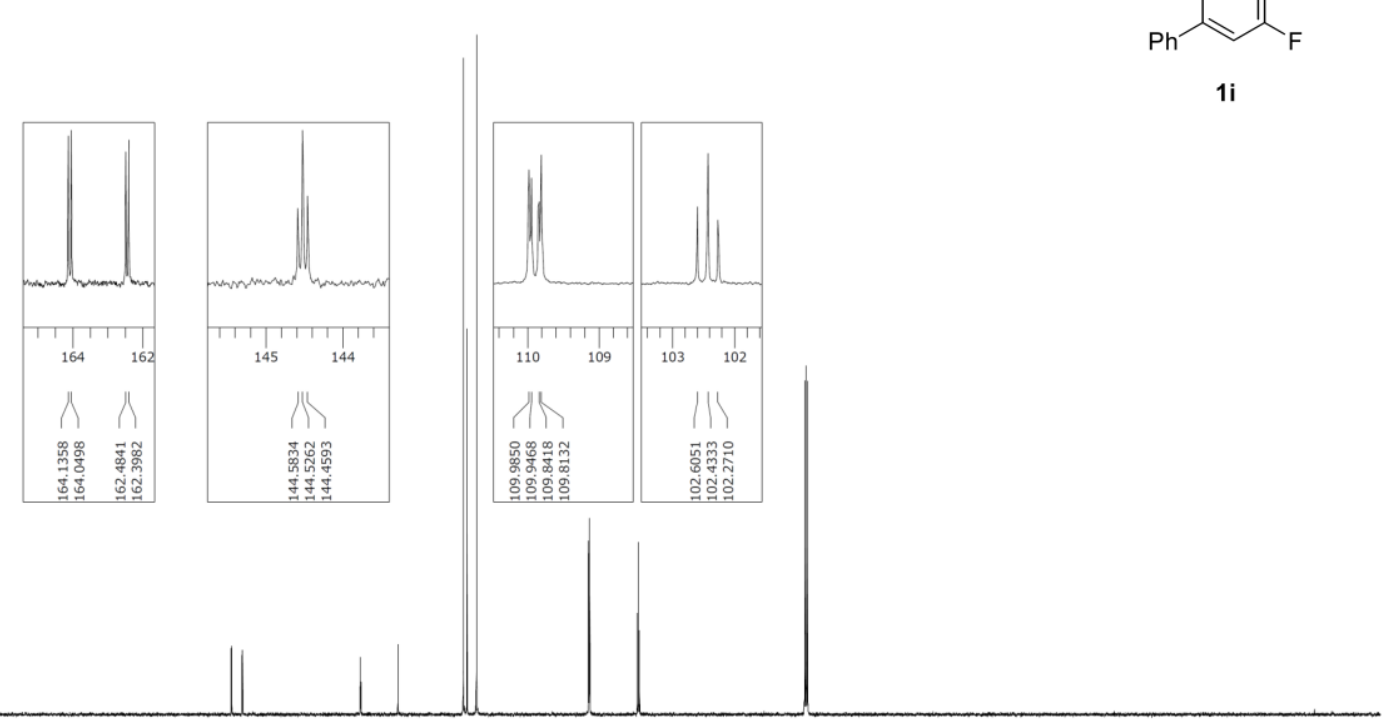

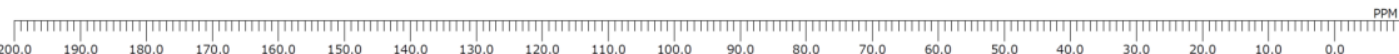

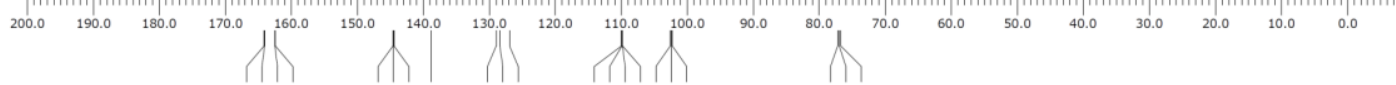

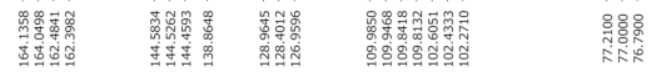


${ }^{19} \mathrm{~F}$ NMR spectra of $1 \mathbf{i}\left(\mathrm{CDCl}_{3}, 565 \mathrm{MHz}\right)$

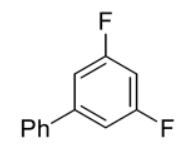

$1 \mathrm{i}$

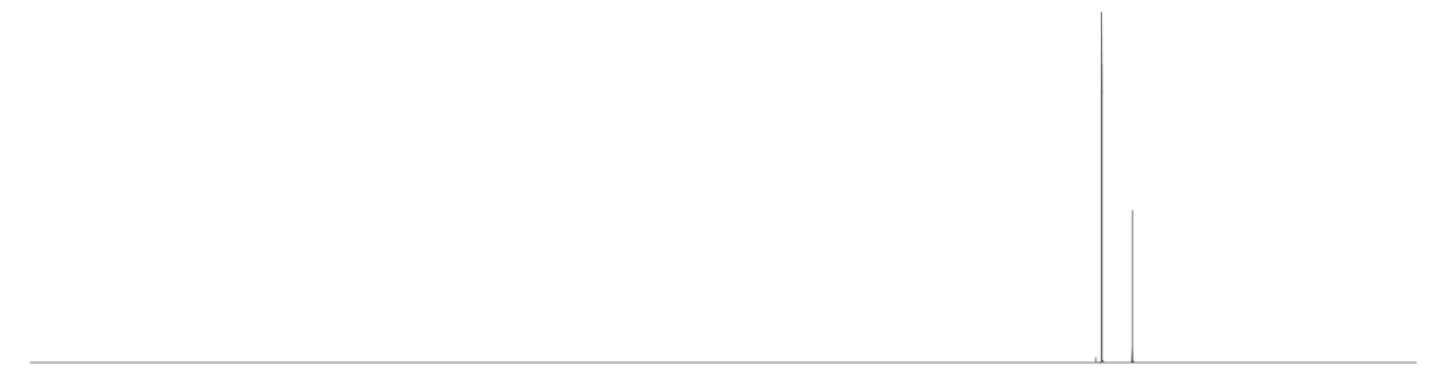

|

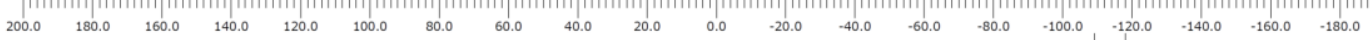

II

${ }^{1} \mathrm{H}$ NMR spectra of $1 \mathbf{j}\left(\mathrm{CDCl}_{3}, 600 \mathrm{MHz}\right)$

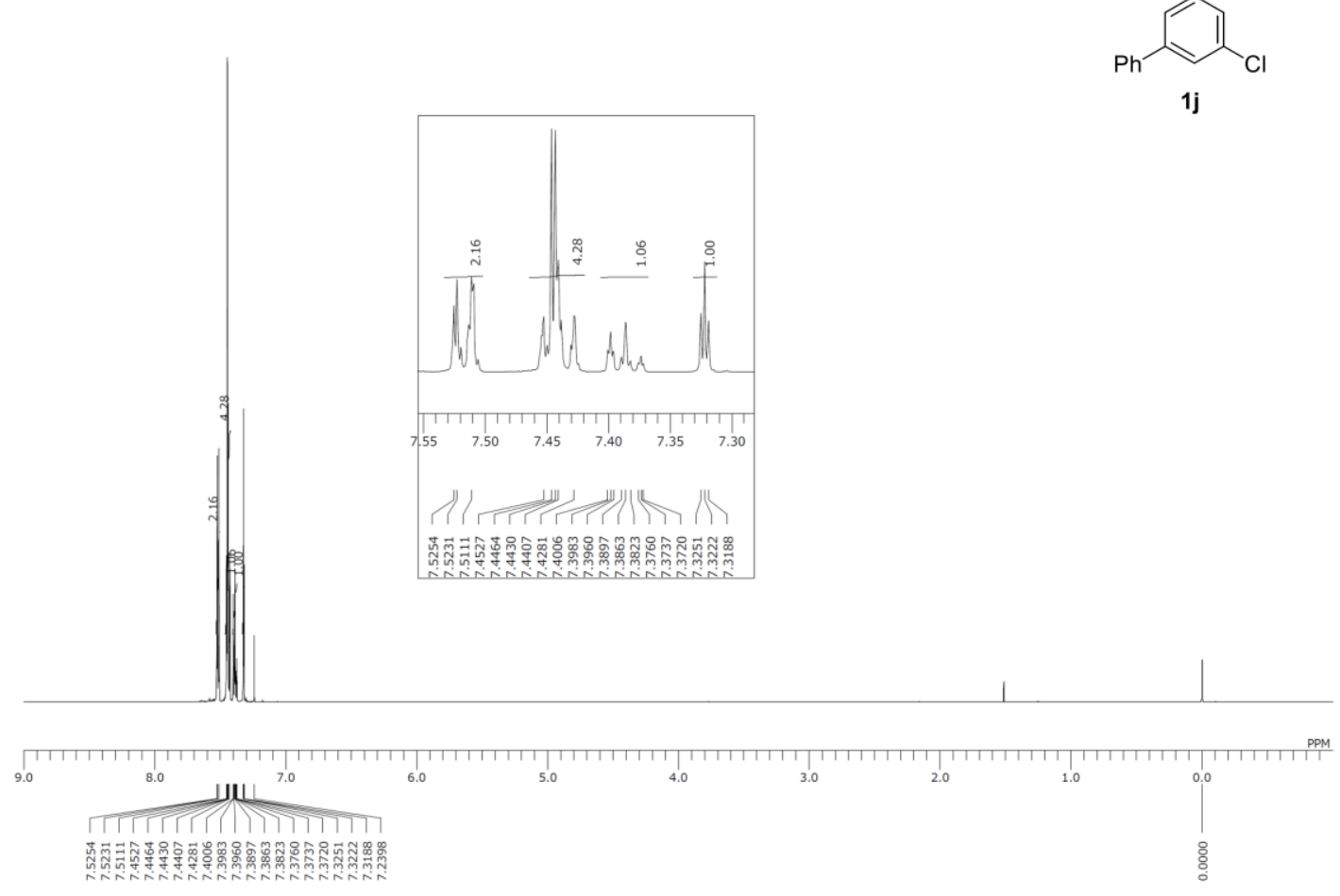


${ }^{13} \mathrm{C} \mathrm{NMR}$ spectra of $1 \mathrm{j}\left(\mathrm{CDCl}_{3}, 150 \mathrm{MHz}\right)$
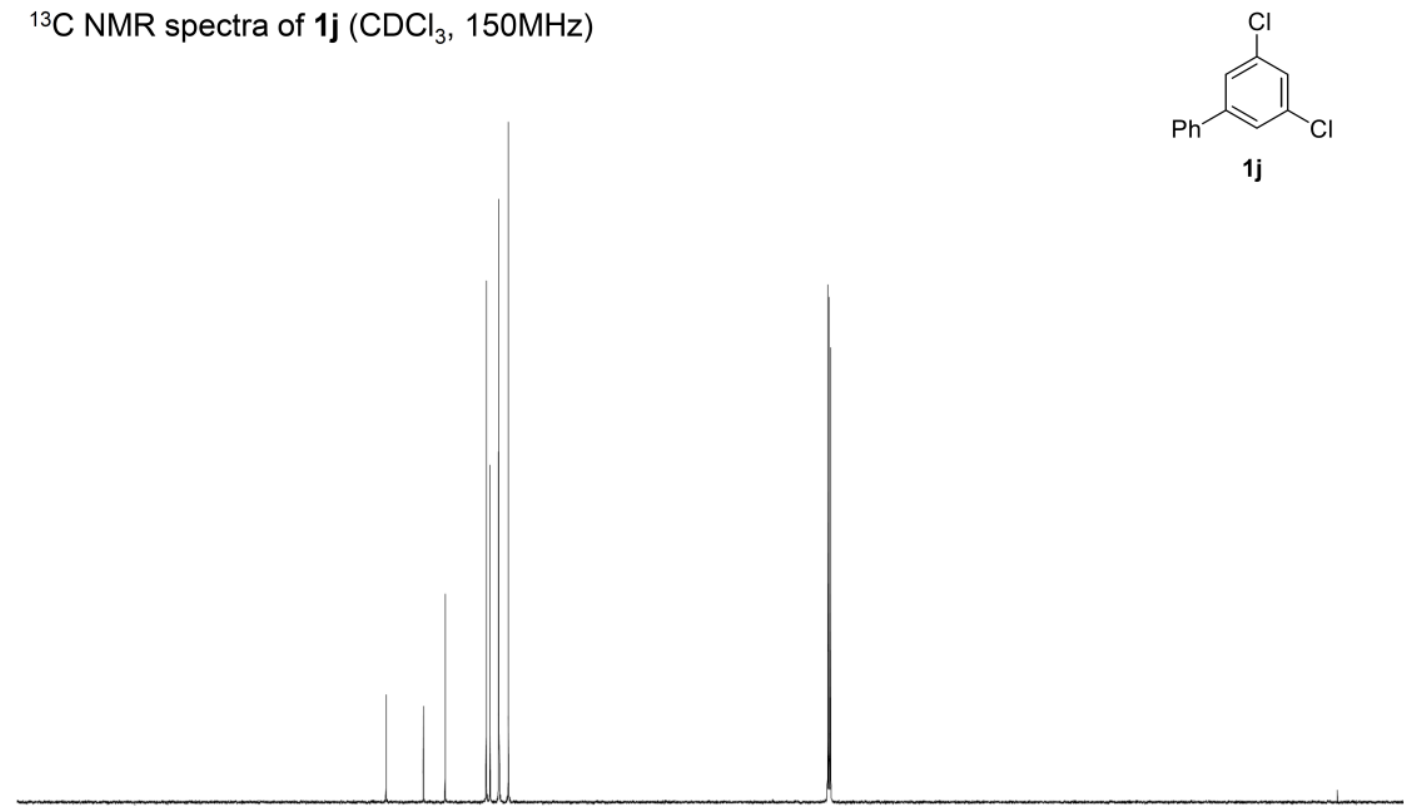

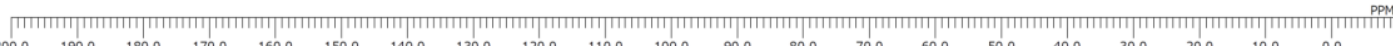

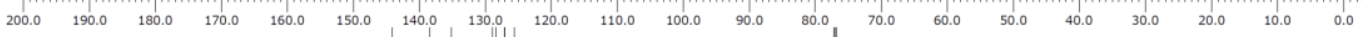

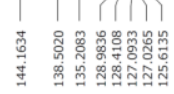

${ }^{1} \mathrm{H}$ NMR spectra of $1 \mathbf{k}\left(\mathrm{CDCl}_{3}, 400 \mathrm{MHz}\right)$
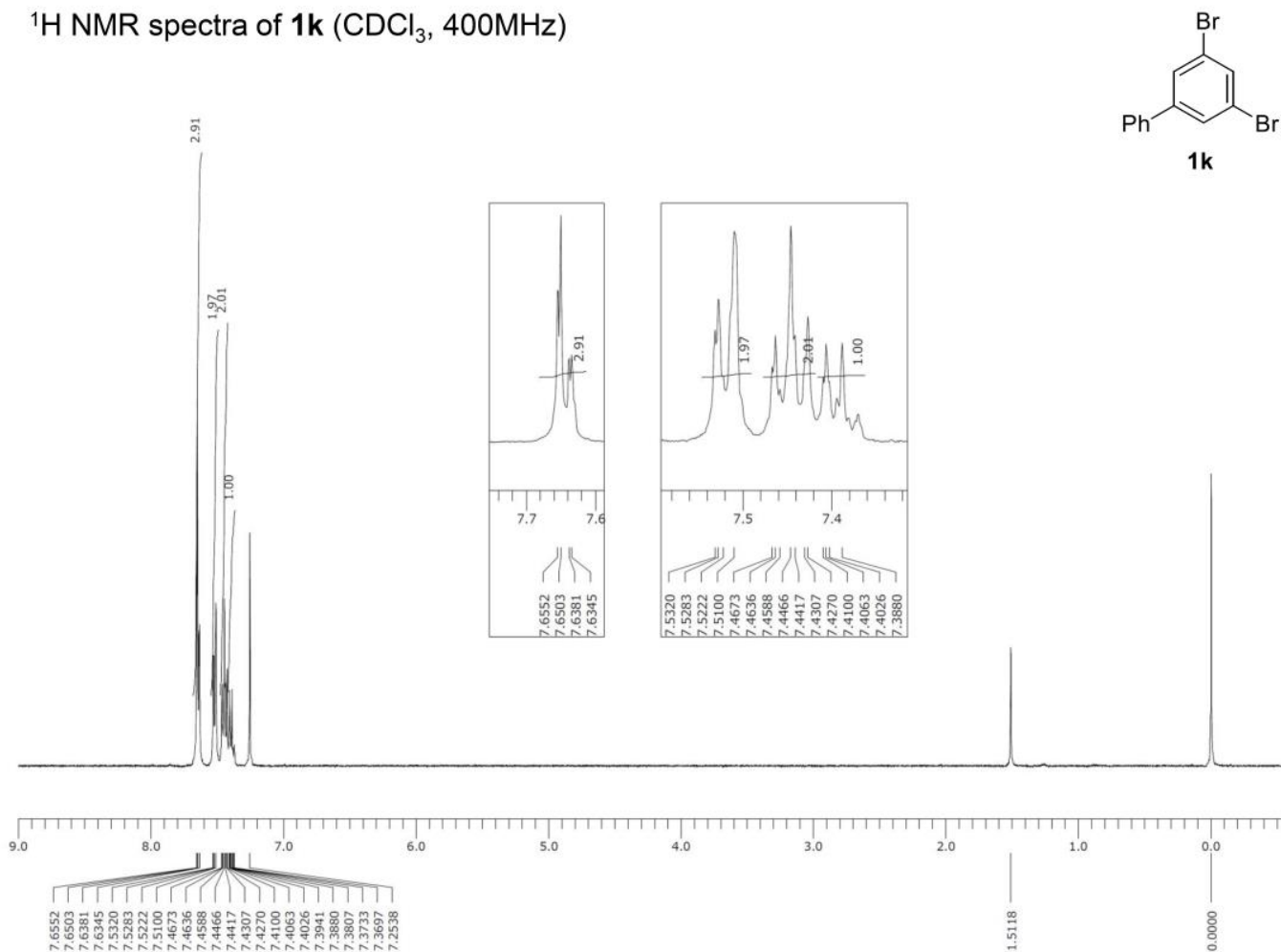

$1 \mathrm{k}$ 
${ }^{13} \mathrm{C}$ NMR spectra of $\mathbf{1 k}\left(\mathrm{CDCl}_{3}, 150 \mathrm{MHz}\right)$
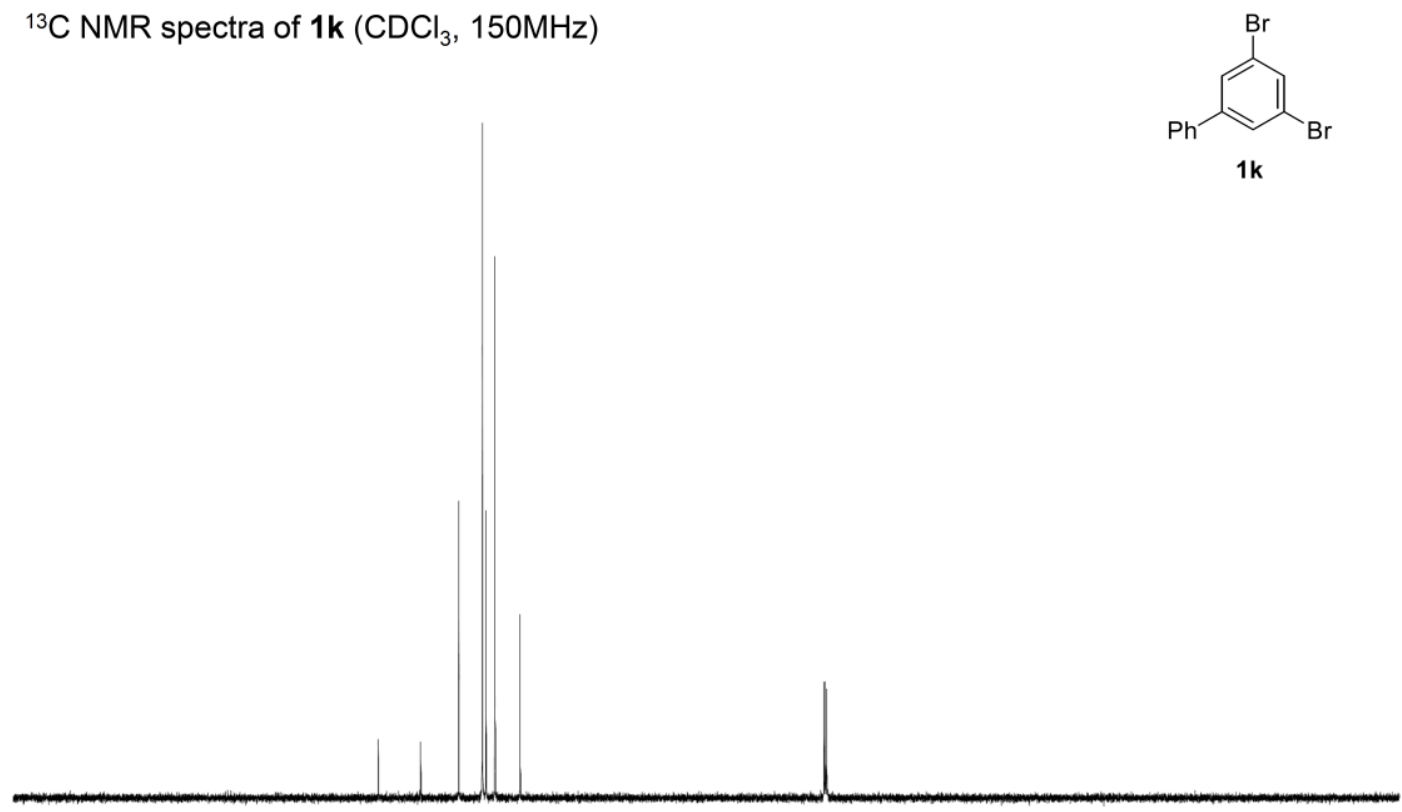

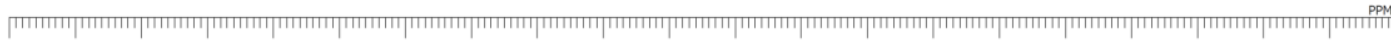

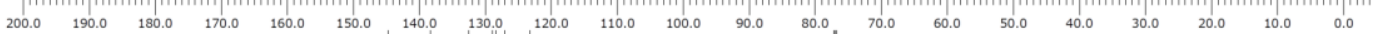

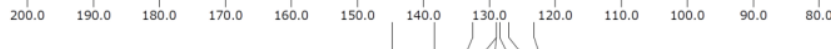

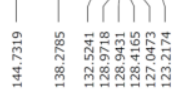

${ }^{1} \mathrm{H}$ NMR spectra of $1 \mathrm{l}\left(\mathrm{CDCl}_{3}, 600 \mathrm{MHz}\right)$

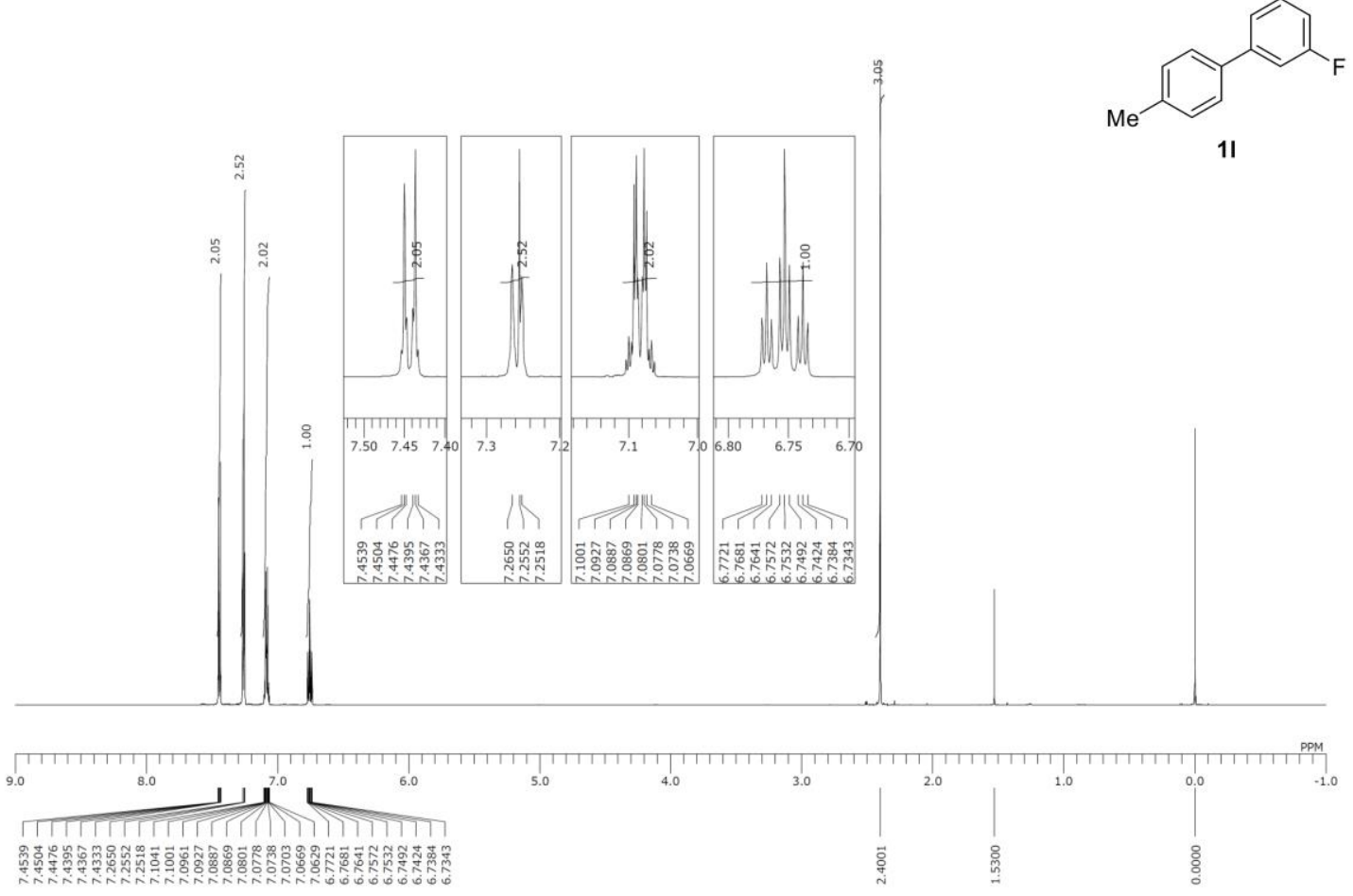


${ }^{13} \mathrm{C} \mathrm{NMR}$ spectra of $1 \mathrm{l}\left(\mathrm{CDCl}_{3}, 150 \mathrm{MHz}\right)$

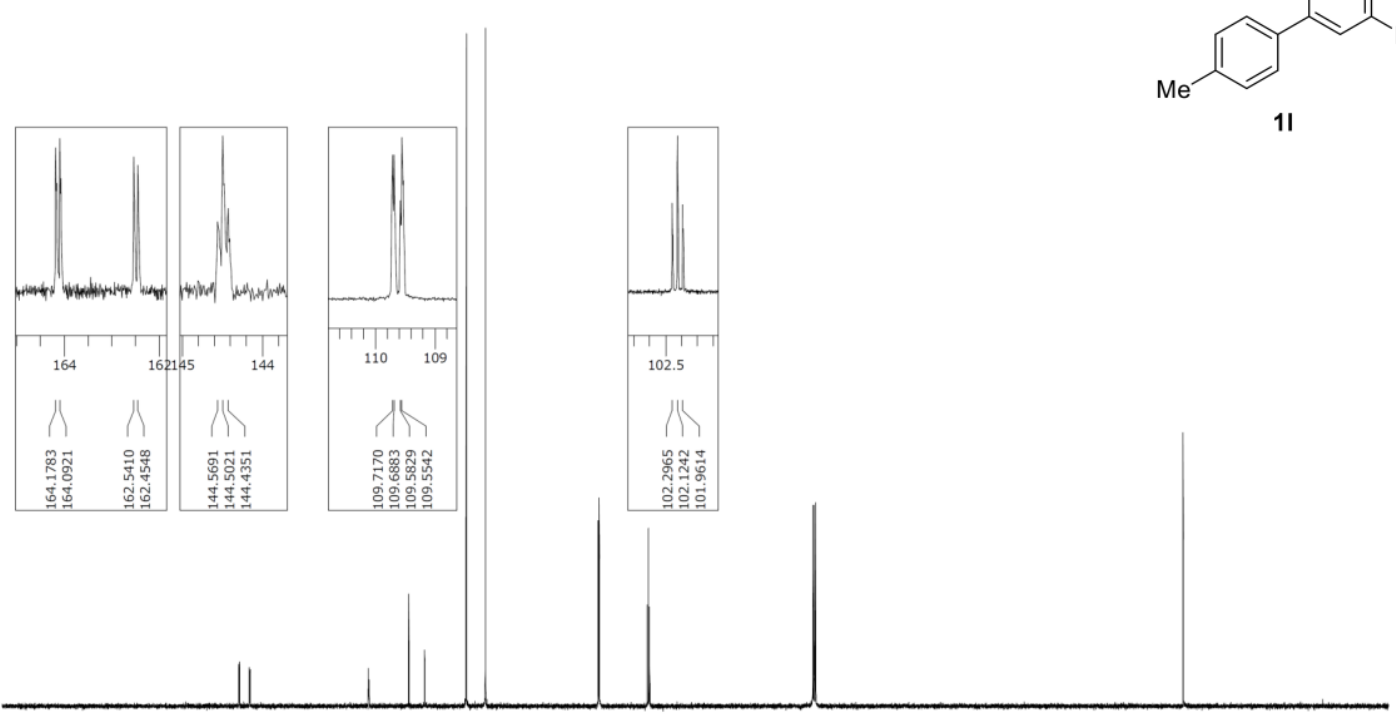

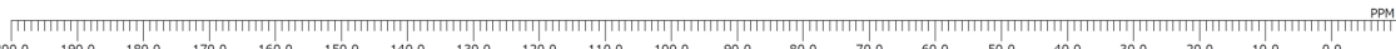

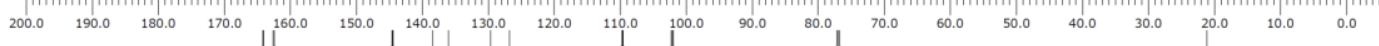
$\left.\lambda \prod_{1}^{160.0}\right|_{\mid} ^{130.0} \prod_{\mid}^{130.0}$

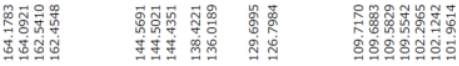

${ }^{19} \mathrm{~F}$ NMR spectra of $11\left(\mathrm{CDCl}_{3}, 565 \mathrm{MHz}\right)$
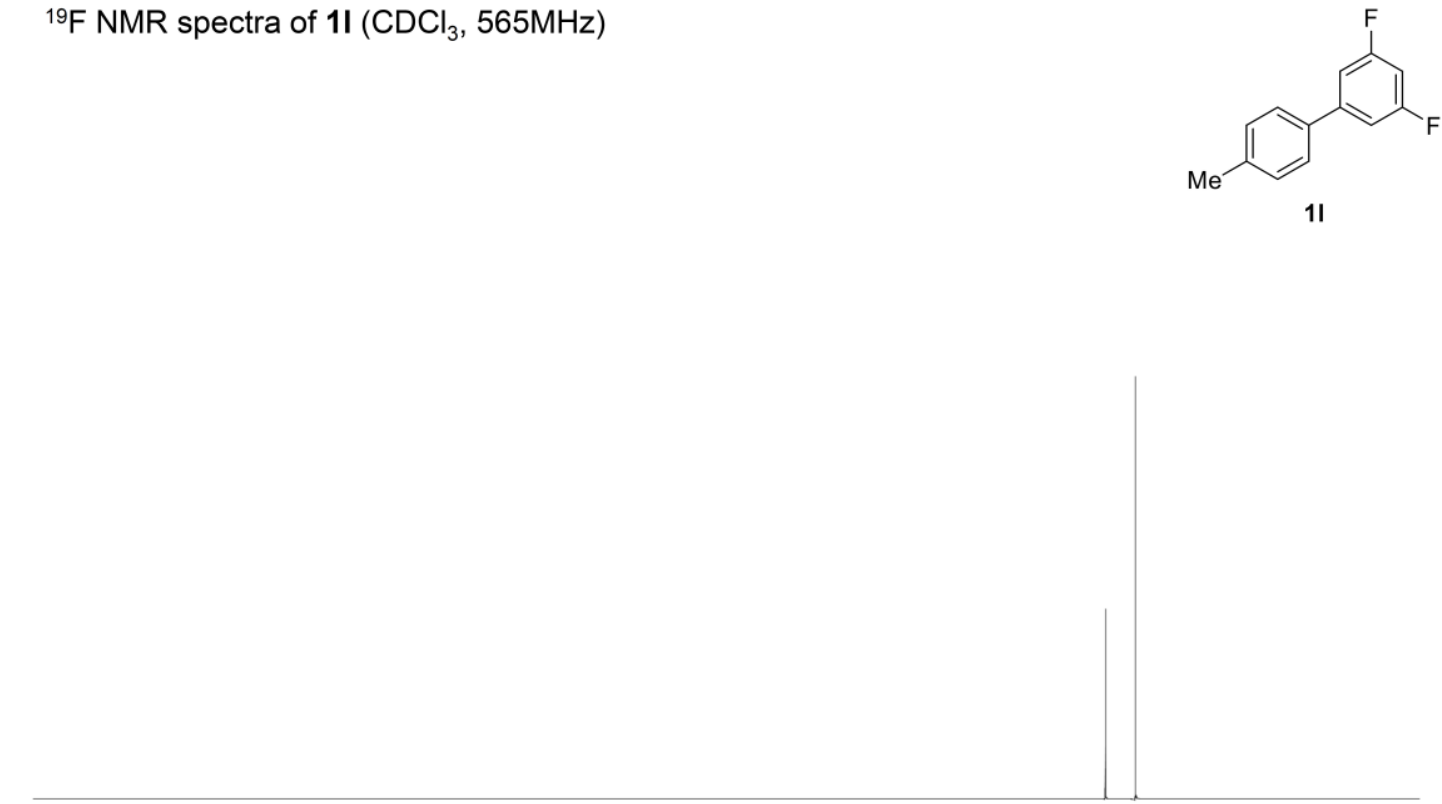

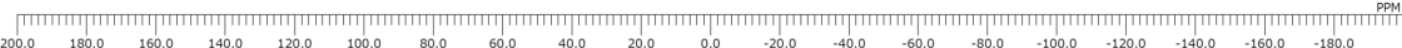

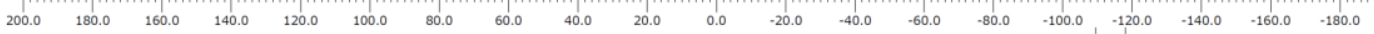


${ }^{1} \mathrm{H}$ NMR spectra of $1 \mathrm{~m}\left(\mathrm{CDCl}_{3}, 600 \mathrm{MHz}\right)$

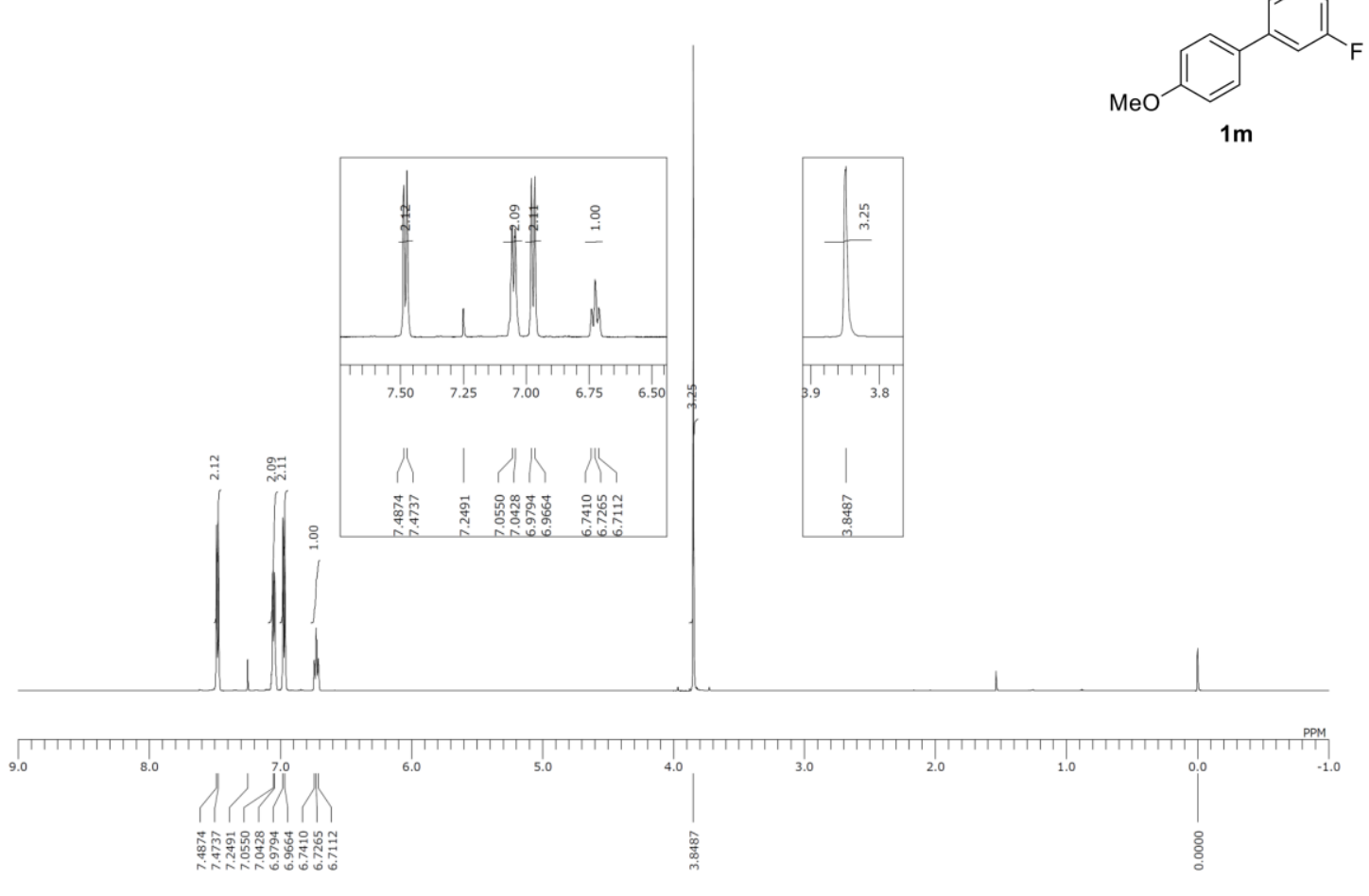

${ }^{13} \mathrm{C} \mathrm{NMR}$ spectra of $1 \mathrm{~m}\left(\mathrm{CDCl}_{3}, 150 \mathrm{MHz}\right)$

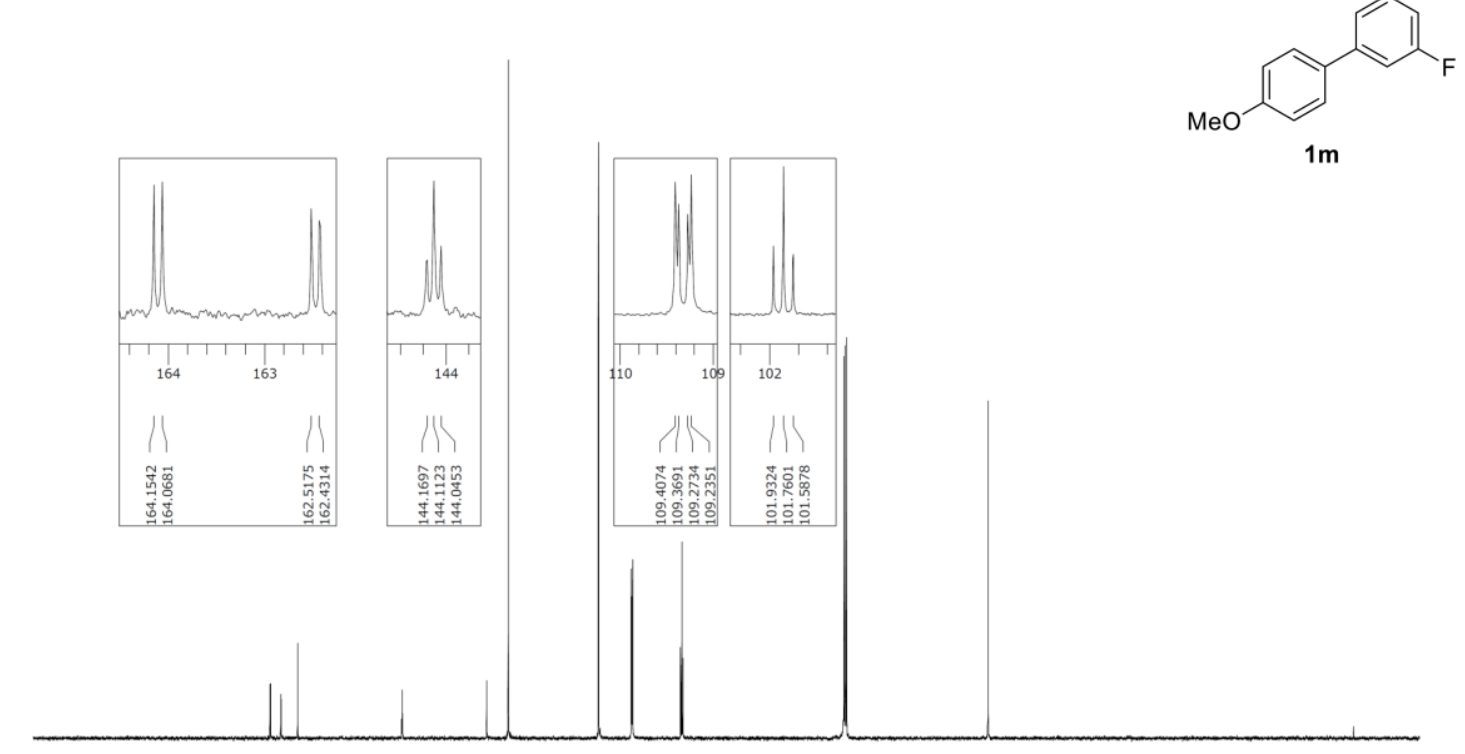

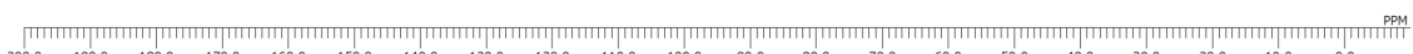

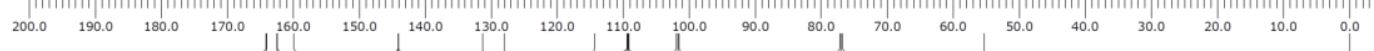
तो A 
${ }^{19} \mathrm{~F}$ NMR spectra of $1 \mathrm{~m}\left(\mathrm{CDCl}_{3}, 565 \mathrm{MHz}\right)$
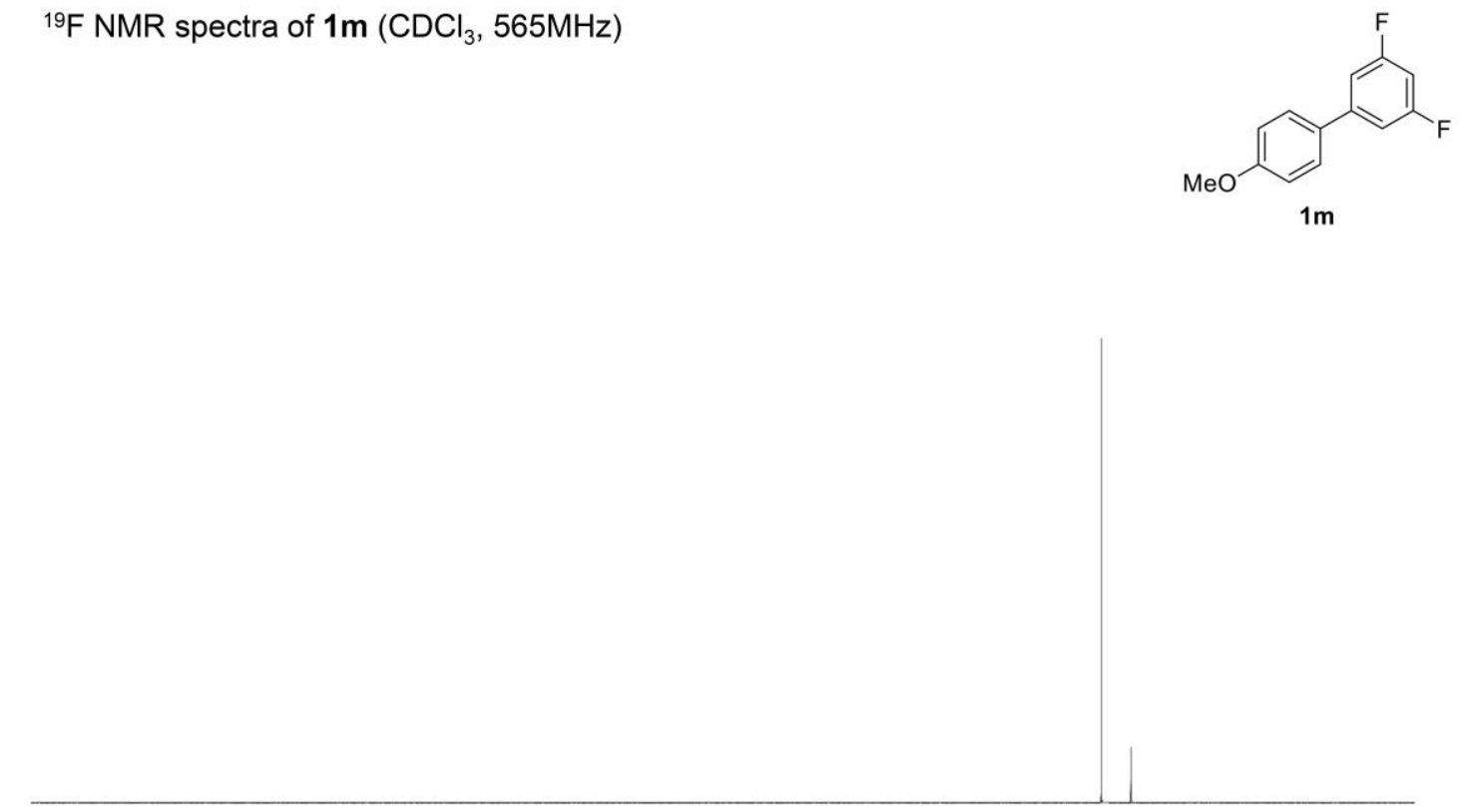

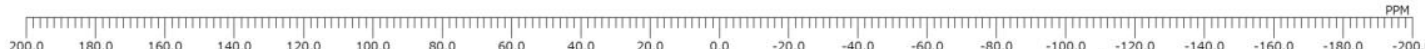
$\begin{array}{lllllllllllllllllllll}200.0 & 180.0 & 160.0 & 140.0 & 120.0 & 100.0 & 80.0 & 60.0 & 40.0 & 20.0 & 0.0 & -20.0 & -40.0 & -60.0 & -80.0 & -100.0 & -120.0 & -140.0 & -160.0 & -180.0 & -200.0\end{array}$ II

${ }^{1} \mathrm{H}$ NMR spectra of $1 \mathrm{n}\left(\mathrm{CDCl}_{3}, 600 \mathrm{MHz}\right)$

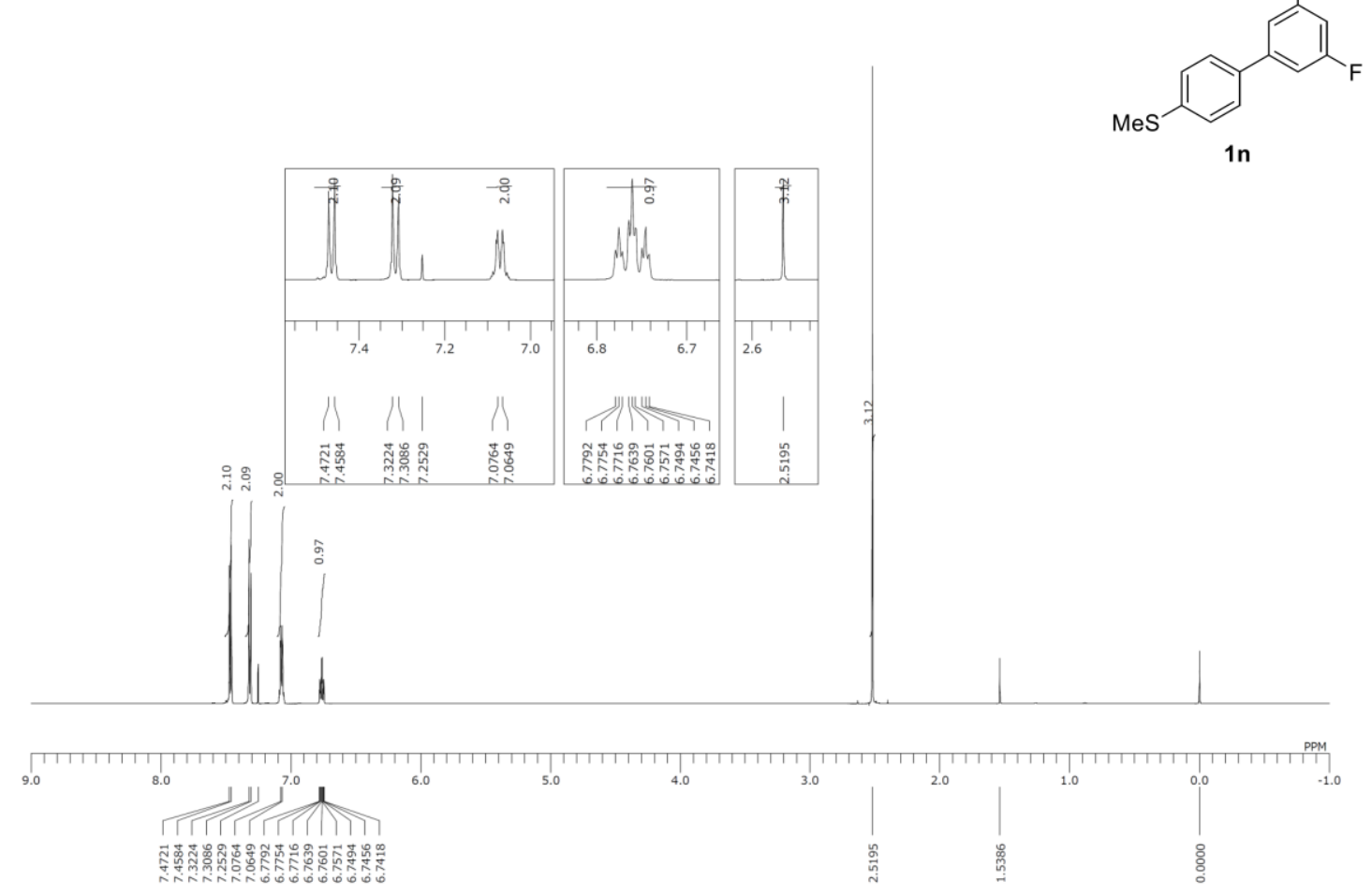


${ }^{13} \mathrm{C}$ NMR spectra of $1 \mathrm{n}\left(\mathrm{CDCl}_{3}, 150 \mathrm{MHz}\right)$
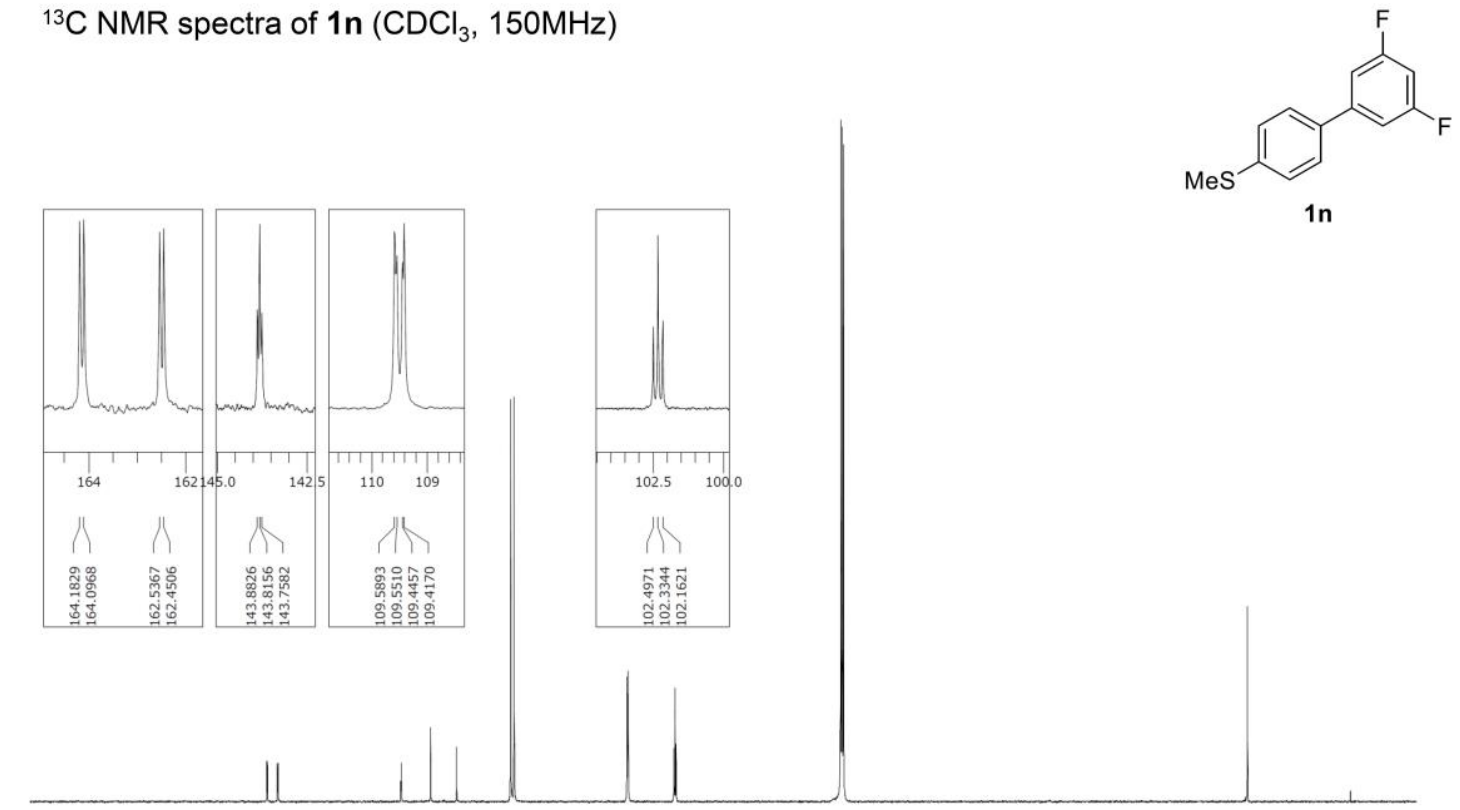

.

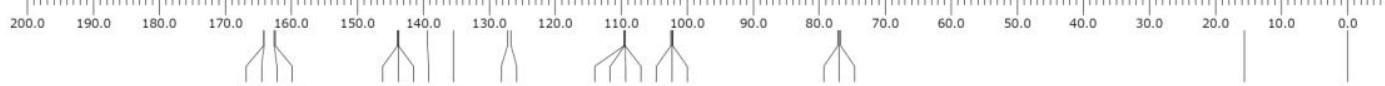

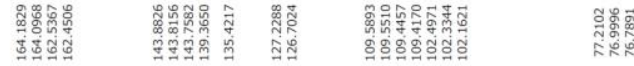

${ }^{19} \mathrm{~F}$ NMR spectra of $1 \mathrm{n}\left(\mathrm{CDCl}_{3}, 565 \mathrm{MHz}\right)$
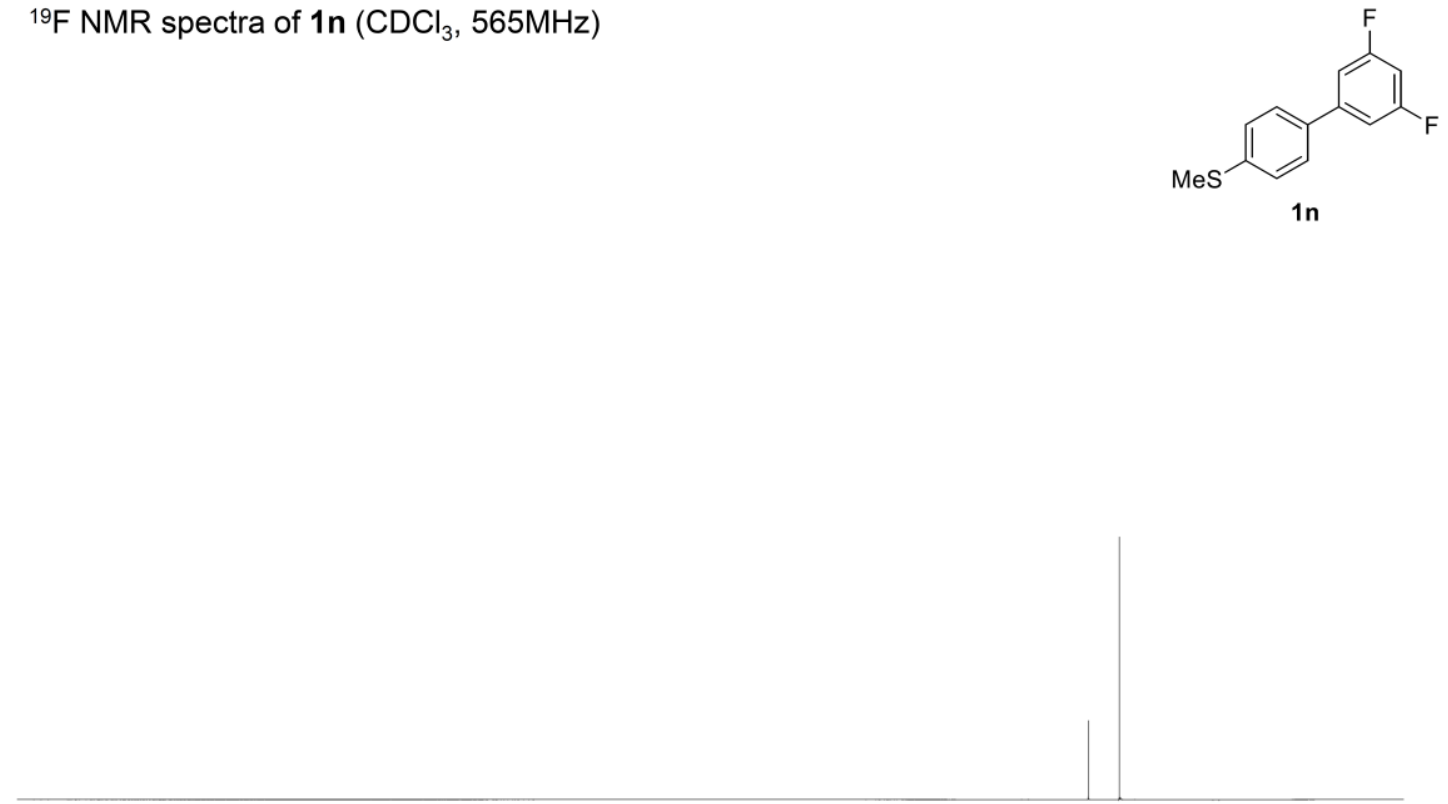

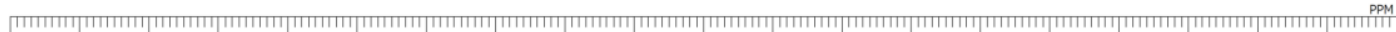

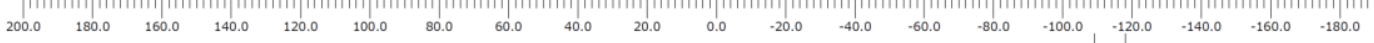


${ }^{1} \mathrm{H}$ NMR spectra of $10\left(\mathrm{CDCl}_{3}, 600 \mathrm{MHz}\right)$
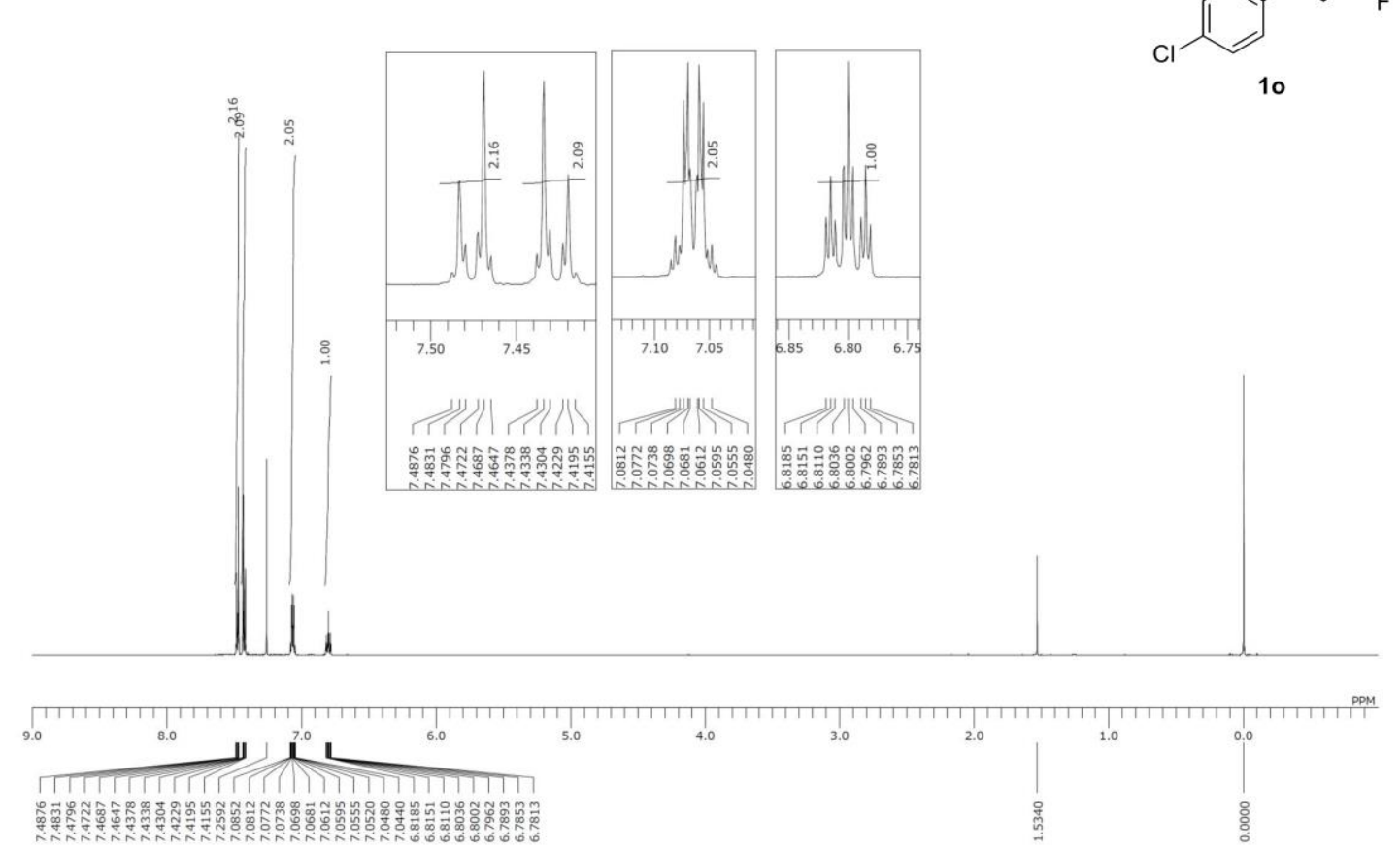

${ }^{13} \mathrm{C}$ NMR spectra of $10\left(\mathrm{CDCl}_{3}, 150 \mathrm{MHz}\right)$
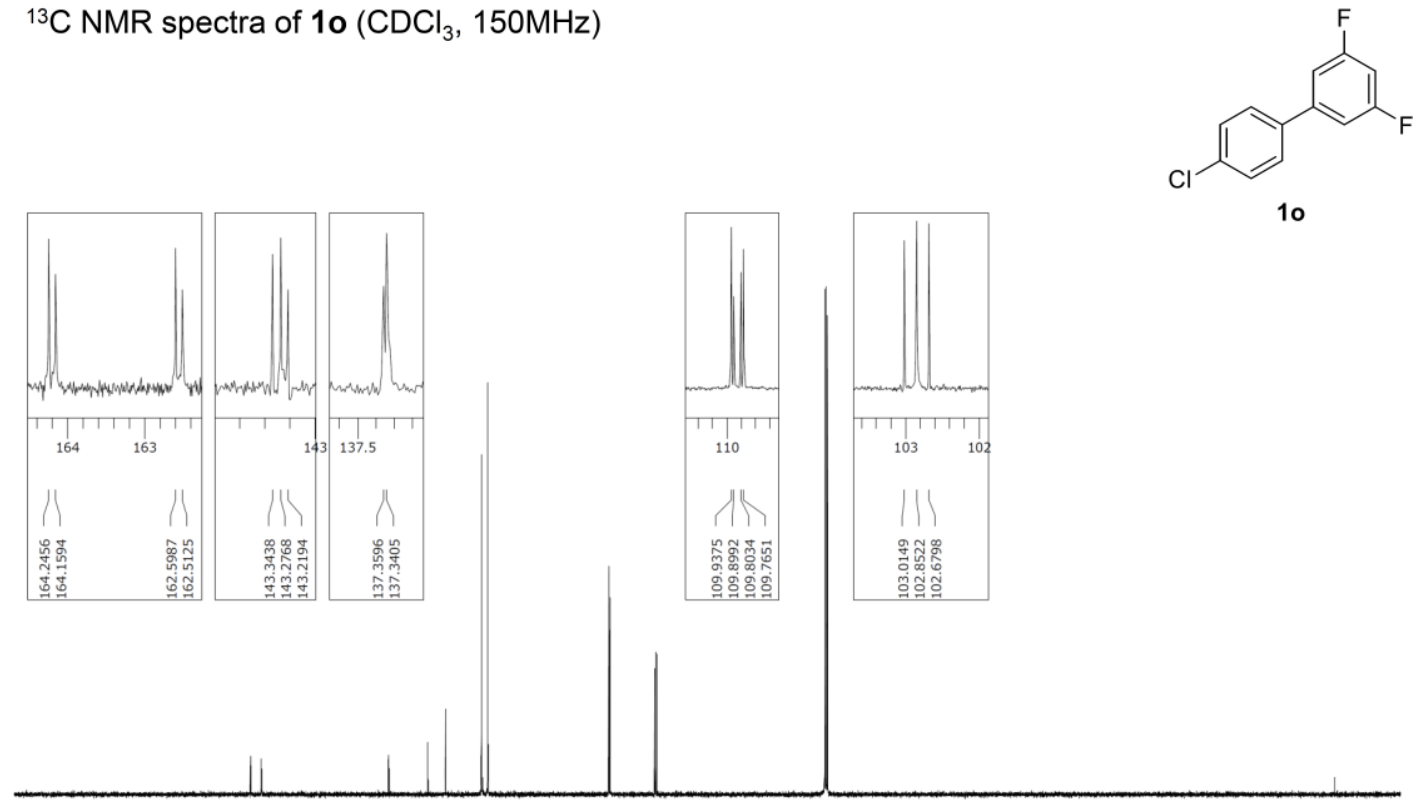

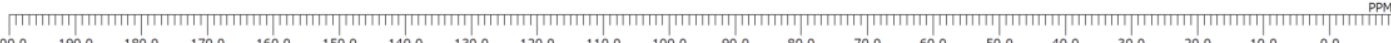

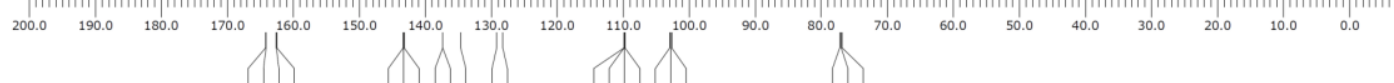

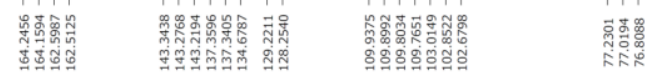


${ }^{19} \mathrm{~F}$ NMR spectra of $10\left(\mathrm{CDCl}_{3}, 565 \mathrm{MHz}\right)$
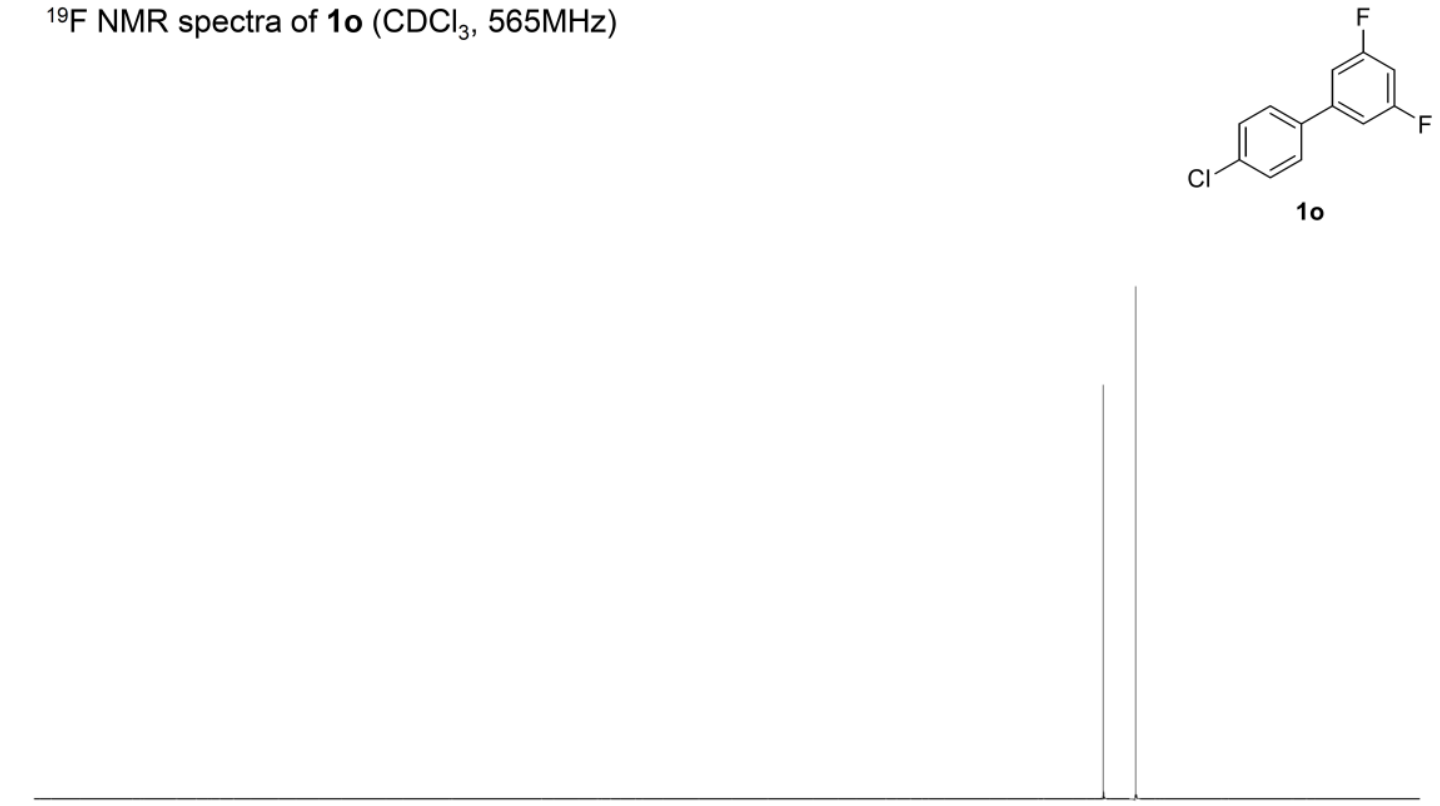

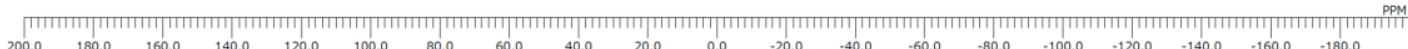

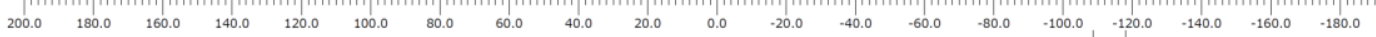

${ }^{1} \mathrm{H}$ NMR spectra of $1 \mathrm{p}\left(\mathrm{CDCl}_{3}, 600 \mathrm{MHz}\right)$

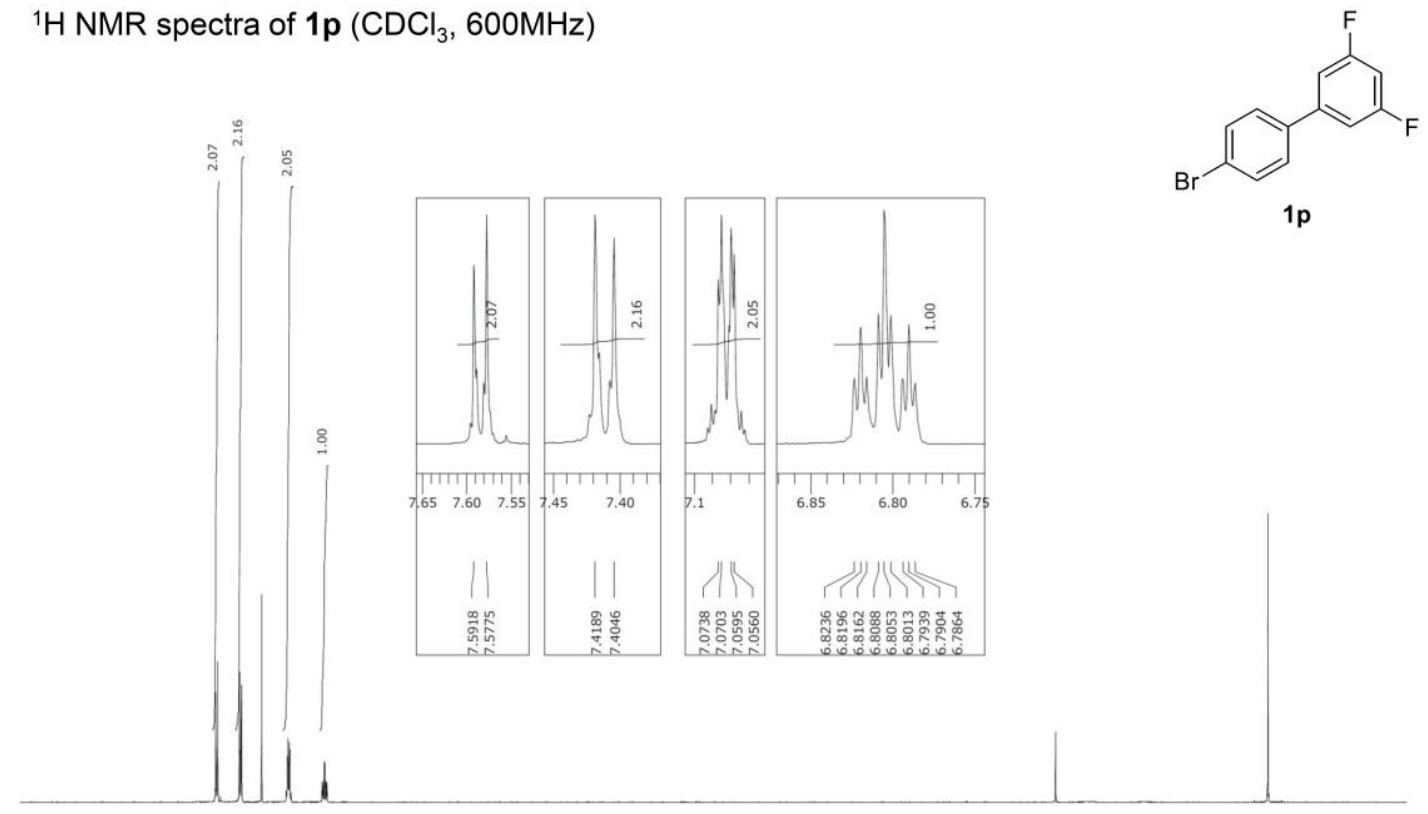

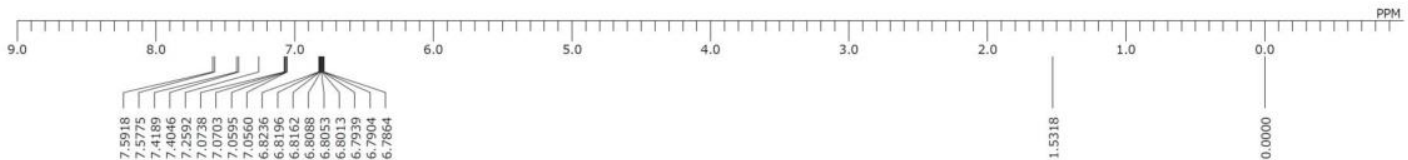


${ }^{13} \mathrm{C}$ NMR spectra of $1 \mathrm{p}\left(\mathrm{CDCl}_{3}, 150 \mathrm{MHz}\right)$

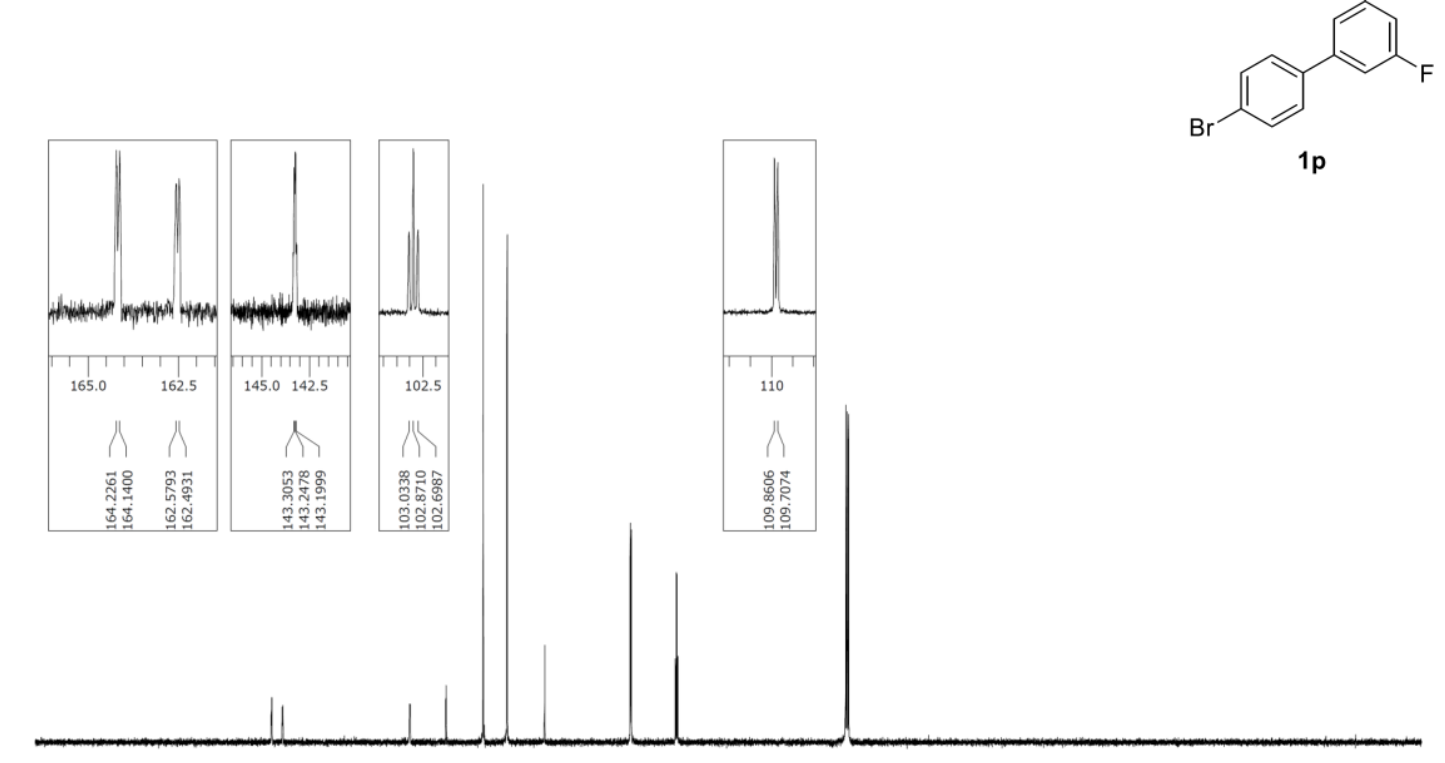

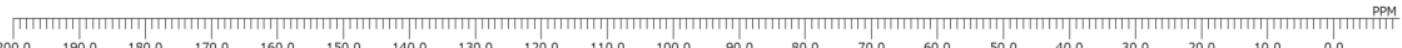

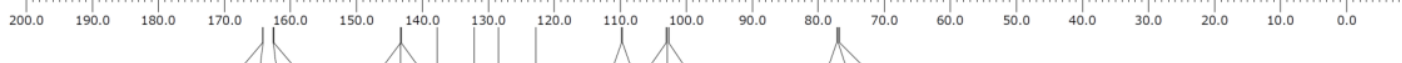

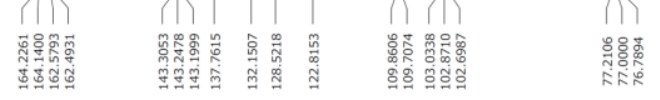

${ }^{19} \mathrm{~F}$ NMR spectra of $1 \mathrm{p}\left(\mathrm{CDCl}_{3}, 565 \mathrm{MHz}\right)$<smiles>Fc1cc(F)cc(-c2ccc(Br)cc2)c1</smiles>

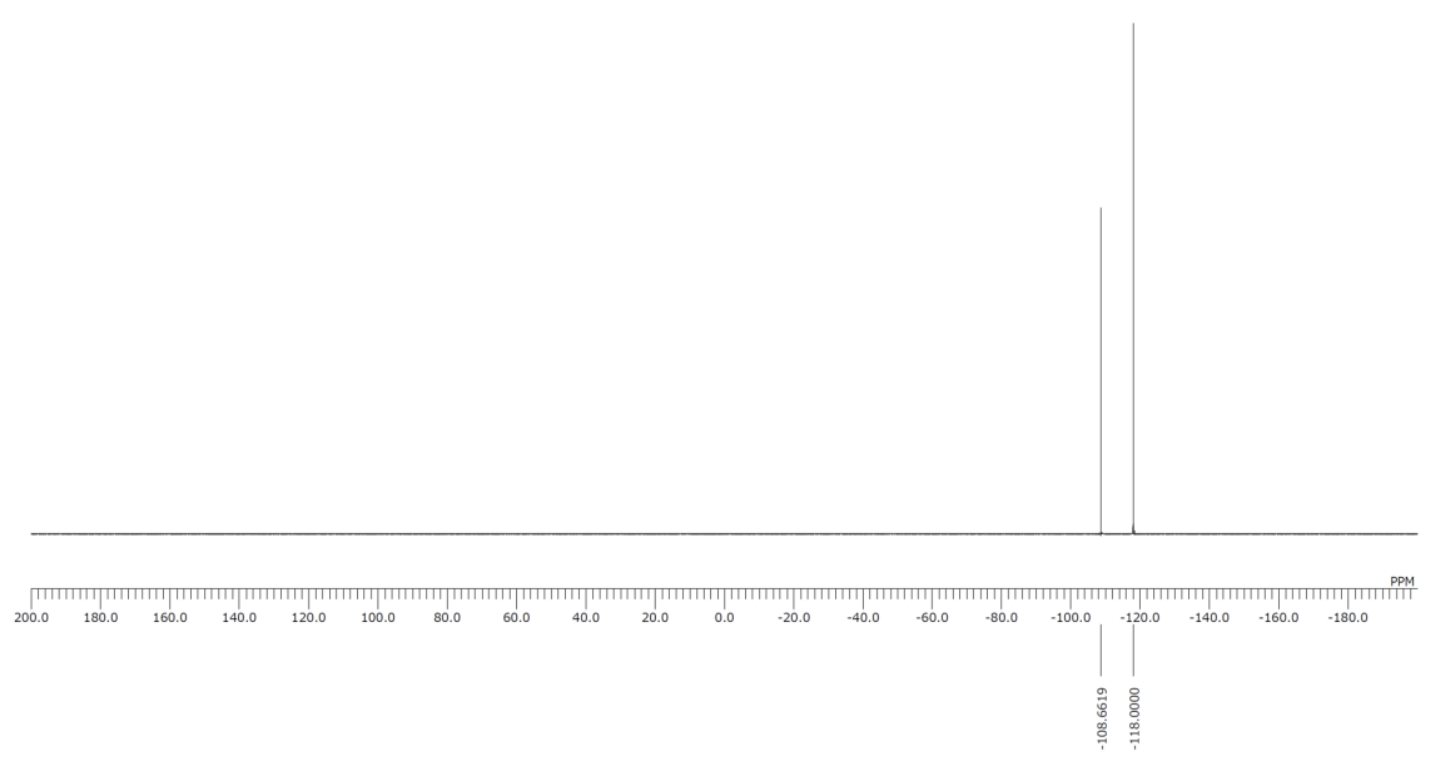


${ }^{1} \mathrm{H}$ NMR spectra of $1 \mathbf{q}\left(\mathrm{CDCl}_{3}, 600 \mathrm{MHz}\right)$

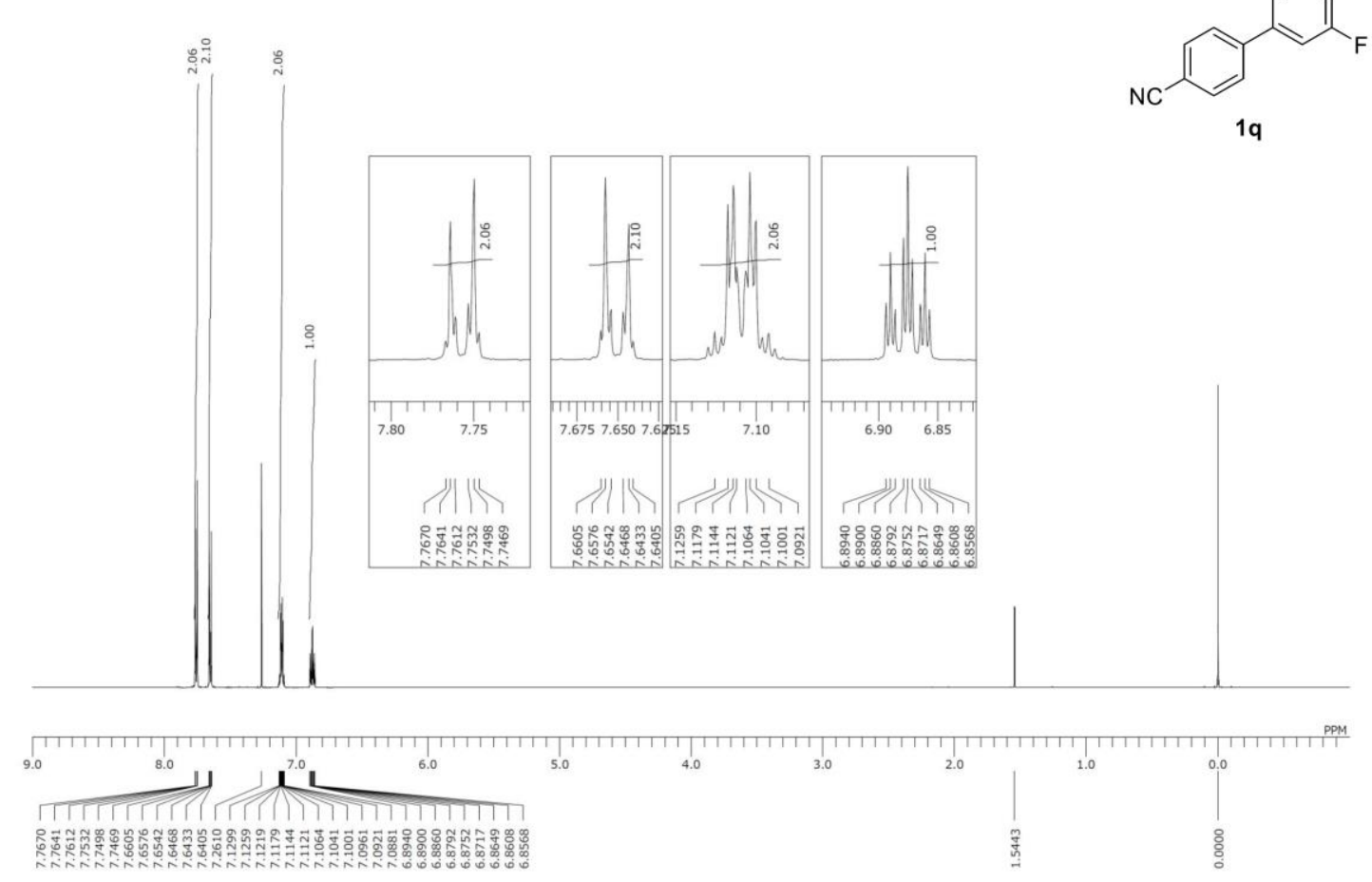

${ }^{13} \mathrm{C}$ NMR spectra of $1 \mathrm{q}\left(\mathrm{CDCl}_{3}, 150 \mathrm{MHz}\right)$

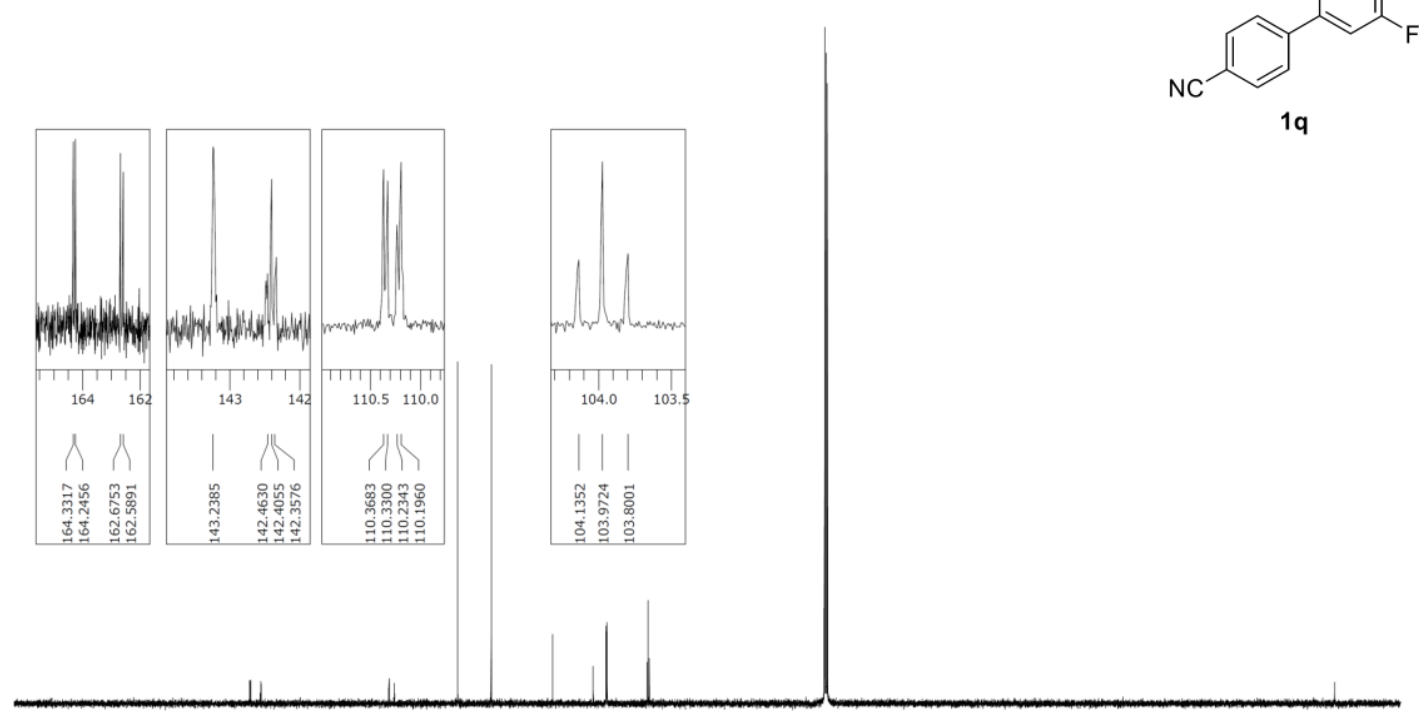

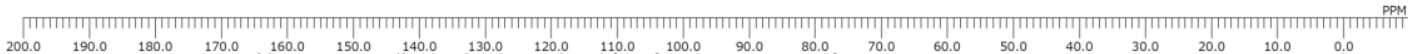

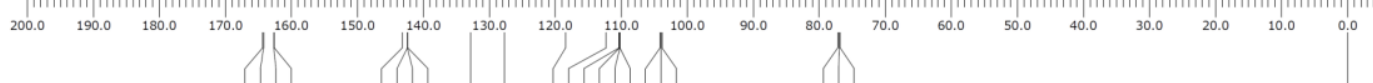

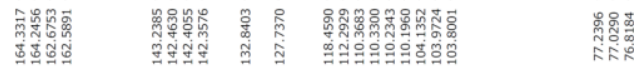


${ }^{19} \mathrm{~F}$ NMR spectra of $1 \mathrm{q}\left(\mathrm{CDCl}_{3}, 565 \mathrm{MHz}\right)$

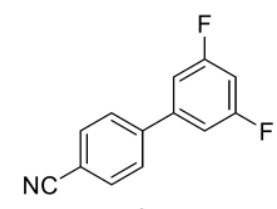

$1 \mathrm{q}$

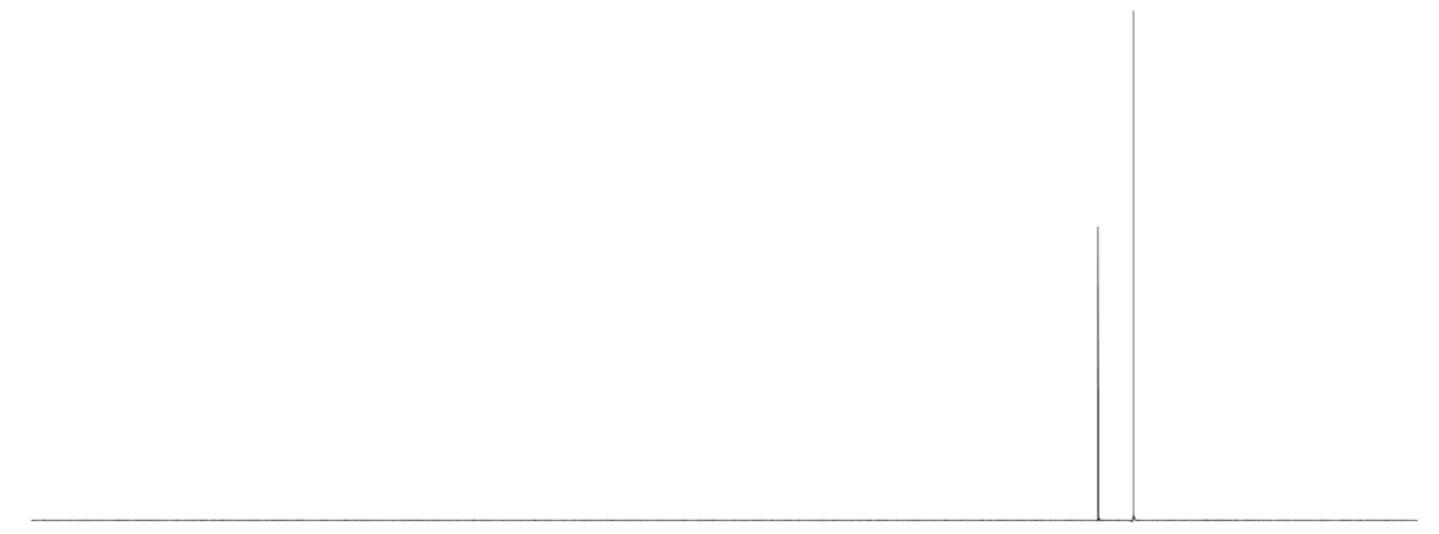

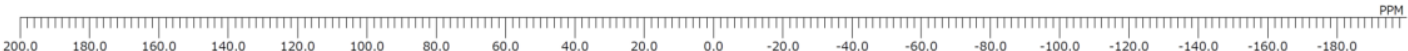

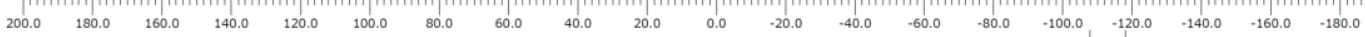

${ }^{1} \mathrm{H} \mathrm{NMR}$ spectra of $\operatorname{1r}\left(\mathrm{CDCl}_{3}, 600 \mathrm{MHz}\right)$

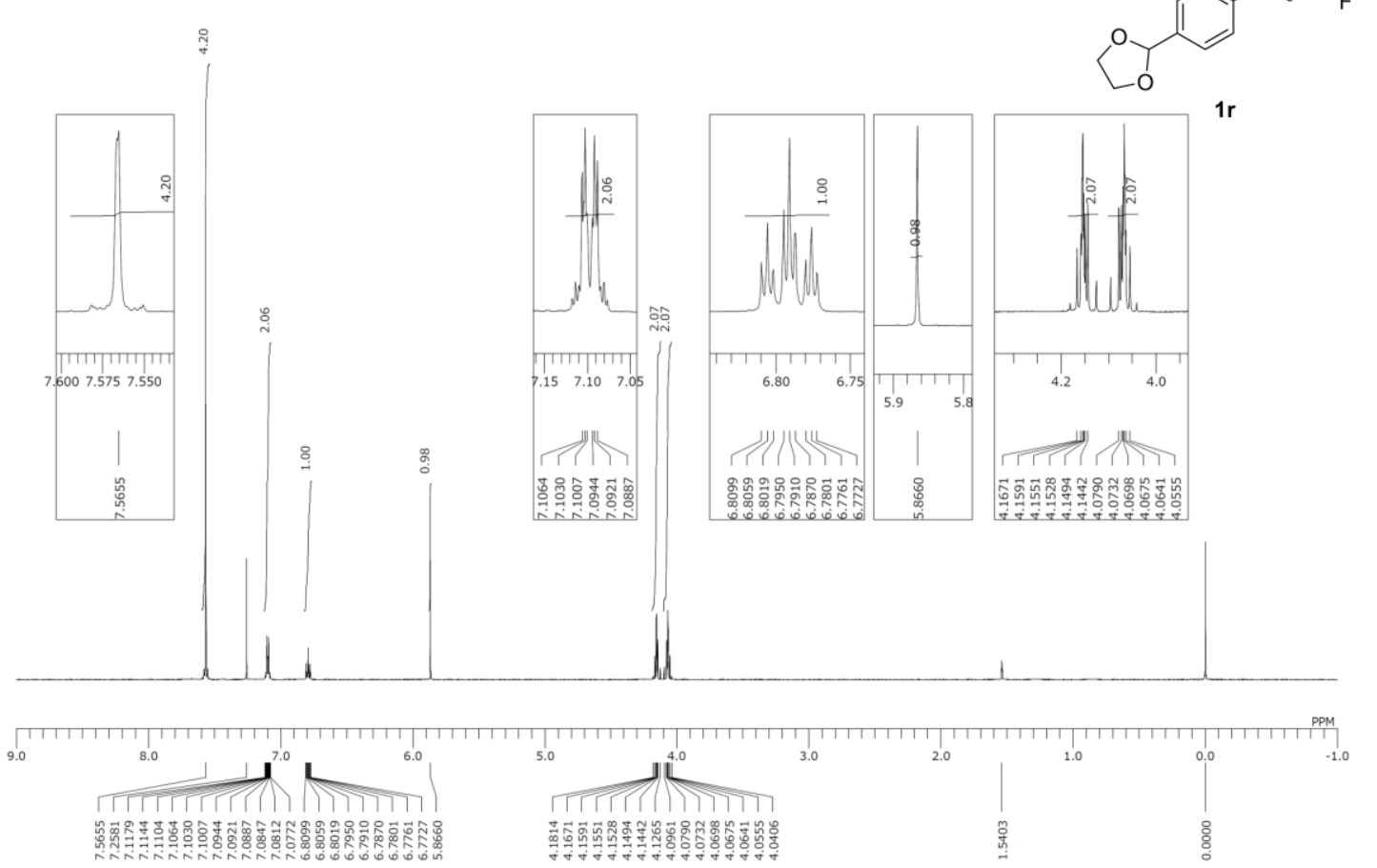


${ }^{13} \mathrm{C}$ NMR spectra of $1 \mathbf{r}\left(\mathrm{CDCl}_{3}, 150 \mathrm{MHz}\right)$
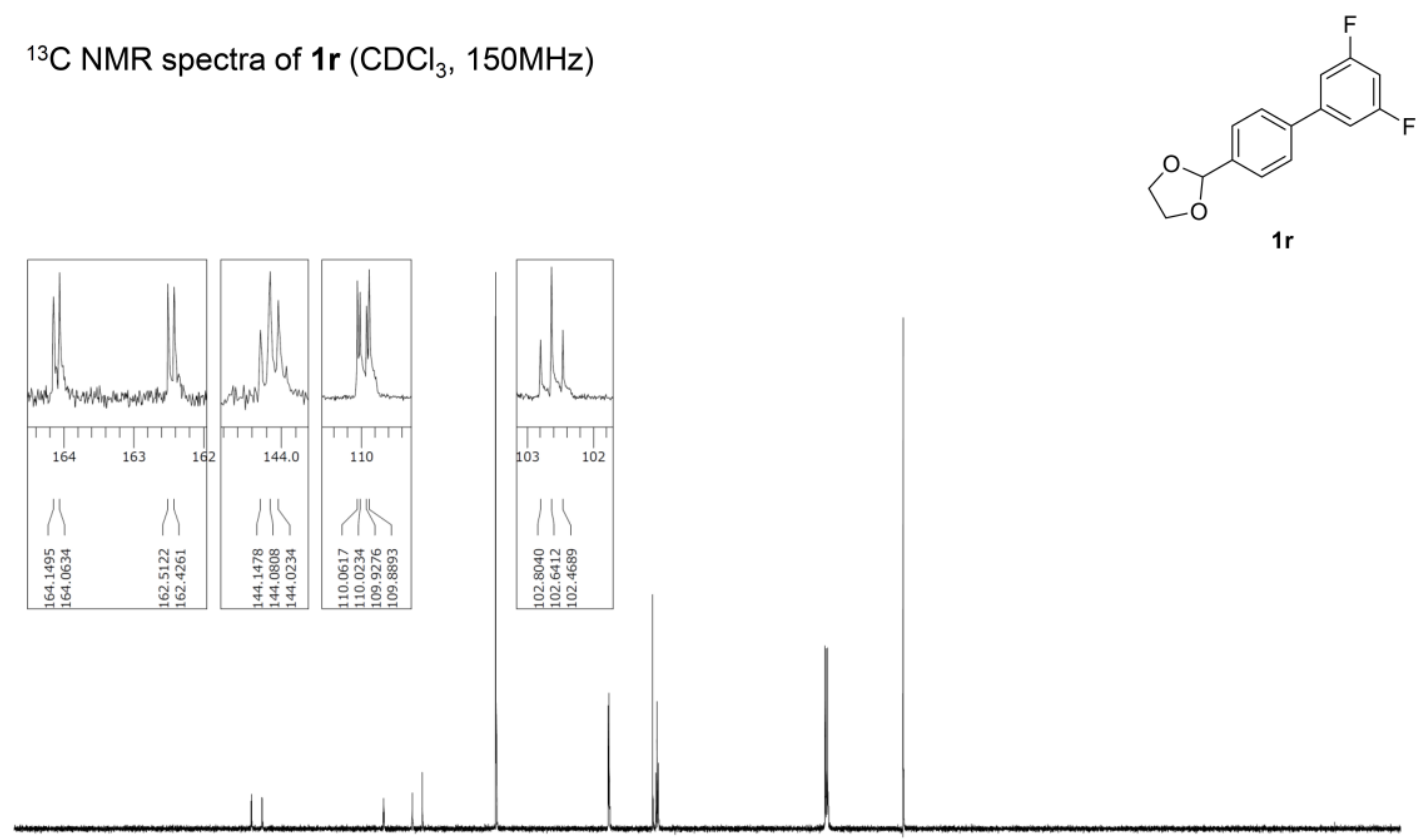

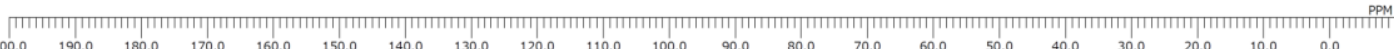

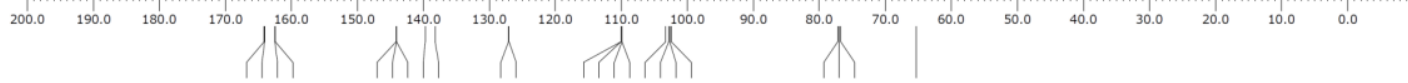

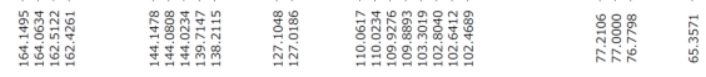

${ }^{19} \mathrm{~F}$ NMR spectra of $1 \mathbf{r}\left(\mathrm{CDCl}_{3}, 565 \mathrm{MHz}\right)$
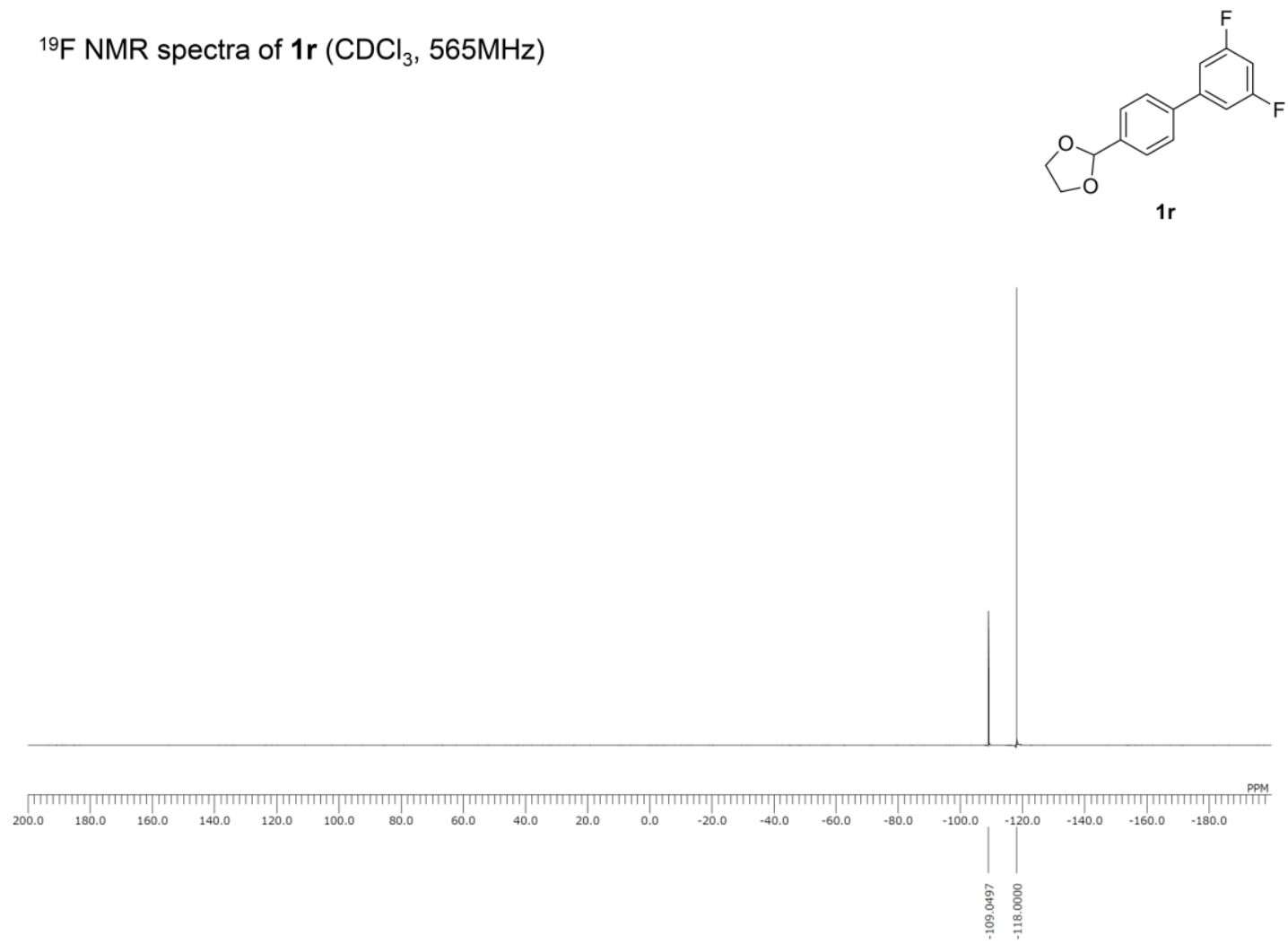
${ }^{1} \mathrm{H}$ NMR spectra of $1 \mathrm{~s}\left(\mathrm{CDCl}_{3}, 600 \mathrm{MHz}\right)$<smiles>O=Cc1ccc(-c2cc(F)cc(F)c2)cc1</smiles>

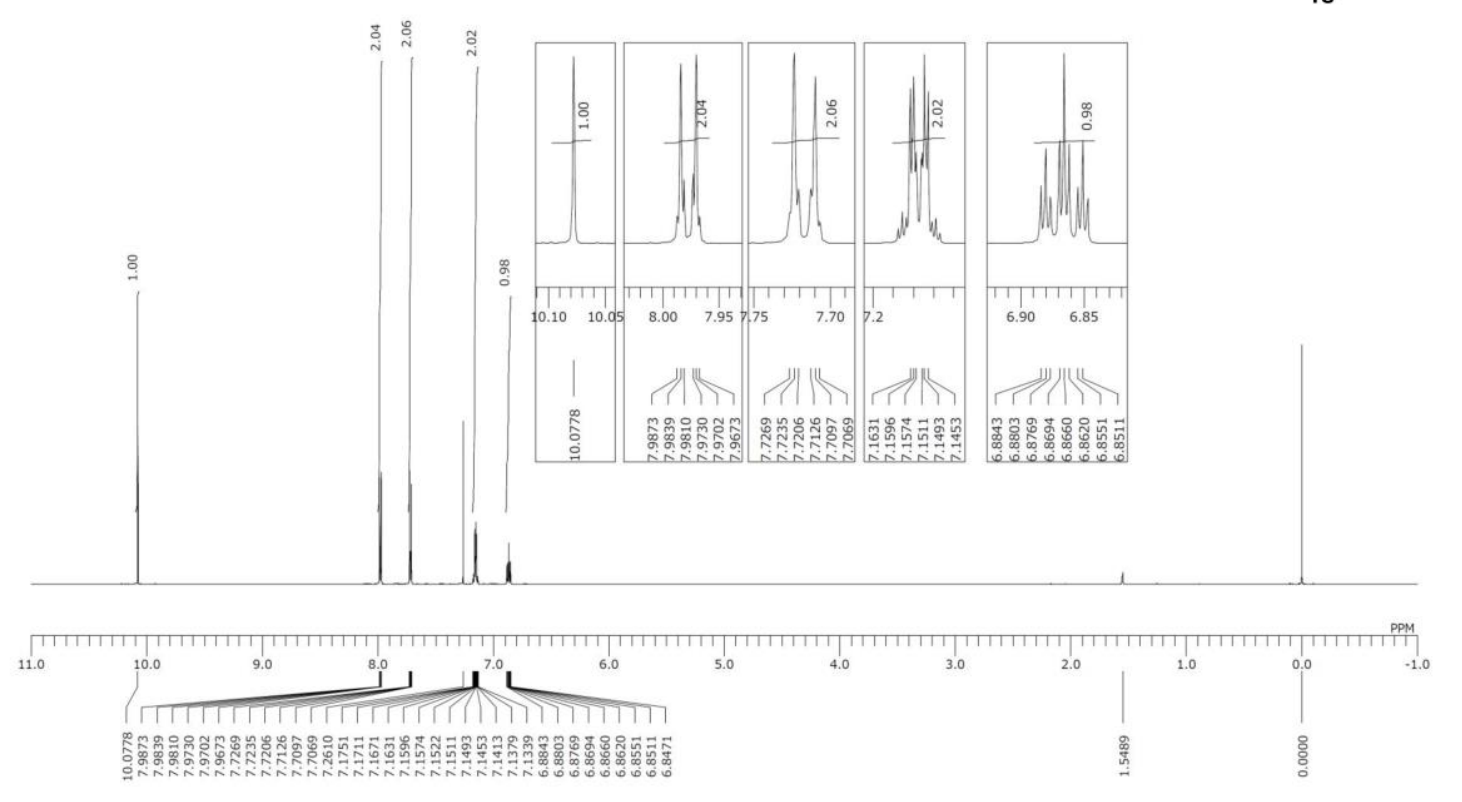

${ }^{13} \mathrm{C}$ NMR spectra of $1 \mathrm{~s}\left(\mathrm{CDCl}_{3}, 150 \mathrm{MHz}\right)$

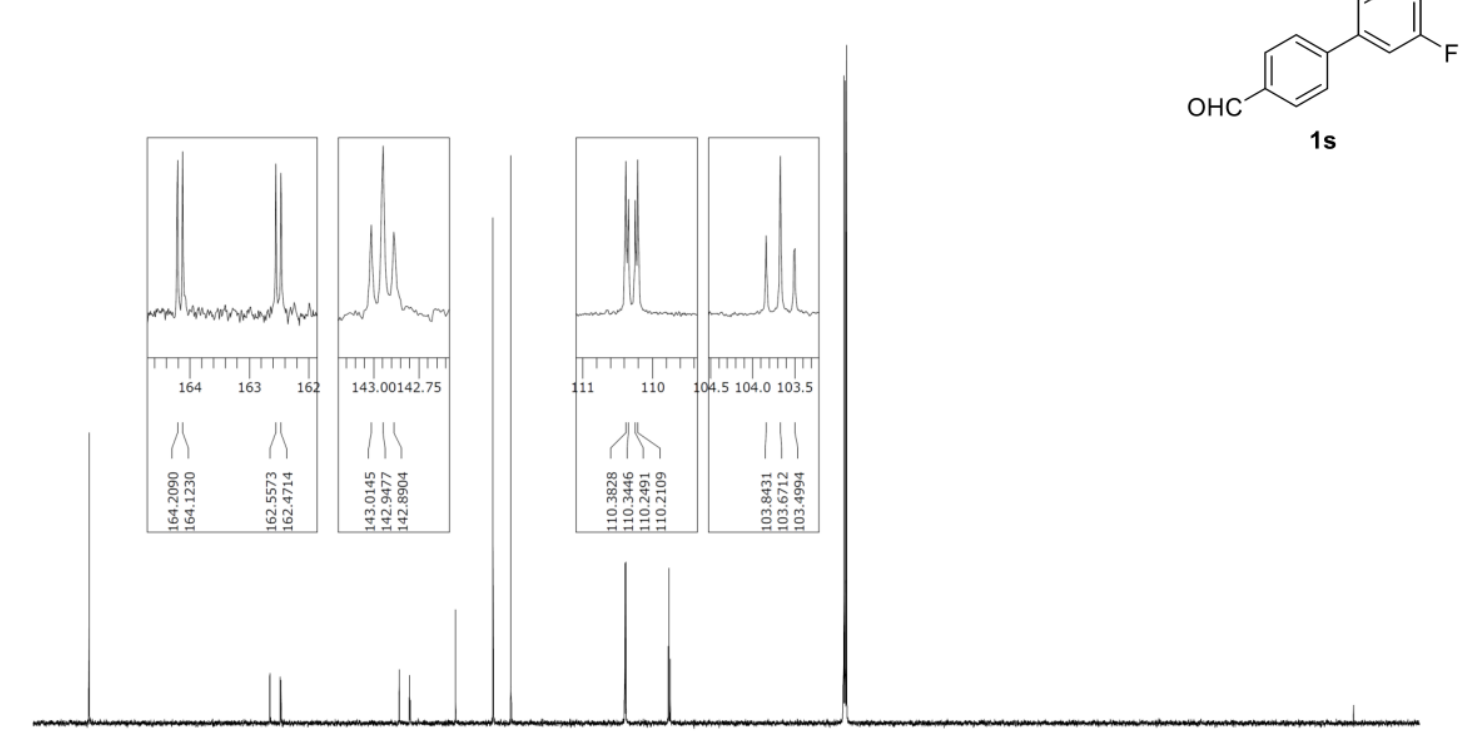

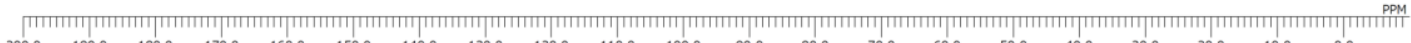
$\left.200.0\right|_{100.0} ^{190.0}$

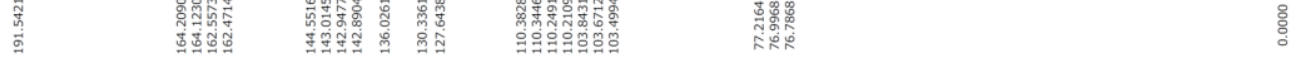


${ }^{19} \mathrm{~F}$ NMR spectra of $1 \mathrm{~s}\left(\mathrm{CDCl}_{3}, 565 \mathrm{MHz}\right)$<smiles>O=Cc1ccc(-c2cc(F)cc(F)c2)cc1</smiles>

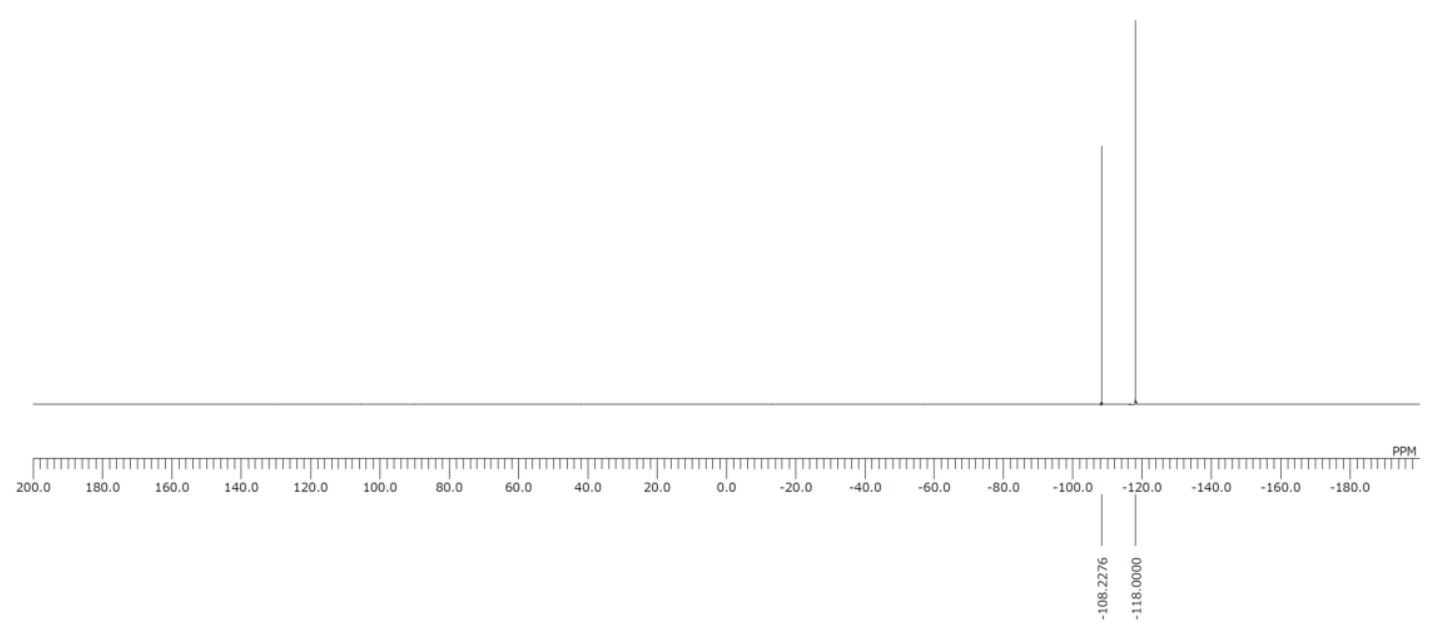

${ }^{1} \mathrm{H}$ NMR spectra of $\mathbf{1 t}\left(\mathrm{CDCl}_{3}, 400 \mathrm{MHz}\right)$

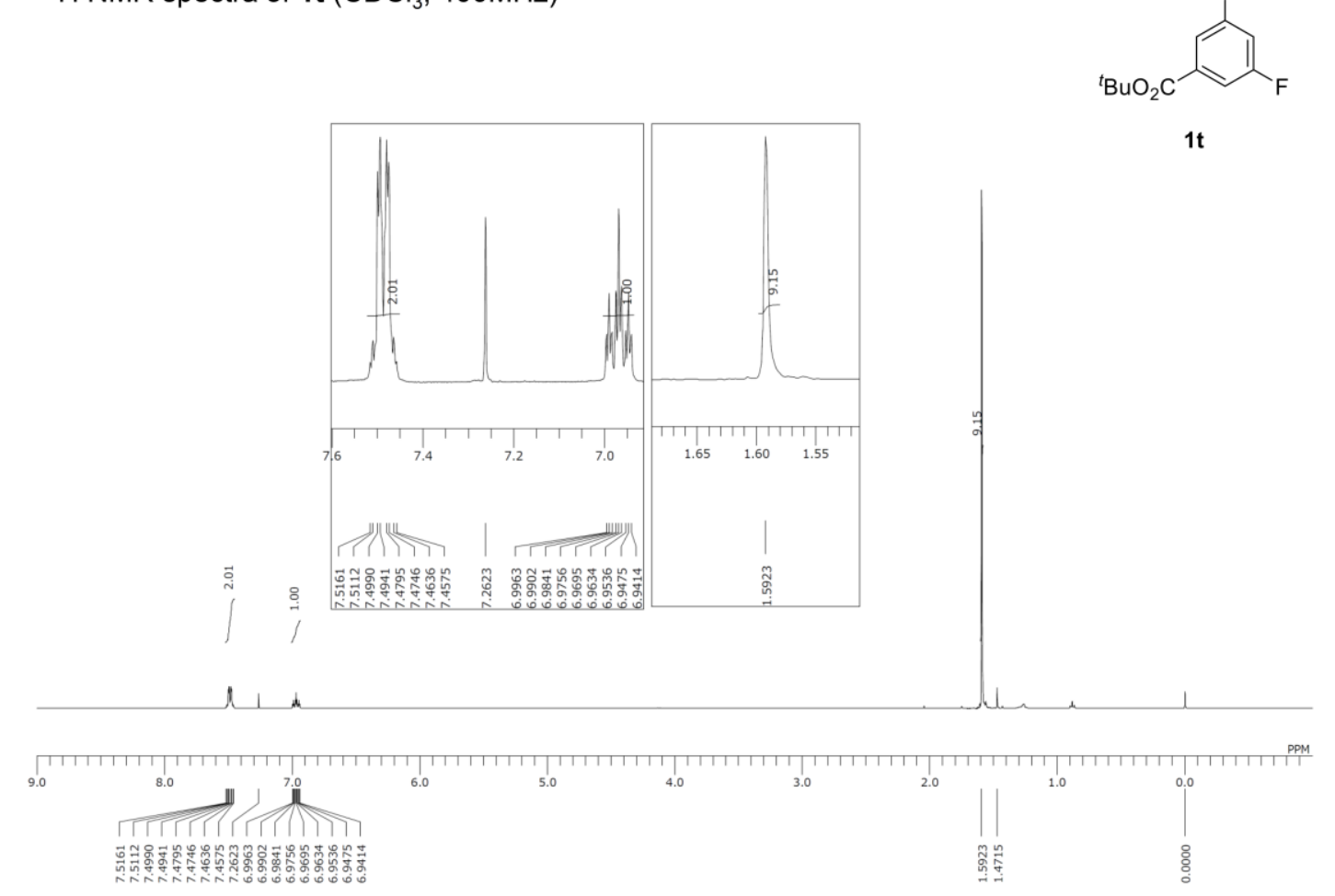


${ }^{13} \mathrm{C}$ NMR spectra of $1 \mathrm{t}\left(\mathrm{CDCl}_{3}, 150 \mathrm{MHz}\right)$

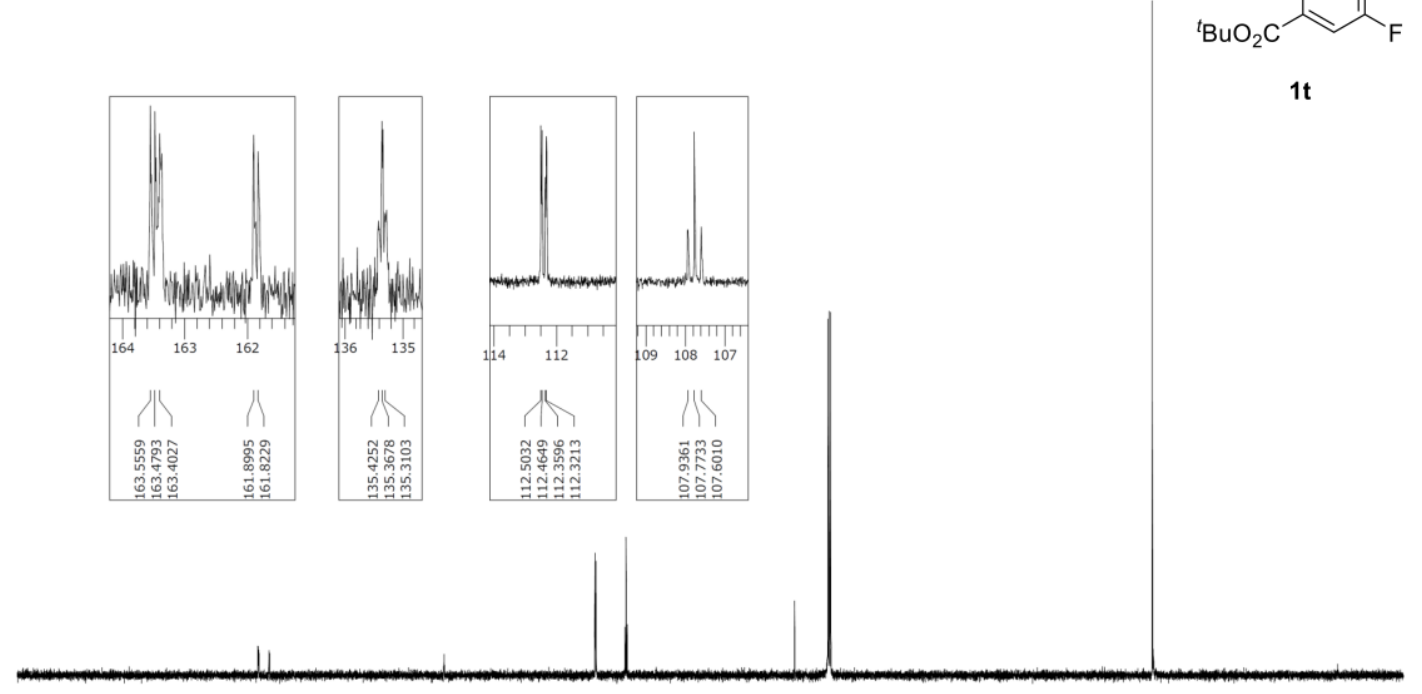

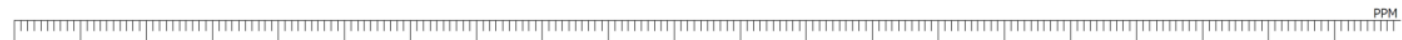

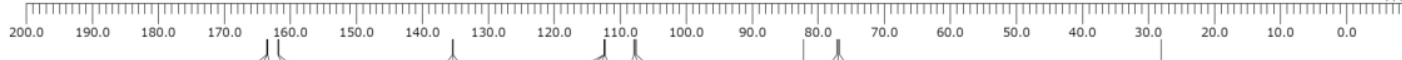

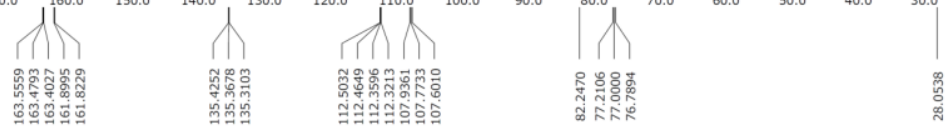

${ }^{19} \mathrm{~F}$ NMR spectra of $1 \mathrm{t}\left(\mathrm{CDCl}_{3}, 565 \mathrm{MHz}\right)$

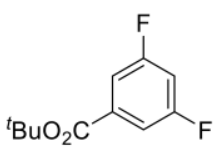

$1 t$

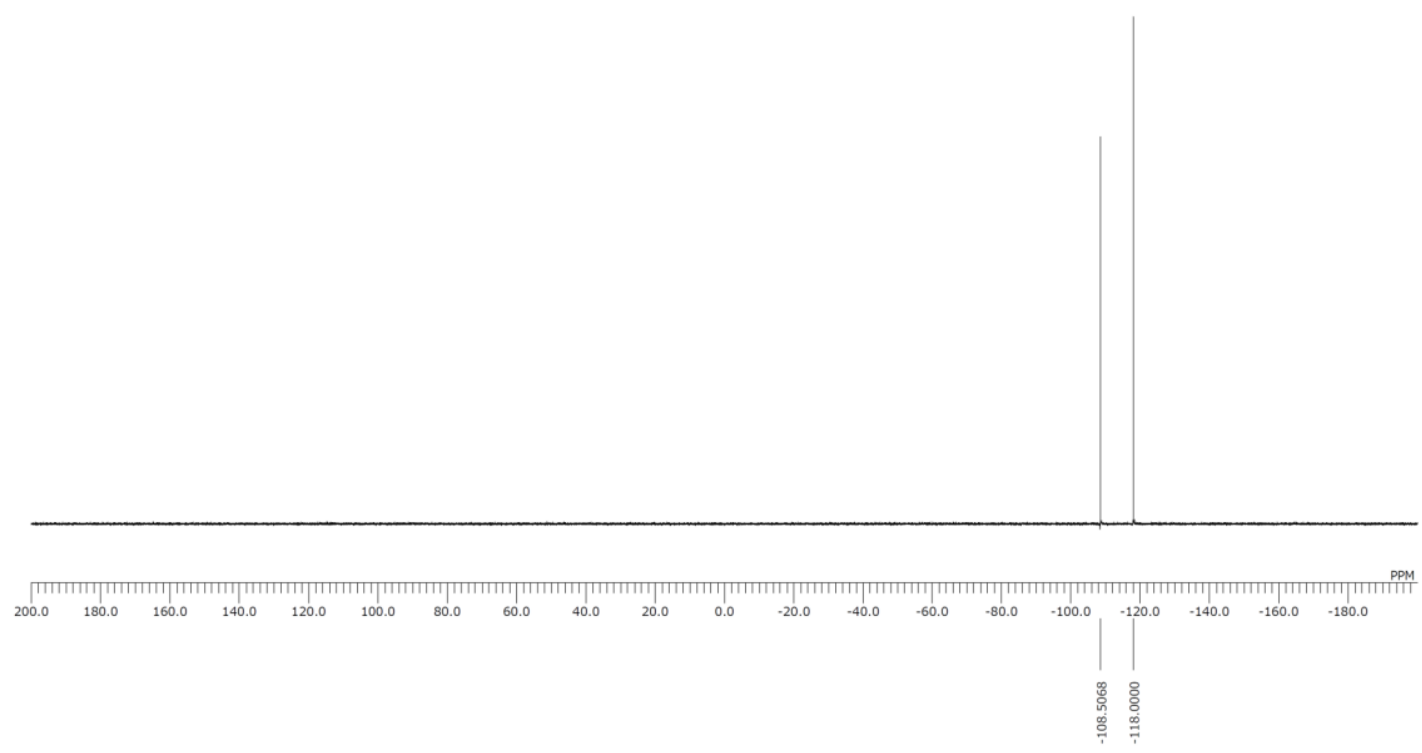


${ }^{1} \mathrm{H}$ NMR spectra of $1 \mathrm{u}\left(\mathrm{CDCl}_{3}, 400 \mathrm{MHz}\right)$

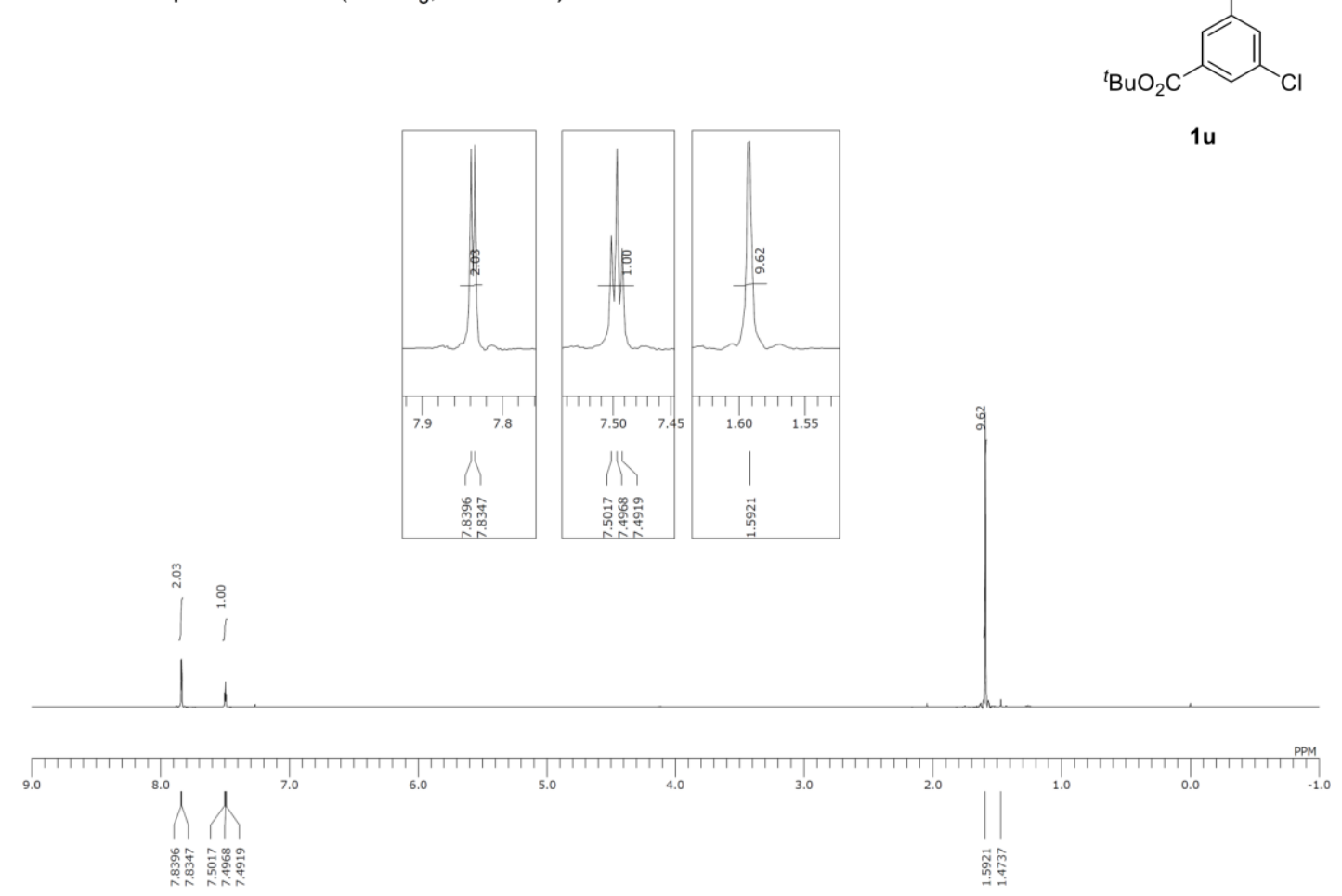

${ }^{13} \mathrm{C}$ NMR spectra of $1 \mathrm{u}\left(\mathrm{CDCl}_{3}, 100 \mathrm{MHz}\right)$

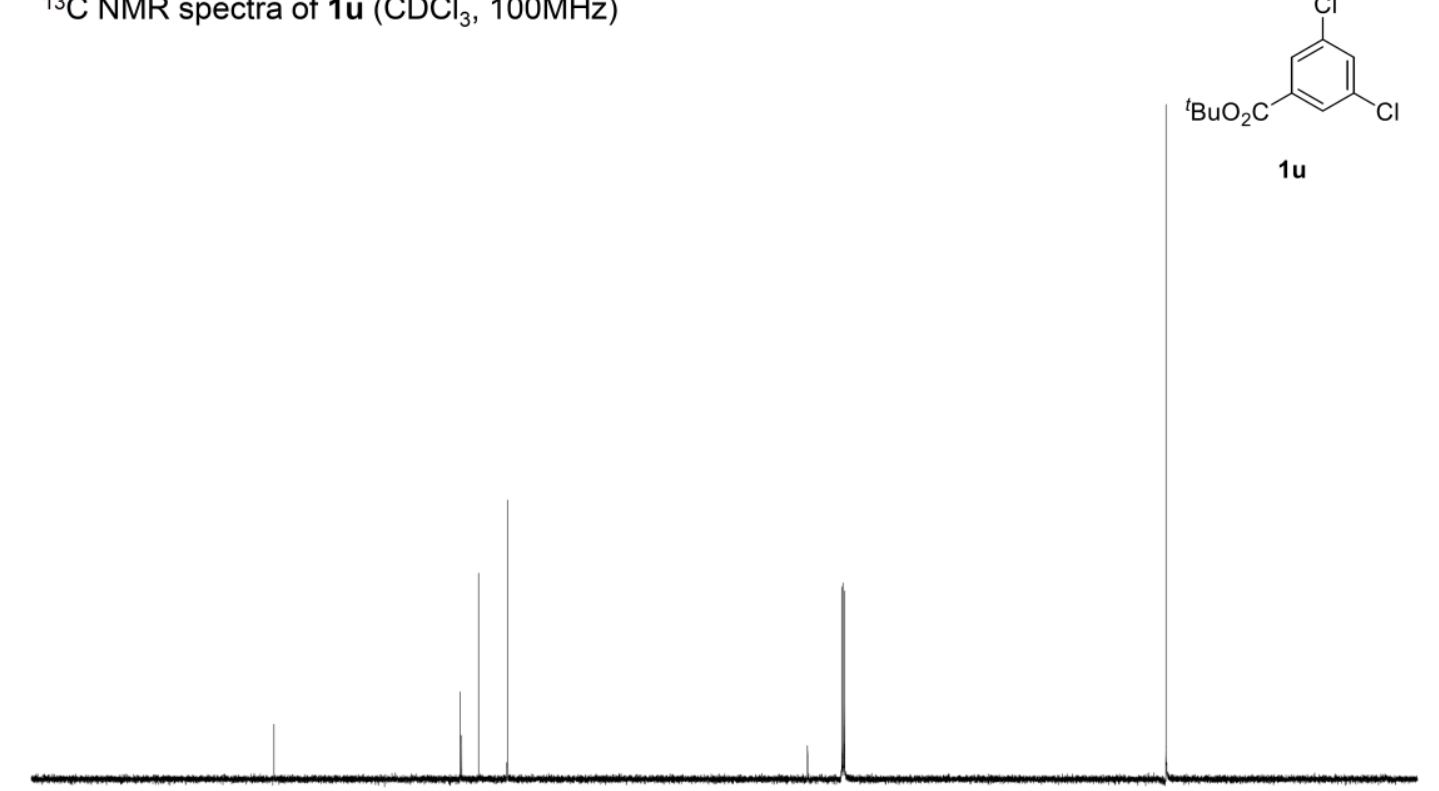

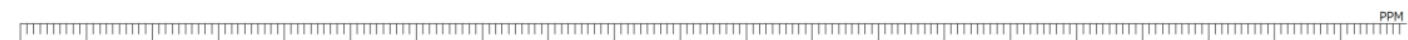

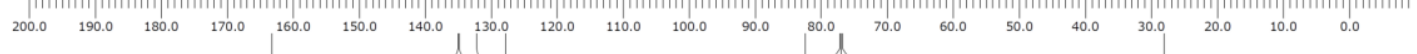

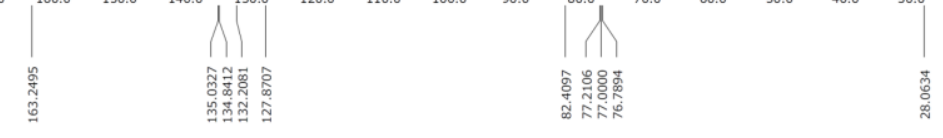


${ }^{1} \mathrm{H}$ NMR spectra of $1 \mathrm{v}\left(\mathrm{CDCl}_{3}, 400 \mathrm{MHz}\right)$

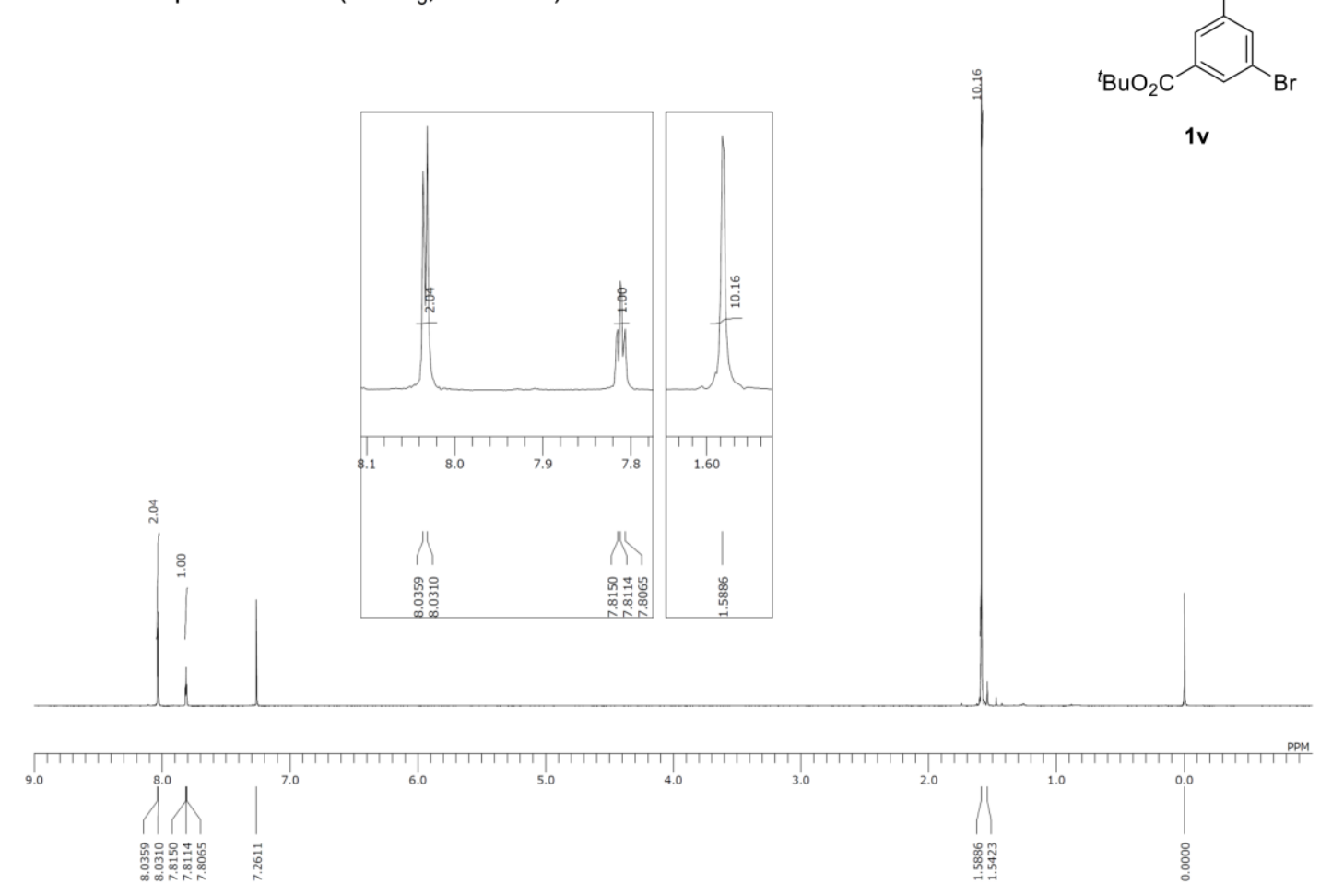

${ }^{13} \mathrm{C}$ NMR spectra of $1 \mathbf{v}\left(\mathrm{CDCl}_{3}, 150 \mathrm{MHz}\right)$

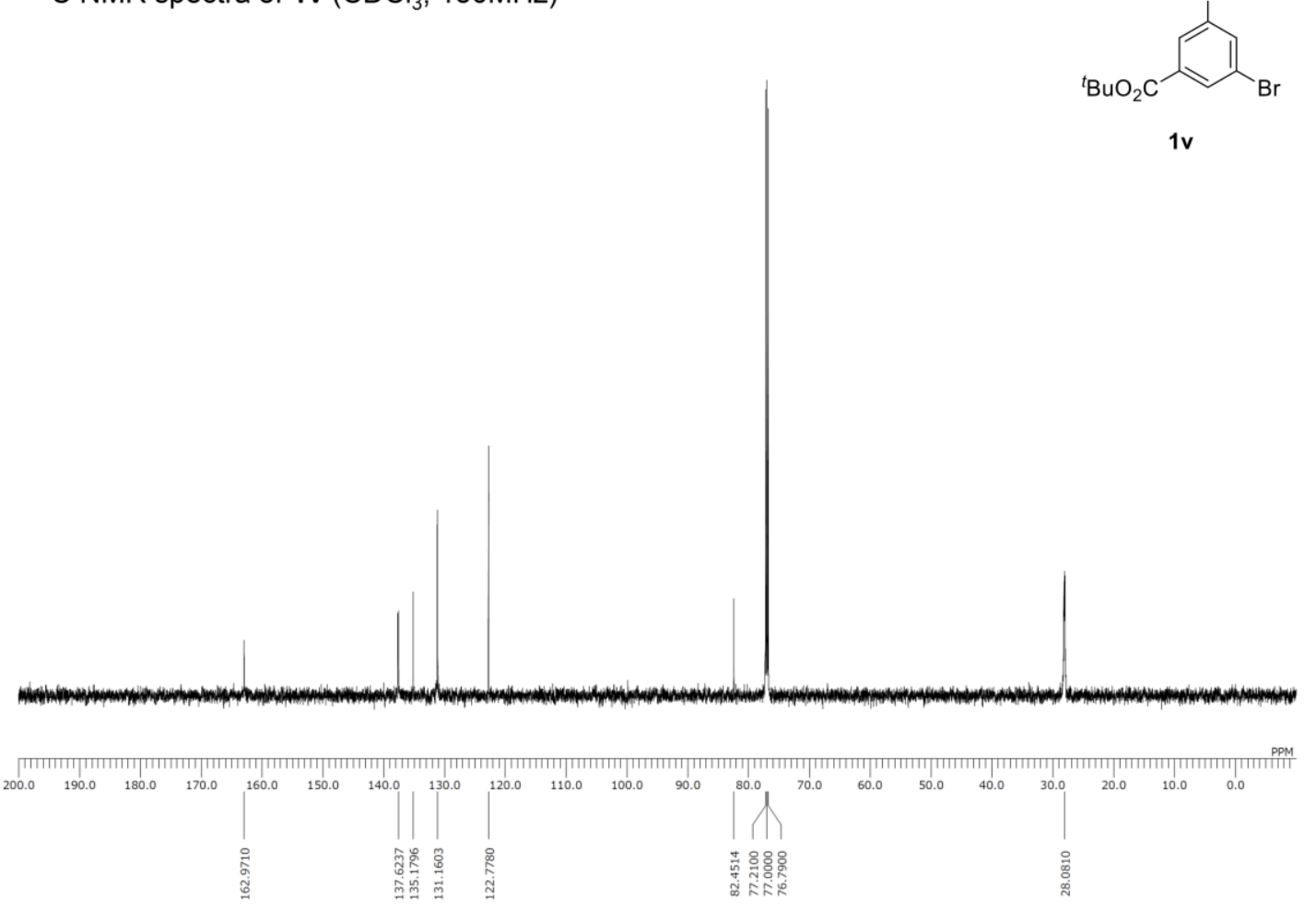


${ }^{1} \mathrm{H}$ NMR spectra of $1 \mathrm{w}\left(\mathrm{CDCl}_{3}, 600 \mathrm{MHz}\right)$

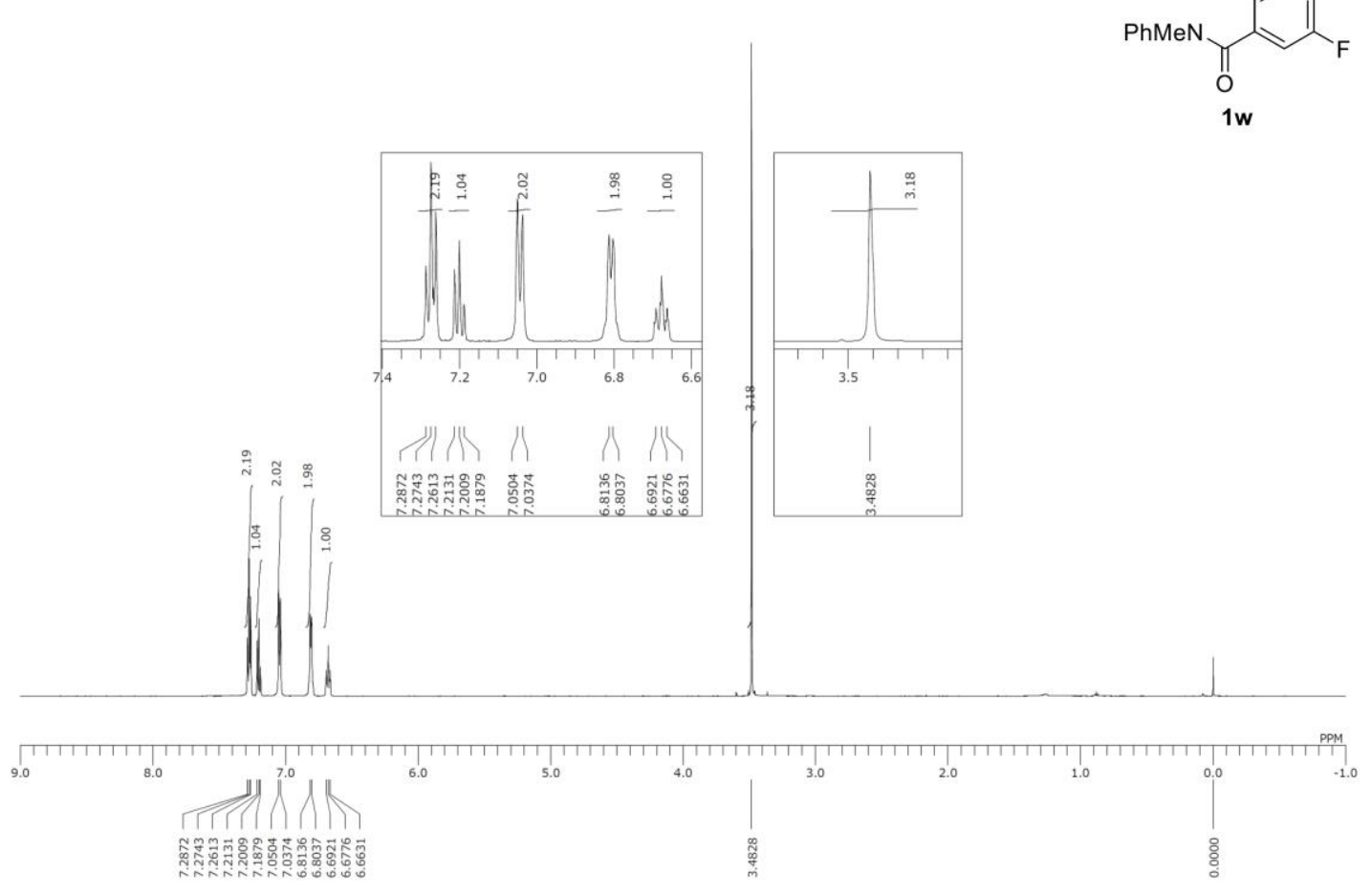

${ }^{13} \mathrm{C} \mathrm{NMR}$ spectra of $1 \mathrm{w}\left(\mathrm{CDCl}_{3}, 150 \mathrm{MHz}\right)$
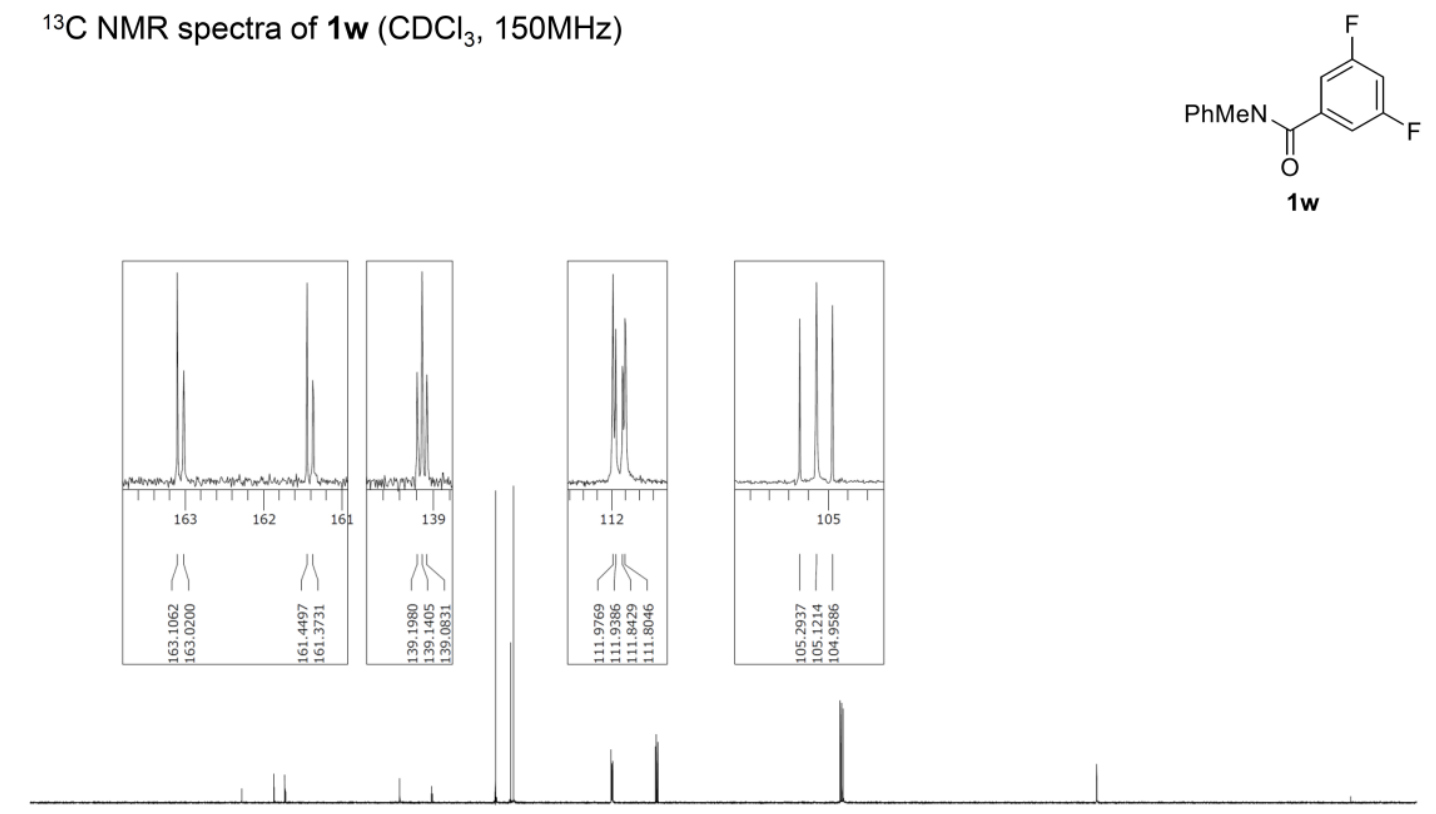

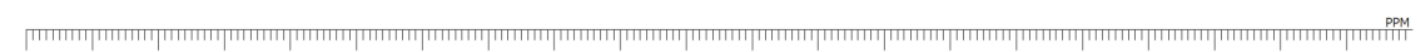

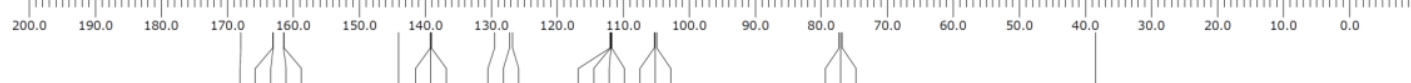

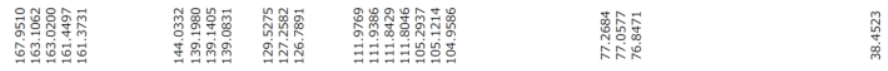


${ }^{19} \mathrm{~F} \mathrm{NMR}$ spectra of $\mathbf{1 w}\left(\mathrm{CDCl}_{3}, 565 \mathrm{MHz}\right)$
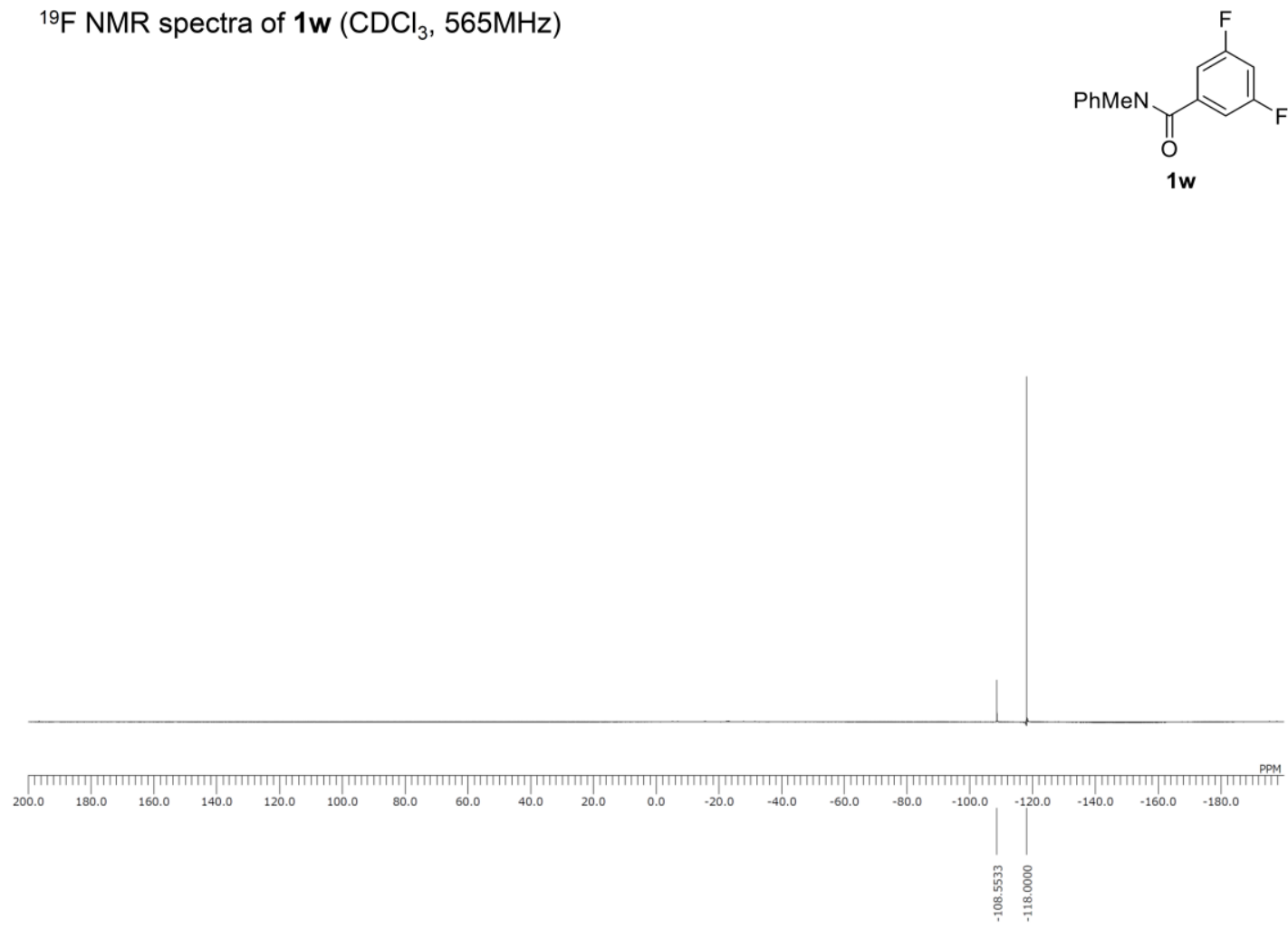

${ }^{1} \mathrm{H}$ NMR spectra of $1 \mathbf{x}\left(\mathrm{CDCl}_{3}, 600 \mathrm{MHz}\right)$

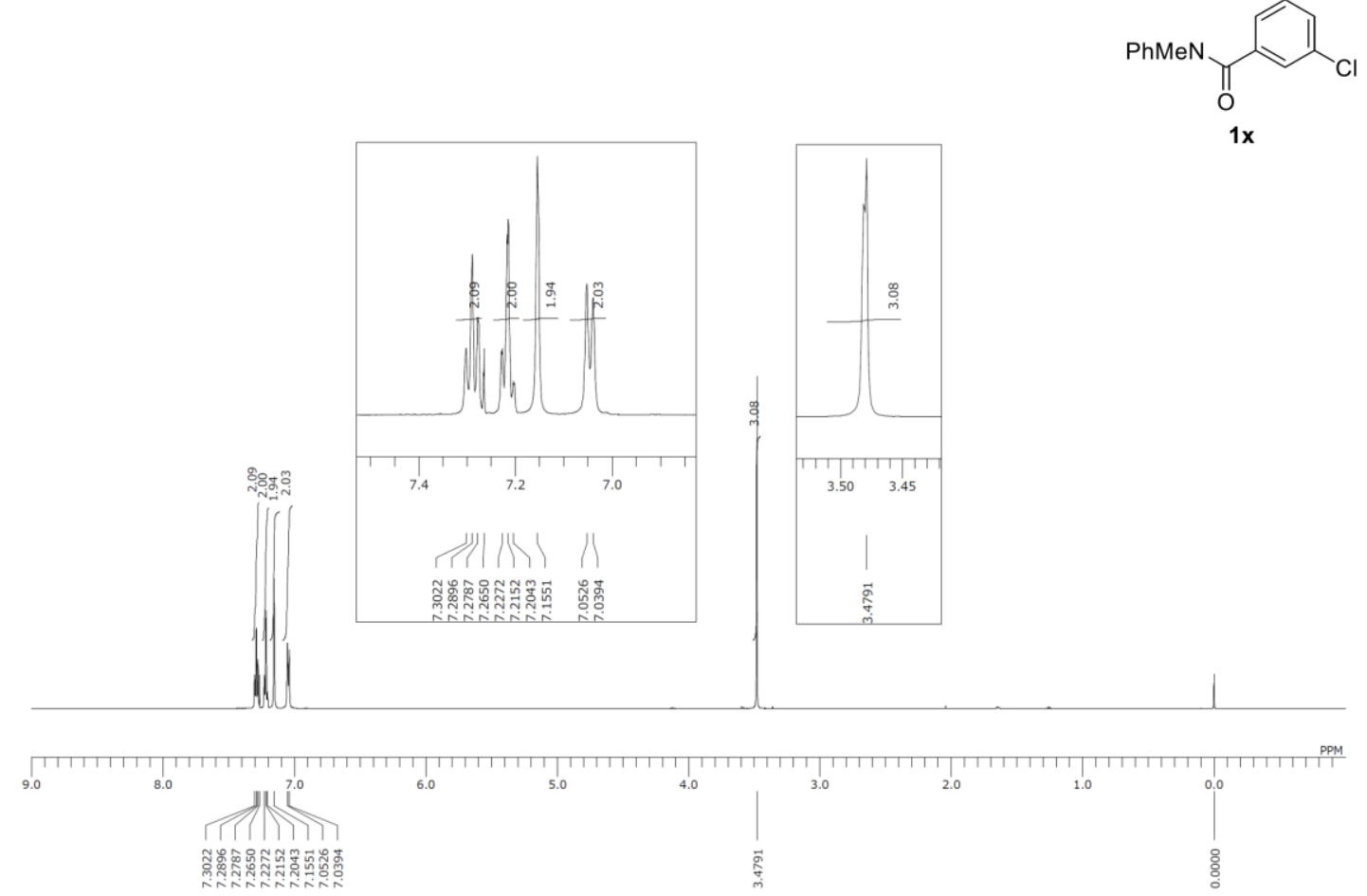


${ }^{13} \mathrm{C}$ NMR spectra of $\mathbf{1 x}\left(\mathrm{CDCl}_{3}, 150 \mathrm{MHz}\right)$
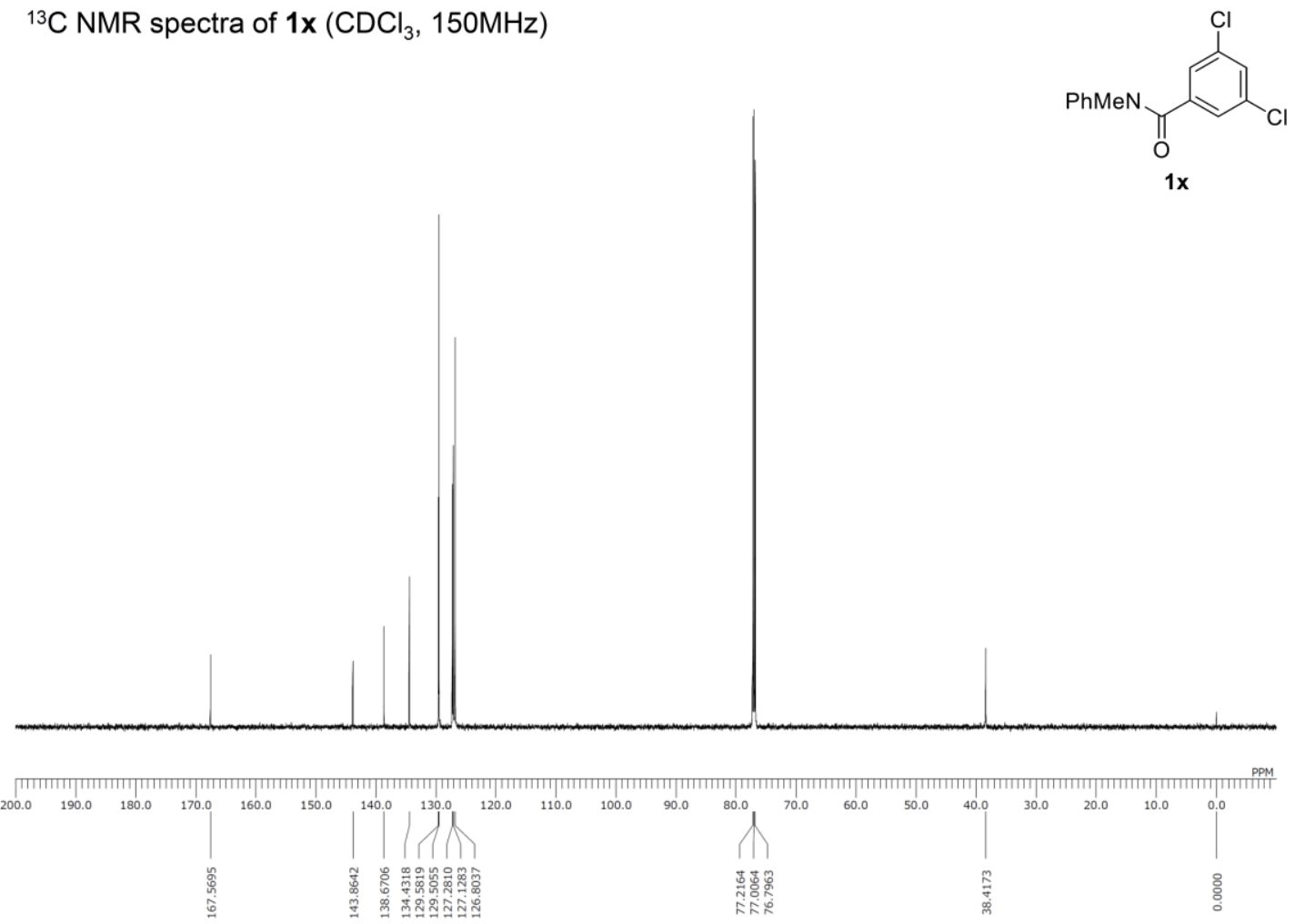

${ }^{1} \mathrm{H}$ NMR spectra of $1 \mathbf{y}\left(\mathrm{CDCl}_{3}, 400 \mathrm{MHz}\right)$

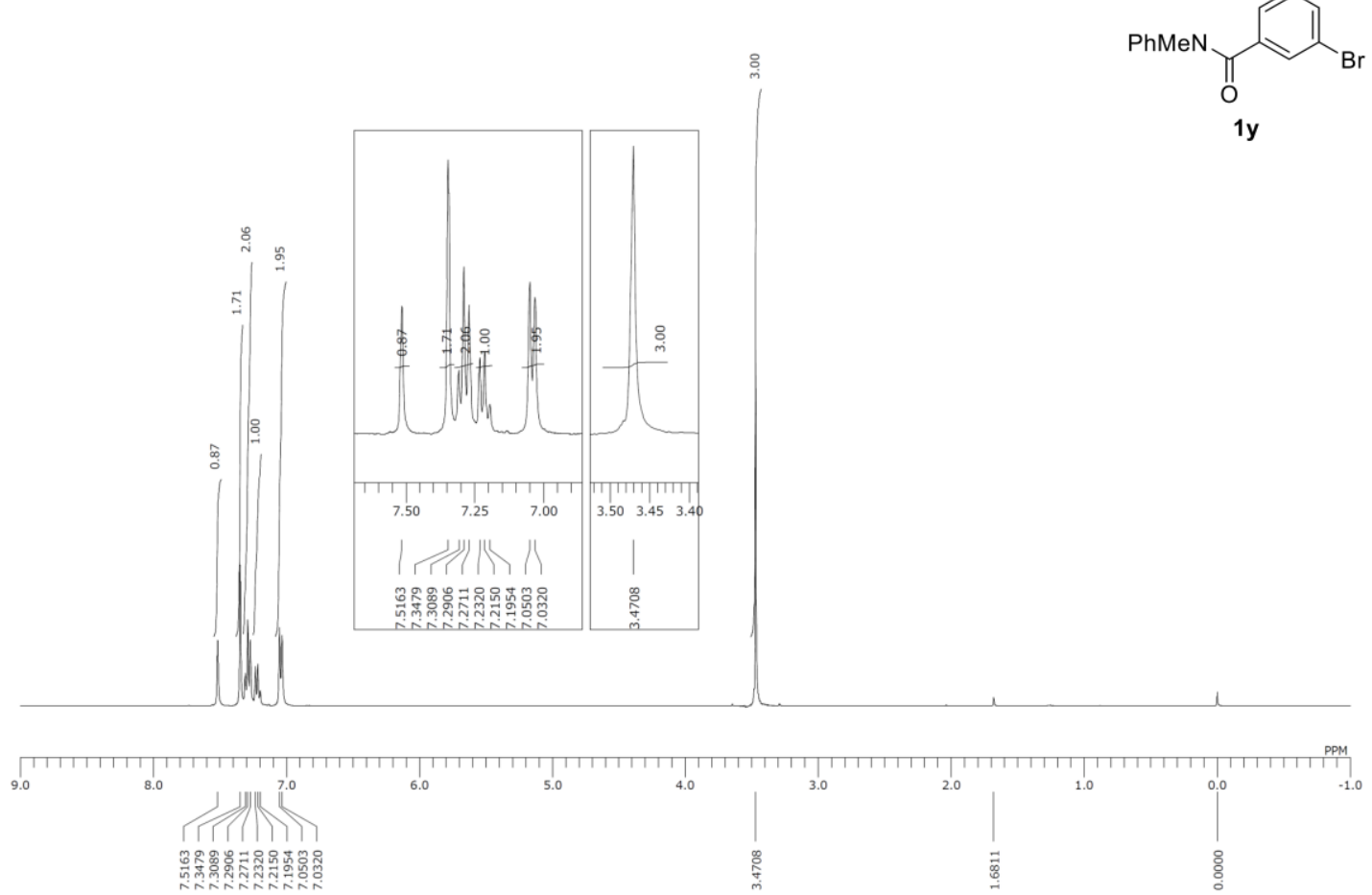


${ }^{13} \mathrm{C}$ NMR spectra of $1 \mathbf{y}\left(\mathrm{CDCl}_{3}, 100 \mathrm{MHz}\right)$<smiles>CNC(=O)c1cc(Br)cc(Br)c1</smiles>

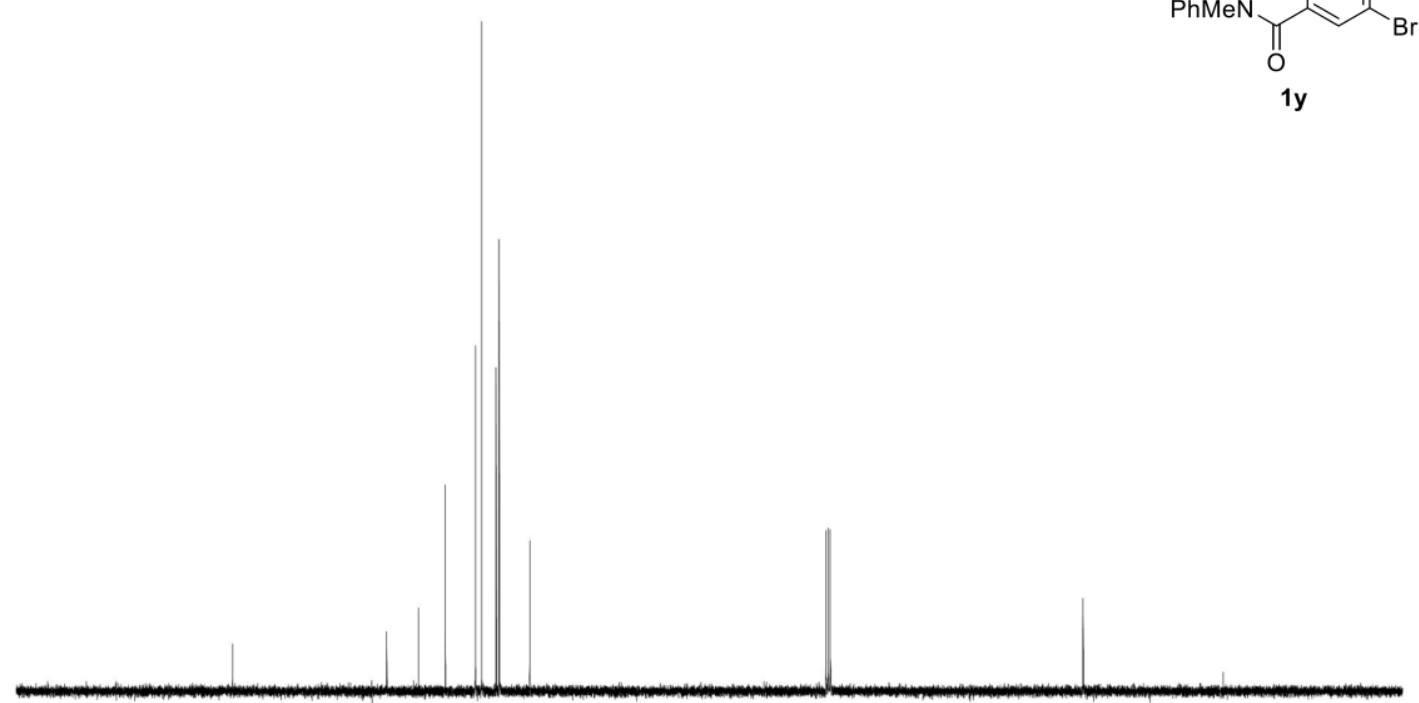

PPM

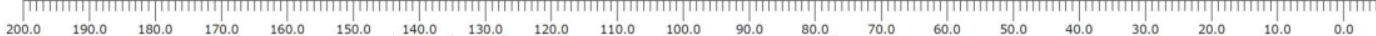

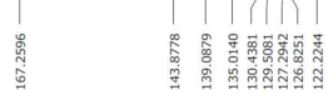

${ }^{1} \mathrm{H}$ NMR spectra of $1 \mathrm{z}\left(\mathrm{CDCl}_{3}, 400 \mathrm{MHz}\right)$

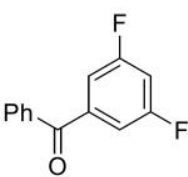

$1 z$

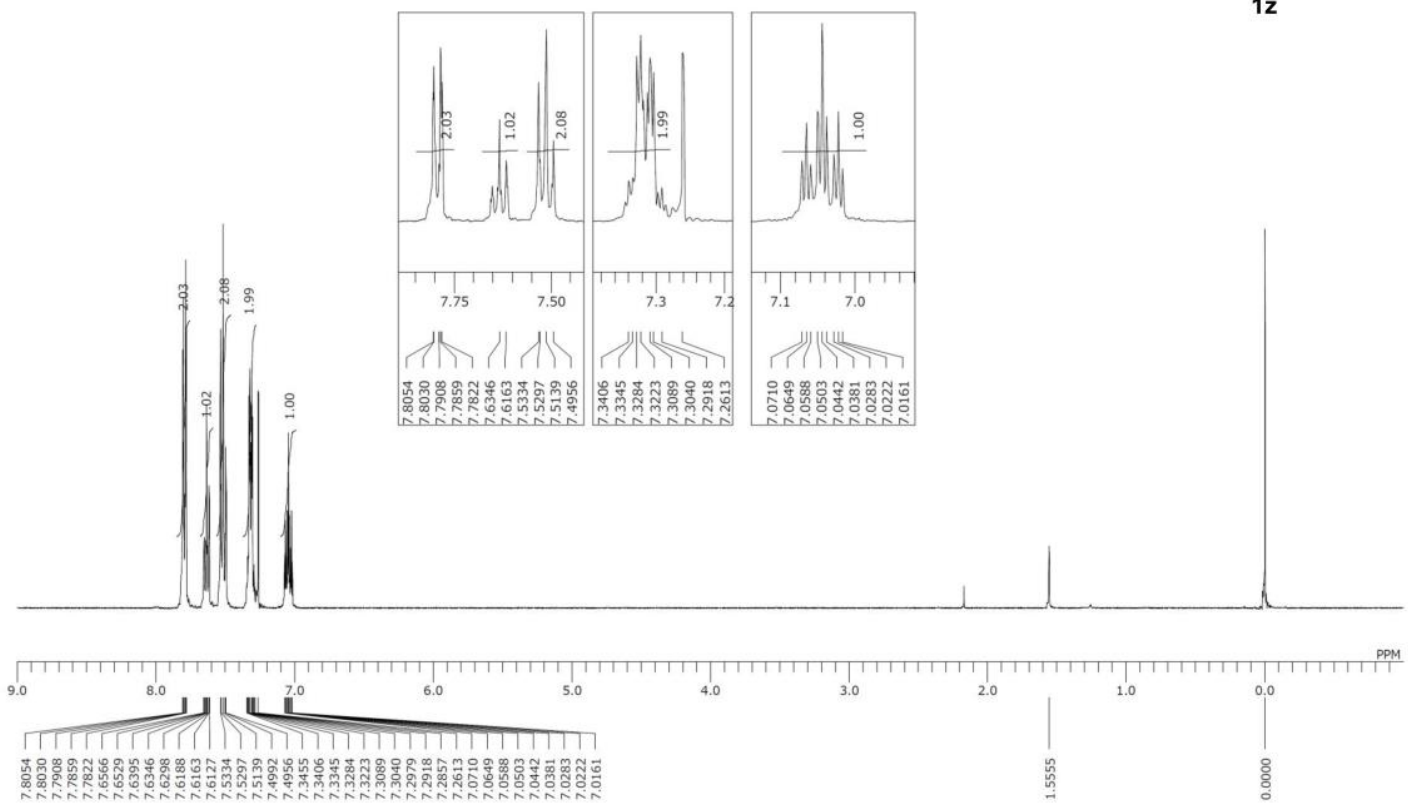


${ }^{13} \mathrm{C}$ NMR spectra of $1 z\left(\mathrm{CDCl}_{3}, 150 \mathrm{MHz}\right)$
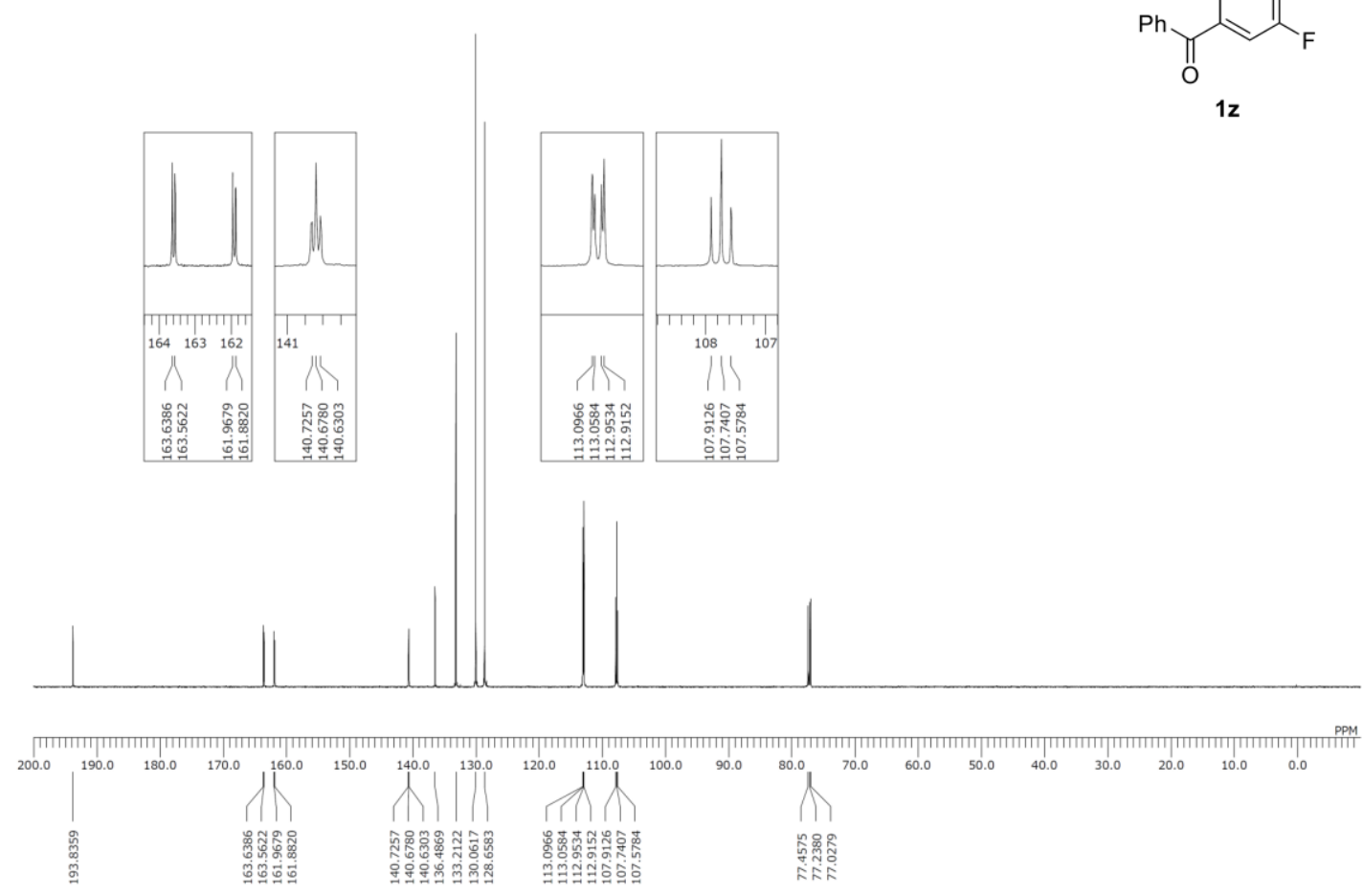

${ }^{19} \mathrm{~F}$ NMR spectra of $\mathbf{1 z}\left(\mathrm{CDCl}_{3}, 565 \mathrm{MHz}\right)$
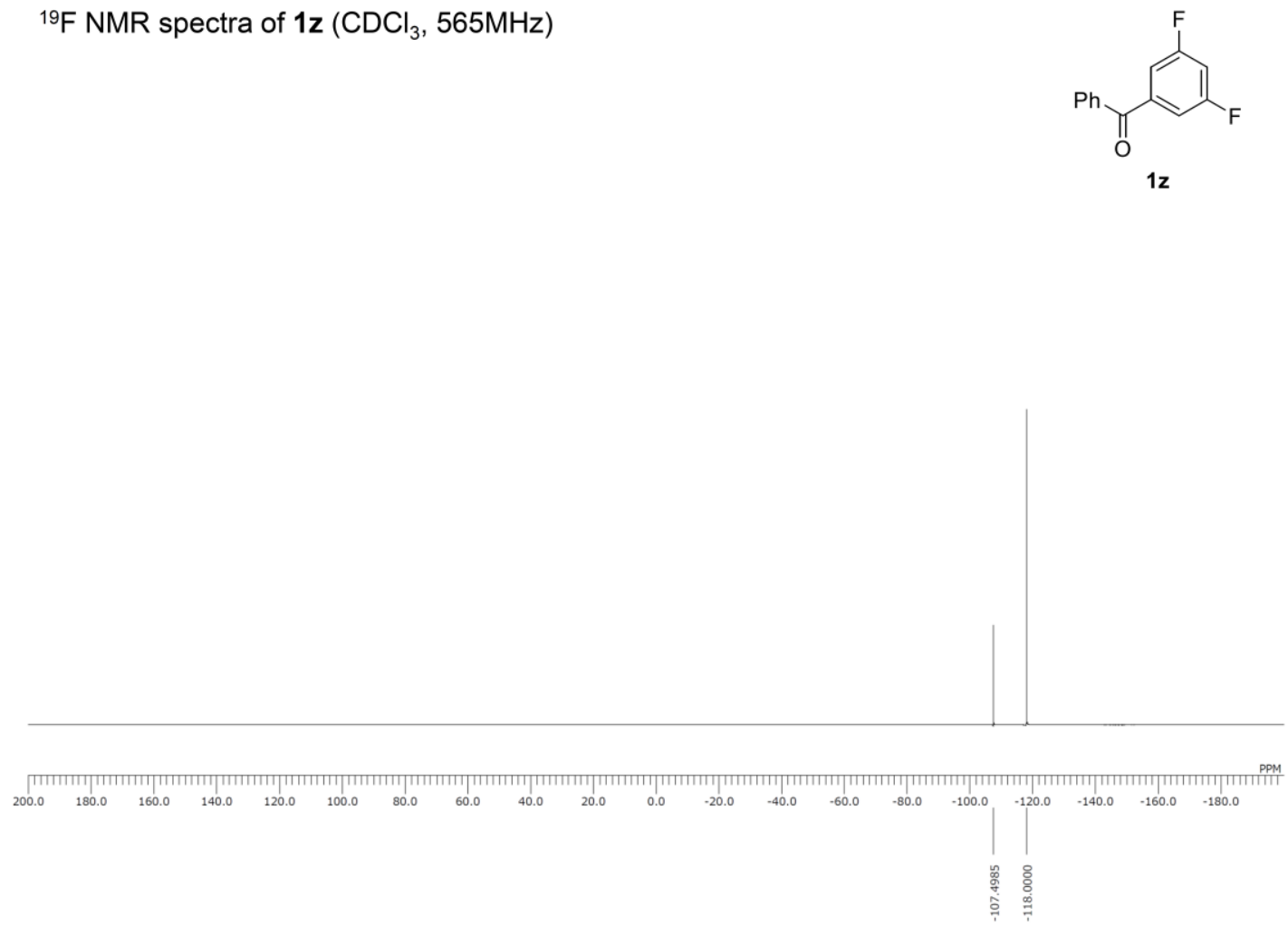
${ }^{1} \mathrm{H}$ NMR spectra of $\mathbf{3 d}\left(\mathrm{CDCl}_{3}, 600 \mathrm{MHz}\right)$
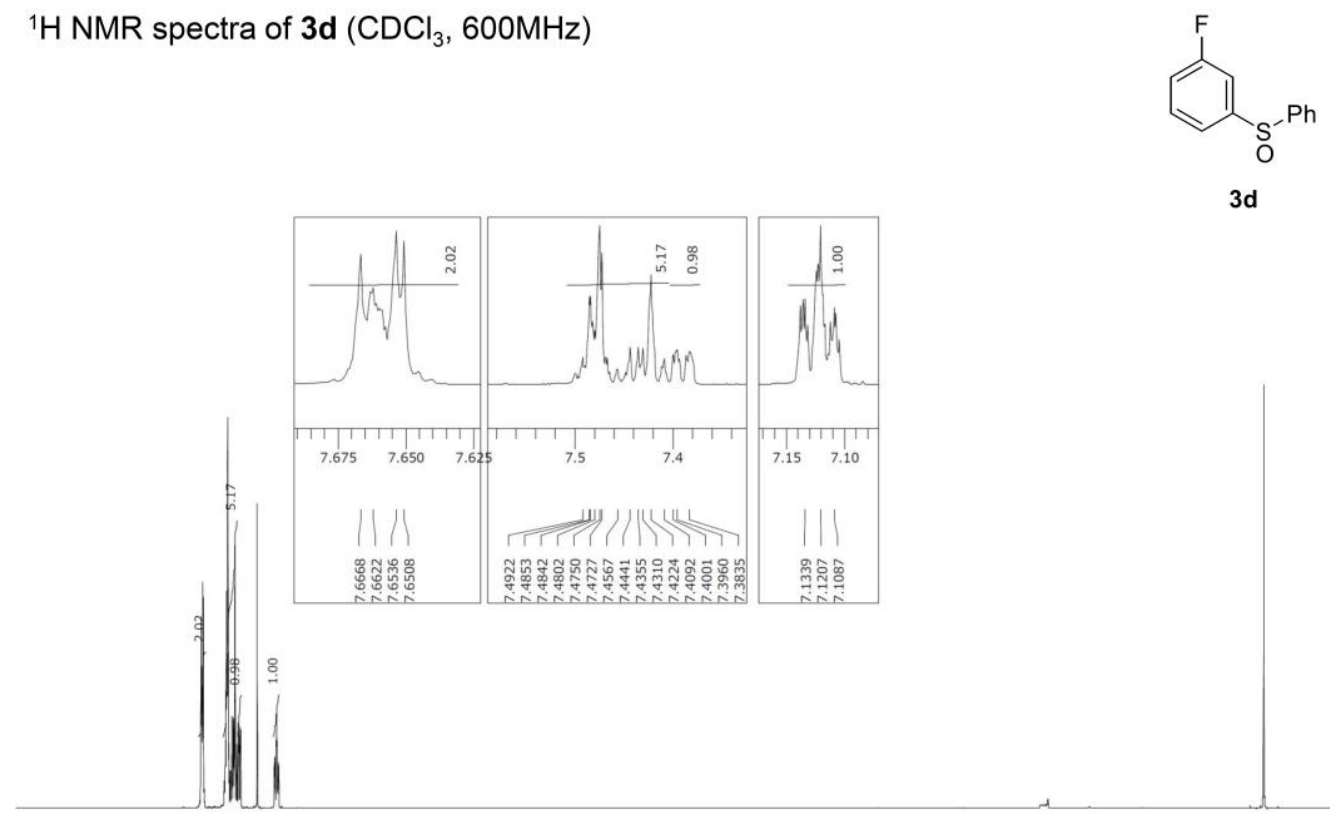

$3 d$

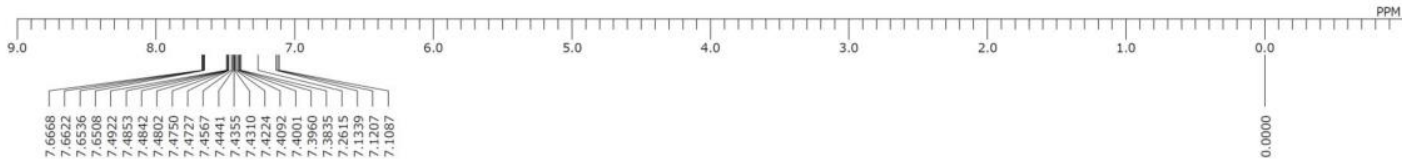

${ }^{13} \mathrm{C}$ NMR spectra of $\mathbf{3 d}\left(\mathrm{CDCl}_{3}, 150 \mathrm{MHz}\right)$

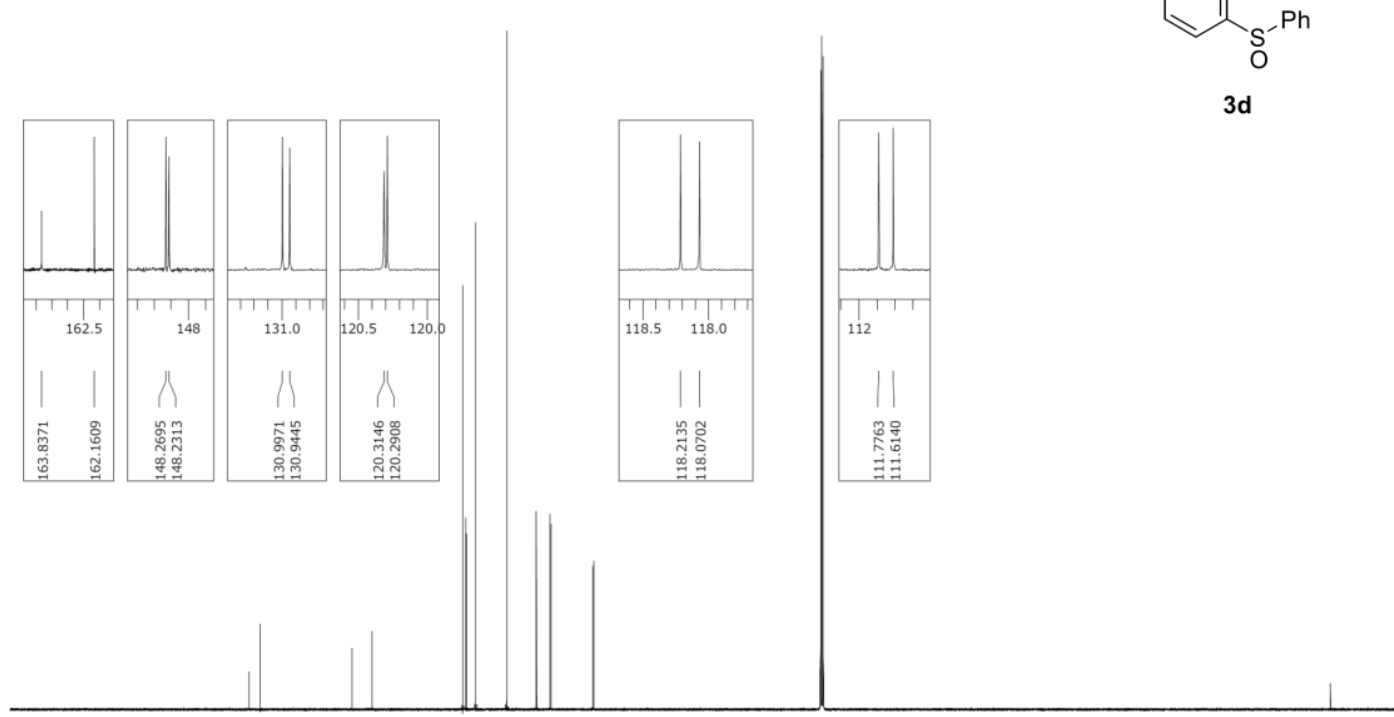

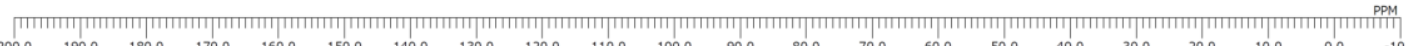

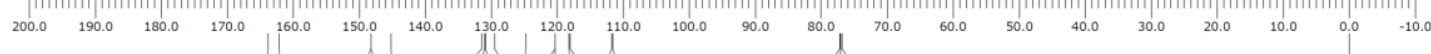

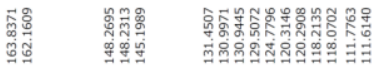


${ }^{19} \mathrm{~F}$ NMR spectra of $\mathbf{3 d}\left(\mathrm{CDCl}_{3}, 565 \mathrm{MHz}\right)$

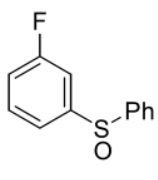

3d

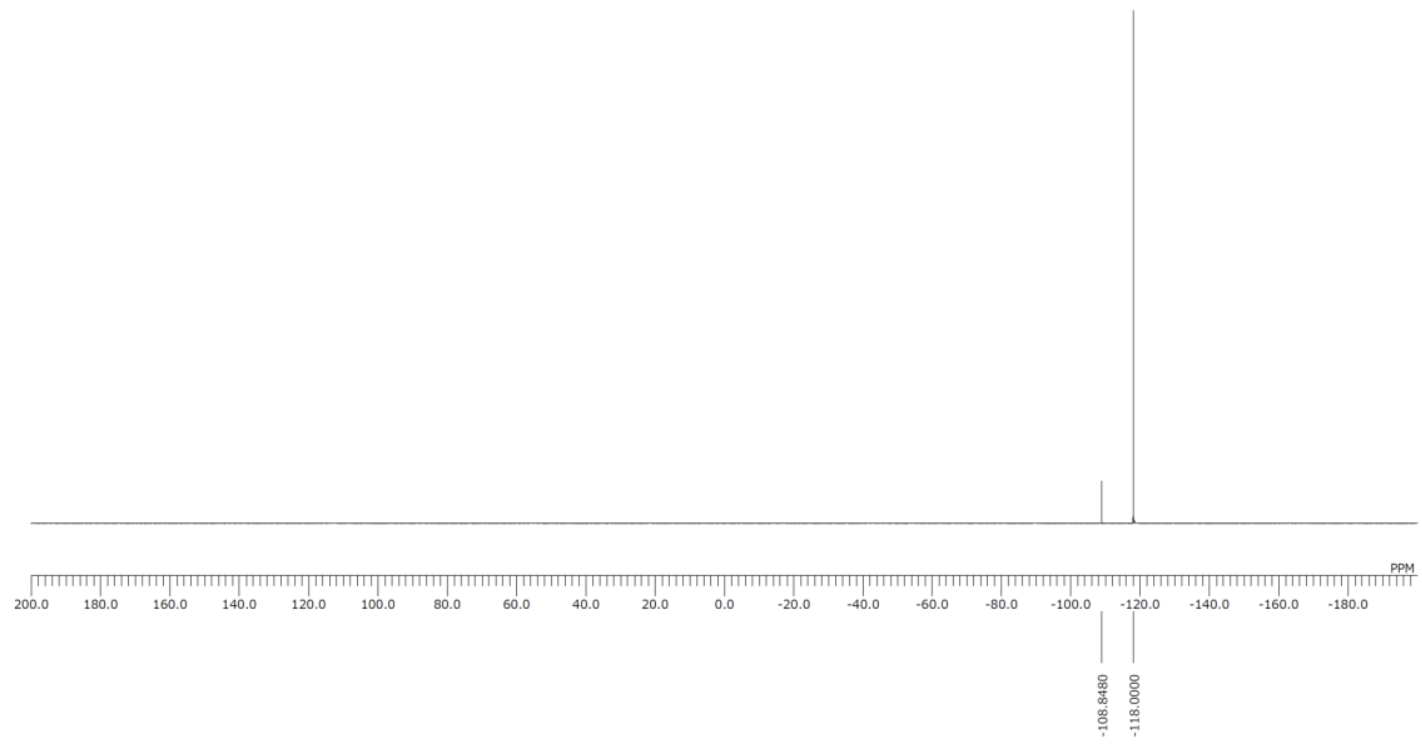

${ }^{1} \mathrm{H}$ NMR spectra of $3 e\left(\mathrm{CDCl}_{3}, 600 \mathrm{MHz}\right)$
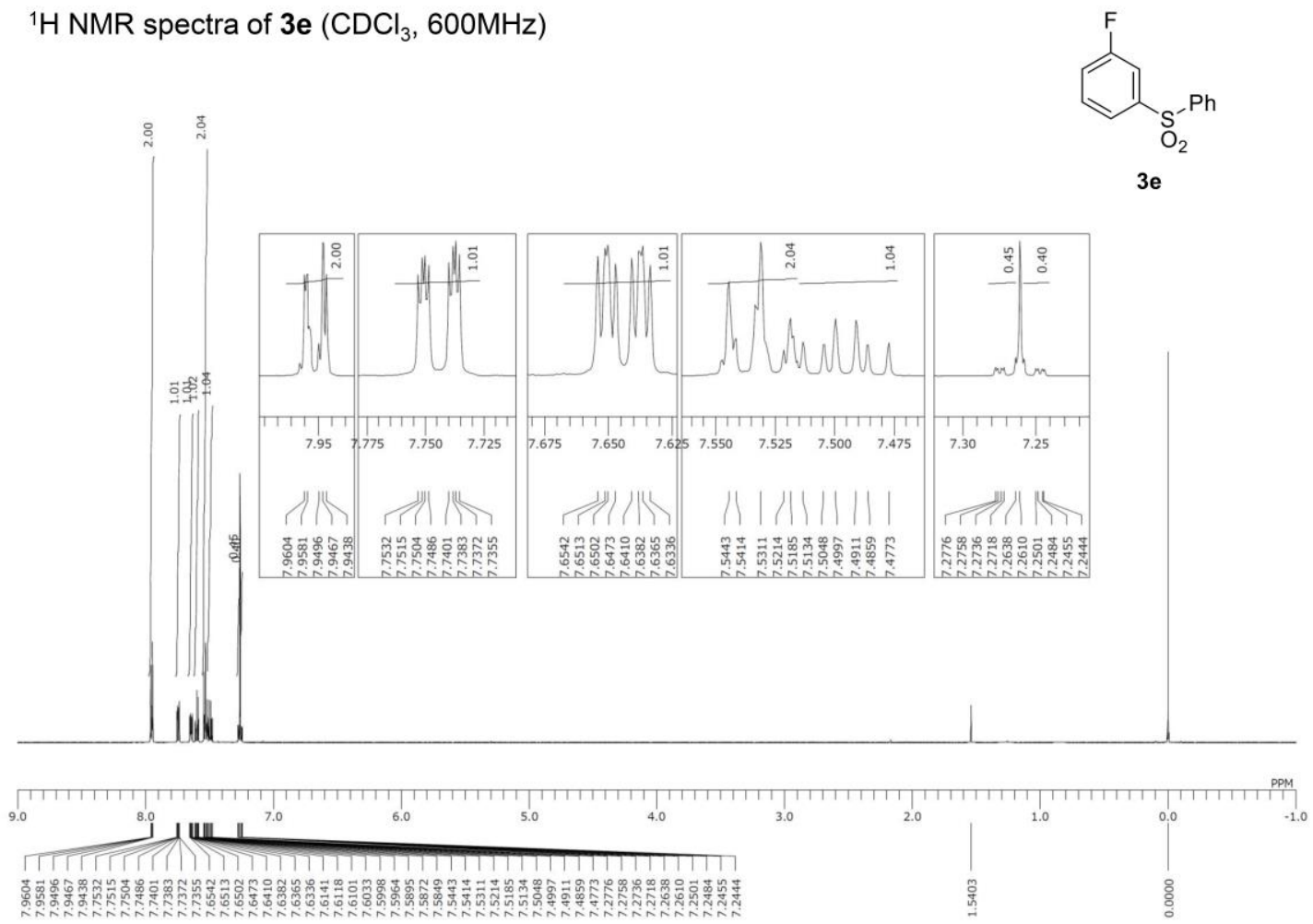
${ }^{13} \mathrm{C}$ NMR spectra of $3 \mathrm{e}\left(\mathrm{CDCl}_{3}, 150 \mathrm{MHz}\right)$

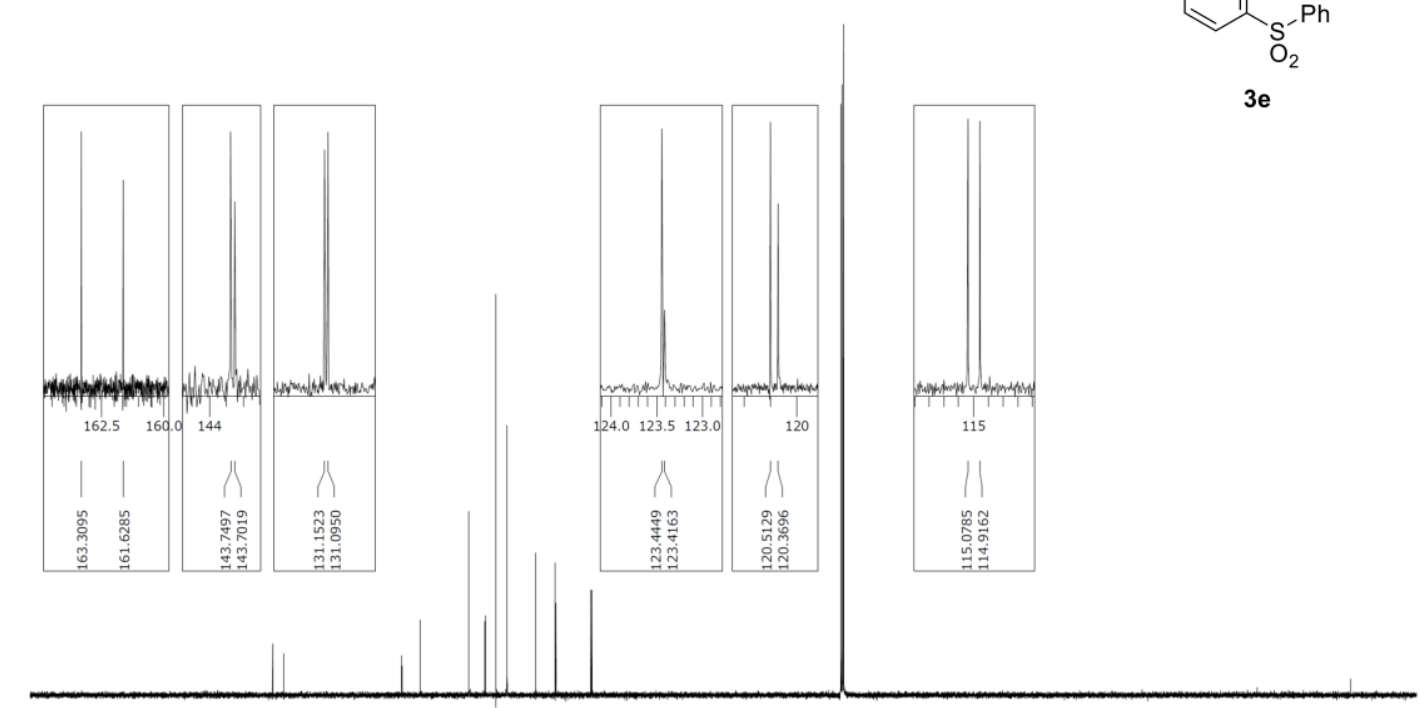

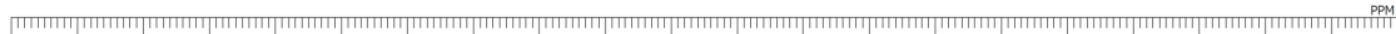

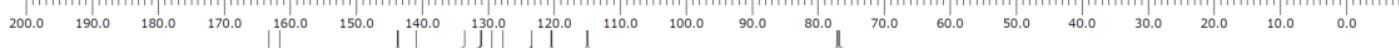

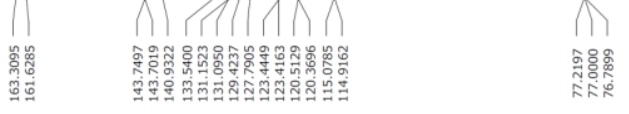

${ }^{19} \mathrm{~F}$ NMR spectra of $3 e\left(\mathrm{CDCl}_{3}, 565 \mathrm{MHz}\right)$

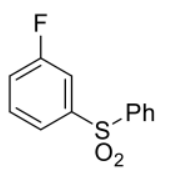

$3 e$

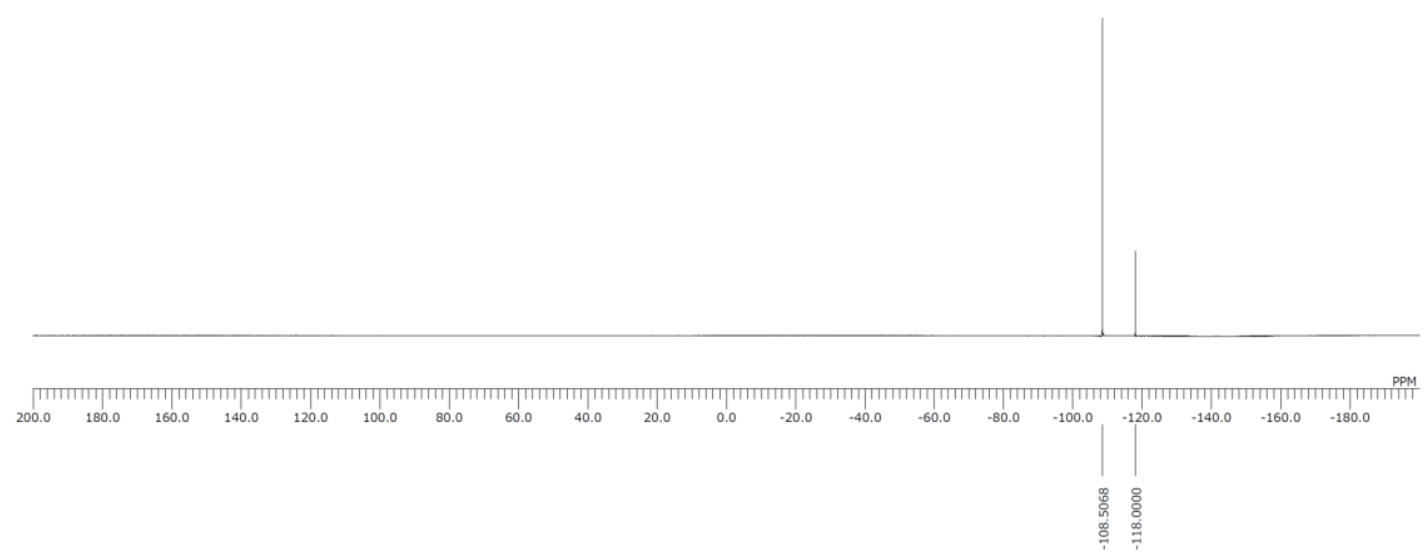


${ }^{1} \mathrm{H}$ NMR spectra of $3 f\left(\mathrm{CDCl}_{3}, 600 \mathrm{MHz}\right)$
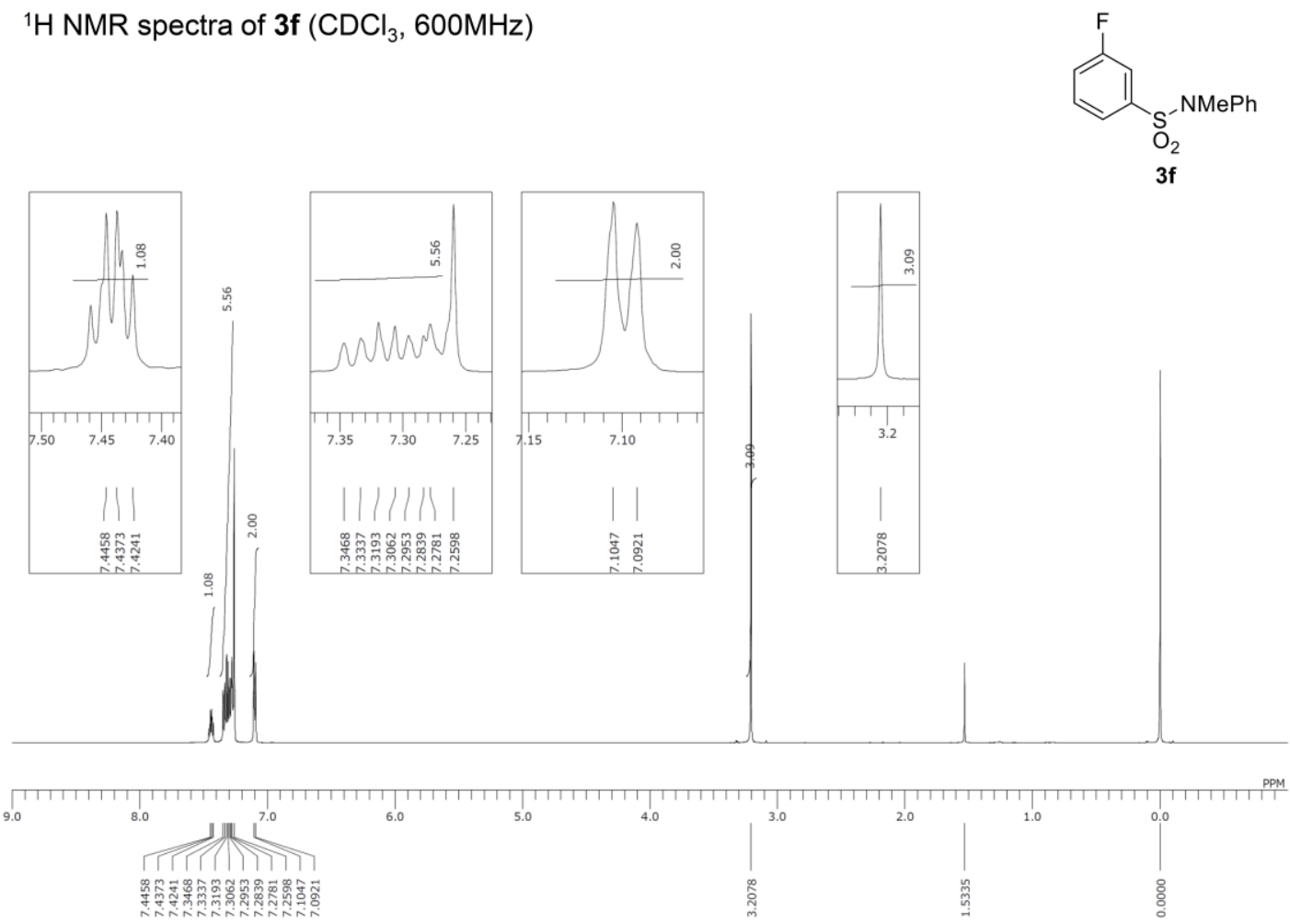

${ }^{13} \mathrm{C}$ NMR spectra of $\mathbf{3 f}\left(\mathrm{CDCl}_{3}, 150 \mathrm{MHz}\right)$

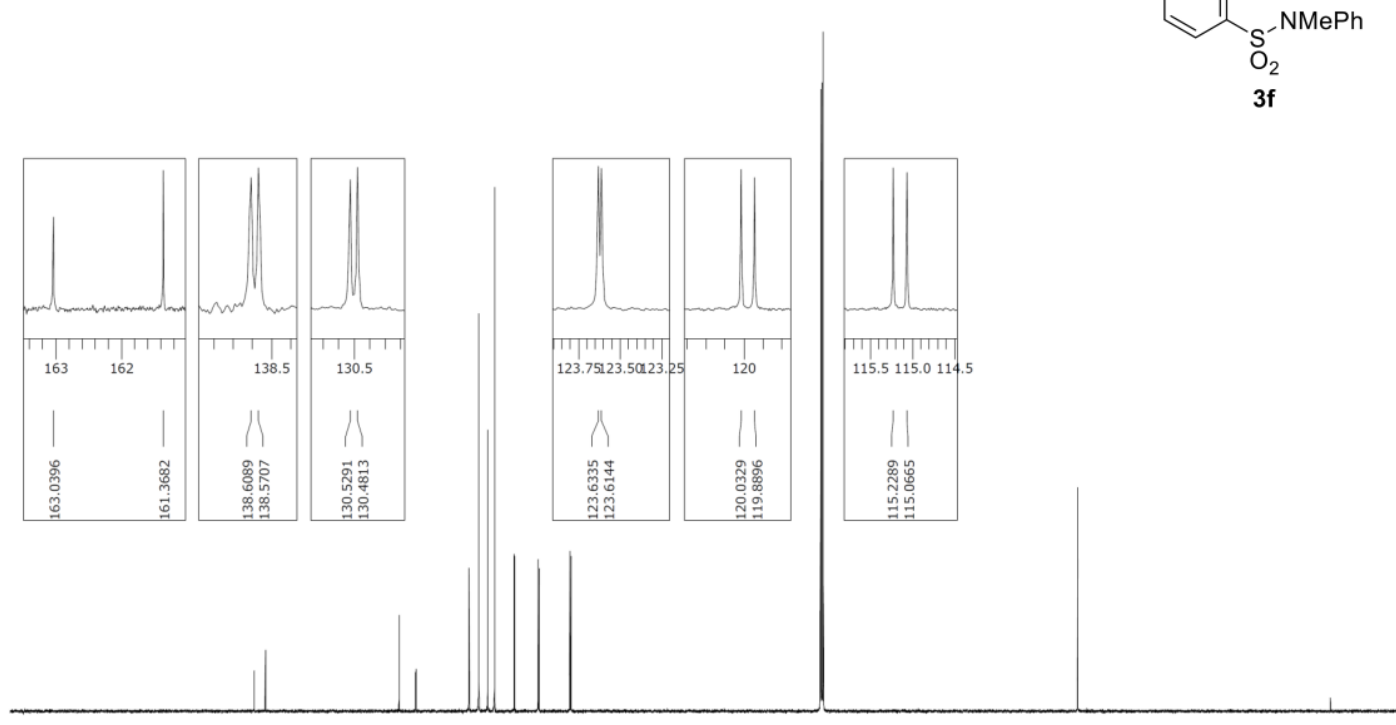

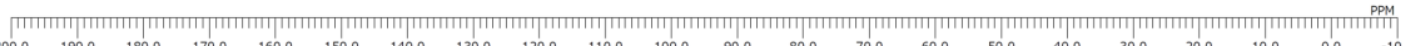

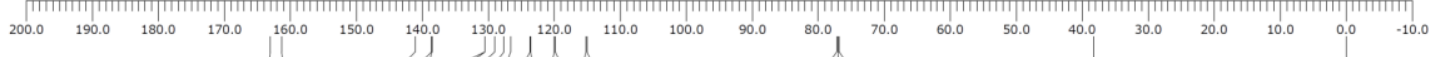

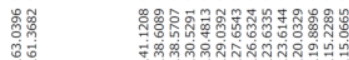


${ }^{19} \mathrm{~F}$ NMR spectra of $\mathbf{3 f}\left(\mathrm{CDCl}_{3}, 565 \mathrm{MHz}\right)$
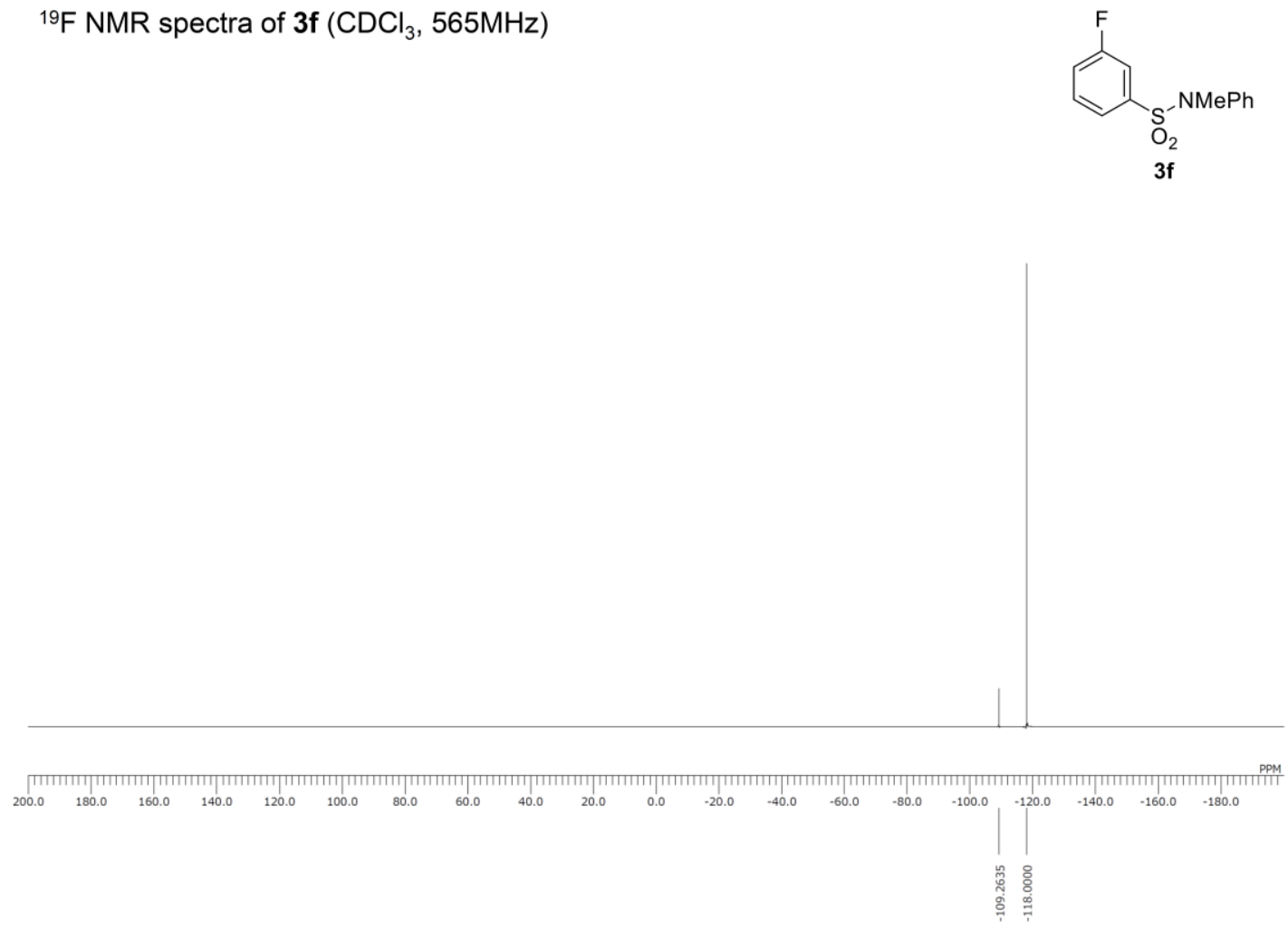

${ }^{1} \mathrm{H}$ NMR spectra of $\mathbf{3 g}\left(\mathrm{CDCl}_{3}, 600 \mathrm{MHz}\right)$

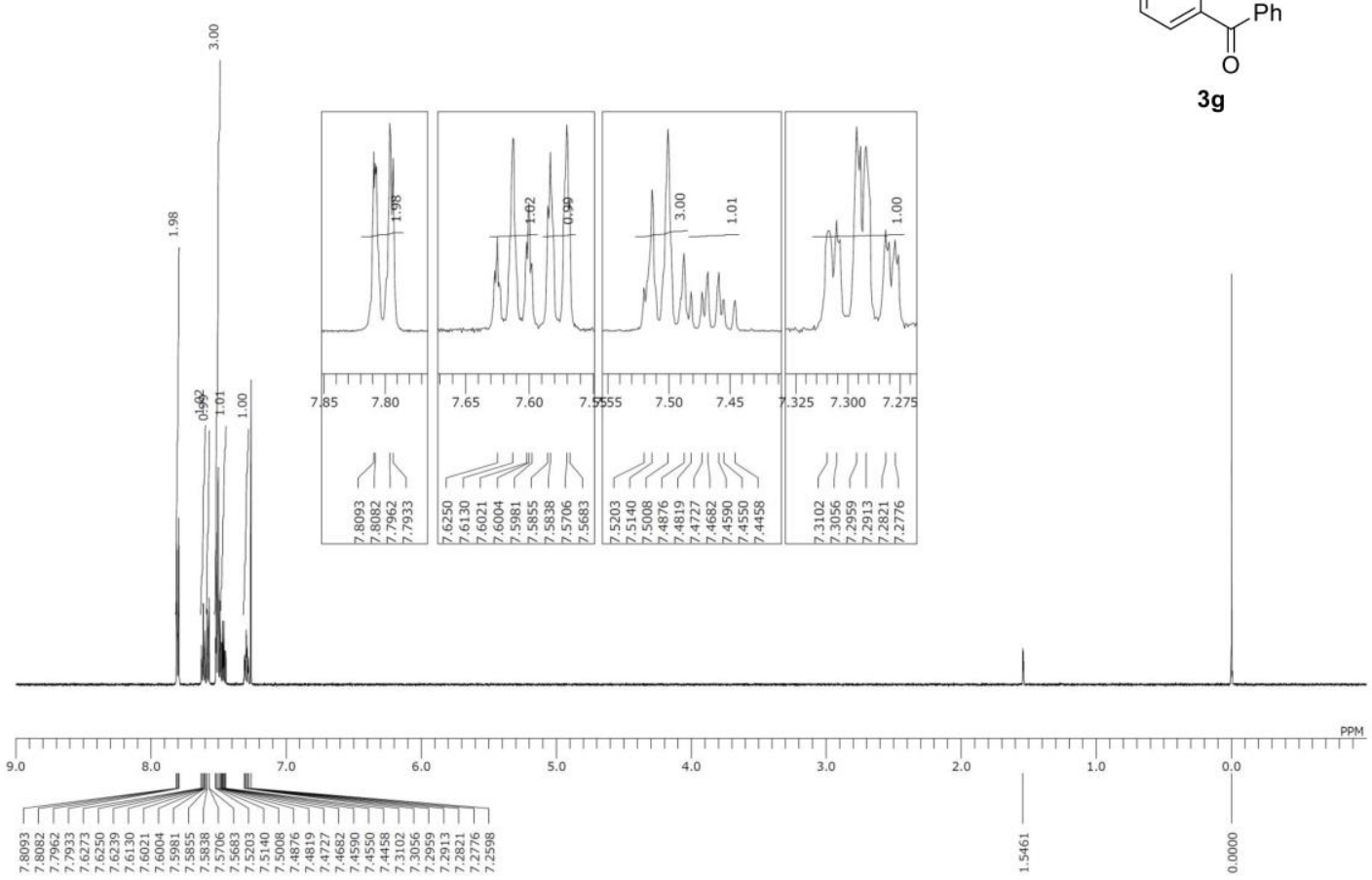


${ }^{13} \mathrm{C}$ NMR spectra of $\mathbf{3 g}\left(\mathrm{CDCl}_{3}, 150 \mathrm{MHz}\right)$

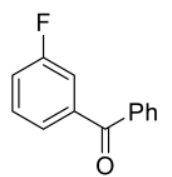

$3 \mathrm{~g}$
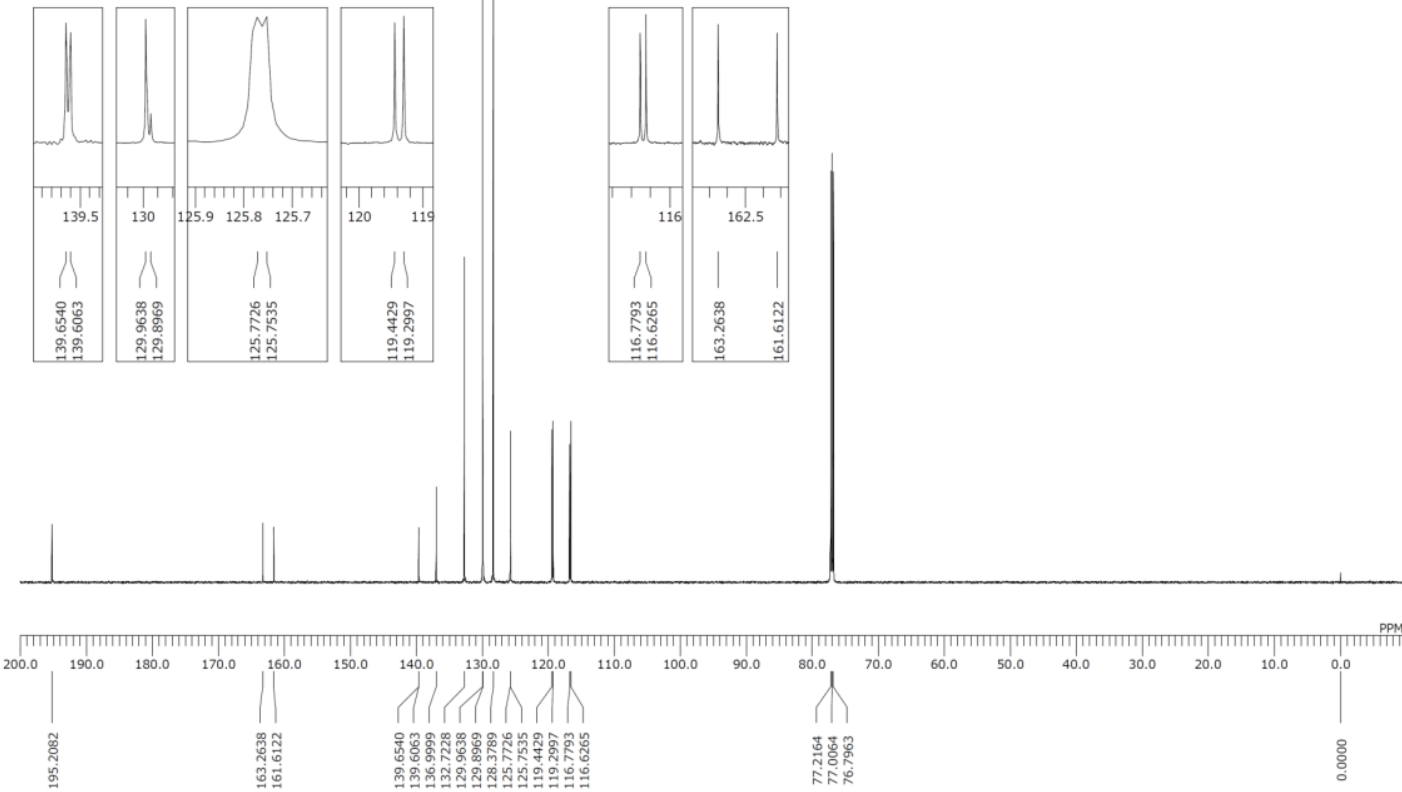

${ }^{19} \mathrm{~F}$ NMR spectra of $\mathbf{3 g}\left(\mathrm{CDCl}_{3}, 565 \mathrm{MHz}\right)$

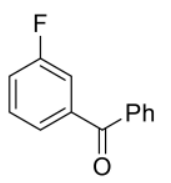

$3 \mathrm{~g}$

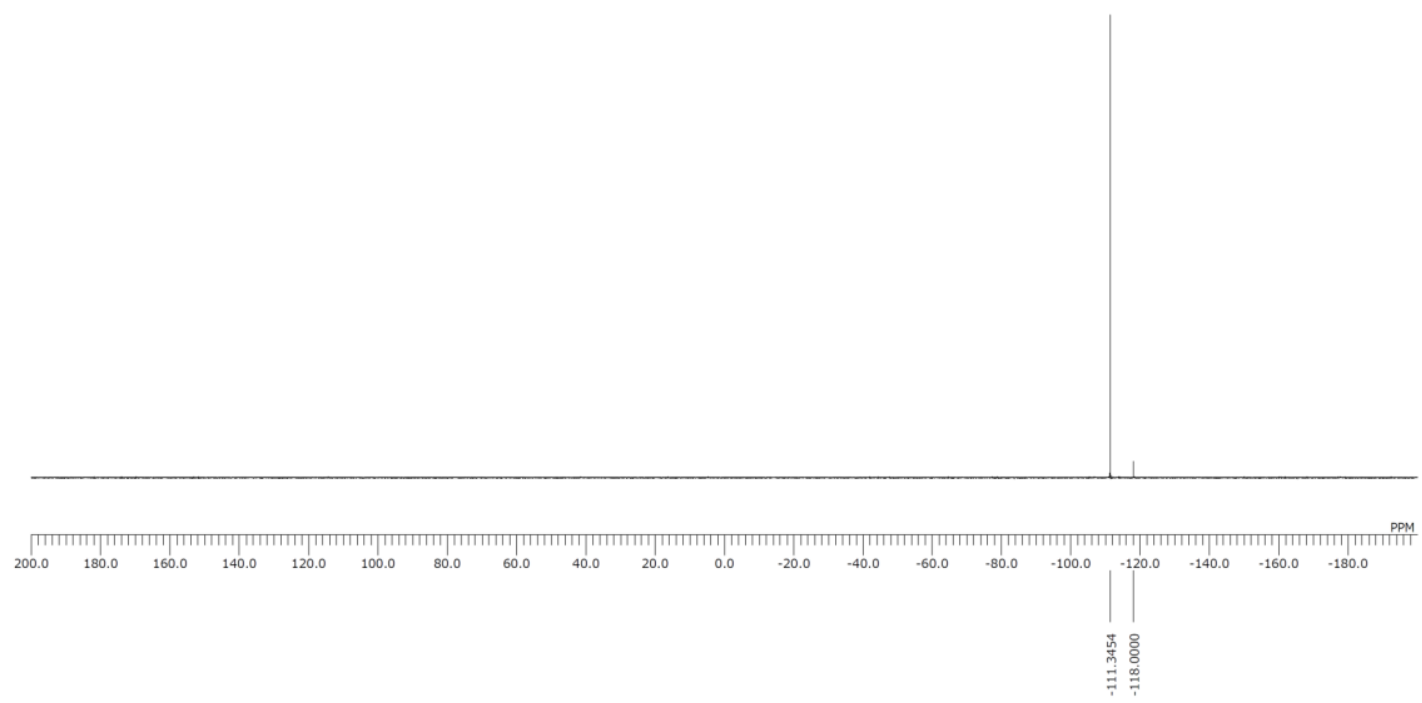


${ }^{1} \mathrm{H}$ NMR spectra of $3 \mathrm{~h}\left(\mathrm{CDCl}_{3}, 400 \mathrm{MHz}\right)$

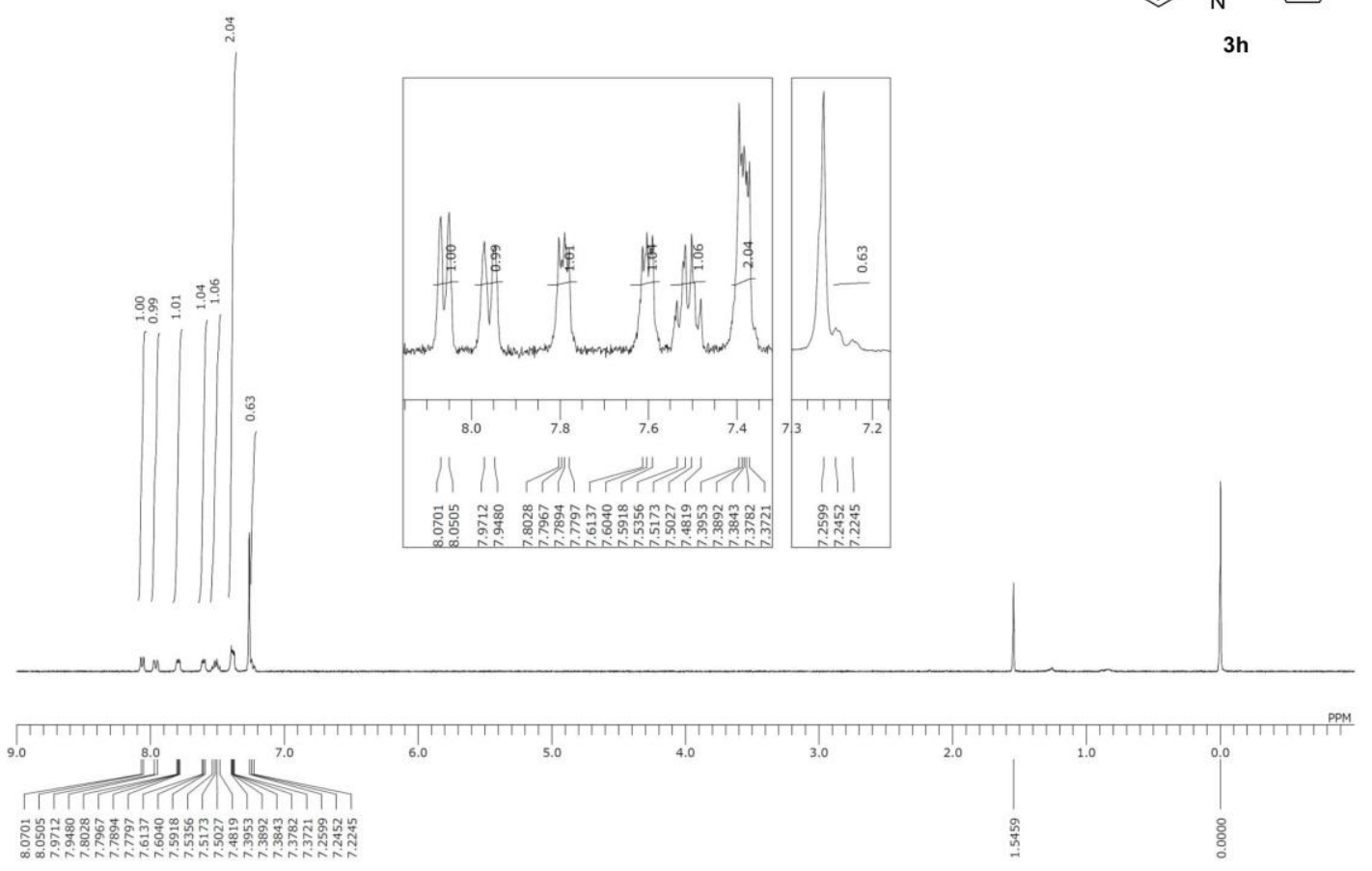

${ }^{13} \mathrm{C}$ NMR spectra of $3 \mathrm{~h}\left(\mathrm{CDCl}_{3}, 150 \mathrm{MHz}\right)$
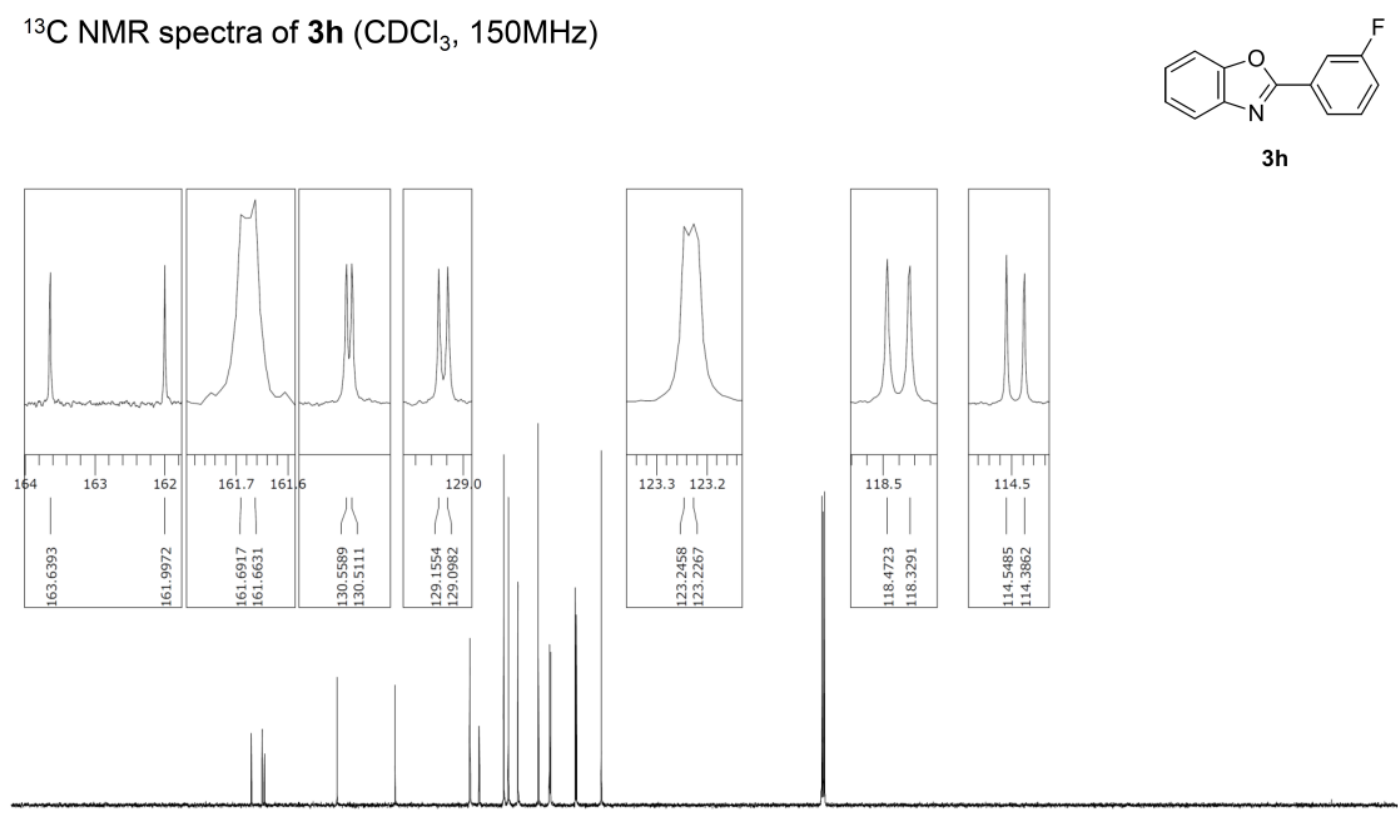

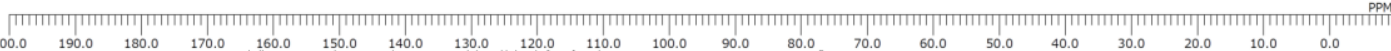

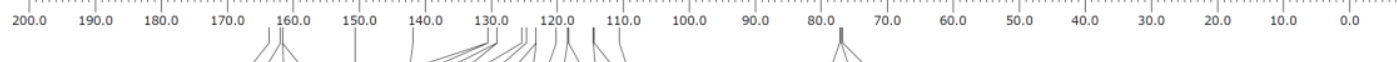

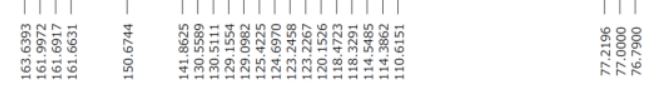


${ }^{19} \mathrm{~F}$ NMR spectra of $3 \mathbf{h}\left(\mathrm{CDCl}_{3}, 565 \mathrm{MHz}\right)$

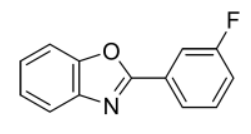

$3 h$

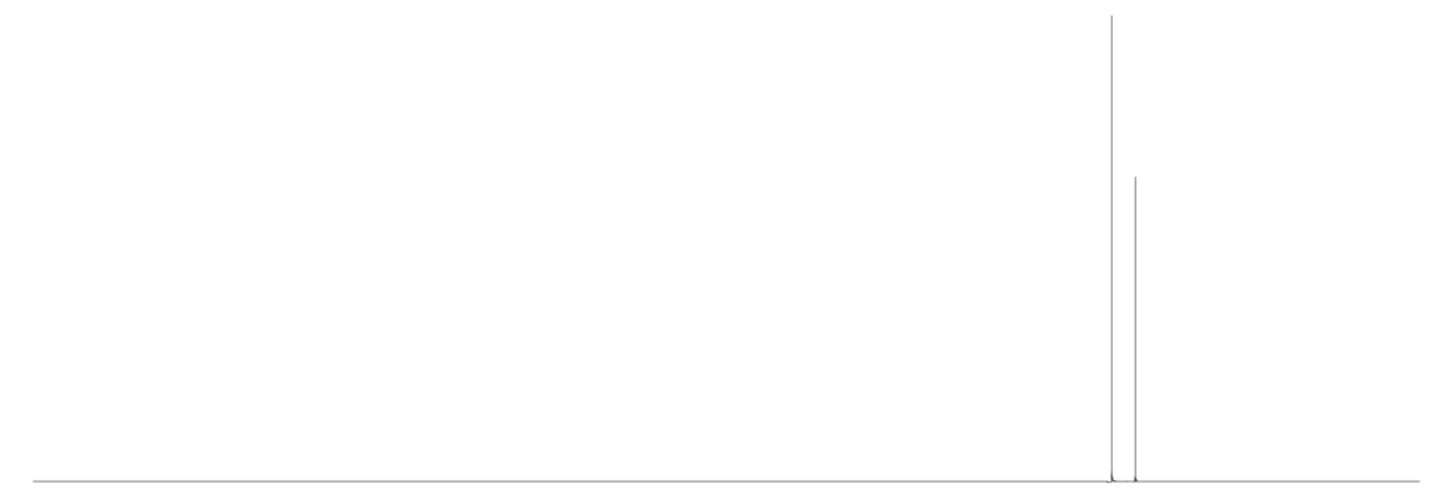

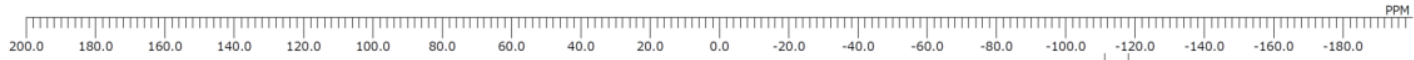

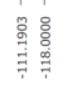

${ }^{1} \mathrm{H}$ NMR spectra of $\mathbf{2 a}\left(\mathrm{CDCl}_{3}, 400 \mathrm{MHz}\right)$

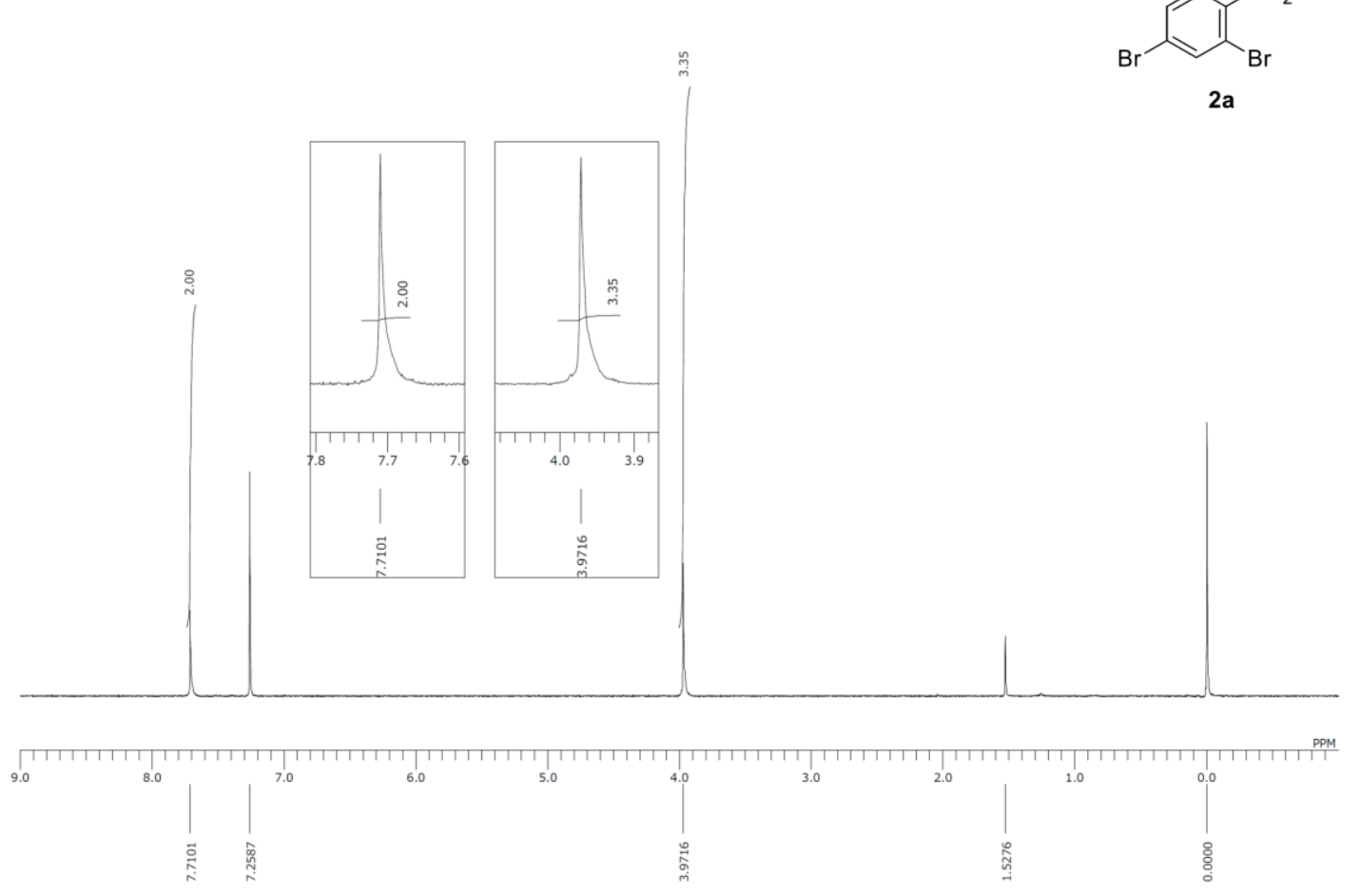


${ }^{13} \mathrm{C}$ NMR spectra of $2 \mathrm{a}\left(\mathrm{CDCl}_{3}, 150 \mathrm{MHz}\right)$
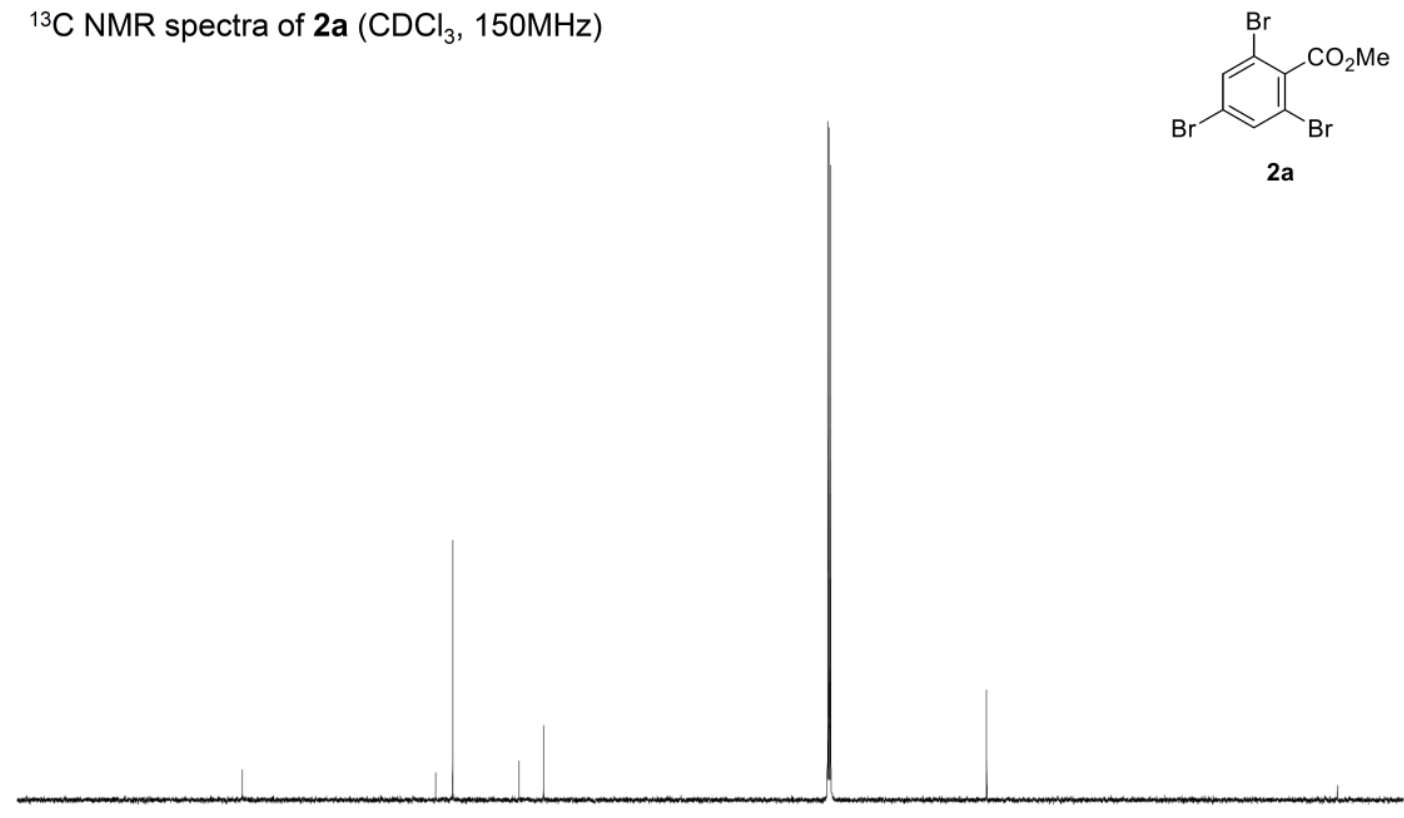

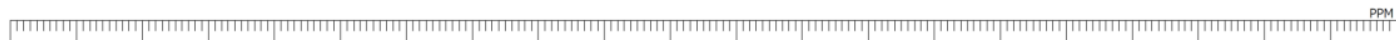

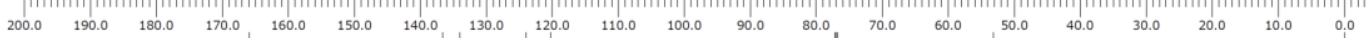

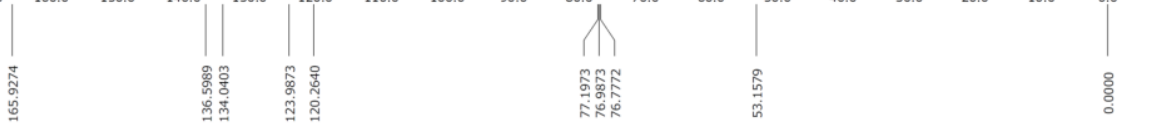

${ }^{1} \mathrm{H}$ NMR spectra of $\mathbf{2 b}\left(\mathrm{CDCl}_{3}, 400 \mathrm{MHz}\right)$

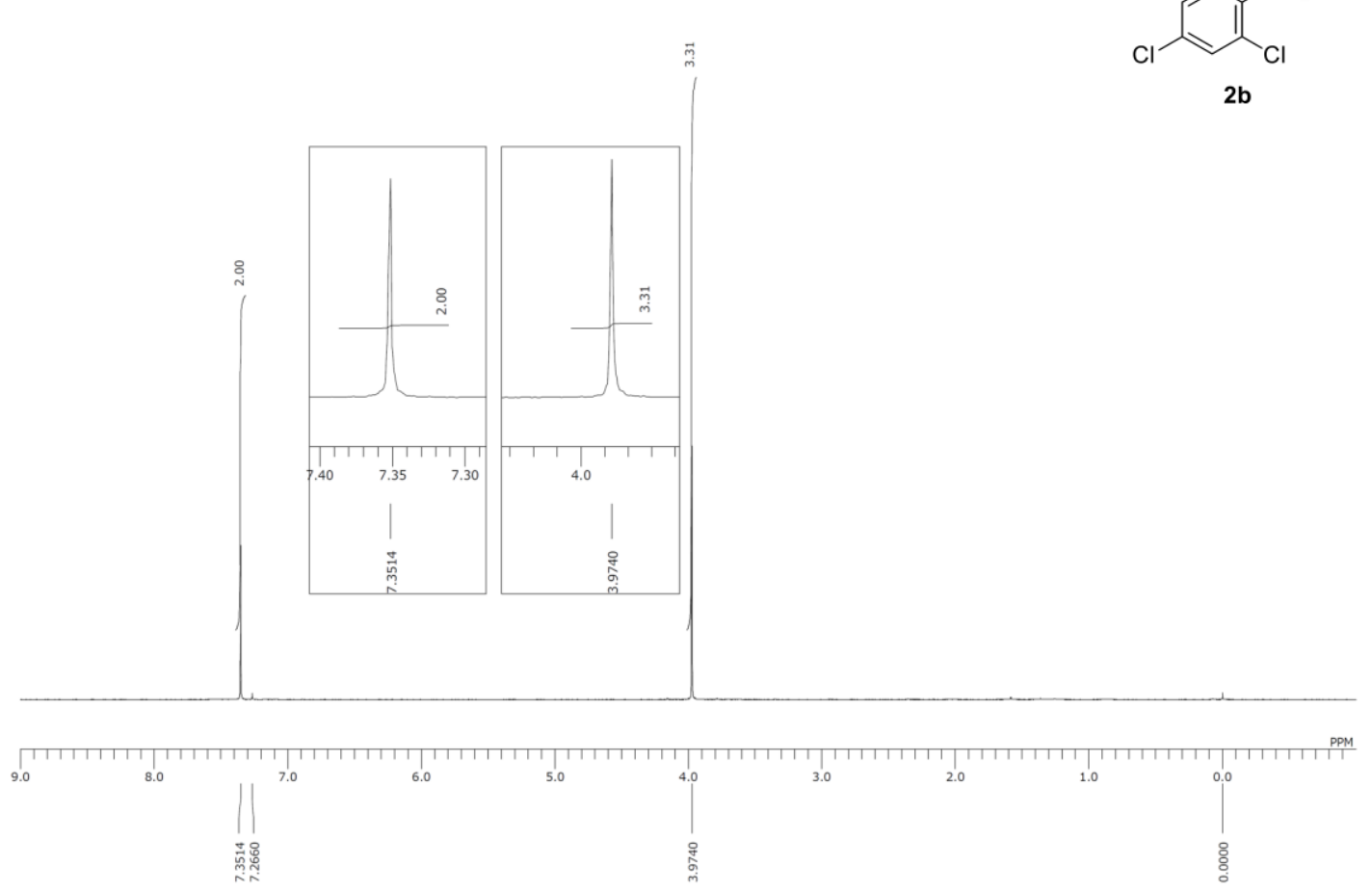


${ }^{13} \mathrm{C}$ NMR spectra of $\mathbf{2 b}\left(\mathrm{CDCl}_{3}, 100 \mathrm{MHz}\right)$

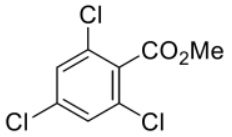

2b

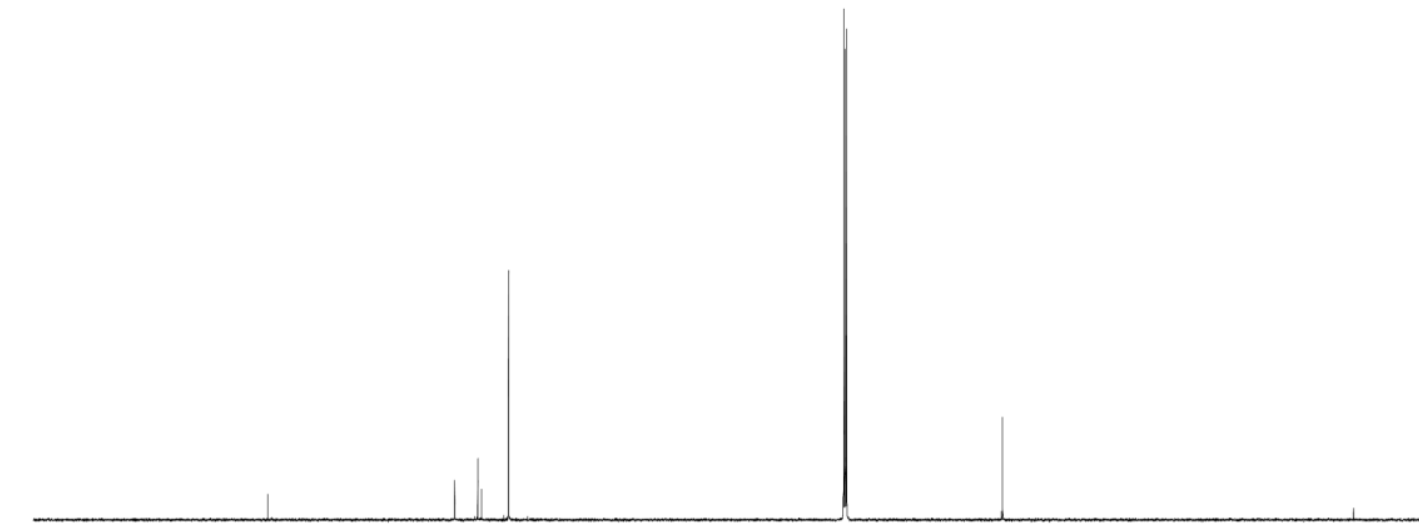

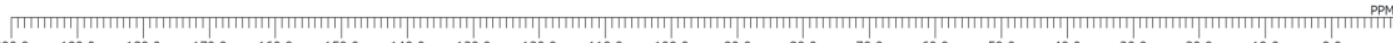

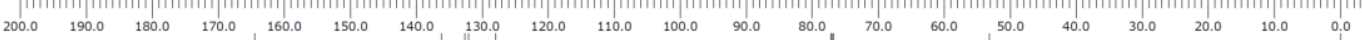

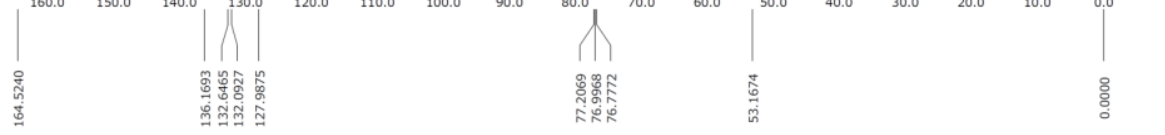

${ }^{1} \mathrm{H}$ NMR spectra of $2 \mathrm{c}\left(\mathrm{CDCl}_{3}, 600 \mathrm{MHz}\right)$

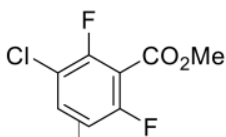

2c

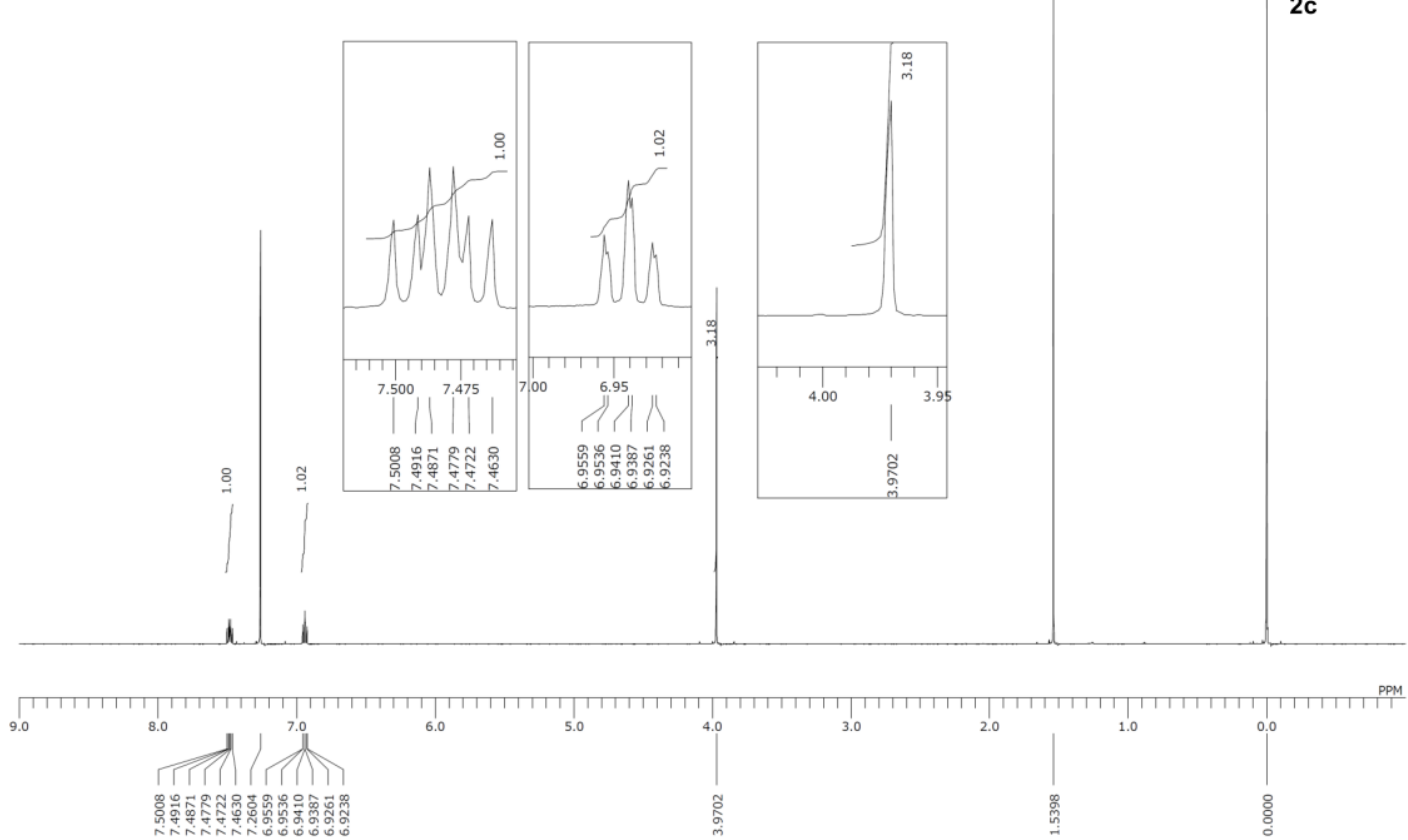


${ }^{13} \mathrm{C}$ NMR spectra of $2 \mathrm{c}\left(\mathrm{CDCl}_{3}, 150 \mathrm{MHz}\right)$

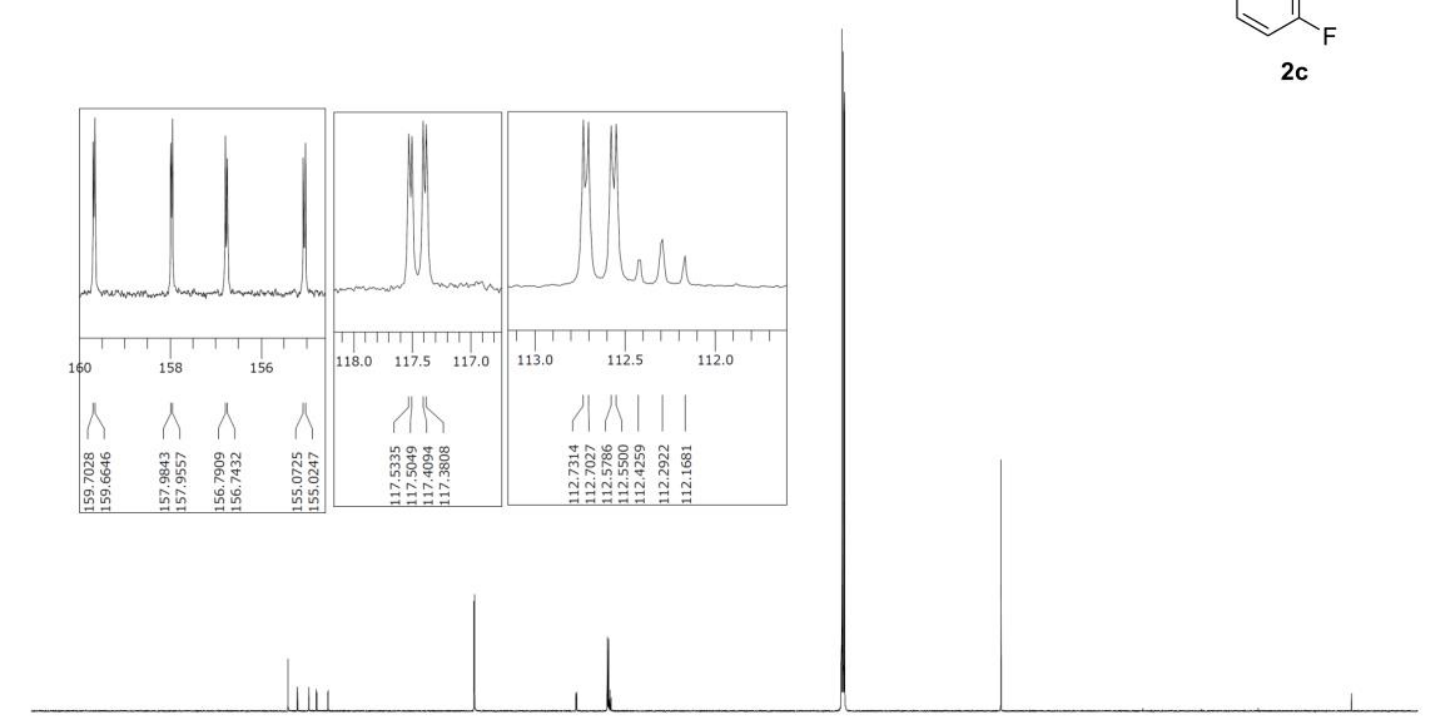

( ता

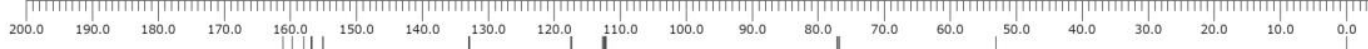

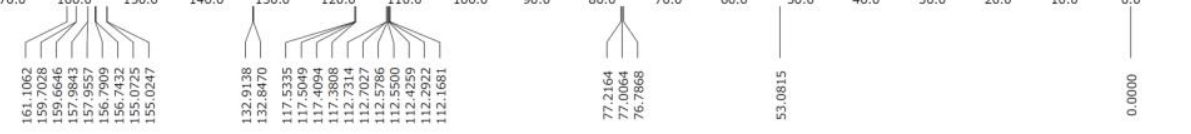

${ }^{19} \mathrm{~F}$ NMR spectra of $2 \mathrm{c}\left(\mathrm{CDCl}_{3}, 565 \mathrm{MHz}\right)$

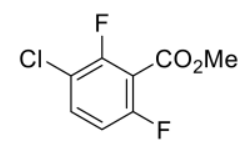

2c

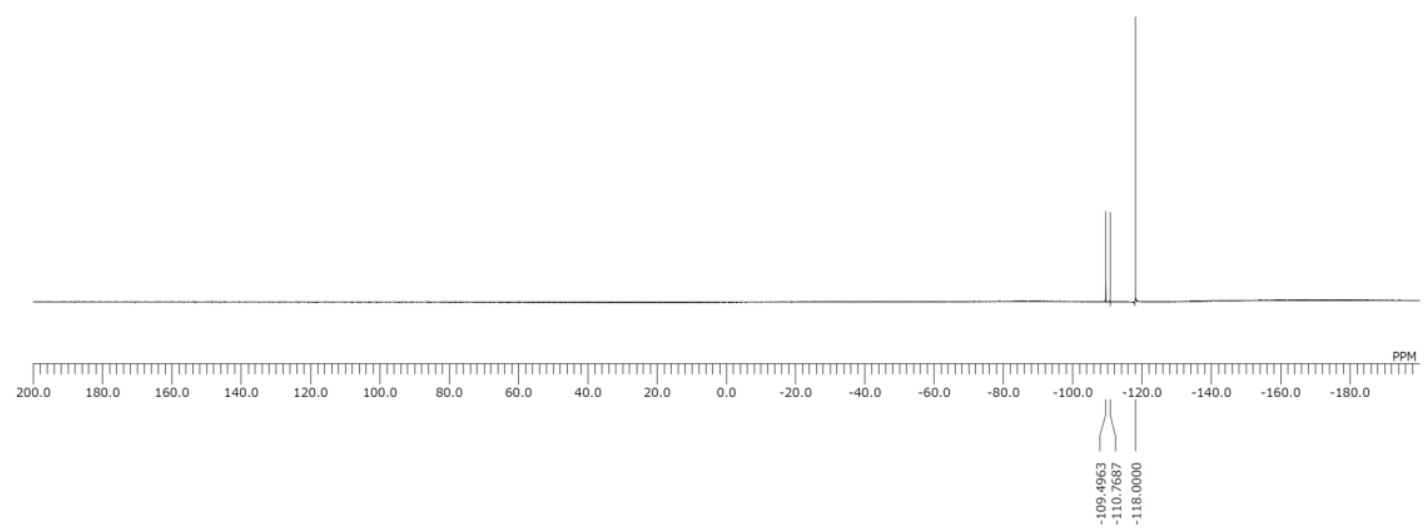


${ }^{1} \mathrm{H}$ NMR spectra of $\mathbf{2 d}\left(\mathrm{CDCl}_{3}, 400 \mathrm{MHz}\right)$
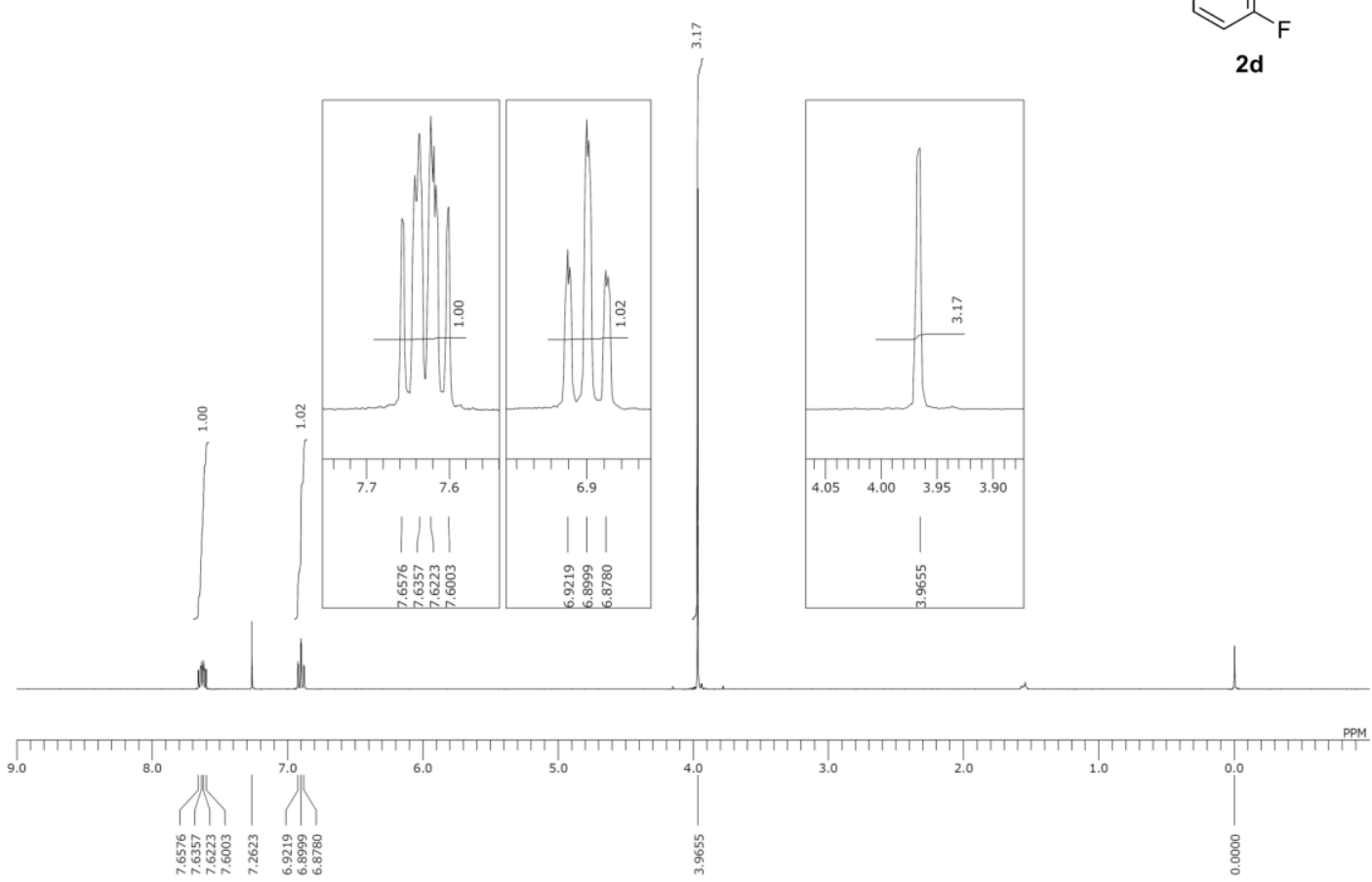

${ }^{13} \mathrm{C}$ NMR spectra of $\mathbf{2 d}\left(\mathrm{CDCl}_{3}, 150 \mathrm{MHz}\right)$

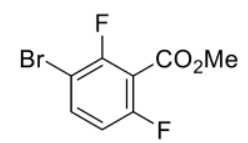

2d

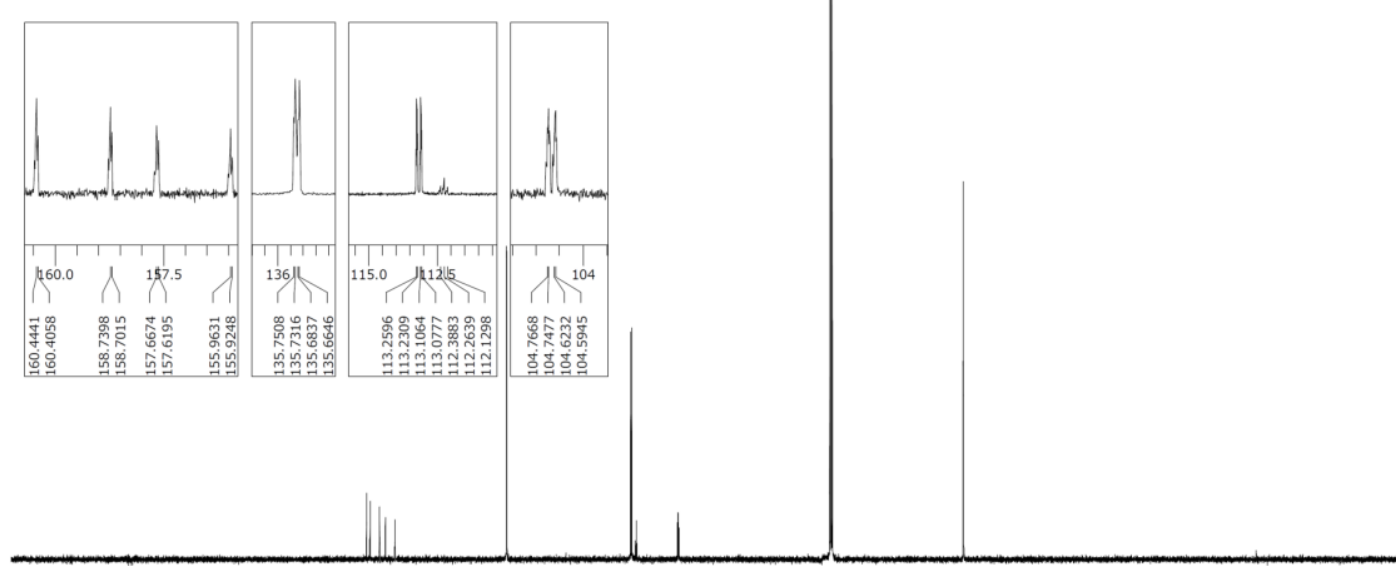

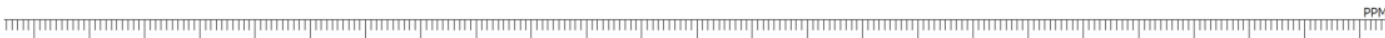

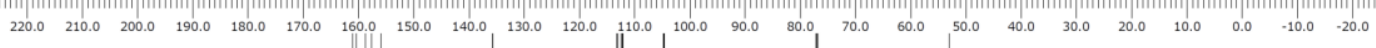
$\prod \prod_{1} \prod_{1} \prod_{n} \prod_{1}$

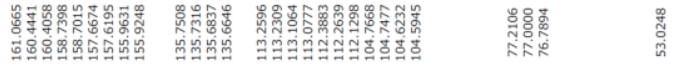


${ }^{19} \mathrm{~F}$ NMR spectra of $\mathbf{2 d}\left(\mathrm{CDCl}_{3}, 565 \mathrm{MHz}\right)$

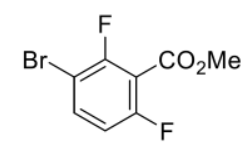

2d

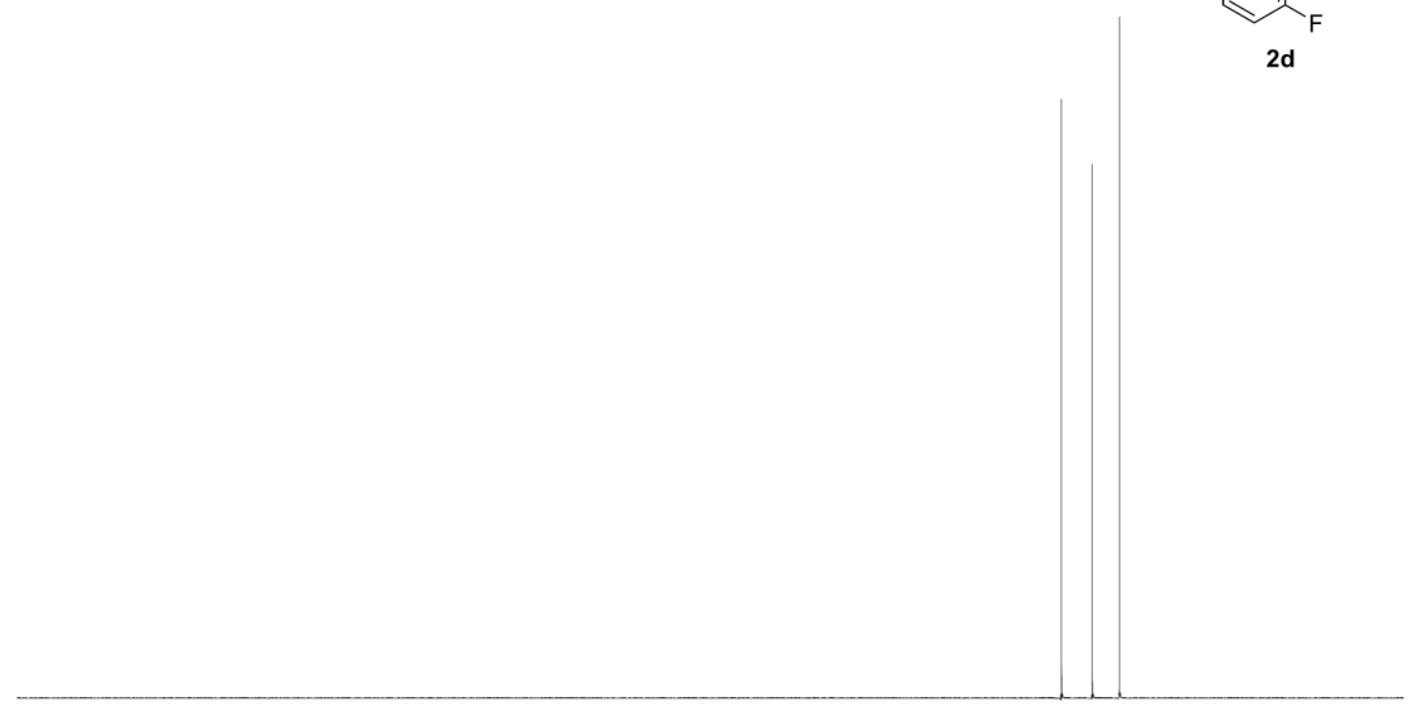

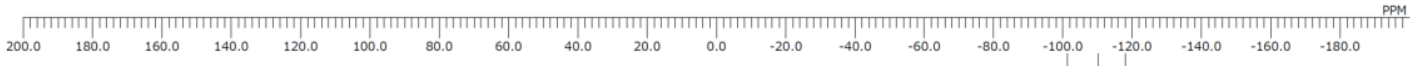

In

${ }^{1} \mathrm{H}$ NMR spectra of $2 \mathrm{e}\left(\mathrm{CDCl}_{3}, 600 \mathrm{MHz}\right)$

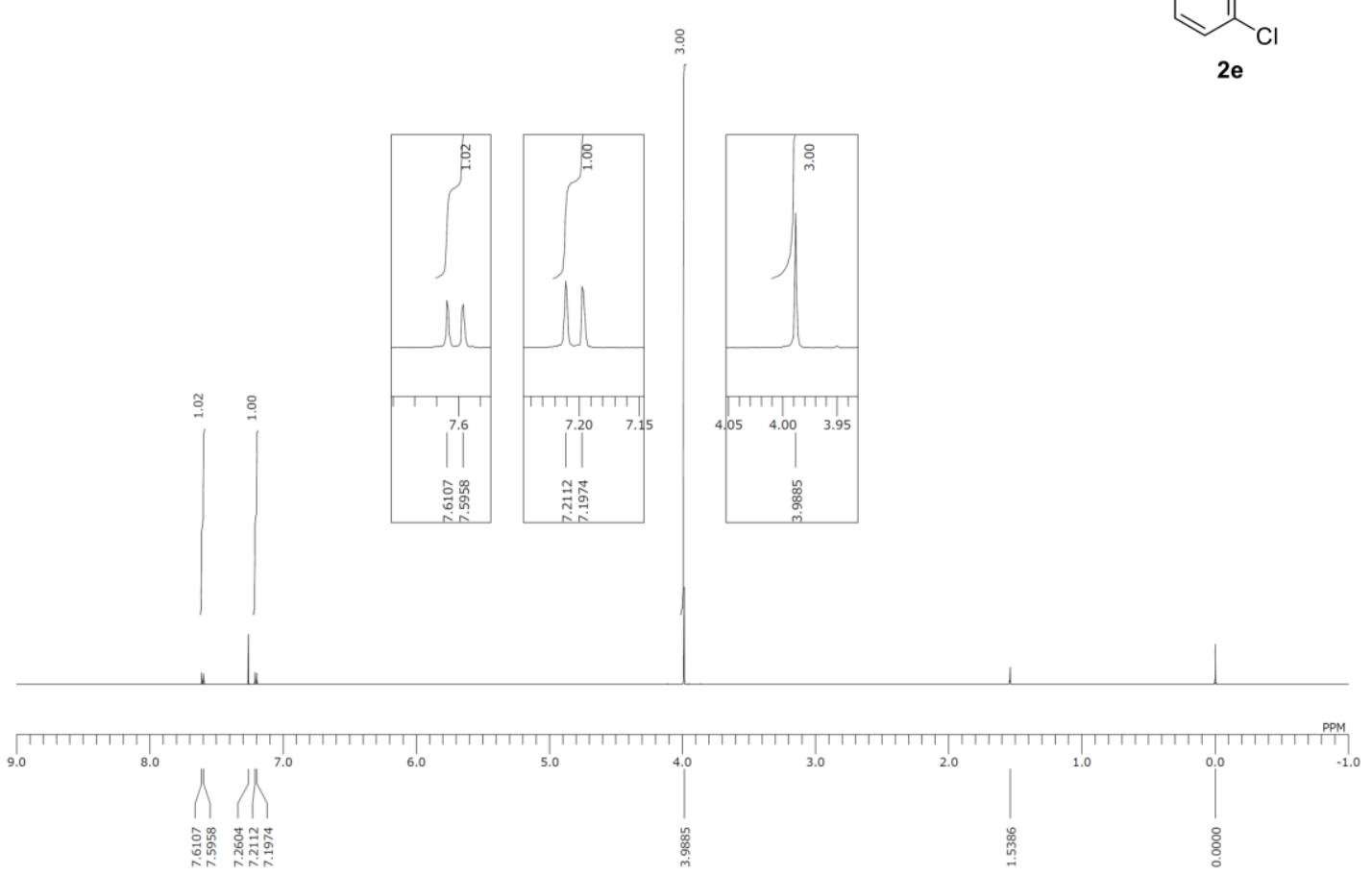


${ }^{13} \mathrm{C}$ NMR spectra of $2 \mathrm{e}\left(\mathrm{CDCl}_{3}, 150 \mathrm{MHz}\right)$
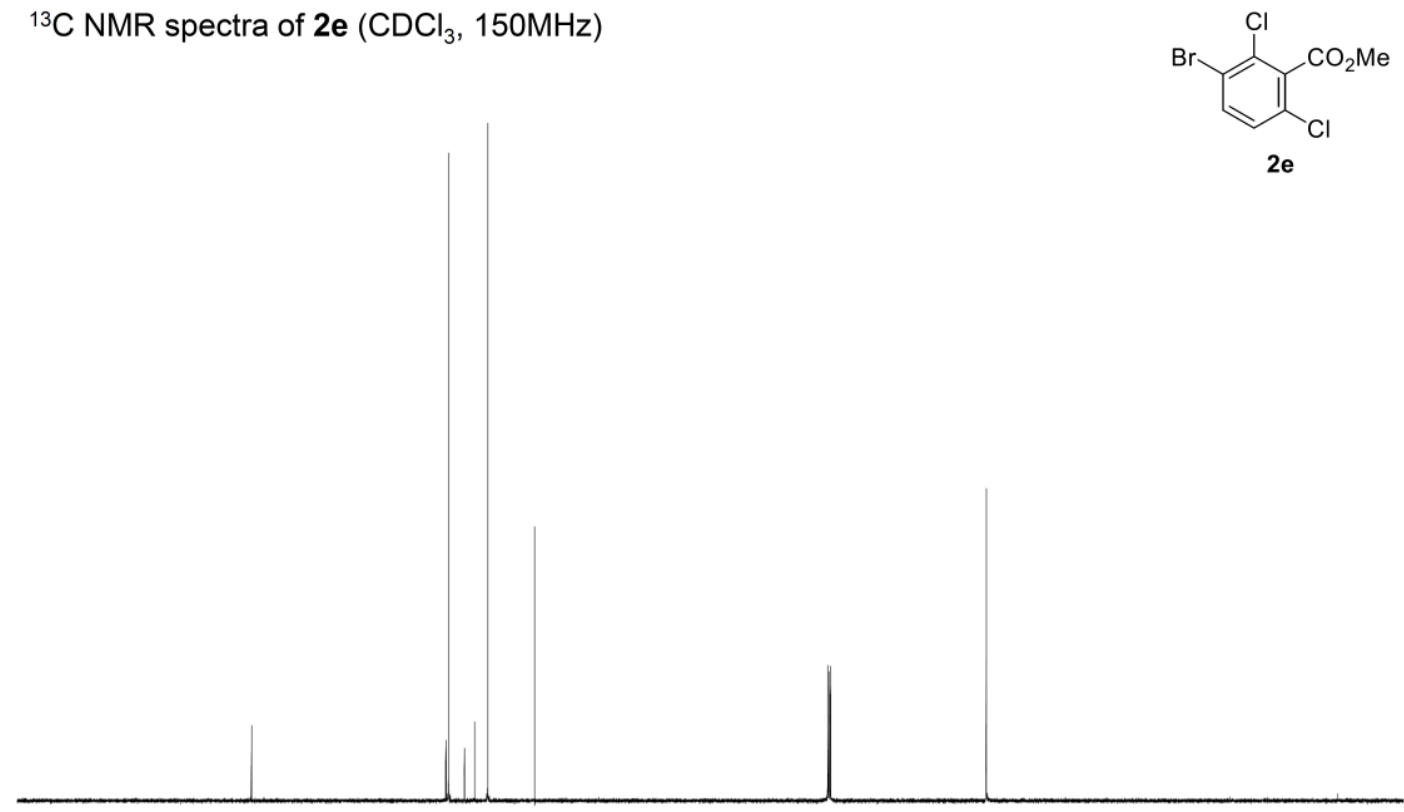

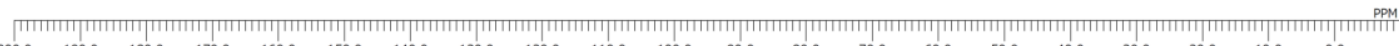

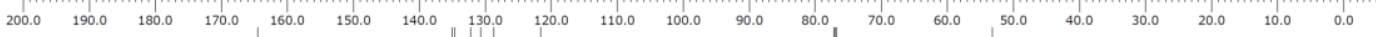

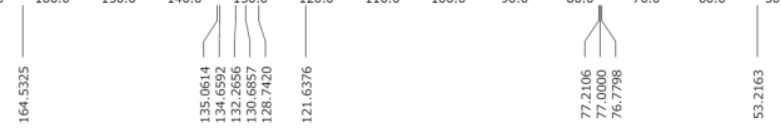

${ }^{1} \mathrm{H}$ NMR spectra of $\mathbf{2} \mathbf{f}\left(\mathrm{CDCl}_{3}, 400 \mathrm{MHz}\right)$

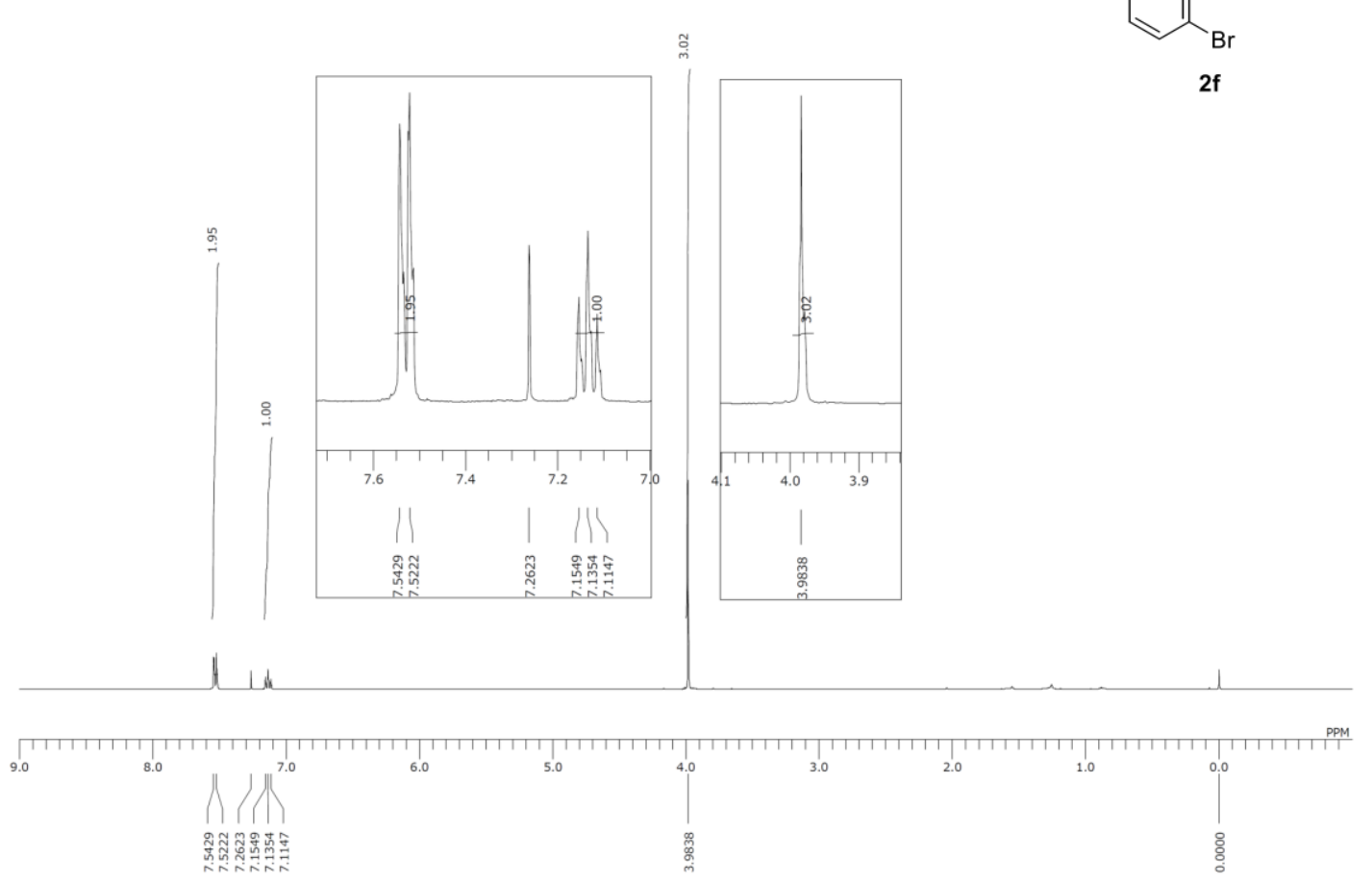


${ }^{13} \mathrm{C} \mathrm{NMR}$ spectra of $2 \mathrm{f}\left(\mathrm{CDCl}_{3}, 100 \mathrm{MHz}\right)$<smiles>CC(=O)c1c(Br)cccc1Br</smiles>

$2 f$

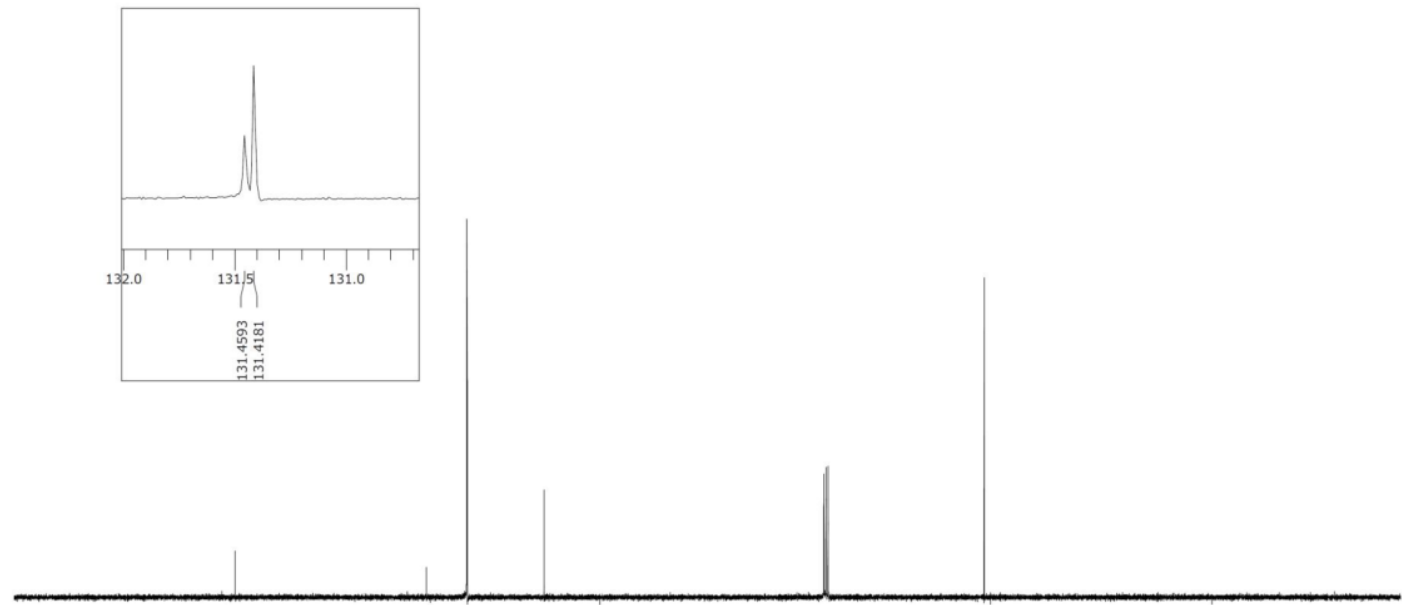

ह

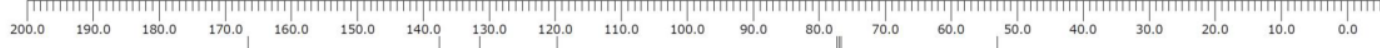

${ }^{1} \mathrm{H}$ NMR spectra of $\mathbf{2} \mathbf{g}\left(\mathrm{CDCl}_{3}, 600 \mathrm{MHz}\right)$

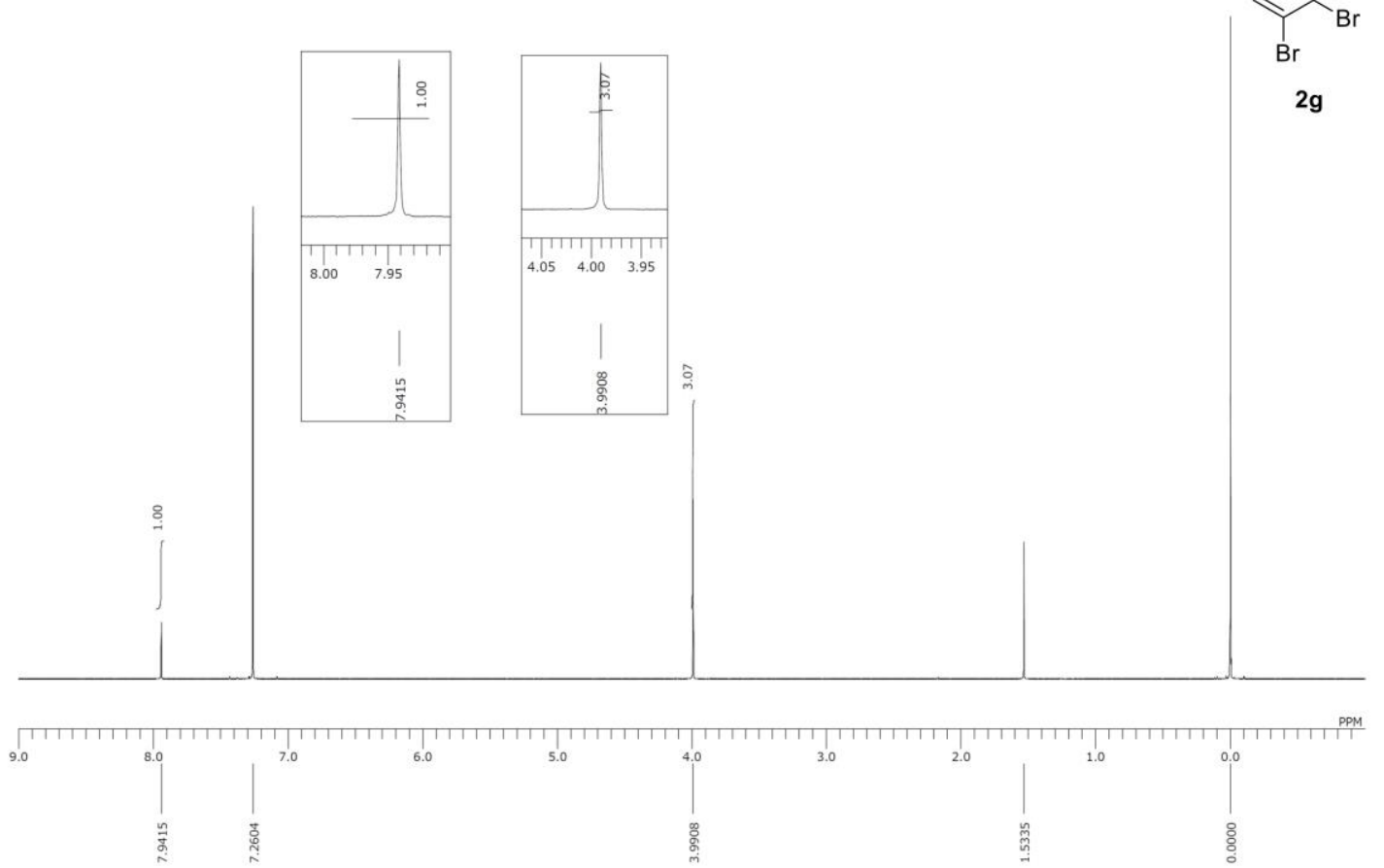


${ }^{13} \mathrm{C}$ NMR spectra of $\mathbf{2 g}\left(\mathrm{CDCl}_{3}, 150 \mathrm{MHz}\right)$

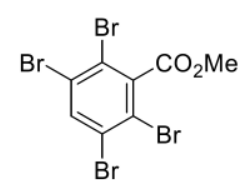

$2 \mathrm{~g}$

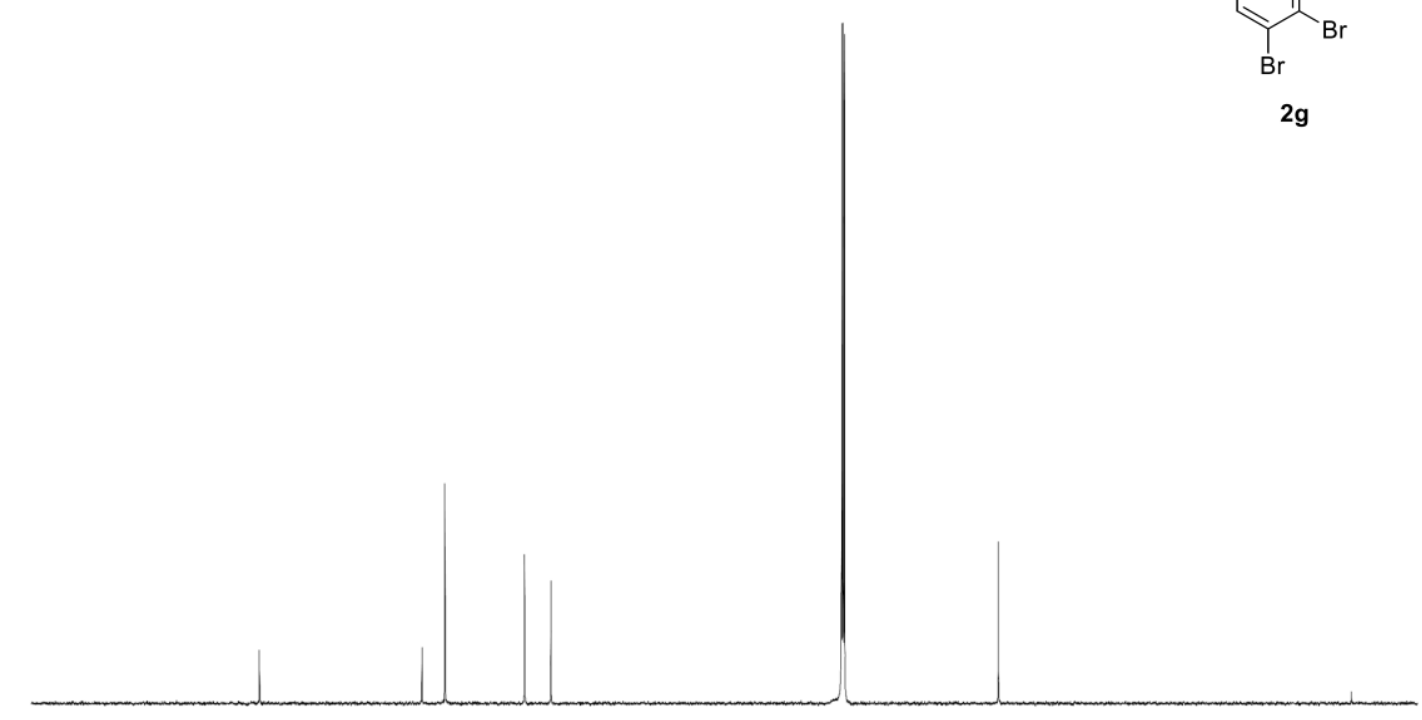

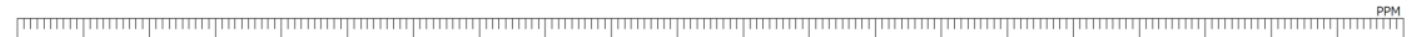

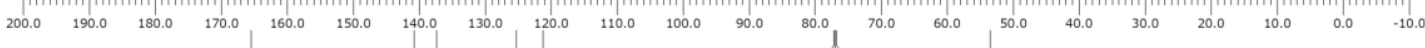

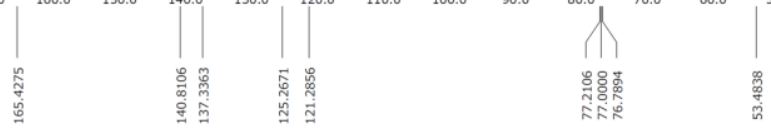

${ }^{1} \mathrm{H}$ NMR spectra of $\mathbf{2 h}-\mathbf{1 / 2} \mathbf{h}-\mathbf{2}\left(\mathrm{CDCl}_{3}, 600 \mathrm{MHz}\right)$
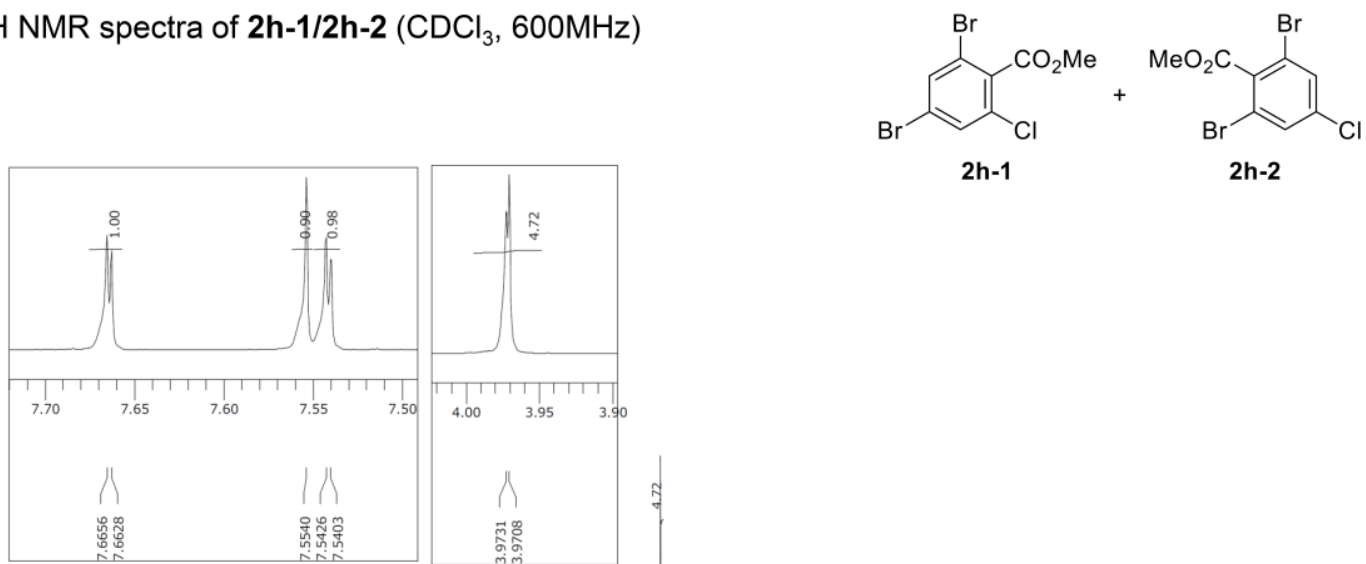

2h-1

2h-2

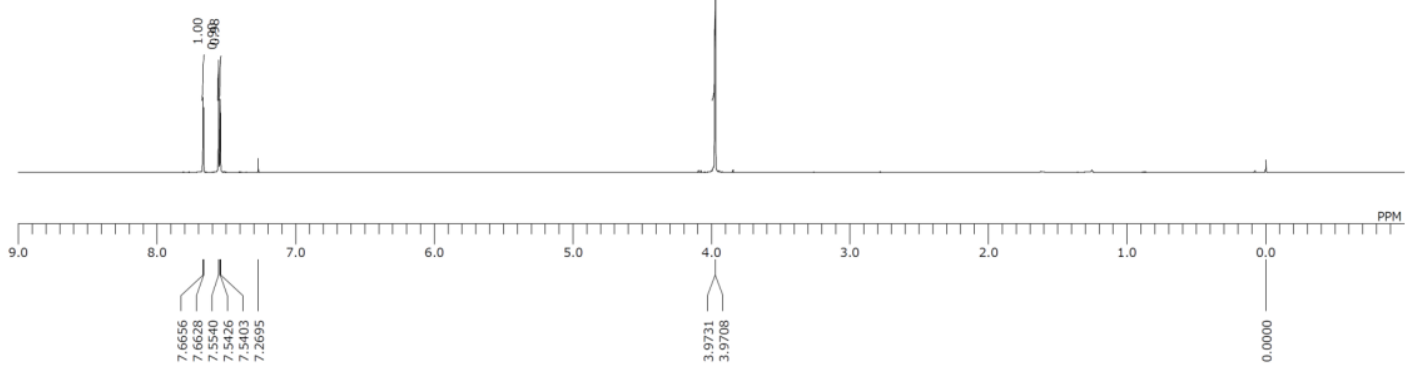


${ }^{13} \mathrm{C}$ NMR spectra of $\mathbf{2 h - 1 / 2 h - 2}\left(\mathrm{CDCl}_{3}, 150 \mathrm{MHz}\right)$
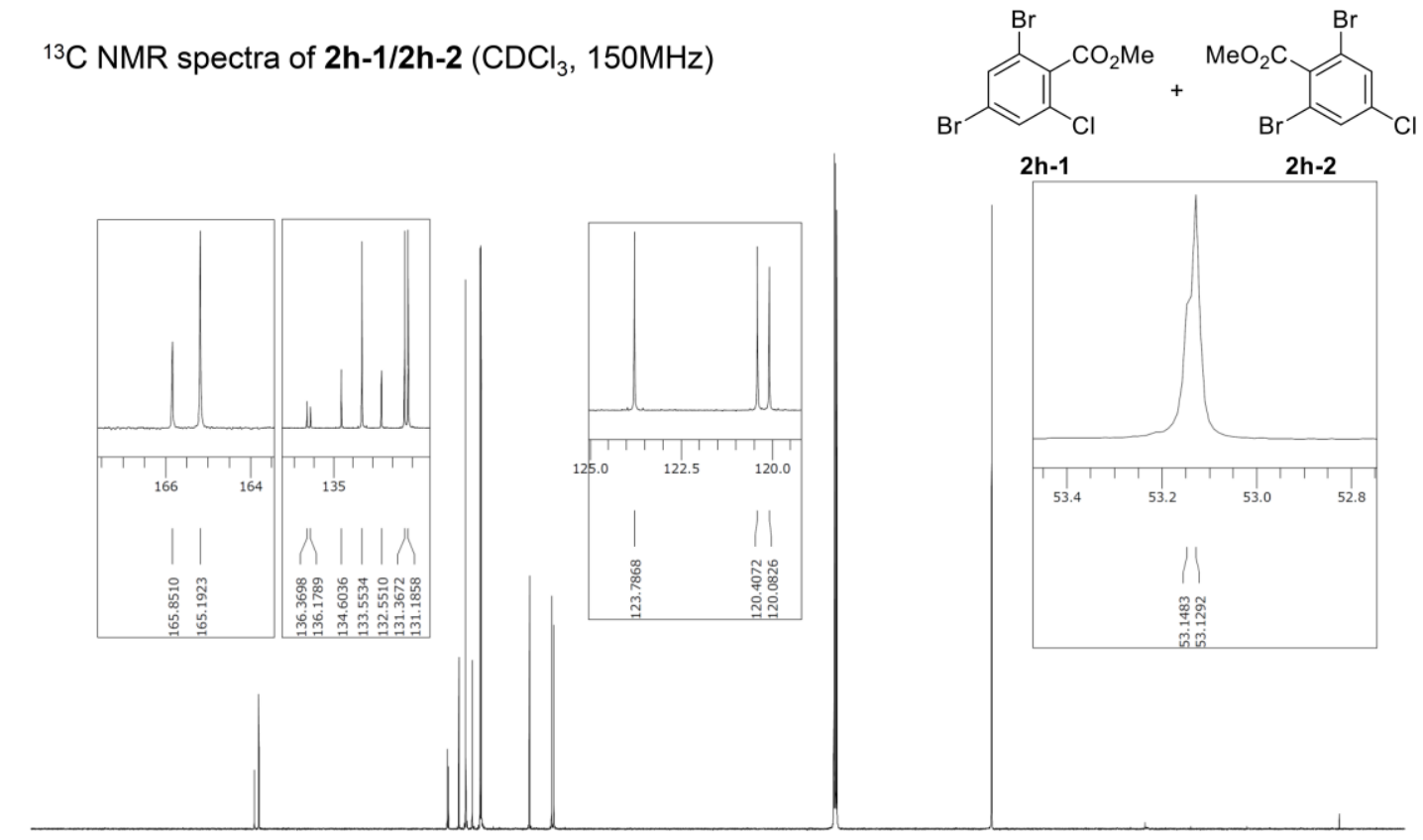

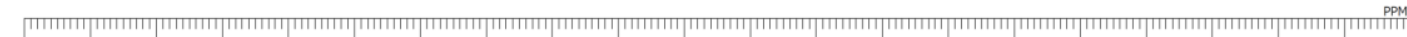

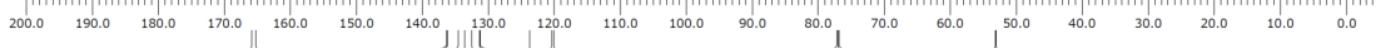

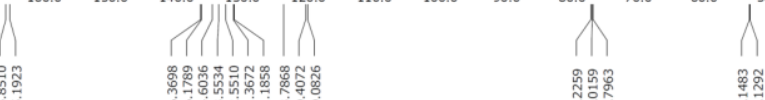

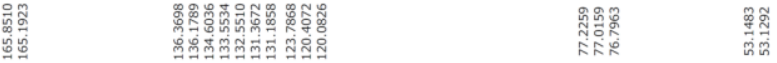

${ }^{1} \mathrm{H}$ NMR spectra of $\mathbf{2} \mathbf{i}(\mathrm{DMSO}-\mathrm{d6}, 600 \mathrm{MHz})$

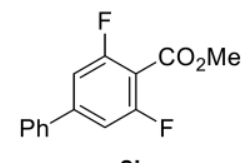

2i

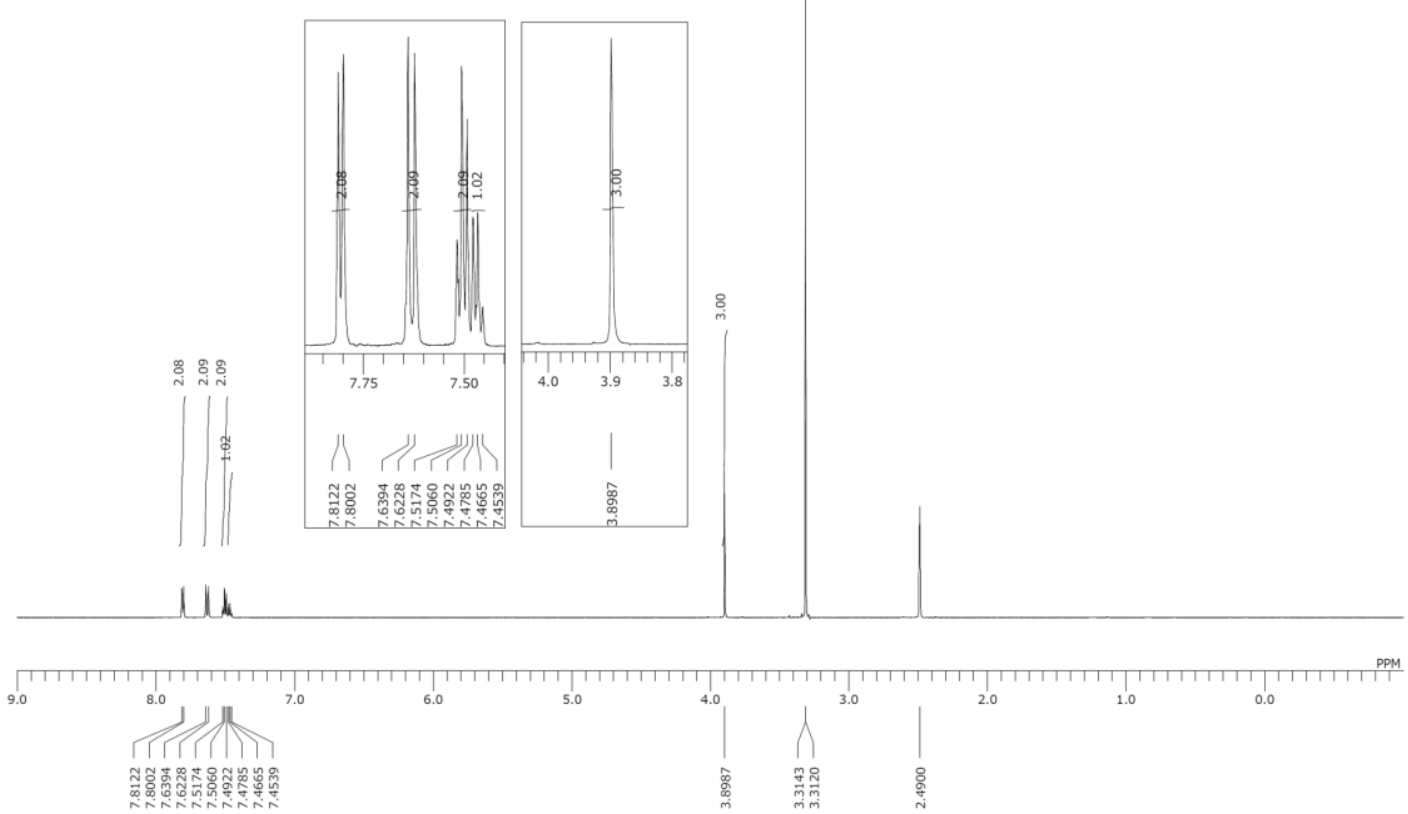


${ }^{13} \mathrm{C}$ NMR spectra of $\mathbf{2} \mathbf{i}(\mathrm{DMSO}-\mathrm{d} 6,150 \mathrm{MHz})$
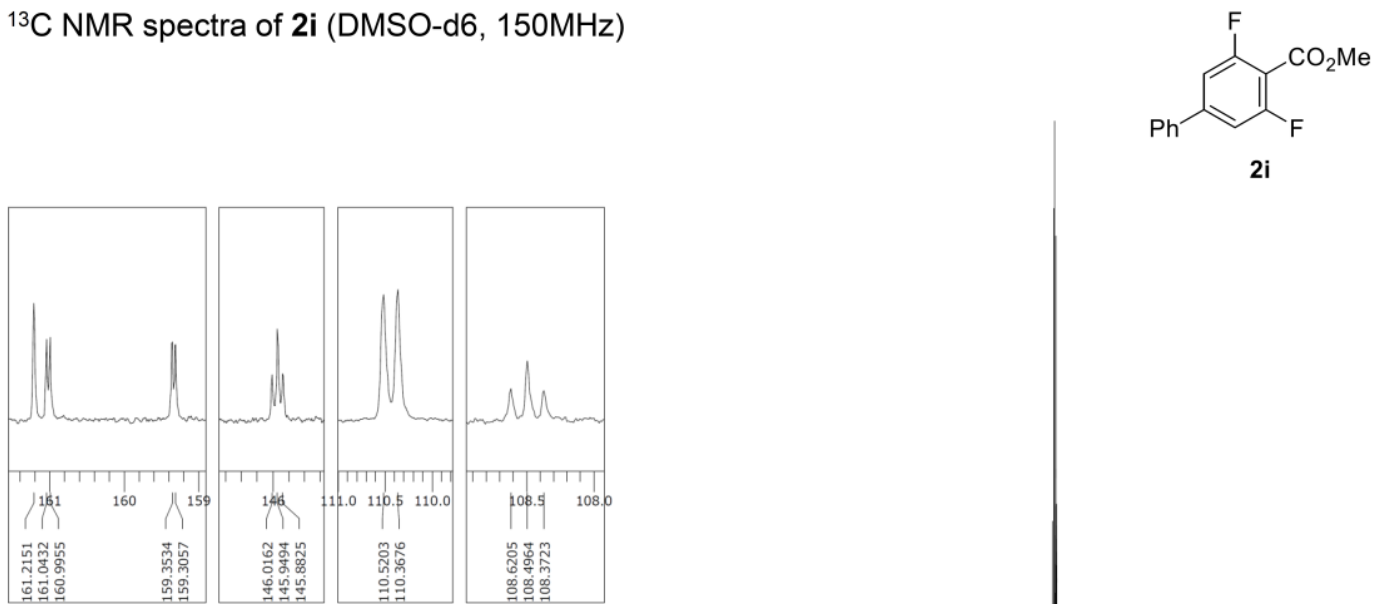

$2 i$

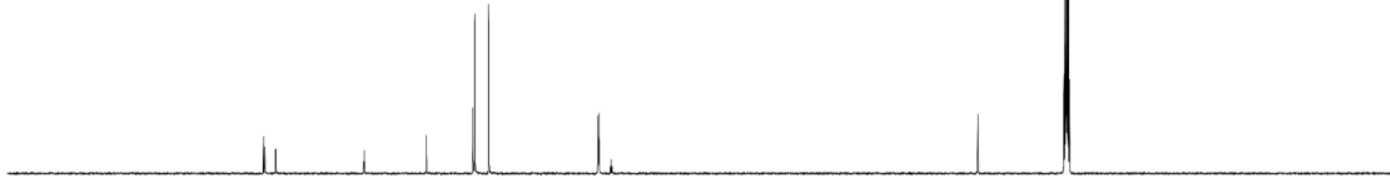

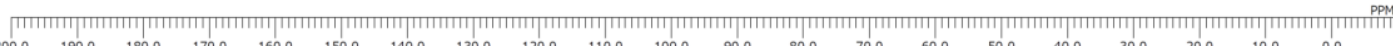

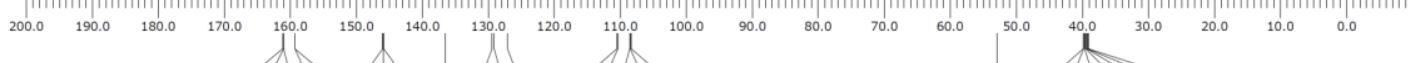
1Th-1

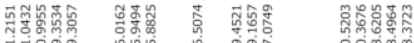

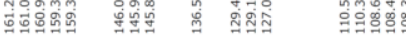

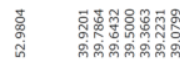

${ }^{19} \mathrm{~F}$ NMR spectra of $\mathbf{2 i}$ (DMSO-d6, 565MHz)

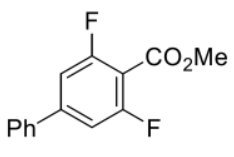

2i

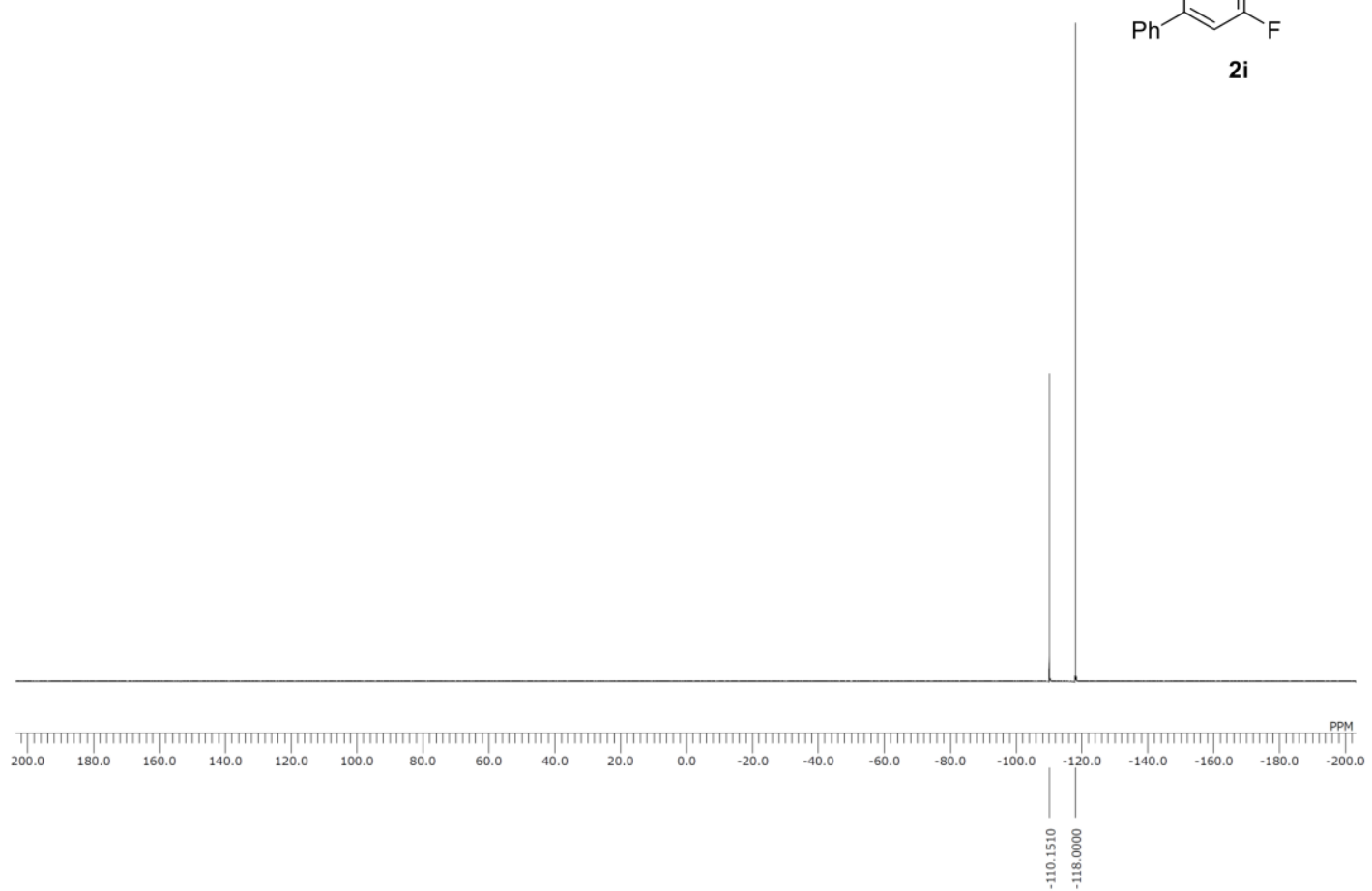


${ }^{1} \mathrm{H}$ NMR spectra of $\mathbf{2} \mathbf{j}\left(\mathrm{CDCl}_{3}, 600 \mathrm{MHz}\right)$

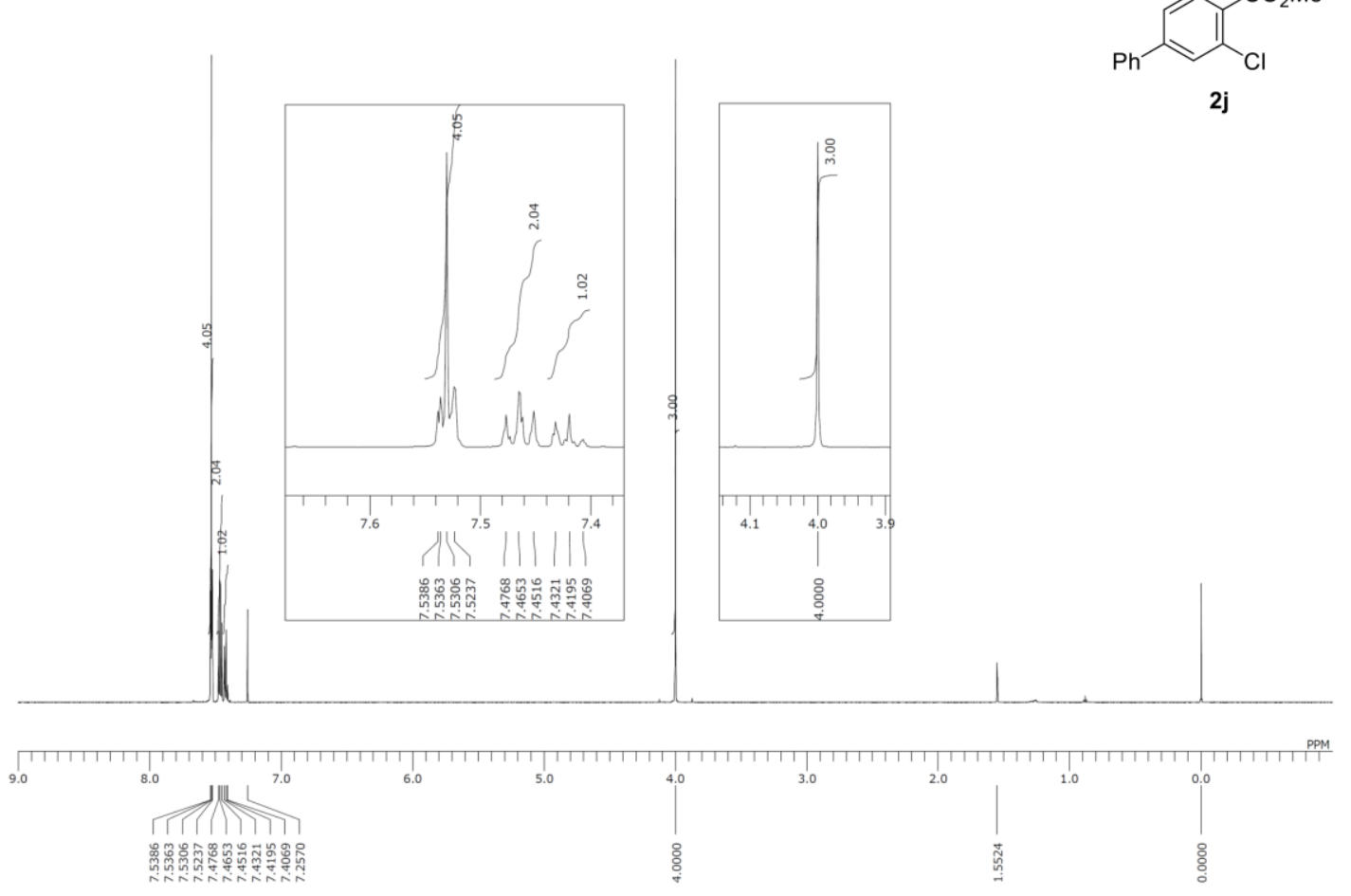

${ }^{13} \mathrm{C} \mathrm{NMR}$ spectra of $\mathbf{2} \mathbf{j}\left(\mathrm{CDCl}_{3}, 150 \mathrm{MHz}\right)$
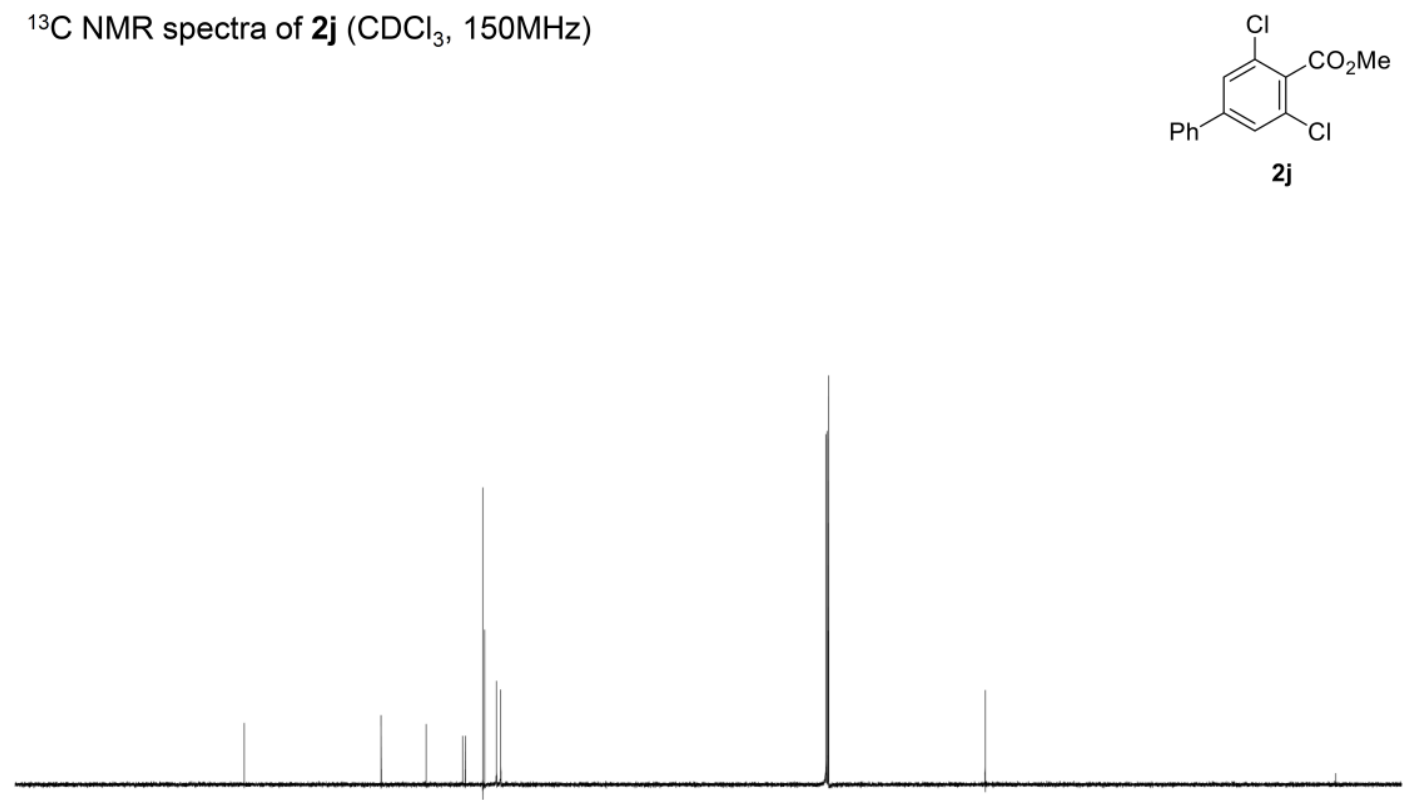

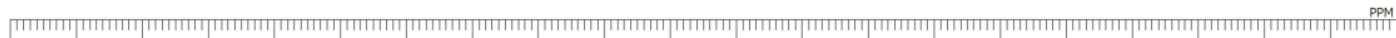

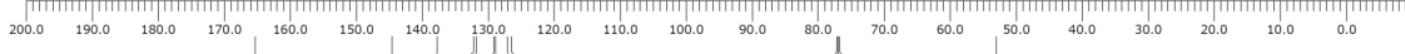

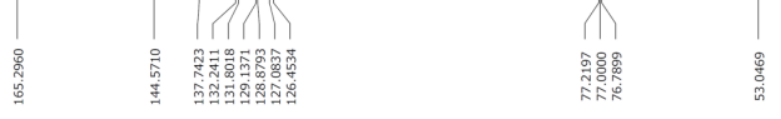


${ }^{1} \mathrm{H}$ NMR spectra of $\mathbf{2} \mathbf{k}\left(\mathrm{CDCl}_{3}, 400 \mathrm{MHz}\right)$
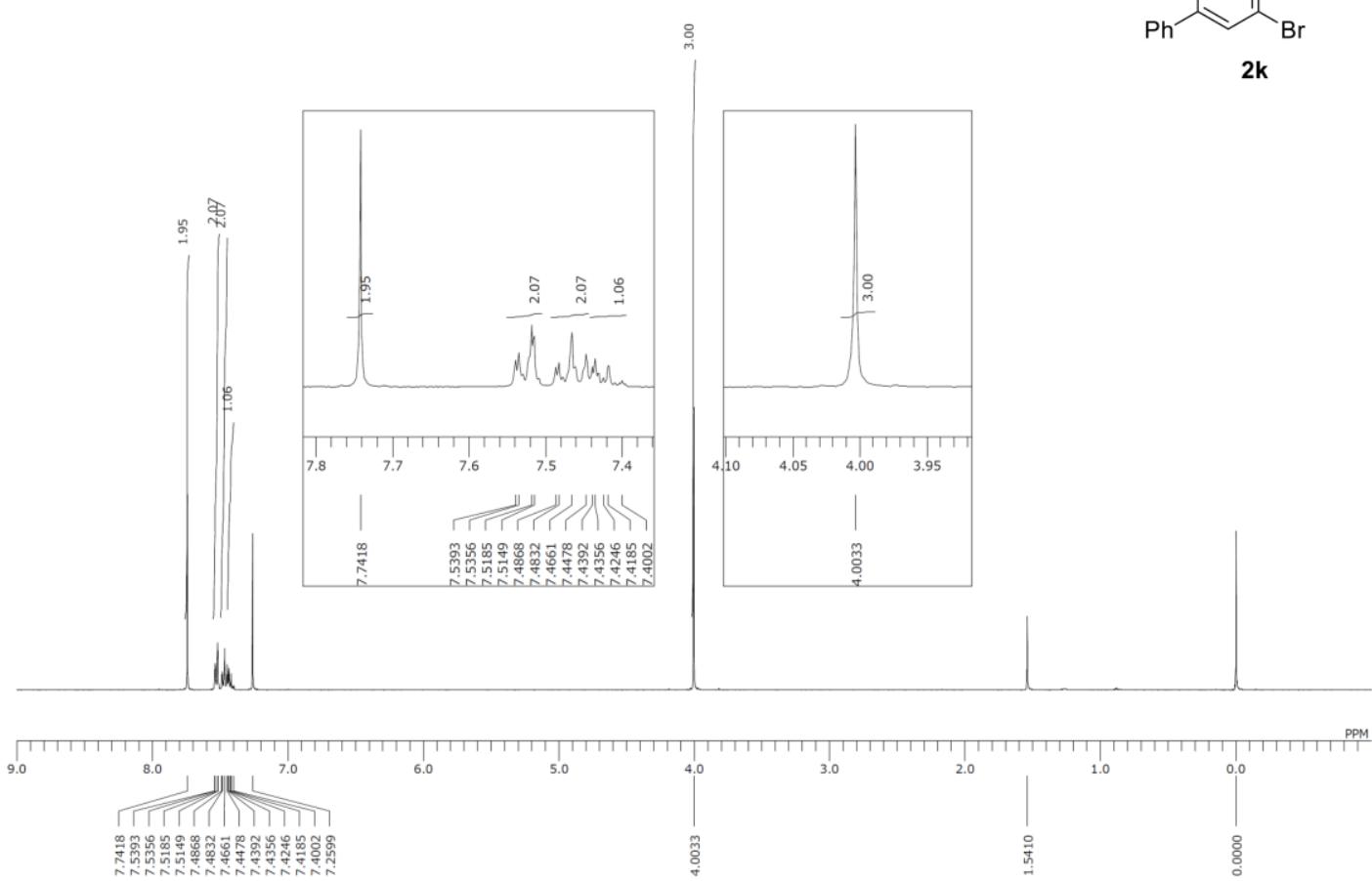

${ }^{13} \mathrm{C}$ NMR spectra of $\mathbf{2 k}\left(\mathrm{CDCl}_{3}, 150 \mathrm{MHz}\right)$

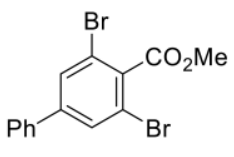

$2 k$

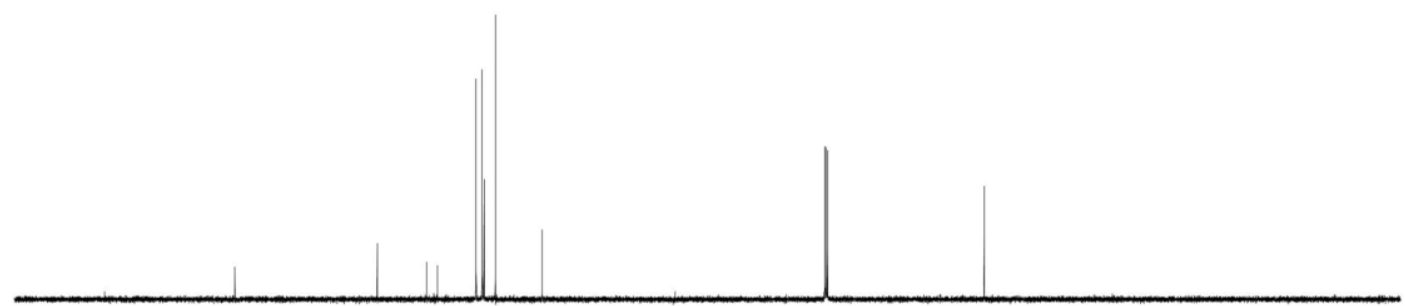

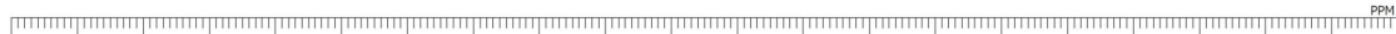

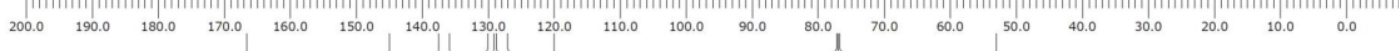

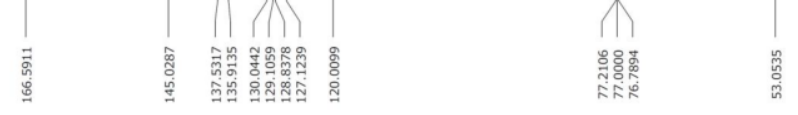


${ }^{1} \mathrm{H}$ NMR spectra of $2 \mathbf{l}\left(\mathrm{CDCl}_{3}, 600 \mathrm{MHz}\right)$

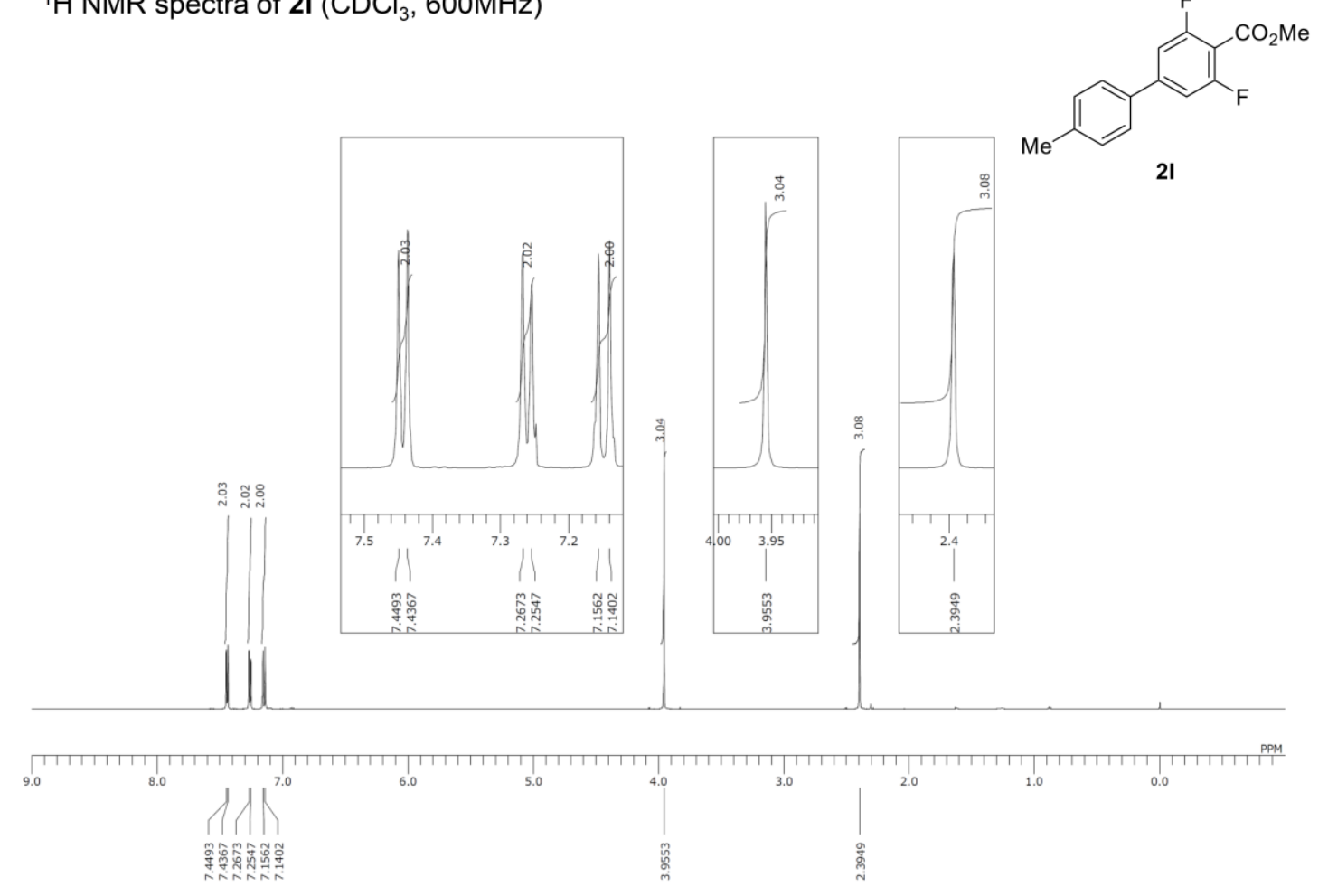

${ }^{13} \mathrm{C}$ NMR spectra of $2 \mathbf{l}\left(\mathrm{CDCl}_{3}, 150 \mathrm{MHz}\right)$

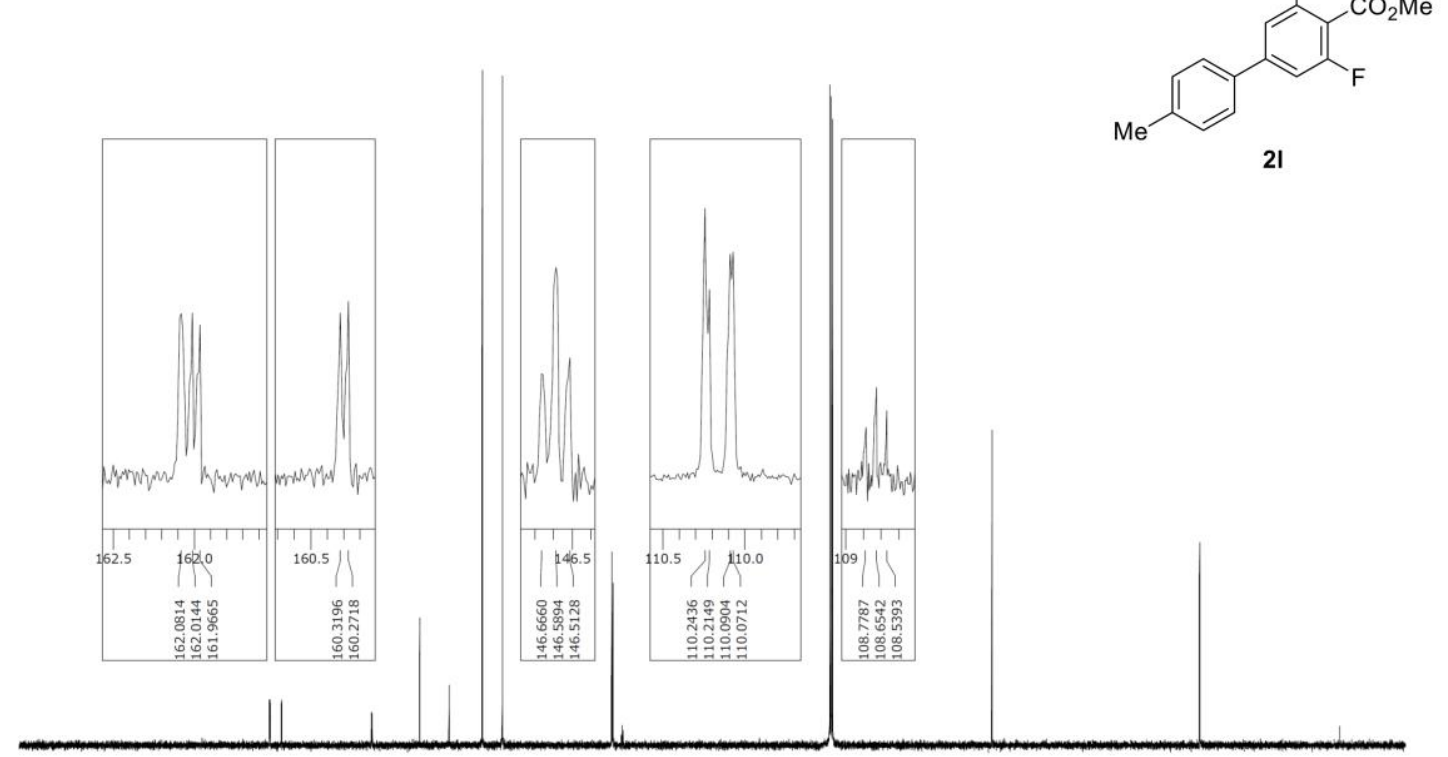

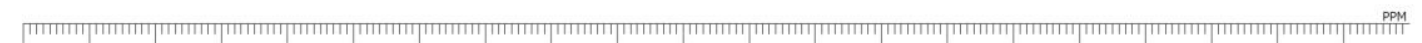

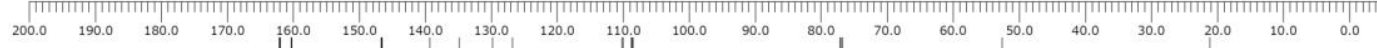

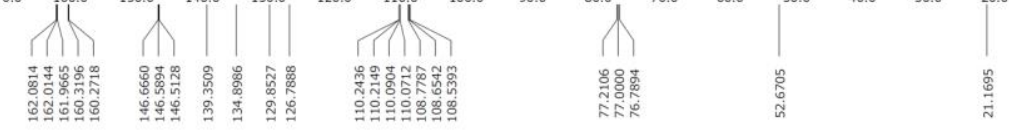


${ }^{19} \mathrm{~F}$ NMR spectra of $2 \mathbf{l}\left(\mathrm{CDCl}_{3}, 565 \mathrm{MHz}\right)$

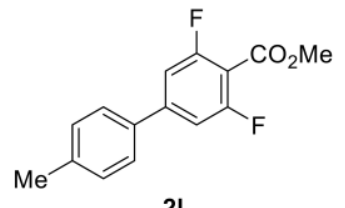

21

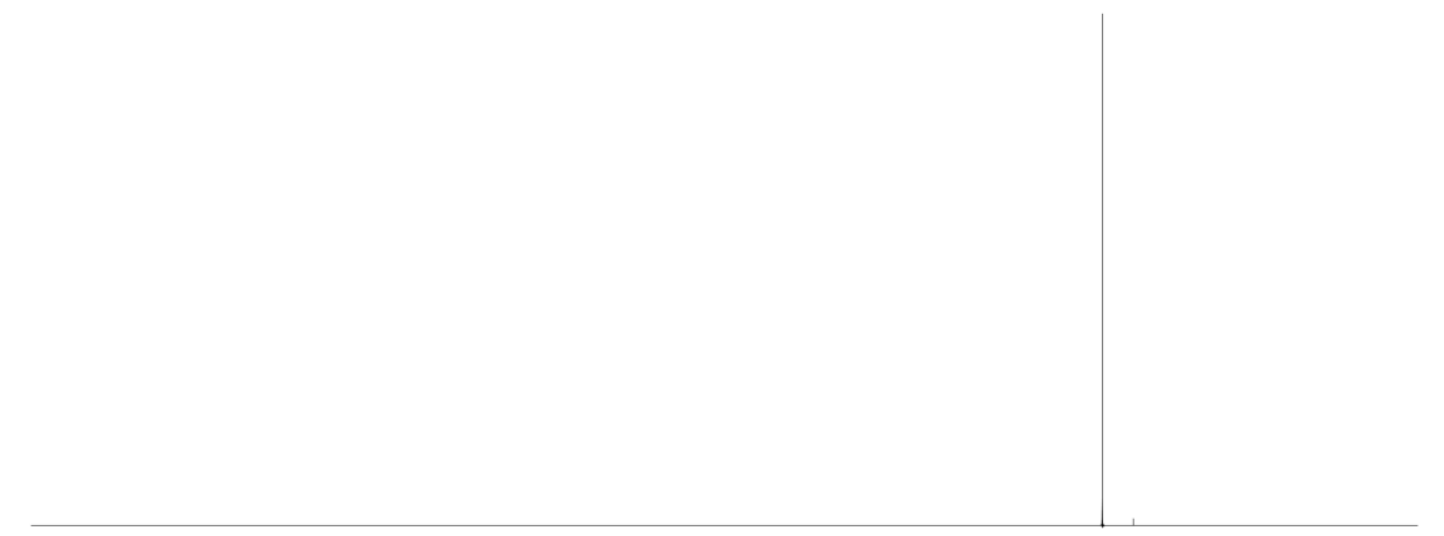

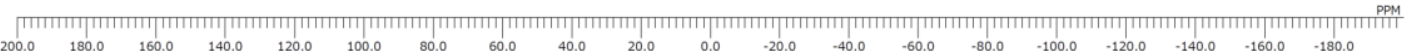

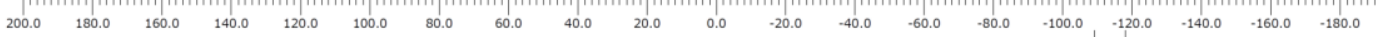

${ }^{1} \mathrm{H}$ NMR spectra of $\mathbf{2 m}(\mathrm{DMSO}-\mathrm{d} 6,600 \mathrm{MHz})$<smiles>COc1ccc(-c2cc(F)c(C(C)=O)c(F)c2)cc1</smiles>
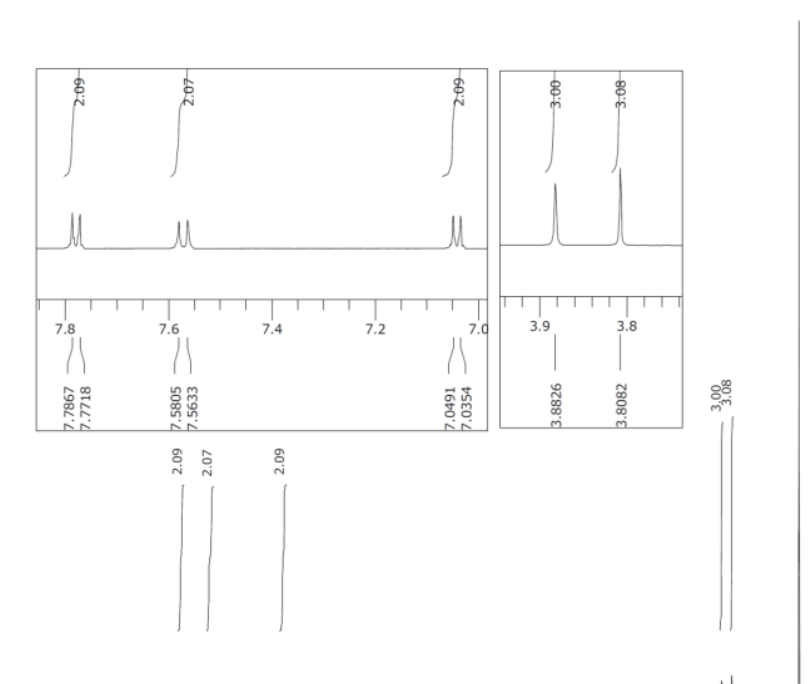

$2 \mathrm{~m}$

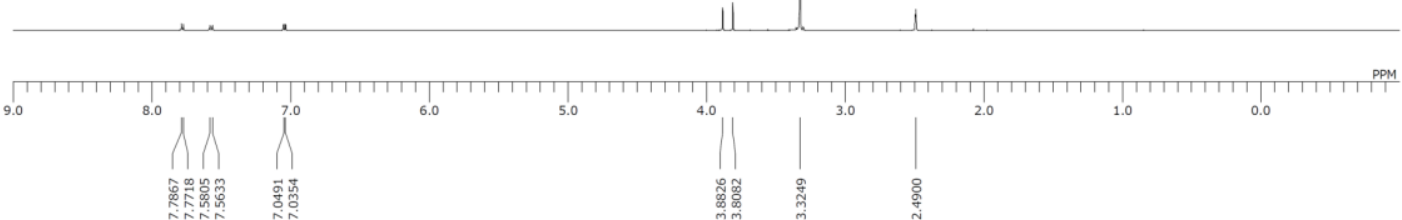


${ }^{13} \mathrm{C}$ NMR spectra of $2 \mathrm{~m}$ (DMSO-d6, 150MHz)
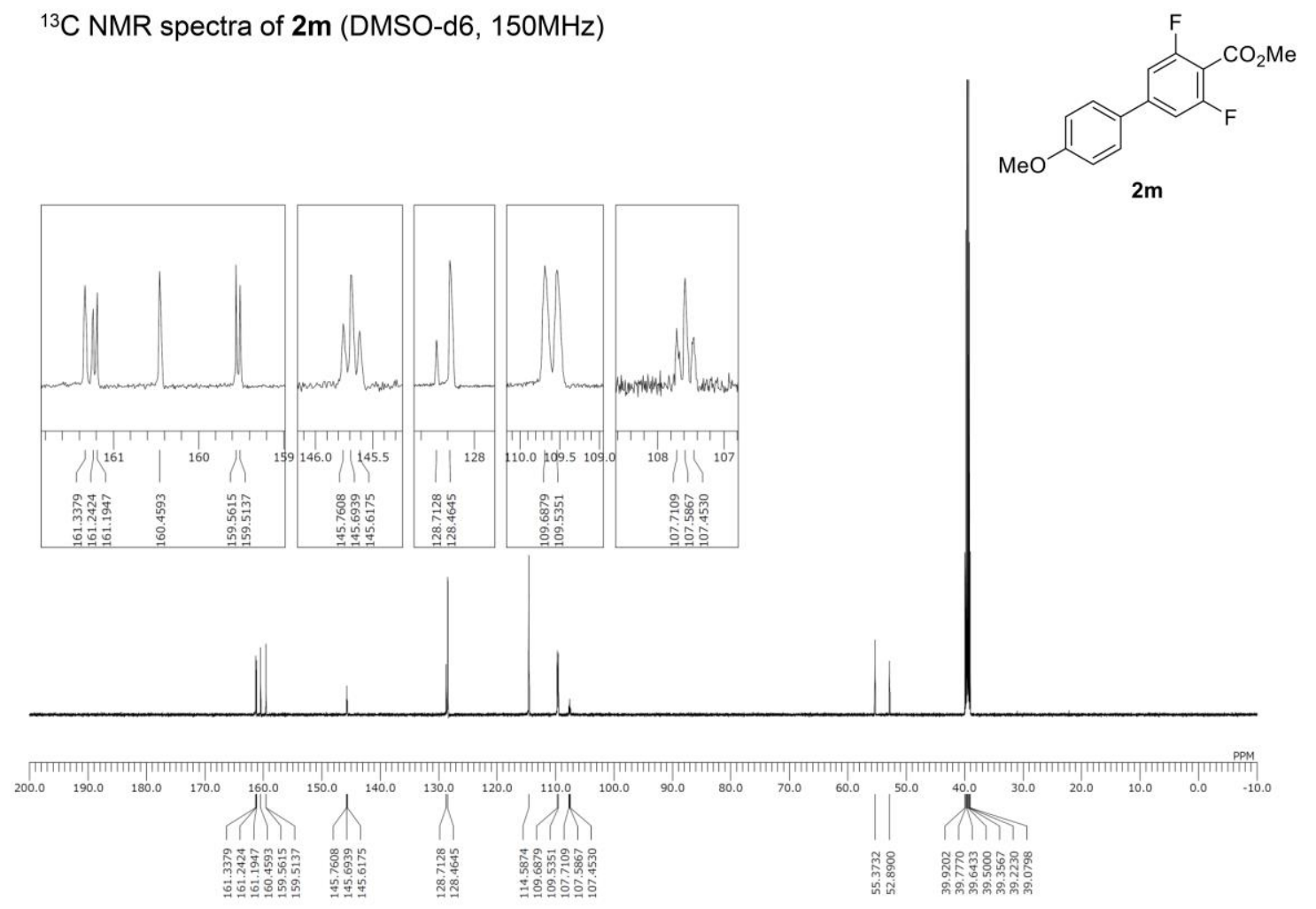

${ }^{19} \mathrm{~F}$ NMR spectra of $2 \mathrm{~m}$ (DMSO-d6, $565 \mathrm{MHz}$ )
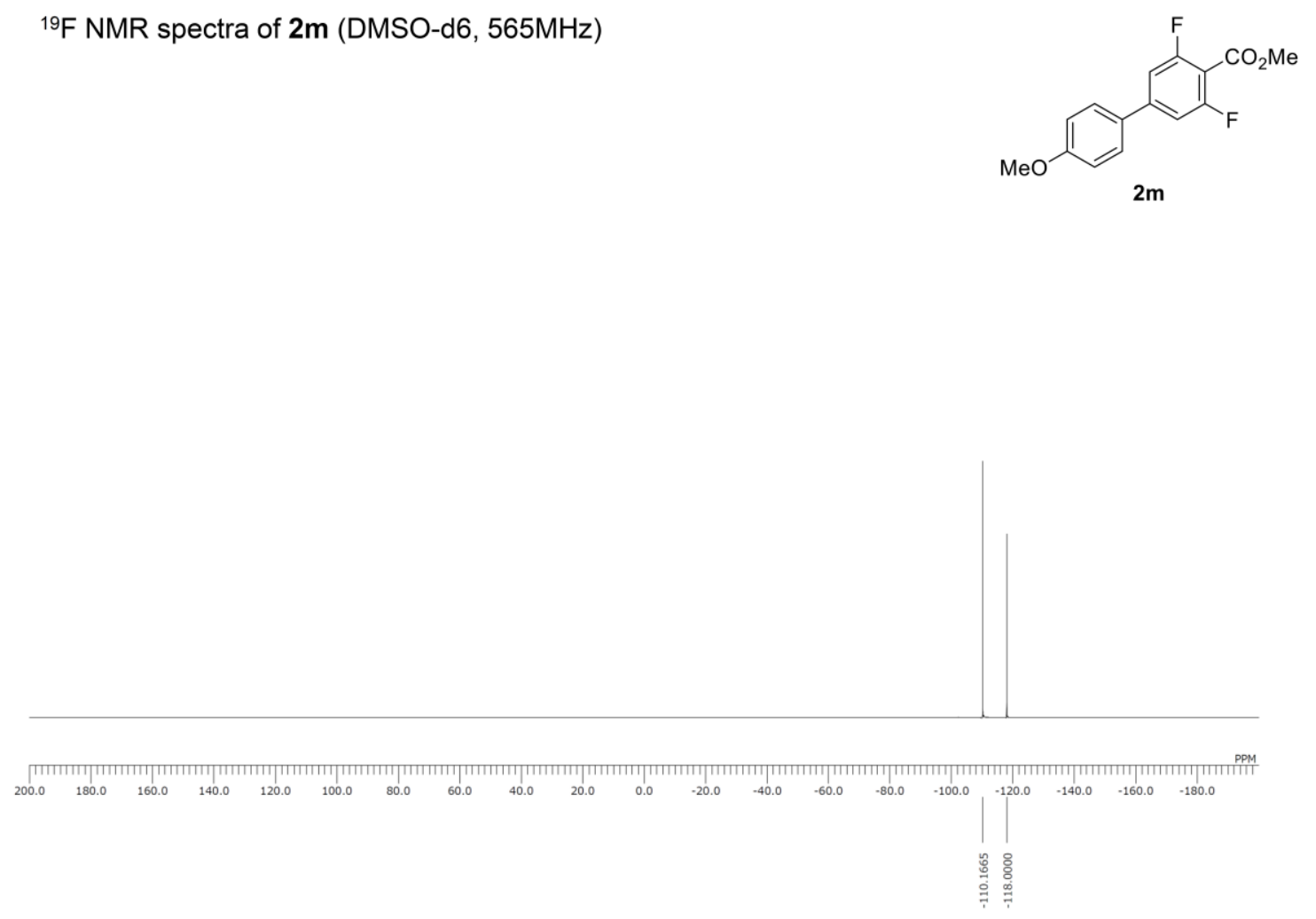
${ }^{1} \mathrm{H}$ NMR spectra of $2 \mathrm{n}\left(\mathrm{CDCl}_{3}, 400 \mathrm{MHz}\right)$

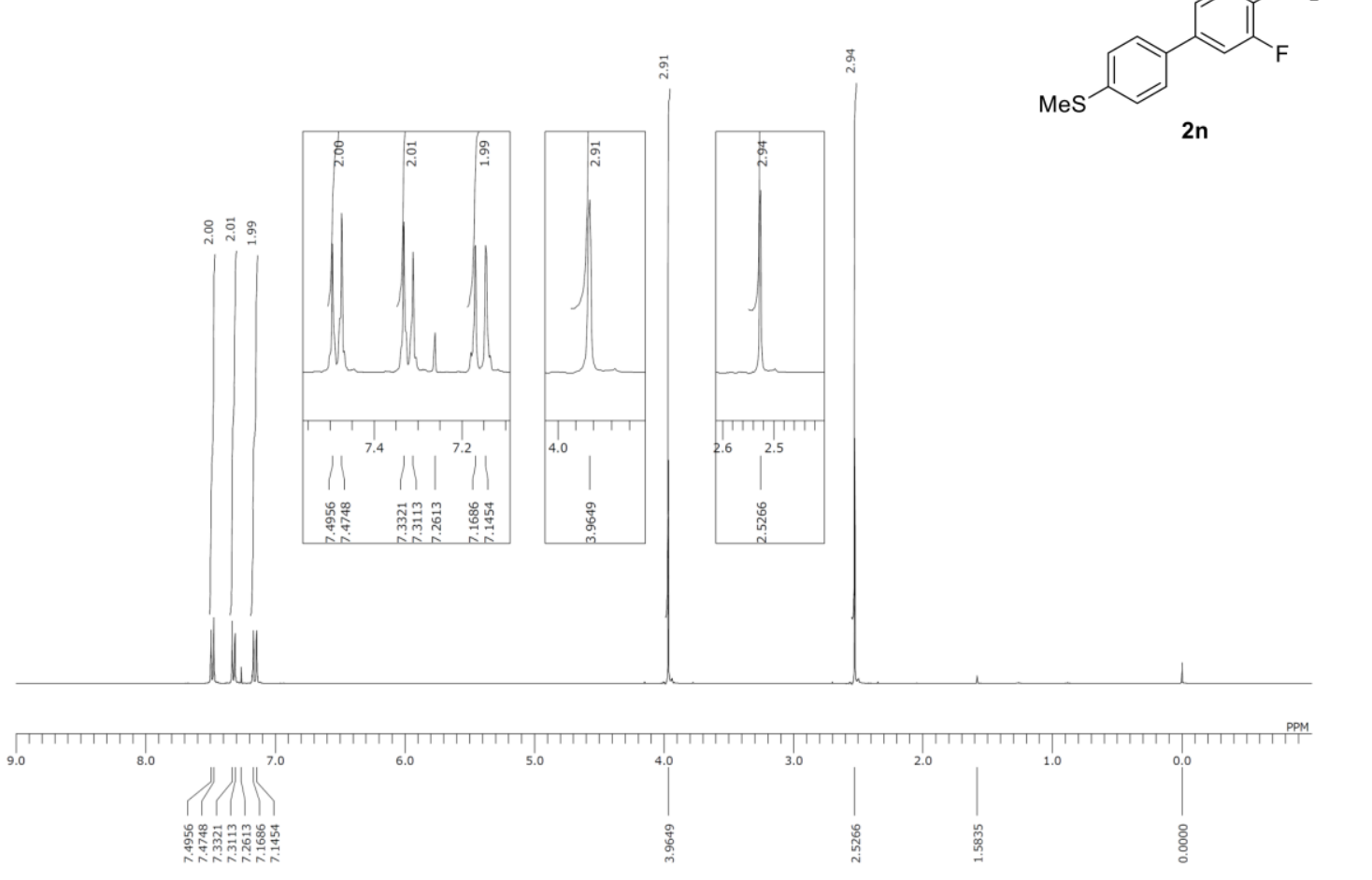

${ }^{13} \mathrm{C}$ NMR spectra of $\mathbf{2 n}\left(\mathrm{CDCl}_{3}, 150 \mathrm{MHz}\right)$

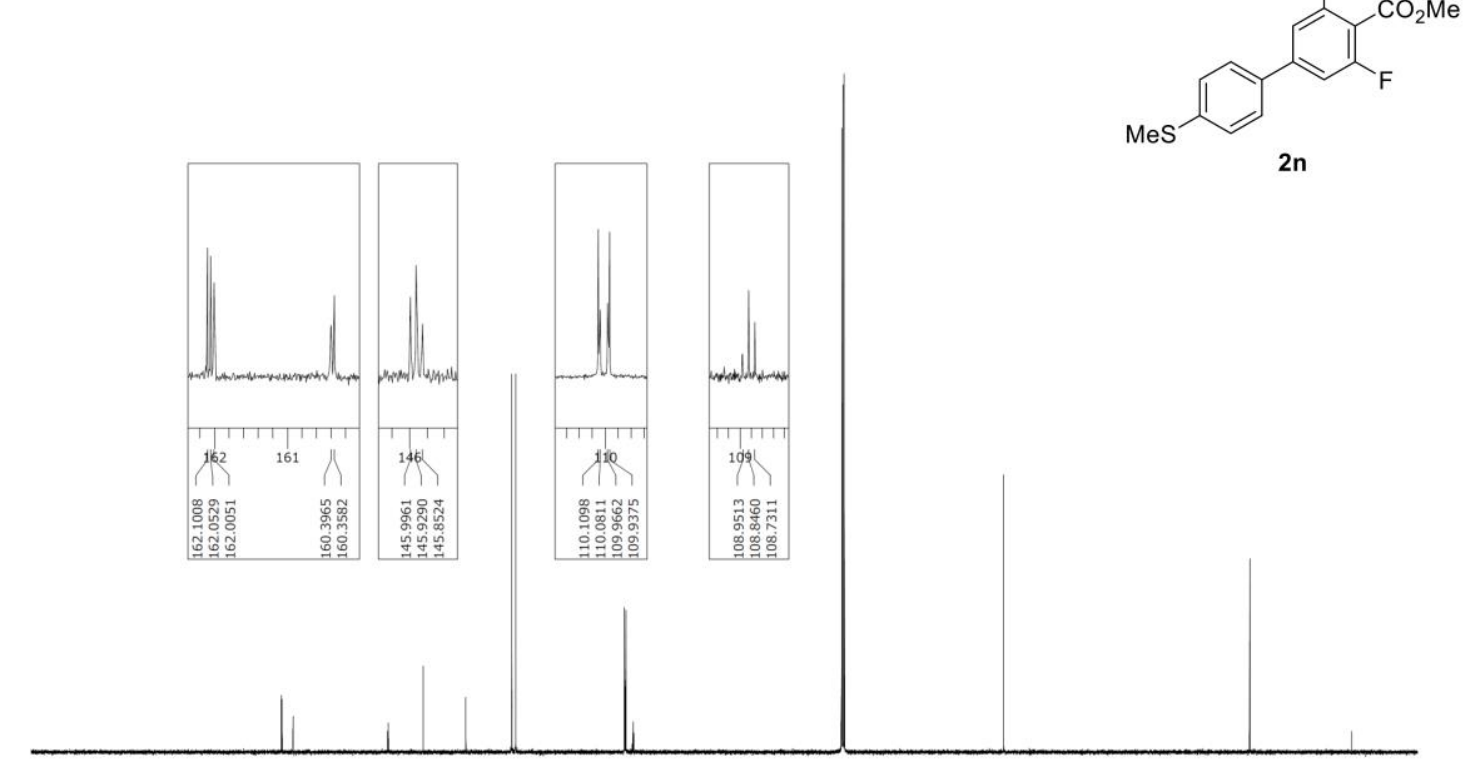

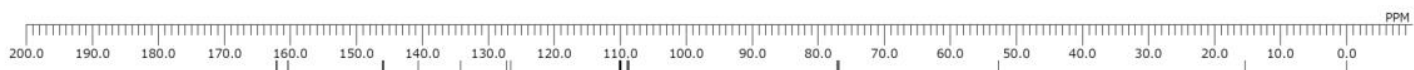

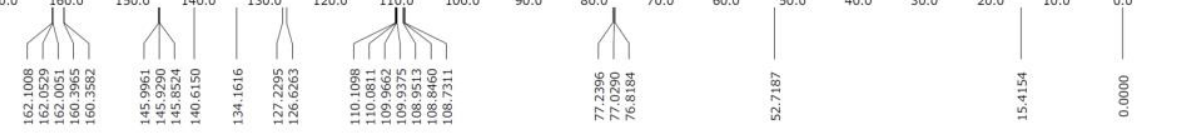


${ }^{19} \mathrm{~F}$ NMR spectra of $\mathbf{2 n}\left(\mathrm{CDCl}_{3}, 565 \mathrm{MHz}\right)$

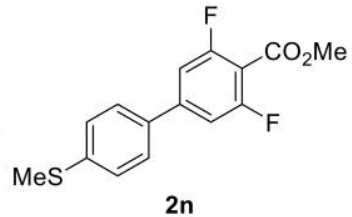

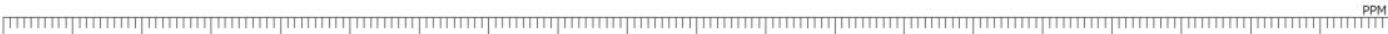

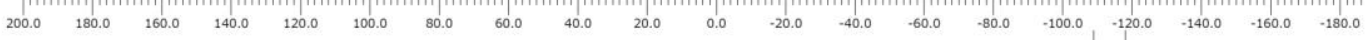

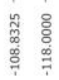

${ }^{1} \mathrm{H}$ NMR spectra of $20\left(\mathrm{CDCl}_{3}, 600 \mathrm{MHz}\right)$

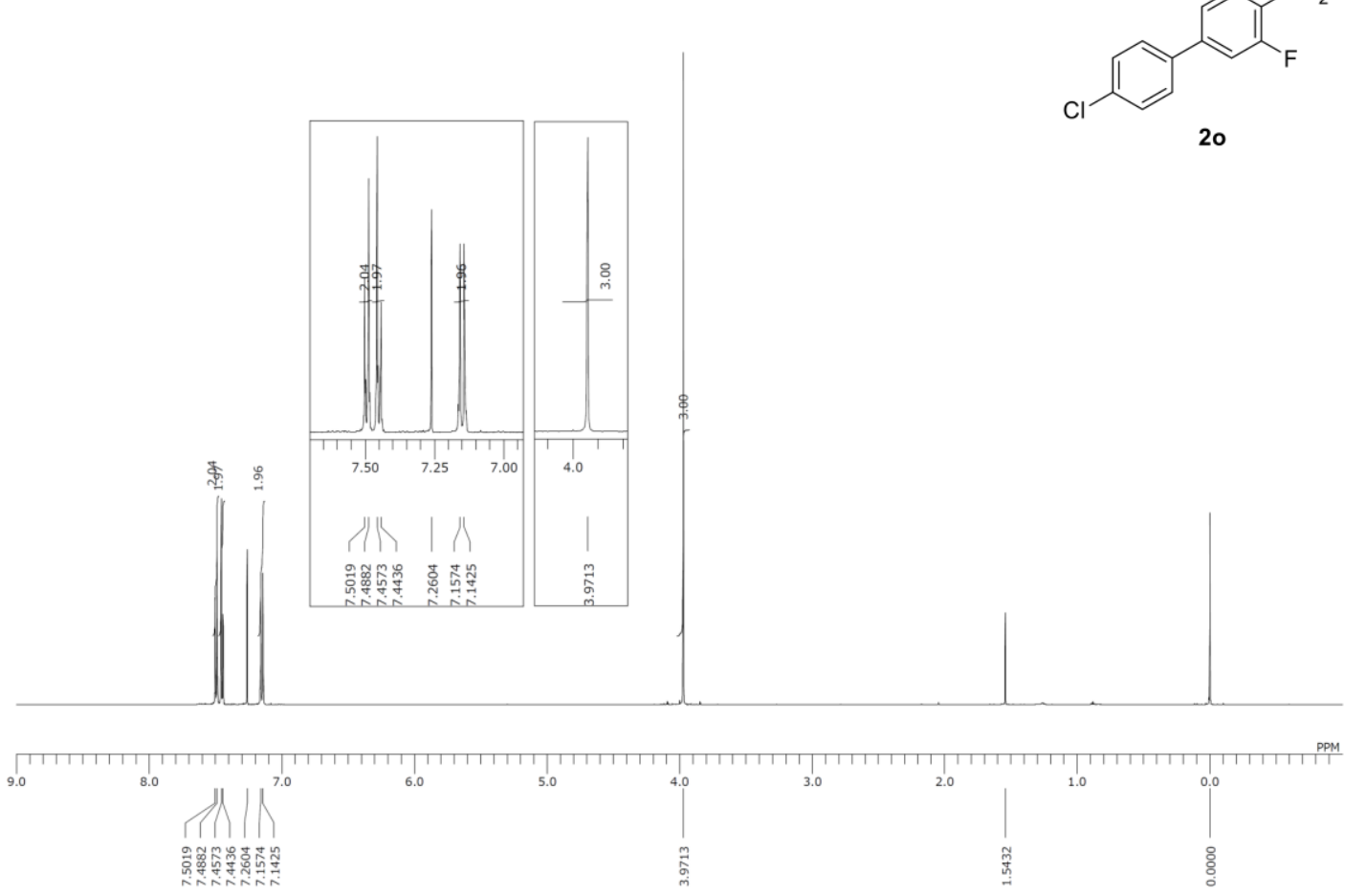


${ }^{13} \mathrm{C}$ NMR spectra of $20\left(\mathrm{CDCl}_{3}, 150 \mathrm{MHz}\right)$

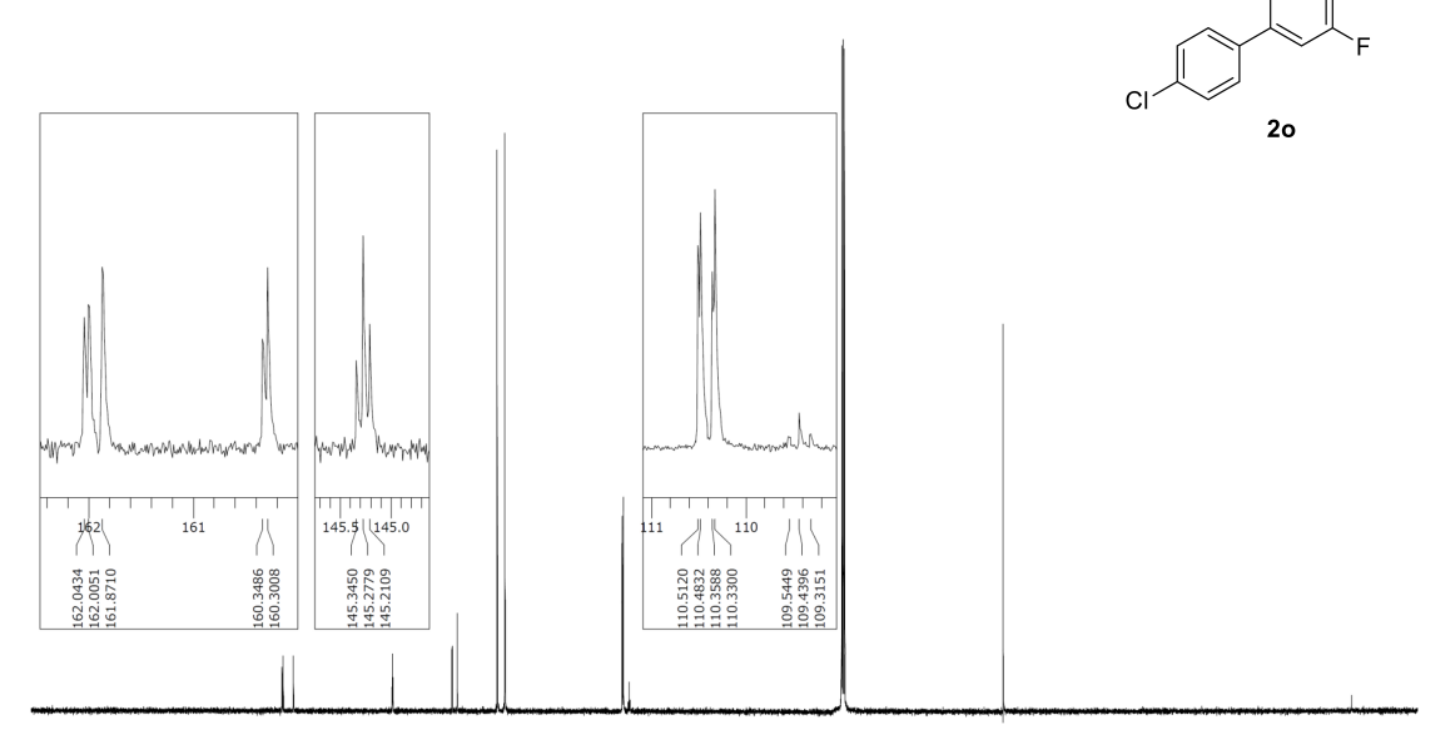

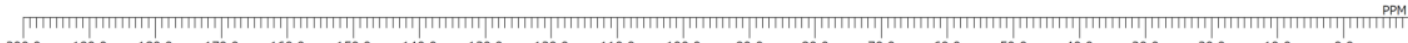

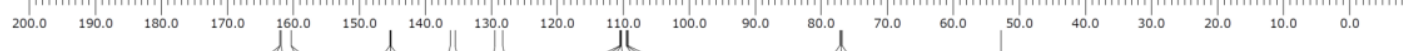

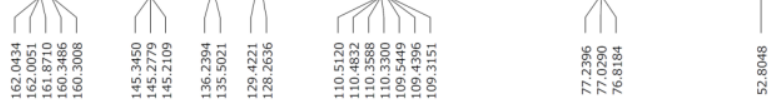

${ }^{19} \mathrm{~F}$ NMR spectra of $20\left(\mathrm{CDCl}_{3}, 565 \mathrm{MHz}\right)$

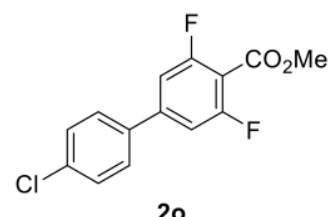

20

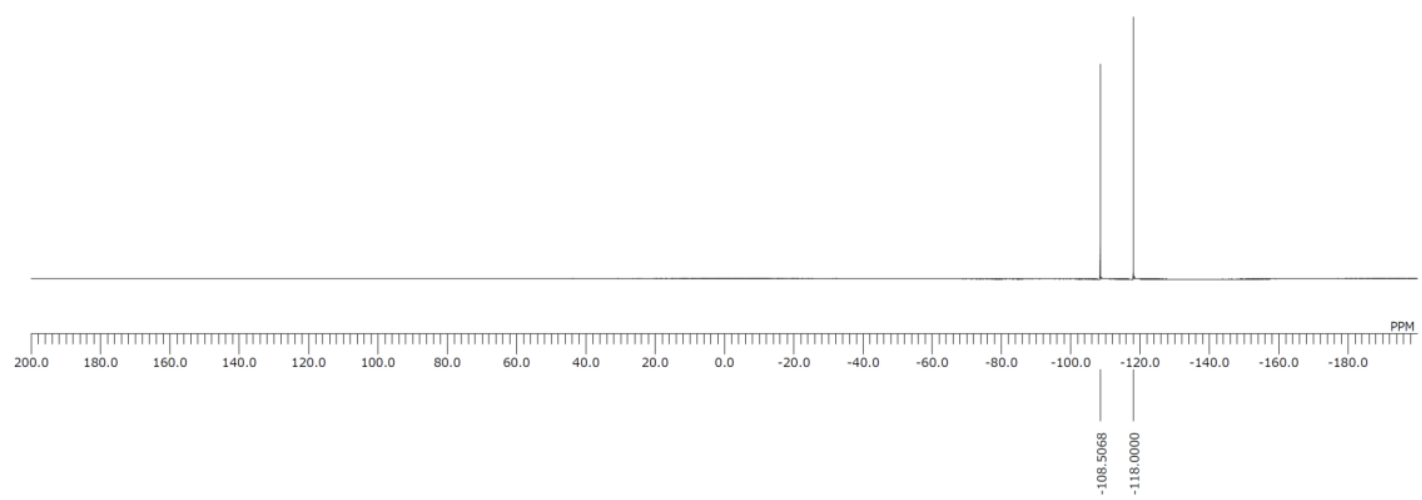


${ }^{1} \mathrm{H}$ NMR spectra of $2 \mathbf{p}\left(\mathrm{CDCl}_{3}, 600 \mathrm{MHz}\right)$

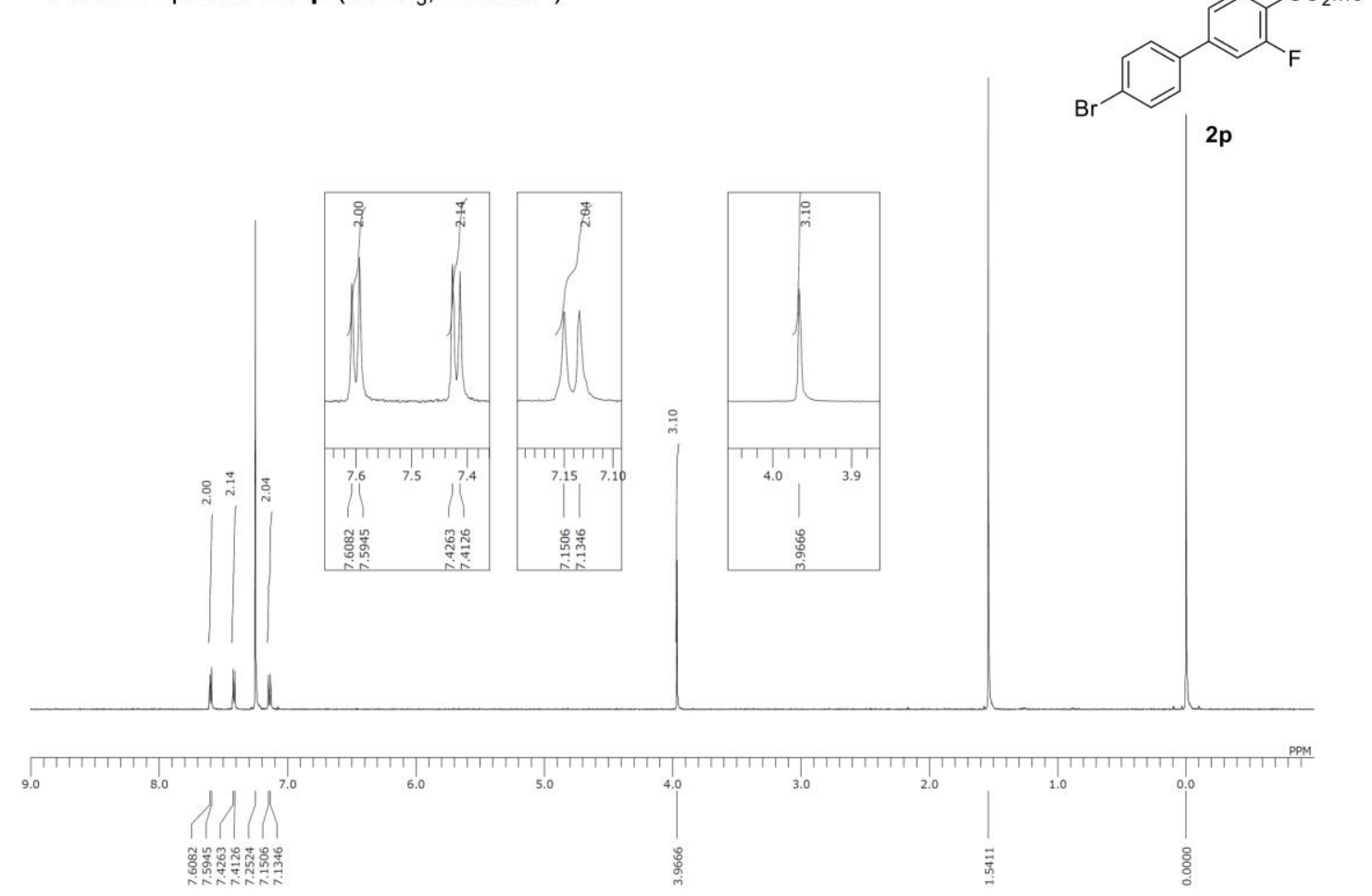

${ }^{13} \mathrm{C}$ NMR spectra of $2 p\left(\mathrm{CDCl}_{3}, 150 \mathrm{MHz}\right)$

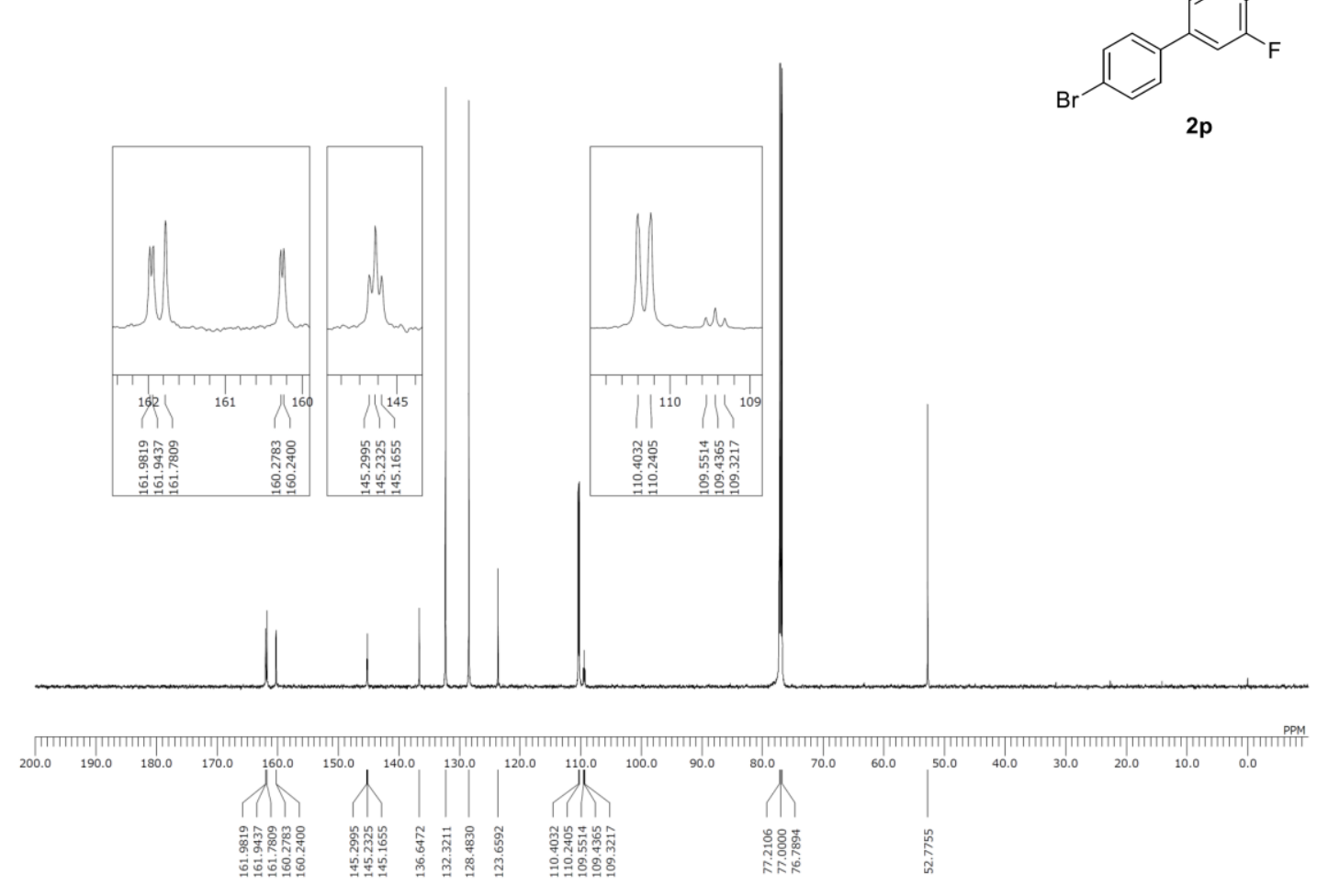


${ }^{19} \mathrm{~F}$ NMR spectra of $\mathbf{2 p}\left(\mathrm{CDCl}_{3}, 565 \mathrm{MHz}\right)$
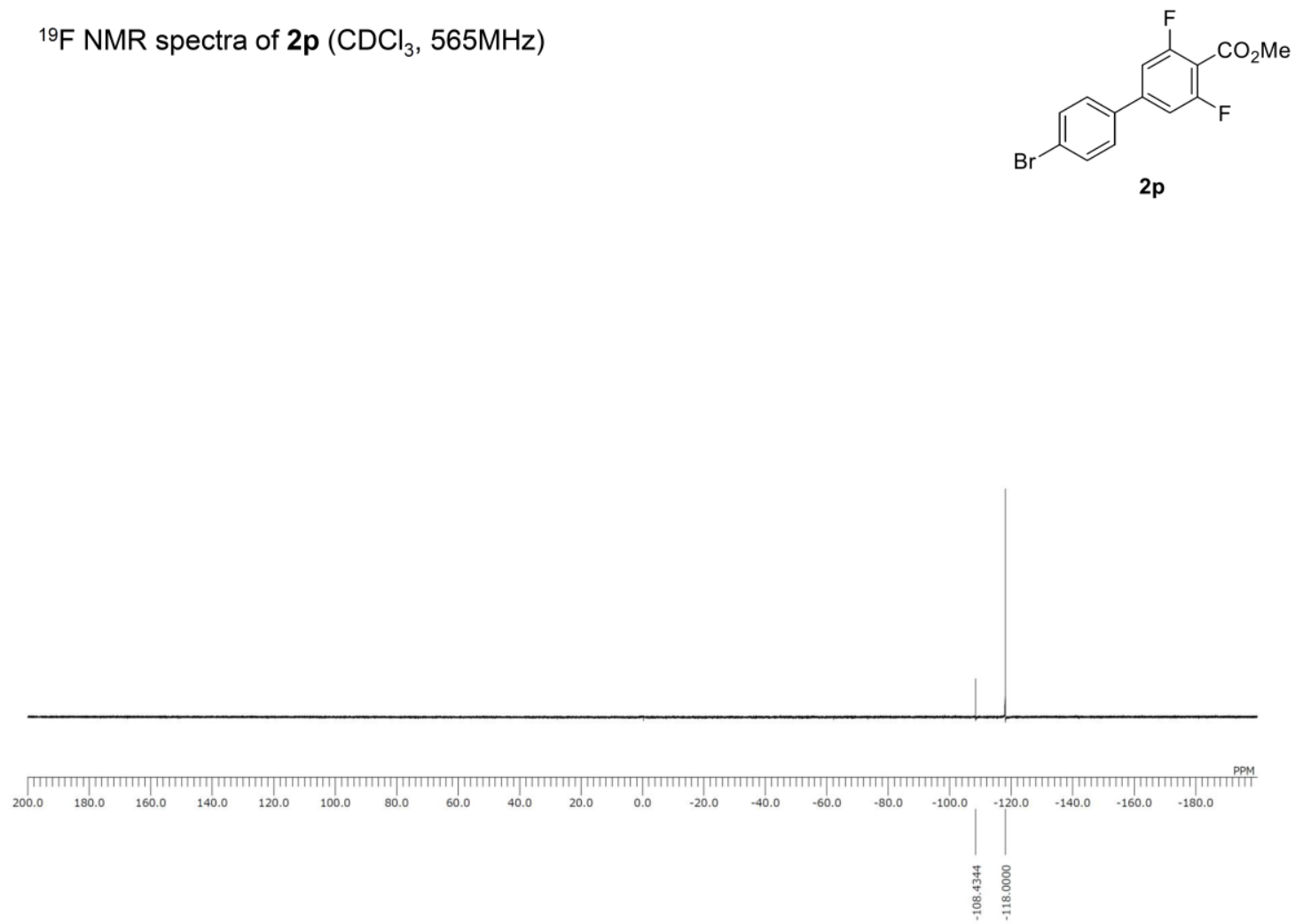

${ }^{1} \mathrm{H}$ NMR spectra of $2 q\left(\mathrm{CDCl}_{3}, 400 \mathrm{MHz}\right)$

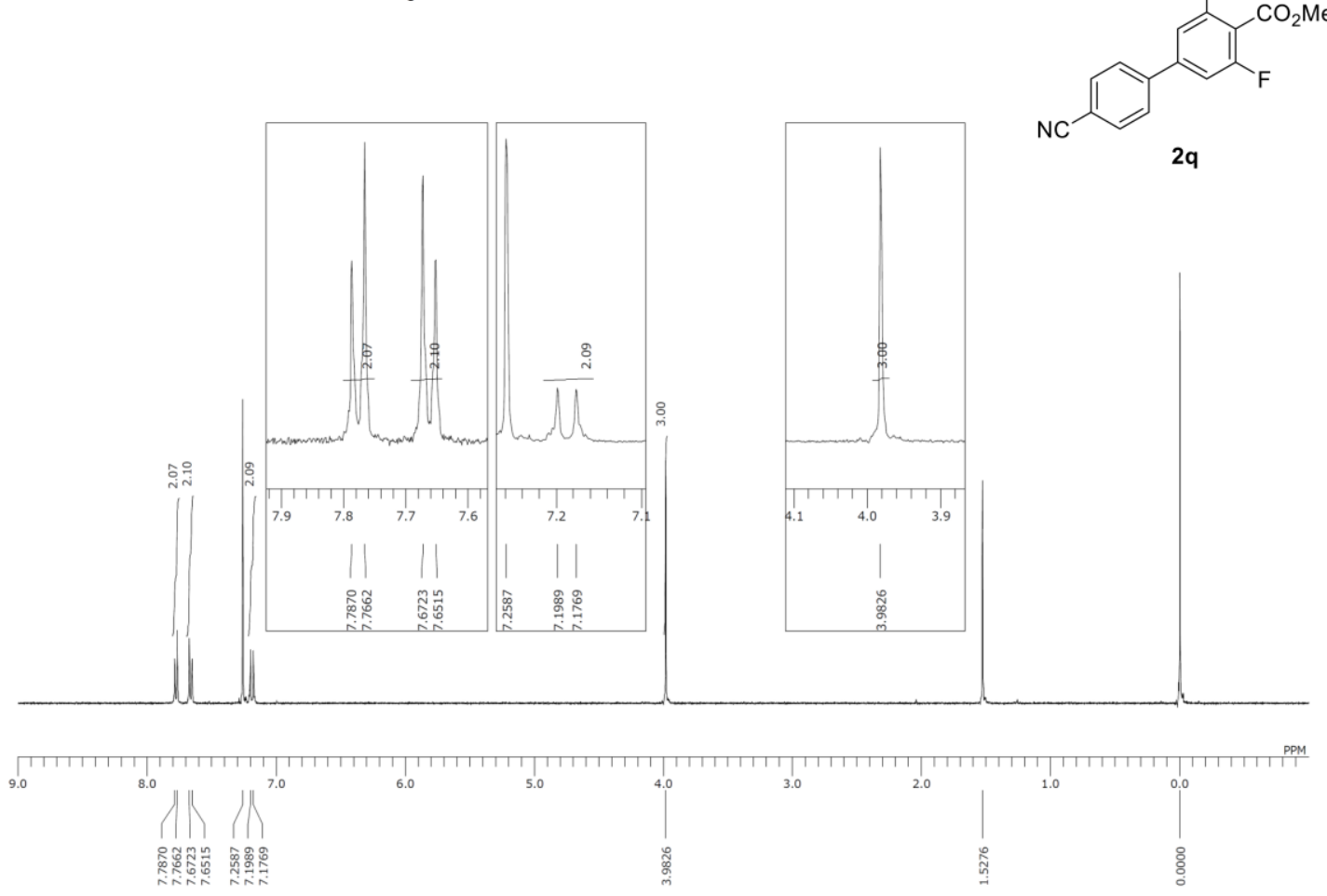


${ }^{13} \mathrm{C}$ NMR spectra of $2 q\left(\mathrm{CDCl}_{3}, 150 \mathrm{MHz}\right)$

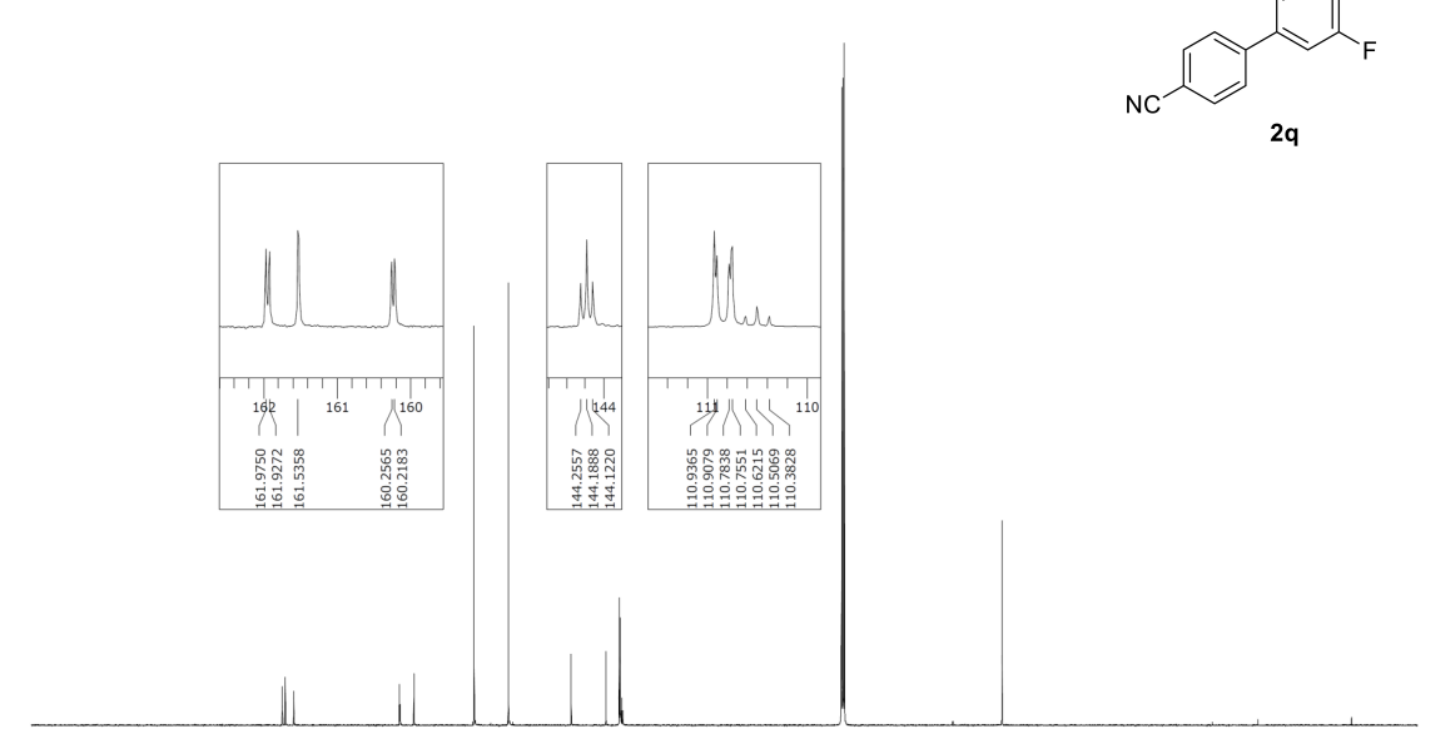

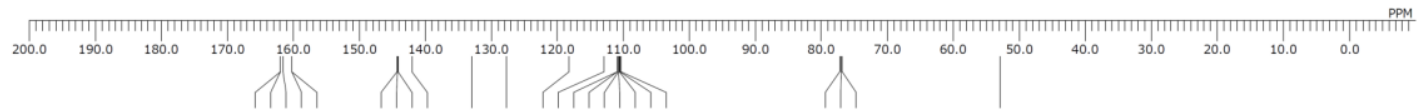

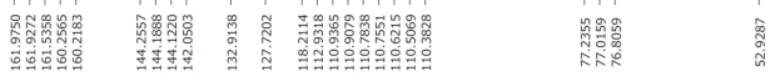

${ }^{19} \mathrm{~F}$ NMR spectra of $\mathbf{2 q}\left(\mathrm{CDCl}_{3}, 565 \mathrm{MHz}\right)$<smiles>CC(=O)c1c(F)cc(-c2ccc(C#N)cc2)cc1F</smiles>

$2 q$

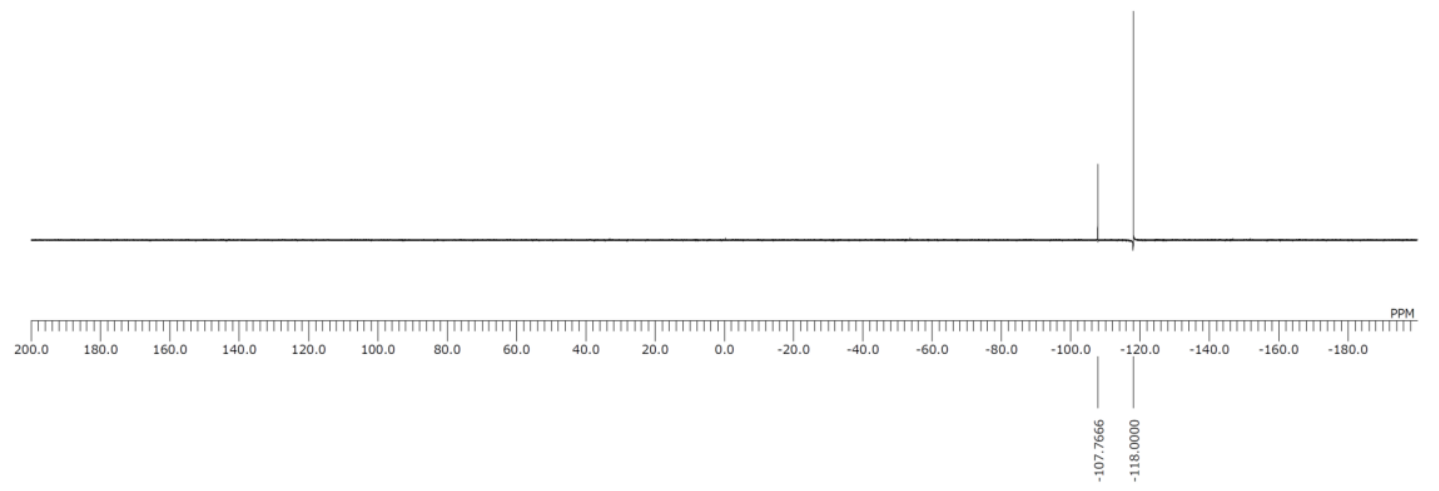


${ }^{1} \mathrm{H}$ NMR spectra of $2 \mathrm{r}(\mathrm{DMSO}-\mathrm{d} 6,600 \mathrm{MHz})$

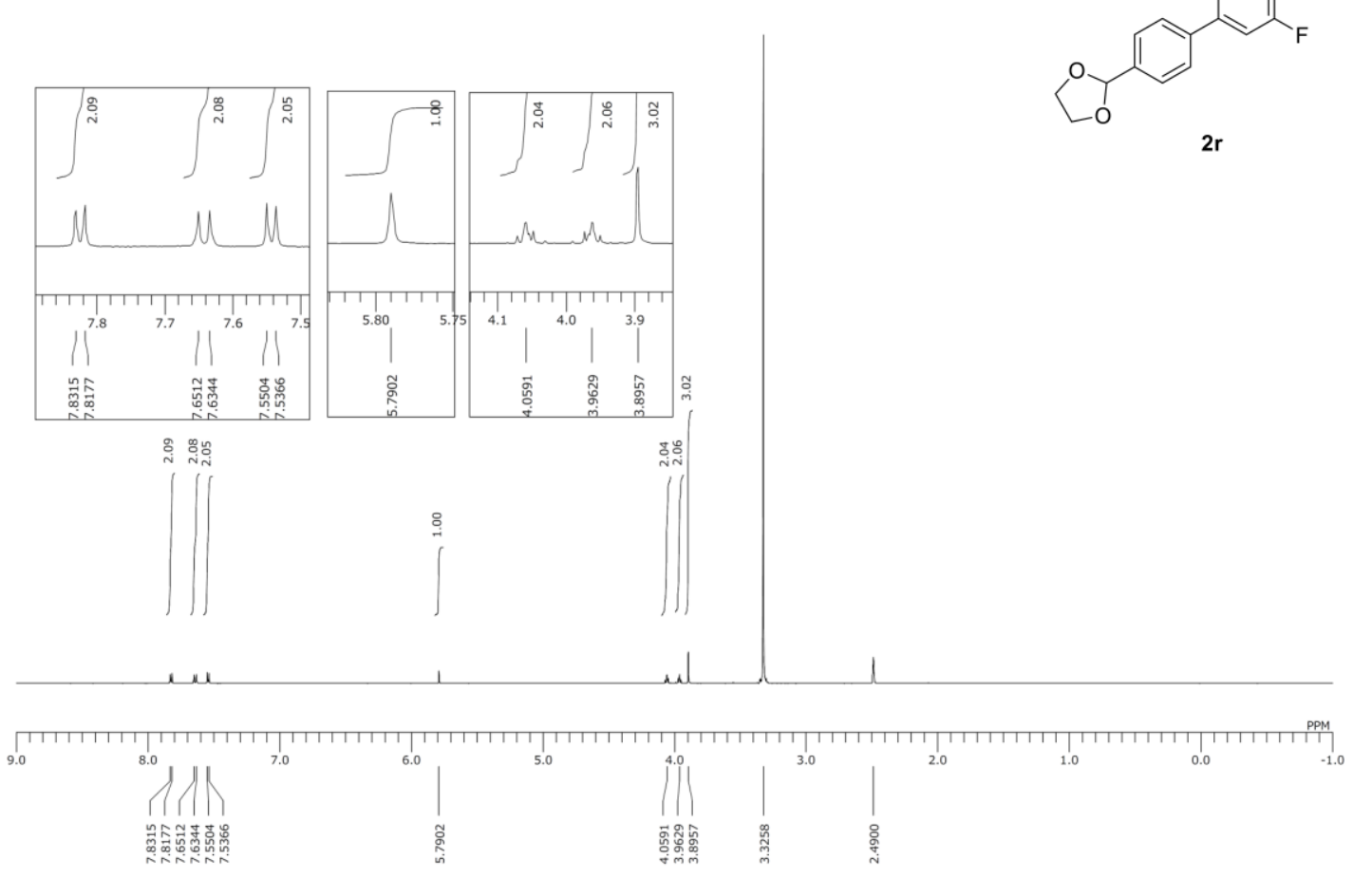

${ }^{13} \mathrm{C}$ NMR spectra of $\mathbf{2 r}$ (DMSO-d6, 150MHz)

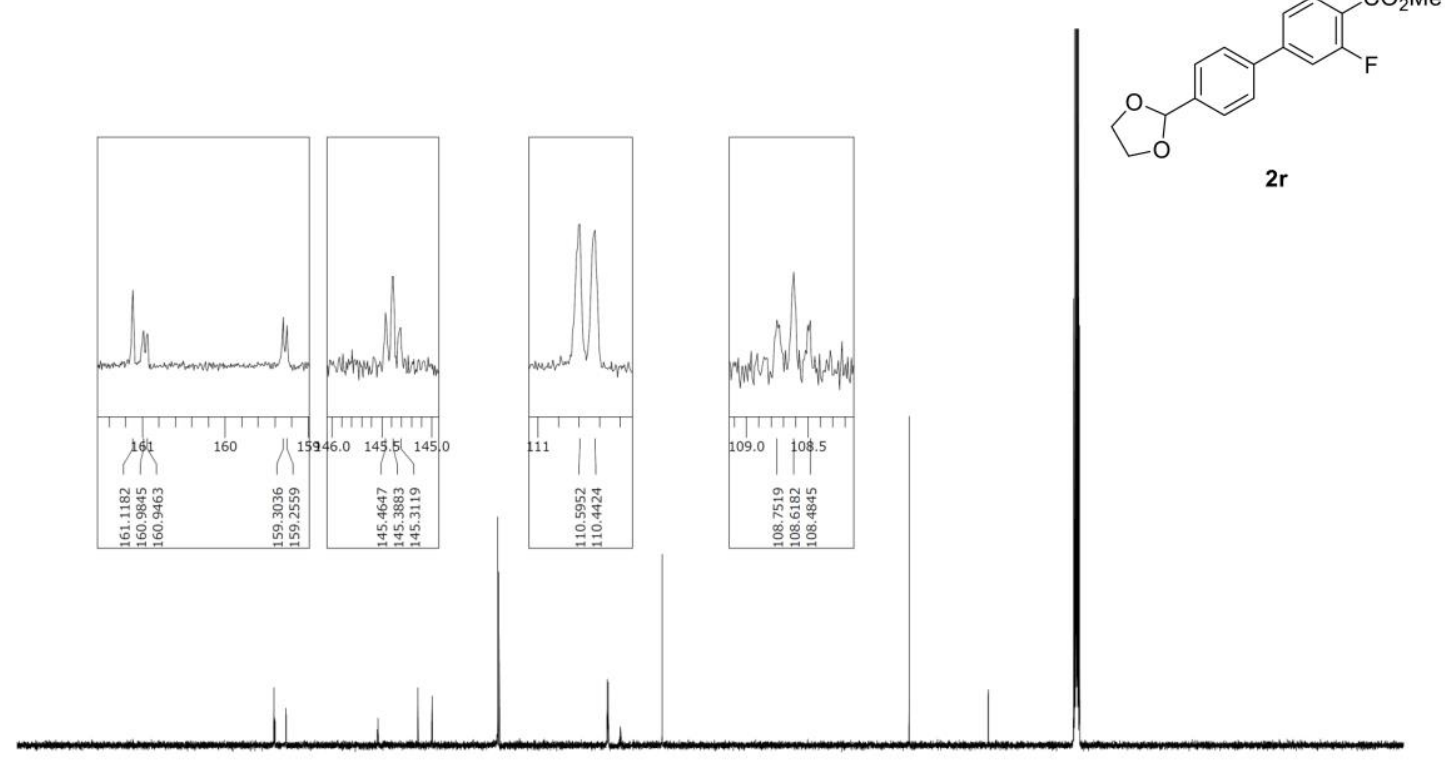

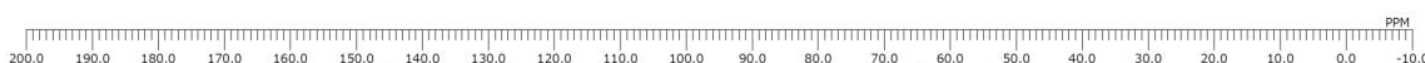

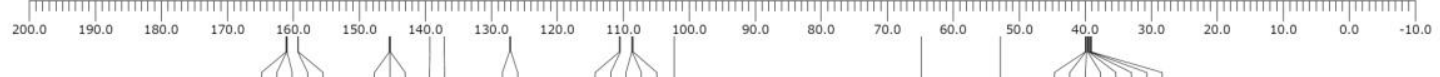

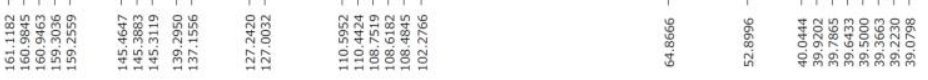


${ }^{19} \mathrm{~F}$ NMR spectra of $2 \mathbf{r}(\mathrm{DMSO}-\mathrm{d} 6,565 \mathrm{MHz}$ )

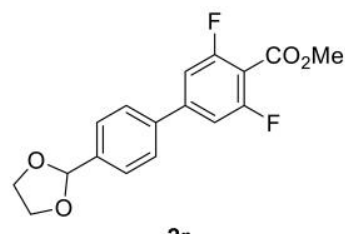

$2 r$

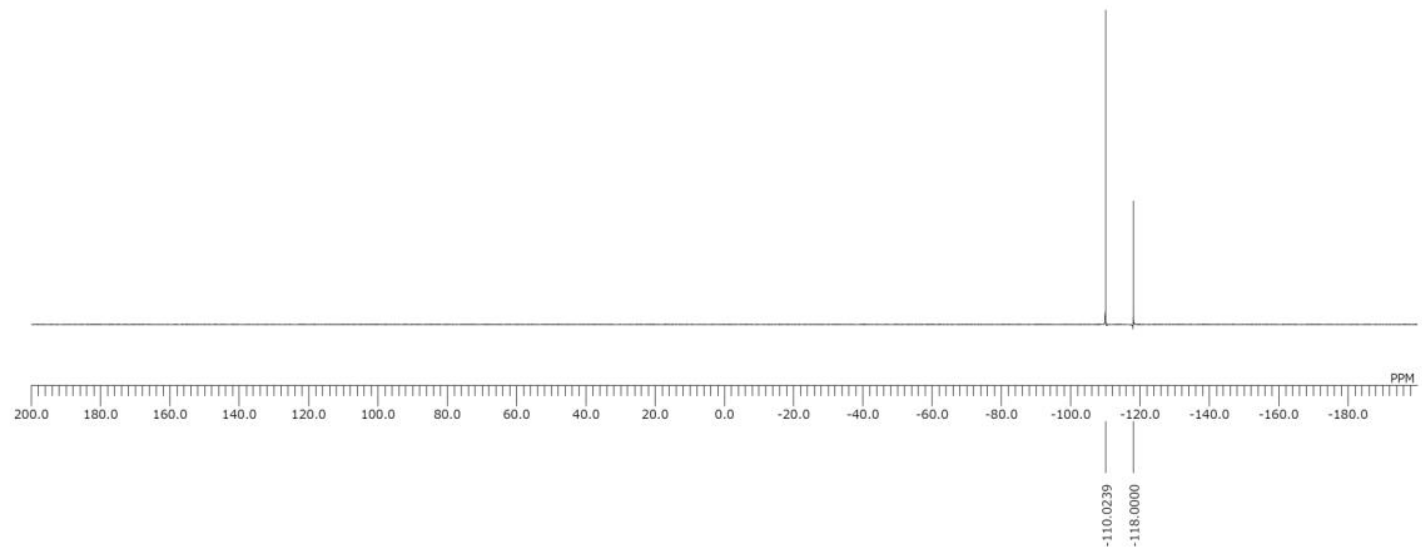

${ }^{1} \mathrm{H}$ NMR spectra of $2 \mathrm{~s}\left(\mathrm{CDCl}_{3}, 600 \mathrm{MHz}\right)$<smiles>CC(=O)c1c(F)cc(-c2ccc(C=O)cc2)cc1F</smiles>

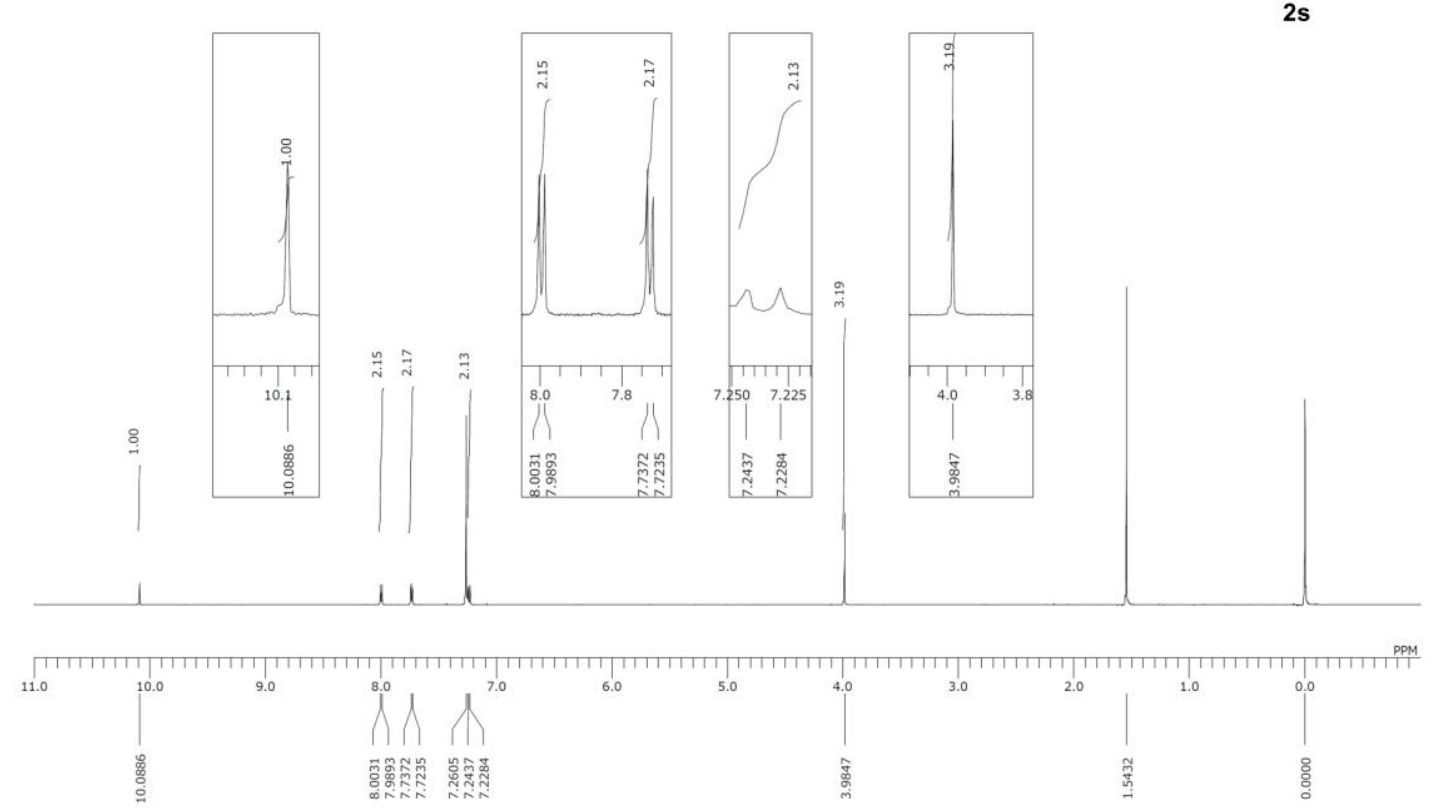


${ }^{13} \mathrm{C}$ NMR spectra of $2 \mathrm{~s}\left(\mathrm{CDCl}_{3}, 150 \mathrm{MHz}\right)$

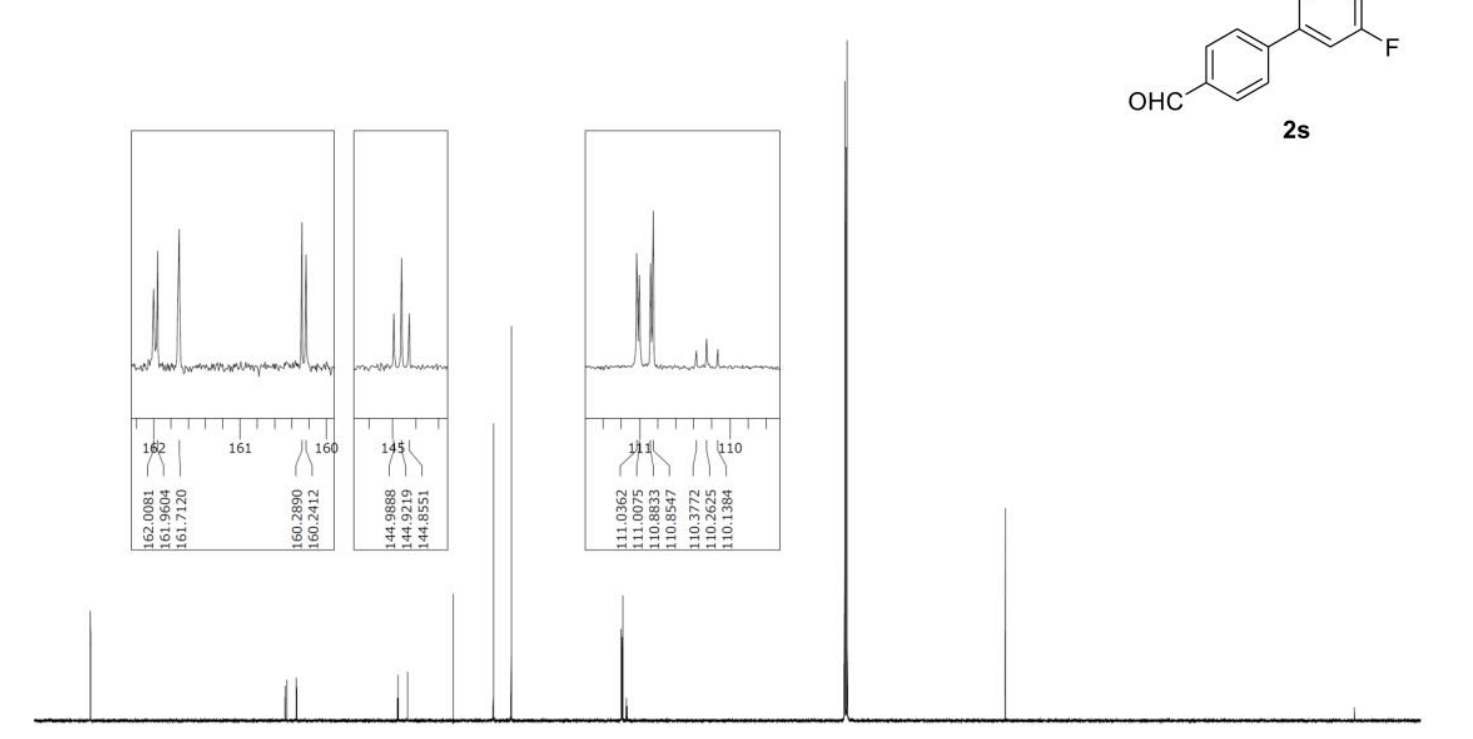

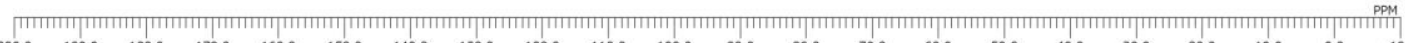

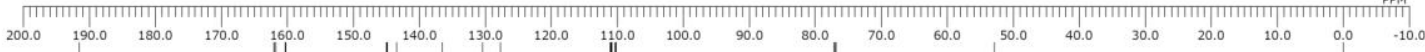

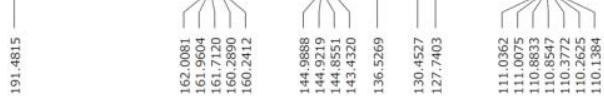

${ }^{19} \mathrm{~F}$ NMR spectra of $2 \mathrm{~s}\left(\mathrm{CDCl}_{3}, 565 \mathrm{MHz}\right)$

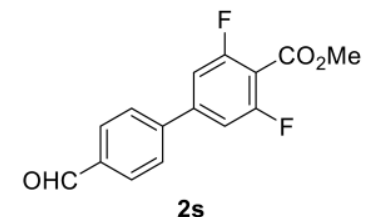

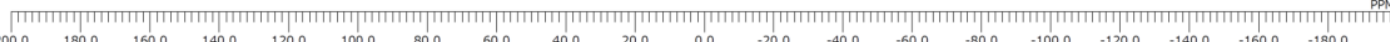

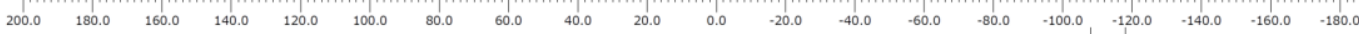


${ }^{1} \mathrm{H}$ NMR spectra of $\mathbf{2 t}\left(\mathrm{CDCl}_{3}, 400 \mathrm{MHz}\right)$
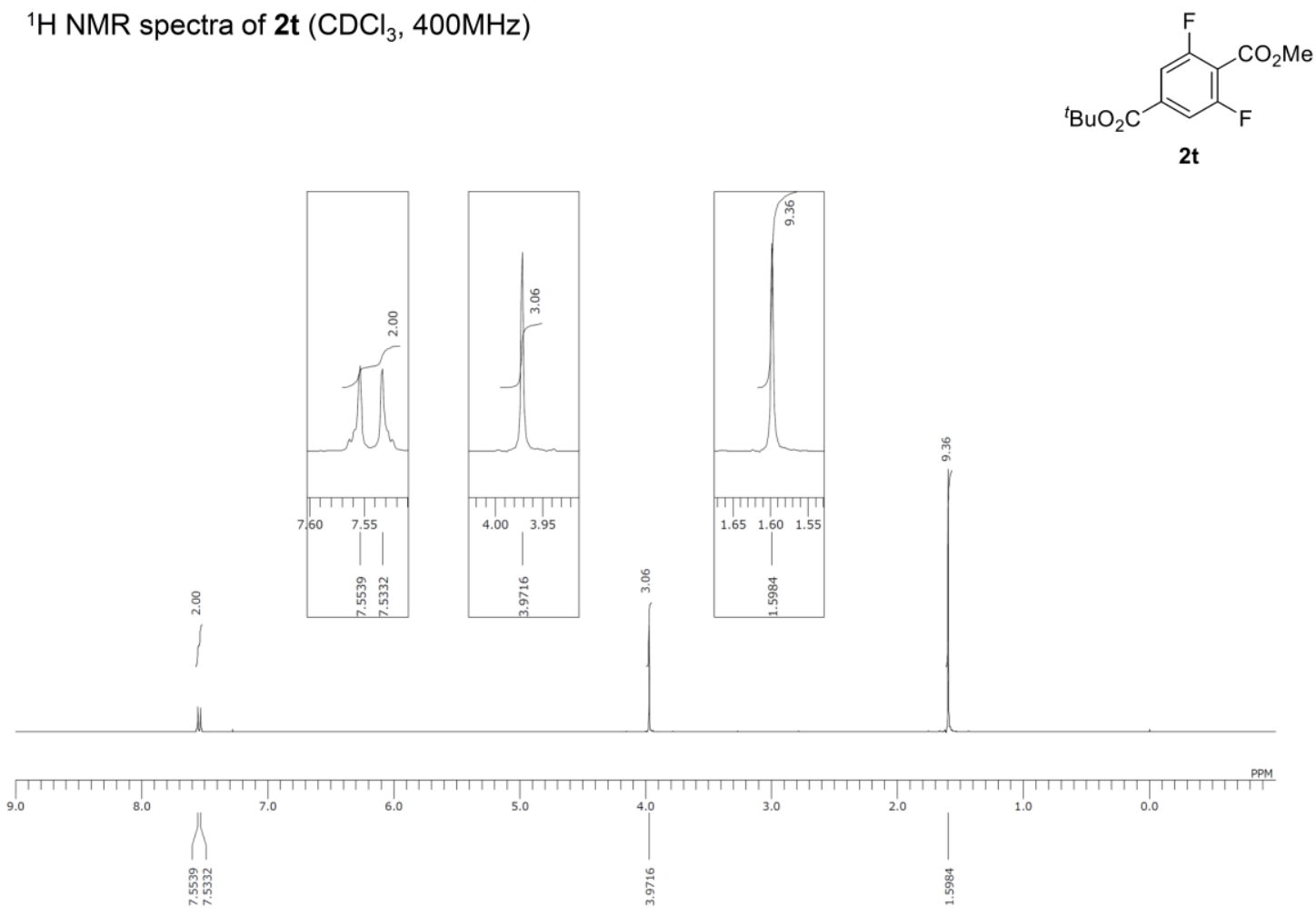

${ }^{13} \mathrm{C} \mathrm{NMR}$ spectra of $2 \mathbf{t}\left(\mathrm{CDCl}_{3}, 150 \mathrm{MHz}\right)$
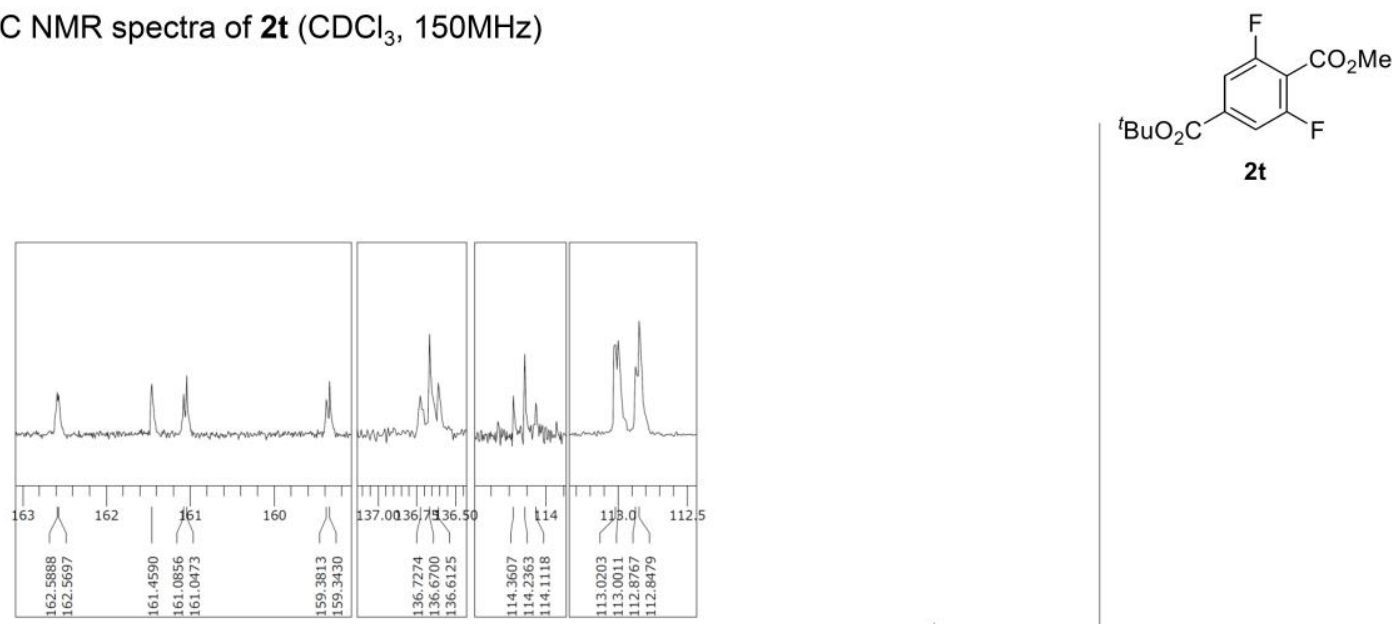

\section{4}

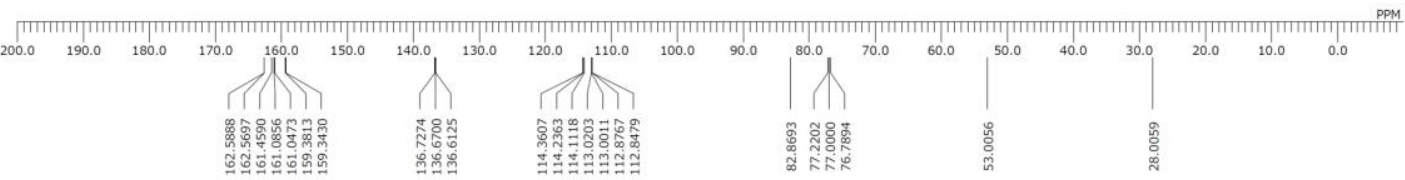


${ }^{19} \mathrm{~F}$ NMR spectra of $\mathbf{2 t}\left(\mathrm{CDCl}_{3}, 565 \mathrm{MHz}\right)$

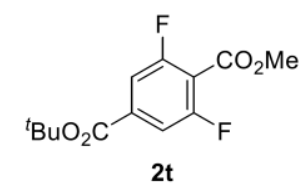

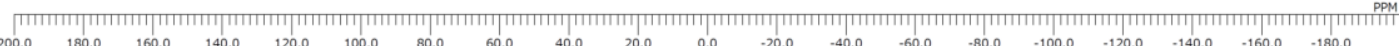

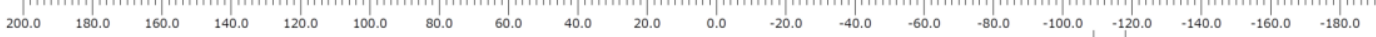

II

${ }^{1} \mathrm{H}$ NMR spectra of $2 \mathbf{u}\left(\mathrm{CDCl}_{3}, 400 \mathrm{MHz}\right)$

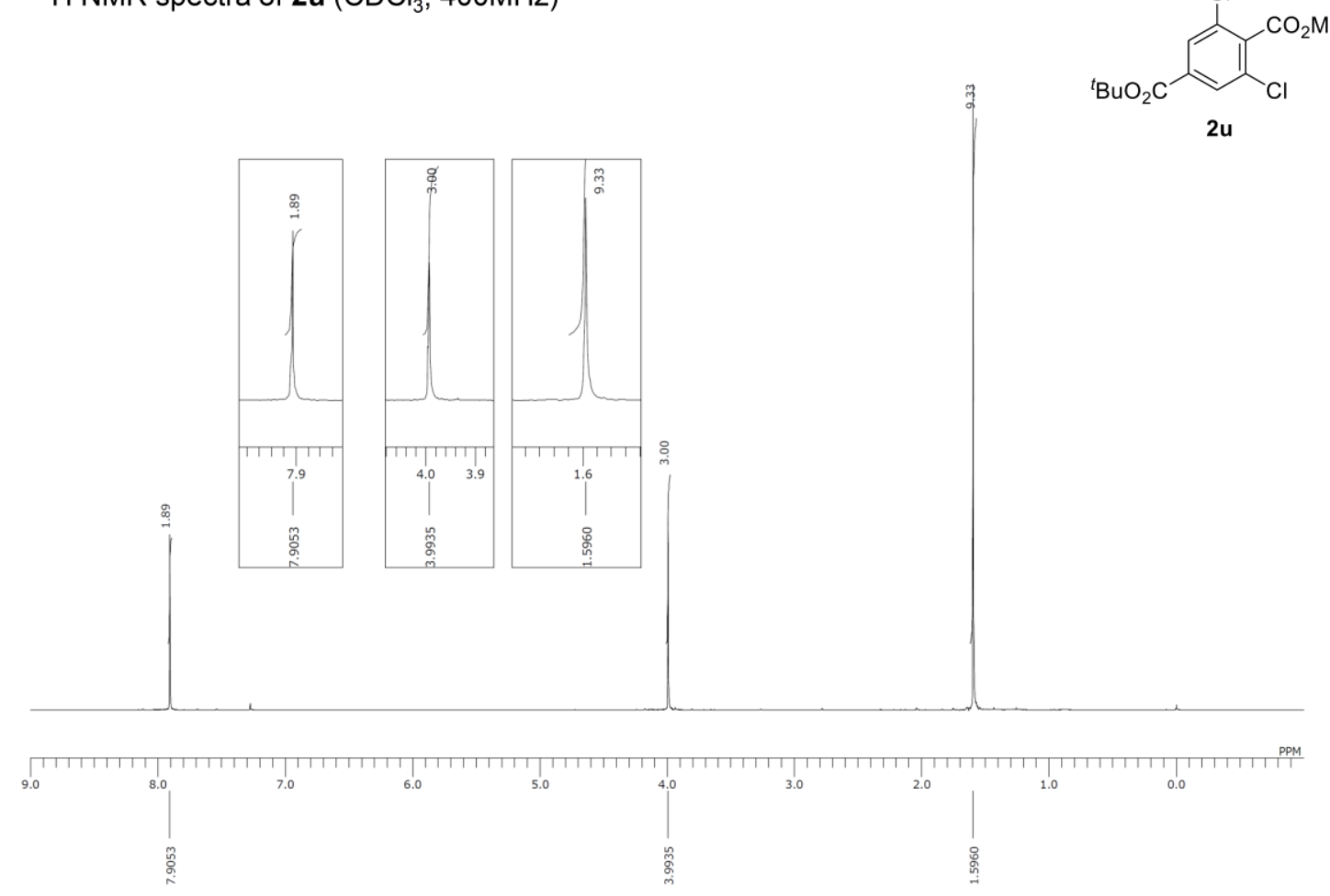


${ }^{13} \mathrm{C}$ NMR spectra of $\mathbf{2 u}\left(\mathrm{CDCl}_{3}, 100 \mathrm{MHz}\right)$
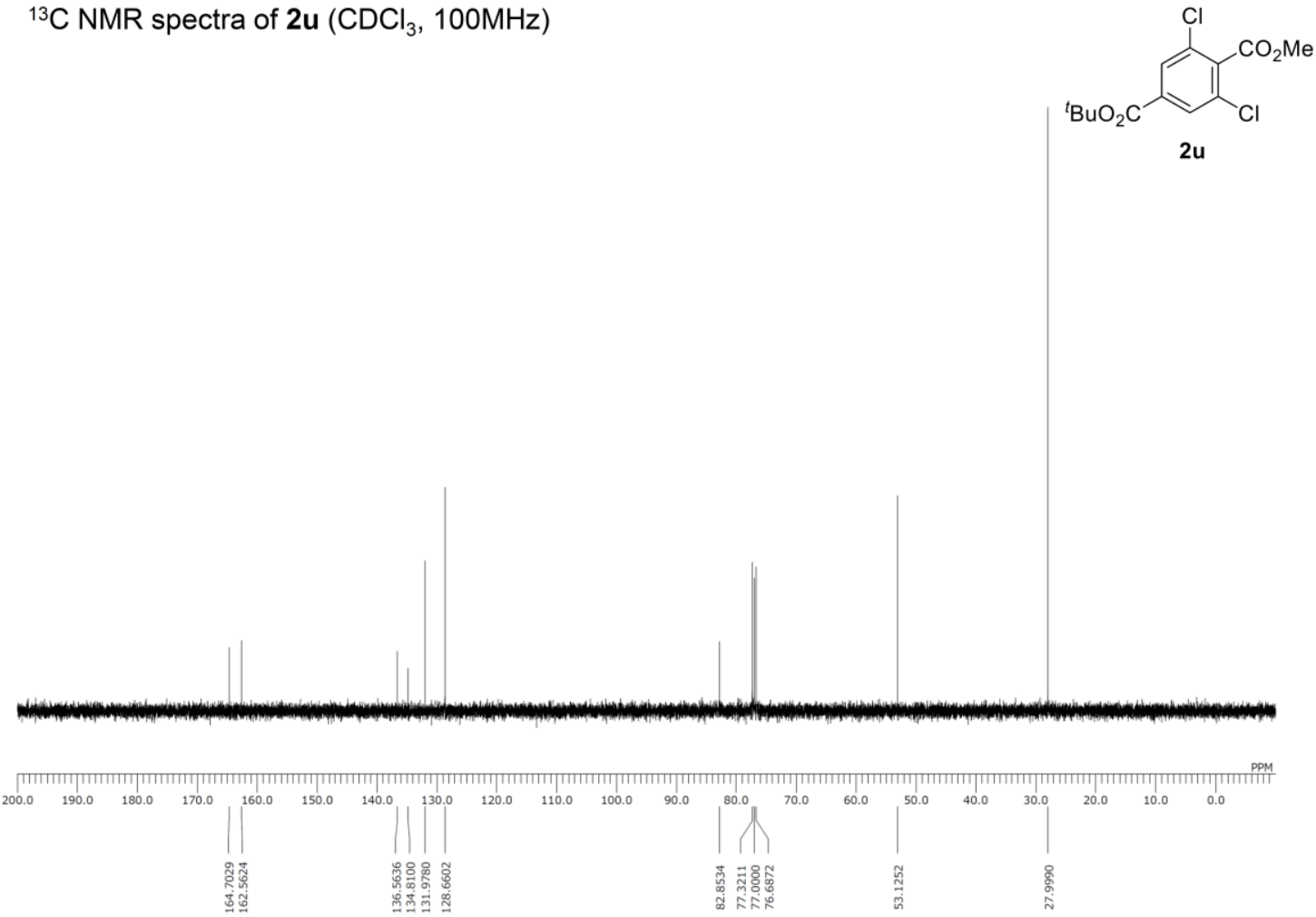

${ }^{1} \mathrm{H}$ NMR spectra of $2 \mathbf{v}\left(\mathrm{CDCl}_{3}, 600 \mathrm{MHz}\right)$

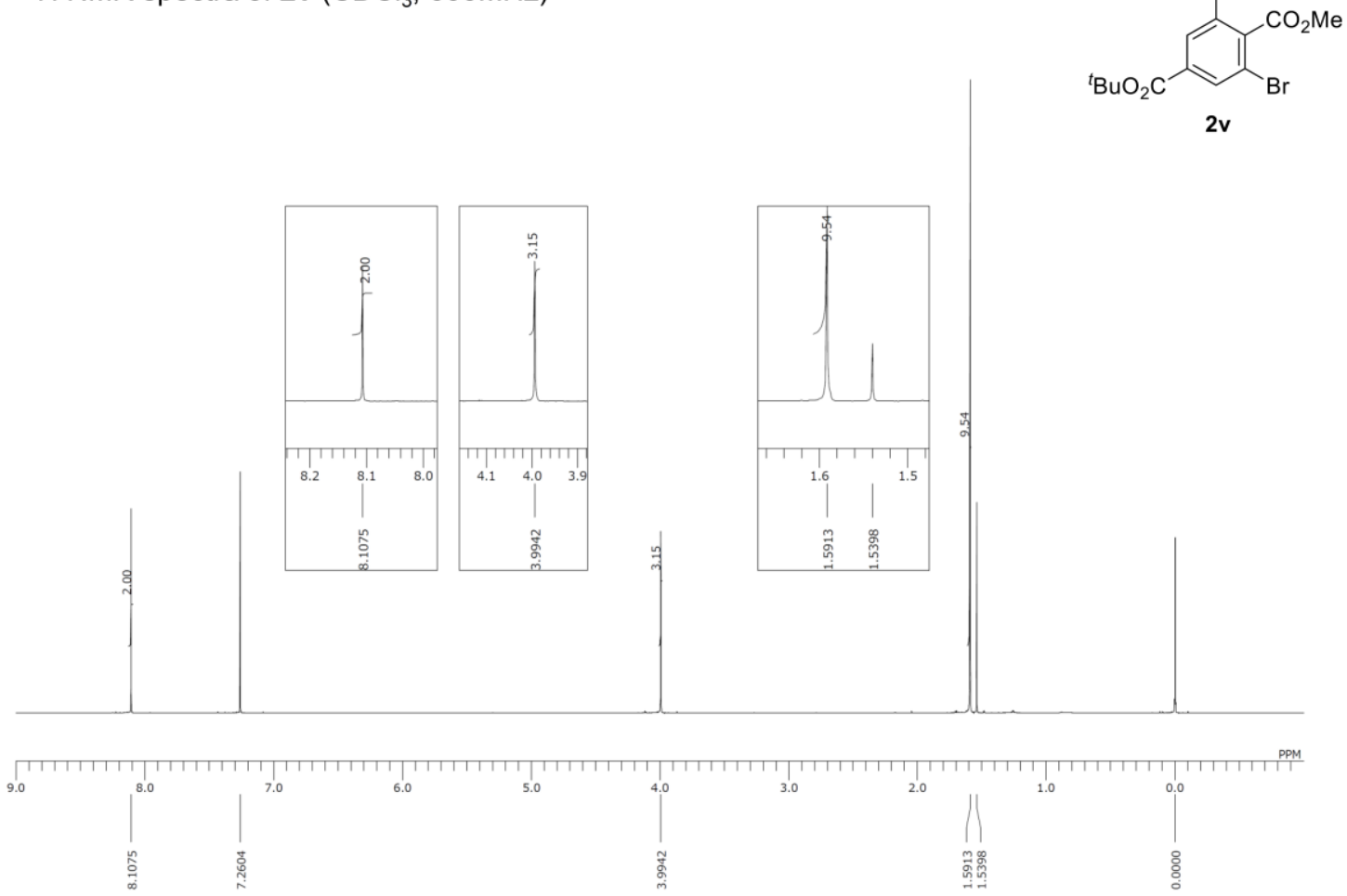


${ }^{13} \mathrm{C}$ NMR spectra of $2 \mathbf{v}\left(\mathrm{CDCl}_{3}, 150 \mathrm{MHz}\right)$

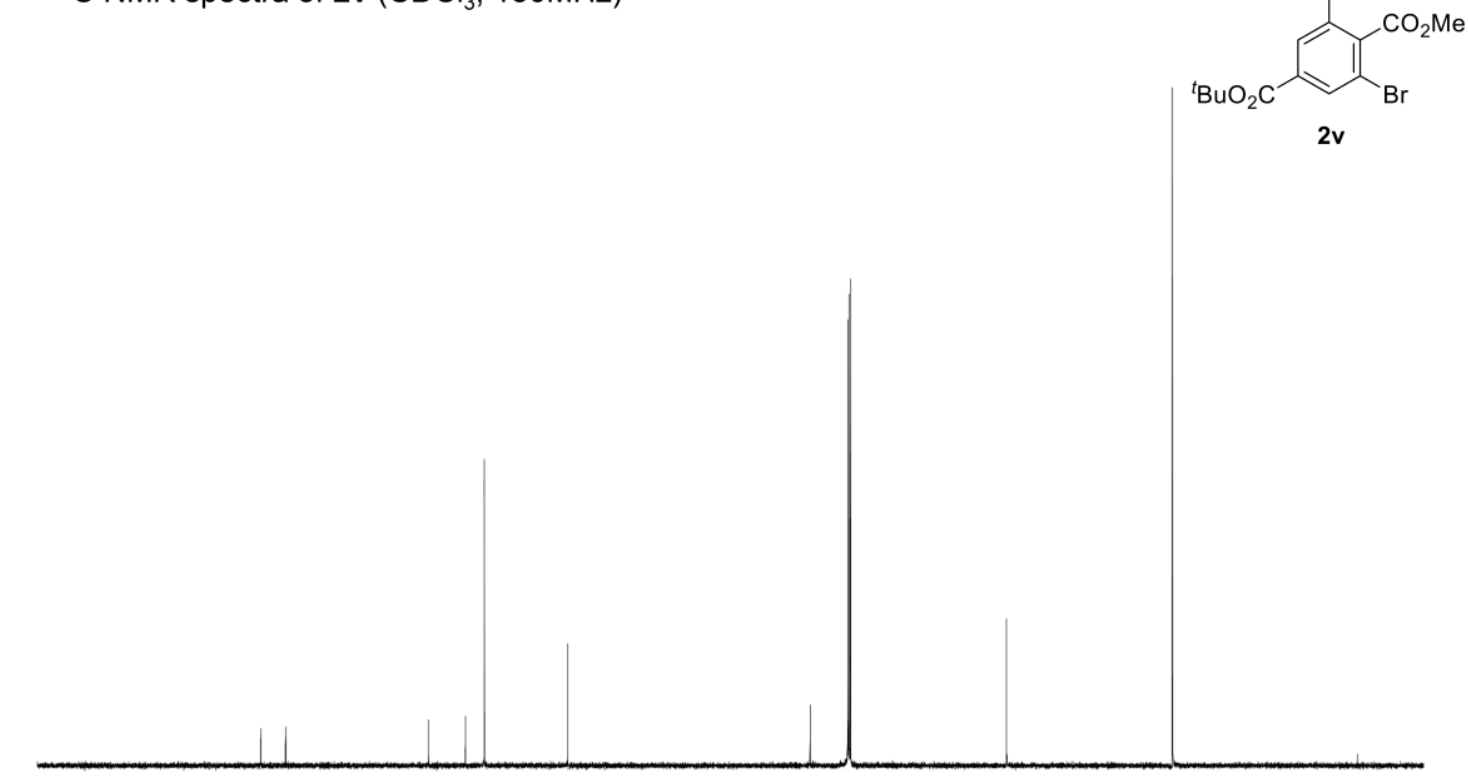

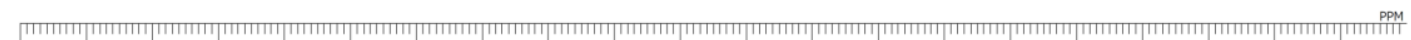

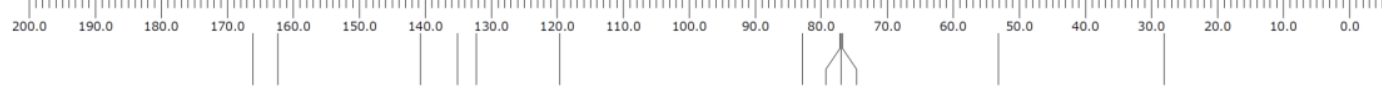

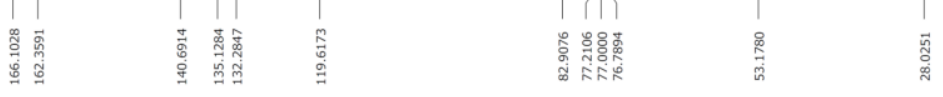

${ }^{1} \mathrm{H}$ NMR spectra of $\mathbf{2} \mathbf{w}\left(\mathrm{CDCl}_{3}, 600 \mathrm{MHz}\right)$

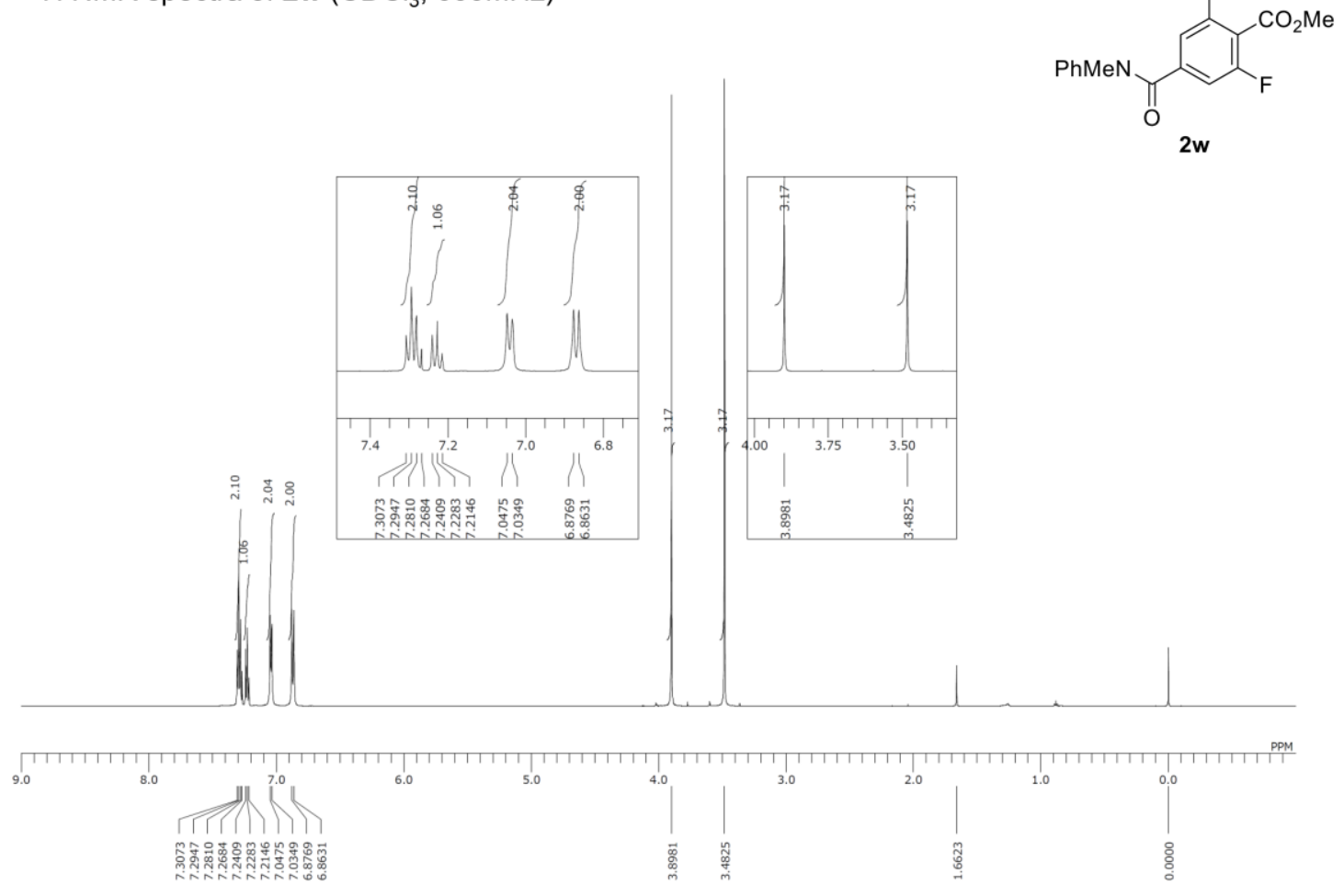


${ }^{13} \mathrm{C}$ NMR spectra of $\mathbf{2} \mathbf{w}\left(\mathrm{CDCl}_{3}, 150 \mathrm{MHz}\right)$<smiles>COc1c(F)cc(C(=O)Nc2ccccc2)cc1F</smiles>

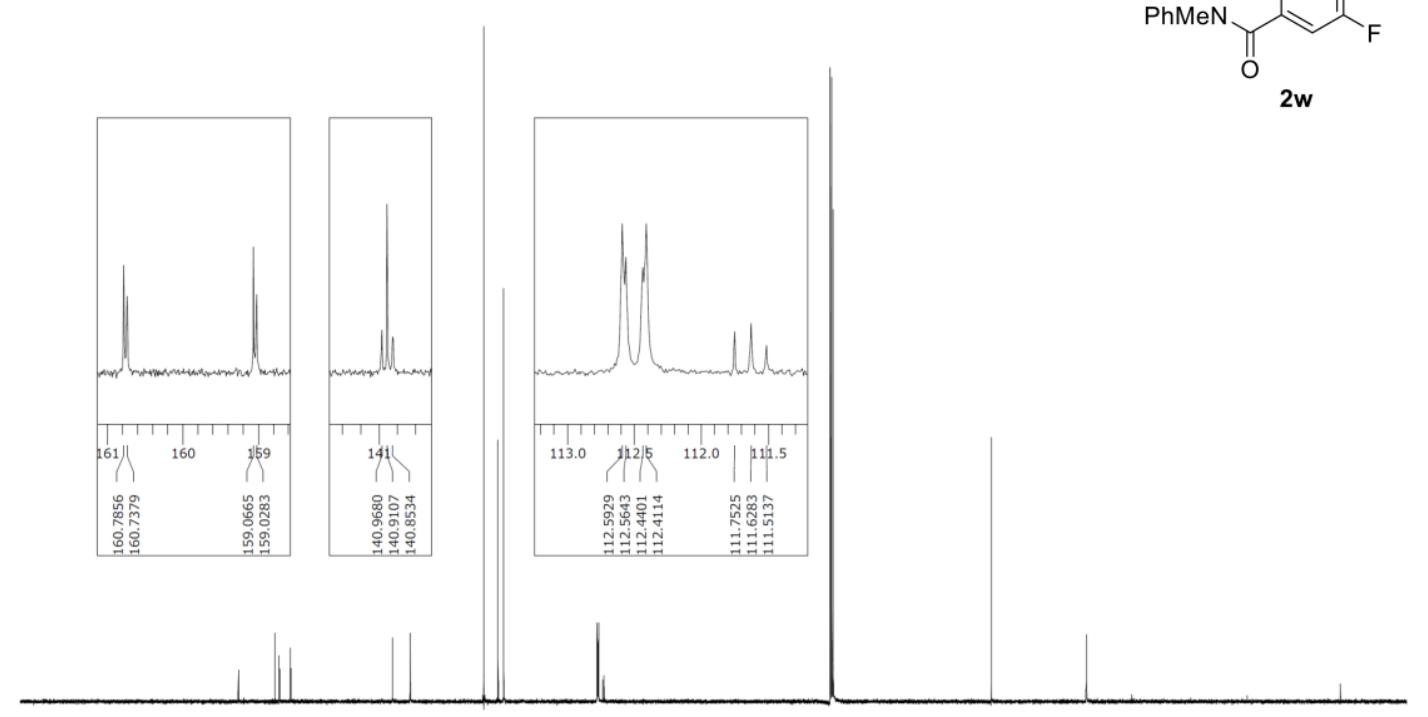

[

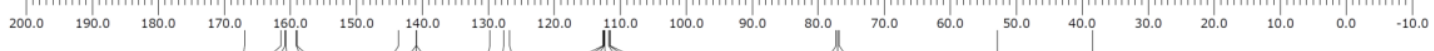
Mh M Mn

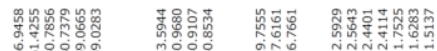

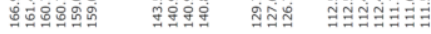

${ }^{19} \mathrm{~F} \mathrm{NMR}$ spectra of $\mathbf{2 w}\left(\mathrm{CDCl}_{3}, 565 \mathrm{MHz}\right)$<smiles>CCNC(=O)c1cc(F)c(C(=O)OCc2ccccc2)c(F)c1</smiles>

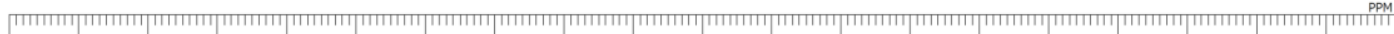
$\begin{array}{llllllllllllllllllll}200.0 & 180.0 & 160.0 & 140.0 & 120.0 & 100.0 & 80.0 & 60.0 & 40.0 & 20.0 & 0.0 & -20.0 & -40.0 & -60.0 & -80.0 & -100.0 & -120.0 & -140.0 & -160.0 & -180.0\end{array}$ 
${ }^{1} \mathrm{H}$ NMR spectra of 2x (DMSO-d6, 600MHz)<smiles>CCCCNC(=O)c1cc(Cl)c(C(=O)OC)c(Cl)c1</smiles>

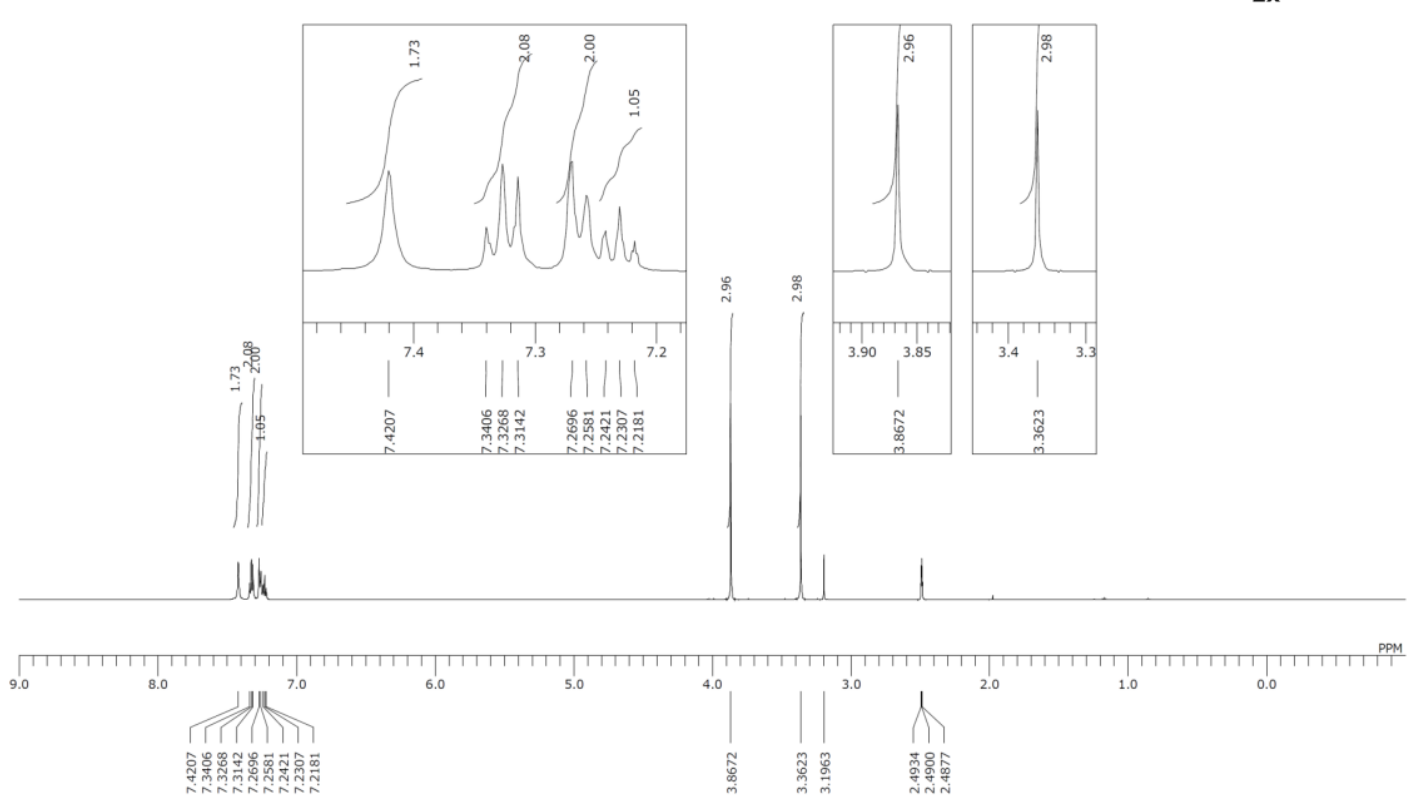

${ }^{13} \mathrm{C}$ NMR spectra of $\mathbf{2 x}\left(\mathrm{CDCl}_{3}, 150 \mathrm{MHz}\right)$
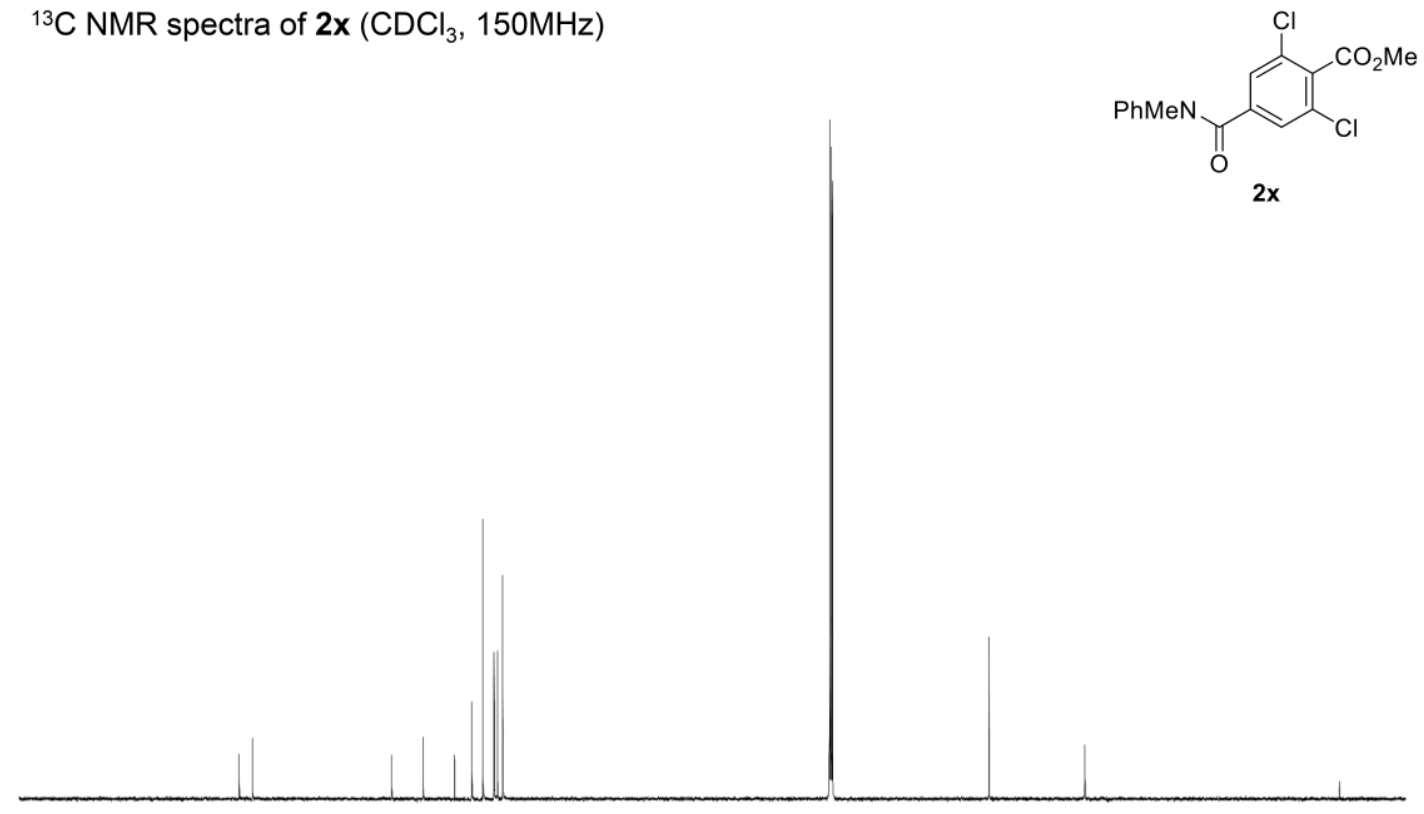

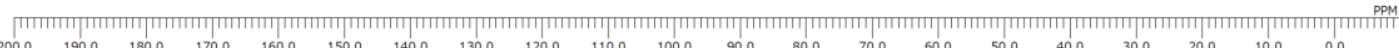

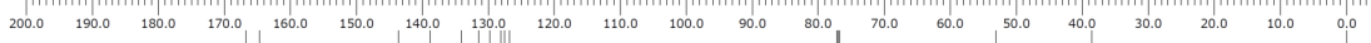

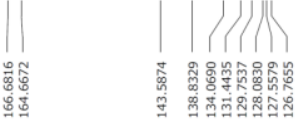

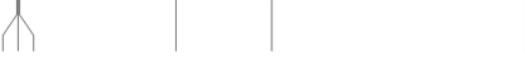

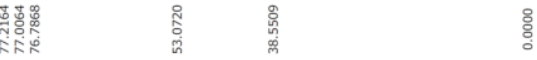


${ }^{1} \mathrm{H}$ NMR spectra of $2 \mathbf{y}\left(\mathrm{CDCl}_{3}, 400 \mathrm{MHz}\right)$

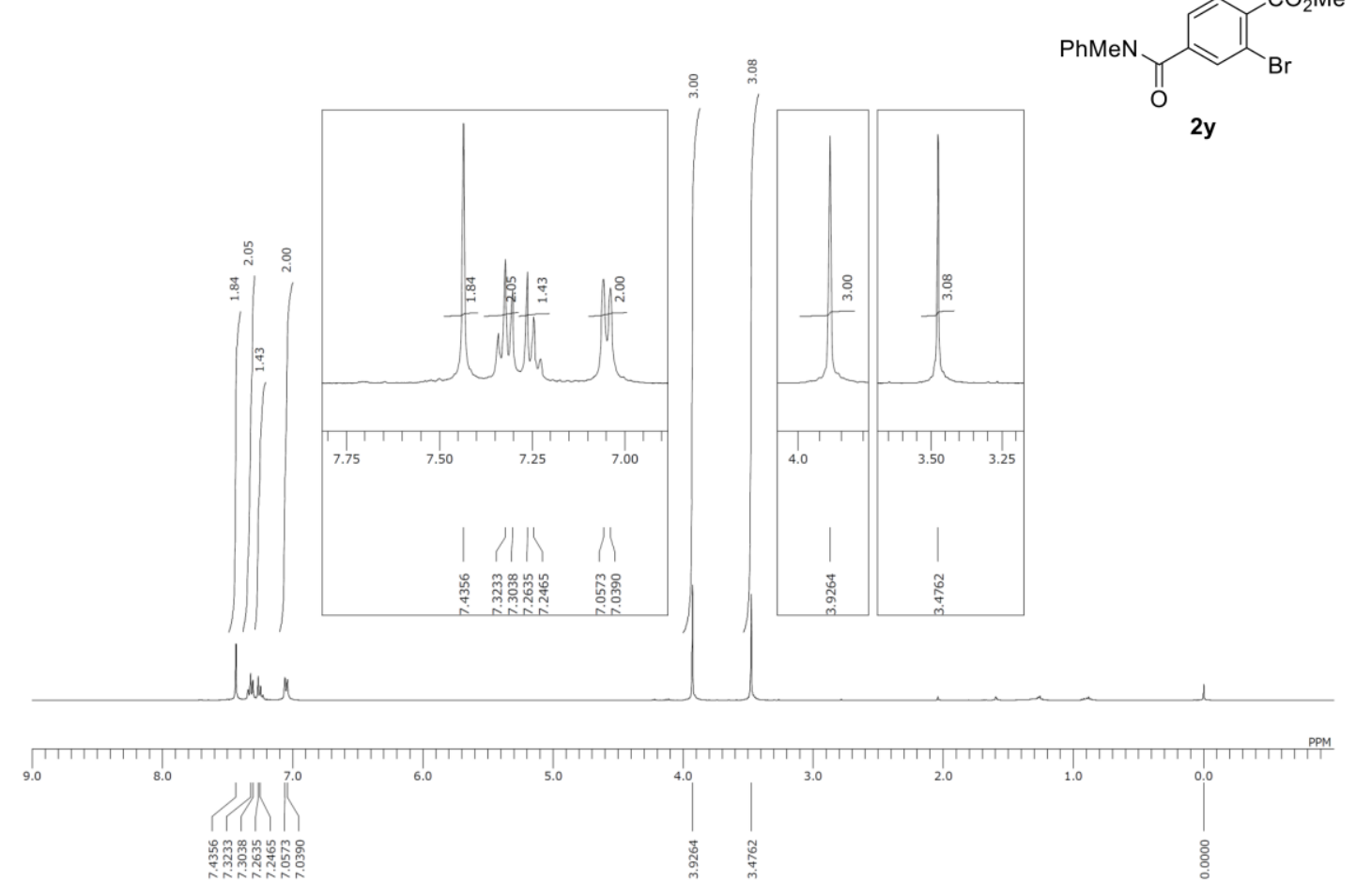

${ }^{13} \mathrm{C}$ NMR spectra of $\mathbf{2 y}\left(\mathrm{CDCl}_{3}, 100 \mathrm{MHz}\right)$
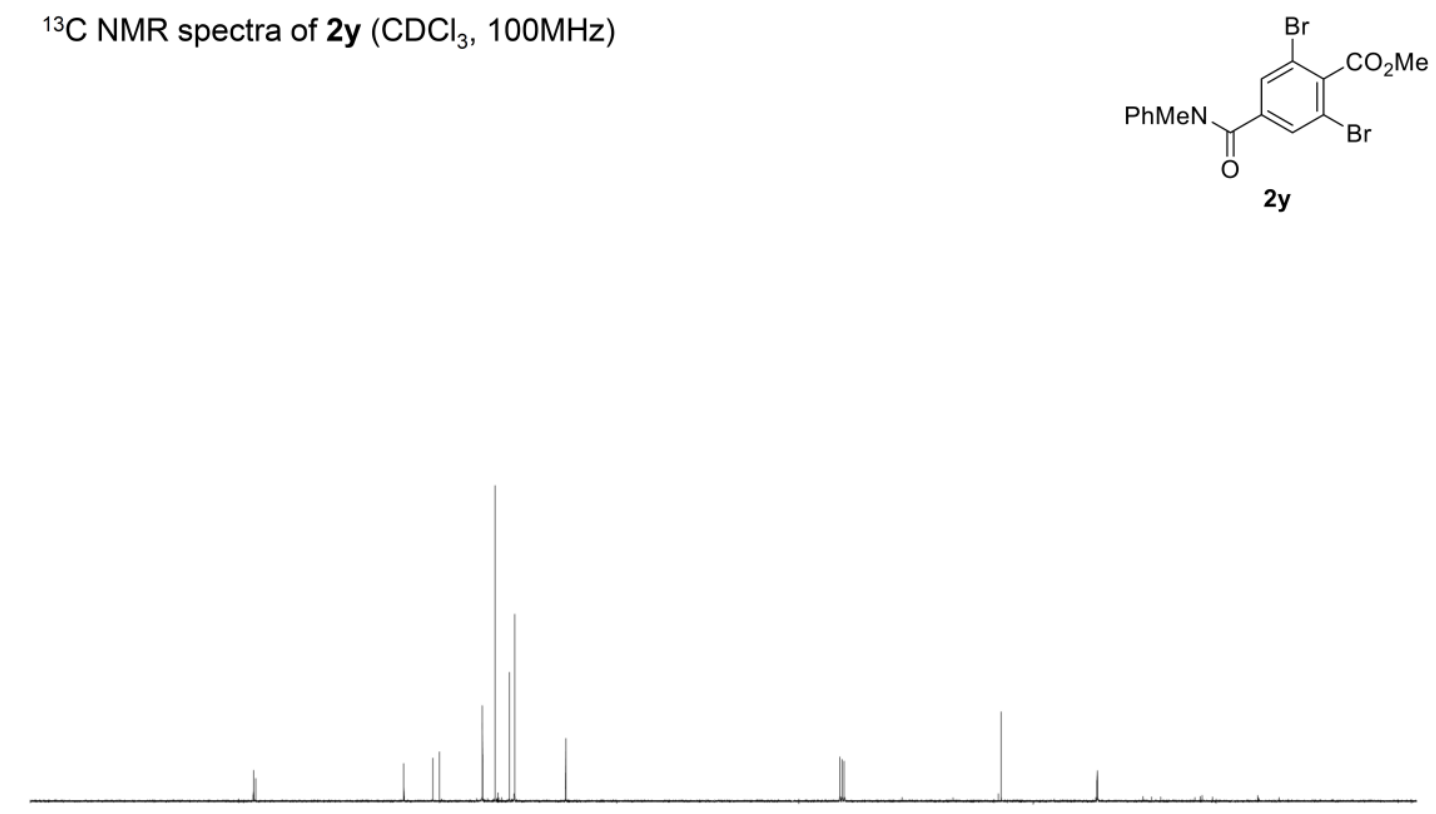

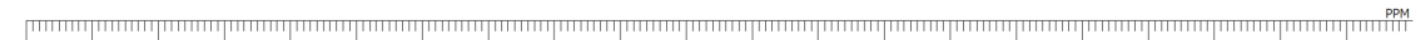

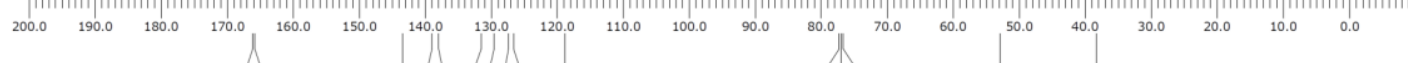

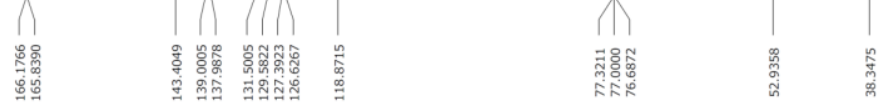


${ }^{1} \mathrm{H}$ NMR spectra of $\mathbf{2 z}\left(\mathrm{CDCl}_{3}, 400 \mathrm{MHz}\right)$

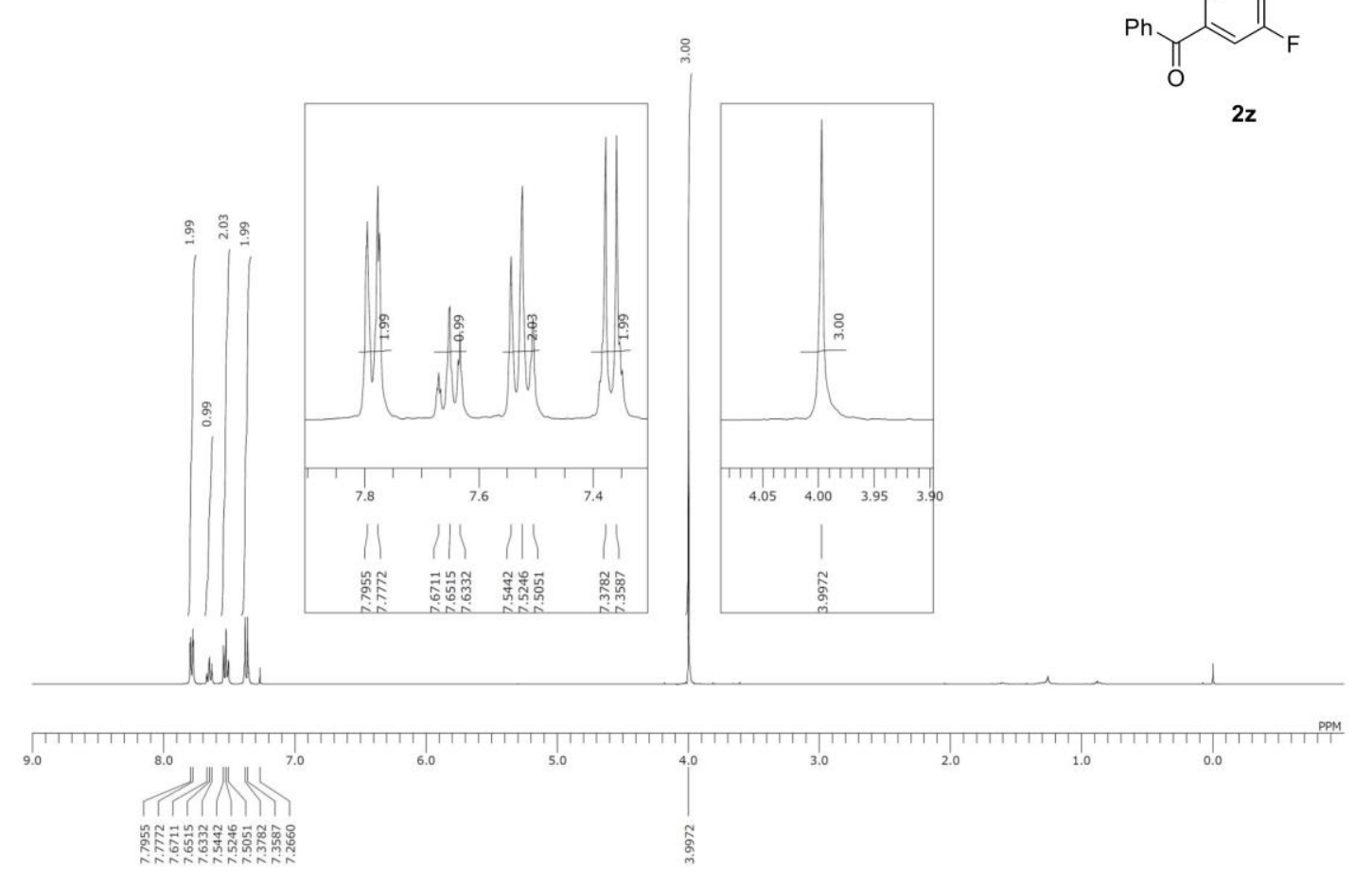

${ }^{13} \mathrm{C}$ NMR spectra of $\mathbf{2 z}\left(\mathrm{CDCl}_{3}, 150 \mathrm{MHz}\right)$

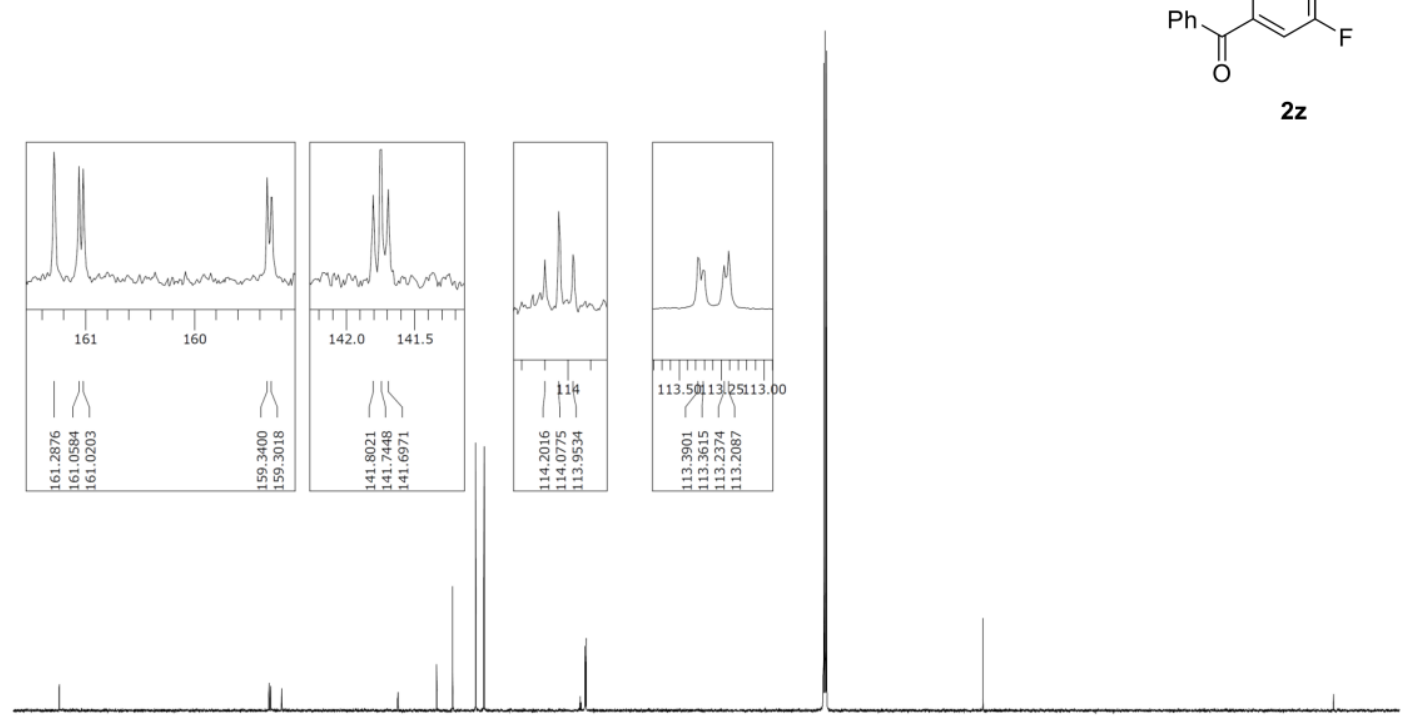

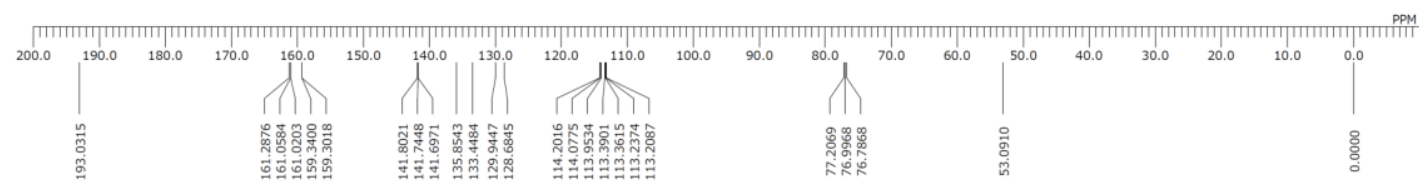


${ }^{19} \mathrm{~F}$ NMR spectra of $\mathbf{2 z}\left(\mathrm{CDCl}_{3}, 565 \mathrm{MHz}\right)$
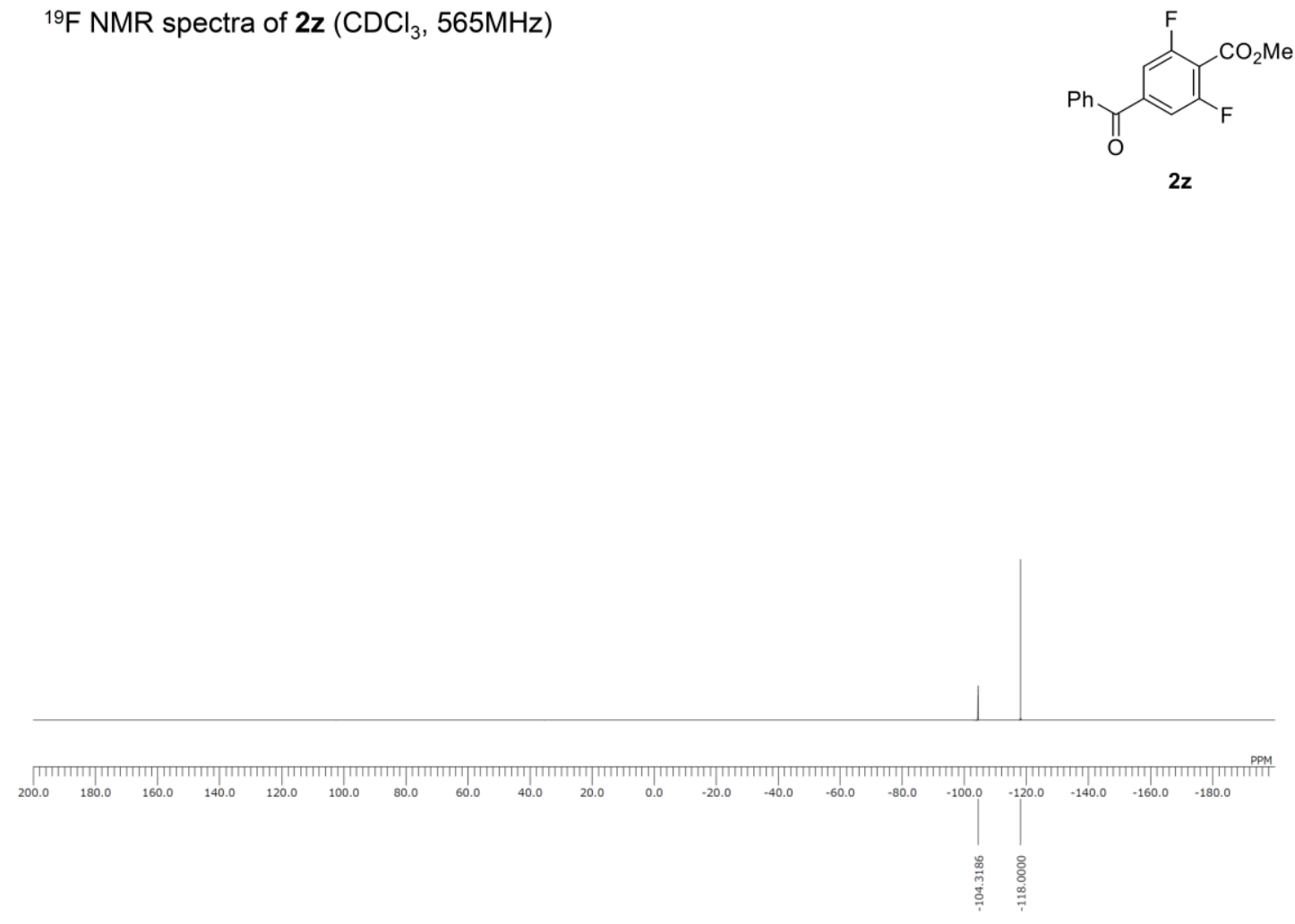

${ }^{1} \mathrm{H}$ NMR spectra of $2 \mathrm{aa}\left(\mathrm{CDCl}_{3}, 400 \mathrm{MHz}\right)$

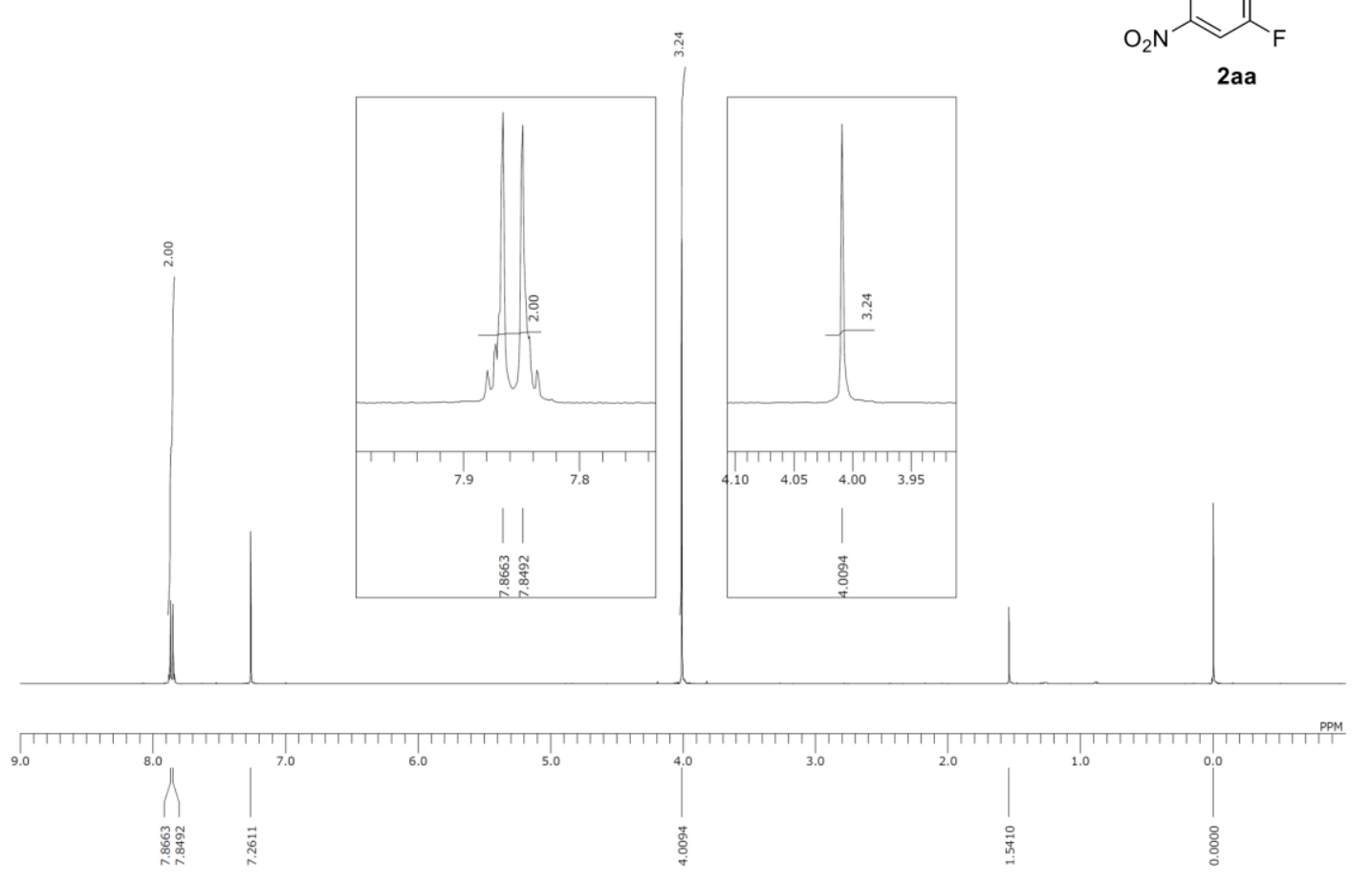


${ }^{13} \mathrm{C}$ NMR spectra of $2 \mathrm{aa}\left(\mathrm{CDCl}_{3}, 150 \mathrm{MHz}\right)$

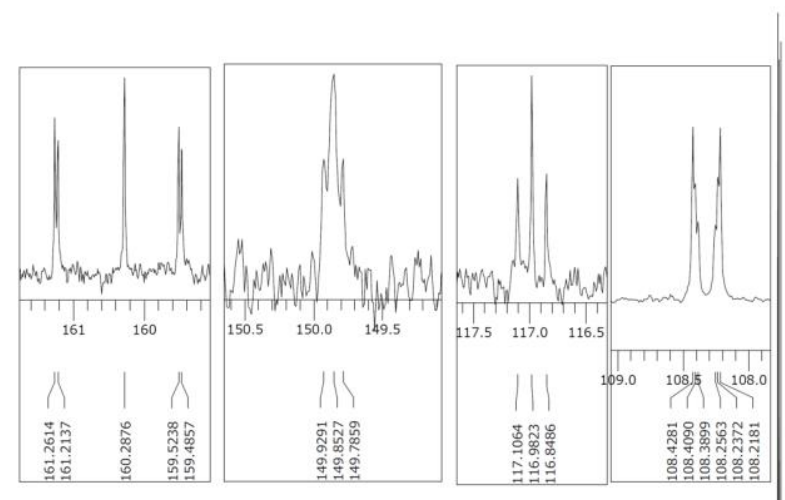<smiles>CC(=O)c1c(F)cc([N+](=O)[O-])cc1F</smiles>

2aa

${ }^{19} \mathrm{~F}$ NMR spectra of $2 \mathrm{aa}\left(\mathrm{CDCl}_{3}, 565 \mathrm{MHz}\right)$<smiles>COC(=O)c1c(F)cc([N+](=O)[O-])cc1F</smiles>

2aa

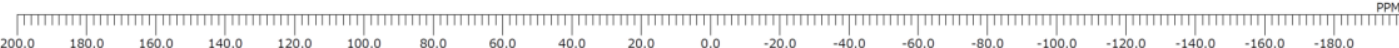

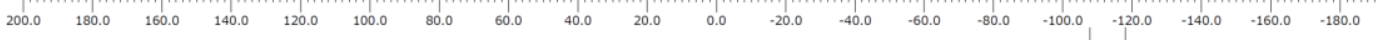


${ }^{1} \mathrm{H}$ NMR spectra of $2 \mathrm{ab}\left(\mathrm{CDCl}_{3}, 600 \mathrm{MHz}\right)$

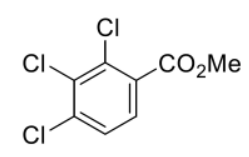

2ab

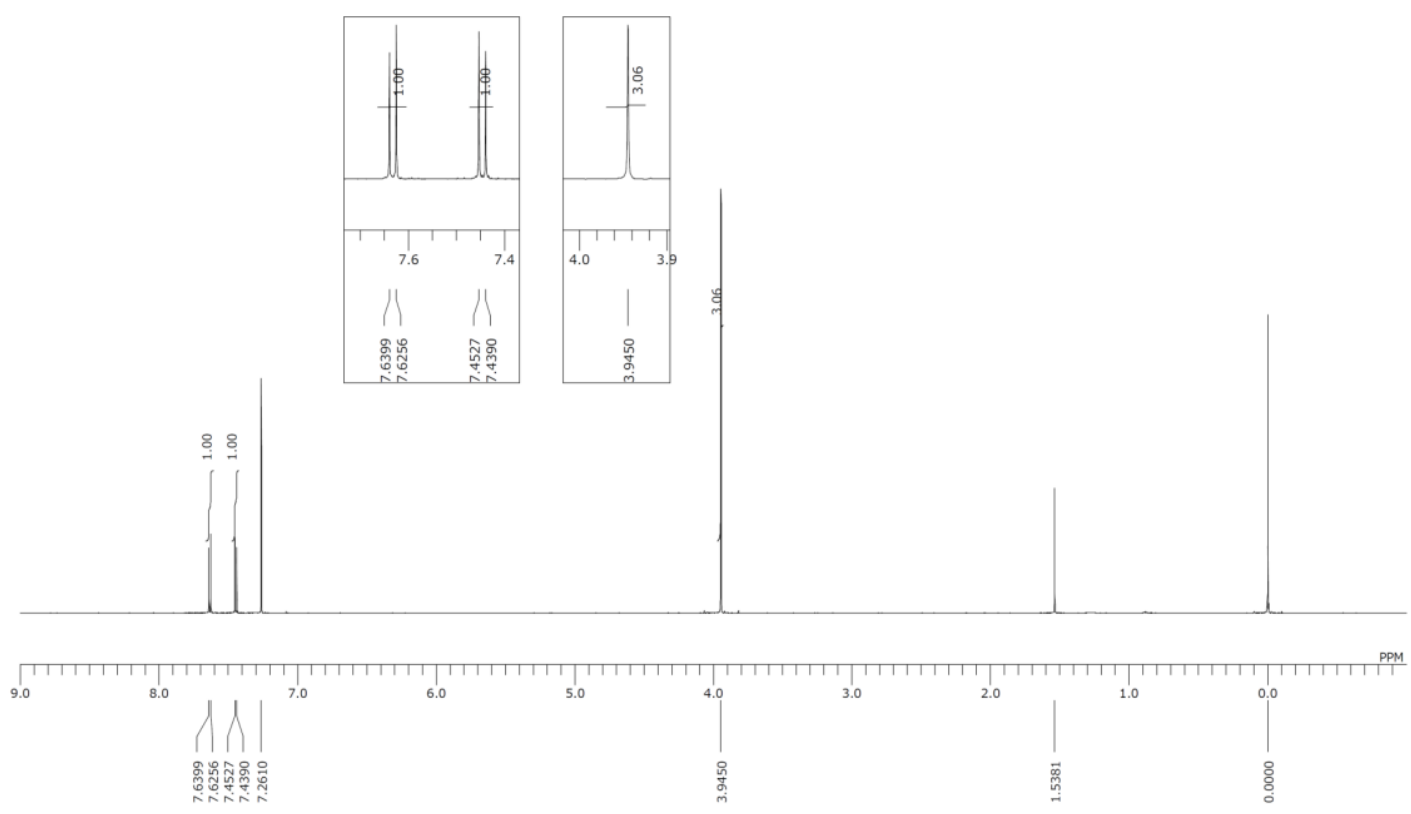

${ }^{13} \mathrm{C}$ NMR spectra of $\mathbf{2 a b}\left(\mathrm{CDCl}_{3}, 150 \mathrm{MHz}\right)$

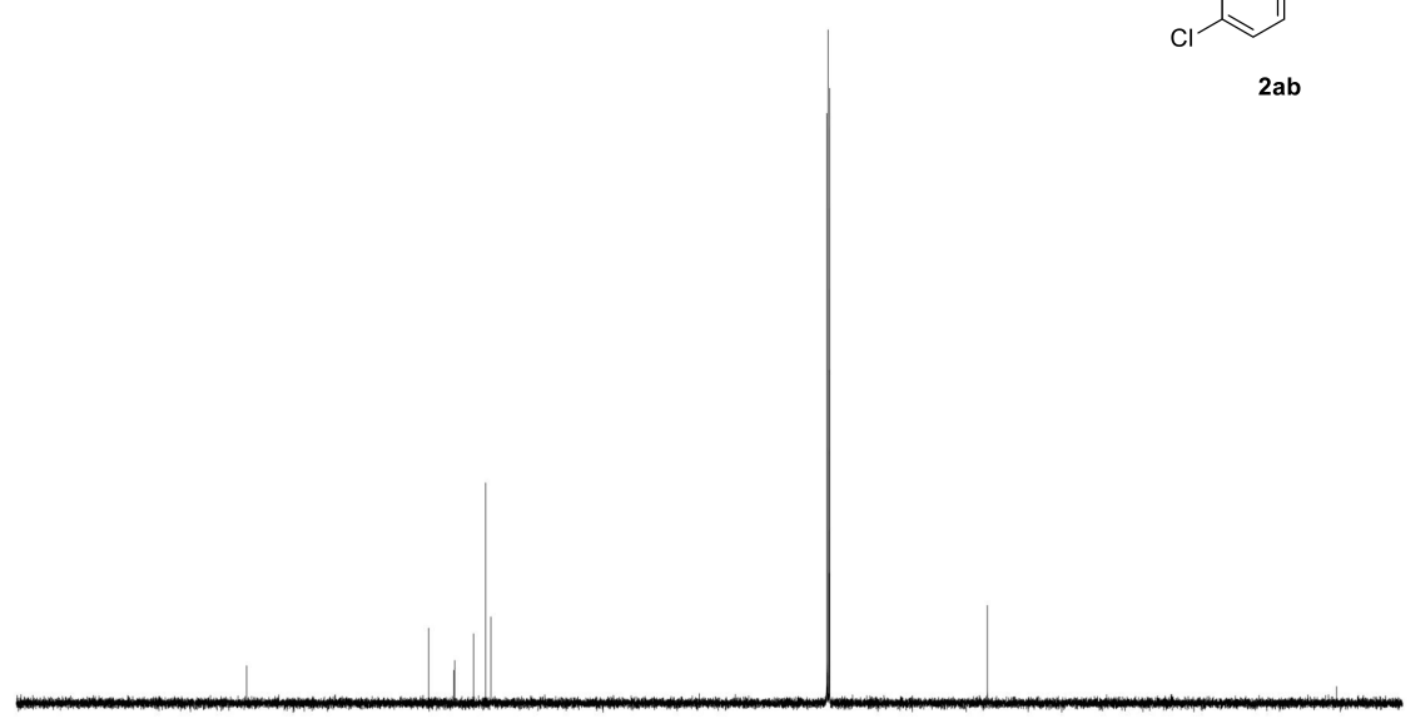

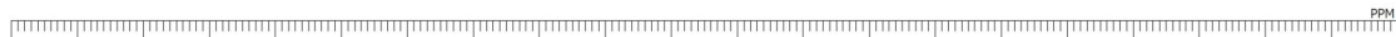

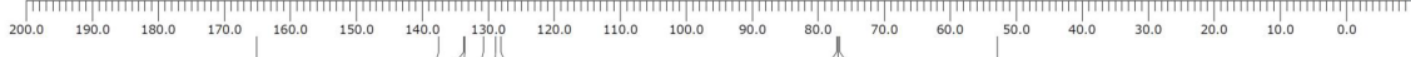

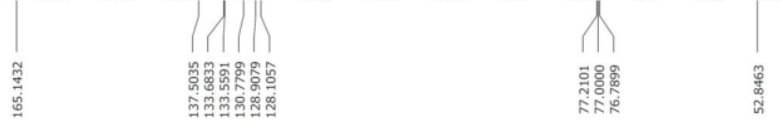


${ }^{1} \mathrm{H}$ NMR spectra of $2 \mathrm{ac}\left(\mathrm{CDCl}_{3}, 400 \mathrm{MHz}\right)$

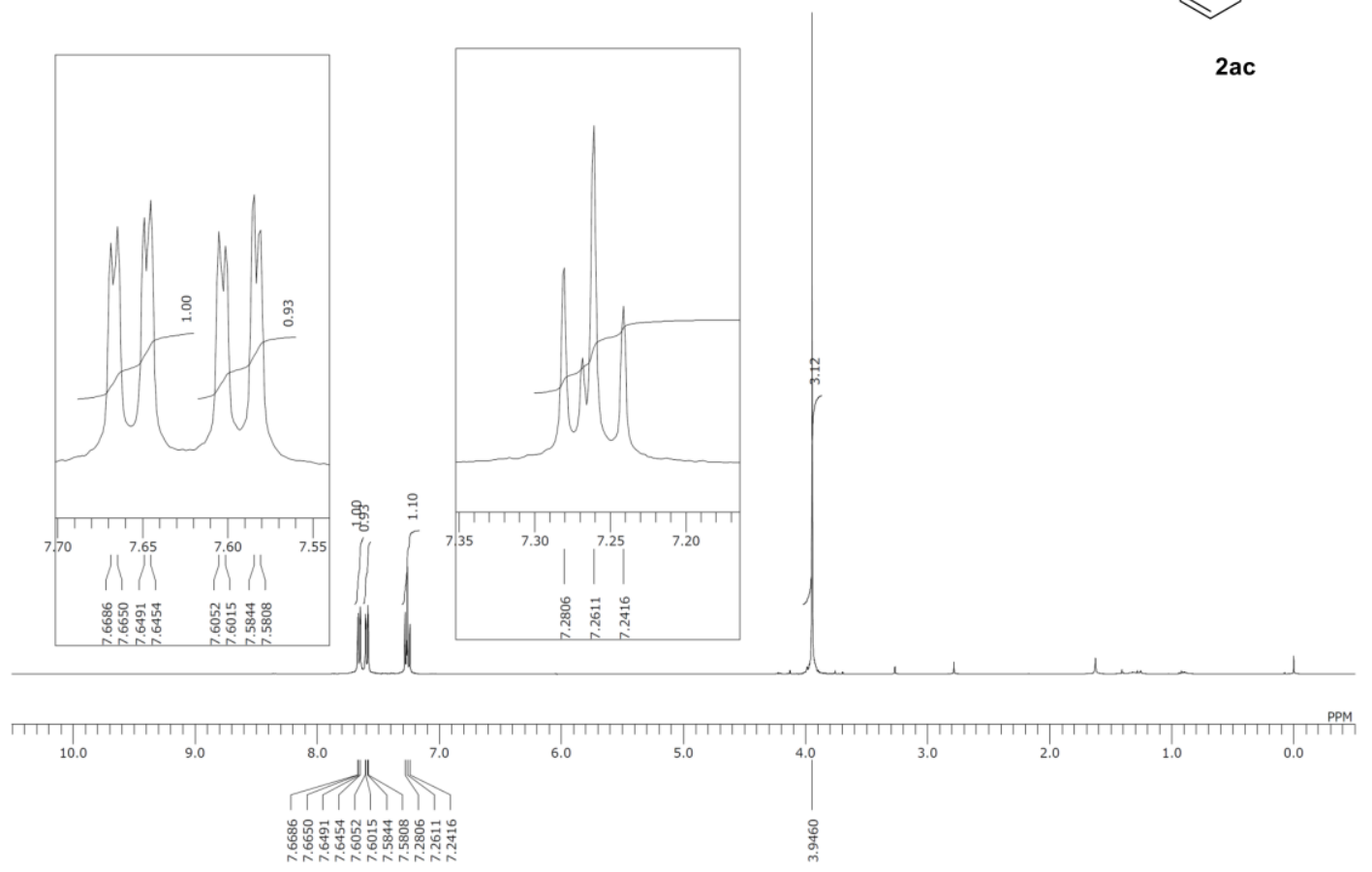

${ }^{13} \mathrm{C}$ NMR spectra of $\mathbf{2 a c}\left(\mathrm{CDCl}_{3}, 100 \mathrm{MHz}\right)$

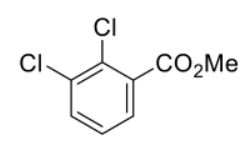

2ac

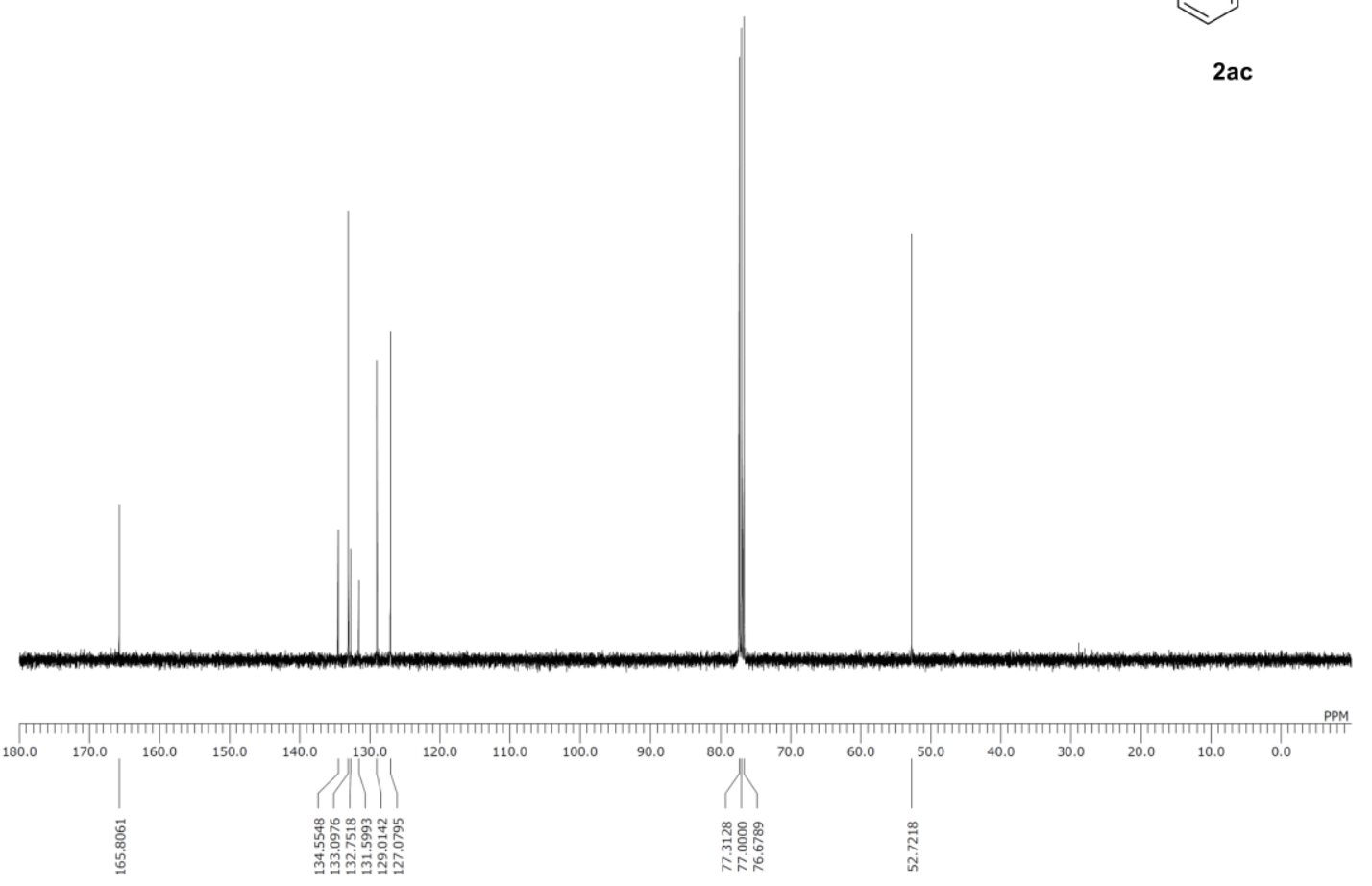


${ }^{1} \mathrm{H}$ NMR spectra of $4 \mathrm{a}\left(\mathrm{CDCl}_{3}, 600 \mathrm{MHz}\right)$

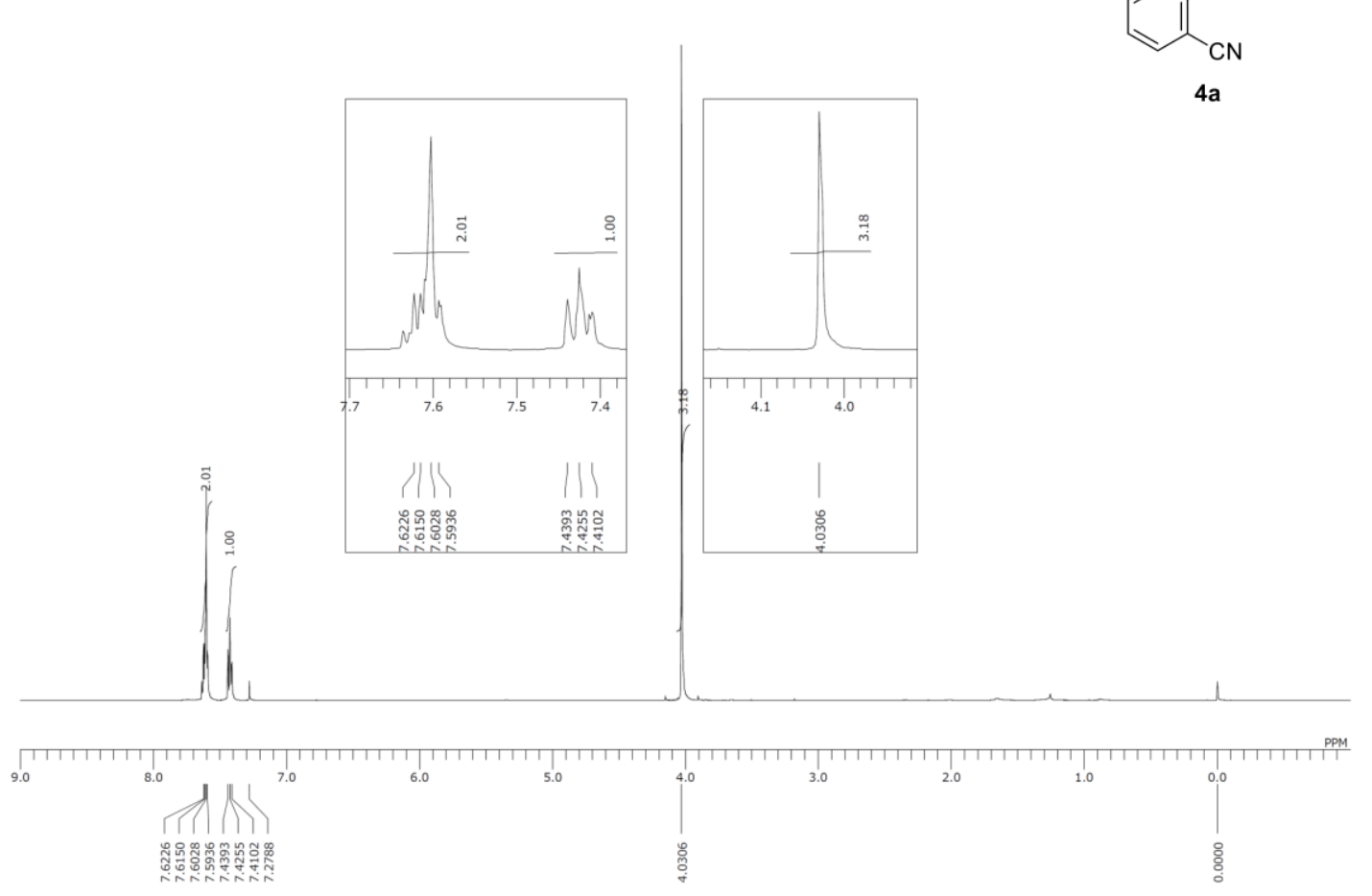

${ }^{13} \mathrm{C}$ NMR spectra of $\mathbf{4 a}\left(\mathrm{CDCl}_{3}, 150 \mathrm{MHz}\right)$

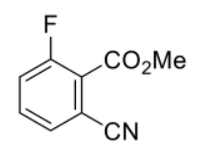

$4 a$

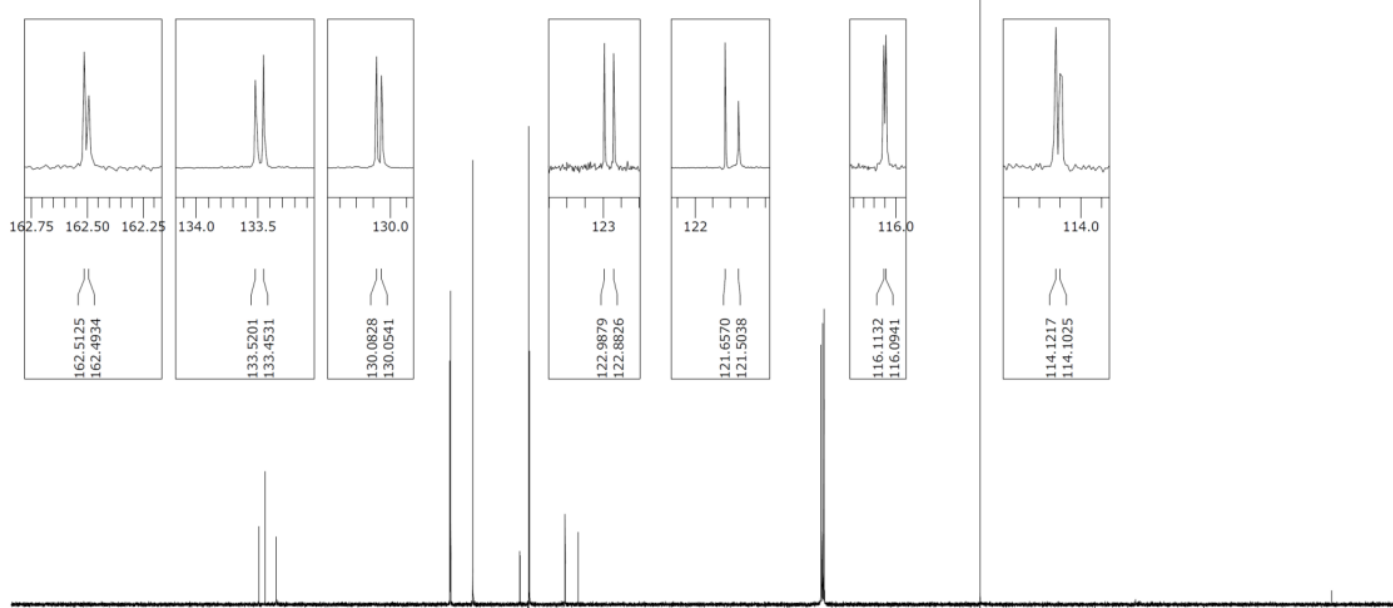

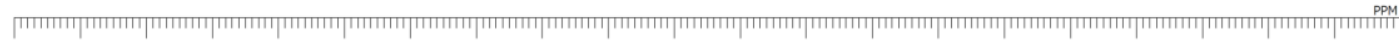

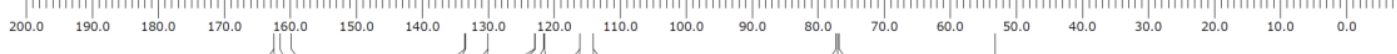

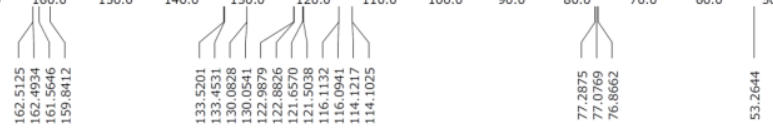


${ }^{19} \mathrm{~F}$ NMR spectra of $4 \mathrm{a}\left(\mathrm{CDCl}_{3}, 565 \mathrm{MHz}\right)$

${ }^{1} \mathrm{H}$ NMR spectra of $\mathbf{4 b}\left(\mathrm{CDCl}_{3}, 400 \mathrm{MHz}\right)$

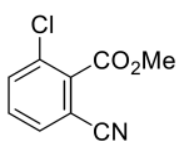

$4 \mathrm{~b}$

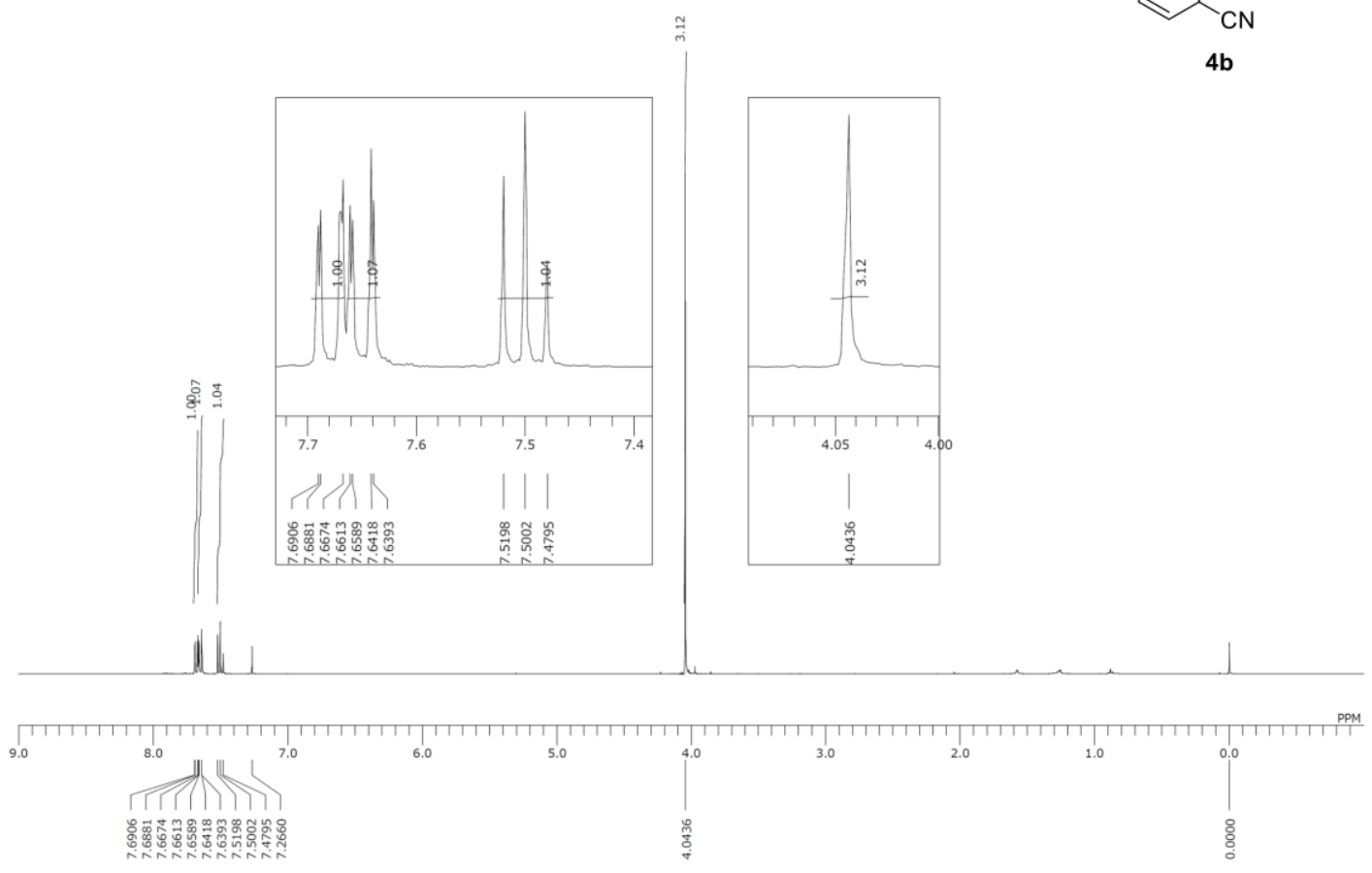


${ }^{13} \mathrm{C}$ NMR spectra of $\mathbf{4 b}\left(\mathrm{CDCl}_{3}, 100 \mathrm{MHz}\right)$

$\overbrace{\mathrm{CN}}^{\mathrm{Cl} O}$

4b

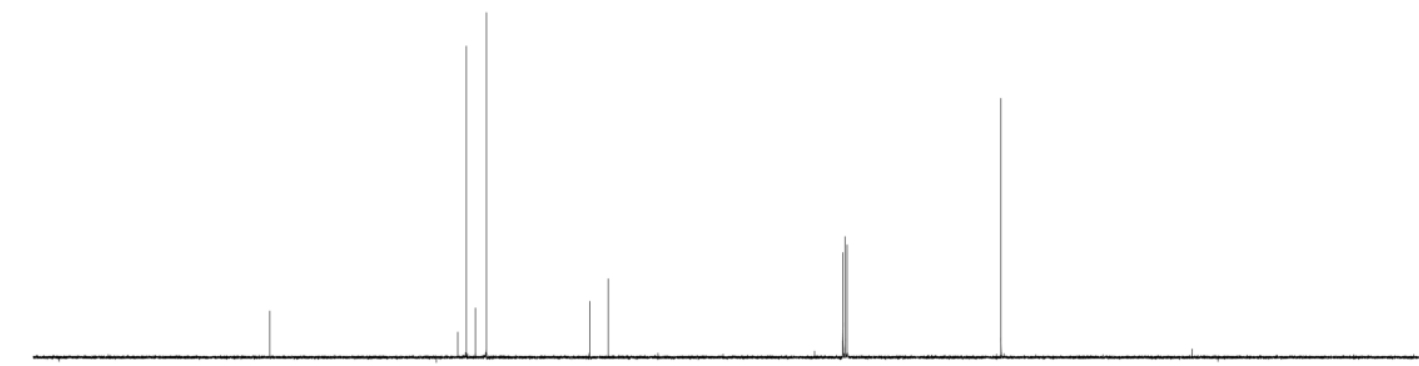

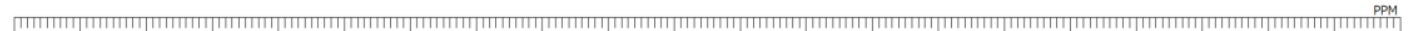

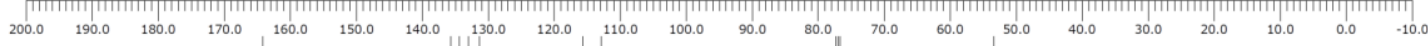

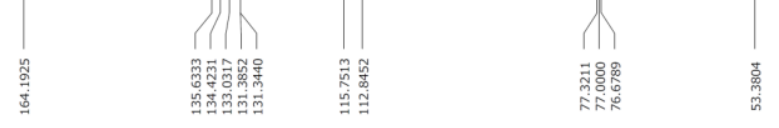

${ }^{1} \mathrm{H}$ NMR spectra of $4 \mathrm{c}\left(\mathrm{CDCl}_{3}, 400 \mathrm{MHz}\right)$
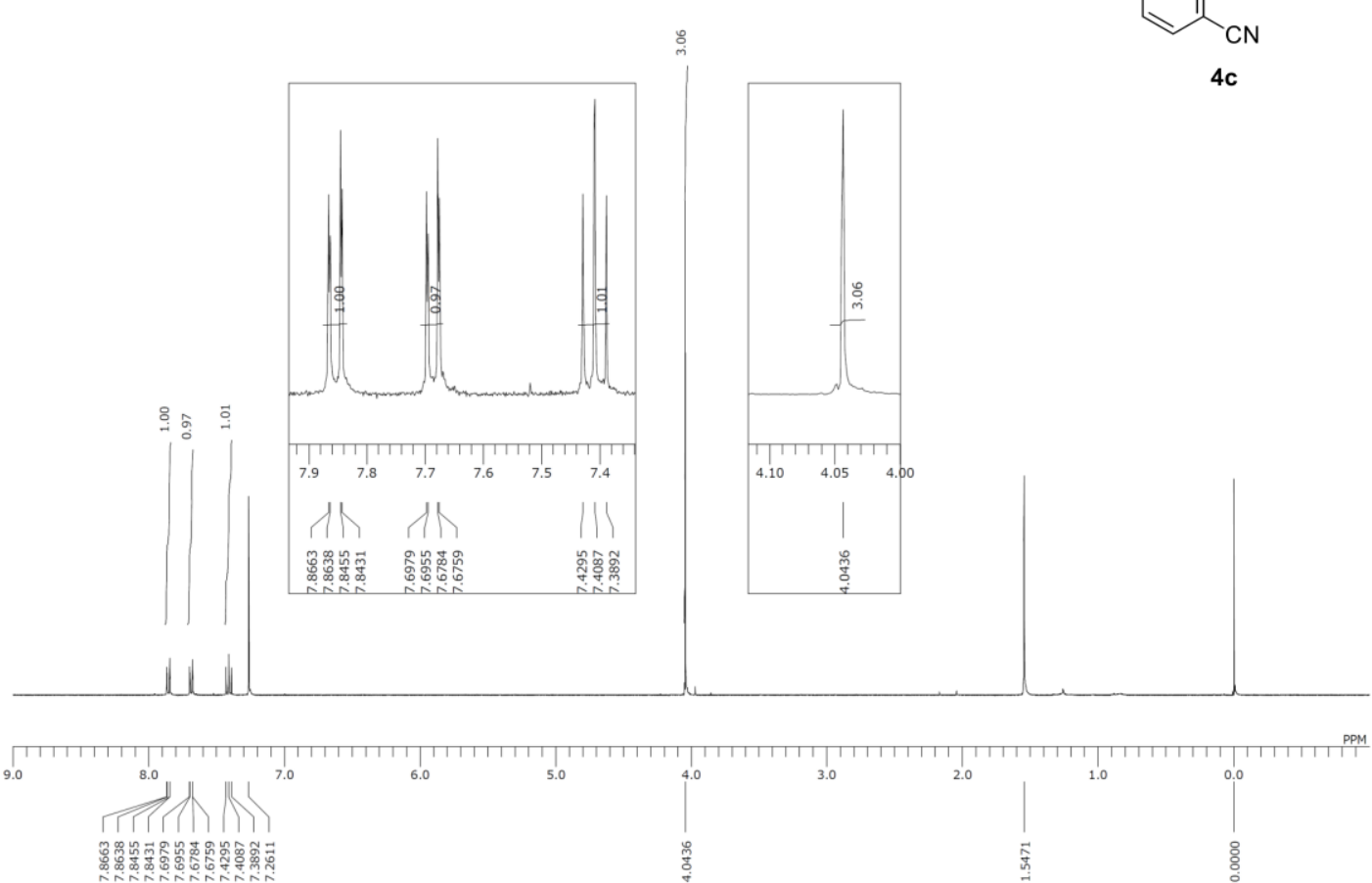
${ }^{13} \mathrm{C}$ NMR spectra of $\mathbf{4 c}\left(\mathrm{CDCl}_{3}, 100 \mathrm{MHz}\right)$<smiles>COC(=O)c1c(Br)cccc1C#N</smiles>

4c

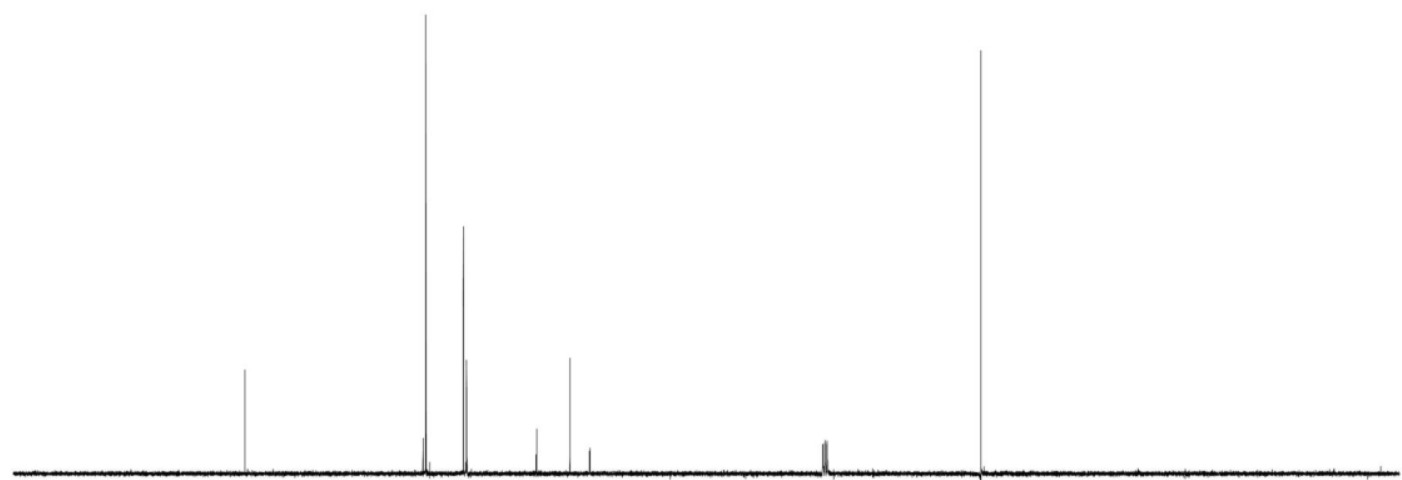

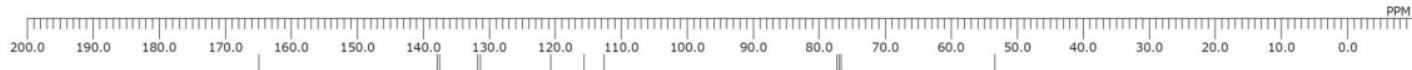

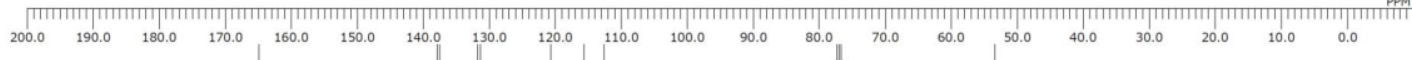

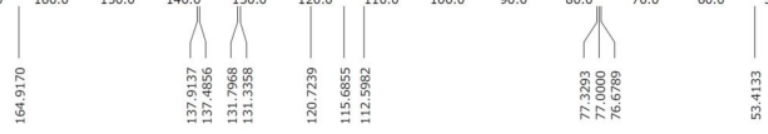

${ }^{1} \mathrm{H}$ NMR spectra of $\mathbf{4 d}\left(\mathrm{CDCl}_{3}, 400 \mathrm{MHz}\right)$
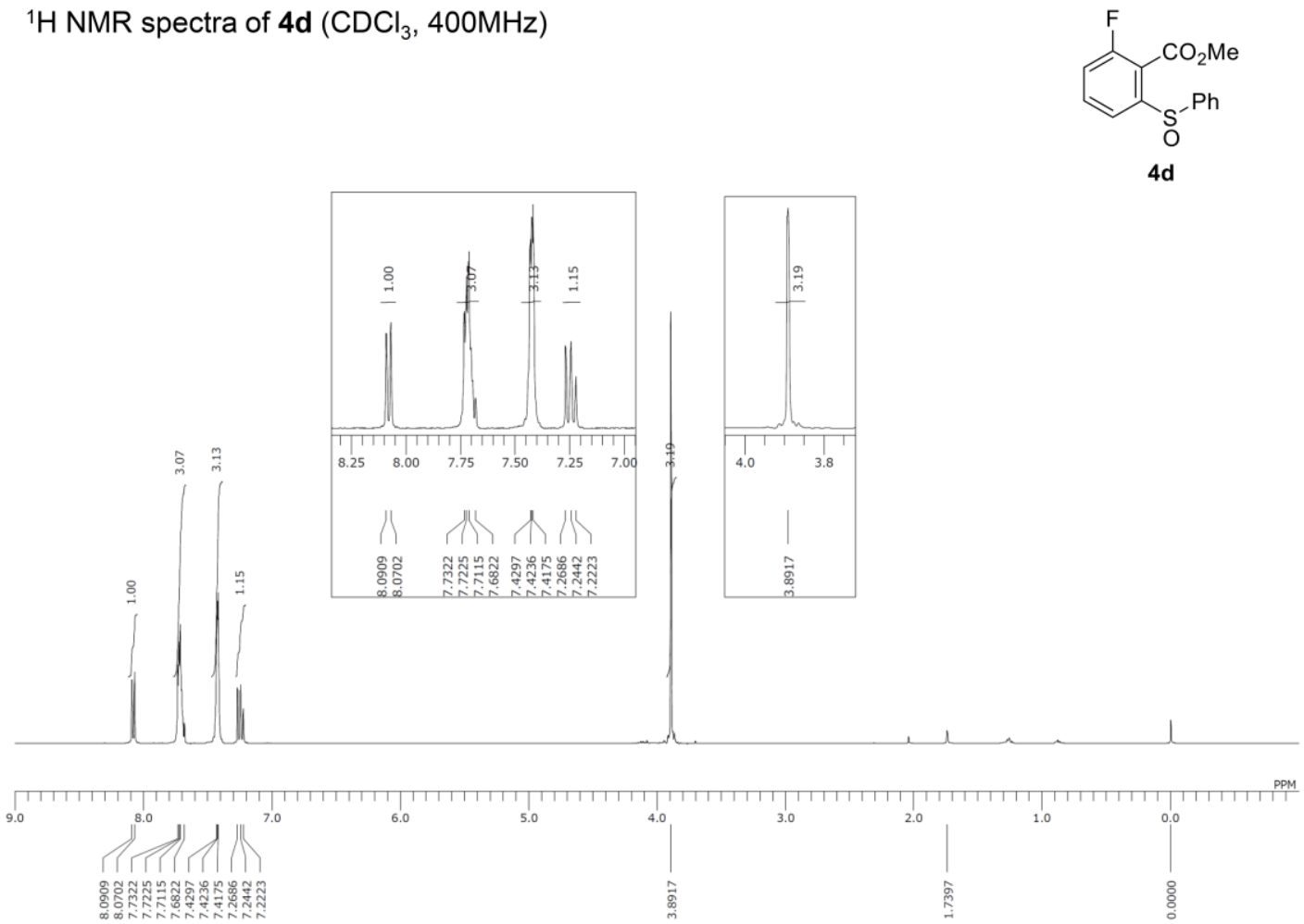
${ }^{13} \mathrm{C}$ NMR spectra of $\mathbf{4 d}\left(\mathrm{CDCl}_{3}, 150 \mathrm{MHz}\right)$
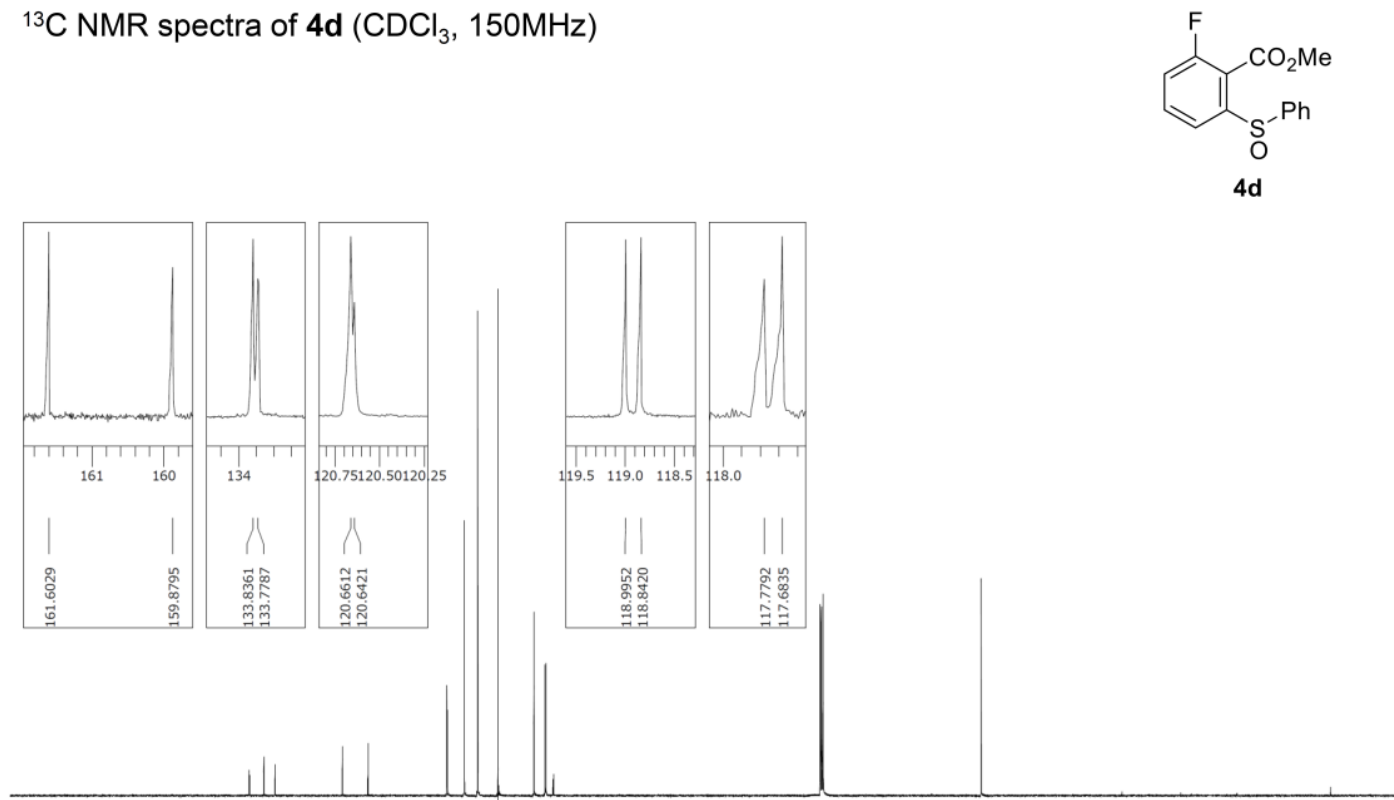

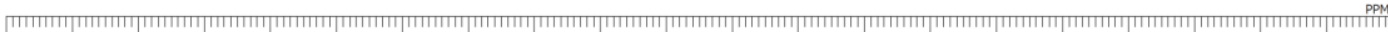

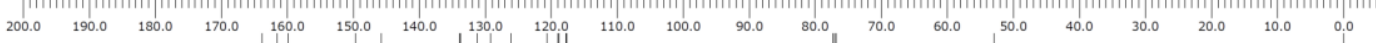
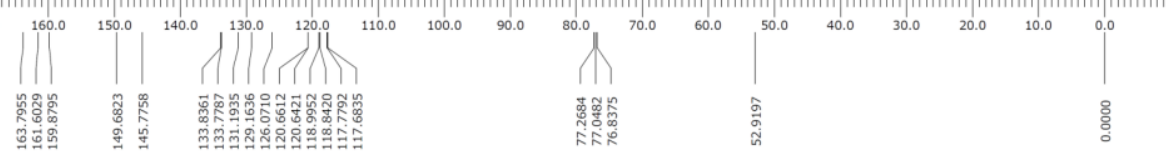

${ }^{19} \mathrm{~F}$ NMR spectra of $\mathbf{4 d}\left(\mathrm{CDCl}_{3}, 565 \mathrm{MHz}\right)$

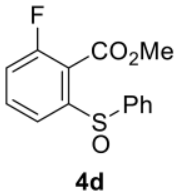

4d

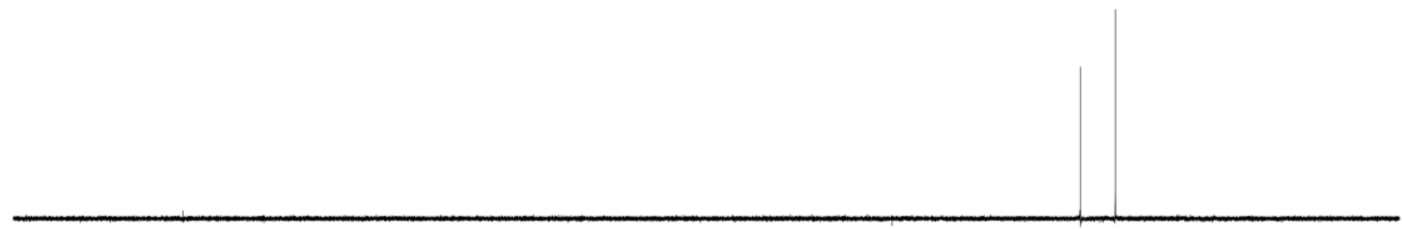

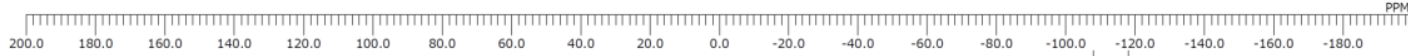


${ }^{1} \mathrm{H}$ NMR spectra of $4 \mathrm{e}\left(\mathrm{CDCl}_{3}, 600 \mathrm{MHz}\right)$

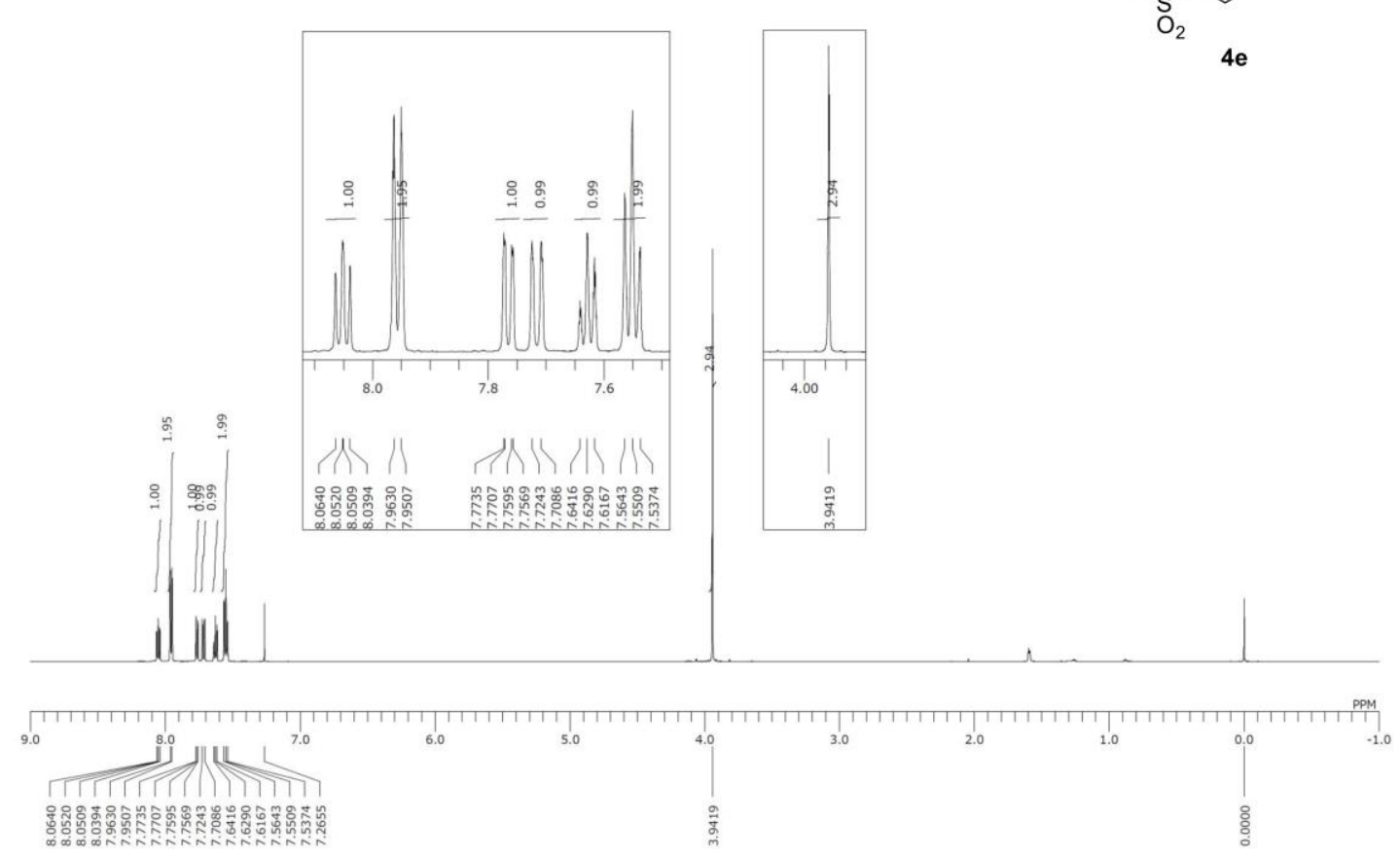

${ }^{13} \mathrm{C}$ NMR spectra of $4 \mathrm{e}\left(\mathrm{CDCl}_{3}, 150 \mathrm{MHz}\right)$

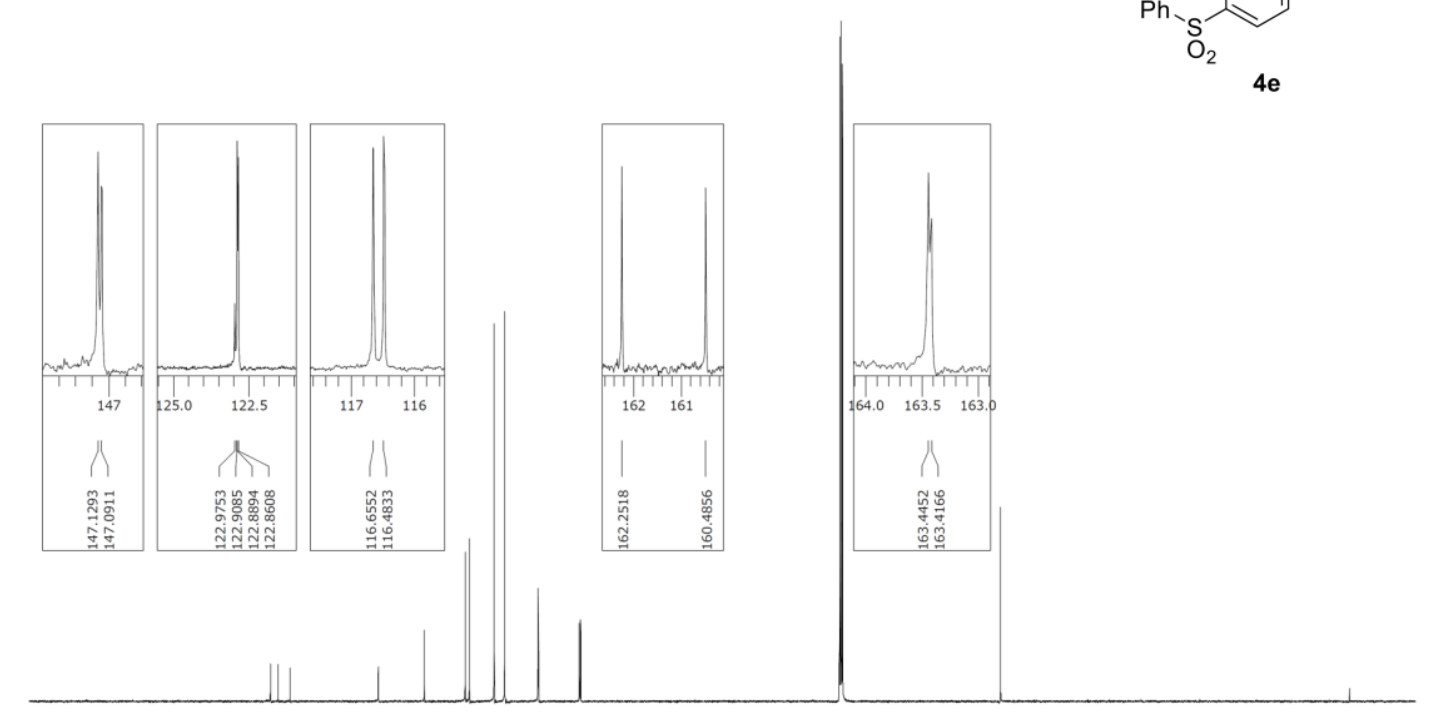

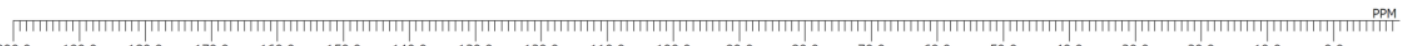

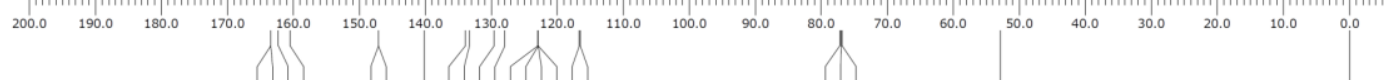

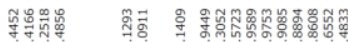

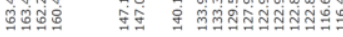

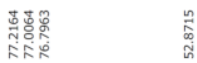


${ }^{19} \mathrm{~F}$ NMR spectra of $4 \mathrm{e}\left(\mathrm{CDCl}_{3}, 565 \mathrm{MHz}\right)$<smiles>COC(=O)c1ccc(OSc2ccccc2)cc1F</smiles>

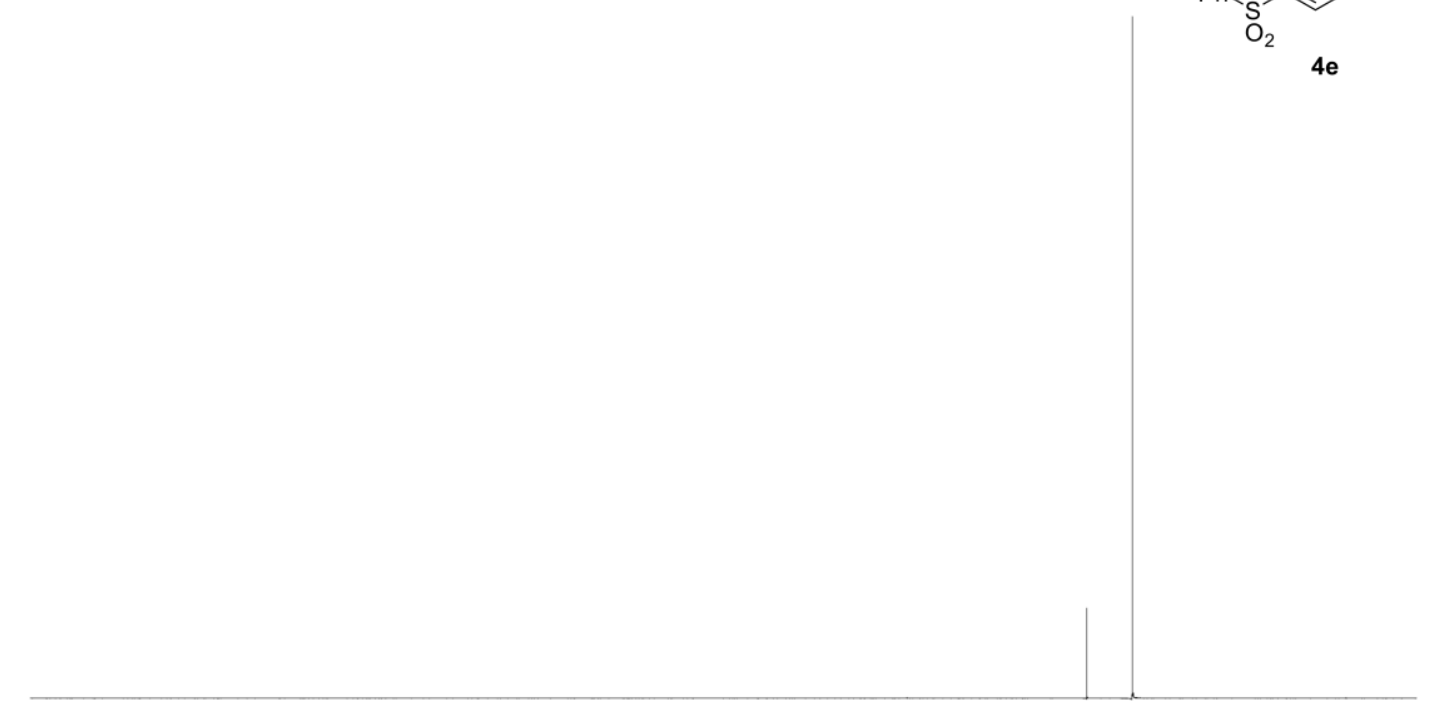

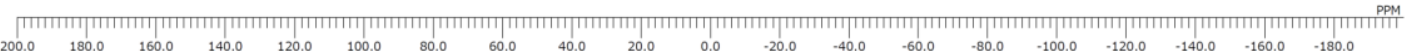

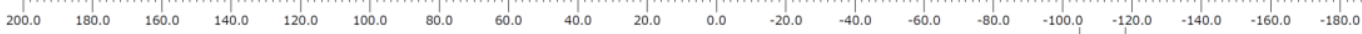
!

${ }^{1} \mathrm{H}$ NMR spectra of $\mathbf{4 f}\left(\mathrm{CDCl}_{3}, 600 \mathrm{MHz}\right)$<smiles>COC(=O)c1ccc(S(=O)N(C)c2ccccc2)cc1F</smiles>

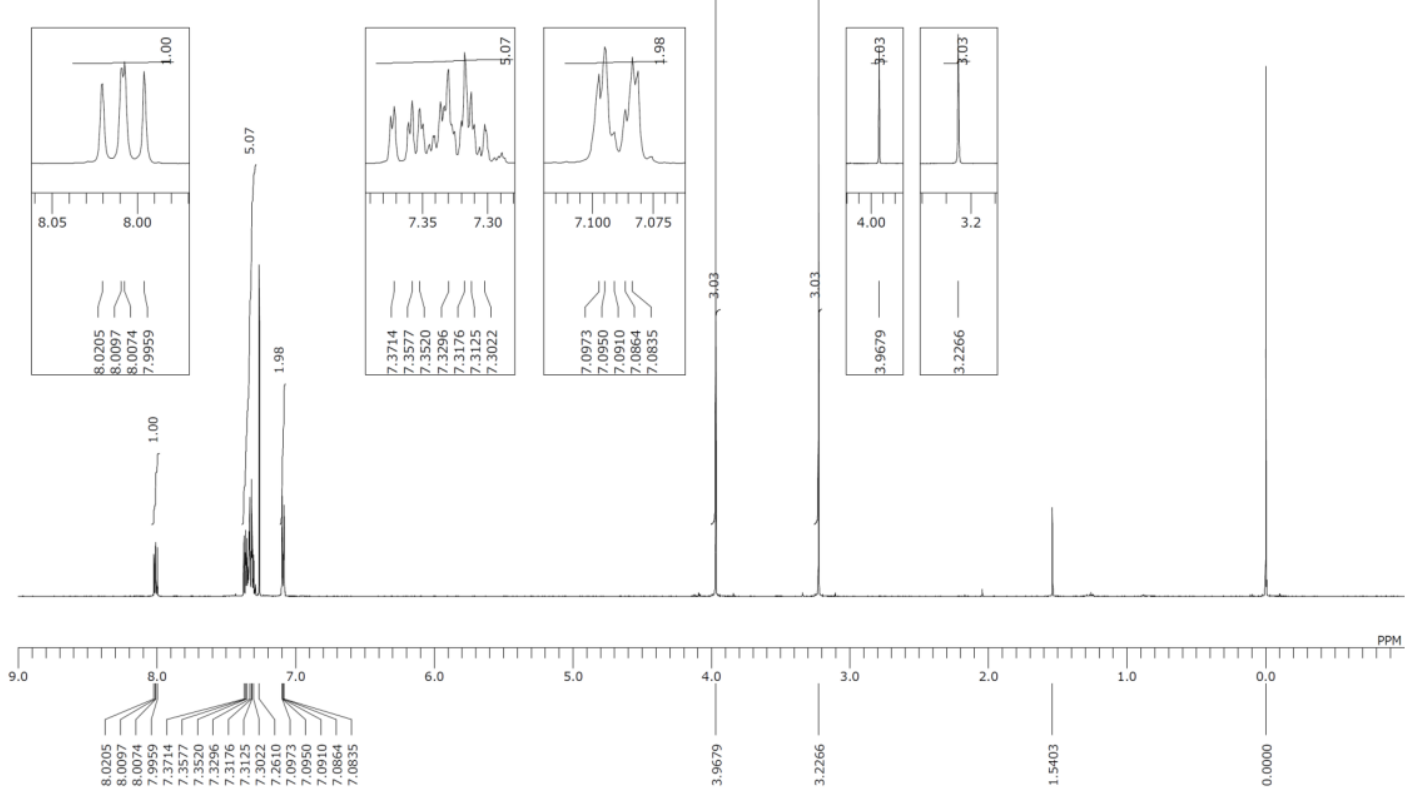


${ }^{13} \mathrm{C}$ NMR spectra of $\mathbf{4 f}\left(\mathrm{CDCl}_{3}, 150 \mathrm{MHz}\right)$

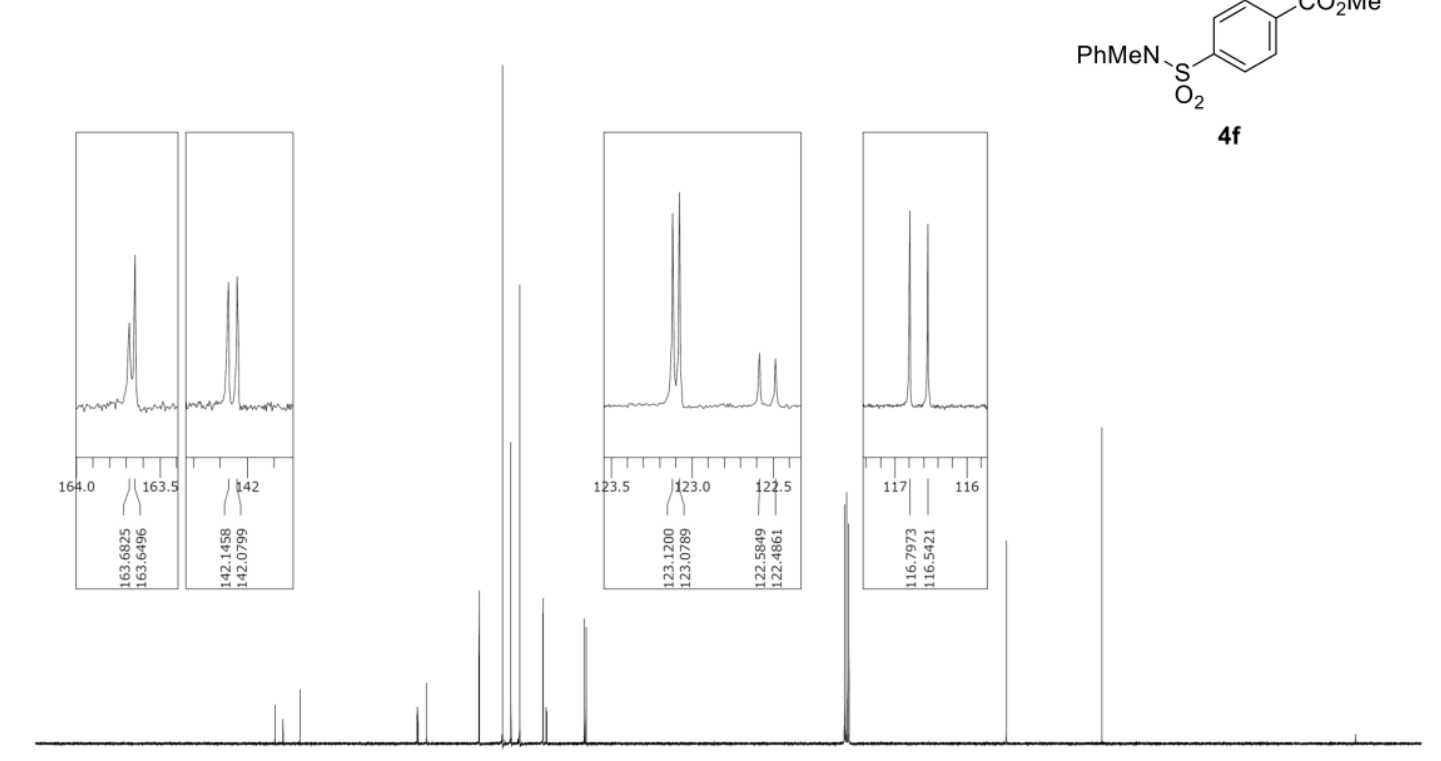

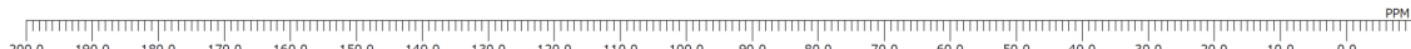

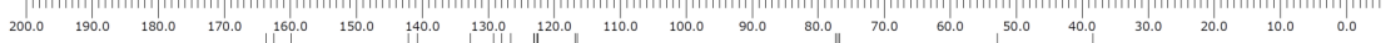

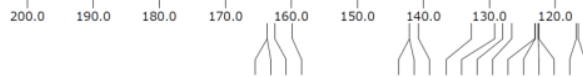

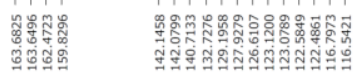

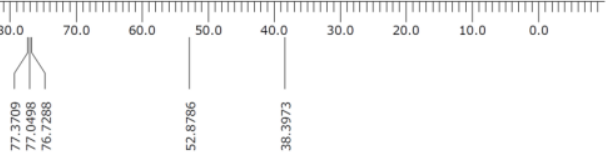

${ }^{19} \mathrm{~F}$ NMR spectra of $\mathbf{4 f}\left(\mathrm{CDCl}_{3}, 565 \mathrm{MHz}\right)$
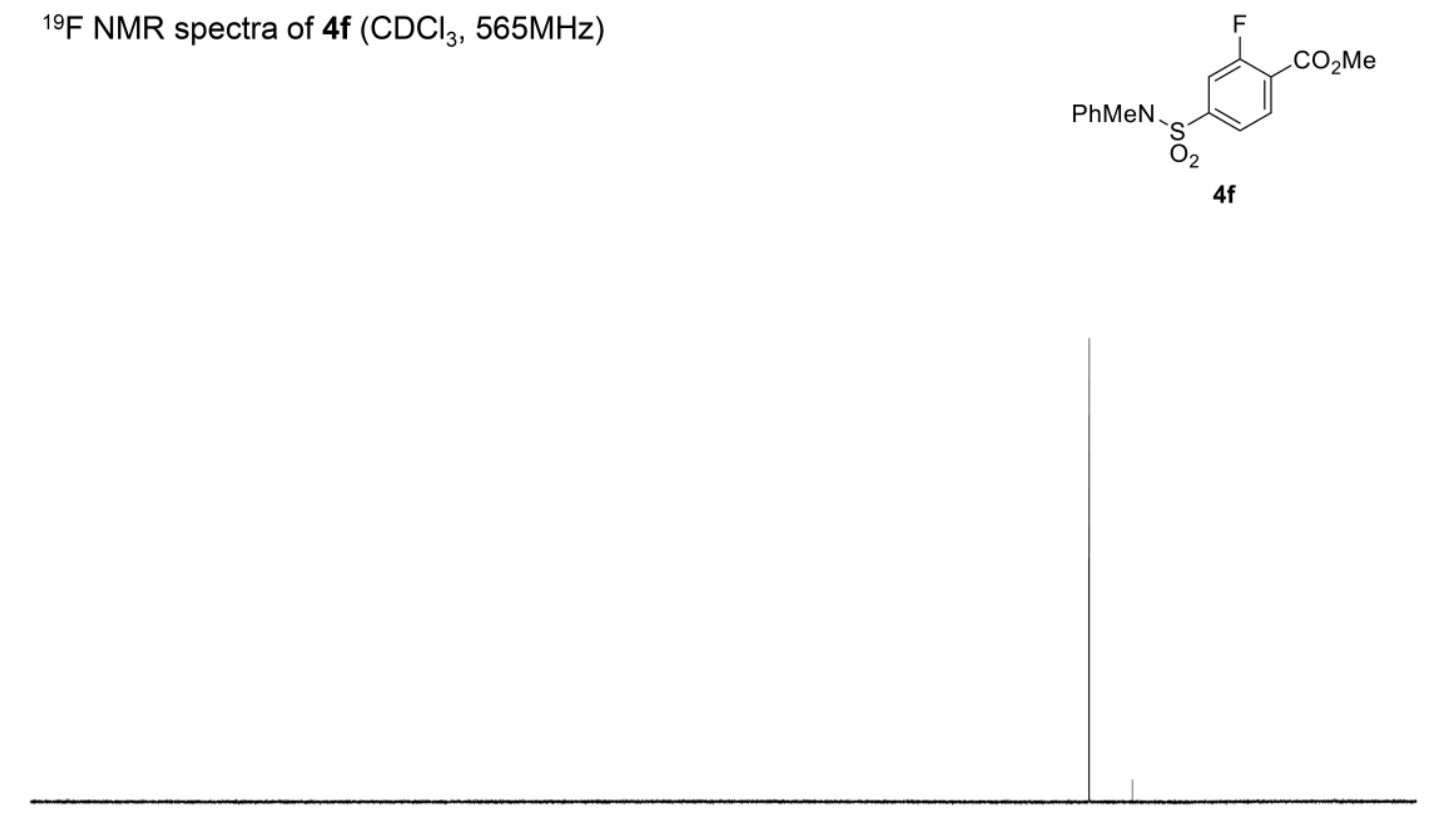

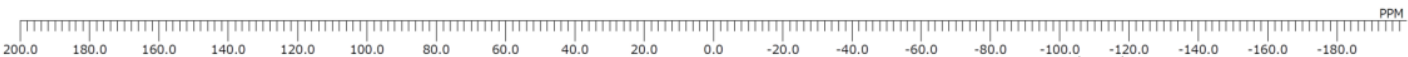

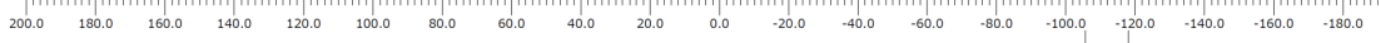


NOESY NMR spectra of $\mathbf{4 f}$ (DMSO-d6, 600MHz)

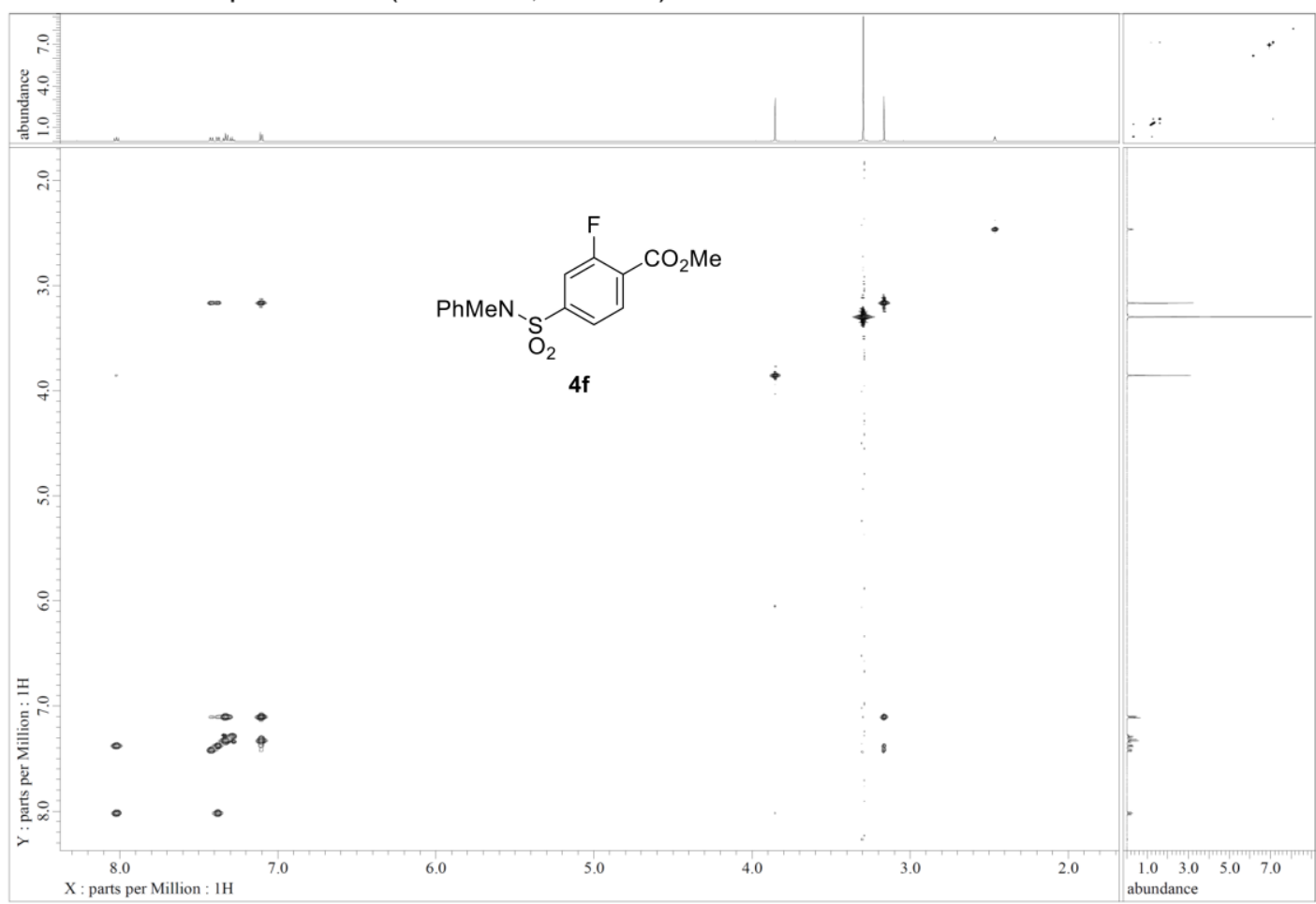

COSY NMR spectra of $\mathbf{4 f}(\mathrm{DMSO}-\mathrm{d} 6,600 \mathrm{MHz})$

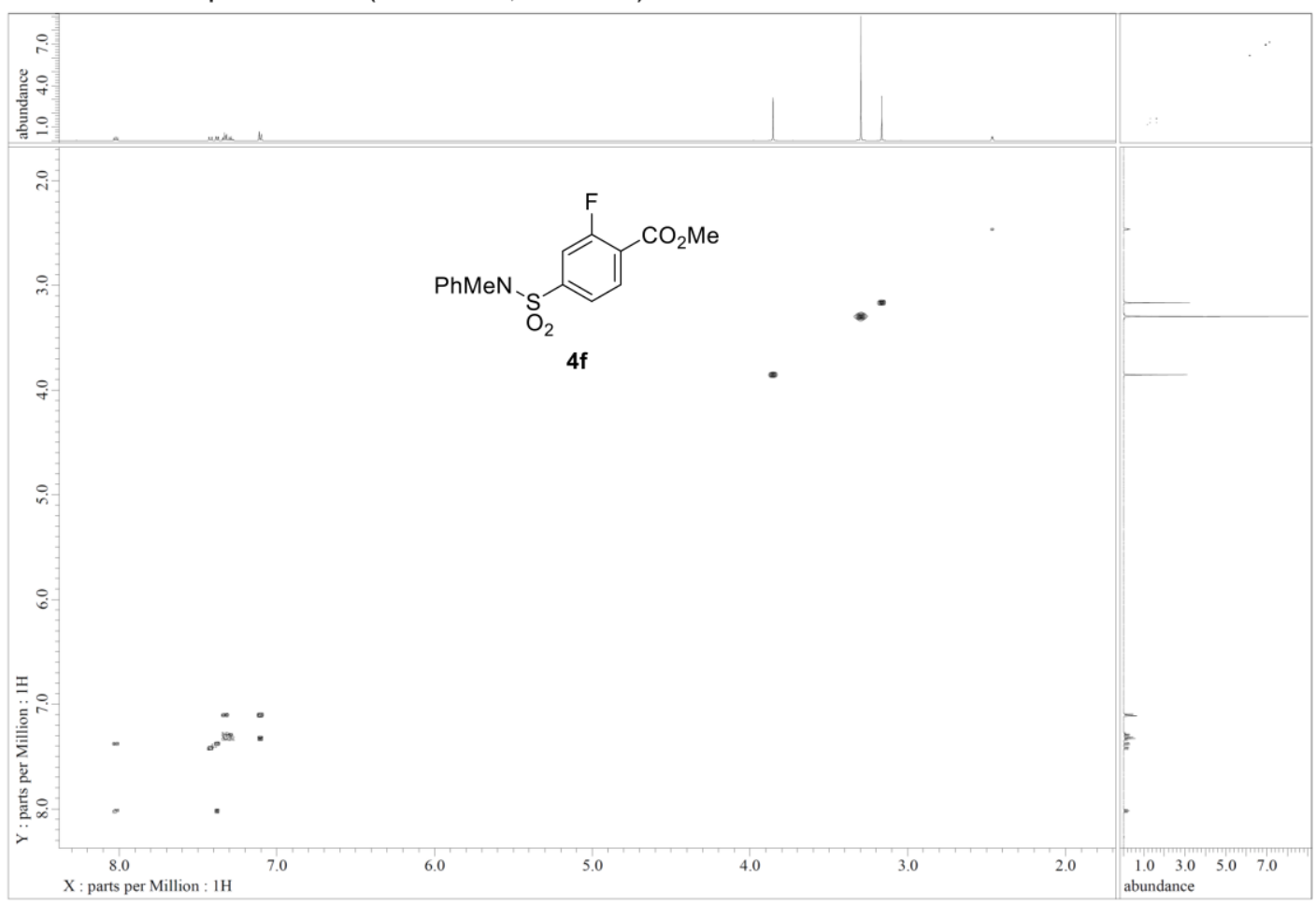


HMQC NMR spectra of $\mathbf{4 f}$ (DMSO-d6, 600MHz for $1 \mathrm{H}$ and $150 \mathrm{MHz}$ for ${ }^{13} \mathrm{C}$ )

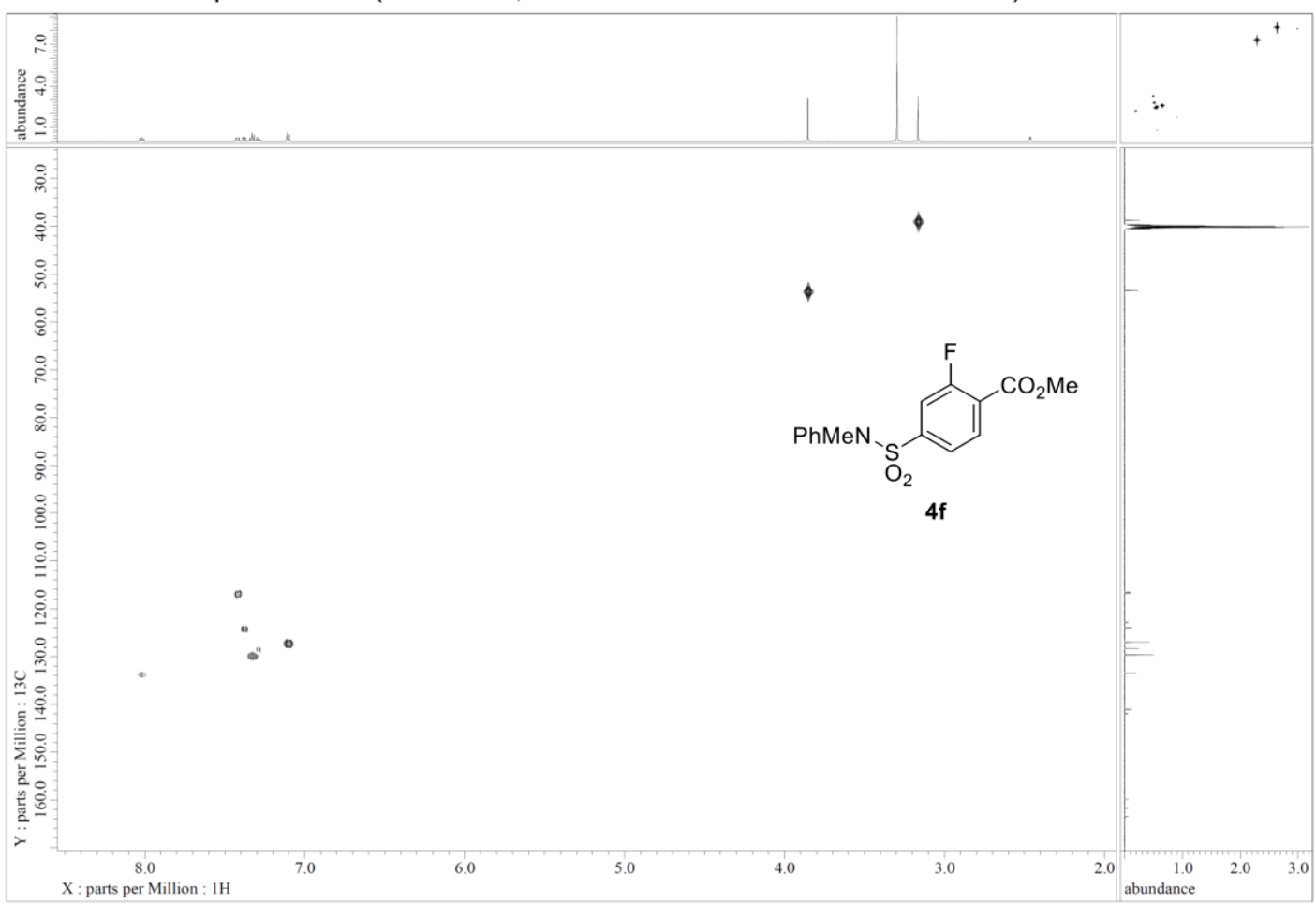

HMBC NMR spectra of $\mathbf{4 f}$ (DMSO-d6, $600 \mathrm{MHz}$ for $1 \mathrm{H}$ and $150 \mathrm{MHz}$ for ${ }^{13} \mathrm{C}$ )

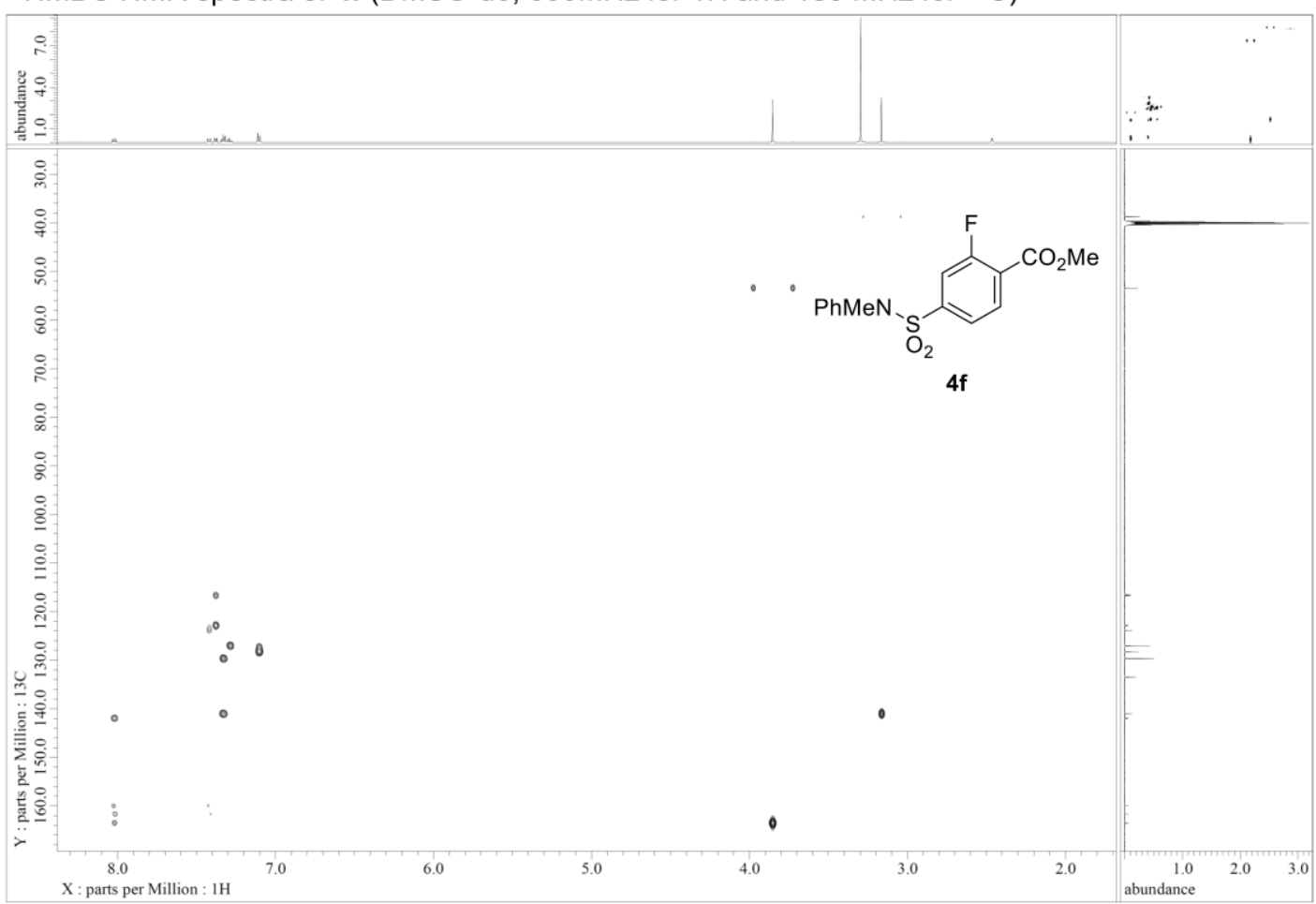


${ }^{1} \mathrm{H}-{ }^{19} \mathrm{~F}$ HETCOR NMR spectra of $\mathbf{4 f}$ (DMSO-d6, 400MHz for $1 \mathrm{H}$ and $376 \mathrm{MHz}$ for ${ }^{19} \mathrm{~F}$ )
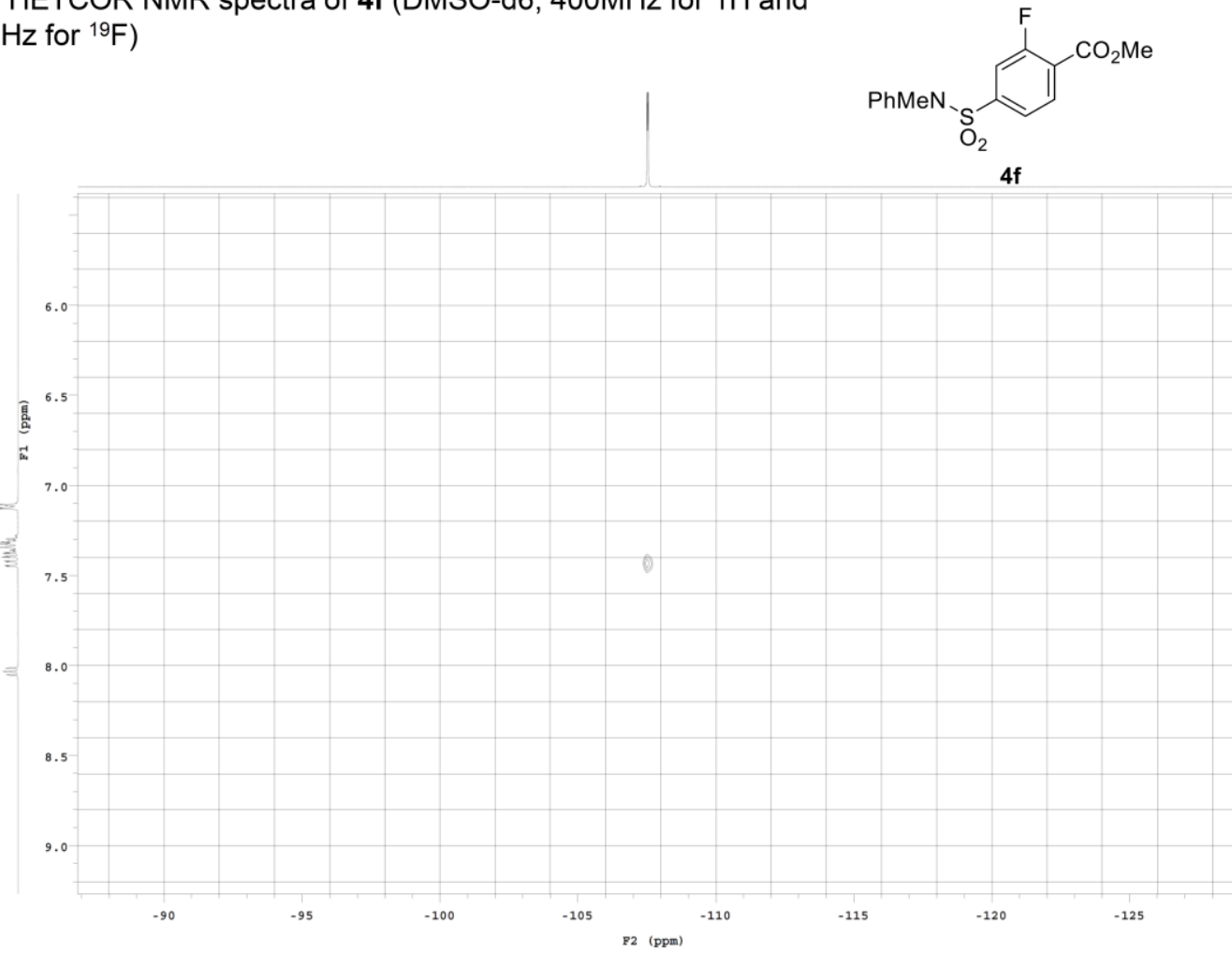

${ }^{1} \mathrm{H}$ NMR spectra of $\mathbf{4 g}\left(\mathrm{CDCl}_{3}, 600 \mathrm{MHz}\right)$
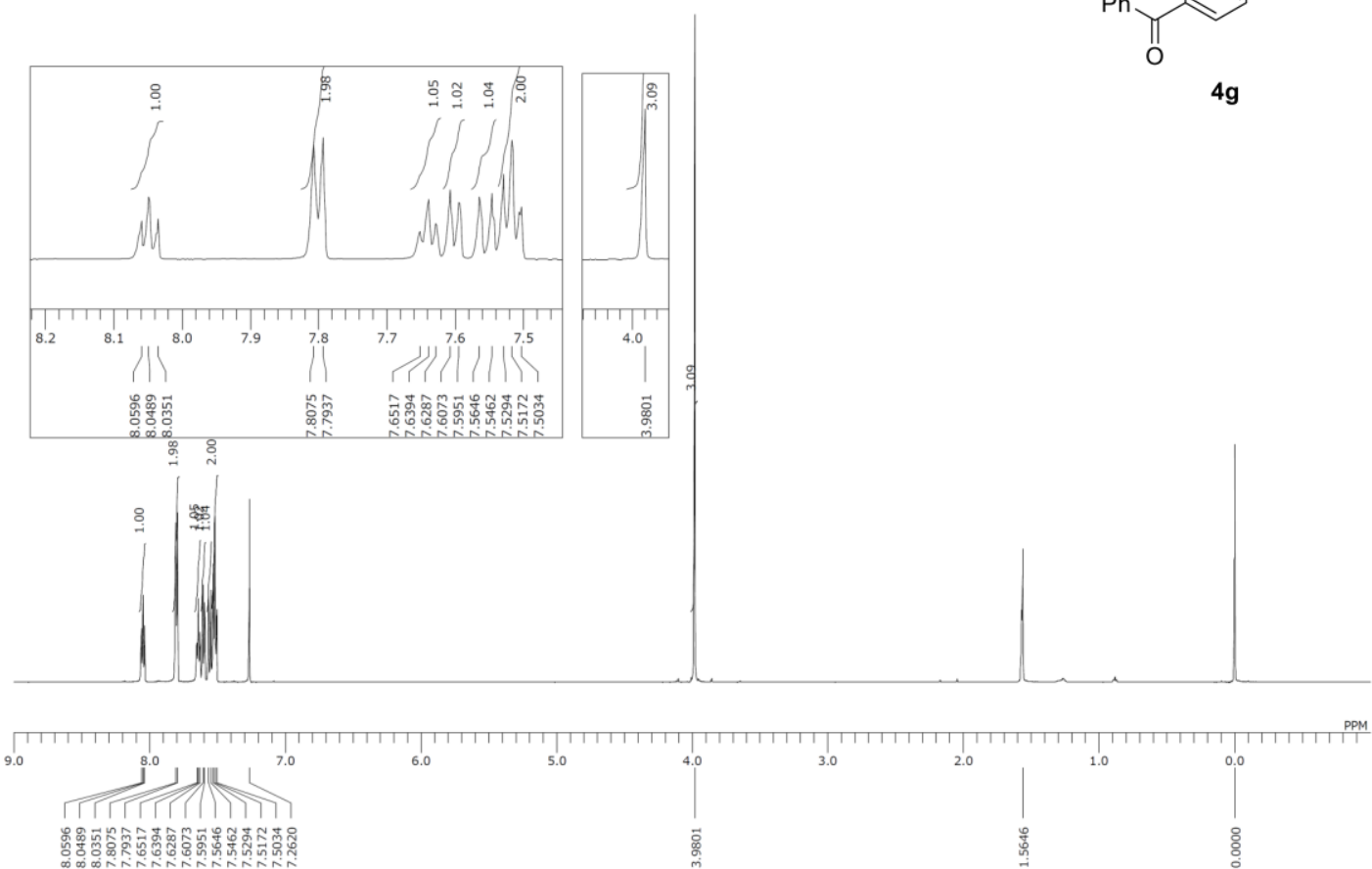
${ }^{13} \mathrm{C}$ NMR spectra of $\mathbf{4 g}\left(\mathrm{CDCl}_{3}, 150 \mathrm{MHz}\right)$

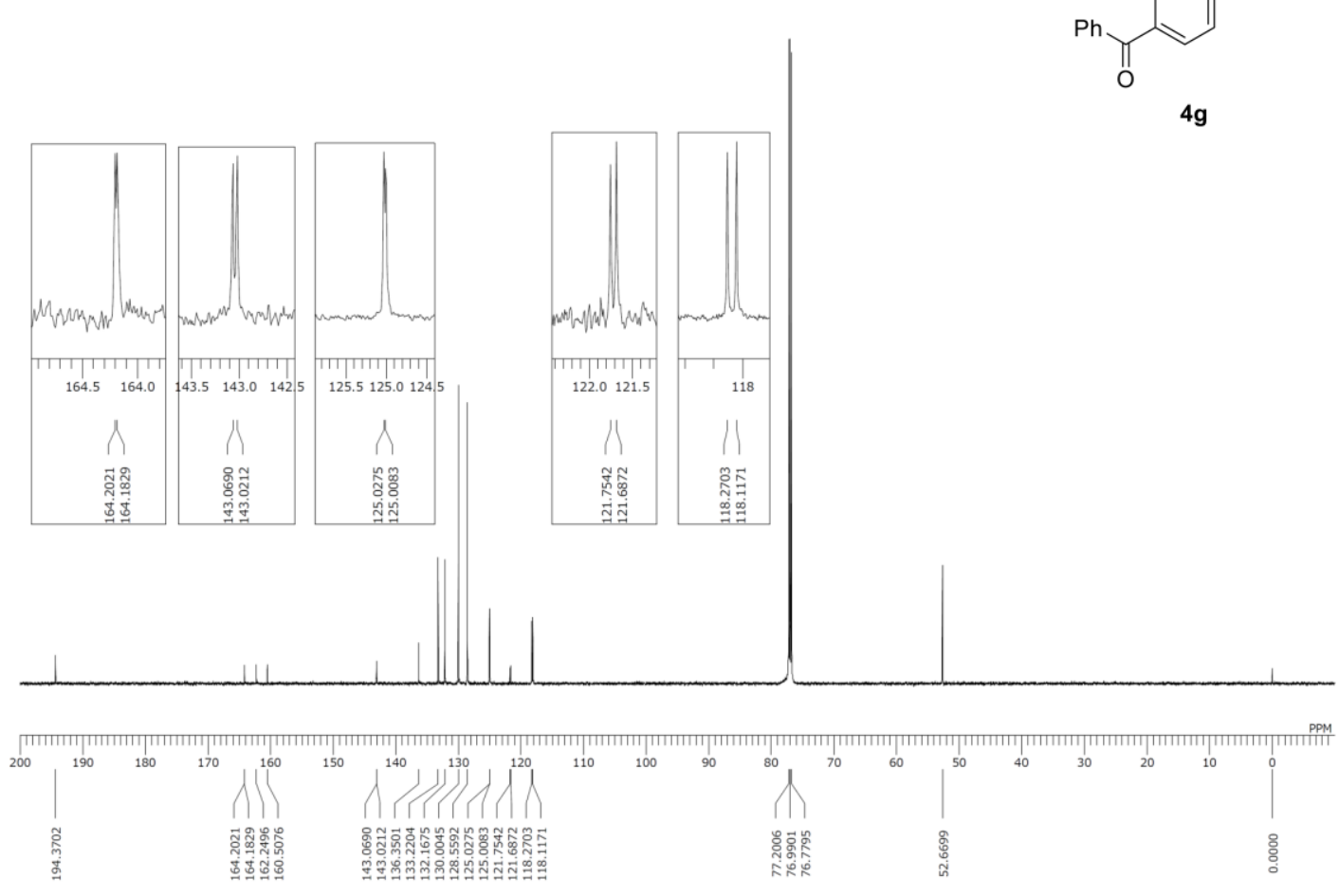

${ }^{19} \mathrm{~F}$ NMR spectra of $\mathbf{4 g}\left(\mathrm{CDCl}_{3}, 565 \mathrm{MHz}\right)$

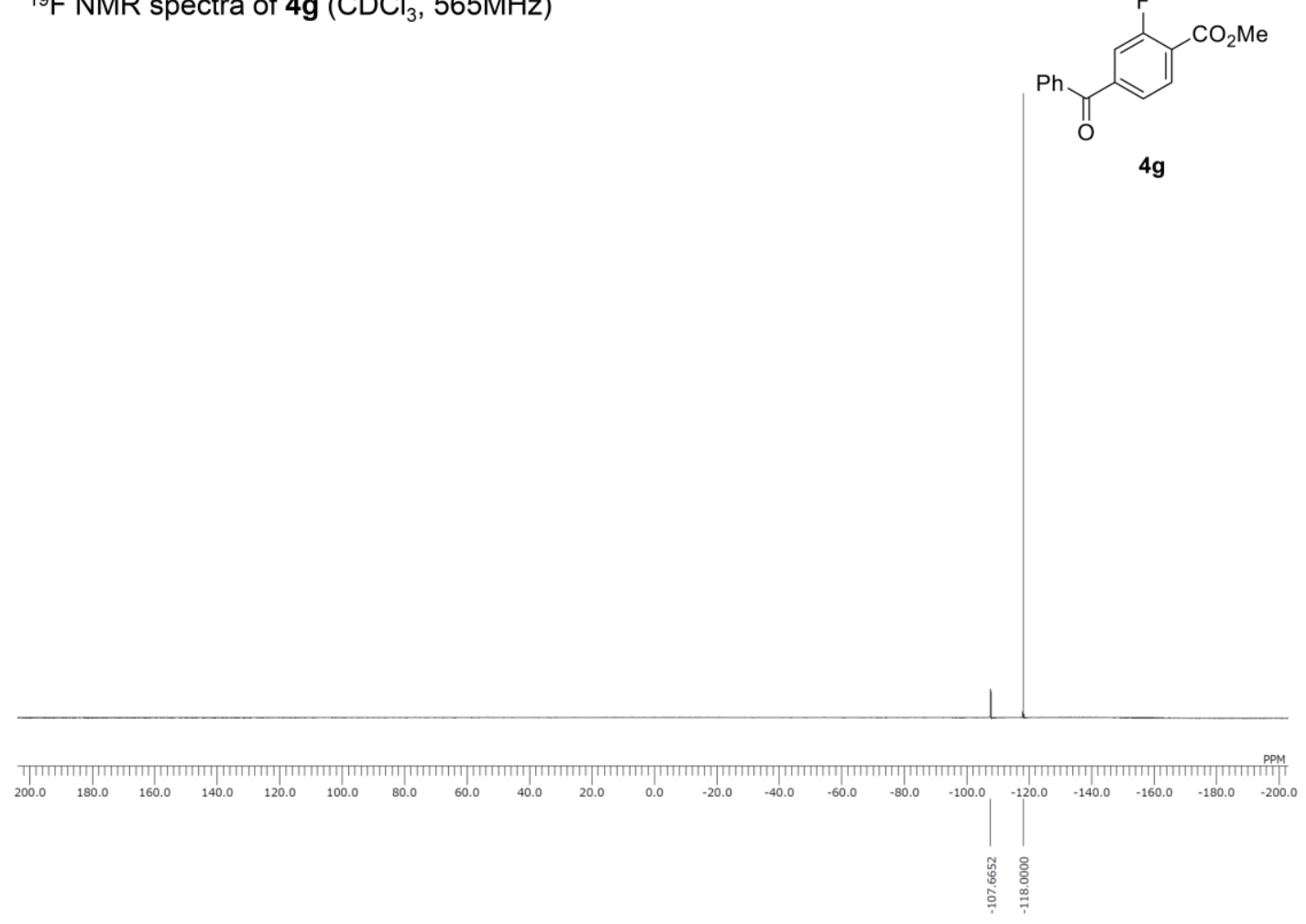


${ }^{1} \mathrm{H}$ NMR spectra of $\mathbf{4 h}(\mathrm{DMSO}-\mathrm{d} 6,600 \mathrm{MHz})$

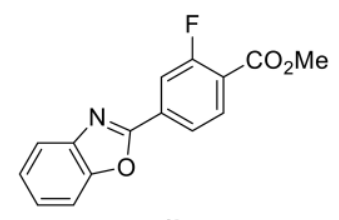

$4 \mathrm{~h}$
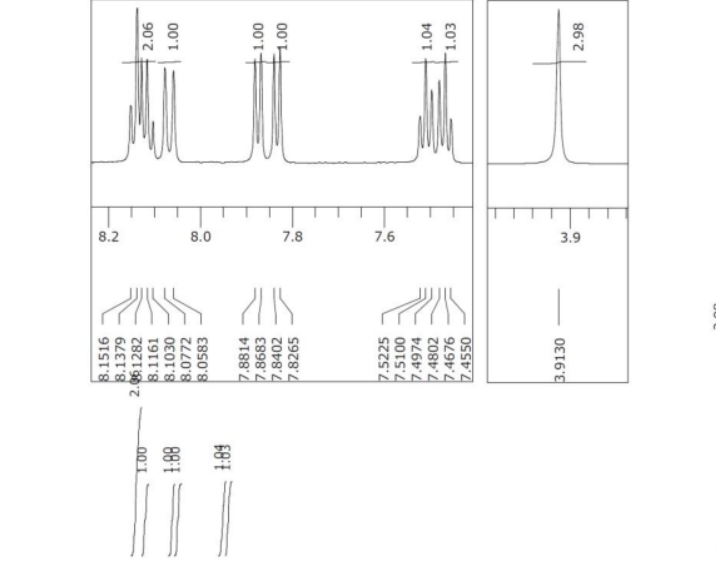
11/

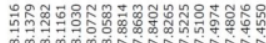

${ }^{13} \mathrm{C}$ NMR spectra of $4 \mathrm{~h}$ (DMSO-d6, 150MHz)

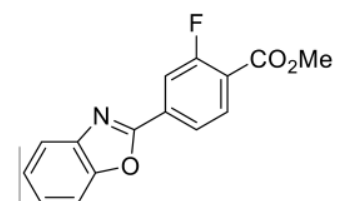

$4 h$
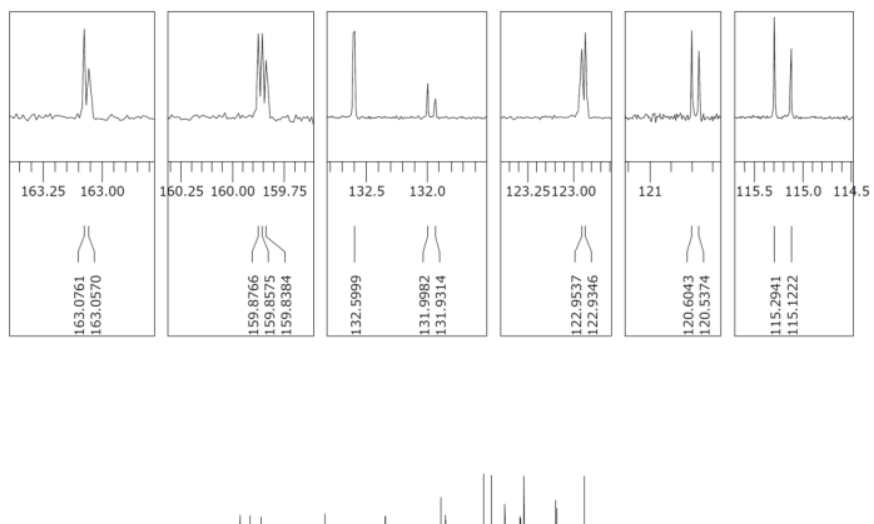

(

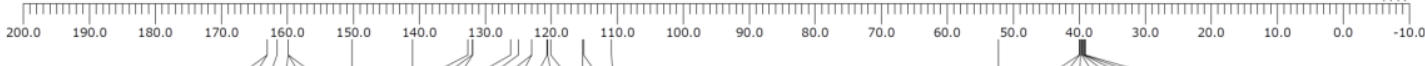
$11111)$

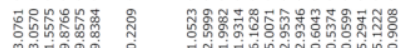

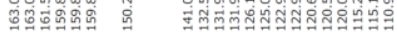

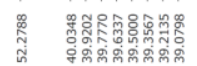


${ }^{19} \mathrm{~F}$ NMR spectra of $\mathbf{4 h}(\mathrm{DMSO}-\mathrm{d} 6,565 \mathrm{MHz})$

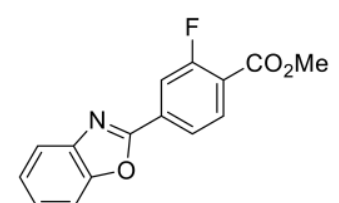

4h

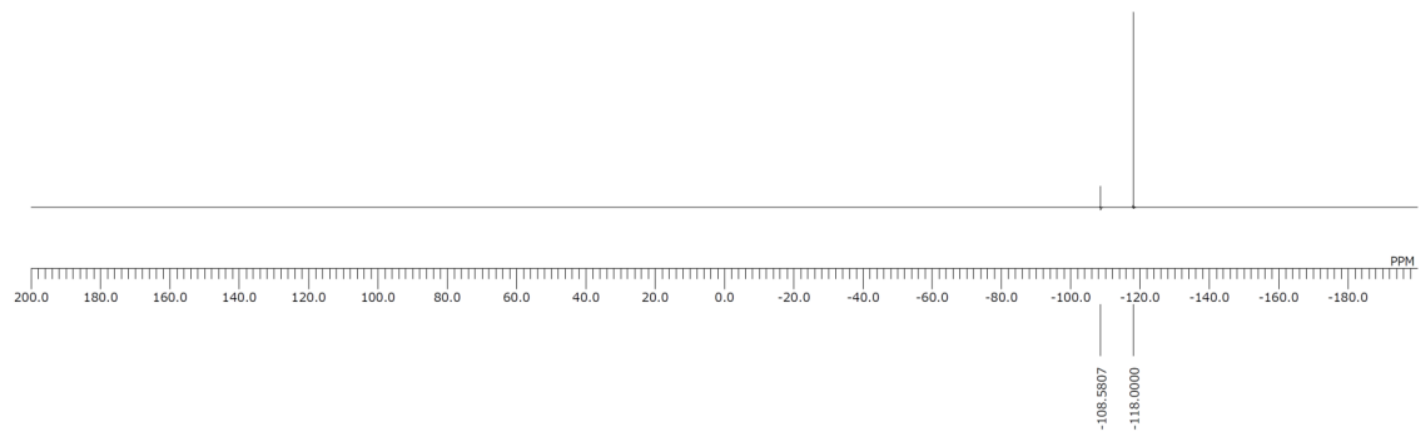

NOESY NMR spectra of $\mathbf{4 h}\left(\mathrm{CDCl}_{3}, 600 \mathrm{MHz}\right)$

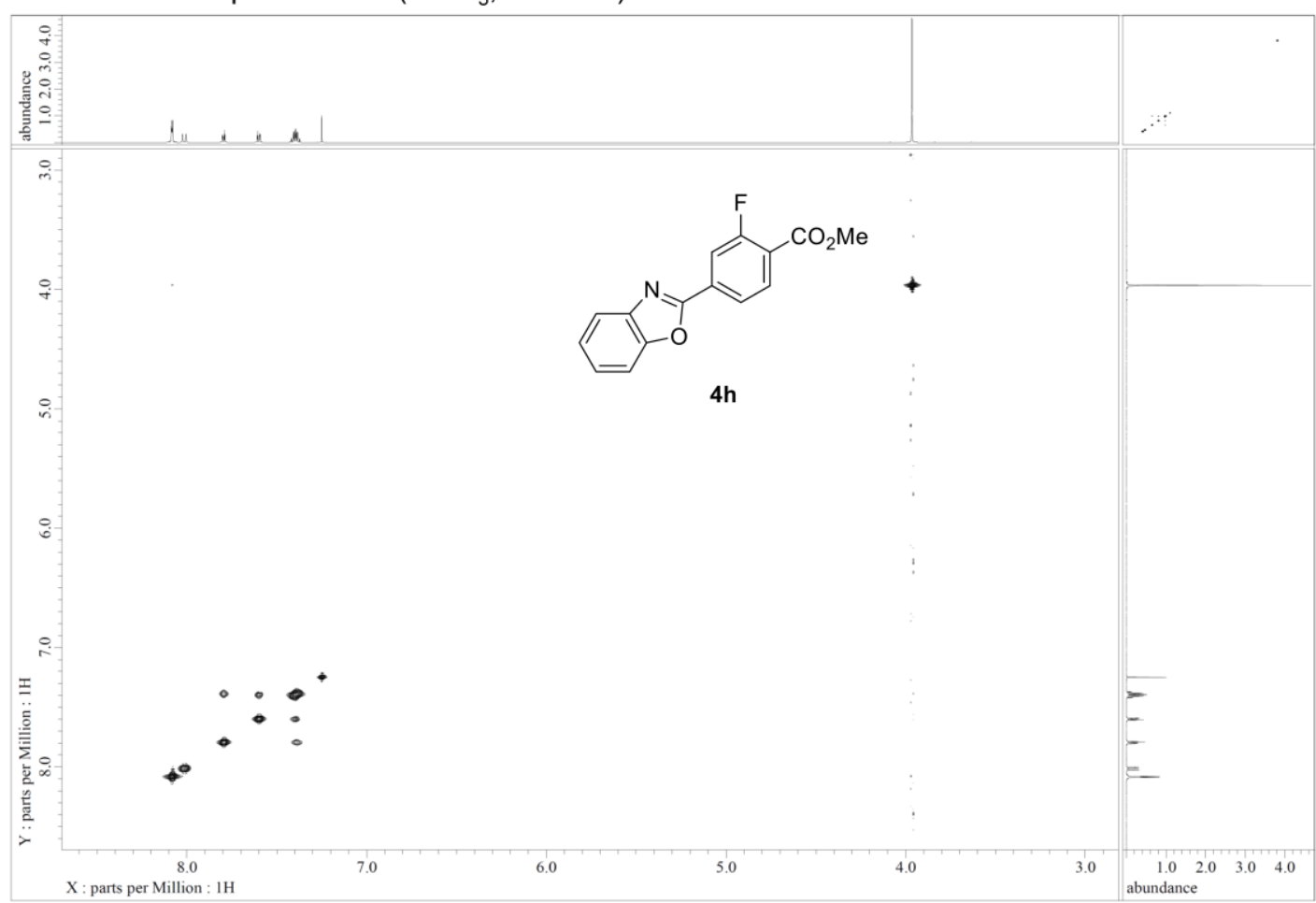


COSY NMR spectra of $\mathbf{4 h}\left(\mathrm{CDCl}_{3}, 600 \mathrm{MHz}\right)$

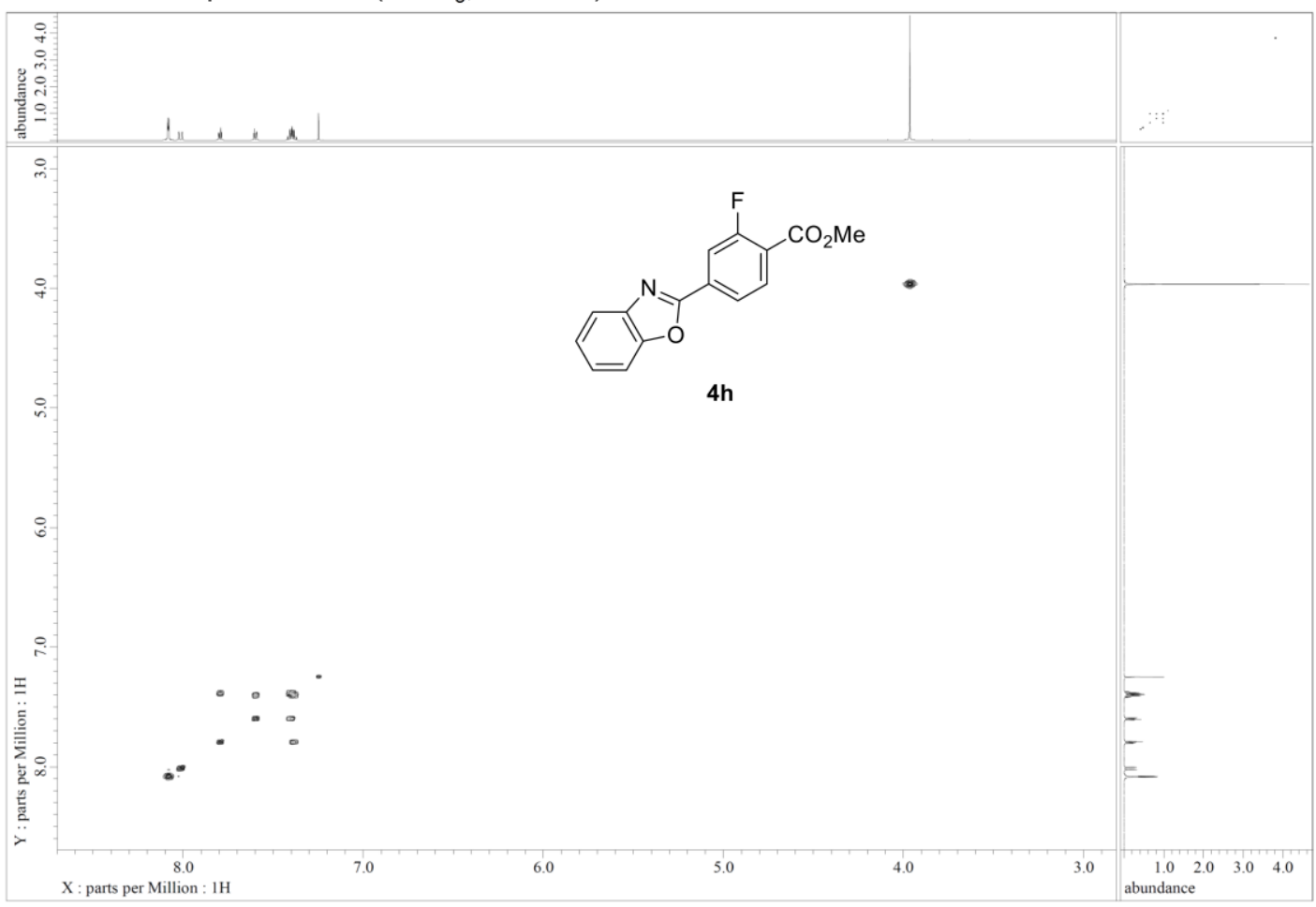

HMQC NMR spectra of $\mathbf{4 h}\left(\mathrm{CDCl}_{3}, 600 \mathrm{MHz}\right.$ for $1 \mathrm{H}$ and $150 \mathrm{MHz}$ for $\left.{ }^{13} \mathrm{C}\right)$

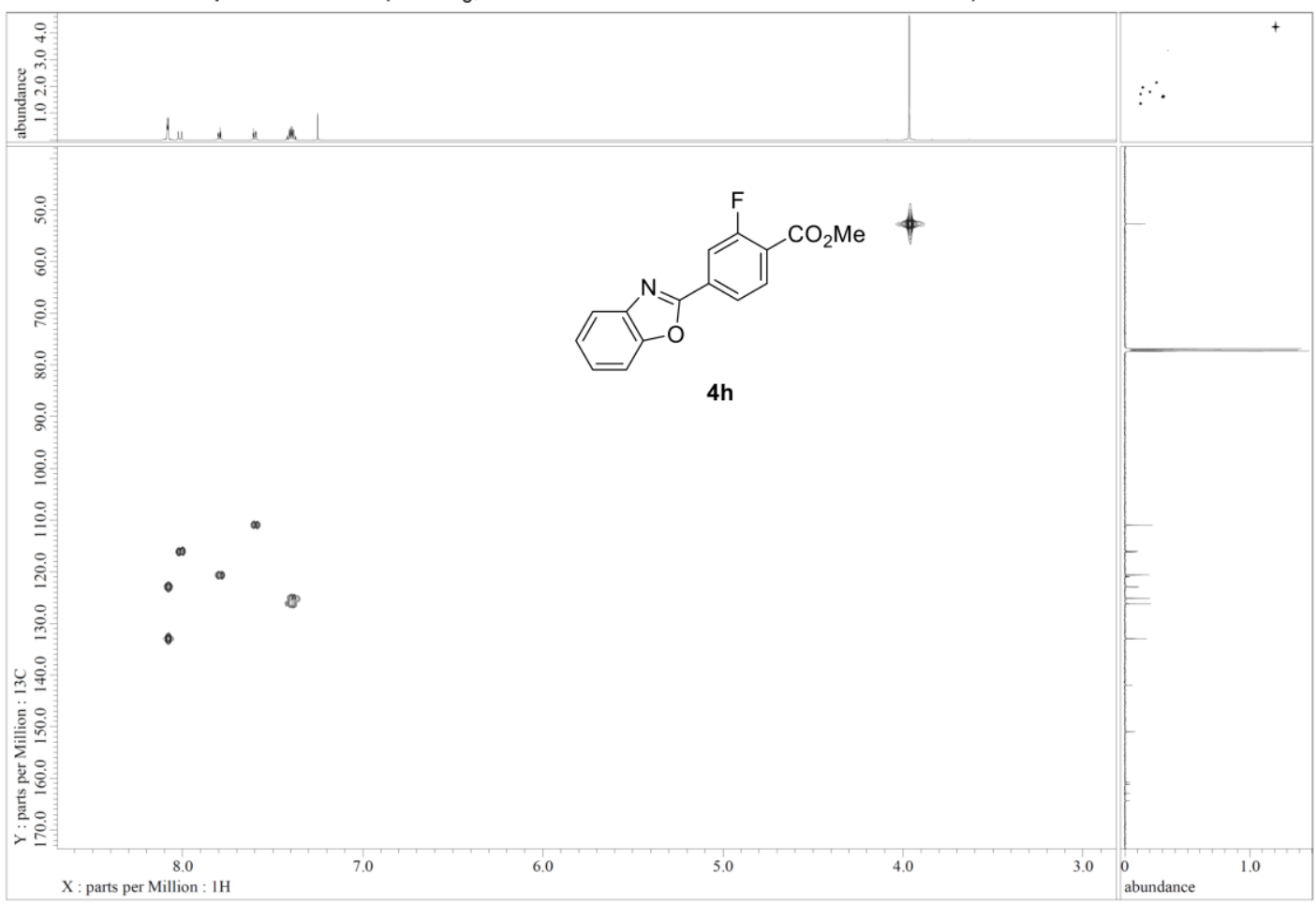


HMBC NMR spectra of $\mathbf{4 h}\left(\mathrm{CDCl}_{3}, 600 \mathrm{MHz}\right.$ for $1 \mathrm{H}$ and $150 \mathrm{MHz}$ for $\left.{ }^{13} \mathrm{C}\right)$

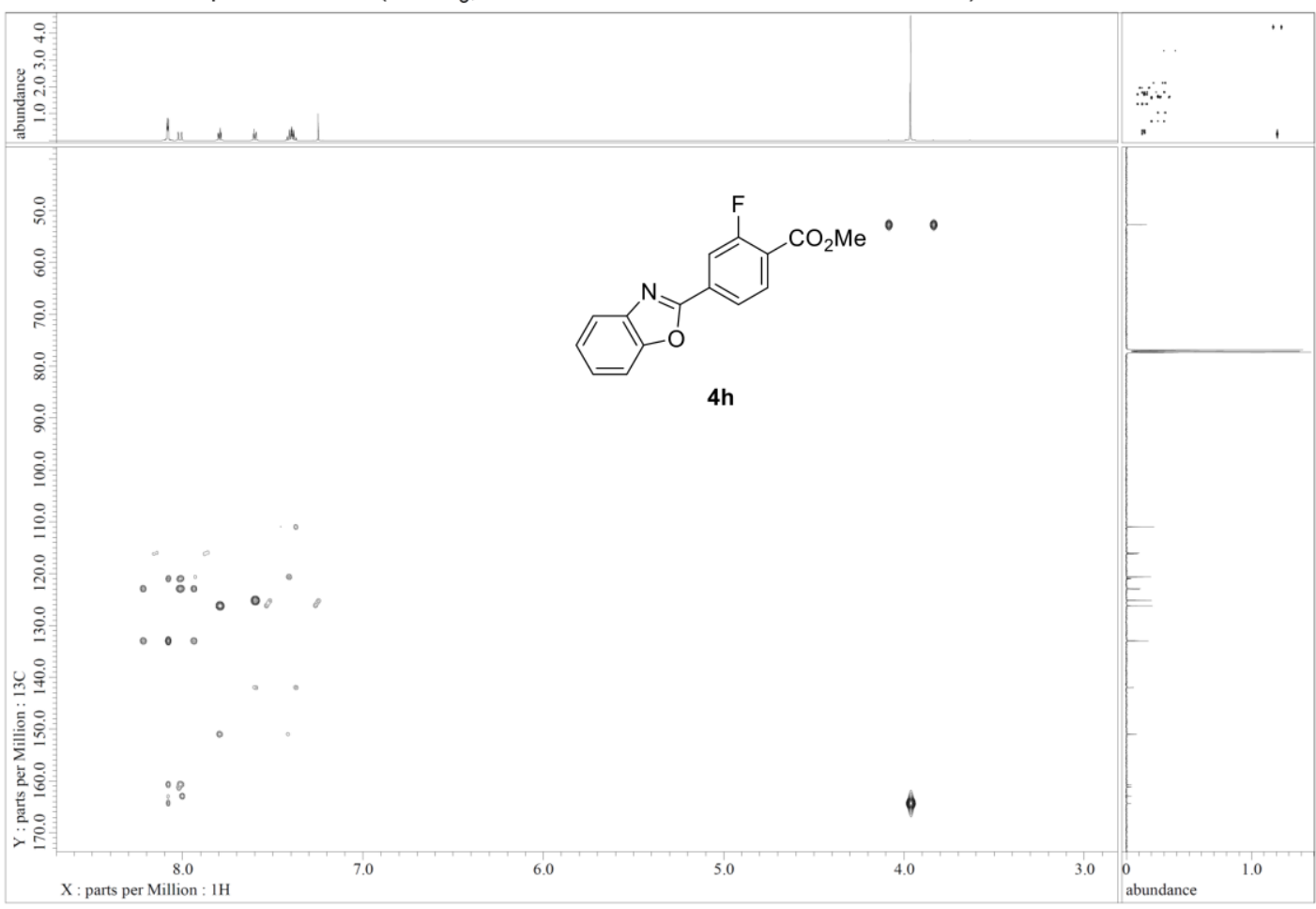

${ }^{1} \mathrm{H}-{ }^{19} \mathrm{~F}$ HETCOR NMR spectra of $\mathbf{4 h}\left(\mathrm{CDCl}_{3}, 400 \mathrm{MHz}\right.$ for $1 \mathrm{H}$ and 376 $\mathrm{MHz}$ for ${ }^{19} \mathrm{~F}$ )

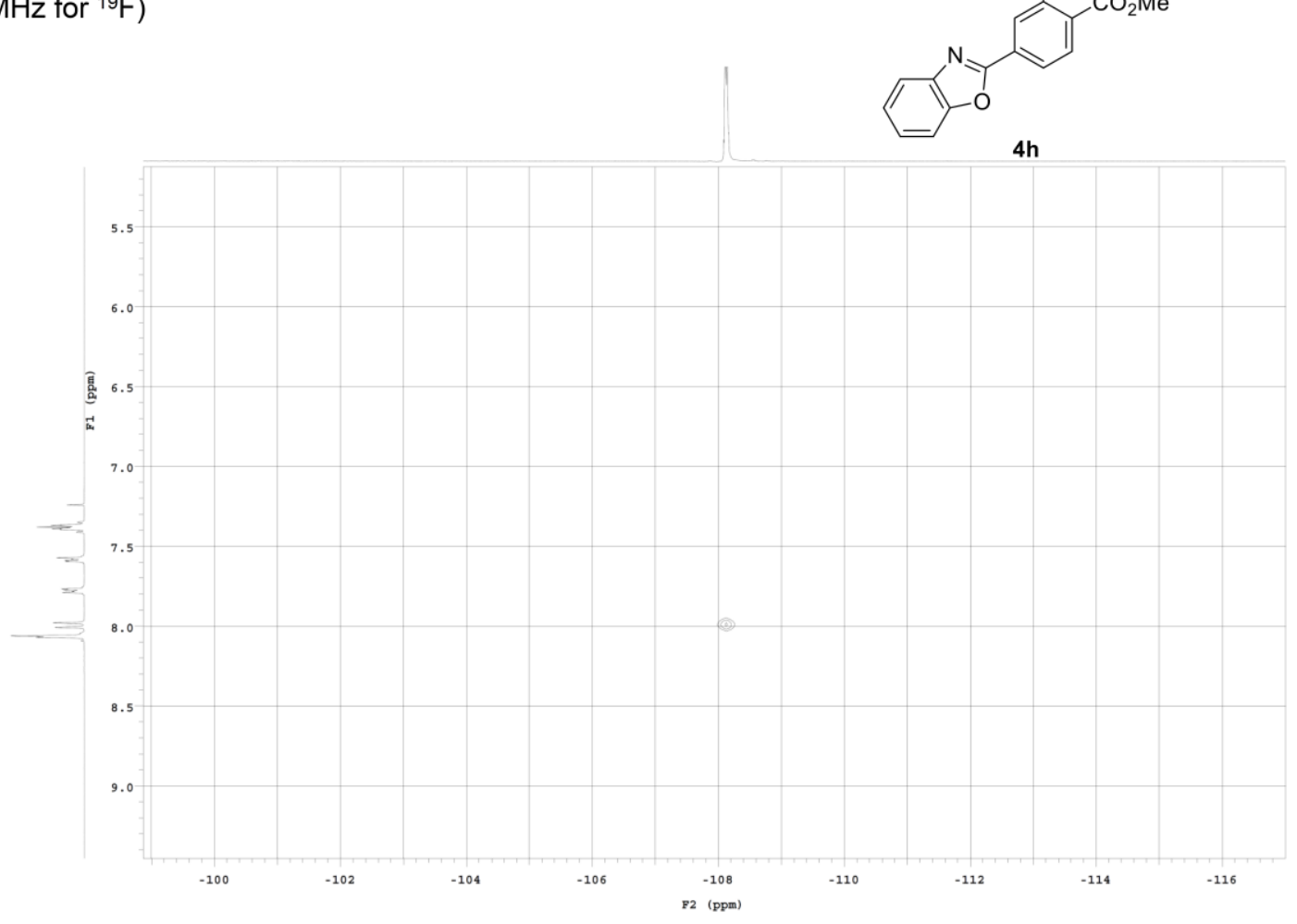


${ }^{1} \mathrm{H}$ NMR spectra of $\mathbf{4 i}\left(\mathrm{CDCl}_{3}, 600 \mathrm{MHz}\right)$

CN<smiles>COC(=O)c1c(C#N)cccc1C#N</smiles>

$4 \mathbf{i}$
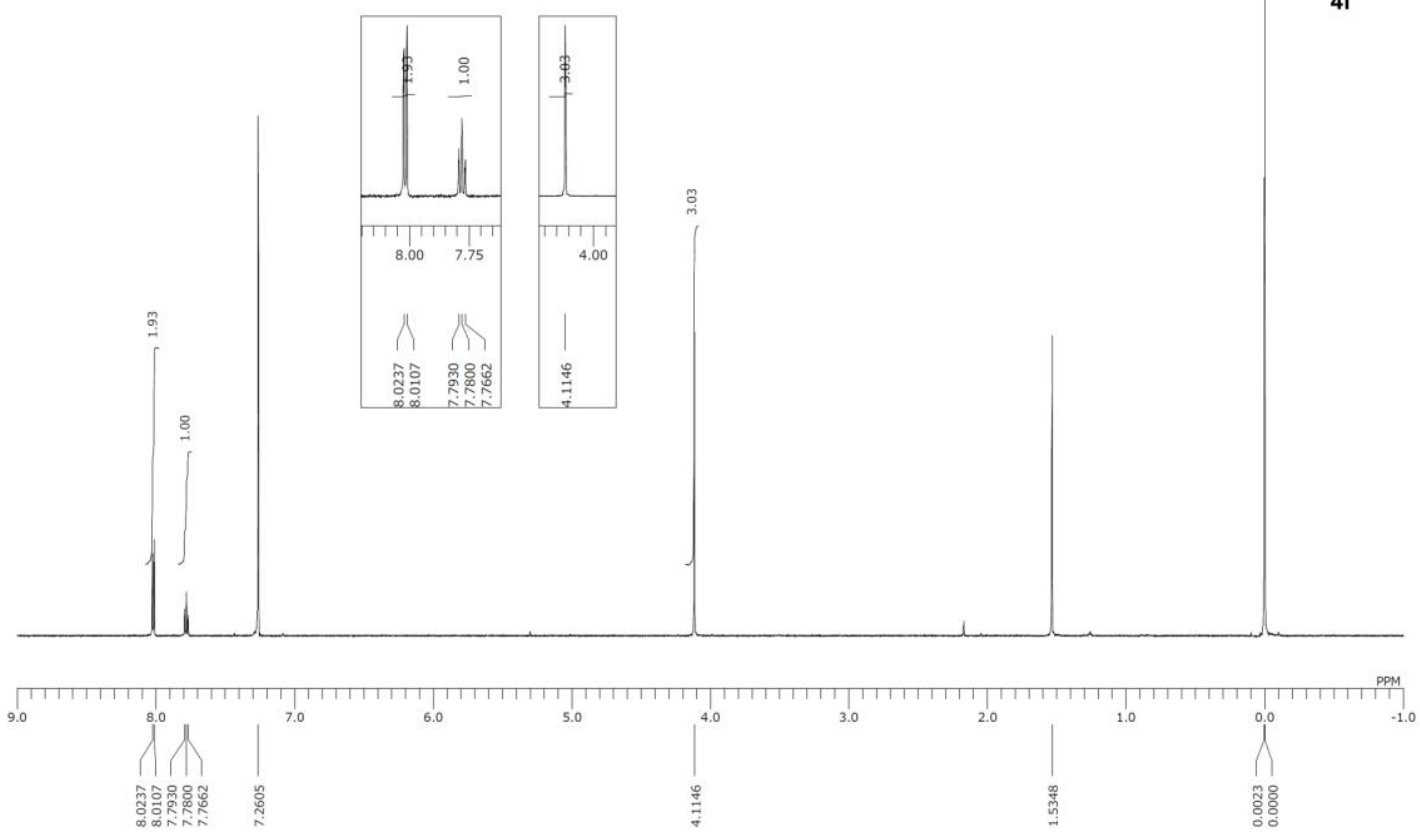

${ }^{13} \mathrm{C}$ NMR spectra of $4 \mathbf{i}\left(\mathrm{CDCl}_{3}, 150 \mathrm{MHz}\right)$
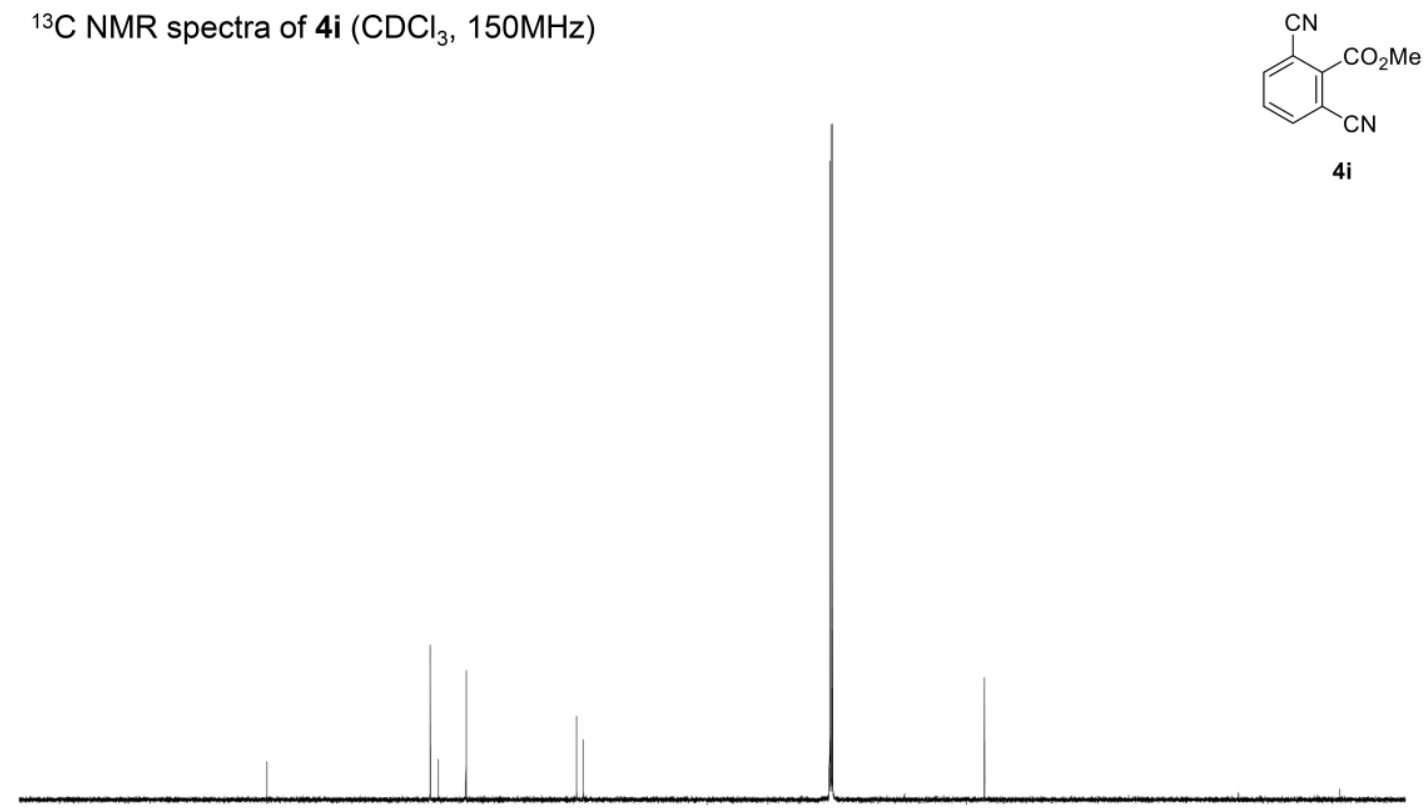

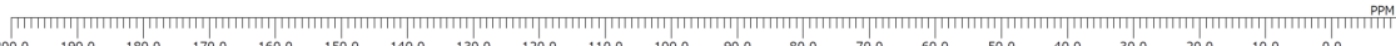

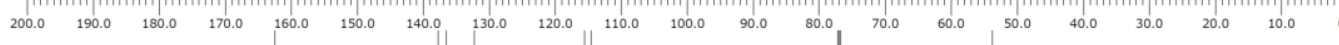

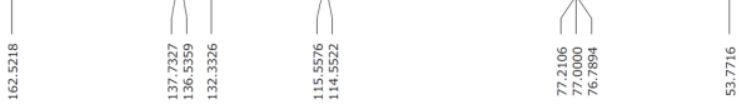


${ }^{1} \mathrm{H}$ NMR spectra of $4 \mathbf{j}\left(\mathrm{CDCl}_{3}, 600 \mathrm{MHz}\right)$

CN

$\overbrace{\mathrm{NO}_{2}}^{\mathrm{CO}_{2} \mathrm{Me}}$

$4 \mathrm{j}$
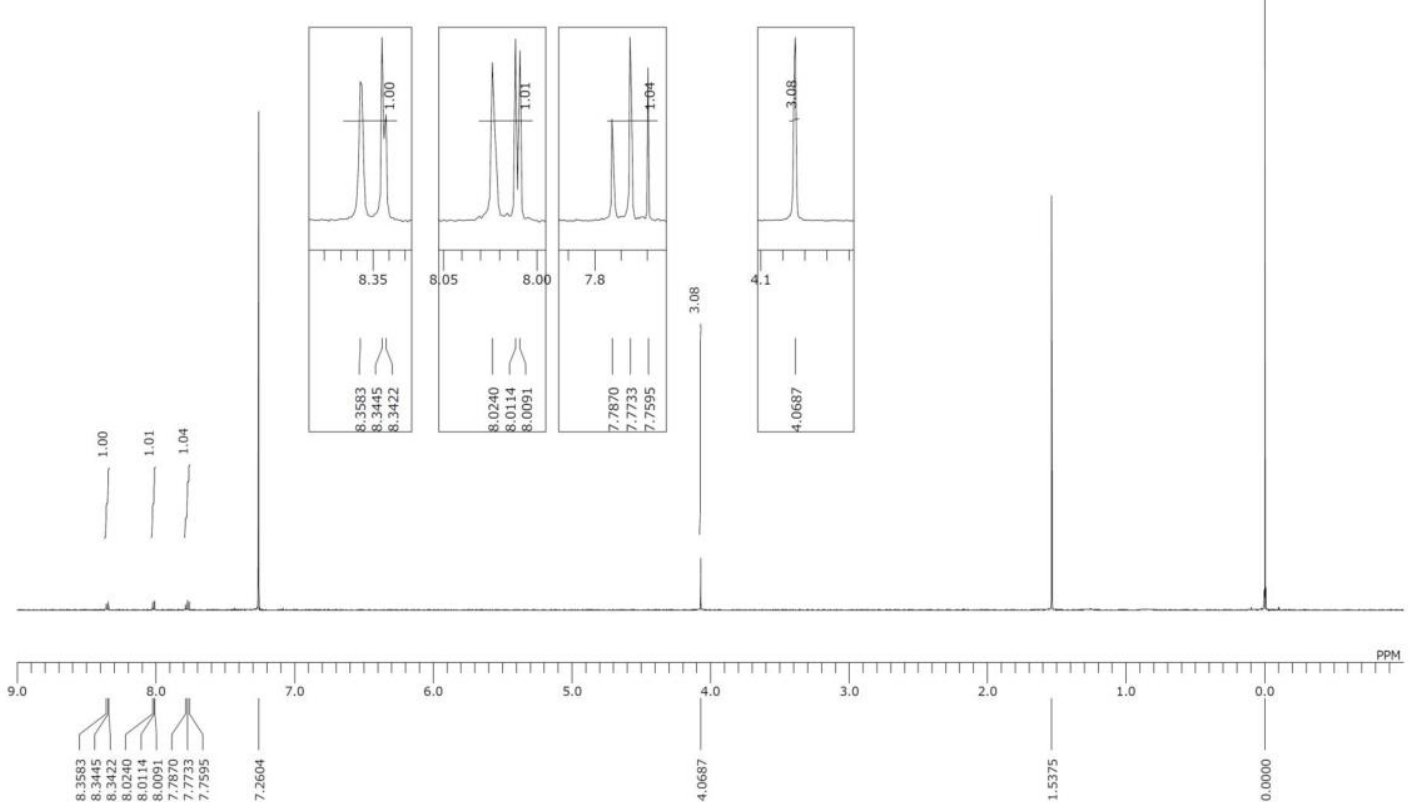

${ }^{13} \mathrm{C}$ NMR spectra of $\mathbf{4 j}\left(\mathrm{CDCl}_{3}, 150 \mathrm{MHz}\right)$

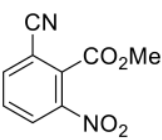

4j

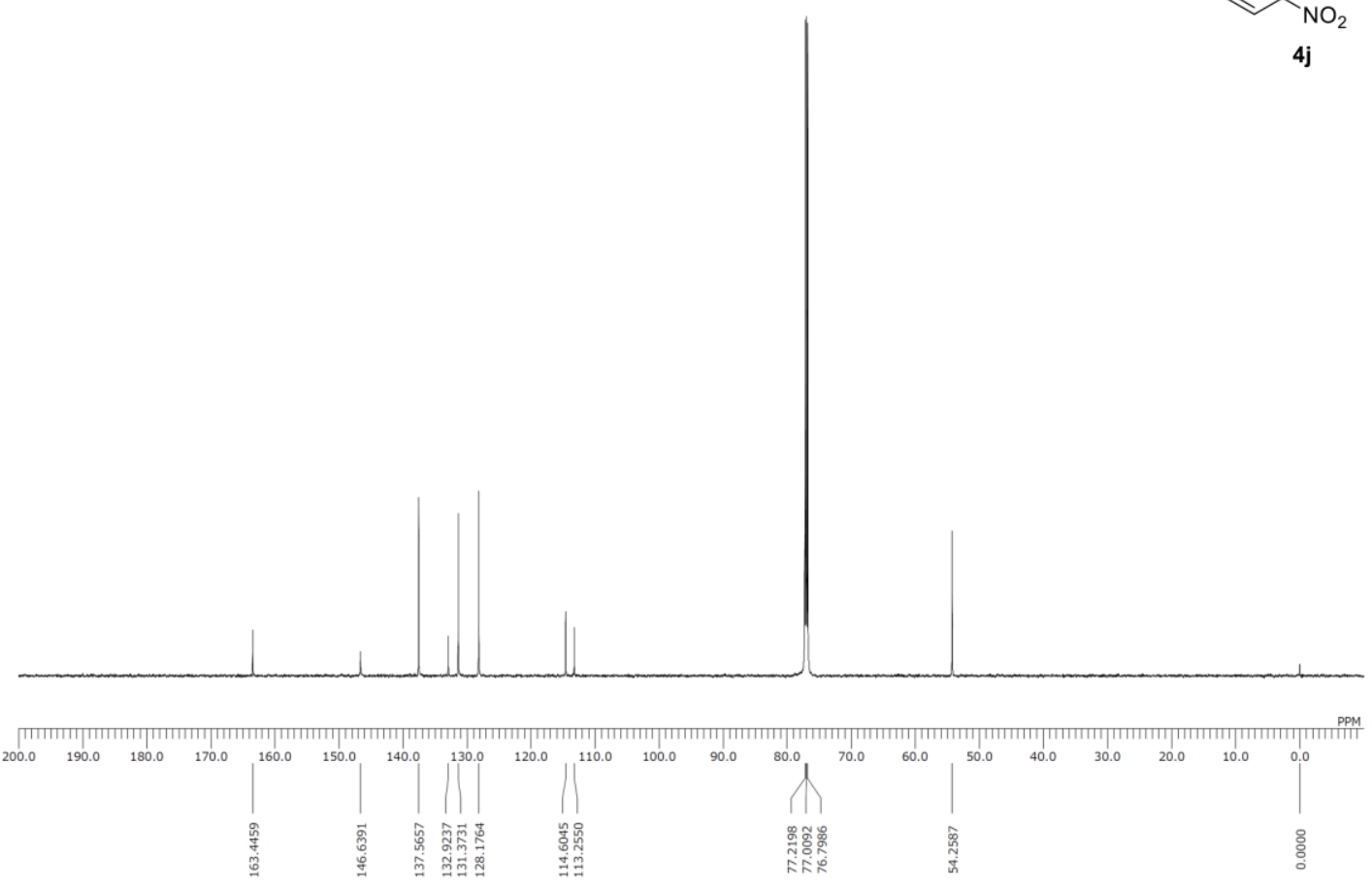


${ }^{1} \mathrm{H} \mathrm{NMR}$ spectra of $\mathbf{4 k}\left(\mathrm{CDCl}_{3}, 600 \mathrm{MHz}\right)$

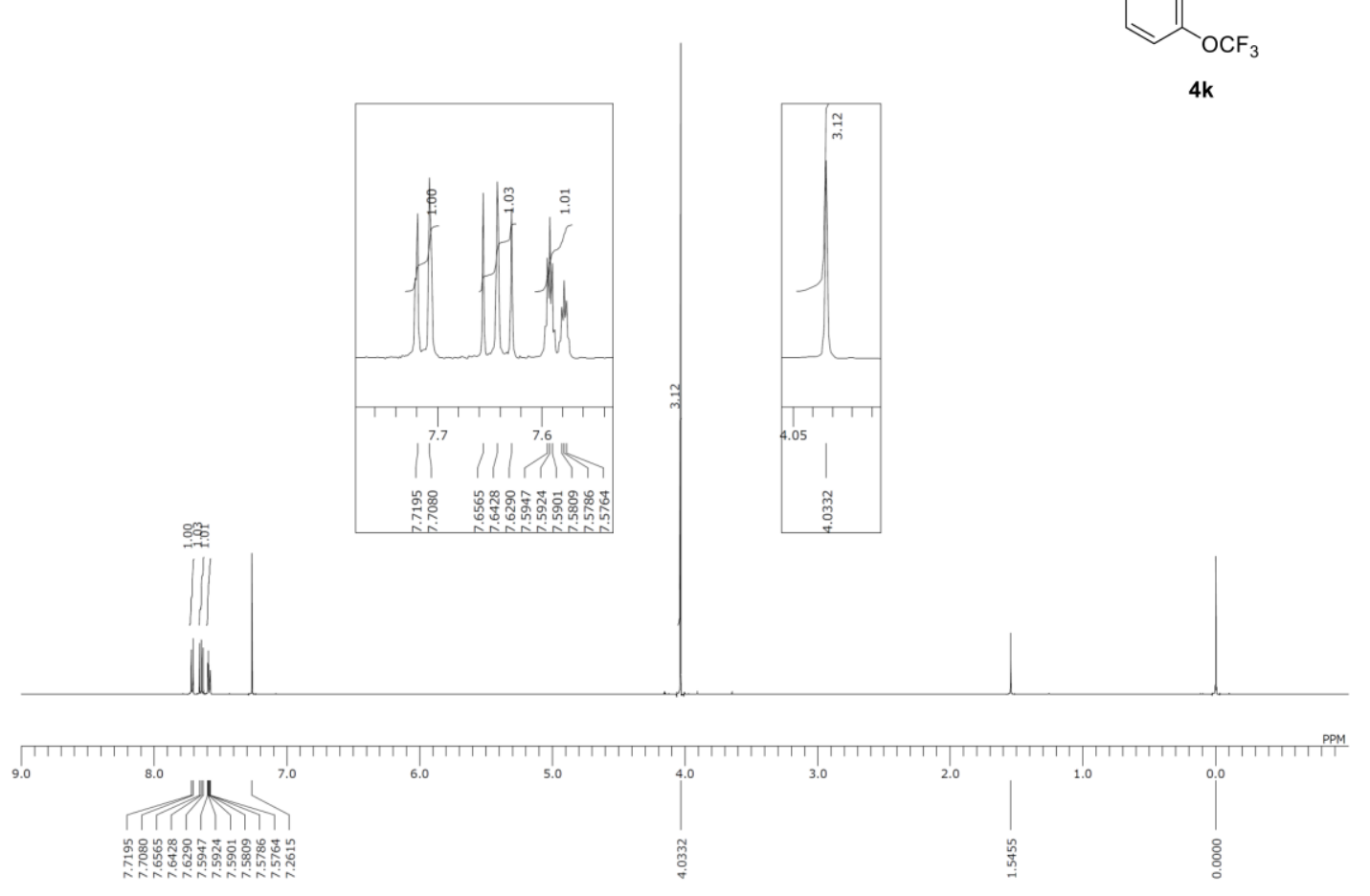

${ }^{13} \mathrm{C}$ NMR spectra of $\mathbf{4 k}\left(\mathrm{CDCl}_{3}, 150 \mathrm{MHz}\right)$

$\mathrm{OCF}_{3}$

$4 k$

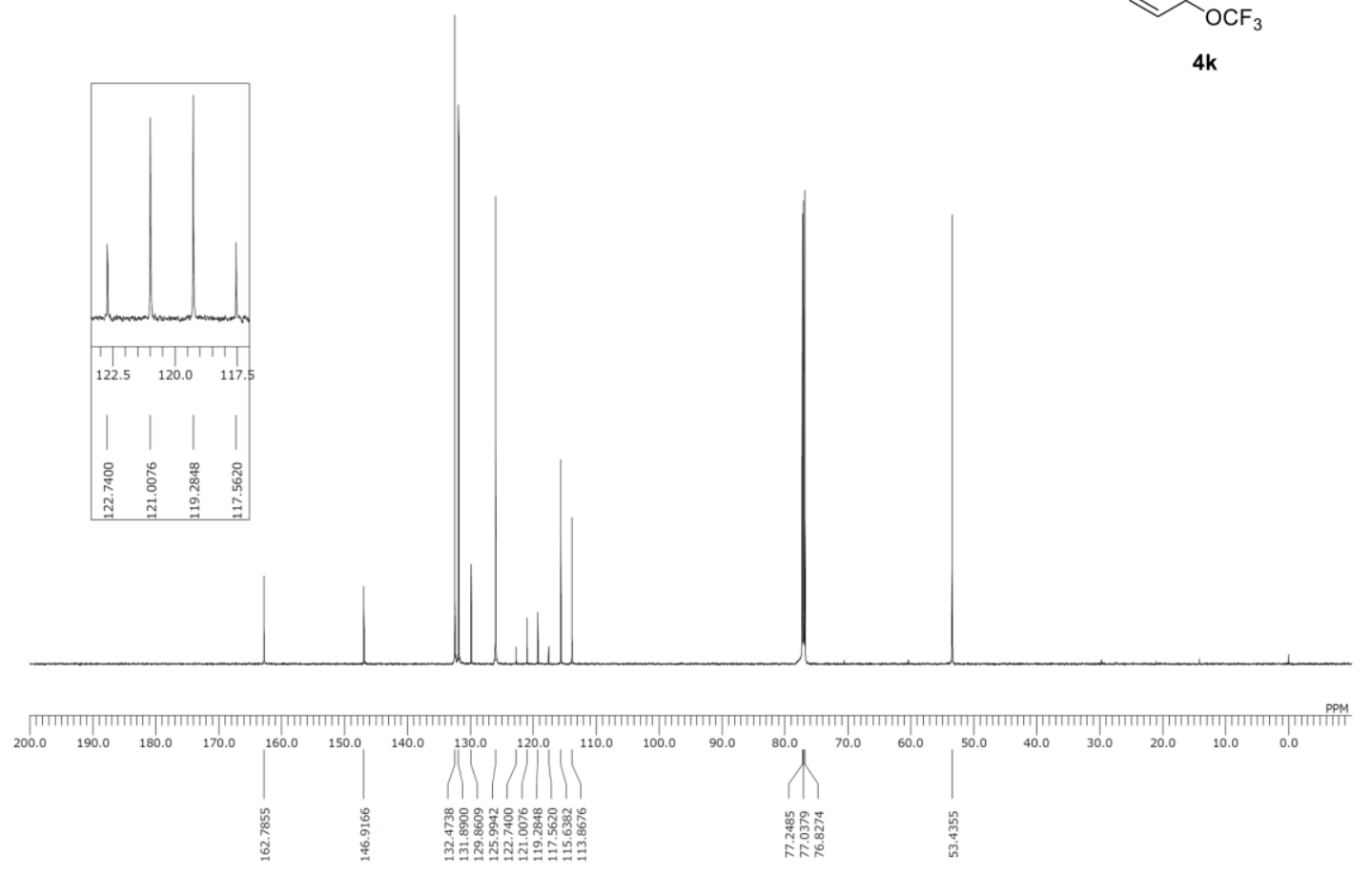


${ }^{19} \mathrm{~F}$ NMR spectra of $\mathbf{4 k}\left(\mathrm{CDCl}_{3}, 565 \mathrm{MHz}\right)$

$\mathrm{OCF}_{3}$

$4 k$

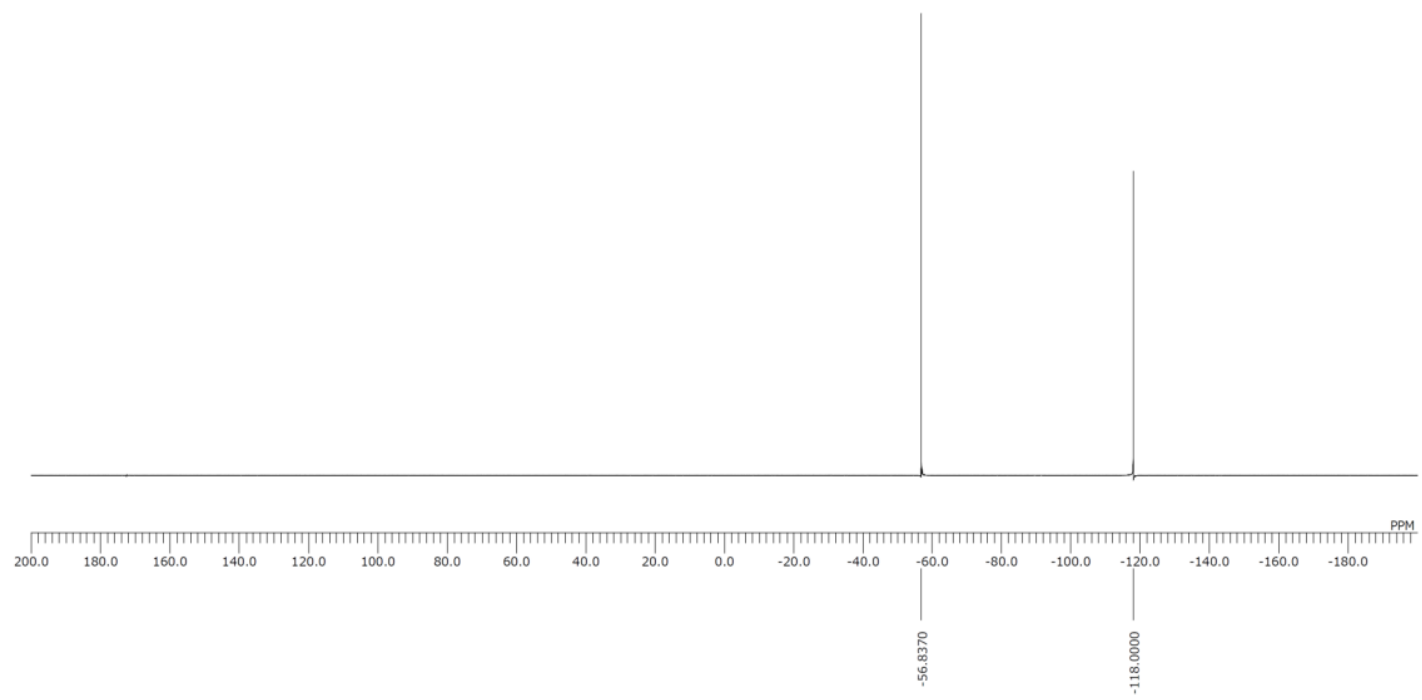

${ }^{1} \mathrm{H}$ NMR spectra of $4 \mathbf{l}\left(\mathrm{CDCl}_{3}, 600 \mathrm{MHz}\right)$

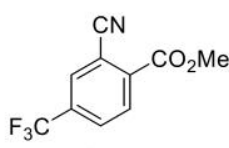

41

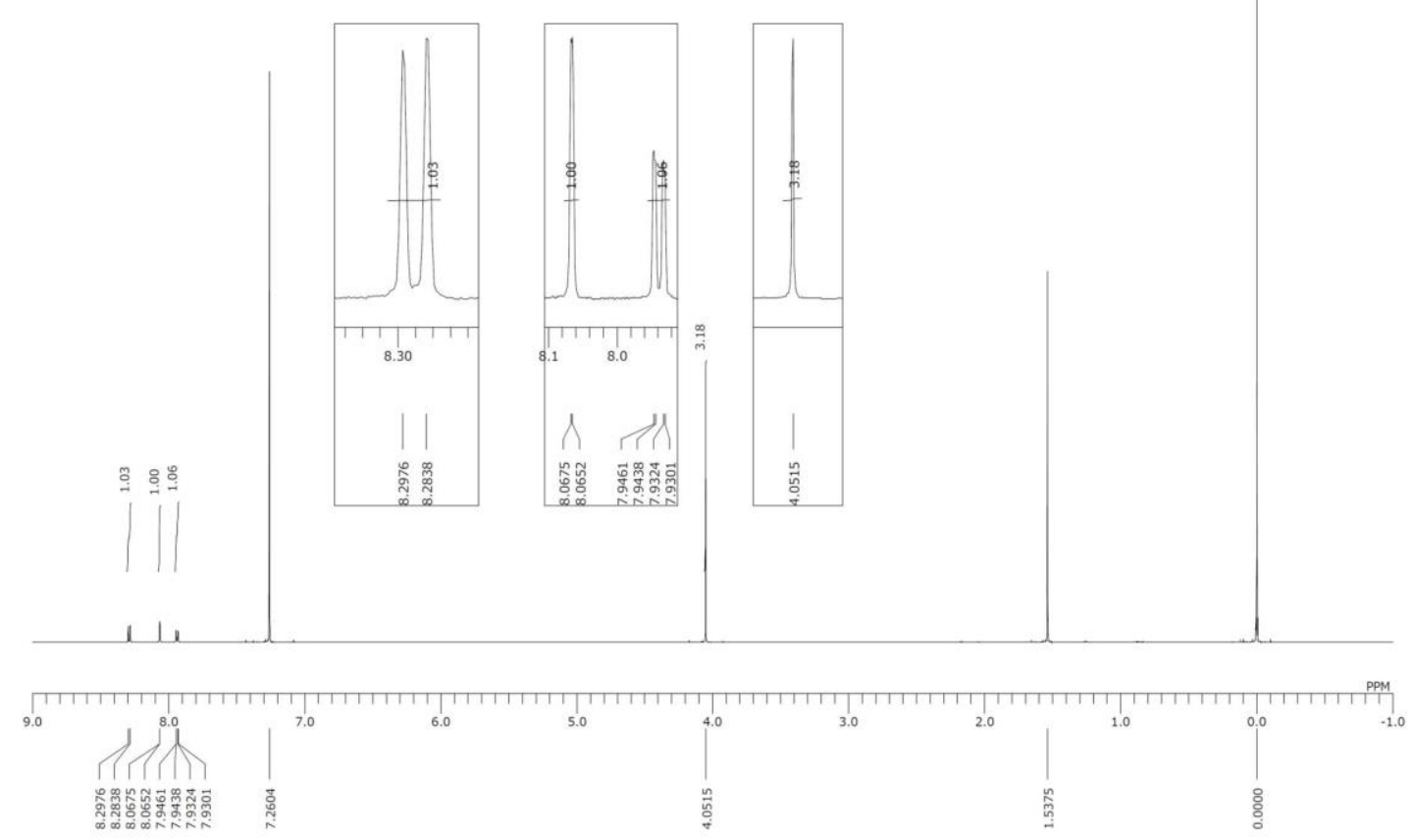


${ }^{13} \mathrm{C}$ NMR spectra of $4 \mathbf{I}\left(\mathrm{CDCl}_{3}, 150 \mathrm{MHz}\right)$

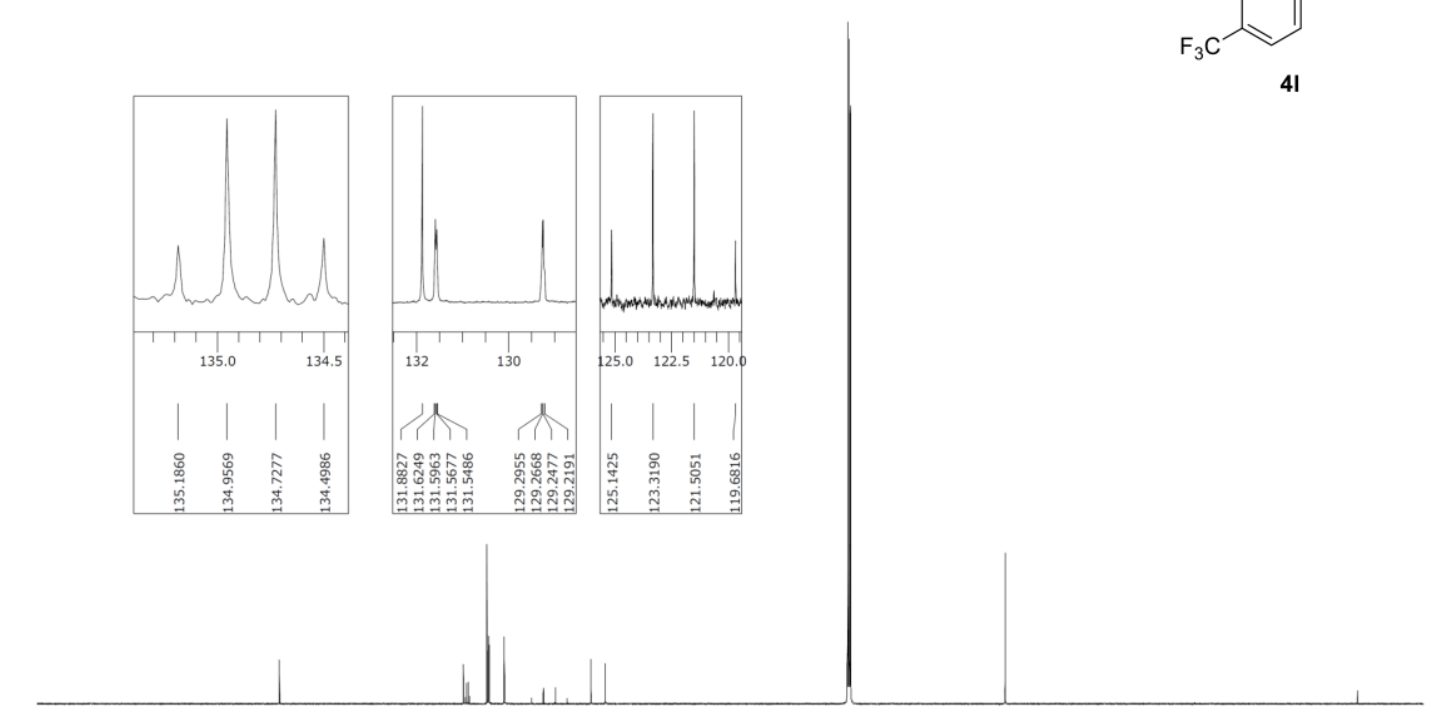

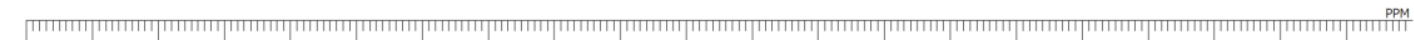

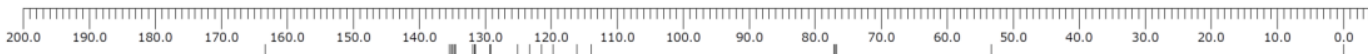

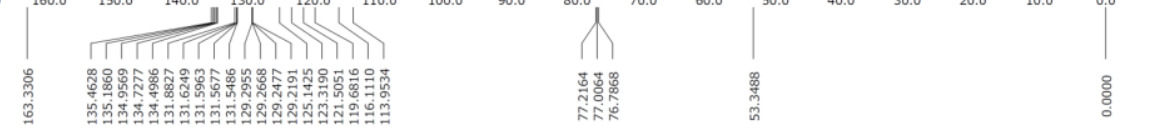

${ }^{19} \mathrm{~F}$ NMR spectra of $\mathbf{4 I}\left(\mathrm{CDCl}_{3}, 565 \mathrm{MHz}\right)$<smiles>COC(=O)c1ccc(C(F)(F)F)cc1C#N</smiles>

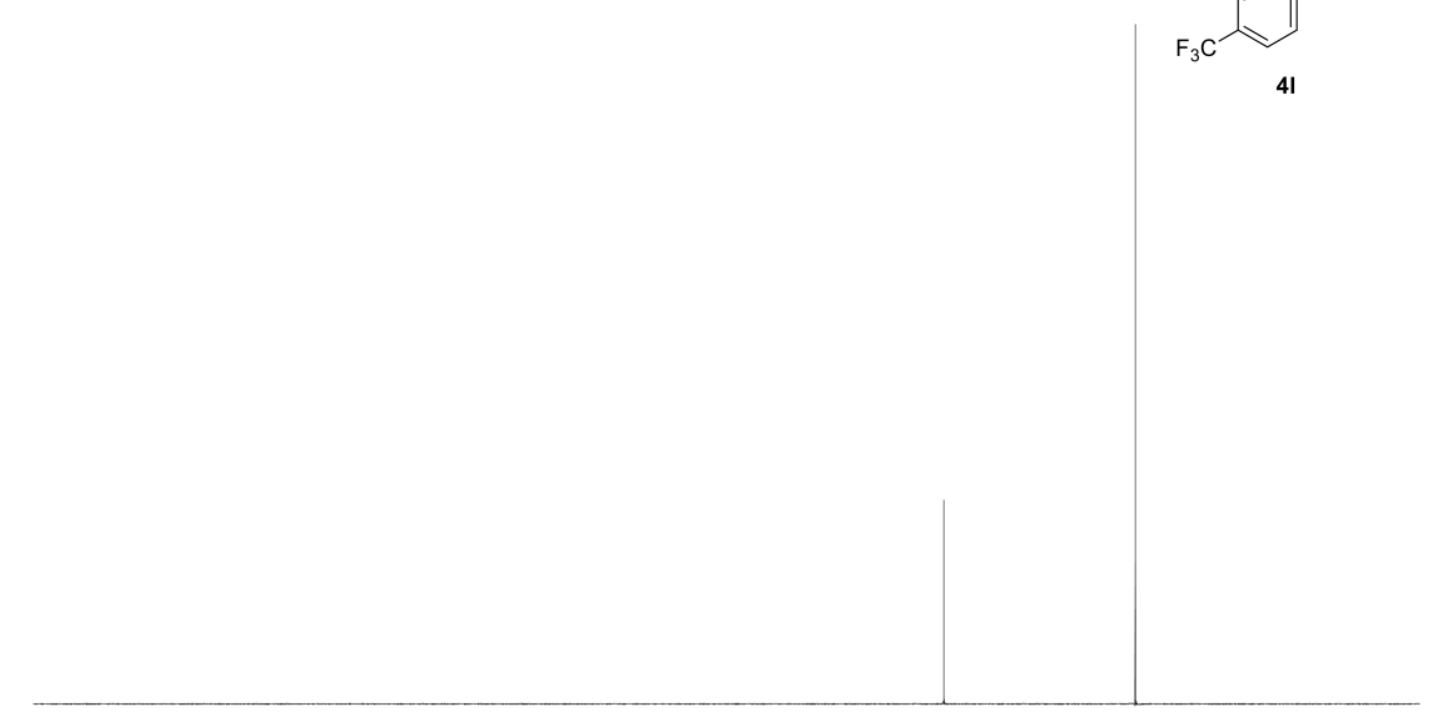

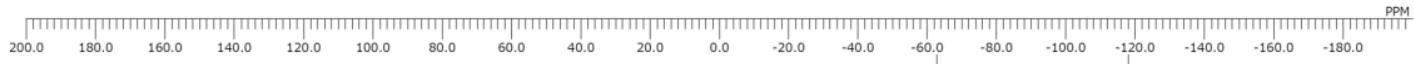

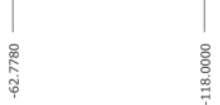


${ }^{1} \mathrm{H}$ NMR spectra of 4I-Bn (DMSO-d6, 600MHz)
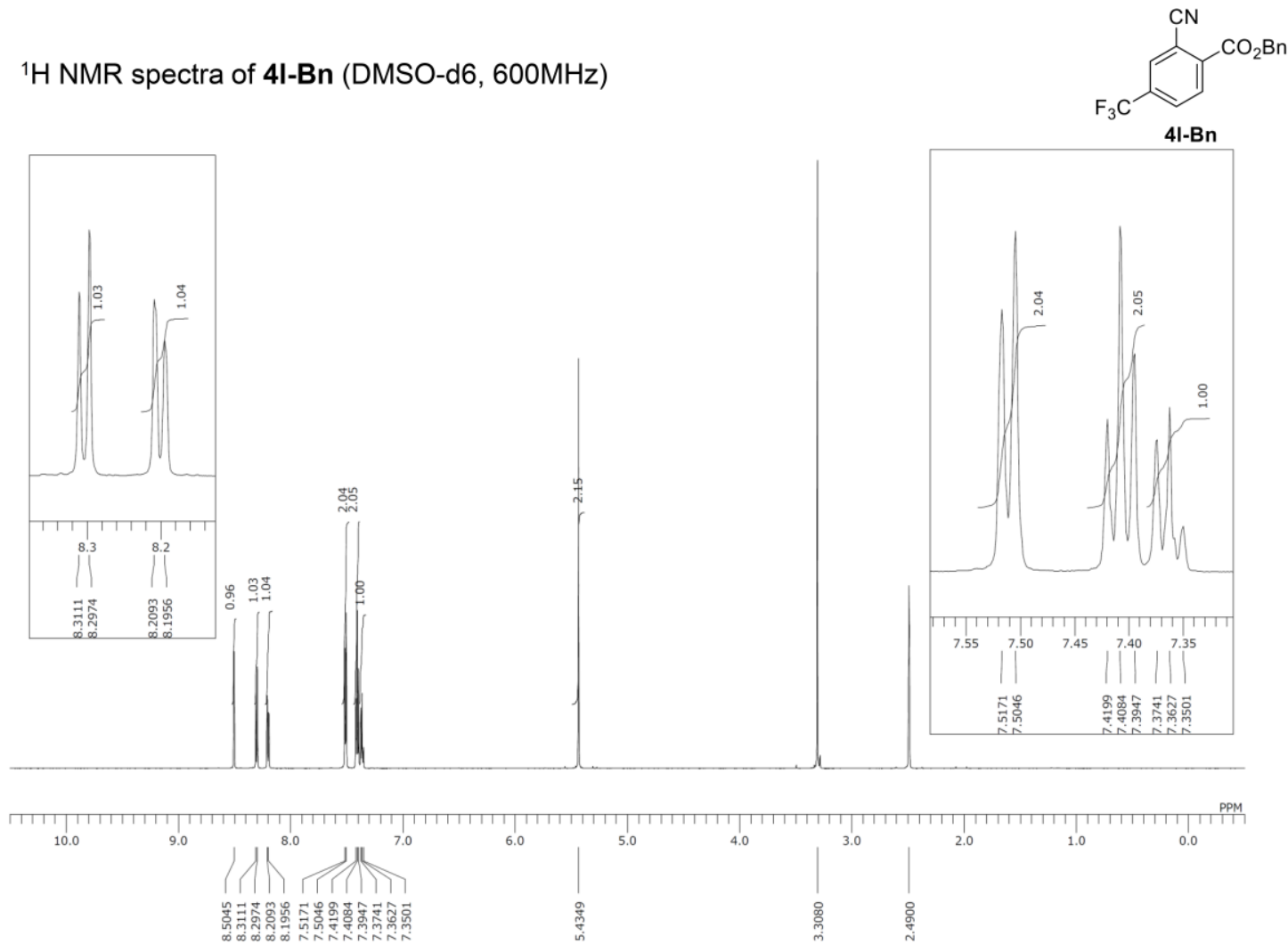

${ }^{13} \mathrm{C}$ NMR spectra of 4I-Bn (DMSO-d6, 150MHz)

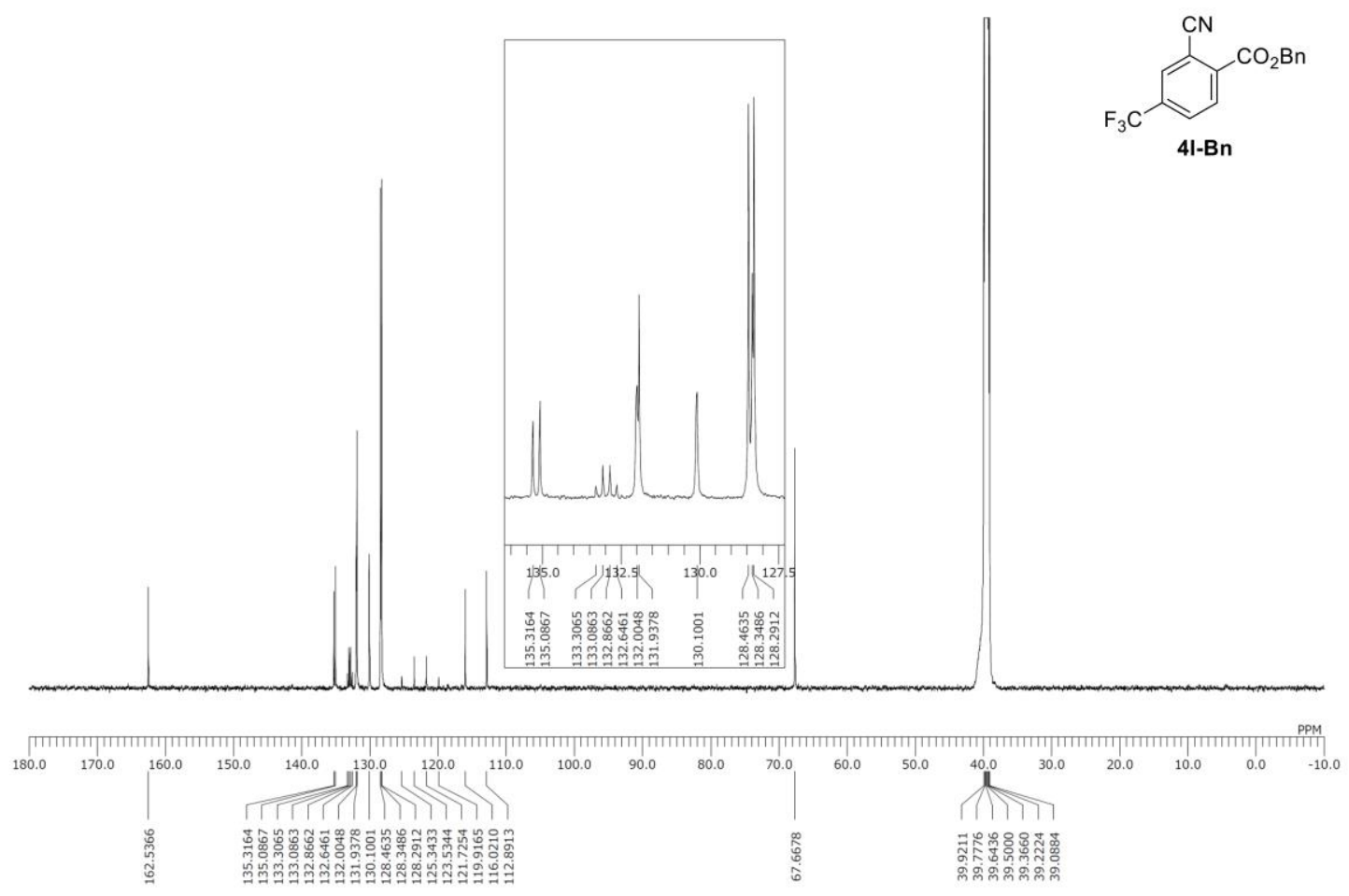


${ }^{19} \mathrm{~F}$ NMR spectra of 4I-Bn (DMSO-d6, 565MHz)

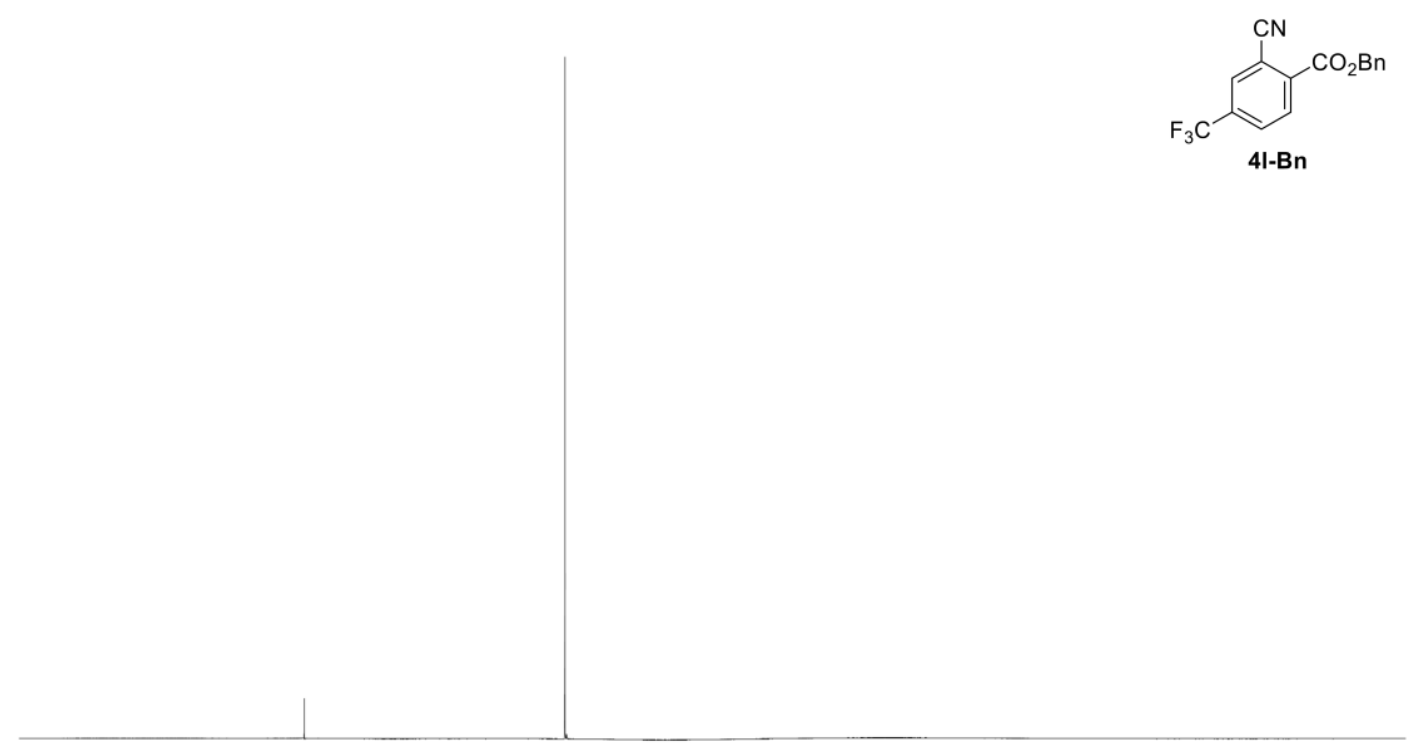

हागा $0.0-10.0-20.0-30.0-40.0-50.0-60.0-70.0-80.0-90.0-100.0-110.0-120.0-130.0-140.0-150.0-160.0-170.0-180.0-190.0-200.0-210.0-220.0-230.0-240.0-250.0-260.0-270.0-280.0-290.0$

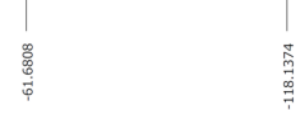

${ }^{1} \mathrm{H} \mathrm{NMR}$ spectra of $4 \mathrm{~m}\left(\mathrm{CDCl}_{3}, 400 \mathrm{MHz}\right)$

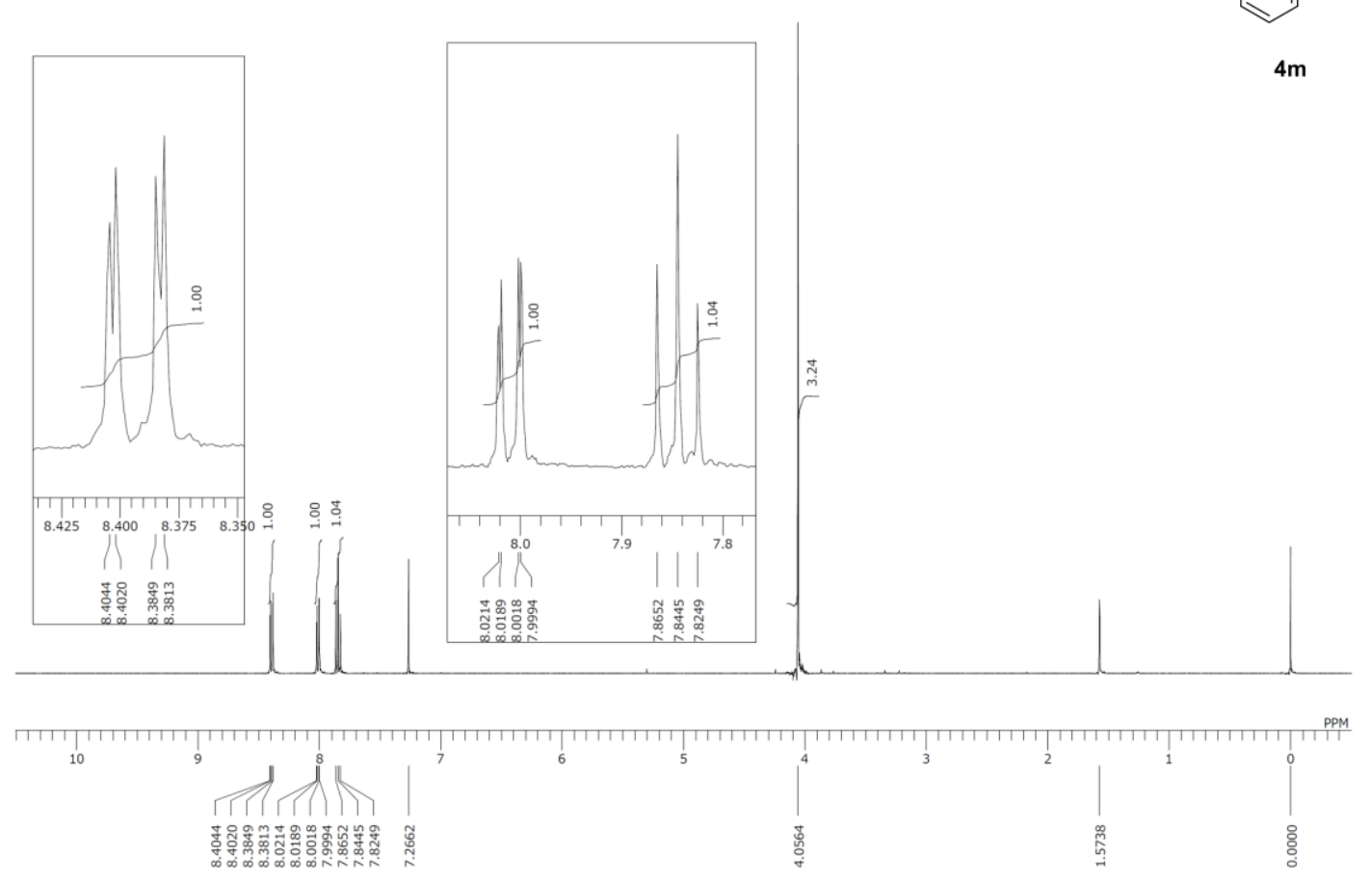


${ }^{13} \mathrm{C} \mathrm{NMR} \mathrm{spectra} \mathrm{of} 4 \mathrm{~m}\left(\mathrm{CDCl}_{3}, 100 \mathrm{MHz}\right)$
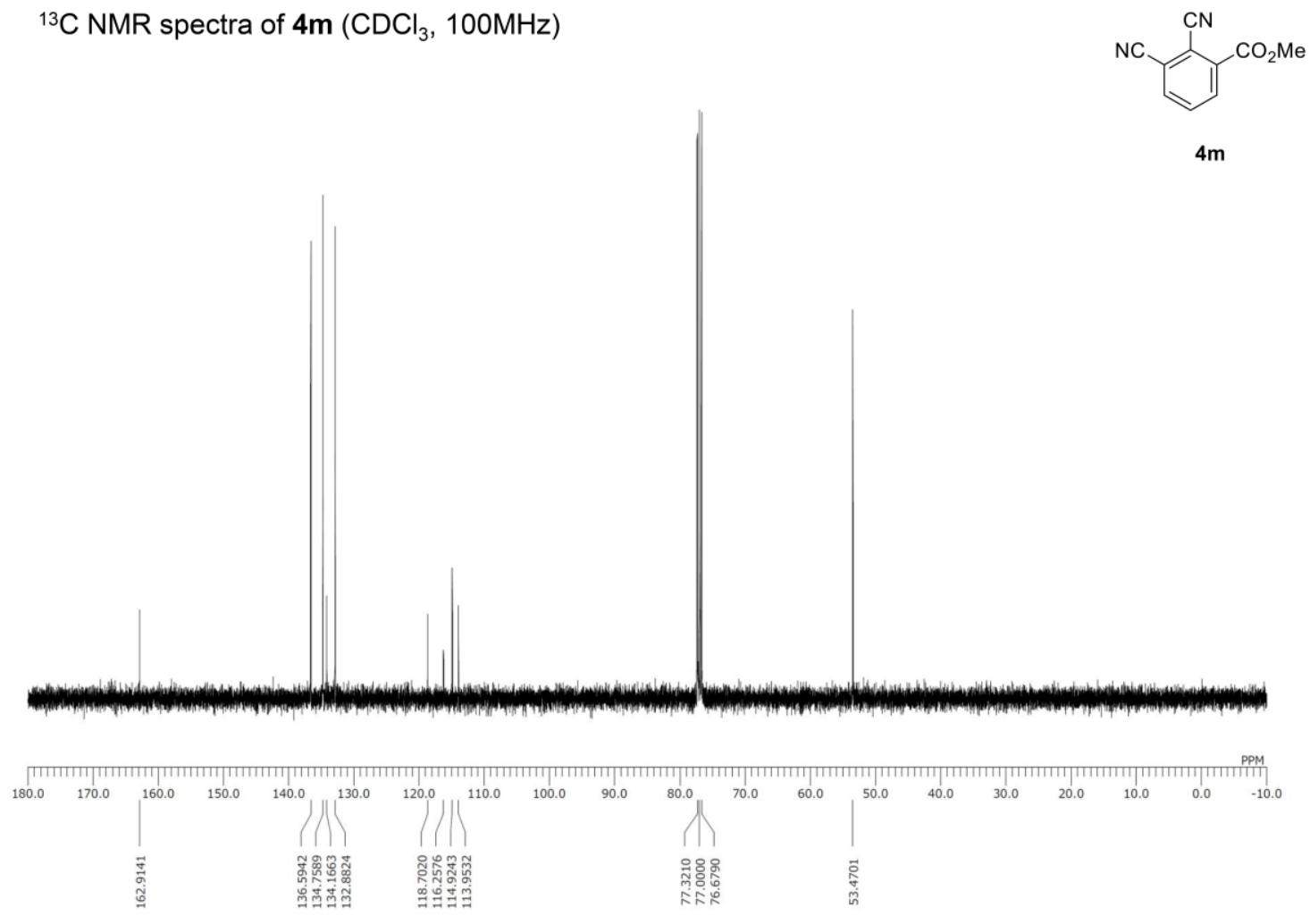

${ }^{1} \mathrm{H}$ NMR spectra of $4 \mathrm{n}\left(\mathrm{CDCl}_{3}, 600 \mathrm{MHz}\right)$
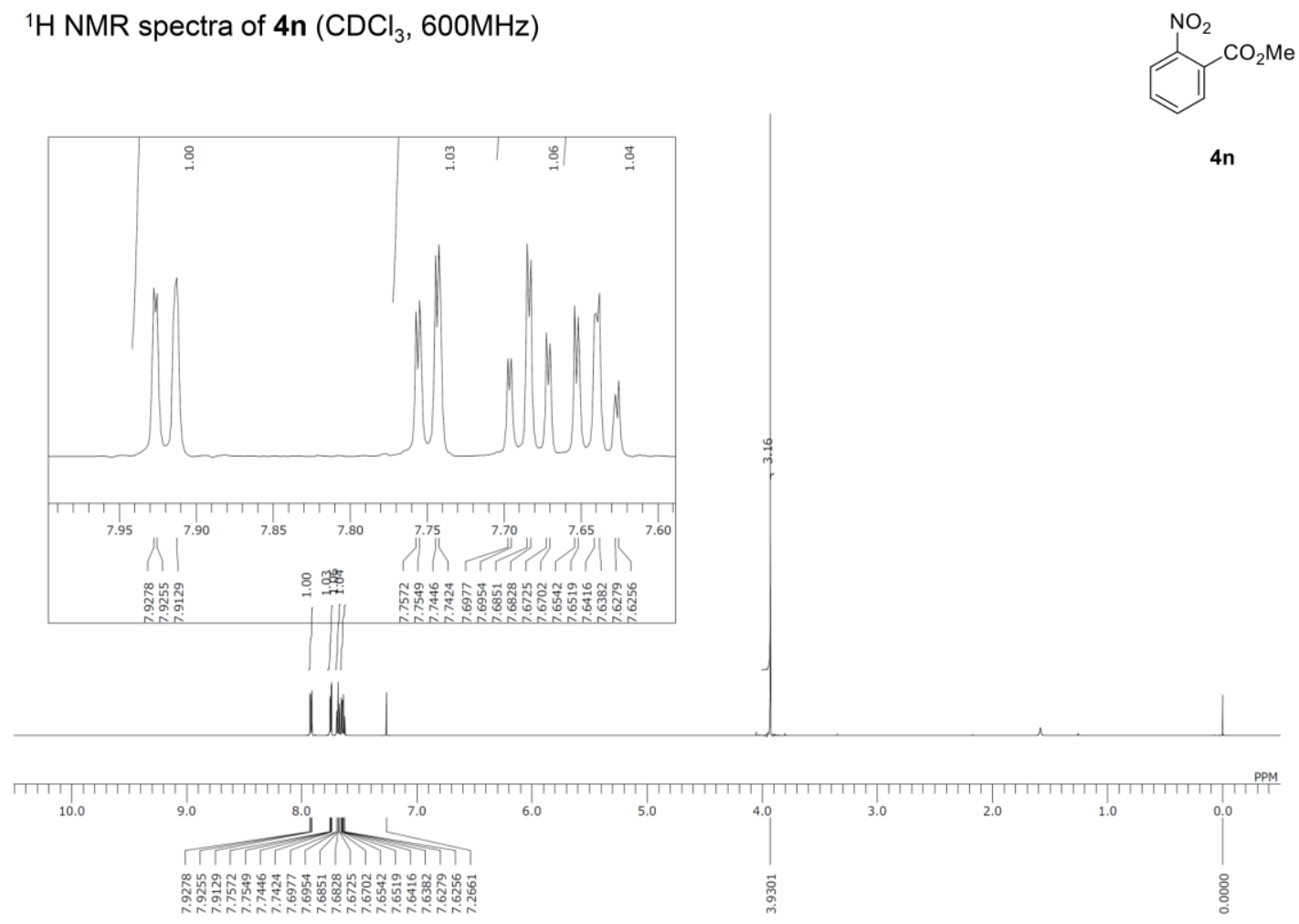
${ }^{13} \mathrm{C}$ NMR spectra of $\mathbf{4 n}\left(\mathrm{CDCl}_{3}, 150 \mathrm{MHz}\right)$

$\mathrm{NO}_{2}$

$\mathrm{CO}_{2} \mathrm{Me}$

$4 n$

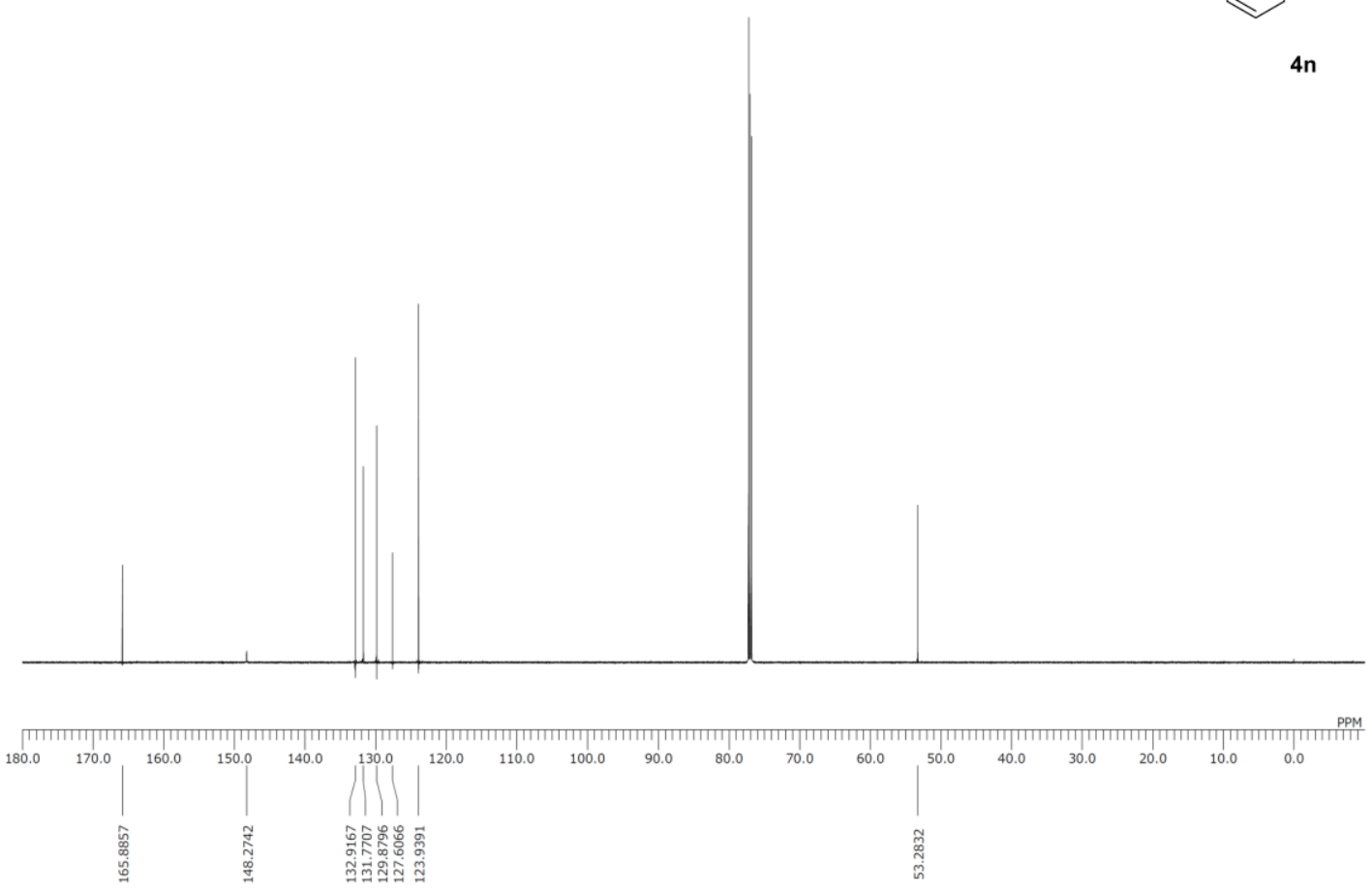

